\title{
Does the Mechanism of the Garratt-Braverman Cyclization Differ with Substrates? A computational Study on Bispropargyl Sulfones, Sulfides, Ethers, Amines and Methanes
}

Saibal Jana ${ }^{\mathrm{a}}$ and Anakuthil Anoop* ${ }^{\mathrm{a}}$

Department of Chemistry, Indian Institute of Technology Kharagpur, Kharagpur 721 302, India

*Corresponding author

Email: anoop@chem.iitkgp.ernet.in

Supporting Information

\section{Table of Contents}

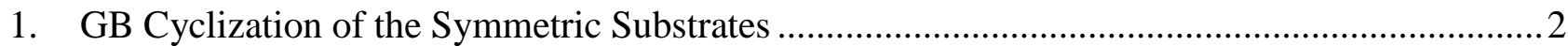

1.1. Various plausible mechanisms for GB cyclization ................................................................... 2

1.2. The two major mechanistic pathways.................................................................................... 3

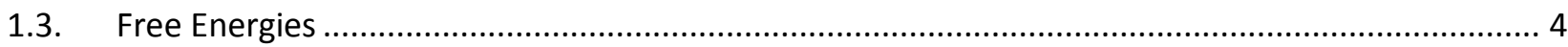

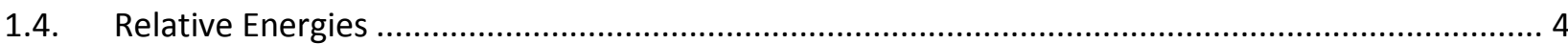

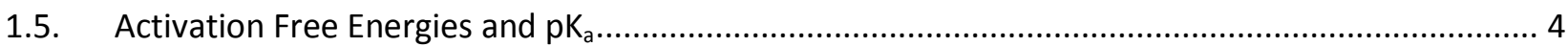

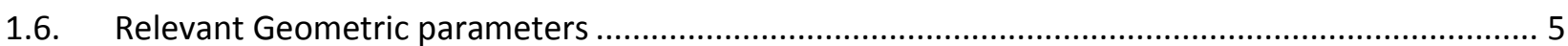

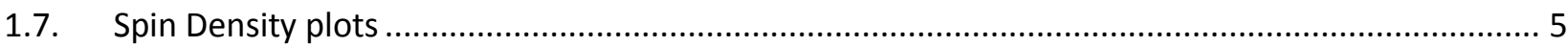

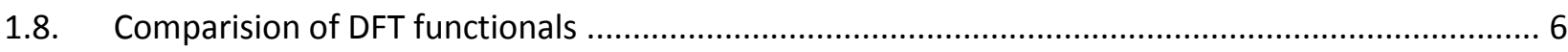

2. GB Cyclization of the Unsymmetric Bispropargyl Ethers …...............................................

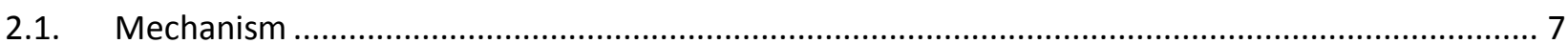

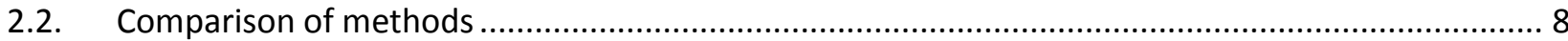

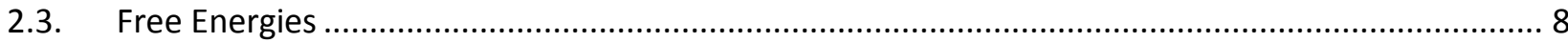

2.4. Activation free energies and $\mathrm{pK}_{\mathrm{a}}$ values for bispropargyl ether .................................................. 9

3. Predicting the selectivity for the GB cyclization of unsymmetric bispropargyl amines, sulfides,

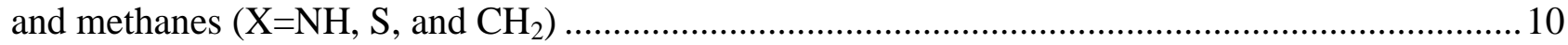

3.1. Comparison of $\mathrm{pK}_{\mathrm{a}}$ values for the bispropargyl substrates $\left(3 ; \mathrm{X}=\mathrm{O}, \mathrm{CH}_{2}, \mathrm{NH}\right.$, and $\left.\mathrm{S}\right) \ldots \ldots \ldots \ldots \ldots \ldots . . . .10$

4. Predicting selectivity for bispropargyl sulfones .................................................................. 12

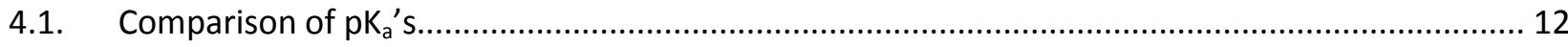

4.2. Comparison of activation free energy for second biradical cyclization ....................................... 12

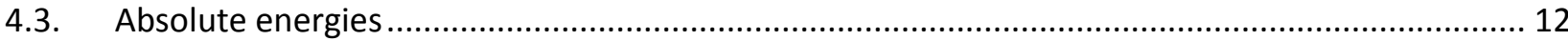

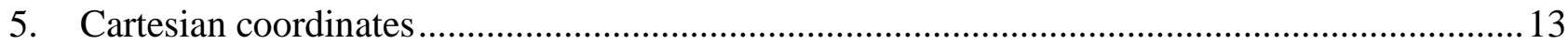




\section{GB Cyclization of the Symmetric Substrates}

\subsection{Various plausible mechanisms for GB cyclization}

We have considered several plausible pathways for the mechanistic investigation of the $G B$ cyclization. These are a) diradical cyclization from bispropargyl system b) anionic IMDA from bispropargyl anion c) IMDA from allenyne system d) diradical cyclization from bisallene system. We have calculated the activation barrier for the above four pathways at M06-2X/def2-SVP.

a) Diradical cyclization of bispropargyl

b) Anionic IMDA of bispropargyl anion

c) IMDA

of allenyne

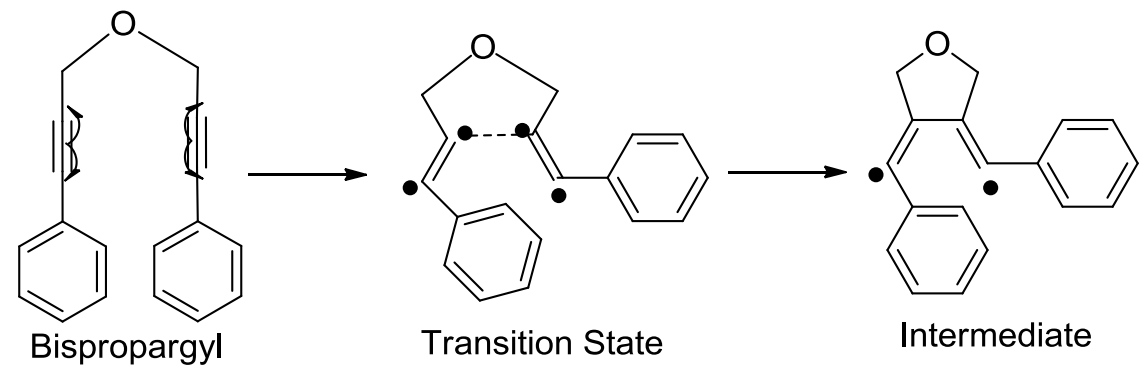

$$
\text { Bispropargyl }
$$

Transition State

Intermediate<smiles>CCOC=CC#Cc1ccccc1</smiles>$$
\mathrm{DG}^{\ddagger}=32.51 \mathrm{kcal} \mathrm{mol}-1
$$

$$
\begin{array}{cc}
\text { Bispropargyl anion } & \begin{array}{c}
\text { Transition State } \\
\mathrm{DG}^{\ddagger}=25.98 \mathrm{kcal} \mathrm{mol}-1
\end{array}
\end{array}
$$

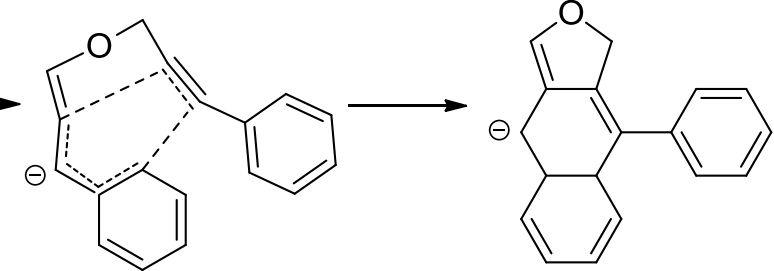<smiles>CCCC#Cc1ccccc1C=COCCC</smiles>
Allenyne<smiles></smiles>
Transition State $\mathrm{DG}^{\ddagger}=20.38 \mathrm{kcal} \mathrm{mol}-1$

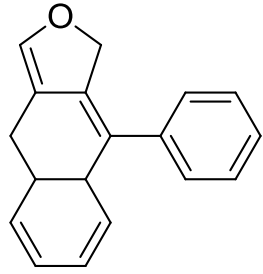

Intermediate

d) Diradical cyclization of bisallene

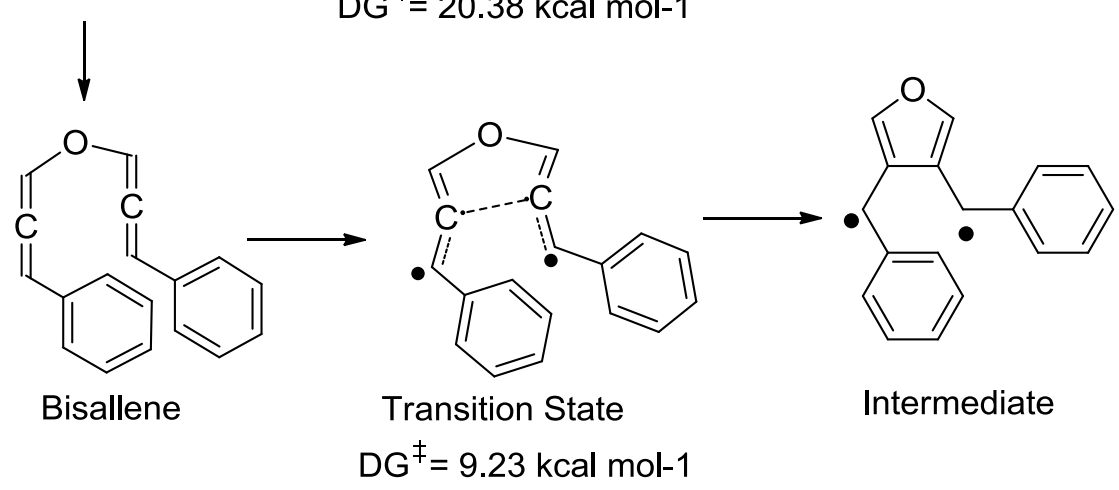

Scheme S 1. Different reaction pathways and their corresponding activation free energy barriers.

From the activation energies, the diradical cyclization from bisallene and the IMDA from allenyne are the most suitable pathways. Therefore, we have excluded the other two pathways, namely, diradical cyclization from bispropargyl system and anionic IMDA from bispropargyl anion. 


\subsection{The two major mechanistic pathways}

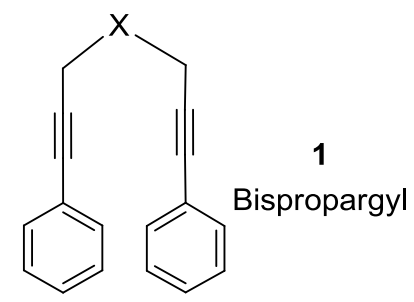<smiles>[X][Y]1=Cc2cccc3cccc(c23)C=C1</smiles>

First isomerization<smiles>[H][Y]([H])=C/C=C/c1ccccc1</smiles>

6

Allenyne<smiles>Cc1ccccc1</smiles>

$\frac{\mathrm{TS}_{6-7}}{\text { IMDA }}$

Intermediate 6-2

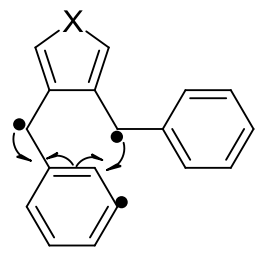

9

Bisallene

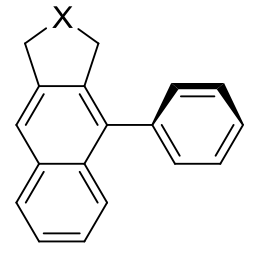

2

Product

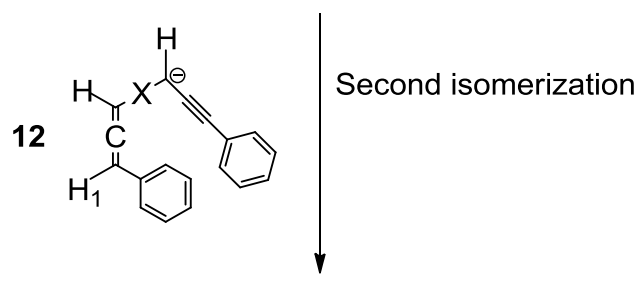<smiles></smiles>

Bisallene

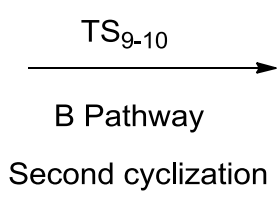

Second cyclization

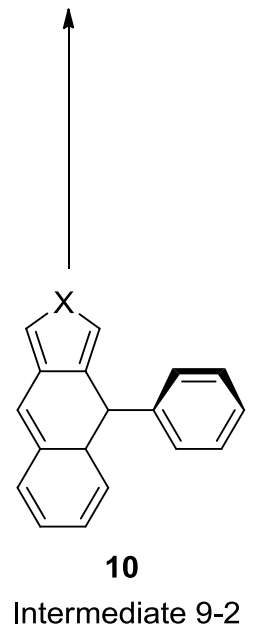

Intermediate 9-2

Scheme $S$ 2. Pathways for the GB cyclization of the symmetric substrates. $X=S O 2, C H 2, S, O$, and NH. 


\subsection{Free Energies}

Table S 1. Free energies (Electronic energy +Correction to the free energy from harmonic vibrational analysis) calculated at M06-2X/def2-SVP level of theory.

\begin{tabular}{|c|c|r|r|r|r|}
\hline \multirow{2}{*}{$\begin{array}{c}\text { species(Compound } \\
\text { Number) }\end{array}$} & \multicolumn{5}{|c|}{$\mathrm{X}$} \\
\cline { 2 - 6 } & $\mathrm{CH}_{2}$ & $\mathrm{NH}$ & $\mathrm{O}$ & \multicolumn{1}{c|}{$\mathrm{S}$} & $\mathrm{SO}_{2}$ \\
\hline Bispropargyl(1) & -732.43496 & -748.45631 & -768.30831 & -1091.24156 & -1241.44236 \\
\hline Allenyne(6) & -732.44052 & -748.46822 & -768.31945 & -1091.2518 & -1241.44247 \\
\hline $\mathrm{TS}_{6-7}$ & -732.39626 & -748.43399 & -768.28661 & -1091.21302 & -1241.39500 \\
\hline Intermediate 6-2 (7) & -732.48798 & -748.52040 & -768.373907 & -1091.3047 & -1241.49802 \\
\hline Bisallene(8) & -732.44327 & -748.47696 & -768.32676 & -1091.25676 & -1241.44260 \\
\hline $\mathrm{TS}_{8-9}$ & -732.41399 & -748.45629 & -768.30933 & -1091.23177 & -1241.40823 \\
\hline Intermeidate 8-2 (9) & -732.44465 & -748.50832 & -768.35217 & -1091.28253 & -1241.43886 \\
\hline TS $_{9-2}$ & -732.43256 & -748.49876 & -768.34154 & -1091.27281 & -1241.42716 \\
\hline Intermediate 9-2 (10) & -732.48526 & -748.55043 & -768.39525 & -1091.33038 & -1241.48002 \\
\hline Product (2) & -732.57161 & -748.59457 & -768.45306 & -1091.38200 & -1241.57930 \\
\hline
\end{tabular}

\subsection{Relative Energies}

Table S 2. Relative free energies (in kcal mol ${ }^{-1}$ calculated at M06-2X/def2-SVP level of theory. The reference is taken as the free energy of the bispropargys (1) for each $\mathrm{X}=\mathrm{CH}_{2}, \mathrm{NH}, \mathrm{O}, \mathrm{S}$, and $\mathrm{SO}_{2}$

\begin{tabular}{|c|r|r|r|r|r|}
\hline \multirow{2}{*}{$\begin{array}{c}\text { Species(Compound } \\
\text { Number) }\end{array}$} & \multicolumn{5}{|c|}{$\mathrm{X}$} \\
\cline { 2 - 6 } & \multicolumn{1}{|c|}{$\mathrm{CH}_{2}$} & \multicolumn{1}{|c|}{$\mathrm{NH}$} & $\mathrm{O}$ & \multicolumn{1}{c|}{$\mathrm{S}_{2}$} \\
\hline Bispropargyl(1) & 0.00 & 0.00 & 0.00 & 0.00 & 0.00 \\
\hline Allenyne(6) & -3.49 & -7.47 & -6.99 & -6.43 & -0.07 \\
\hline $\mathrm{TS}_{6-7}$ & 24.28 & 14.01 & 13.62 & 17.91 & 29.72 \\
\hline Intermediate 6-2 (7) & -33.27 & -40.22 & -41.16 & -39.62 & -34.93 \\
\hline Bisallene(8) & -5.21 & -12.96 & -11.58 & -9.54 & -0.15 \\
\hline $\mathrm{TS}_{8-9}$ & 13.16 & 0.01 & -0.64 & 6.14 & 21.42 \\
\hline Intermeidate 8-2 (9) & -6.08 & -32.64 & -27.52 & -25.71 & 2.20 \\
\hline TS $_{9-2}$ & 1.51 & -26.64 & -20.85 & -19.61 & 9.54 \\
\hline Intermediate 9-2 (10) & -31.56 & -59.06 & -54.56 & -55.74 & -23.63 \\
\hline Product (2) & -85.75 & -86.76 & -90.83 & -88.13 & -85.93 \\
\hline
\end{tabular}

\subsection{Activation Free Energies and $\mathrm{pK}_{\mathrm{a}}$}

Table S 3.Stepwise activation free energies (calculated at M06-2X/def2-SVP level) for the cyclization steps: $\triangle G \neq A$ : [4+2] IMDA from allenyne. $\triangle G \neq B 1$ and $\triangle G \neq B 2$ : biradical pathways from bisallene. The value of the vibrational frequency of the transition state mode are given in the parenthesis. Energies are in hartrees. $\mathrm{pKal}$ and $\mathrm{pKa} 2$ : first and second $\mathrm{pKa}$ values.

\begin{tabular}{|c|c|c|c|c|c|}
\hline \multirow{2}{*}{$\mathrm{X}$} & \multicolumn{5}{|c|}{$\mathrm{X}$} \\
\cline { 2 - 6 } & $\mathrm{CH}_{2}$ & $\mathrm{NH}$ & $\mathrm{O}$ & $\mathrm{S}$ & $\mathrm{SO}_{2}$ \\
\hline$\Delta G_{\mathrm{A}}^{\ddagger}$ & $27.77\left(-418.04 \mathrm{~cm}^{-1}\right)$ & $21.48\left(-368.21 \mathrm{~cm}^{-1}\right)$ & $20.61\left(-313.92 \mathrm{~cm}^{-1}\right)$ & $24.33\left(-404.51 \mathrm{~cm}^{-1}\right)$ & $29.79\left(-463.42 \mathrm{~cm}^{-1}\right)$ \\
\hline$\Delta G_{\mathrm{B} 1}^{\ddagger}$ & $18.37\left(-516.76 \mathrm{~cm}^{-1}\right)$ & $12.97\left(-460.43 \mathrm{~cm}^{-1}\right)$ & $10.94\left(-460.38 \mathrm{~cm}^{-1}\right)$ & $15.68\left(-494.91 \mathrm{~cm}^{-1}\right)$ & $21.56\left(-497 \mathrm{~cm}^{-1}\right)$ \\
\hline$\Delta G_{\mathrm{B} 2}^{\ddagger}$ & $7.58\left(-645.27 \mathrm{~cm}^{-1}\right)$ & $6.01\left(-632.42 \mathrm{~cm}^{-1}\right)$ & $6.67\left(-636.92 \mathrm{~cm}^{-1}\right)$ & $6.10\left(-628.15 \mathrm{~cm}^{-1}\right)$ & $7.34\left(-556.48 \mathrm{~cm}^{-1}\right)$ \\
\hline $\mathrm{pK}_{\mathrm{a} 1}$ & 42 & 41.51 & 39.34 & 32.86 & 22.48 \\
\hline $\mathrm{pK}_{\mathrm{a} 2}$ & 44.81 & 41.16 & 41.83 & 36.81 & 19.12 \\
\hline
\end{tabular}




\subsection{Relevant Geometric parameters}

Table S 4. Bond distance between propargyl carbon and the link atoms (C1-X and C2-X) in $\AA$.

\begin{tabular}{|c|c|c|c|c|c|c|c|c|c|c|}
\hline $\mathrm{X}$ & $\mathrm{SO}_{2}$ & & $\mathrm{CH}_{2}$ & & $\mathbf{O}$ & & $\mathbf{S}$ & & NH & \\
\hline Bonds & $\mathrm{C} 1-\mathrm{S}$ & $\mathrm{C} 2-\mathrm{S}$ & $\mathrm{C} 1-\mathrm{C}$ & $\mathrm{C} 2-\mathrm{C}$ & $\mathrm{C} 1-\mathrm{O}$ & $\mathrm{C} 2-\mathrm{O}$ & $\mathrm{C} 1-\mathrm{S}$ & $\mathrm{C} 2-\mathrm{S}$ & $\mathrm{C} 1-\mathrm{N}$ & $\mathrm{C} 2-\mathrm{N}$ \\
\hline Bispropargyl & 1.82 & 1.83 & 1.54 & 1.53 & 1.41 & 1.41 & 1.84 & 1.85 & 1.45 & 1.45 \\
\hline Allenyne & 1.80 & 1.83 & 1.51 & 1.53 & 1.36 & 1.41 & 1.78 & 1.84 & 1.39 & 1.44 \\
\hline Bisallene & 1.79 & 1.80 & 1.51 & 1.51 & 1.37 & 1.37 & 1.77 & 1.78 & 1.39 & 1.39 \\
\hline Biradical intermediate & 1.77 & 1.78 & 1.49 & 1.49 & 1.35 & 1.35 & 1.72 & 1.73 & 1.36 & 1.36 \\
\hline
\end{tabular}

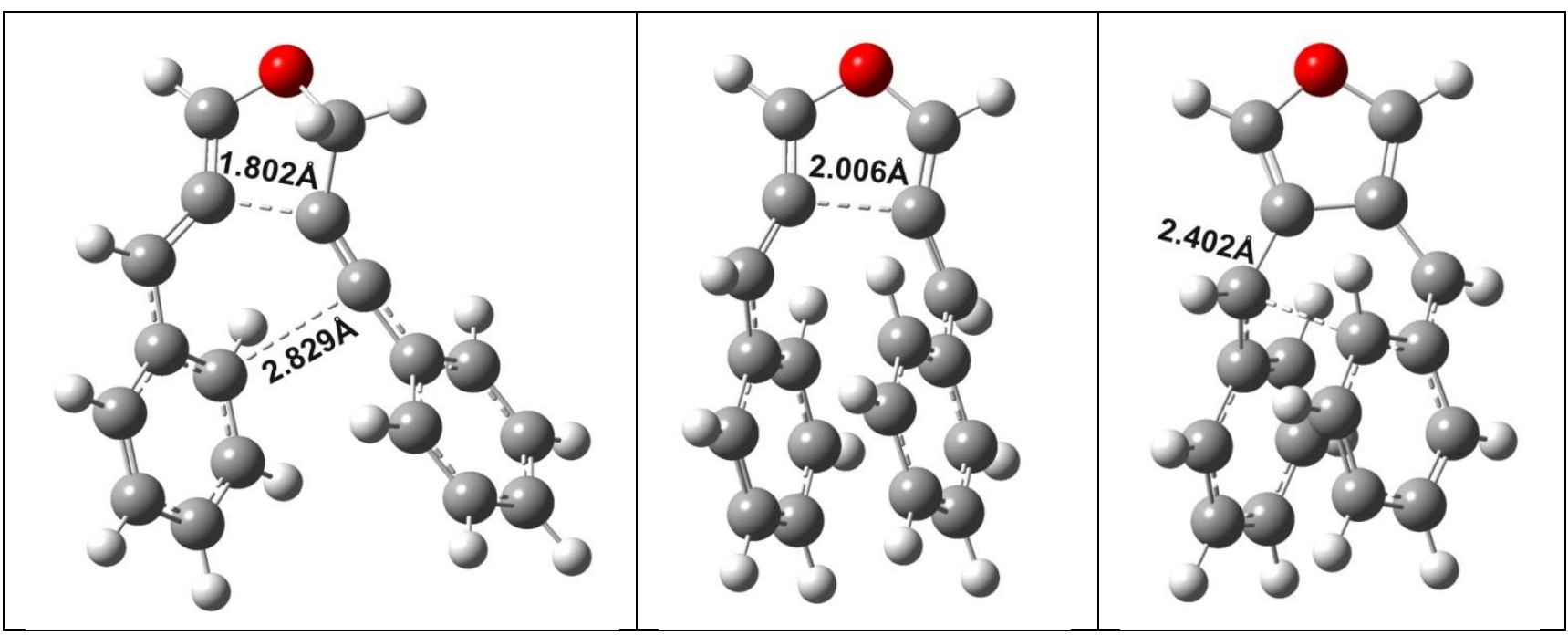

Figure S 1. Transition state geometries are for 4+2 IMDA, stepwise biradical first and second bond formation respectively for the GB cyclization reaction of symmetric bispropargyl ether.

\subsection{Spin Density plots}

All the open-shell specieses were calculated using open-shell formalism. The spin densities are plotted from the population analysis

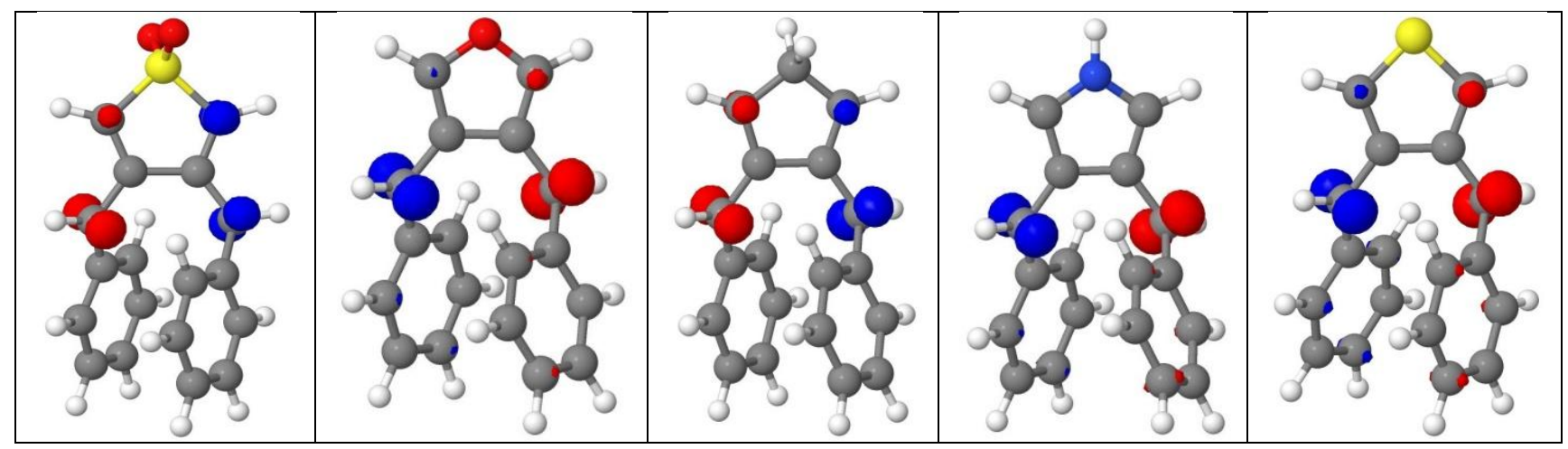

Figure $S$ 2. Spin density plots for biradical intermediates $(X=S O 2, O, C H 2, N H, S)$. 


\subsection{Comparision of DFT functionals}

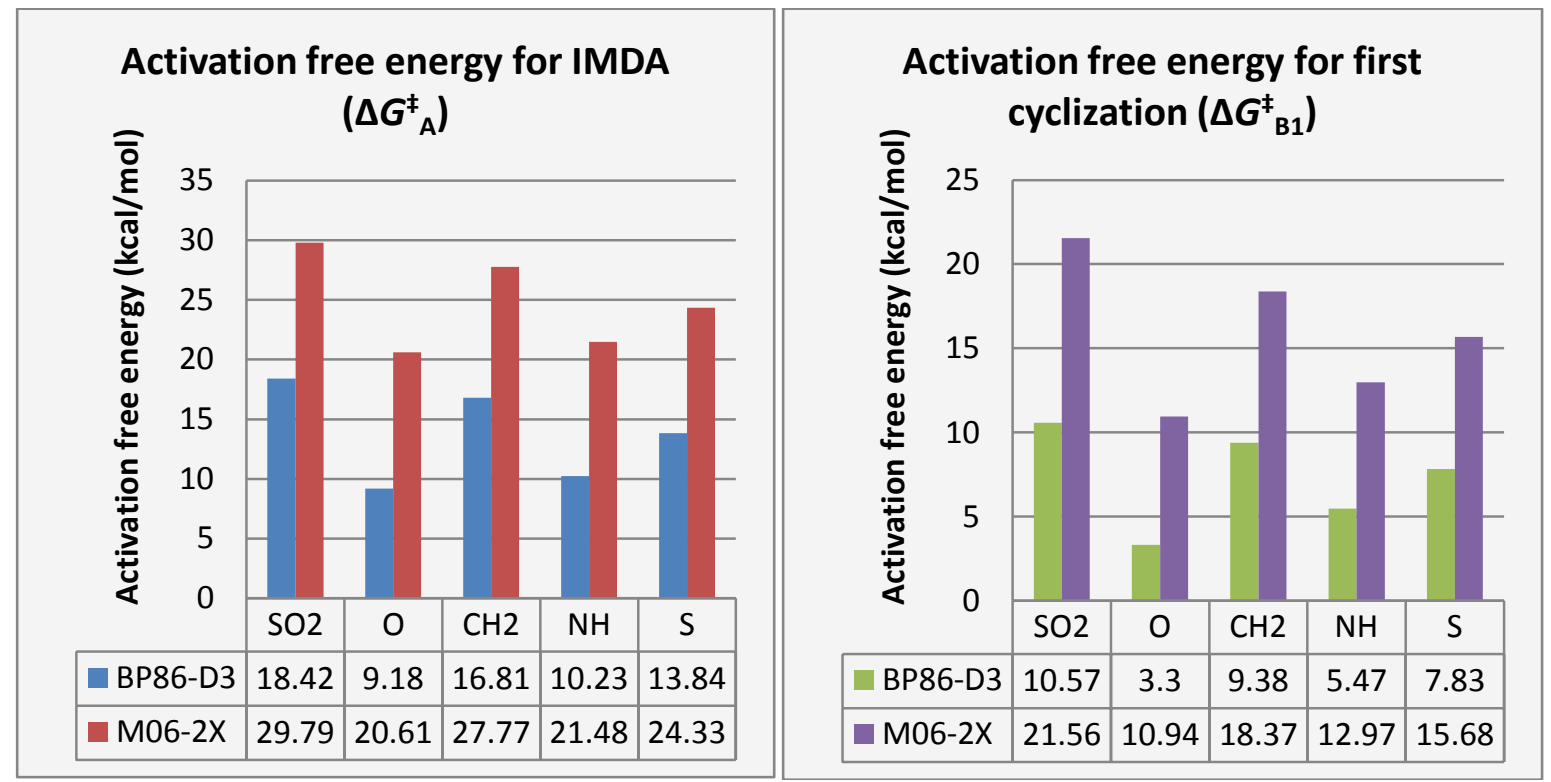

We calculated the activation energies for cycloaddition and cyclization steps in the GB cyclization of symmetric bispropargyl substrates (1) two popular density functionals (M06-2X and BP86-D3). Double-zeta basis set was used. Although barriers are higher with hybrid functionals, the preference for the major pathway among the competing ones are reproduced in all the methods. Thus we used less expensive BP86/def2-SVP method for the studies on unsymmetric systems 


\section{GB Cyclization of the Unsymmetric Bispropargyl Ethers}

\subsection{Mechanism}
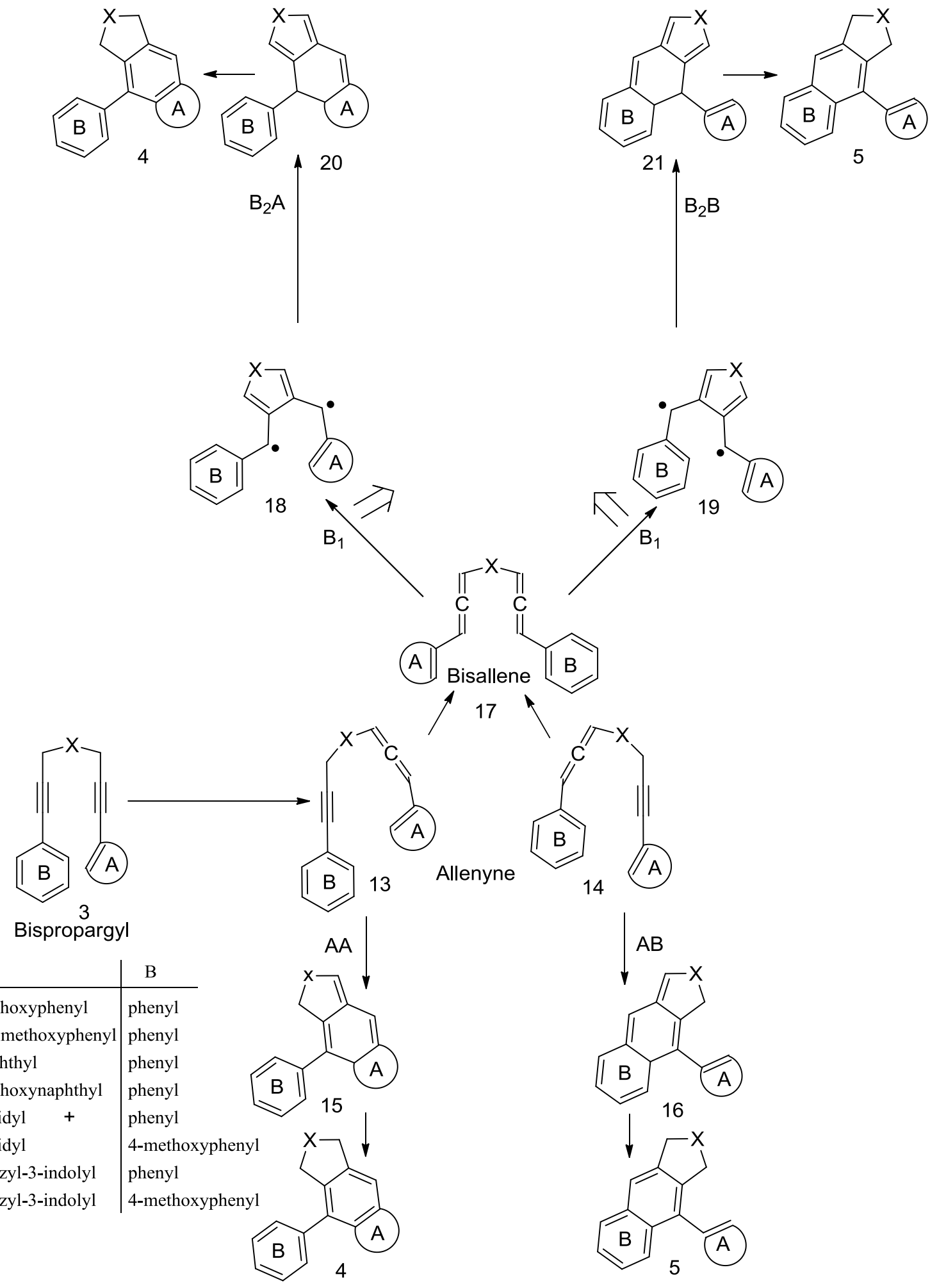


\subsection{Comparison of methods}

We calculated the activation energies for cycloaddition and cyclization steps in the GB cyclization of unsymmetric bispropargyl ether (3) using two popular hybrid functional (M06-2X and B3LYP) and two pure functionals (BP86 and BPW91), and two basis sets of double-zeta and triple-zeta quality (def2-SVP and def2-TZVP). Although barriers are higher with hybrid functionals, the preference for the major pathway among the competing ones are reproduced in all the methods. Thus we used less expensive BP86/def2-SVP method for the studies on unsymmetric systems

Table S 5. Comparison of activation energy with three DFT functionas and double and triple-zeta basis sets for unsymmetric bispropargy ether.Free energy corrections are not added.

\begin{tabular}{|c|c|c|c|c|c|c|c|c|}
\hline & $\begin{array}{l}\text { Level of } \\
\text { theory }\end{array}$ & $\begin{array}{c}\text { BP86- } \\
\text { D3/def2- } \\
\text { SVP }\end{array}$ & $\begin{array}{c}\text { BP86- } \\
\text { D3/def2- } \\
\text { TZVP }\end{array}$ & $\begin{array}{c}\text { BPW91/def2- } \\
\text { SVP }\end{array}$ & $\begin{array}{c}\text { BPW91/def2- } \\
\text { TZVP }\end{array}$ & $\begin{array}{c}\text { B3LYP- } \\
\text { D3/def2- } \\
\text { TZVP }\end{array}$ & $\begin{array}{c}\text { M06- } \\
\text { 2X/def2- } \\
\text { SVP }\end{array}$ & $\begin{array}{c}\text { M06- } \\
\text { 2X/def2- } \\
\text { TZVP }\end{array}$ \\
\hline & $\Delta E_{\mathrm{A}}^{\ddagger}$ & 8.89 & 8.54 & 9.23 & 9.89 & 16.62 & 18.47 & 19.3 \\
\hline & $\Delta E_{\mathrm{AB}}^{\ddagger}$ & 10.05 & 9.58 & 10.69 & 11.44 & 18.15 & 20.05 & 20.95 \\
\hline & $\Delta \Delta E_{(\mathrm{AA}-\mathrm{AB})}^{\ddagger}$ & -1.16 & -1.04 & -1.46 & -1.55 & $-1,53$ & -1.58 & -1.65 \\
\hline \multirow{4}{*}{$\begin{array}{r}\mathrm{OCH}_{3} \\
3.1\end{array}$} & $\Delta E_{\mathrm{B} 1}^{\dagger}$ & 2.38 & 2.03 & 2.83 & 3.04 & 6.7 & 7.46 & 7.89 \\
\hline & $\Delta E_{\mathrm{B} 2 \mathrm{~A}}^{\ddagger}$ & 5.77 & 4.41 & 8.26 & 9.37 & 5.14 & 5.05 & 3.75 \\
\hline & $\Delta E_{\mathrm{B} 2 \mathrm{~B}}^{\ddagger}$ & 8 & 6.26 & 8.6 & 9.62 & 7.13 & 6.39 & 5.05 \\
\hline & $\Delta \Delta E_{(\mathrm{B} 2 \mathrm{~A}-\mathrm{B} 2 \mathrm{~B})}^{\dagger}$ & -2.83 & -1.85 & -0.34 & -0.25 & -1.99 & -1.34 & -1.3 \\
\hline
\end{tabular}

\subsection{Free Energies}

Table S 6. Free energies (Electronic energy + Correction to the Gibbs free energy from harmonic vibrational analysis) calculated at BP86-D3/def2-SVP level of theory.

\begin{tabular}{|c|c|c|c|c|c|c|c|c|}
\hline Species (Compound Number) & 1 & 2 & 3 & 4 & 5 & 6 & 7 & 8 \\
\hline Bispropargyl (3) & -883.11340 & -997.52470 & -922.19558 & -1036.61474 & -784.72691 & -899.15257 & -1170.24635 & -1284.66391 \\
\hline Allenyne_A (13) & -883.13099 & -997.54928 & -922.21321 & -1036.63093 & -784.74970 & -899.16677 & -1170.26230 & -1284.68113 \\
\hline $\mathbf{T S}_{13-15}$ & -883.11613 & -997.53672 & -922.19856 & -1036.61850 & -784.73258 & -899.15103 & -1170.25239 & -1284.67061 \\
\hline Allenyne_B (14) & -883.12713 & -997.54673 & -922.20956 & -1036.62823 & -784.74434 & -899.16594 & -1170.26211 & -1284.68838 \\
\hline $\mathbf{T S}_{14-16}$ & -883.11081 & -997.53139 & -922.19350 & -1036.61174 & -784.72894 & -899.15210 & -1170.24455 & -1284.67191 \\
\hline Bisallene (17) & -883.13923 & -997.56147 & -922.22238 & -1036.63909 & -784.76044 & -899.18001 & -1170.27622 & -1284.69716 \\
\hline $\mathbf{T S}_{17-18}$ & -883.13470 & -997.55773 & -922.21594 & -1036.63473 & -784.75522 & -899.17542 & -1170.27460 & -1284.69555 \\
\hline Biradical intermediate (18) & -883.17865 & -997.59818 & -922.25975 & -1036.67708 & -784.79784 & -899.21962 & -1170.31365 & -1284.73534 \\
\hline TS $_{18-20 \_A}$ & -883.16957 & -997.58927 & -922.24316 & -1036.65980 & -784.78826 & -899.20695 & -1170.31015 & -1284.72983 \\
\hline $\mathrm{TS}_{19-21}$ & -883.16591 & -997.58591 & -922.24898 & -1036.66595 & -784.78797 & -899.20995 & -1170.29862 & -1284.71957 \\
\hline
\end{tabular}

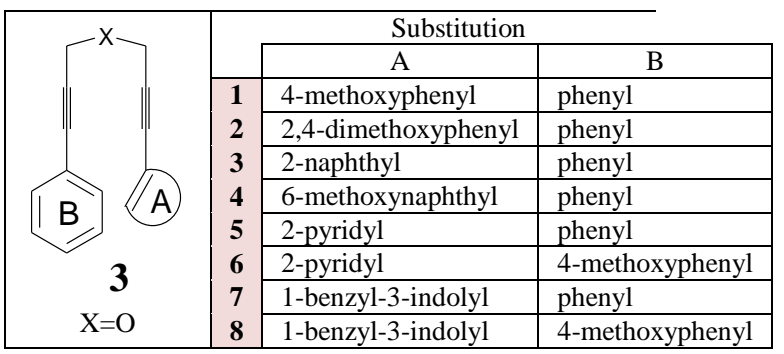




\subsection{Activation free energies and $\mathrm{pK}$ a values for bispropargyl ether}

Table S 7.Stepwise activation free energies (total energy + free energy correction_calculated at BP86-D3/def2-SVP for unsymmetric bispropargyl ethers. The two pathways for the formation of products through the participation of ring $A$ and $B$ are shown with subscripts $A$ and $B . \triangle G \neq A A$ and $\triangle G \ddagger A B:[4+2]$ IMDA from allenyne intermediate. $\triangle G \neq B 1, \triangle G \neq B 2 A$ and $\triangle G \neq B 2 B$ : barrier for th

\begin{tabular}{c|c|c|c|c|c|c|c|c|}
\hline & 1 & 2 & 3 & 4 & 5 & 6 & 7 & 8 \\
\hline$\Delta G_{\mathrm{AA}}^{\ddagger}$ & 9.32 & 7.88 & 9.19 & 7.8 & 10.74 & 10.02 & 6.21 & 6.59 \\
\hline$\Delta G_{\mathrm{AB}}^{\ddagger}$ & 10.24 & 9.62 & 10.07 & 10.35 & 9.66 & 8.69 & 13.65 & 10.33 \\
\hline$\Delta G_{\mathrm{B} 1}^{\ddagger}$ & 2.84 & 2.35 & 4.04 & 2.73 & 3.28 & 2.88 & 1.02 & 1.01 \\
\hline$\Delta G_{\mathrm{B} 2 \mathrm{~A}}^{\ddagger}$ & 5.70 & 5.6 & 10.41 & 10.84 & 6.02 & 7.95 & 2.2 & 3.46 \\
\hline$\Delta G_{\mathrm{B} 2 \mathrm{~B}}^{\ddagger}$ & 8.00 & 7.71 & 6.76 & 6.98 & 6.2 & 6.07 & 9.43 & 9.9 \\
\hline $\mathrm{pK}_{\mathrm{aA}}$ & 40.23 & 38.94 & 35.79 & 38.67 & 32.19 & 33.86 & 44.57 & 44.88 \\
\hline $\mathrm{pK}$ & 37.47 & 37.73 & 35.85 & 37.67 & 36.32 & 39.92 & 36.01 & 40.74 \\
\hline Expt (A:B)* & $01: 08$ & $01: 08$ & $1.6: 1$ & $01: 01.3$ & Only A & Only A & Only B & $01: 04$ \\
\hline
\end{tabular}




\section{Predicting the selectivity for the GB cyclization of unsymmetric bispropargyl amines, sulfides, and methanes $\left(\mathrm{X}=\mathrm{NH}, \mathrm{S}\right.$, and $\left.\mathrm{CH}_{2}\right)$}

\begin{tabular}{|c|c|c|c|}
\hline & & Substit & tion \\
\hline & & $\mathrm{A}$ & $\mathrm{B}$ \\
\hline & 1 & 4-methoxyphenyl & phenyl \\
\hline & 2 & 2,4-dimethoxyphenyl & phenyl \\
\hline & 3 & 2-naphthyl & phenyl \\
\hline & 4 & 6-methoxynaphthyl & phenyl \\
\hline & 5 & 2-pyridyl & phenyl \\
\hline & 6 & 2-pyridyl & 4-methoxyphenyl \\
\hline 3 & 7 & 1-benzyl-3-indolyl & phenyl \\
\hline & 8 & 1-benzyl-3-indolyl & 4-methoxyphenyl \\
\hline
\end{tabular}

\subsection{Comparison of $\mathrm{pK}_{\mathrm{a}}$ values for the bispropargyl substrates $\left(3 ; \mathrm{X}=0, \mathrm{CH}_{2}\right.$, NH, and S)}

Table $S$ 8. The relative $p K a$ values of $H_{A}$ and $H_{B}$ of the unsymmetric bispropargyl ether $(Z=O)$.

\begin{tabular}{|c|c|c|c|c|c|}
\hline $\mathrm{X}=\mathrm{O}$ & $\mathrm{A}$ & $\mathrm{B}$ & $\mathrm{pK}_{\mathrm{a}}$ for A & $\mathrm{pK}_{\mathrm{a}}$ for B & Expt (A:B) \\
\hline 1 & 4-methoxyphenyl & phenyl & 40.23 & 37.47 & $1: 8$ \\
\hline 2 & 2,4-dimethoxyphenyl & phenyl & 38.94 & 37.73 & $1: 8$ \\
\hline 3 & 2-naphthyl & phenyl & 35.79 & 35.85 & $1.6: 1$ \\
\hline 4 & 6-methoxynaphthyl & phenyl & 38.67 & 37.67 & Only A \\
\hline 5 & 2-pyridyl & phenyl & 32.19 & 36.32 & Only A \\
\hline 6 & 2-pyridyl & 4-methoxyphenyl & 33.86 & 39.92 & Only B \\
\hline 7 & 1-benzyl-3-indolyl & phenyl & 44.57 & 36.01 & $1: 4$ \\
\hline 8 & 1-benzyl-3-indolyl & 4-methoxyphenyl & 44.88 & 40.74 & \\
\hline
\end{tabular}

Table $S$ 9. The relative $p K a$ values of $H A$ and $H B$ of the unsymmetric bispropargyl ether $(Z=O)$

\begin{tabular}{|c|c|c|c|c|}
\hline $\mathrm{X}=\mathrm{CH}_{2}$ & $\mathrm{~A}$ & $\mathrm{~B}$ & $\mathrm{pK}_{\mathrm{a}}$ for A & $\mathrm{pK}_{\mathrm{a}}$ for $\mathrm{B}$ \\
\hline 1 & 4-methoxyphenyl & phenyl & 41.02 & 37.63 \\
\hline 2 & 2,4-dimethoxyphenyl & phenyl & 41.88 & 37.46 \\
\hline 3 & 2-naphthyl & phenyl & 37.66 & 39.16 \\
\hline 4 & 6-methoxynaphthyl & phenyl & 39.64 & 39.68 \\
\hline 5 & 2-pyridyl & 4-methoxyphenyl & 34.40 & 39.26 \\
\hline 6 & 2-pyridyl & phenyl & 45.00 & 41.43 \\
\hline 7 & 1-benzyl-3-indolyl & 4-methoxyphenyl & 45.15 & 37.38 \\
\hline 8 & 1-benzyl-3-indolyl & & & 41.47 \\
\hline
\end{tabular}

Table $S$ 10. The relative $p K a$ values of $H A$ and $H B$ of the unsymmetric bispropargyl amine $(Z=N H)$

\begin{tabular}{|c|c|c|c|c|}
\hline $\mathrm{X}=\mathrm{NH}$ & $\mathrm{A}$ & $\mathrm{B}$ & $\mathrm{pK}_{\mathrm{a}}$ for A & $\mathrm{pK}_{\mathrm{a}}$ for $\mathrm{B}$ \\
\hline 1 & 4-methoxyphenyl & phenyl & 40.59 & 36.47 \\
\hline 2 & 2,4-dimethoxyphenyl & phenyl & 41.33 & 36.38 \\
\hline 3 & 2-naphthyl & phenyl & 37.04 & 37.04 \\
\hline 4 & 6-methoxynaphthyl & phenyl & 38.72 & 37.68 \\
\hline 5 & 2-pyridyl & phenyl & 32.34 & 37.11 \\
\hline 6 & 2-pyridyl & 4-methoxyphenyl & 33.08 & 39.45 \\
\hline 7 & 1-benzyl-3-indolyl & phenyl & 45.16 & 37.95 \\
\hline 8 & 1-benzyl-3-indolyl & 4-methoxyphenyl & 45.08 & 40.78 \\
\hline
\end{tabular}


Table $S$ 11. The relative $p K a$ values of $H A$ and $H B$ of the unsymmetric bispropargyl sulfide $(X=S)$

\begin{tabular}{|c|c|c|c|c|}
\hline $\mathrm{X}=\mathrm{S}$ & $\mathrm{A}$ & $\mathrm{B}$ & $\mathrm{pK}_{\mathrm{a}}$ for $\mathrm{A}$ & $\mathrm{pK}_{\mathrm{a}}$ for $\mathrm{B}$ \\
\hline 1 & 4-methoxyphenyl & phenyl & 34.13 & 29.63 \\
\hline 2 & 2,4-dimethoxyphenyl & phenyl & 34.09 & 31.07 \\
\hline 3 & 2-naphthyl & phenyl & 29.31 & 29.29 \\
\hline 4 & 6-methoxynaphthyl & phenyl & 32.83 & 28.79 \\
\hline 5 & 2-pyridyl & phenyl & 27.19 & 29.56 \\
\hline 6 & 2-pyridyl & 4-methoxyphenyl & 26.99 & 31.57 \\
\hline 7 & 1-benzyl-3-indolyl & phenyl & 35.74 & 27.98 \\
\hline 8 & 1-benzyl-3-indolyl & 4-methoxyphenyl & 35.41 & 32.91 \\
\hline
\end{tabular}




\section{Predicting selectivity for bispropargyl sulfones}

\subsection{Comparison of $\mathrm{pK}_{\mathrm{a}}$ 's}

Table $S$ 12. The relative $\mathrm{pKa}$ values of $H A$ and $H B$ of the unsymmetric bispropargyl sulfones $\left(\mathrm{X}=\mathrm{SO}_{2}\right)$

\begin{tabular}{|c|c|c|c|c|}
\hline $\mathrm{X}=\mathrm{SO}_{2}$ & $\mathrm{~A}$ & $\mathrm{~B}$ & $\mathrm{pK}_{\mathrm{a}}$ for A & $\mathrm{pK}_{\mathrm{a}}$ for B \\
\hline 1 & 4-methoxyphenyl & phenyl & 26.11 & 23.09 \\
\hline 2 & 2,4-dimethoxyphenyl & phenyl & 23.22 & 20.19 \\
\hline 3 & 2-naphthyl & phenyl & 23.23 & 23.14 \\
\hline 4 & 6-methoxynaphthyl & phenyl & 27.16 & 24.91 \\
\hline 5 & 2-pyridyl & phenyl & 21.64 & 26.59 \\
\hline 6 & 2-pyridyl & 4-methoxyphenyl & 22.81 & 26.26 \\
\hline 7 & 1-benzyl-3-indolyl & phenyl & 27.39 & 25.00 \\
\hline 8 & 1-benzyl-3-indolyl & 4-methoxyphenyl & 26.97 & 26.72 \\
\hline
\end{tabular}

\subsection{Comparison of activation free energy for second biradical cyclization}

Table $S$ 13. The activation free energy for the formation of both products from bisallene active intermediate of unsymmetric bispropargyl sulfone.

\begin{tabular}{|c|c|c|c|c|}
\hline $\mathrm{X}=\mathrm{SO}_{2}$ (bisallene) & $\mathrm{A}$ & $\mathrm{B}$ & $\Delta G^{\ddagger}{ }_{\mathrm{B} 2 \mathrm{~A}}$ & $\Delta G^{\ddagger}{ }_{\mathrm{B} 2 \mathrm{~B}}$ \\
\hline 1 & 4-methoxyphenyl & phenyl & 14.47 & 16.28 \\
\hline 2 & 2,4-dimethoxyphenyl & phenyl & 13.78 & 15.86 \\
\hline 3 & 2-naphthyl & phenyl & 11.66 & 13.00 \\
\hline 4 & 6-methoxynaphthyl & phenyl & 10.82 & 12.62 \\
\hline 5 & 2-pyridyl & phenyl & 12.30 & 12.75 \\
\hline 6 & 2-pyridyl & 4-methoxyphenyl & 14.85 & 13.38 \\
\hline 7 & 1-benzyl-3-indolyl & phenyl & 6.61 & 12.63 \\
\hline 8 & 1-benzyl-3-indolyl & 4-methoxyphenyl & 10.58 & 14.68 \\
\hline
\end{tabular}

\subsection{Absolute energies}

Table S 14. Free energies (Electronic energy +Correction to the Gibbs free energy from harmonic vibrational analysis) calculated at BP86-D3/def2-SVP level of theory.

\begin{tabular}{|c|c|c|c|c|c|c|c|c|}
\hline Species( & $\mathbf{1}$ & $\mathbf{2}$ & $\mathbf{3}$ & $\mathbf{4}$ & $\mathbf{5}$ & $\mathbf{6}$ & $\mathbf{7}$ & $\mathbf{8}$ \\
\hline Bisallene (17) & -1356.370908 & -1470.792057 & -1395.446204 & -1509.865279 & -1257.983951 & -1372.407335 & -1643.497814 & -1757.922577 \\
\hline $\mathrm{TS}_{18-20}(\mathrm{~A})$ & -1356.347844 & -1470.770102 & -1395.425178 & -1509.844666 & -1257.964346 & -1372.383668 & -1643.487284 & -1757.905714 \\
\hline $\mathrm{TS}_{19-21}(\mathrm{~B})$ & -1356.34497 & -1470.766791 & -1395.432615 & -1509.851499 & -1257.963633 & -1372.386009 & -1643.477688 & -1757.899183 \\
\hline
\end{tabular}

Table S 15. The free energy (total energy + free energy correction) of unsymmetric bispropargyl systems and the corresponding anions calculated at BP86-D3(COSMO)/DEF2-svp. Energyies are in hartrees.

\begin{tabular}{|c|c|c|c|c|c|c|c|c|c|}
\hline$X$ & pecies & 1 & 2 & 3 & 4 & 5 & 6 & 7 & 8 \\
\hline \multirow[b]{3}{*}{$\mathrm{CH}_{2}$} & Bispropargyl & -847.24201 & -961.66188 & -886.32366 & -1000.74479 & -748.85976 & -863.28397 & -1134.37870 & -1248.80306 \\
\hline & Anion 22 & -846.71631 & -961.13432 & -885.80529 & -1000.22210 & -748.34847 & -862.77137 & -1133.84353 & -1248.26840 \\
\hline & Anion 23 & -846.72368 & -961.14392 & -885.80201 & -1000.22202 & -748.33790 & -862.75740 & -1133.86094 & -1248.27640 \\
\hline \multirow[b]{3}{*}{$\mathrm{NH}$} & Bispropargyl & -863.26991 & -977.69005 & -902.35136 & -1016.77243 & $\begin{array}{l}-764.88705 \\
\end{array}$ & -879.31010 & -1150.40686 & -1264.83130 \\
\hline & Anion 22 & -862.74516 & -977.16368 & -901.83434 & -1016.25175 & -764.38024 & -878.80167 & -1149.87216 & -1264.29680 \\
\hline & Anion 23 & -862.75411 & -977.17446 & -901.83434 & -1016.25400 & -764.36986 & -878.78783 & -1149.88784 & -1264.30614 \\
\hline \multirow[b]{3}{*}{$\mathrm{O}$} & Bisp & -883. & $-997 .$. & -922 & -102 & -784 & -899. & 286 & -128 \\
\hline & Anion 22 & -882.60179 & -997.01993 & -921.69233 & -1036.10725 & -784.23518 & -898.65690 & -1169.72945 & -1284 \\
\hline & Anion 23 & -882.60779 & -997.02258 & -921.69221 & -103 & -784.22619 & 4372 & -1169.74806 & -128 \\
\hline \multirow[b]{3}{*}{ S } & Bispropargyl & -1206.09116 & -1320.51315 & -1245.17200 & -1359.59352 & -1107.70718 & -1222.13070 & -1493.22815 & -1607.65250 \\
\hline & Anion 22 & -1205.58045 & -1320.00252 & -1244.67179 & -1359.08564 & -1107.21158 & -1221.63553 & -1492.71394 & -1607.13901 \\
\hline & Anion 23 & -1205.59025 & -1320.00910 & -1244.67183 & -1359.09443 & -1107.20641 & -1221.62556 & -1492.73083 & -1607.14444 \\
\hline & Bispropargyl & -1356.38215 & -1470.79548 & -1395.46144 & -1509.88391 & -1257.99674 & -1372.42044 & -1643.52049 & -1757.94340 \\
\hline & Anion 22 & -135 & -1470.30850 & -1394.97445 & -1509.38 & -1257 & -1371.93435 & -1643.02444 & -1757.44826 \\
\hline & Anion 23 & -1355.8955 & -1470.3151 & -1394.9746 & -1509.39324 & -1257.5024 & -1371.9268 & -1643.0296 & -1757.4488 \\
\hline
\end{tabular}




\section{Cartesian coordinates}

Cartesian coordinates of the optimized geometries calculated at M062X/ def2-SVP for symmetric systems and BP86-D3/def2-SVP level for unsymmetric systems.

\section{Symmetric bispropargyltitle}

\section{$\mathrm{X}=-\mathbf{C H 2}$}

\section{Bispropargyl}

35

Free energy $=-732.434964$ Hartree

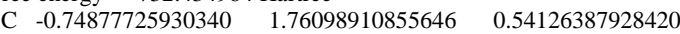

$\begin{array}{llll}\text { C } & 0.30088570699747 & 2.41239334011407 & -0.12645672998120\end{array}$

C $0.00267199204705 \quad 3.41700328801031 \quad-1.06182329812513$

$\begin{array}{llll}\text { C } & -1.31999940625524 & 3.76012412213225 & -1.32696404454392\end{array}$

C $-2.35967093633128 \quad 3.10737729914314 \quad-0.66361093019724$

$\begin{array}{llll}\text { C } & -2.07026405335595 & 2.10967607933152 & 0.27102518952570\end{array}$

$\begin{array}{llll}\text { C } & 1.67203127559904 & 2.10009245232471 & 0.17201199564430\end{array}$

$\begin{array}{llll}\text { C } & 2.83971441258019 & 1.92860344297120 & 0.44801727232415\end{array}$

C $4.23942499849745-1.89629186495831-0.88426423667005$

$\begin{array}{llll}\text { C } 4.55570492841588 & 3.03555062660814 & 1.86412937880133\end{array}$

$\begin{array}{llll}\text { C } & 4.31254670149466 & 4.44422853443397 & 1.28452225415298\end{array}$

$\begin{array}{llll}\text { C } & 2.91245152899869 & 4.86978903379125 & 1.38244823042534\end{array}$

$\begin{array}{llll}\text { C } & 1.74752883436943 & 5.16374734913139 & 1.54526383872632\end{array}$

$\begin{array}{llll}\text { C } & 0.36602212737442 & 5.44384297023742 & 1.83024737220522\end{array}$

$\begin{array}{llll}\text { C } & -0.33687912548910 & 4.59450954053139 & 2.70289746359699\end{array}$

$\begin{array}{llll}\text { C } & -1.64918112341288 & 4.88661947895463 & 3.06036004284305\end{array}$

$\begin{array}{llll}\text { C } & -2.28078369730799 & 6.01815470053074 & 2.54080004275514\end{array}$

$\begin{array}{llll}\text { C } & -1.60395960836793 & 6.84216493305087 & 1.64002099835628\end{array}$

$\begin{array}{llll}\text { C } & -0.28855743643981 & 6.55844669295429 & 1.28205418932154\end{array}$

$\begin{array}{llll}\mathrm{H} & -3.29579494207700 & 6.27034183567485 & 2.85278340069872\end{array}$

H $\quad-3.39242517362709 \quad 3.38901726218440 \quad-0.88112139397158$

$\begin{array}{lllll}\mathrm{H} & 4.46195525357914 & 0.93211979750709 & 1.36879435070259\end{array}$

$\begin{array}{llll}\mathrm{H} & 4.64799708090340 & 4.48118335772481 & 0.23518167343232\end{array}$

$\begin{array}{llll}\mathrm{H} & -0.51329746267187 & 0.98935837282956 & 1.27594221415749\end{array}$

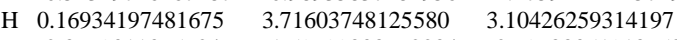

$\begin{array}{llll}\mathrm{H} & -2.87693113657046 & 1.59799838672084 & 0.79788351918752\end{array}$

$\begin{array}{llll}\mathrm{H} & -2.18182165651702 & 4.22665828622459 & 3.74718363157601\end{array}$

$\begin{array}{llll}\mathrm{H} & -1.54481251490961 & 4.54761117821360 & -2.04740679981984\end{array}$

$\begin{array}{llll}\mathrm{H} & -2.10394708587329 & 7.71428550468604 & 1.21557597321836\end{array}$

$\begin{array}{llll}\mathrm{H} & 0.25121463480707 & 7.20631158130627 & 0.58970944197786\end{array}$

$\begin{array}{llll}\text { H } & 4.93404644843319 & 5.17155681976551 & 1.82607245094052\end{array}$

$\begin{array}{lllll}\mathrm{H} & 0.82294835739612 & 3.93107539836063 & -1.56317394786677\end{array}$

$\begin{array}{llll}\mathrm{H} & 4.89793848641463 & 1.96561028629477 & 0.00309019537801\end{array}$

$\begin{array}{llll}\text { H } & 5.60943278447945 & 2.95122853617912 & 2.16824739088808\end{array}$

H $3.94487717962650 \quad 2.91173025981109 \quad 2.76931955297736$

\section{Allenyne}

35

Free energy $=-732.4405186$ Hartree

C $-2.21317700625021 \quad 0.91175857165506$

C $-1.41107391691306 \quad 2.05473800222044$

$\begin{array}{lll}\text { C } & -2.01487026881029 & 3.32357652725147\end{array}$

C $-3.39680512795196 \quad 3.44471537752834$

$\begin{array}{lll}\text { C } & -4.19109963181187 & 2.30199433283647\end{array}$

C $-3.59642159162700 \quad 1.04082146314684$

C $0.00924959212683 \quad 1.93596726681600$

C $1.20211235832522 \quad 1.86721599320907$

$\begin{array}{lll}\text { C } & 2.64000869306811 & 1.84831294129505\end{array}$

$\begin{array}{lll}\text { C } & 2.96219807766513 & 1.44719908000420\end{array}$

$\begin{array}{lll}\text { C } 2.76519994858759 & -0.02576478377197\end{array}$

C $2.14705918732531 \quad-0.84903107808181$

C $1.47044632464016-1.64263005678511$

C 0.02253992781023

C -0.62479316952292

C -1.97834293189218

C -2.69579787764530

C -2.06644386420800

C -0.71828463474845

H -3.74346157979070

H -5.27602146708272

H 3.04478503251613

H 3.17413436933445

H 2.00105584710850

Н -1.38472890085492

H -0.21594190558737

H -3.86262385937412

H $\quad-2.63333855419878$

H -4.21159474297078

H -2.46686757916999

H -0.05351156078059

$-1.89747256568752$

$-2.72334906440659$

$-3.02929066987938$

$-2.51055451809349$

$-1.65776513280214$

$-1.35259327827487$

2.7823536564147

2.39979670072145

2.84930883836015

0.42336624303104

$-2.17163800538246$

4.20823789217010

$-0.69417700581534$

4.43156355682035

$-1.22507161851273$

0.14454708533432

$-3.68437290167269$

$-3.14567389834002$

1.15299808814137
$-0.77966528619962$ $-0.63498665199222$ 0.63828037442671 $-0.83858581511502$ $-0.86641443103749$ $-0.43572765707937$ $-0.22683836428293$ 0.06422472342689 1.51148910182285 1.78749503656456 0.97978308654816 0.30879520414533 $-0.62118534171074$ $-0.47573813459518$ 1.51581118922518 1.36998895612682 0.74479082803949 $-0.9050087918042$ $-0.14572388876435$ 2.72437513510799 $-0.61940609169137$ $-0.54132559254074$ 2.08063537098355 $-0.73918500886416$ 2.3410401935227 $-0.95789992372685$ $-1.19877212285985$ $-1.4508247456459$ $-0.62219518357350$ $-0.74325653285324$ 0.17885153521858 0.60338555049142
H -1.74079588146272

H 4.0008645775130

H 2.32984252570342

0.07103284616414 1.72003199505687 2.02874061054846

$-0.81254327881112$ 1.75598707263007 2.19940523372099

Allenyne2Cyclization

Free energy $=-732.396259$ Hartree

C $-2.11015476194934 \quad 0.89806917893296$

C -1.41860186317285

C -2.06705208381907

C -3.34348504398996

C -4.00073469151302

C -3.38327830744416

C -0.08590314949074

C 1.15969014416179

C 2.44111448629021

C 3.17648596315095

C 2.89410939012704

C 1.9229830242722

C 1.39657665312740

C -0.00509309445710

C -0.61402048023165

C -1.98508391225641

C -2.80150141435879

C -2.23287731324996

C -0.83983231420558

H -3.88468099119062

H -4.98629849897802

H 2.23652471842976

H 3.42313244363035

H 2.04369438754983

H -1.54109898421370

H $\quad-0.38249825456147$

H -3.82892137418020

H -2.86846580540587

H -3.90450368066315

H -2.44806479841222

H 0.02377023361976

H 3.03043798730821

H -1.62408945796223

H 4.25238298654343

H 2.77524885749518

1.52893103724268

2.55092614276187

2.96598412306693

2.36649160679252

.32499372672929

1.14053771640318

1.02624096815665

1.80732963022259

1.63250494243490

0.20311140600712

0.39625355824198

$-1.62102650185182$

$-1.84621642617988$

$-2.86543051789615$

$-3.00128936937976$

$-2.13603716387904$

$-1.14788031355721$

$-0.98388939777044$

$-2.27234367770354$

2.72007760079819

2.85020361952303

$-0.31095372762784$

$-2.32752787973934$

3.03157885501697

$-0.28706567117331$

3.76740580364520

$-0.49585654167071$

0.83343552127805

$-3.77825021269396$

$-3.53158368089550$

1.33806004569913

0.07673563830286

1.84463252055359

2.31973855669275

$-1.42395430623965$

$-0.36844120269942$

0.35721007222317

$-0.00573803903298$

$-1.08374985929076$

. 7809827723456

$-0.05722087013025$

0.07202762562903

$-0.10389398838892$

1.22693811260865

60471420718963

0.90644838263469

0.54532889994245

0.56982487221669

$-0.25598479281503$

0.5049202031059

1.2914806738902

1.32241838779130

0.49264808644878

$-1.38995690040270$

$-0.37533676259904$

2.40918476293306

0.01682678989139

1.18266776011320

2.02450988552183 0.55353005013389 1.89242659360445 $-2.6035283695283$ $-0.86644794496242$ $-0.80277269208731$ $-0.90634470935849$

$-1.95247830257272$

14385816734968

1.98998181870885

Free energy $=-732.4879839$ Hartree

C $\quad-2.09794004890829 \quad 1.21733969143824$

$\begin{array}{lll}\text { C } & -1.51591460369759 & 1.40422068071140\end{array}$

C $-1.89922854664026 \quad 2.51743076054493$

$\begin{array}{lll}\text { C } & -2.84538927368754 & 3.41975980570749\end{array}$

C $-3.42894237356237 \quad 3.21432900509007$

C $-3.05410000601314 \quad 2.10595218074593$

$\begin{array}{lll}\text { C } & -0.51676459012631 & 0.41119765765324\end{array}$ $\begin{array}{lll}\text { C } & 0.78783430612219 & 0.71244537059409\end{array}$

C 1.4960

C 2.9593297772616

C 3.01816729741323

C 1.80437247939884

C 1.40446861039195

C 0.09649906106146

C -0.32342531192752

C -1.60322254011760

C -2.62096262576650

C -2.33147870977456

C -0.99243356304384

H $\quad-3.62298678991696$

$-4.17116795142378$

H 1.01401904208508

H 3.9407903529118

2.17298831326067

H -1.45753994588781

H -1.18009354755985

H -3.13678314035210

H -3.10676027000950

H -3.50824254431974

H -1.88789887616592

$\begin{array}{ll}H & 0.44716613873067\end{array}$

H 1.4496363930187

H -1.78655248723575 2.0370770236378 1.77141010894017 0.27895235905314 $-0.29872474180002$ $-1.69181255520807$ 2.03383498169673 $-3.42478619493545$ $-3.76503211830063$ 
H $\quad 3.21684156772682 \quad 2.31564777761875 \quad 1.60710246242067$

Bisallene

35

Free energy $=-732.4432685$ Hartree

C $1.99908102215760 \quad 8.47552266659058 \quad-3.45214803644035$

C 3.30109190843291

$\begin{array}{llll}\text { C } & 4.03891247829268 & 7.25827200064759 & -3.87501183151482\end{array}$

$\begin{array}{llll}\text { C } 3.47231868138030 & 6.71067384847885 & -5.02035948851716\end{array}$

$\begin{array}{llll}\text { C } & 2.17205142694605 & 7.05651881395597 & -5.39954539315630\end{array}$

$\begin{array}{llll}\text { C } & 1.44157516302712 & 7.94842113910517 & -4.61673001366792\end{array}$

$\begin{array}{llll}\text { C } 3.86196164875116 & 8.70315293756957 & -1.82269623776903\end{array}$

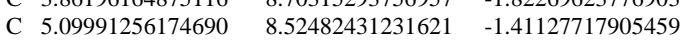

$\begin{array}{llll}\text { C } & 6.35845524137479 & 8.39070124725874 & -1.07804417362246\end{array}$

$\begin{array}{llll}\text { C } & 6.98885697635414 & 7.30667741721785 & -0.23571767255786\end{array}$

$\begin{array}{llll}\text { C } & 6.11842316491311 & 6.13039359891854 & 0.14628317456242\end{array}$

$\begin{array}{llll}\text { C } & 5.12979768071305 & 5.63641304082102 & -0.55771375469261\end{array}$

$\begin{array}{llll}\text { C } & 4.17775715054737 & 5.08967205155799 & -1.28621048103041\end{array}$

$\begin{array}{llll}\text { C } & 2.75010994087210 & 5.46239092071121 & -1.29591394792088\end{array}$

$\begin{array}{llll}\text { C } & 1.94810825589765 & 4.98648294638390 & -2.34247486465696\end{array}$

$\begin{array}{llll}\text { C } & 0.63165858236540 & 5.41416281080226 & -2.48260456583859\end{array}$

$\begin{array}{llll}\text { C } & 0.09419425162816 & 6.31431602343131 & -1.56392456367074\end{array}$

$\begin{array}{llll}\text { C } & 0.86559470691019 & 6.75428464763992 & -0.48587659649263\end{array}$

$\begin{array}{llll}\text { C } & 2.18835224836589 & 6.33567326222977 & -0.35158108278474\end{array}$

$\begin{array}{llll}\mathrm{H} & -0.92508078962154 & 6.68223284482686 & -1.69469425458973\end{array}$

$\begin{array}{llll}\mathrm{H} & 1.73054865807752 & 6.62694800902696 & -6.29996948701743\end{array}$

$\begin{array}{llll}\mathrm{H} & 7.04945723717810 & 9.15927882483873 & -1.44584750342782\end{array}$

$\begin{array}{llll}\mathrm{H} & 6.37834874628131 & 5.62399743949848 & 1.08366199134543\end{array}$

H $3.18188998939310 \quad 9.29420534189490 \quad-1.19888234206449$

$\begin{array}{lllll}\mathrm{H} & 4.48156155586130 & 4.33731885015661 & -2.02343508848568\end{array}$

$\begin{array}{llll}\mathrm{H} & 5.05317304924215 & 6.98780265541277 & -3.57371777827887\end{array}$

$\begin{array}{llll}\mathrm{H} & 2.80623538452494 & 6.70304227785613 & 0.47071698230591\end{array}$

$\begin{array}{llll}\mathrm{H} & 4.05344288972948 & 6.01946204517314 & -5.63391084529112\end{array}$

$\begin{array}{lllll}\mathrm{H} & 0.43747801978087 & 7.44150083233369 & 0.24590460509136\end{array}$

$\begin{array}{lllll}\mathrm{H} & 0.43607871136438 & 8.24649045674137 & -4.91994432622377\end{array}$

$\begin{array}{llll}\mathrm{H} & 0.03192496956241 & 5.06106525453178 & -3.32227898084315\end{array}$

$\begin{array}{llll}\mathrm{H} & 1.41213515202880 & 9.14500161419376 & -2.81830632834527\end{array}$

$\begin{array}{llll}\text { H } & 2.38083911056329 & 4.30882287292132 & -3.08048103369609\end{array}$

H $7.87420696013910 \quad 6.92944466943019 \quad-0.77496656108504$

$\begin{array}{llll}\mathrm{H} & 7.39369726521827 & 7.76328258290967 & 0.68195123062994\end{array}$

Bisallene2Cyclization_TS

35

Free energy $=-732.4139862$ Hartree

C $2.04295942112968 \quad 8.43313334695785$

C $3.33844644051613 \quad 8.04409698657800$

C $4.06820011458991 \quad 7.15709789407813$

C $3.48484377957937 \quad 6.61659239824653$

C $2.19208467010873 \quad 6.98394858798579$

C $1.48296929617194 \quad 7.91712175106354$

$\begin{array}{lll}\text { C } 3.87034857981386 & 8.48911912974265\end{array}$

C $5.04145569110388 \quad 8.01486891287529$

C $6.26236247943822 \quad 8.35555526900773$

C $7.06726639089611 \quad 7.34350454704093$

C $6.22635109858933 \quad 6.10250899395808$

C $5.12207158600744 \quad 6.10412115032633$

C $4.17001674589821 \quad 5.33697469877767$

C $2.75796619787552 \quad 5.58295812502692$

C $1.88608524421723 \quad 4.99475919944328$

$\begin{array}{lll}\text { C } & 0.56331891596760 & 5.38389588218309\end{array}$

C $0.06665757614748 \quad 6.35780437096333$

C $0.88473381340360 \quad 6.88529181679916$

$\begin{array}{lll}\text { C } 2.22880153174763 & 6.50817784426162\end{array}$

$\mathrm{H} \quad-0.96798164171993 \quad 6.69443338712008$

H $1.73617635728219 \quad 6.55337372177798$

H $6.67832899733933 \quad 9.33078991387730$

$\begin{array}{lll}\text { H } & 6.48946183072690 & 5.23952077483328\end{array}$

H $3.24286693704793 \quad 9.14633652261355$

$\mathrm{H} \quad 4.50586859382510 \quad 4.62048162814469$

H $5.09107149388604 \quad 6.90142439297625$

$\begin{array}{lll}\mathrm{H} & 2.87809591856813 & 6.91306461241469\end{array}$

$\begin{array}{lll}\mathrm{H} & 2.87809591856813 & 6.91306461241469 \\ \mathrm{H} & 4.05033542489829 & 5.91701837974663\end{array}$

$\begin{array}{lll}\mathrm{H} & 0.48025212754885 & 7.60746952699029\end{array}$

$\begin{array}{lll}\mathrm{H} & 0.48845202590929 & 8.23912765371040\end{array}$

H $-0.08945282085065 \quad 4.95547857876192$

$\begin{array}{lll}\mathrm{H} & 1.47351355700117 & 9.12089857674541\end{array}$

$\begin{array}{lll}\text { H } & 2.29351053992963 & 4.27582150638427\end{array}$

H $8.03866381145513 \quad 7.16344781774503$

H $7.30898427395072 \quad 7.68301910084225$

$-3.46106305623745$

$-3.05052391335962$

$-3.87191319122455$

$-5.01575021056756$

$-5.38763207087186$

$-4.61889535120254$

$-1.78956309978590$

$-1.25271864002362$

$-0.85017881252650$

$-0.09335575827588$

$-0.09218497700491$

$-0.83791464167171$

$-1.45746955500449$

$-1.36519886379839$

$-2.31374299793632$

$-2.39214215917150$

$-1.50979770565603$

$-0.51595490377254$

$-0.43942303192365$

$-1.59979486829482$

$-6.28047592294054$

$-1.11412577277026$

0.52290050071619

$-1.17928089873976$

$-2.21313354418639$

$-3.59390323630905$

0.33734596031619

$-5.63425820527002$

0.19521241304930

$-4.93327974727027$

$-3.15338758998722$

$-2.83051531262886$

$-3.02747393573176$

$-0.58657127104474$

0.92836237110703

Bisallene2Cyclization_INT1

35

Free energy $=-732.4446454$ Hartree

$\begin{array}{llll}\text { C } & 2.11838584948803 & 8.54792884781423 & -3.46302201833600 \\ \text { C } & 3.35561463895558 & 8.02687003120690 & -3.01734080371899 \\ \text { C } & 3.97985717529987 & 7.03867248958295 & -3.81761259994890 \\ \text { C } & 3.35892738861289 & 6.54713265690104 & -4.95829602242832 \\ \text { C } & 2.11884694610695 & 7.04862381435200 & -5.35995994920387 \\ \text { C } & 1.51231110486705 & 8.06644748992086 & -4.61611492702631 \\ \text { C } & 3.94316756045953 & 8.49445555655528 & -1.79389319253311\end{array}$

C 5.03135115783400

C 6.16284669830394

C 7.13244859972177

C 6.38027654985478

C 5.15012726539090

C 4.15928863641216

C 2.73872869223412

C 1.93378305279698

C 0.6106160282306

C 0.03799962817384

C 0.78453640334502

C 2.12244862341604

H -0.98745075686263

H 1.62822678640880

H 6.41040013522686

H $\quad 6.74491236473838$

H 3.57551112826836

H 4.51951140266208

H 4.96150567637757

H 2.71241247860633

H 3.85273053430958

H 0.32510582037438

H $\quad 0.55667293475650$

H 0.02277667210809

H 1.62095486489069

H 2.39452841775279

H 8.05292641475273

H 7.47077512612540

7.82207114418087 .39973095245493 7.35991733506808 6.08290122132071 6.3330400232600 5.35560189382441 5.59040492558632 4.90166437832415 5.26262943643169 6.28139280318074 6.89360722187353 6.55517990158411 6.60068491333887 6.64856773603496 9.46092611097632 5.09977968649131 9.43449474505024 4.38897804783398 6.66143323497960 7.04410323521169 5.77547473668654 7.65167187952539 8.48143910494880 4.76481640029738 9.31003257813102 4.13819607276942 7.40683730734396 7.49656308695771

$-1.10898069228369$ $-0.62459334014992$ $-0.15582193879718$ $-0.37231079312965$ $-0.91298309147622$ $-1.26812708756082$ $-1.28376668599109$ $-2.21836962908614$ $-2.41850930560987$ $-1.64817845160775$ $-0.63934007169283$ $-0.45476478949354$ $-1.84523304849537$ $-6.24838758031011$ $-0.67788918724987$ $-0.07523300001220$ $-1.37658746075524$ $-1.63071973469209$ $-3.52942418304144$ 0.32246738903270 $-5.55149728496946$ $-0.00278975791370$ $-4.94157669813351$ $-3.19073150935792$ $-2.85745999379714$ $-2.84861540701107$ $-0.76545326015919$ 0.88681110693983

Bisallene2Cyclization_TS2

Free energy $=-732.4325599$ Hartree

$\begin{array}{lll}\text { C } & 2.10800253296847 & 8.48449974948042\end{array}$

$\begin{array}{lll}\text { C } 3.36808360264648 & 8.04868117981767\end{array}$

$\begin{array}{lll}\text { C } 4.08889413678451 & 7.05458965158818\end{array}$

$\begin{array}{lll}\text { C } 3.50048522529975 & 6.44437373857585\end{array}$

$\begin{array}{lll}\text { C } & 2.24088020800992 & 6.82684946073145\end{array}$

C $1.56233536796123 \quad 7.89000385829687$

C $3.80693643205926 \quad 8.46886348655148$

C $4.96380414535868 \quad 7.85124393801275$

C $6.06129645205965 \quad 8.39980198905100$

C $7.02408780453667 \quad 7.34159214679405$

$\begin{array}{lll}\text { C } 6.30807595348041 & 6.07110487595266\end{array}$

$\begin{array}{lll}\text { C } 5.13083579394734 & 6.33230404409063\end{array}$

$\begin{array}{lll}\text { C } & 4.15348332364425 & 5.44493567324693\end{array}$

$\begin{array}{lll}\text { C } & 2.77732434213010 & 5.63259428066712\end{array}$

C $1.82692356576468 \quad 4.97139729981892$

C $0.51602924846046 \quad 5.37623454051271$

C $0.07871405231368 \quad 6.42620590144063$

$\begin{array}{lll}\text { C } & 0.95042417107222 & 7.00419457467324\end{array}$

$\begin{array}{lll}\text { C } & 2.30402601137566 & 6.63043893595875\end{array}$

H $\quad-0.96006067363102 \quad 6.75899564906398$

$\begin{array}{lll}\text { H } & 1.77854297539367 & 6.33206881782229\end{array}$

$\begin{array}{llll}\mathrm{H} & 6.29088272234484 & 9.46515644302434\end{array}$

H $\quad 6.67432076750747 \quad 5.07692995669416$

H $3.28786282178257-9.28586601875036$

H $4.49036987411010 \quad 4.60282171082570$

$\begin{array}{lll}\mathrm{H} & 5.13735614803378 & 6.87163772612585\end{array}$

H $\quad 2.96573529270919 \quad 7.00142312407276$

H $4.04854641893700 \quad 5.67209304011346$

$\begin{array}{lll}\mathrm{H} & 0.58662116225748 & 7.76311210365987 \\ \mathrm{H} & 0.59795274433072 & 8.22956121315239\end{array}$

$\begin{array}{lll}\mathrm{H} & 0.0 .18931799823965 & 4.9062314561413\end{array}$

H $\quad 1.56544096292892 \quad 9.26406833188414$

H $2.18235402224029 \quad 4.19196352963053$

H $7.97998164684462 \quad 7.44994531484502$

H $7.28582974257657 \quad 7.40048623893250$

$-3.52088969540237$

$-3.00016236463914$

$-3.73178301590178$

$-4.85093464479899$

$-5.27766377481493$

$-4.63093048411259$

$-1.73491315378446$

$-1.11934728050944$

$-0.55215977382371$

$-0.10626335759202$

$-0.46477980266184$

$-1.07638480379498$

$-1.66810526819499$

$-1.45415159213370$

$-2.29575328935685$

$-2.31953371353534$

$-1.47501555614995$

$-0.56873583266901$

$-0.55070924060734$

$-1.51856813284265$

$-6.13319930054371$

$-0.50255671457147$

$-0.20825909889171$

$-1.22883489761716$

$-2.27643584033762$

$-3.50245415054780$

0.22976568849482

$-5.39391768256845$

0.12604067983464

$-5.01251784362849$

$-3.0063078546522$

$-2.98123599029270$

$-2.97375585120943$

$-0.64897165485095$

0.96512128870833

Bisallene2Cyclization_INT2

Free energy $=-732.4852565$ Hartree

$\begin{array}{ll}\text { C } & 2.11136199302389 \\ \text { C } 3.18867150556699\end{array}$

C 3.94788358971420

C 3.58877108671546

C 2.4810731966891

C 1.75496773294732

C 3.40509230922772

C 4.79820628701727

C 5.87079495281477

C 7.02404680582470

C 6.45554727482187

C 5.15723452701281

C 4.1599746336326

C 2.85140870932957

C 1.82771318035768

C 0.54340894028274

C 0.11683281137597

C 0.99657102276877

C 2.45208535996780

8.59433690687759 7.89073298705556 
H $\quad-0.93476525245059 \quad 6.56895074311812 \quad-1.56434813862132$

H 2.17219687465877

H 5.93358275245829

H 7.00927743032141

H 3.13364186847059

H 4.47222363193299

H 4.81103949096215

H 2.66169245176090

H 4.17661783465999

H 0.65813466047805

H 0.90155388377652

H -0.19282093938196

H 1.51894193517030

H 2.14448818364036

H 7.87532121776971

H 7.42029005668125

7.38056954674095 9.40579114140673 5.12809847906395 8.91903924950571 4.10049575870045 6.52865503123184 6.95819858530819 6.22864697099635 8.17710386879380 8.97786801014512 4.26598203431882 9.25008415188562 3.57055484127468 7.59502767408682 7.62912531099206

$-6.70279621427134$ $-0.75015312077616$ $-0.29677464494128$ $-1.08552112658985$ $-1.44405158569991$ $-3.41373570639820$ 0.28810807938700 $-5.77869165047752$ $-0.66995182252372$ $-5.26875274568202$ $-2.27553677950505$ $-2.88304310896830$ $-2.05245535643563$ $-1.04773276919733$ 0.64624334757200

\section{Product}

35

Free energy $=-732.5716079$ Hartree

C $-1.68289541271886 \quad 1.85992072095447$

C -1.62323870868233

C -2.61342745569796

C -3.62992738974632

C -3.65321765062392

C -2.69275168779742

C -4.58647699264757

C -3.78063365281216

C -2.83239777879437

C -0.80754897282489

C -0.91722772997792

C -1.89730566387871

C -2.75863177260297

C -0.50488572230406

C -0.73737095809799

C 0.32362804538512

C 1.63611340901869

C 1.88418109639758

C 0.82398454007933

H -0.25472793056247

H 2.46302226350000

H -1.89777747681926

H -5.46804622840468

H -4.41555540556944

H 1.01893665281673

H 2.90678502038966

H -0.06608417093219

H 0.11983019605253

H -1.98377864872113

H -3.53023765359166

H -3.33382436400760

H -1.75959140244579

H -4.41900941280261

H -3.1890603473314

H -4.95912963324387 1.2717655555091
1.5851583352910 2.53437415657087 3.1727778131355 2.82979999240676 2.65852536775708 2.10132146390765 1.06129343353070 1.46597186001116 2.0149145681125 3.0020293447906 3.4015186137800 0.36340113476844 $-0.89126789259295$ $-1.73953124637167$ $-1.34775560571103$ $-0.08881292535135$ 0.75827335623475 1.67348959822042 $-2.01417130003181$ 0.90195481770812 2.02875936274248 3.92011973794752 .74133629276079 0.24084849000264 0.68748956592574 2.71270943232056 3.43420272895608 4.14622896226012 0.08015457223435 $-1.21565253927581$ 1.67512483870826 2.91297234508394 3.67943091234402

43501927666462 $-0.11910018953366$ 0.79125916647997 0.47883855798316 $-0.73099613839232$ $-1.72605895404004$ 1.63972880162650 2.82654925918299 2.19983609596866 $-2.49152355467778$ $-3.74796867199872$ $-4.01853843233067$ $-3.02628250933633$ 0.25195239212707 0.83100602643914 1.14087035302986 0.88297479739549 0.33550156601699 0.02858052117589 $-4.54498489490283$ 1.12789600352267 2.75566075514044 1.43562862089605 $-0.96114587052940$ $-0.40265157335924$ 0.13966068016395 $-2.30785591892104$ 1.59021134513608 $-5.01680029159558$ $-3.23315870380272$ 2.15783815594177 1.03551100572411 3.61103510609586 3.27556328185360 1.80058348818469

\section{$\mathrm{X}=$-NH-}

\section{Bispropargy}

Free energy $=-748.4563053$ Hartre

$\begin{array}{lll}\text { C } & -0.77699850763547 & 1.81090385521240\end{array}$

$\begin{array}{lll}\text { C } & 0.32210580481931 & 2.43057121723514\end{array}$

$\begin{array}{lll}\text { C } & 0.09914841209448 & 3.45647252657427\end{array}$

C $-1.19788379899498 \quad 3.84731632995666$

C $-2.28758313377534 \quad 3.22548259327575$

$\begin{array}{lll}\text { C } & -2.07319129182904 & 2.20972492671377\end{array}$

C 1.6747

133230

C 2.84228379792189

C 4.27436059910948

N 4.69152201143275

C 4.29867108504642

C 2.89053640658189

C 1.74063222976418

C 0.36654688552381

C -0.36963026181452

C -1.6830811694133

C -2.28767549493422

C -1.57617438281495

C -0.25922376012580

H -3.30598776171158

H $\quad-3.29982564161799$

H 4.53533298987663

H 4.51416918885435

H $\quad-0.60057947258906$

H 0.10302658924746

H -2.91768302308955

H -2.24174093301801

H -1.36414014835505
2.20972492671377
2.07451579693627

1.88893072126045

1.91880058343164

3.10042870219535 40198072722251 4.79493120050347 5.08145813089764

4.51781978941309 4.82648748328128 5.98240640065130 6.82186382542115 6.52437622554628 6.23987591784238 3.54355742835753 1.03832373671137 4.42366265700508 1.02267210439418 3.61117653778490 1.72022172634454 4.15518544265470 4.64768112444404
0.52620469947063 $-0.08836440201148$ $-1.02238775158573$ $-1.34134749821849$ $-0.73001975702496$ 0.20503449909676 0.24794887291909 0.51880409669300 0.88451616843885 1.63067666378297 1.10077553462958 1.34495175790619 1.60976583158222 1.92970321188848 2.76454837990082 3.10890581786132 2.61293678929568 1.75415667019281 1.41389674967166 2.91059306104240 $-0.98954490734956$ 1.48994051357790 0.02132532617176 1.26014534325788 3.14338538228282 0.69230556026256 3.76387400227770 $-2.06377788056250$

H -2.05067462903488 H 0.30713974512095

H 4.94819621584382

H 0.95697361538542

H 4.8763206496668

H 4.36658445313119

7.71645568394595 7.18507477404573 5.16500270312678 3.95068226149123 1.84300201301077 3.0260868744827

Allenyne

Free energy $=-748.4682195$ Hartree

C -2.16456225648124

C -1.504746337016

C -2.18826728061940

C -3.49426052194097

C -4.12872013728568

C -3.46952094068966

C -0.11197058801778

C 1.08510746900954

C 2.55633926706972

N 3.08526560670160

C 2.74075712283745

C 2.08351116867443

C 1.43041078847196

C -0.02942849030291

C -0.59595397831211

C -1.96228937085837

C -2.77757075637221

C -2.22708803833027

C -0.86464694635471

H -3.84003449495260

H -5.13754788148633

H 2.97076057182012

H 3.10486776924702

H 1.99329820141716

H -1.67565832314105

H -0.42135701290970

H -4.01650005057644

H -2.87084848966134

H -3.96853721925608

H -2.38976186624287

H 0.05176971299235

H 2.93272138444274

H -1.63620428204127

H 3.02492320016497

0.96260539062828

2.00843674124476

3.20963327423966

3.37942045641830 


$\begin{array}{llcc}\text { C } & -3.39988152388587 & 3.18633937394847 & -1.26444413828476 \\ \text { C } & -3.21377350133878 & 1.92935058980676 & -1.84197740327063 \\ \text { C } & -0.57173120585688 & 0.41116566713019 & 0.39867154914782 \\ \text { C } & 0.72849193374210 & 0.73699330728783 & 0.50361393059071 \\ \text { C } & 1.42228028477248 & 2.05879064481600 & 0.23922162381948 \\ \text { N } & 2.84184741677833 & 1.70241592678281 & 0.33430832897850 \\ \text { C } & 2.96986662196980 & 0.39796543669287 & 0.75476365644199 \\ \text { C } & 1.76858604833331 & -0.23769159183266 & 0.84431215933470 \\ \text { C } & 1.41740158515930 & -1.63260966552982 & 0.89815173733182 \\ \text { C } & 0.11526342539550 & -2.00612763361334 & 0.82547020740334 \\ \text { C } & -0.26096365042698 & -3.39862137788324 & 0.66039559746742 \\ \text { C } & -1.53279040296044 & -3.77051710969126 & 0.40361452574375 \\ \text { C } & -2.59099596125077 & -2.77140577199286 & 0.28512817636736 \\ \text { C } & -2.34455903711570 & -1.46953280269554 & 0.48977689733868 \\ \text { C } & -0.99789013725112 & -0.96304317174640 & 0.93924051544594 \\ \text { H } & -3.59528144849010 & -3.10590833714229 & 0.01941301060342 \\ \text { H } & -4.10420860954863 & 3.89574111311219 & -1.70014293294508 \\ \text { H } & 1.15912313682792 & 2.80551511828399 & 1.00446180624596 \\ \text { H } & 3.96119546747090 & -0.02313168897246 & 0.92868820504989 \\ \text { H } & 2.20690438730894 & -2.38998263996818 & 0.89339863150871 \\ \text { H } & -1.18928798709069 & 2.91382560645468 & 1.31486697129238 \\ \text { H } & -1.13413264746652 & -0.79465042089994 & 2.03289141620780 \\ \text { H } & -2.81196756985966 & 4.51653453619354 & 0.32672884840443 \\ \text { H } & -3.15711345246517 & -0.74288522153304 & 0.43145768676783 \\ \text { H } & -3.76844909805579 & 1.64825507218862 & -2.73857990330147 \\ \text { H } & -1.78175220229308 & -4.82078096915524 & 0.24157258941962 \\ \text { H } & 0.53921683300049 & -4.14266652139015 & 0.69859335344229 \\ \text { H } & 1.16397922620483 & 2.49284771496396 & -0.74051285161886 \\ \text { H } & -2.13768594280499 & 0.06031093768488 & -1.75132339583473 \\ \text { H } & 3.50681416681511 & 2.39785524835325 & 0.64605345056278\end{array}$

\section{Bisallene}

34

Free energy $=-748.4769631$ Hartree

C $2.13195808526092 \quad 8.48783747467726$ $\begin{array}{lll}\text { C } 3.43775828910331 & 8.20048702132357\end{array}$ C $4.23635225941641 \quad 7.37218379058160$ $\begin{array}{lll}\text { C } 3.72027844261894 & 6.81616632621947\end{array}$ C 2.41346554032718 $\begin{array}{lll}\text { C } & 2.41346554032718 & 7.10157845041380\end{array}$ $\begin{array}{lll}\text { C } & 1.62454216745878 & 7.95349916793801\end{array}$ $\begin{array}{lll}\text { C } & 3.89360442128613 & 8.71334172706083\end{array}$ $\begin{array}{lll}\text { C } & 5.06546840815764 & 8.51932777194138\end{array}$ $\begin{array}{lll}\text { C } & 6.20235239927129 & 8.40203903072798\end{array}$ N $6.57670590502456 \quad 7.33186112653876$ C $6.03039003832494 \quad 6.05293521560860$ C $5.13384686203985 \quad 5.66357418467971$ C 4.14171439569247 C 2.71841471252705 C 1.72583179531252 C 0.37888767894736 C 0.00576747194847 C 0.99015001475409 C 2.33319812941665 H -1.04602447146465 H 2.01353540779576 H 6.94784493192493 H $\quad 6.41154245011437$ H 3.12720294797668 H 4.35446324438285 H 5.25497416963898 H 3.10491879808242 H 4.34591710975133 H 0.70321497057279 H 0.61634410954202

H -0.37908097489248

H $\quad 1.50333104964140$

H 2.01990884875031

Н 7.48956739129472 5.29512744246408 5.54975209856544 5.04233107362473 5.29035340962500 6.06419688781990 6.59243521917448 6.33553127427871 6.26864626505722 6.66320194638412 9.20200975418203 5.33667363661326 9.24168454279244 4.82096974773191 7.14671586399075 6.75513270656410 6.16368970182207 7.20982131959910 8.21515086350496 4.87589697767788 9.12500960998179 4.45226296522993 7.39351240560509

Bisallene2Cyclization_TS

34

Free energy $=-748.4562907$ Hartree

$\begin{array}{lll}\text { C } & 2.22489009174529 & 8.51134567244534 \\ \text { C } & 3.53197299195317 & 8.15902029659222\end{array}$ C 3.53197299195317

C 3.53197

C 4.25441342475111

C 3.66929832215371

C 1.65491636541649

C 4.05747480345677

C 5.16591594100796

C 6.17667632830572

N 6.57643847900335

C 5.95793994318325

C 5.09941541955713

C 4.12165885206842

C 2.72371181469234

C 1.70769916654976

C 0.37023679764136

C 0.00966698567397

C 1.00142098574852

7.25195121161957

6.68107804203340

7.02190383736048

7.95693822236571

8.63901553091963

8.14173476744163

8.37031484103398

7.32354868941637

6.12876508064126

6.11588814864529

5.45431467203951

5.61330629563271

4.95455302939668

5.18942852179979

6.09639471654805

6.75132570048292
$-3.71009369521606$ $-3.29107064949377$ $-4.09448449151775$ $-5.26180750200604$ $-5.66433791323069$ $-4.89090752280736$ $-1.98726241996468$ $-1.41732402171370$ $-0.76120010247054$ 0.05093219664779 $-0.05844995904566$ $-0.94352153457069$ $-1.72722206413848$ $-1.43167316940610$ $-2.27970557744776$ $-2.02177200381488$ $-0.92183851886522$ $-0.08456920173753$ $-0.33472472767279$ $-0.71025693683996$ $-6.58004092784401$ $-0.82239247390284$ 0.67694813374869 $-1.40535929285433$ $-2.69179628592958$ $-3.77132023420823$ 0.31195010949838 $-5.87422652369851$ 0.76813773632980 $-5.21679200114962$ $-2.68692568244069$ $-3.08438995177412$ $-3.15128988404601$ 0.47723809358293

$-3.71765030678702$ $-3.31594080453390$ $-4.11436476949120$ $-5.61463840018463$ $-4.85144535054518$ $-2.05681861367470$ $-1.45400444511850$ $-0.59769340733815$ 0.19744990192558 $-0.13086824430067$ $-1.15472993076936$ $-1.82941363678437$ $-1.47365643281108$ $-2.19910640502598$ $-1.90939280577025$ $-0.90306617611775$ $-0.17685741625049$ $-5.24749783118590$

C 2.34470777322458 H -1.04045642316305 H 1.91178009658133 H $\quad 6.74721089070711$

H 6.21735532897896

H 3.39469998500019

H 4.33934522536635

H 5.27901798290771

H 3.1231229795233

H 4.23802950814540

H $\quad 0.72458230788452$

H 0.65147084768369

H -0.40243386362834

H 1.65261665554431

H 1.98723945180112

H 7.46480247997699

6.51403815423893 6.2946380250969 6.56508001058479 9.29847951870718 5.24122381229959 9.28625318879958 4.95816115584697 7.00614135167193 7.02427994139527 5.97226693986266 7.45513301604903 8.25619233763832 4.67012073100205 9.20594922522180 4.26888083976272 7.33963747540768

$-0.45717067393363$ $-0.67727606566027$ $-6.49280925646820$ $-0.55276310832629$ 0.44635401947480 $-1.4704337480205$ $-2.77900916454124$ $-3.83183285943304$ 0.10762922868849 $-5.85248221564025$ 0.60964406014876 $-5.15879048701548$ $-2.47794248856286$ $-3.09845224014843$ $-3.0027825576555$ 0.67715663185719

Bisallene2Cyclization_INT

Free energy $=-748.5083244$ Hartree

C $2.29672054282674 \quad 8.67609238226220$

C $3.50512383928492 \quad 8.13114687809555$

C $4.12800649095541 \quad 7.11658094678287$

C $3.53356899523771-6.62322666197504$

C $2.31882377912550 \quad 7.14689295676336$

$\begin{array}{ll}\text { C } 1.71395498071451 & 8.18799186201512\end{array}$

$\begin{array}{lll}\text { C } 4.07295585009477 & 8.59871361659414\end{array}$

C $5.04345857472191 \quad 7.85531189335693$

C $6.08859496135544 \quad 8.36462863278744$

N $6.76565379978766 \quad 7.31627610368497$

$\begin{array}{lll}\text { C } & 6.19537460302927 & 6.13074581926241\end{array}$

C $5.10688000974132 \quad 6.40822109550680$

$\begin{array}{lll}\text { C } 4.11242280338415 & 5.45893852553271\end{array}$

C $2.71388743177004 \quad 5.63821624256819$

C $1.76799052064735 \quad 4.88200171990194$

$\begin{array}{lll}\text { C } & 0.40721943487153 & 5.06289126451969\end{array}$

C $-0.05038989083532 \quad 5.99485673777602$

$\begin{array}{lll}\text { C } & 0.86757104975530 & 6.74412091260398\end{array}$

C $2.23011894958524 \quad 6.57216462143442$

$\begin{array}{lll}\mathrm{H} & -1.11960609385270 & 6.13812341447284\end{array}$

$\begin{array}{lll}\mathrm{H} & 1.84921024454058 & 6.74675659946358\end{array}$

H $6.42065483386431 \quad 9.38750710215128$

H $\quad 6.57101637309291 \quad 5.18159219322836$

H $3.71825641323040 \quad 9.55356125481706$

H $4.42924156953941 \quad 4.59613540169988$

H $5.09883920732962 \quad 6.73599913814717$

H $2.94501556624450 \quad 7.14593545621149$

H $4.03483512771524 \quad 5.83988349936777$

$\begin{array}{lll}\mathrm{H} & 0.51035286716644 & 7.46287121669723\end{array}$

H $\quad 0.77915615830304 \quad 8.61965216351053$

$\begin{array}{lll}\text { H } & -0.30713984969151 & 4.47728593091029\end{array}$

$\begin{array}{lll}\mathrm{H} & 1.80152912006823 & 9.46391941840207\end{array}$

H $2.12882559194337 \quad 4.16936836085567$

$\begin{array}{lll}\mathrm{H} & 7.59918614445268 & 7.40908597664095\end{array}$

$-3.77816631490516$ $-3.28335356177821$ $-4.05224820408648$ $-5.20549490502248$ $-5.65410109561229$ 4.94099274549230 $-2.05162688993395$ $-1.27739149919481$ $-0.51632597021748$ 0.04086302275304 $-0.33426208847777$ $-1.13682940502233$ $-1.62019666564692$ $-1.38644462220371$ $-2.11798835952534$ $-1.91358470290616$ $-0.97426634049952$ $-0.23463963892596$ $-0.43552521841814$ $-0.8077580703338$ $-6.55373066461297$ $-0.35175378916996$ 0.03660555075716 $-1.65647830065342$ $-2.21072256672043$ $-3.73533123508688$ 0.15410387768684 $-5.77709141227655$ 0.50475440448748 $-5.30236623578310$ $-2.4932416895706$ $-3.20598292846272$ $-2.86345994745243$ 0.60373121230739

Bisallene2Cyclization_TS2

Free energy $=-748.4987546$ Hartree

$\begin{array}{lll}\text { C } & 2.33423562591667 & 8.65102898484639\end{array}$

C 3.58615991892587

C 4.17124605102317

C 3.50387102228769

C 2.2686362937757

C 1.69009004350480

C 4.15544817473326

C 5.12263161673348

C 6.10609483732647

N 6.67658797172504

C 6.08324395197177

C 5.1125715974506

C 4.11579200299686

C 2.72523382537896

C 1.75958591917726

C 0.40547673735800

C -0.02794861263230

C 0.90837145846165

C 2.26804068597097

H -1.09285449891203 
$\begin{array}{llll}\text { H } & 2.09719569792757 & 4.29131573081200 & -3.07070879246107\end{array}$

H $7.47061992510636 \quad 7.28192084875415 \quad 0.77080043681878$

Bisallene2Cyclization_INT2

34

Free energy $=-748.5504299$ Hartree

$\begin{array}{lll}\text { C } & 2.55331364805721 & 8.94685389172534\end{array}$

C 3.61083641036357

C 4.02484384154742

C 3.24565580000665

C 2.29104282894695

C 1.92239764584511

C 4.20997874770564

C 5.12219479018227

C 6.07139210157972

N 6.57527060237757

C 5.97177101765601

C 5.06437285923820

C 4.06834797130734

C 2.67490420247098

C 1.78342211798973

C 0.46714472046873

C 0.02092388592845

C 0.89922359504377

C 2.21821314238725

H -1.00805859995885

H 1.76436097510812

H 6.43916010809566

H 6.25117272880933

H 3.93611293980377

H 4.42257551696786

H 5.08240371733346

H 2.90859589548610

H 3.52723525679126

H 0.55631099400063

H 1.12534768126951

H $\quad-0.21816937391557$

H 2.25053492027892

H 2.12321154252535

H 7.32126576830185

6.87337386446591

6.29994255220940

6.98642054558951

8.34095177181658

8.85270973410552

8.04797528415411

8.37326717941036

7.20299663513219

6.12494911966039

6.61309767185390

5.95989400254849

5.81501921685595

4.87978319118816

4.80011866020221

5.66473758158801

6.59669730797562

6.66391033928447

5.60861977722677

6.53500725500435

9.33112435763588

5.11172342298163

9.86223267844476

4.96825109799937

6.91905041768783

7.38060760749184

5.29518245887571

7.27761991445424

8.85770796300456

4.07278283102778

9.94270159564145

4.21574589661622

7.13095863382318

Product

34

Free energy $=-748.5945664$ Hartree

C $-1.62397813236134 \quad 2.02026611277734$

C -1.55550423749415

C -2.54064468433571

C -3.58563610884611

C -3.67197670042926

C -2.69112056836325

C -4.42963374874680

N -4.08267443471376

C -2.68949439952264

C -0.66825472856931

C -0.76219354856310

C -1.8197988318928

C -2.76153312875949

C -0.44861553902171

C -0.72011959333772

C $\quad 0.26339848000012$

C 1.53743935744288

C 1.82591751924627

C 0.84015156802649

H -0.02581922214038

H 2.30277852182263

H -1.95747202202538

H -4.14281549315180

H -4.47345922100862

H 1.06054982437215

H 2.81533670353416

H 0.13509199694405

H 0.02479444825815

H $\quad-1.90068813320467$

H $\quad-3.58946673884102$

H $\quad-2.49868895692776$

H -1.71872246335828

H -4.3098006182444

1.3748480342235

1.64150576531394

2.56419956574873

3.20432168749740

2.9369713730425

2.69120730673054

1.47142879191880

1.14326428540990

1.76905486284371

2.39882171181872

3.30552549373790

3.56275923745974

0.46801455561247

$-0.87197242231346$

$-1.68726756529516$

$-1.17560464045395$

0.15043082531845

0.96676450977838

2.19046477303721

. 80788297414291

1.6725718038423

3.62102540316558

3.91419928208734

2.01015775879812

0.56314426167050

1.05625180611844

$-2.72071096515514$

3.78778823503483

4.2456534301750

0.06802373999227

$-1.26988817341007$

1.49008076062941

2.7482803669875

$-3.92878964958458$

18379926094865

$-3.57927280719116$

$-4.72503525885015$

37103886982882

.95675104027517

.10949470674721

$-31105343834048$

$-0.35790608444784$

0.13557627672175

0.47179255588998

$-1.38247749820511$

$-2.30717792128291$

. 71003050571631

2.24873440846968

.79566330645744

$-0.79621380338740$

$-0.24597552018577$

0.69346304556839

$-0.43712696912661$

$-6.21365234812664$

$-0.00114384388982$

19007235677125

1.79197869781362

$-2.62772239616718$

$-3.90680073135996$

$-0.24985162760792$

$-5.05403012414271$

0.53502538890694

$-5.49274872361268$

$-2.23219087231318$

5.5606601720460

$-3.04658273081151$

0.81273845469604

1.62245112603617

$-0.34220371366692$

0.57785878761221

0.31387964216245

$-0.89089357418826$

$-1.89025352803252$

1.55622987024407

2.2745625152721

1.99675901505287

$-2.6481968538656$

$-3.86811741154078$

4.12983728112584

$-3.16390619633015$

0.07647387787003

0.38116855192897

0.93441034431224

1.18411016053928

0.86136451603029

0.30966122502691

$-4.64591037174340$

1.63510572067382

2.6427090579331

2.09267608183832

$-1.10613252747838$

0.07330052920409

1.06570451605728

$-2.45297022188084$

1.19253244114423

$-5.10478444715863$

$-3.36820135307052$

2.11708842592134

0.1879349843272

3.26234250027580

1.33350884269141

\section{$\mathrm{X}=\mathbf{- O}-$}

Bispropargyl

33

Free energy $=-768.3083071$ Hartree

C $-0.27387049461917 \quad 4.67621412839313 \quad 3.23281501744627$

$\begin{array}{llll}\text { C } & 0.32179425186054 & 5.26518259503194 & 2.10422008603530\end{array}$

$\begin{array}{llrr}\text { C } & -0.45442968135850 & 6.08642577537323 & 1.26969627276165\end{array}$

C -1.77929010003992

C -2.35409828843717

C -1.60300895703732

C 1.71046393407147

C 2.87529402626792

C 4.26984646047528

O 4.57367921758913

C 4.24677687738991

C 2.84994611866250

C 1.70402759491407

C 0.35365180428883

C 0.11367477554999

C -1.19040200984056

C -2.26926883354857

C -2.03840290001894

C -0.73627192121696

H -3.37998806028865

H $\quad-3.28698465632187$

H 4.45632323791597

H 4.47048465391839

H -0.54573739902562

H 0.33006460626075

H -2.87285556260426

H -2.05408339528802

H -1.37242972620126

H $\quad-2.37121398694340$

H -0.00540100970861

H 4.95710873931328

H $\quad 0.96043726734440$

Н 4.91244741667629

4.94251989886935

5.01777095615977

4.7621818954967

4.36464137934801

3.05898680501906

2.02783263604275

2.06561547019058

2.26451176953295

2.60382880337864

3.57362675863112

3.93965349076359

3.34771491405958

2.38395323084154

2.01137987795007

6.05108866154256

3.64827652071800

1.08768157473779

4.46423003288405

1.26682742065656

4.02635693145338

1.91778289920515

.48486949360562

7.0138042182029

6.51250013795419

5.04462386509374

4.03991346051952

2.07320439908370

1.5976892396831

2.73922449316969

3.54572863192526

1.82327828026682

1.60633692062868

1.32947018608352

1.78074110390740

0.86741573920732

0.39525500358658

0.05184448098920

0.31550463117554

$-1.30398440548183$

$-1.62775888384415$

$-0.96888552744769$

0.01587414786797

0.34235723653853

3.01490579263271

$-1.22902262786982$

1.39660073396734 


$\begin{array}{clcc}\text { H } & -3.87554041327893 & -2.45493403663159 & 0.41259466532051 \\ \text { H } & -5.09841499762245 & 2.60501673016266 & -1.43456797826159 \\ \text { H } & 2.42413484368810 & 2.81992332698476 & -0.47490612050495 \\ \text { H } & 3.52373098910870 & 0.13100617952676 & 2.37256788570891 \\ \text { H } & 2.04201761729725 & -2.28713372669245 & 0.54263723926760 \\ \text { H } & -1.62451414847123 & 3.13062871493420 & 1.06311910768550 \\ \text { H } & -0.64248491774260 & -0.05559773153021 & 1.97403792709627 \\ \text { H } & -3.95822712881832 & 3.73642063684060 & 0.47048021957767 \\ \text { H } & -3.08679366300124 & -0.44862653948918 & 1.66038821350924 \\ \text { H } & -3.94317679243479 & 0.75998183923463 & -2.64494476994434 \\ \text { H } & -2.23306809207759 & -4.04520706854689 & -0.55203410006646 \\ \text { H } & 0.19864526429103 & -3.63186776746993 & -0.29044324730630 \\ \text { H } & 2.93534621172108 & 1.19414677989348 & -1.05726501507488 \\ \text { H } & -1.61693637707335 & 0.13292312293387 & -2.03780531957980\end{array}$

Allenyne2Cyclization_INT

33

Free energy $=-768.3739072$ Hartree

$\begin{array}{llll}\text { C } & -0.45880879645823 & -3.11823746959552 & 1.68367459703431\end{array}$

C $0.02922772960123-1.82617913029500$

C $-1.06364666297552-0.8952925407642$

$\begin{array}{lll}\text { C } & -2.06277764196947 & -1.65061846728626\end{array}$

$\begin{array}{lll}\text { C } & -2.33928662193761 & -2.93309655095177\end{array}$

C $-1.60060181260871 \quad-3.63612367838448$

C $1.34300864984444-1.50203524806932$

$\begin{array}{lll}\text { C } & 1.76593326710713 & -0.26544841398711\end{array}$

C $2.96709632345122 \quad 0.30113785052794$

$\begin{array}{lll}\text { O } & 2.90196009954848 & 1.49119564304899\end{array}$

C 1.51346486766430

C 0.75234604644755

C -0.56287479898265

C -1.59207373859322

C -2.30765243274254

C -3.27527742449240

C -3.55010620714817

C -2.85227435808337

C -1.87955889478965

H -3.09303053502929

H $\quad-4.30811232329386$

H 1.33825687512091

H 3.96916028639663

H 2.07957327754382

H -2.08166876624654

H -1.64052954237076

H -3.81736496226295

H -2.59236476360339

H $\quad-3.07684461122317$

H -1.95094519363659

H 0.16355735863186

H 1.29810073991136

.86606386606938

0.64372586272631

0.39042179934033

1.39049535465046

2.05255777802177

3.00382943323658

3.30481284056850

2.6479415447940

1.69485822964042

$-3.47889522512442$

4.04703487834018

2.71935245964633

$-0.05291817277922$

$-2.19097663871809$

1.81736957127605

0.6053529130855

3.51470560240125

$-1.10630575334874$

2.88582800498684

$-4.61543456660469$

$-3.69848625186776$

2.19147686019713

1.17041244138965

20340548375719

0.70614817603300

$-0.15214160099992$

0.12747141187059

1.18043062137138

1.18373756070803

0.56164249181926

0.30948698011509

$-0.33873123172909$

43390019941353

0.02354618797287

0.07198317643871

$-0.3336958384795$

0.67456015771418

0.35773659742929

0.97704241060503

$-1.98950034244428$

$-1.67129694077003$

0.44246572075627

$-1.23107330275821$

0.24188642726987

0.55243602167012

60371850878392

1.71831391049902

1.61481868357324

1.15416901898369

$-0.93714252098874$

$-3.03184137802587$

1.51041141763179

2.37032504980442

$-1.46059243934323$

$-2.46188055416628$

Bisallene

33

Free energy $=-768.3267632$ Hartree

C $1.97550713084395 \quad 5.01113048906061$

C $2.77794954543033 \quad 5.46455141053418$

C 2.18701029607393

C 0.82570488171162

C 0.04629701213259

C 0.62050761796244

C 4.22758948136094

C 5.18382697568411

C 6.20886589214413

O 6.93465764205089

C 6.38262588301174

C 5.12715969852554

C 3.89836583027150

C 3.31739108823162

C 4.05253442424553

C 3.46266322565699

C 2.14003782127887

C 1.41056228395556

C 1.99135873439685

H -1.01079029377438

H 1.67930109673411

H 7.13955327558961

H 6.61491188127106

H 3.22111612184913

H 4.54061169312250

H 5.08465695779540

H 2.80873979420622

H 4.04029067636715

H 0.36589372939269

H 0.38686418693071

H 0.01461910237352

H 1.40583284059892

H 2.43517447257418

.21258800894008

6.51267821536348

08360011386648

5.32442382500558

5.20095406129045

5.72060972247979

6.15097923616605

7.29762042422420

8.31256598696926

8.47050028966368

8.70516632015427

8.21146595610720

7.38884025927445

6.87210689650260

7.19289500148605

8.03108073989750

8.52453922394344

6.35246944441890

6.78764741569670

9.05758097949843

.58906802566449

9.27151863604640

4.56537114821833

7.14181989057790

6.56739912295218

6.22316705327537

7.09476229492488

8.30831888389529

4.98499193643466

14837057849822

4.43985740896885
$-2.47352930643388$

.41570414810097 $-0.38434096619136$ $-0.42904948516135$ $-1.50563686570449$ $-2.52522290474384$ $-1.48770602270506$ $-0.74148378135585$ $-0.03983980180372$ $-0.25567141224345$ $-0.99347530717246$ $-1.34766141679645$ $-1.76758508744094$ $-3.03138550485604$ $-3.90081502667545$ $-5.04924348691944$ $-5.36718361394095$ $-4.52494806199635$ $-3.35703755680112$ $-1.55146523092563$ $-6.26953113641133$ $-1.24990640212954$ 0.80504110736346 $-1.11594872618454$ $-2.32596540001416$ $-3.64376787386891$ 0.44066038797516 $-5.71047432100232$ 0.37176668071936 $-4.78441977840429$ $-3.36645847913780$ $-2.67673129362076$ $-3.28242177731550$

Free energy $=-768.3093249$ Hartree

C 1.93320093704909

C 2.77408984859337

C 2.21824952911984

C 0.87987949586676

C 0.09573787633915

C 0.61731709088952

C 4.1971331799102

C 5.14185128748256

6.28783549137872

7.01107065617076

C 6.30438902034691

C 5.05861717192900

C 3.88098917467015

C 3.33245915683372

C 4.03007021560870

C 3.4250932248841

C 2.13867469454364

C 1.45701304943192

C 2.03968749804946

H -0.92929421669991

H 1.66598397706642

H 6.85918750921146

H 6.71128010847654

H 3.22198397240939

H 4.5305896578702

H 5.04048403357743

H 2.84701584516555

H 3.96407538704799

H 0.45624798919450

H 0.46593940895971

H -0.00782113195387

H 1.48981239774914

H 2.35854946282776

4.97298245829754 5.5480487126957 6.47108517523037 6.85649912228842 6.34648125491658 5.37563240675681 5.3133145877521 6.08879291401142 6.20552332630427 7.3597070256950 8.28163408791187 8.02454158436674 8.47766100777251 8.05197293143127 7.14843035911495 6.63087944153030 7.03504961763656 7.97230574711644 8.46474673713561 6.6983712763199 6.62337285027439 9.19275096607783 5.46983469897695 9.06772745788711 4.62664765596784 6.84985172534016 6.88071003655305 5.91626438043335 7.57736393119799 8.31746677161129 4.9606254825439 9.15731708436619 4.25705318448545

$-2.28391179409098$ $-1.30095837761745$ $-0.39213478044706$ $-0.51359934682572$ . 54456778915421 2.4137301410054 $-1.37657240407435$ $-0.77477887545428$ $-0.10456105703918$ $-0.19819200377223$ $-0.9132083432743$ $-1.29441936563897$ $-1.80877675388365$ $-3.07676095854089$ $-3.9057164984564$ $-5.04790040297429$ $-5.40886631293344$ $-4.62436920375928$ $-3.46488313350112$ $-1.67877101103878$ $-6.30199625065311$ $-1.13031660304119$ 0.57858565807783 1.16263756677847 $-2.16248089037857$ $-3.62373397454942$ 0.39987746929185 $-5.67266770812285$ 0.18755303909233 $-4.92502380785969$ $-3.20537786728039$ - 82218766873204 


$\begin{array}{llll}\text { C } & 3.94899357542230 & 7.05492148644204 & -3.85134668814853 \\ \text { C } & 3.36753280277794 & 6.56953579353488 & -5.01689794442403 \\ \text { C } & 2.10918314956654 & 7.01798150060403 & -5.42286637084484 \\ \text { C } & 1.44493431101263 & 7.98923277489061 & -4.66535589582141 \\ \text { C } & 2.00299858660249 & 8.45546503193719 & -3.48219733099721 \\ \text { H } & -0.79957672818113 & 6.87805846911566 & -1.75279402166027 \\ \text { H } & 1.65036846673753 & 6.61527970748319 & -6.32736055214022 \\ \text { H } & 6.41383618356449 & 9.35152537999466 & -0.62587646588057 \\ \text { H } & 7.02406828146159 & 5.32488452283269 & -0.10347467107859 \\ \text { H } & 3.22068964438434 & 9.11625060695674 & -1.17919314581100 \\ \text { H } & 4.55628138881193 & 4.41189492686589 & -1.95300135335629 \\ \text { H } & 4.94545198670907 & 6.72330968010610 & -3.56061601224960 \\ \text { H } & 2.97577591864827 & 7.02928464644086 & 0.31692054028151 \\ \text { H } & 3.90583063699968 & 5.84069383080631 & -5.62599528362205 \\ \text { H } & 0.66452844148730 & 7.91589307892785 & -0.04122257530072 \\ \text { H } & 0.47955353021618 & 8.37301587096043 & -5.00087711372578 \\ \text { H } & -0.02309714920773 & 4.87372797109681 & -3.01989782039767 \\ \text { H } & 1.45440567494223 & 9.16999710716803 & -2.86326219662790 \\ \text { H } & 2.27534067907814 & 4.01735003869317 & -2.71884344143058\end{array}$

Bisallene2Cyclization_INT2

33

Free energy $=-768.3952512$ Hartree
C 1.92999360242455
C 2.92338254695268
C 2.47405593562234
C 1.01950775953535
C 0.17631610351073
C 0.63845991378347
C 4.23898727829584
C 5.20069088934164
C 6.51887030753709
O 6.96956797627047
C 5.94070125539547
C 4.82213466923951
C 3.41630477755952
C 3.16380626208477
C 3.79363275220474
C 3.36932650350335
C 2.32239018192813
C 1.72923214473767
C 2.15119719274174
H -0.87295041889266
H 1.96078623458214
H 6.16673364628356
H 7.23680411017772
H 3.13020158307407
H 4.57886292201859
H 4.60161177827292
H 2.62317455048713
H 3.85088083720686
H 0.64682616585482
H 0.92245945386148
H -0.07350238758413
H 1.65571069366879
4.45167901953810
5.43271156013856
$-1.60007710416985$
$\begin{array}{ll}7.11029905584887 & -1.14166044097070\end{array}$
$6.15519198590862 \quad-1.55627183069715$
$4.78715930543770 \quad-1.78908681848716$
$5.11992082342751-1.11944949803064$
$6.18516061681339-0.89515305634569$
$\begin{array}{ll}6.20457506486778 & -0.53289852288141\end{array}$
$7.47622761371248 \quad-0.47488535631144$
$8.29518492708166 \quad-0.81898770169854$
$7.56854748949194 \quad-1.09192588901912$
$\begin{array}{ll}7.92282752549069 & -1.51940782038205\end{array}$
$7.93839524594473 \quad-3.02198118244094$
$7.03438870264958-3.88579591423969$
$6.90868609136068 \quad-5.20843119761806$
$\begin{array}{ll}7.69258615835770 & -5.69163377240040\end{array}$
$8.64112694508579 \quad-4.85947644918594$
$8.75975091852092 \quad-3.53578398449922$
$6.39477310635993 \quad-1.73888448331707$
$\begin{array}{ll}7.55625453457497 & -6.71245621340396\end{array}$
$9.35817914882658 \quad-0.81166881207730$
$\begin{array}{ll}5.43013632303895 & -0.28305065244894\end{array}$
$8.90300565742965-1.11011064074968$
$4.09859221848859-1.30378410177123$
$6.40179239717031-3.51511079016821$
$\begin{array}{ll}6.98233728345492 & 0.22928751096725\end{array}$
$6.18259850475638 \quad-5.86431108650938$
$8.12207117201842 \quad-0.96109051642728$
$9.27325367074920 \quad-5.23443323582510$
$\begin{array}{ll}4.04166974902520 & -2.14317295801416\end{array}$
$9.47588720128151 \quad-2.87666178001413$

$3.43923302834047-1.81422066760763$

\section{Product}

33

Free energy $=-768.4530578$ Hartree

$\begin{array}{llll}\text { C } & -0.19406211816071 & -2.94908830315652 & 2.10056409170397 \\ \text { C } & 0.18117264412191 & -1.94764596238575 & 1.19707198216940 \\ \text { C } & -0.80632479915208 & -1.30386650056956 & 0.44009571618299 \\ \text { C } & -2.14684607036826 & -1.65914602585893 & 0.58733136621436 \\ \text { C } & -2.51349239214139 & -2.65239532289641 & 1.49833925430371 \\ \text { C } & -1.53670996158078 & -3.29903887034986 & 2.25447303121234 \\ \text { C } & 1.61330608935611 & -1.57776507956907 & 1.00617160072870 \\ \text { C } & 2.18949116068718 & -0.43553822383392 & 1.65997381061564 \\ \text { C } & 3.54146728669484 & -0.06963614466099 & 1.37421695709439 \\ \text { C } & 4.31590530825875 & -0.85830304734330 & 0.47261761827291 \\ \text { C } & 3.75224407140691 & -1.96728767129109 & -0.09556997863915 \\ \text { C } & 2.40525469739060 & -2.31753388198705 & 0.16235016535019 \\ \text { C } & 1.45649376595580 & 0.35041552254730 & 2.59402124164721 \\ \text { C } & 2.03956292668185 & 1.42167293466427 & 3.23105398570005 \\ \text { C } & 3.37150842634964 & 1.79589231529785 & 2.93072263315471 \\ \text { C } & 4.09634468515173 & 1.07338897710543 & 2.01262220386935 \\ \text { C } & 2.08507569206534 & -3.54856900999967 & -0.65347721894216 \\ \text { O } & 3.18456312904025 & -3.68850758217137 & -1.54231219255277 \\ \text { C } & 4.31677714413890 & -2.99999145120187 & -1.03594502602739 \\ \text { H } & 1.46611168890157 & 1.99625919249397 & 3.96007862307131 \\ \text { H } & -3.56057697659830 & -2.94006258506817 & 1.61320701587892 \\ \text { H } & 1.16310165186615 & -3.45291588307315 & -1.24577000227754 \\ \text { H } & 5.34096874883617 & -0.55787824181622 & 0.24151126550539 \\ \text { H } & -0.50919263502247 & -0.52587653429286 & -0.26671975783785 \\ \text { H } & -2.90604657671349 & -1.15853234103963 & -0.01478364293635 \\ \text { H } & 0.42651384091774 & 0.07141896444286 & 2.82331084802447 \\ \text { H } & -1.82399244956907 & -4.08183265202004 & 2.95798891539005 \\ \text { H } & 3.82343461762641 & 2.65150061635315 & 3.43465288772869 \\ \text { H } & 5.11306958281090 & 1.37185514108133 & 1.74636183422424 \\ \text { H } & 1.96680088151411 & -4.43511206309786 & -0.00003745894052\end{array}$

H $\quad 0.57740404298747 \quad-3.45312358624801$

$\begin{array}{ll}0.57740404298747 & -3.45312358624801\end{array}$

H $\quad 4.99102692434573 \quad-3.68857713054217$

.68499477253445

1.87338944701005

$-0.48974909541363$

$\mathbf{X}=\mathbf{- S}-$

Bispropargyl

Free energy $=-1091.241559$ Hartree

C $3.27053128653235 \quad 2.22390845378497$

C $2.81349778778955 \quad 2.81652630075026$

C $1.59907223950440 \quad 2.37626176338998$

C $0.87278478074973 \quad 1.35832216334593$

C $1.34103171684069 \quad 0.76735760179919$

$\begin{array}{lll}\text { C } 2.53918907311197 & 1.20764150254185\end{array}$

C $3.56616510673128 \quad 3.90184607143612$

C $4.18564911342279 \quad 4.85826517186750$

C $4.81789601145920 \quad 6.11953862973193$

$\begin{array}{lll}\text { S } & 6.38878613525433 & 6.56608311763216\end{array}$

C $5.89736744133716 \quad 6.11571285700182$

C $4.50212986460860 \quad 6.39318668887427$

C $3.30557326888836 \quad 6.47033468002992$

C $1.86974146603457 \quad 6.44610132666639$

C $1.11485290171675 \quad 7.63037711582749$

$\begin{array}{lll}\text { C } & -0.27550153998974 & 7.57338744636189\end{array}$

C $-0.92677092360666 \quad 6.34112585478289$

C $-0.18533636501590 \quad 5.15963518082419$

$\begin{array}{lll}\text { C } & 1.20570643632856 & 5.20796340824226\end{array}$

$\begin{array}{lll}\mathrm{H} & -2.01753481617883 & 6.30130441562473\end{array}$

$\mathrm{H} \quad 0.76258673280544 \quad-0.02118166846060$

H $\quad 6.59392259794802 \quad 6.66636485394549$

$\begin{array}{lll}\mathrm{H} & 1.21940808304186 & 2.85208656458416\end{array}$

$\begin{array}{lll}\mathrm{H} & -0.06425477042787 & 1.01393287928003\end{array}$

H $2.90755368291986 \quad 0.75459474430457$

H $\quad 1.79182327504679 \quad 4.28982415618688$

H $\quad-0.85758643651763 \quad 8.49572505248320$

$\mathrm{H} \quad-0.69543740993780 \quad 4.19613483805573$

$\begin{array}{lll}\text { H } & 1.62955454625391 & 8.58945629691008\end{array}$

$\begin{array}{lll}\text { H } & 4.19110429229929 & 2.59852405410388\end{array}$

H $5.08428689363704 \quad 6.11202888933849$

$\begin{array}{lll}\mathrm{H} & 6.08230096567690 & 5.04027487378594\end{array}$

$\mathrm{H} \quad 4.09219156173506 \quad 6.92833171496638$

2.66090778771221 1.47083708214165 0.91595952278015 1.53419145955857 2.70881638776204 3.27356952601463 0.88771989899157 0.46535587132385 0.06109612493941 0.91680986534658 2.65214310864999 2.99620060461297 3.19224721658018 3.32791092323201 3.31896243377087 3.36981282895821 3.42736571871723 3.45506144327856 3.41451887745900 3.45547646318938 3.19349192780882 3.29740184824868 0.01108542663149 1.0923069221674 4.19522731593988 3.44187844480690 3.35649573217218 3.51074005400793 3.25847297836455 3.11055622175934 $-1.00619019187951$ 2.79330393508668 0.23109323986652

Allenyne

33

Free energy $=-1091.251797$ Hartree

C $-1.97721258575186 \quad 1.03874523444055$

$\begin{array}{lll}\text { C } & -1.28524570674450 & 1.90145744224112\end{array}$

$\begin{array}{lll}\text { C } & -1.99255969193137 & 2.94367619383359\end{array}$

C $-3.34034444792073 \quad 3.15880128744038$

C $-4.00365184340834 \quad 2.32447435605142$

$\begin{array}{lll}\text { C } & -3.32290646505947 & 1.25305586935609\end{array}$

$\begin{array}{lll}\text { C } & 0.17265160076436 & 1.77218360301441\end{array}$

$\begin{array}{lll}\text { C } & 0.99451038382772 & 1.00903911795048\end{array}$

C $1.82751386449758 \quad 0.31835543515498$

$\begin{array}{lll}\text { S } & 2.42360289292124 & -1.34764576707345\end{array}$

C $1.66995217626614 \quad-1.64185645738801$

$\begin{array}{lll}\text { C } & 0.21529709854867 & -1.80156459702665\end{array}$

$\begin{array}{lll}\text { C } & -0.99035135480537 & -1.94282876845717\end{array}$

$\begin{array}{lll}\text { C } & -2.41215304364258 & -2.16986540181770\end{array}$

$\begin{array}{lll}\text { C } & -3.23883533167327 & -1.33287873936451\end{array}$

C $-4.59216859370879 \quad-1.62997766901812$

C $\quad-5.14167630175198 \quad-2.74230523490476$

C $-4.33882056163283 \quad-3.54736912783826$

C $-2.98179326951282 \quad-3.26618922509524$

$\begin{array}{lll}\mathrm{H} & -5.04512829788511 & 2.52197755216937\end{array}$

H $-6.19449686349628-2.99440466279546$

H $2.16140872081459 \quad-2.53920244511429$

H $2.25442142166435 \quad 0.77933404927735$

$\mathrm{H} \quad 0.59422425975210 \quad 2.41919856132463$

H $-2.33909091508591 \quad-3.90551330878027$

$\begin{array}{rrr}\mathrm{H} & -2.33909091508591 & -3.90551330878027 \\ \mathrm{H} & -1.43665078601248 & 0.20837851295027\end{array}$

$\begin{array}{rrr}\mathrm{H} & -1.43665078601248 & 0.20837851295027 \\ \mathrm{H} & -4.77100456058871 & -4.40445625014294\end{array}$

H $-3.84699356469362 \quad 0.58257432928136$

H $-5.22595807940013 \quad-0.98729477927519$

H $\quad-3.87563101718700 \quad 3.98287426084503$

H $-1.47175646723303 \quad 3.61629201554936$

H $1.95471693183266-0.79487394394283$

H $\quad-2.80935860176324 \quad-0.45793044284554$

$-1.28331465966329$ $-0.41803533054162$ 0.20432260883843 $-0.08082205279846$ $-0.98022402697490$

$-1.5653549525110$ $-0.19580970591901$ $-0.89418784907908$ $-1.63712532914210$ $-1.38894432139959$ 0.26401368703730 0.26282234192159 0.2918075109327 0.36376955865146 1.13413177657465 1.27258783758365 0.63272053665391 $-0.17697555741557$ $-0.3076718890353$ $-1.24333902731655$ 0.77684780517155 0.66326596131180 $-2.53400560860290$ 0.58335842116761 $-0.91342162001615$ $-1.74248967488465$ $-0.69564680595208$ $-2.24795635882765$ 1.88530347771207 0.39385683781197 0.88931068543973 0.90682314079595 1.6236495824755

Allenyne2Cyclization_TS

Free energy $=-1091.21302$ Hartree

$\begin{array}{lll}\text { C } & -1.98477202803693 & 0.71331488742667\end{array}$

C $-1.32228484971382 \quad 1.68490368958259$

C $-2.11001592496308 \quad 2.59064711241562$

C $\quad-3.48787946650108 \quad 2.47298297878264$

$\begin{array}{lll}\text { C } & -4.12557346092986 & 1.46700791788440\end{array}$

C $-3.37965196378950 \quad 0.61949825239133$

$-1.58185156511780$ $-0.8003583041563$ $-0.04224304931782$ $-0.01542748129740$ $-0.76646998961448$ $-1.57369915203033$ 
C $\quad 0.10977613209784$

C 0.86238197099994

C 2.02496384693308

S 2.79677284333275

C 1.56743296246377

C 0.23262017199306

C -0.98462361138312

C -2.33664749970856

C -3.06785222209642

C -4.38573063336187

C -5.00696976183807

C -4.30740230437021

C -2.98639310739782

H $\quad-5.21186195622357$

H -6.03169031163998

H $\quad 1.50209768597603$

H 2.50180212570658

H 0.59354557391605

H $\quad-2.43639067919199$

H -1.39162638104900

H $\quad-4.79617622309420$

H $\quad-3.88326561086818$

H -4.93951655397213

H -4.08687835051857

H -1.60806366509193

Н 1.89989766887520

H $\quad-2.58551741655444$

68581491541590 0.55309153348185 0.14593809851711 $-1.33377894908344$ $-1.45241067909513$ $-0.94344558697075$

$-1.23451153014903$ $-1.61596612345770$ $-1.03950230426449$ $-1.42013555326812$ $-2.35727496904122$ 2.89931879044820 $-2.53746986417080$ 1.36033259381556 $-2.67254355029920$ .48791184694198 0.67434431385226 2.55808236361400 $-2.9649762709107$ 0.06375284678707 $-3.61354727124107$ $-0.14328198321698$ $-0.96873877399012$ 3.15299678619713 3.36124561586813 $-0.80531192381392$ $-0.28956493566938$

Allenyne2Cyclization_INT 33 Free energy $=-1091.304703$ Hartree

C -2.06741577743681

C -1.16379386006793

C -1.78196305375752

C -3.12057444684468

C -4.00863236350482

C -3.52825363064469

C 0.17642838935434

C 0.75397920364173

C 2.06579475003991

S 2.42930259217605

C 0.62530553550318

C -0.14956405976021

C -1.48917150275153

C -2.45913456398372

C -3.26070626601556

C -4.2343999958021

C -4.42313049786017

C -3.62840247873295

C -2.65230972729765

H -5.08365685115058

H $\quad-5.18263004393755$

H $\quad 0.33185426424721$

H 2.89288683428105

H 0.82922044627205

H -2.0487266466614

H -2.03819336008832

H -3.77908315865998

H $\quad-4.21476898353559$

H -4.84732539034940

H -3.56138307568635

H -1.12597747508566

H 0.48652491380380

H -3.1170337197041

0.75876103658978 1.96607690764373

3.2856023079365

3.44423334055122

2.28645900644322

1.03801209264042

1.79036169054534

0.45924862669556

0.11639814274089 $-1.58148989897325$

$-1.92599299232642$

$-0.6707212880612$

$-0.52353010127853$

$-1.54202917535482$

$-1.21290325456559$

08963605793610

$-3.32013413576478$

$-3.66215973956205$

2.77887576772438

2.45270716642698

$-4.01439153320005$

2.78737547247851

0.81451703127533

2.66442780202594

$-3.03800776938986$

0.55390978370004

$-4.61897200894006$

0.18996028493711

$-1.81072168107126$

4.44058165078139

4.15860016985229

$-19406878042328$

$-0.24474138373559$

.01091319103408 $-0.80912531182433$ 0.76359605971172 $-0.69258927053684$ $-0.66273895082576$ $-0.76297956289431$ $-0.71757657909221$ $-61133372815910$ $-0.57711159936209$ $-0.26407744920038$ 0.03838771384229 $-0.34267141511953$ $-0.39124953913919$ 0.09109323001152 1.19695662457543 1.66322558981547 1.03006946496262 $-0.06284486586800$ $-0.53168111793953$ $-0.56567415418640$ 1.39160208652308 $-0.57030105768598$ $-0.71361870213309$ $-0.65438362361533$

.40412069054094 $-2.10599406193111$ $-0.56439971863865$ $-0.77964714909923$ 2.52183539071258 $-0.62200839812062$ $-0.73509405249682$ 1.09636369826754 1.68089845044469

Bisallene

33

Free energy $=-1091.256763$ Hartree

C 1.94021698800799

C 3.25544300374268

C 3.96682579707301

C 3.36382386531120

C 2.05274731356573

C 1.34811215654858

C 3.85333909318239

C 5.11399635977122

C 6.38300322698264

S 7.34843776614580

C 6.25369019299875

C 5.23572619654897

C 4.27493878499576

C 2.82642157450867

C 2.04072840176997

C 0.69264445085029

C 0.10324889819283

C 0.86053563344662

C 2.21580171960291

H -0.94657953148092

H 1.58256506919232

H 6.99674891796838

8.52304571814535

8.16231401771808

7.33613876772396

6.86329591639172

7.23260460272232

8.07547570784626

8.64333953201948

8.52127738744132

8.53691320866348

7.40501556094189

6.00508781724821

5.63545633730283

5.15144933305235

5.44283119736716

5.00949270541659

5.34458370414698

6.10619046547719

6.50684701361072

6.18357594737044

6.39683427536740

6.86068909741778

9.38591071581612

$-3.35918291831066$

$-3.03744552815505$

$-3.92245416389361$

$-5.08309765634500$

$-5.39781201049577$

$-4.54006286004168$

$-1.77709211249745$

$-1.40688455636299$

$-1.07895060306104$

$-0.10273826535624$

0.08286013266871

$-0.65909535634715$

$-1.42671087070756$

$-1.39178600382716$

$-2.47193941742663$

$-2.55660141623355$

$-1.54769524588206$

$-0.44514217388924$

$-0.36699073970439$

$-1.62351587626141$

$-6.30979684073921$

$-1.40037474445669$

H 6.54187477847336

H 3.16665163177530

H 4.59340442890034

H 4.98920337414693

H 2.81561707708097

H 3.91969366102163

H $\quad 0.38941403902826$

H 0.33444173295303

H 0.1038380341385

H 1.37322032437442

H 2.50761803918141

5.38964931688314 9.14704152544324 4.49238790042395 7.04911569965212 6.51587962389699 6.20820610442916 7.08523099547316 8.39113593179903 5.02357575062323 9.15287793150686

4.4371851906615

Bisallene2Cyclization_TS1

Free energy $=-1091.231774$ Hartree

C $2.02918130493910 \quad 8.44578670091005$

C $3.32389923992316 \quad 8.01690713734478$

$\begin{array}{lll}\text { C } 4.00050482044041 & 7.11279939001291\end{array}$

C $3.37235358438415 \quad 6.60720653706501$

$\begin{array}{lll}\text { C } 2.08545051631667 & 7.02758247638832\end{array}$

C $1.42617011821984 \quad 7.96939381718880$

C $3.89514578055506 \quad 8.45024179794424$

C $5.09274298137440 \quad 8.03894469746196$

C $6.30065592781726 \quad 8.45241671746938$

$\begin{array}{lll}\text { S } 7.37008238561875 & 7.47187659316396\end{array}$

$\begin{array}{lll}\text { C } & 6.27616012597311 & 6.08701659248177\end{array}$

$\begin{array}{lll}\text { C } 5.17708138236797 & 6.08708496502957\end{array}$

C $4.22425814934979 \quad 5.33085876858658$

C $2.80124148054655-5.56304540250403$

C $1.95561590309783 \quad 4.97594030653340$

C $0.63621951260597 \quad 5.36810432194134$

$\begin{array}{lll}\text { C } & 0.11323638672734 & 6.34266771771841\end{array}$

$\begin{array}{lll}\text { C } & 0.89954298573087 & 6.86201462692719\end{array}$

C $2.24099357927350 \quad 6.48383464130146$

$\begin{array}{lll}\mathrm{H} & -0.91459256471511 & 6.68818365730682\end{array}$

$\begin{array}{lll}\mathrm{H} & 1.59539753241698 & 6.62653018346465\end{array}$

H $\quad 6.68871920225725 \quad 9.42461215204564$

H $\quad 6.53123686889932 \quad 5.23699985827128$

H $3.24935952855677 \quad 9.06086270071398$

$\mathrm{H} \quad 4.56149989047303 \quad 4.64319248823585$

$\mathrm{H} \quad 5.01378241461378 \quad 6.80548369051286$

H $2.86779658354466 \quad 6.89708920262640$

H $3.89211883341862 \quad 5.88999281212202$

$\begin{array}{lll}\mathrm{H} & 0.47472911033115 & 7.58361201975804\end{array}$

$\begin{array}{lll}\mathrm{H} & 0.43499345829909 & 8.32747248620850\end{array}$

H $\quad 0.00754371767834 \quad 4.94377715157024$

H $1.49540637297860 \quad 9.14150132698778$

Н $\quad 2.37887088598579 \quad 4.25762706220280$

$-3.45720509217526$

$-3.08769677170927$

$-3.93360685996578$

$-5.06962872541789$

- 41086143072094

4.61293481960742

$-1.83307630507483$

$-1.31513935452680$

$-0.94014971882444$

06468583083080

$-0.00594672640228$ 
C 3.23452342443308

C 3.89220030859876

C 3.29959359488188

C 2.07058887432510

C 1.44422245275297

C 3.70290119083646

C 4.96409638510604

C 6.04601915429116

S 7.38107585126821

C 6.47828108212557

C 5.21950887713151

C 4.24641331024502

C 2.87458919825951

C 1.96475405454876

C 0.69821780324548

C 0.22957712812985

C 1.02881858730746

C 2.36891068761126

H -0.77486873457030

H 1.60633521148768

H 6.14305771177780

H 6.90561834717359

H 3.22939902026306

H 4.59709143061304

H 4.86419245086550

H 2.94938558702063

H 3.80482145030083

H 0.64100154237060

H 0.50150375153703

H 0.04236001953586

H 1.49532172408285

H 2.33124316988432

Bisallene2Cyclization_INT2

33

Free energy $=-1091.330377$ Hartree

C $2.22967083989145 \quad 8.83705138462264$

C 3.12698867912360

C 3.69591497906581

C 3.34598183312890

C 2.43465777705337

C 1.88251352874547

C 3.37461026583376

C 4.80469150392052

C 5.82375183456735

S 7.32554064523213

C 6.56777994789491

C 5.22743095707329

C 4.25484124422695

C 2.92745869259900

C 1.92371086843694

C 0.61687761567171

C 0.14818224373989

C 0.99934030184887

C 2.45245765543033

H -0.90386659283672

H 2.14667883064575

H 5.77834451443013

H 7.13939275927196

H 3.09431784985945

H 4.60318077344199

H 4.40778716497769

H 2.57387135866051

H 3.78734976904993

H 0.63606536030778

H 1.17856655149016

H -0.10860785049615

H 1.78094199283465

7.89589417763356

6.95723603868277

6.94667963793933

7.87726289303065

8.83317709439000

7.86063937639013

7.55813095580886

8.44962537994799

7.67905756005231

6.12016283786589

6.19434727143607

5.10946164346992

5.38214521074880

4.34957712785202

4.65113509995029

6.03522953196188

7.03525410299847

6.81005932291921

6.24716963388872

7.85750952525892

9.52956961038225

5.22103666093789

84409678631975

4.07702621033589

6.22323989694353

6.98056025298675

6.20617866704204

8.06575372361104

.57139108479856

3.86009985835493

9.57473944066236

3.31597600077660

$-3.57345749591930$

$-3.05258670470599$

$-3.92321334985163$

$-5.27390239816400$

$-5.77419137157154$

$-4.92426778143006$

$-1.54952316318943$

$-1.15625555380249$

$-0.99085198294752$

$-0.58630990767513$

.64422119792837

0.94263580664696

$-1.06594118885002$

$-1.10780851419318$

. 32920441516631

$-1.47754246525814$

$-1.43065201246873$

$-1.17201143755755$

$-0.86403894843835$

$-1.63153951548675$

$-6.82667186039109$

$-1.11688718859043$

$-0.42106000060969$

$-1.14493076755593$

$-1.15444885919056$

$-3.54199134116779$

0.22558947581781

$-5.94183473762782$

$-1.13893437623411$

$-5.31169601956225$

$-1.67234903684655$

$-2.90515908851790$

$-1.41909098827224$

\section{Product}

33

Free energy $=-1091.382003$ Hartree

C $-2.04428901143906 \quad 1.56027934452860$

C $-1.14684000738343 \quad 2.03505107095689$

$\begin{array}{lll}\text { C } & -1.65950735786914 & 2.62094010550874\end{array}$

$\begin{array}{lll}\text { C } & -3.03765897629042 & 2.70656784467014\end{array}$

C $-3.91924467983896 \quad 221992254220071$

$\begin{array}{lll}\text { C } & -3.41969661584082 & 1.65249766470409\end{array}$

C 0.31339916685925

C 1.06905070420003

C 2.43739749901588

C 3.03210586575130

C 2.28900639051831

C 0.92980745119186

C 3.16469903151150

C 2.56778062033403

C 1.20700870870182

C 0.47906780944758

C 0.31340071656191

841872

0.97631901841341

0.70423574895861

1.26636422252828

2.05873842764254

2.36326309320087

$-0.18941951402765$

$-0.55929543474045$

0.30798498725013

3.24795215575905

S 1.15483602512457

C 2.71766579092726

H $\quad 0.72011224487447$

H $\quad-4.99558391595620$

H -0.77196834906634

H 4.06220198526306

H -1.63892365471745

H -4.10061581359768

H -0.57393260489018

H $\quad-3.43196428191441$

H 3.14034927137713

H 4.20590339187679

H 0.54837350649368

H $\quad-0.97095876333784$

H 3.45604620802981

H 3.13976864408169

2.75690387365799 .63366284772794 $-1.0754311921871$ 2.27645351170860 3.15402414153039 0.99260183039516

1.09694924682850 1.26702973041982 0.47422890969013 3.1503412335717 $-1.49770164998608$ $-0.40790960497597$ 4.30443275889009 2.99188193266377 2.01583330200748

3.64593285613879

\section{$\mathrm{X}=$-SO2-}

Bispropargyl

Free energy $=-1241.442359$ Hartree

C 3.8730651101672

C 3.2517710057316

C 2.20025957792783

C 1.76936926918332

C 2.3821744194485

C 3.43493229000707

C 3.72478774506096

C 4.16648926964077

C 4.68879058806313

S 4.90841509195729

O 5.12068673286140

C 3.27566576161391

C 2.16816881874757

C 1.26205441269168

C 0.2103352389257

C -1.13982725299394

C -2.13742136867656

C -1.80160034961896

C -0.46221558344540

C 0.54057691403698

O 5.86402465605700

H 2.02874562968362

H $\quad-2.58677171745008$

H 5.70207315297260

H -1.40026653104594

H -3.18398461759026

H -0.19180932369806

H 1.72694279234073

H 3.92717856810938

H 0.96013733729102

H 4.69274098188562

H 1.5888805906718

H 3.34636624903407

H 4.03313565257284

H 3.24187888783534

1.34524402677640 1.98421113176268 1.34335648695089 0.08798484934918 $-0.53789721639478$ 0.09081158319430 3.26815457518406 4.31963408129438 5.57783242860297 6.84889696351379 8.1022003689068 6.89971385439753 6.95552283514583 6.94423052953755 6.91620834734714 6.8635939177676 6.8339963808981 6.8517010338144 6.90049411432912 6.93289014850538 6.37863693346558 $-1.51339532703328$ 6.82527352810477 5.48442355444158 6.84603855707796 6.79341938698486 6.9162398088393 1.84229862755655 $-0.40559655215599$ $-0.41094575689468$ 1.84853732639816 6.97816529294362 7.79384994156918 6.04612401090824 5.9989552269106

2.14901424209548 1.06381914279870 0.38793510327708 0.80482513420015 1.89238717481427 2.56048473274628 0.62172932735171 0.21217751445847 $-0.28846600792414$ 1.00597923865146 0.29421163547692 1.83184482606953 0.88702403577306 0.08065736193584 $-0.90531107533946$ $-0.52279128068069$ $-1.49249345495296$ $-2.84630550145567$ $-3.23274851791835$ $-2.27015796915192$ 2.00011287150165 22945051177125 $-3.60349352999652$ $-0.70654219805825$ 0.53600153420242 


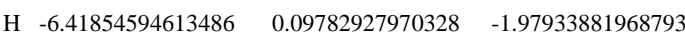
$\begin{array}{llll}\mathrm{H} & -4.73769538632385 & -4.02526986707436 & -0.87045126406413\end{array}$ $\begin{array}{llll}-2.35911396664911 & -3.49141951569807 & -1.34631279984216\end{array}$ H $0.79898441844838 \quad 1.16698279845614 \quad-0.52710608678051$ $\begin{array}{llll}\mathrm{H} & -3.99926552300754 & -0.18579202522462 & -1.42119839643097\end{array}$ $\begin{array}{llll}\text { O } & 2.07002832672738 & 1.70527903673025 & 1.98722345424257\end{array}$

$\begin{array}{llll}\text { O } & -0.33245074741546 & 1.76476972391699 & 2.78705015187362\end{array}$

Allenyne2Cyclization_TS

35

Free energy $=-1241.395004$ Hartree

C $-2.81844989324092-3.02709175138739$

C $-2.27316681162391-2.03565414272735$

C $-3.15151419128095-1.23933469975121$

C $-4.53728369201188-1.44224515851315$

C $-5.04634638039057 \quad-2.39136850193699$

C $-4.18204811436713-3.19227020711076$

C $-0.88021711563173-1.77699252943888$

C $\quad-0.38420285197256 \quad-0.57187637406634$

$\begin{array}{lll}\text { C } & 0.56640827605148 & -0.03754521855858\end{array}$

$\begin{array}{lll}\text { S } & 1.02276885417149 & 1.63777347553453\end{array}$

C $0.00413769347002 \quad 1.70046942050191$

$\begin{array}{lll}\text { C } & -1.20488283868989 & 0.88245658443148\end{array}$

C $\quad-2.45332664404203 \quad 0.92343540298003$

C $-3.79176368010695 \quad 1.27992430936405$

C $-4.45156270400915 \quad 2.31557001084620$

$\begin{array}{lll}\text { C } & -5.72861214581780 & 2.70549648749058\end{array}$

C $-6.36707906243392 \quad 2.06934552534145$

C $-5.73341839606608 \quad 1.01784596588418$

C $-4.46102171631169 \quad 0.61233290060795$

$\begin{array}{lll}\mathrm{H} & -6.12393680103936 & -2.55238754314047\end{array}$

$\begin{array}{lll}\mathrm{H} & -7.35278188825296 & 2.40466272117230\end{array}$

H $-0.22751946487672 \quad 2.75104450871743$

H $1.10569490407532 \quad-0.52743478648119$

$\begin{array}{lll}\mathrm{H} & -0.18616210228600 & -2.44228334020278\end{array}$

$\begin{array}{lll}\mathrm{H} & -3.93530469943542 & 2.82288108665682\end{array}$

H $-2.73808058888823 \quad-0.57161179119462$

H $-6.22971313250974 \quad 3.51555187042127$

H $\quad-5.21103417625159 \quad-0.84629589407978$

$\begin{array}{lll}\mathrm{H} & -6.23981940523840 & 0.50185092343260\end{array}$

H $-4.60025494552573 \quad-3.94439899808196$

H $-2.13949150151433 \quad-3.64419412466977$

\begin{tabular}{ll}
-3.64419412466977 \\
\hline
\end{tabular}

H $\quad-3.96036104457078 \quad-0.21667489669531$

$\begin{array}{lll}\text { O } & 2.43208643719483 & 1.68196765884007\end{array}$

$\begin{array}{lll}\text { O } & 0.50354014056306 & 2.55813167152458\end{array}$

Allenyne2Cyclization_INT

35

Free energy $=-1241.498021$ Hartree

C $-2.62061000000000 \quad-3.62033100000000$

C $-2.20071000000000-2.23379200000000$

C $-3.30237900000000-1.20376200000000$

C $-4.50624600000000-1.74855300000000$

C $-4.77018300000000 \quad-3.07040700000000$

C $-3.84813900000000 \quad-4.01645200000000$

C $-0.89509000000000 \quad-1.85908300000000$

C $-0.48547900000000 \quad-0.48714800000000$

$\begin{array}{lll}\text { C } & 0.76525400000000 & 0.01573400000000\end{array}$

$\begin{array}{lll}\text { S } & 0.83614400000000 & 1.77239700000000\end{array}$

C $-0.91445200000000 \quad 1.85063600000000$

C $-1.50545600000000 \quad 0.49340600000000$

C $-2.81432600000000 \quad 0.17318800000000$

C $-3.85257100000000 \quad 1.17578000000000$

C $-4.58378400000000 \quad 1.84095700000000$

C $-5.58054000000000 \quad 2.76041300000000$

C $-5.86483200000000 \quad 3.02090000000000$

C $-5.14476600000000 \quad 2.36121000000000$

C $-4.14405400000000 \quad 1.44466500000000$

H $\quad-5.67941200000000 \quad-3.45366700000000$

$\mathrm{H} \quad-6.64775600000000 \quad 3.73492200000000$

$\mathrm{H}-1.41238500000000 \quad 2.67266200000000$

H $1.66228700000000 \quad-0.51094700000000$

H $-0.12705200000000 \quad-2.60640200000000$

H $-4.35976800000000 \quad 1.63955400000000$

$\mathrm{H}-3.70052700000000-1.03826400000000$

$\mathrm{H}-6.13767100000000 \quad 3.27524000000000$

H $\quad-5.19844100000000 \quad-1.03408500000000$

$\mathrm{H} \quad-5.36857900000000 \quad 2.56119800000000$

H $-4.13816200000000 \quad-5.06936200000000$

$\mathrm{H}-1.89563100000000 \quad-4.35232800000000$

H $\quad-0.85934100000000 \quad 2.07996900000000$

H $\quad-3.58577200000000 \quad 0.92117600000000$

$\begin{array}{lll}\text { O } & 1.77137300000000 & 2.09950200000000\end{array}$

O 0.95406900000000

2.09950200000000
2.48590700000000

Bisallene

35

Free energy $=-1241.442598$ Hartree

$\begin{array}{lll}\text { C } 2.04074680086596 & 8.23836107798646 & -3.43476563640838\end{array}$

0.09310194877077

1.23798163001470

2.11194469346738

0.5663717337327

1.88361700762589

$-2.51685237012960$

$-1.05142667916875$

$-0.87320861088065$

$-0.88178331545050$

$-1.81904585048586$

1.05055275295736

2.41895504738734

0.99517400000000 0.88314900000000 0.70011800000000 $-0.04312700000000$ $-0.04610800000000$ 0.56801400000000 0.97122000000000 0.76765400000000 0.92711700000000 .65079300000000 .04614200000000 0.34138400000000 0.27165400000000 $-0.10622900000000$ 0.89183700000000 0.54991500000000 $-0.79466300000000$ $-1.79569500000000$ 45443500000000 $-0.51770900000000$ $-1.06423800000000$ 0.57737300000000 1.25340200000000 1.18850400000000 1.94357600000000 1.73369300000000 1.33645600000000 .49211100000000 $-2.84731600000000$ 0.62930900000000 1.36192500000000 $-1.02815800000000$ $-2.23530600000000$ $-0.43282700000000$ 1.93066900000000
C 3.39616299461572

C 4.13392207395590

C 3.51736048417186

C 2.16505509369546

C 1.43313220116766

C 4.03453482975697

C 5.21095155770479

C 6.37255612260435

S 6.49373947879840

C 6.07609655066912

C 5.19399911224657

C 4.23028706623548

C 2.81986132353992

C 1.79123288915380

C 0.45965618882671

C 0.14575249436391

C 1.17091718320995

C 2.50048858576848

H -0.89336062893052

H 1.68443029427277

H 7.33859923830015

H 6.50786590011828

H 3.50267182136945

H 4.46962299899760

H 5.20055179936863

H 3.29966004267566

H 4.10343304069567

H $\quad 0.92850318628091$

H 0.38906525219890

H $\quad-0.33107441522186$

H 1.46001869475084

H 2.04169919175809

O 7.89200314557229

O 5.45431940644206

8.01670077143912

7.18283739580828

6.55057840614156

6.77677556649678

7.63439918896260

8.69188271608825

8.38399133247383

8.07273196603873

7.08743161931258

5.39421157310314

5.14522312146833

5.01771509959044

5.29454281398653

4.79447388499047

5.06780097802667

5.86517531764036

6.38292055951848

6.09860440743650

6.09493340538446

6.29571710306031

8.44952836729356

4.66715034240705

9.52438685311682

4.70937449216033

7.04187955250434

6.52063170466191

5.90372928941451

7.01217220233136

7.85410269463974

4.65771559159633

8.88547180510513

4.19099394208114

7.10336864968949

7.52876520804439

Bisallene2Cyclization_TS1

Free energy $=-1241.408234$ Hartree

C $1.56394740341150 \quad 4.72067018371909$

C $2.51795957130919 \quad 5.31428605353749$

C $2.11656762205406 \quad 6.36010646228862$

C $\quad 0.79245784234625 \quad 6.75682529663621$

C $\quad-0.15672245908153 \quad 6.12894795078366$

$\begin{array}{lll}\text { C } & 0.23227037721558 & 5.13345004453807\end{array}$

C $3.85985292964385 \quad 4.80904103855428$

C $4.23274541655131 \quad 3.64474320557476$

C $5.06522696850805 \quad 3.17410406500272$

S $5.30046664131941 \quad 1.41886730596885$

$\begin{array}{lll}\text { O } & 4.95258033576949 & 0.93476402824630\end{array}$

C $4.01291080512648 \quad 1.02790671330550$

C $3.25198112768505 \quad 2.02506367178027$

$\begin{array}{ll}\text { C } 2.09126625705051 & 2.28149129749514\end{array}$

C $2.06136605385908 \quad 3.07546192202481$

$\begin{array}{ll}\text { C } 3.26408356286336 & 3.48795293751767\end{array}$

C $3.23117280186433 \quad 4.38285624292605$

C $2.01373254216940 \quad 4.85440017000110$

C $0.80996914096595 \quad 4.40688383961197$

C $0.82949879396848 \quad 3.52992440581871$

H $1.99129478681266 \quad 5.55388875264329$

$\begin{array}{lll}\text { O } & 6.59660592297958 & 1.07661251707700\end{array}$

$\begin{array}{lll}\mathrm{H} & -1.19806908281606 & 6.45235895091263\end{array}$

H $\quad 5.60905564561499 \quad 3.75772303153264$

$\begin{array}{lll}\text { H } & 3.95711818616195 & -0.00180567375113\end{array}$

H $4.60987648391539 \quad 5.33232818458312$

H $\quad 1.15103292366455 \quad 1.92444226216536$

H $\quad 1.88615258207001 \quad 3.96095642903630$

H $4.20682770158922 \quad 3.06742057387685$

$\mathrm{H} \quad-0.50305707221978 \quad 4.67840526009457$

H $4.16104563581789 \quad 4.71521354421119$

H $0.48099321876515 \quad 7.54485575821437$

H $-0.13762327946732 \quad 4.77962021668619$

$\begin{array}{lll}\mathrm{H} & 2.85788028432239 & 6.81968728645343\end{array}$

H $\quad-0.09554867181045 \quad 3.20079007093291$

$-3.16327647123447$ 4.01598894355513 $-5.09199378661897$ $-5.35647476723766$ $-4.53350447747838$ $-2.01891818431896$ $-.52174069409513$ $-1.01872138485630$ 0.48172701260889 0.01622254558444 $-0.90786655619507$ $-1.79356001880293$ 1.45468670217282 $-2.26089353156172$ $-1.95180967365284$ 0.85051302117573 $-0.05577810141383$ $-0.34823285835268$ $-0.6041942897118$ 20940839346303 1.36489132640017 0.71194306156671 $-1.54596239580152$ $-2.81523862785740$ $-3.82490388777733$ 0.26670155576776 $-5.74744743349266$ 0.80126268107568 4.76331920090634 $-2.58080786252886$ $-2.77367724230784$ $-3.13612037831119$ 0.88598168469702 1.40642330638861

$-1.99277062428963$ 1.13880623323374 $-0.27426112724117$ $-0.22572971184267$

$-1.04709386802700$ $-1.94087629243208$ $-1.09477736485878$ $-1.71685066592077$ $-2.62901280330317$ $-69290512277565$ $-4.02000362284495$ $-1.52826097337684$ $-1.1111076252965$ 0.42515651734572 


$\begin{array}{llll}\text { C } & 2.23710364521946 & 2.25172803861771 & -0.44679850254891 \\ \text { C } & 2.09101488194869 & 3.09672190314423 & 0.71561016774514 \\ \text { C } & 3.21959687268076 & 3.60958352008452 & 1.39061902608880 \\ \text { C } & 3.06250396716491 & 4.48567203685864 & 2.46050870479631 \\ \text { C } & 1.78448592036315 & 4.85986252690443 & 2.88174695383583 \\ \text { C } & 0.65786099656686 & 4.32424670170299 & 2.25155721517003 \\ \text { C } & 0.80624216922636 & 3.43803732678573 & 1.19127277603500 \\ \text { H } & 1.66113724970221 & 5.55743797152516 & 3.71209552169174 \\ \text { O } & 6.60159382853211 & 0.76439633591669 & -1.86150922095155 \\ \text { H } & -1.12388068788213 & 6.58142994262997 & -0.40814523833560 \\ \text { H } & 6.05785350263133 & 3.74843626364870 & -2.88871518503920 \\ \text { H } & 3.63698123803492 & 0.04865313799383 & -1.47117525124638 \\ \text { H } & 4.58482607379455 & 5.53325908879986 & -1.67544551324433 \\ \text { H } & 1.45316265970700 & 1.52133303708533 & -0.66294090815508 \\ \text { H } & 1.45979661090940 & 3.94366866482366 & -2.65583134745949 \\ \text { H } & 4.21287340142078 & 3.28584445383420 & 1.07269043261118 \\ \text { H } & -0.82030277460570 & 4.76706919402782 & -2.11776281106632 \\ \text { H } & 3.93723670653466 & 4.88140347230755 & 2.97870930183019 \\ \text { H } & 0.86072125670677 & 7.60317004579873 & 0.66283375084202 \\ \text { H } & -0.33808492079349 & 4.62429964482838 & 2.58509511974198 \\ \text { H } & 3.13721876673271 & 6.80758433755575 & 0.09373265008596 \\ \text { H } & -0.06939452571890 & 3.03538162491166 & 0.67645142134270\end{array}$

Bisallene2Cyclization_TS2

35

Free energy $=-1241.427156$ Hartree

C $1.43188413430098 \quad 4.60210739043206 \quad-1.94914327264587$

$\begin{array}{llll}\text { C } & 2.50178866343100 & 5.33168438076661 & -1.34339327116996\end{array}$

$\begin{array}{llll}\text { C } & 2.17716639209607 & 6.42050049939209 & -0.47494066132620\end{array}$

$\begin{array}{llll}\text { C } & 0.87325362871190 & 6.70771634169244 & -0.16784703176155\end{array}$

C $-0.18232360267068 \quad 5.96678617022352 \quad-0.76266096433370$

$\begin{array}{llll}\text { C } & 0.09585825596295 & 4.96288319988828 & -1.67040109190856\end{array}$

$\begin{array}{llll}\text { C } & 3.83019265531788 & 4.89373581081504 & -1.44898503488252\end{array}$

C $4.17607316408334 \quad 3.55077142208347 \quad-1.79261089571990$

$\begin{array}{llll}\text { C } 5.33256791915332 & 3.12758250165874 & -2.35092116715708\end{array}$

S $5.42582985036735 \quad 1.36024356139720 \quad-2.39585795239111$

$\begin{array}{lllll}\text { O } & 5.50325446897007 & 0.90548716174209 & -3.77571280552846\end{array}$

$\begin{array}{llll}\text { C } 3.77895153320127 & 1.18566601656031 & -1.71784178136346\end{array}$

$\begin{array}{llll}\text { C } 3.25229887683630 & 2.38097514153259 & -1.41155102416256\end{array}$

$\begin{array}{llll}\text { C } 2.00865326484746 & 2.58717022706678 & -0.68602420695972\end{array}$

$\begin{array}{llll}\text { C } & 2.02522756465882 & 3.20255869269479 & 0.60661708287071\end{array}$

$\begin{array}{llll}\text { C } & 3.24830311414755 & 3.58415212170304 & 1.20931416401111\end{array}$

$\begin{array}{llll}\text { C } & 3.24704497445013 & 4.37362069503179 & 2.35427142779896\end{array}$

$\begin{array}{llll}\text { C } 2.04077876964199 & 4.76554841474075 & 2.93538200744761\end{array}$

$\begin{array}{llll}\text { C } & 0.82657516770335 & 4.31753851185155 & 2.40038508132219\end{array}$

$\begin{array}{llll}\text { C } & 0.81208983271146 & 3.53846649749240 & 1.25394496047463\end{array}$

$\begin{array}{llll}\text { H } & 2.04537554284064 & 5.39329541592807 & 3.82720448576672\end{array}$

$\begin{array}{llll}\text { O } & 6.38503538491741 & 0.85929797570010 & -1.41747790058605\end{array}$

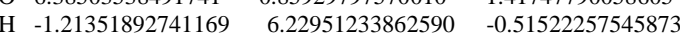

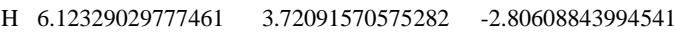

H $3.40029323352894 \quad 0.19988723166967 \quad-1.44949733351001$

$\begin{array}{llll}\mathrm{H} & 4.63781547150316 & 5.56357870971719 & -1.14619947518992\end{array}$

$\begin{array}{llll}\mathrm{H} & 1.12888161776029 & 2.02215142098062 & -1.00258663161448\end{array}$

$\begin{array}{llll}\text { H } & 1.64662071487745 & 3.93429174671994 & -2.78350652807175\end{array}$

H $\quad 4.18652804898182 \quad 3.22758721272918 \quad 0.78299985945583$

$\begin{array}{llll}\mathrm{H} & -0.71519540832601 & 4.43851993593684 & -2.17824950017948\end{array}$

$\begin{array}{llll}\mathrm{H} & 4.19173755672606 & 4.68796441632484 & 2.80117702988505\end{array}$

$\begin{array}{llll}\mathrm{H} & 0.64041046945911 & 7.49757830202095 & 0.54744956135057\end{array}$

$\begin{array}{llll}\mathrm{H} & -0.11004976209843 & 4.61327274541991 & 2.8756058231364\end{array}$

$\begin{array}{llll}\text { H } & 2.99085774313978 & 6.96904997424600 & 0.00206791973352\end{array}$

$\begin{array}{llll}\mathrm{H} & -0.12827561159567 & 3.20788410946241 & 0.80725014261313\end{array}$

Bisallene2Cyclization_INT2

35

Free energy $=-1241.480022$ Hartree

$\begin{array}{llll}\text { C } & 1.34519045100911 & 2.69681973253659 & 1.97374500056789\end{array}$

$\begin{array}{llll}\text { C } 2.28093446769842 & 2.90274438830703 & 0.87002304419188\end{array}$

$\begin{array}{llll}\text { C } & 3.13280829798459 & 4.15777986197082 & 0.92419228830472\end{array}$

$\begin{array}{llll}\text { C } & 2.44339159186889 & 5.29184997576740 & 1.64043253162020\end{array}$

$\begin{array}{llll}\text { C } & 1.46004466094532 & 5.07386936247174 & 2.52571657268316\end{array}$

$\begin{array}{llll}\text { C } & 0.97122789988903 & 3.71709825386611 & 2.77291407277525\end{array}$
C 2.41565406021680

C 3.36849498827397

C 4.07801747356753

C 4.9829744030553

S 5.04800872266087

C 3.74806146731280

C 3.6779957372654

C 4.80235138442539

C 6.08123006790394

C 7.12905911141396

C 6.90911110653909

C 5.63664642735804

C 4.59125536058636

O 6.32415609707173

O 4.60482459854181

H 1.00043683053947

H 7.73155027211012

H 5.63870318868625

H 3.39715547517960

H 2.83304203921626

H 1.84000662153672

H 6.26830884015653

H 4.01490555258647

H 8.11594150139974

H 2.81495793912468

H 5.45295943327106

H 0.25000726114475

H 3.60381294279319

H $\quad 0.91550572666679$

Product

Free energy $=-1241.579299$ Hartree

C $1.00809888404755 \quad 3.22379951655413$

C $2.31465323239561 \quad 2.89682578423758$

C $3.39138211073291 \quad 3.5481485471237$

C $3.16416523220237 \quad 4.51912031170959$

C $1.86043509493095 \quad 4.84590912436799$

$\begin{array}{lll}\text { C } & 0.78197260862896 & 4.19359118199385\end{array}$

C $2.55336091209960 \quad 1.95868596185489$

C $3.29512114249480 \quad 0.73845603969640$

$\begin{array}{lll}\text { C } 3.57289355589254 & -0.05667812668133\end{array}$

$\begin{array}{lll}\text { C } 3.06601916345478 & 0.34039831832404\end{array}$

$\begin{array}{lll}\text { C } & 2.35267559196109 & 1.50419501383769\end{array}$

$\begin{array}{lll}\text { C } 2.12072969621253 & 2.33293538178196\end{array}$

C $3.81089958855614 \quad 0.31107013286358$

C $4.61007388348677 \quad-0.80575823641233$

C $4.92316280025494 \quad-1.56525372947039$

C $4.40252377708468 \quad-1.20551009974285$

C $1.53722106210377 \quad 3.68720986697091$

$\begin{array}{lll}\text { S } & 2.03390367707373 & 3.91093486697912\end{array}$

C $1.87278503101016 \quad 2.11569541228137$

$\begin{array}{lll}\text { O } & 3.45016303745743 & 4.25007530519235\end{array}$

$\begin{array}{lll}\text { O } & 1.08013405995224 & 4.72501752068803\end{array}$

H $5.01542750648909-1.11655672354060$

$\begin{array}{lll}\text { H } & 1.68118595281381 & 5.61255227340153\end{array}$

$\begin{array}{lll}\mathrm{H} & 3.30193577270360 & -0.27331300171649\end{array}$

$\begin{array}{lll}\mathrm{H} & 4.40645480556431 & 3.30440030517885\end{array}$

H $4.00586524146446 \quad 5.03876166435073$

H $3.56620205757803 \quad 0.89357356778209$

$\begin{array}{lll}\mathrm{H} & -0.23523562619928 & 4.45160178739807\end{array}$

H $\quad 5.57354627859500 \quad-2.43707339516857$

$\begin{array}{lll}\mathrm{H} & 0.17092300309763 & 2.70677729713566\end{array}$

H $4.63943185933094 \quad-1.78942338502825$

H $2.48569833549328 \quad 1.87540545947670$

$\begin{array}{lll}\mathrm{H} & 0.80871077091266 & 1.92804891521378\end{array}$

$\begin{array}{lll}\mathrm{H} & 0.43903850377679 & 3.75740358745958\end{array}$

H 1.98112239634612
$-0.11229843625595$

$-1.18980386724083$ $-1.32703765191507$ $-2.30721516021478$ $-3.12935590042879$ $-2.09957763461733$ $-0.45118835103177$ $-0.31096992822729$ 0.08892192462683 0.18463087289763 $-0.09780551336528$ $-0.46581494582464$ $-0.57706529785312$ $-2.84769569415952$ $-4.51120896748446$ 3.06447218935918 $-0.02802276741390$ $-2.62747899136472$ $-2.23944153327593$ $-0.96523061704967$ $-0.07297917910392$ 0.30589982195198 1.54307451090175 0.49074555605592 1.43883343166618 $-0.69320337126458$ 3.57317969766272 $-0.89292057885779$ 2.11015387168407

1.25477362216142 0.86551114403049 1.48094080255794 2.45588079055360 2.83126360971965 2.23353628359845 $-0.26854625696590$ $-0.11790875544777$ $-1.2705123601021$ $-53888777068565$ $-2.66724364905703$ $-1.52423813548043$ 1.13779406126640 1.23088355355410 0.07797633921438 $-1.14063864497455$ $-1.85363829294402$ $-3.59517158152051$

$-3.95650747313116$ $-3.62678788919666$ $-4.33023169968838$ 2.19525971872399 3.58642075911473 $-3.41096943192875$ 1.15811384975956 2.91483418105493 2.02639612744074 2.53674558625264 0.16247204139244 0.77868121932058 $-2.03217320193534$ $-4.83254717607495$ $-4.16950679610429$ $-1.82974304481241$ $-1.27227052966612$

\section{Coordinates of Solvated optimized geometries}

\section{$\mathrm{X}=-\mathrm{CH} 2$ -}

\section{Bispropargyl}

35

Free energy $=-732.4448362$ Hartree
C $-0.78809767047724 \quad 1.75216198074073$
$\begin{array}{lll}\text { C } & 0.27636436403899 & 2.40487195379835\end{array}$
0.54285656853490
$3.40825563384321-1.04571316813427$
$\begin{array}{lll}3.75116189405488 & -1.3396320614766\end{array}$
$\begin{array}{llll}\text { C } & -2.37455356445157 & 3.10138831829011 & -0.69463403111619\end{array}$
$\begin{array}{llll}\text { C } & -2.10432316332497 & 2.10270458945065 & 0.24599133556539\end{array}$
$\begin{array}{llll}\text { C } & 1.64306977444784 & 2.09523596908033 & 0.22917457797763\end{array}$

$\begin{array}{llll}\text { C } & 2.80935306482009 & 1.93253427791178 & 0.52243424415537\end{array}$

C 4.21371820316083

C 4.5733567715124

C 4.3313675066725

C 2.9310269259988

C 1.76456947478475

C 0.38095602339210

C -0.31961276386404

C -1.63002532238258

C -2.26313161102834

C -1.58873194862086

C -0.27314536795118

H -3.27740320108099

H -3.40355149093409

H 4.42602445266581
1.89761645832873 3.07346040020904 4.46327043021367 4.89169852569649 5.18187578385093 5.44934639458797 4.5632686915555 4.84168276840558 5.99587009025372 6.85682950890458 6.58927610301262 6.23397083420681 3.38177190458392 0.95340007838230
0.94927303820889 1.86995831835115 1.24739081275096 1.34556364600155 1.51189720558334 1.80436810265604 2.64218333709196 3.02037471284813 2.55266196935971 1.68376797613550 1.30955464139960 2.87855290501537 $-0.93250435676557$ 1.47439427855441 


$\begin{array}{clcc}\text { H } & 4.65820081897671 & 4.46989270410011 & 0.19492152310700 \\ \text { H } & -0.57129383546846 & 0.97859965896286 & 1.28198845088667 \\ \text { H } & 0.18526149079559 & 3.66467893480891 & 3.00005790874251 \\ \text { H } & -2.92037906413751 & 1.59010597805878 & 0.75769012542139 \\ \text { H } & -2.15738157505957 & 4.15383414985680 & 3.68403136910942 \\ \text { H } & -1.53028847891524 & 4.53439693517177 & -2.06950895027903 \\ \text { H } & -2.08919065896375 & 7.74701301262154 & 1.29924254359805 \\ \text { H } & 0.26381165335264 & 7.26954862658728 & 0.64625827703258 \\ \text { H } & 4.95803296462952 & 5.20543997129726 & 1.76171030694194 \\ \text { H } & 0.82573949169644 & 3.92424354859153 & -1.53327532874795 \\ \text { H } & 4.85824830897638 & 1.91245470244413 & 0.05637910301083 \\ \text { H } & 5.63724499742318 & 2.98603768531053 & 2.13305661866102 \\ \text { H } & 3.99868253930387 & 2.99383070533164 & 2.80354948095738\end{array}$

Bispropargyl_anion

34

Free energy $=-731.9170274$ Hartree

C $-0.77007461874188 \quad 1.63104007530859 \quad 0.56033662229190$

$\begin{array}{llll}\text { C } & 0.34414864613174 & 2.23828215278894 & -0.08953494951643\end{array}$

$\begin{array}{llll}\text { C } & 0.03955938119736 & 3.25157235164203 & -1.04985474894749\end{array}$

$\begin{array}{llll}\text { C } & -1.26868549255995 & 3.61173394654115 & -1.33733824950730\end{array}$

$\begin{array}{llll}\text { C } & -2.35098082898495 & 2.99177168589231 & -0.69718452635540\end{array}$

$\begin{array}{llll}\text { C } & -2.07527223525026 & 2.00402526804278 & 0.25768820144437\end{array}$

$\begin{array}{llll}\text { C } & 1.66915263174871 & 1.87477572610009 & 0.17855600251165\end{array}$

$\begin{array}{llll}\text { C } & 2.87832488803931 & 1.76827699480891 & 0.49067133187434\end{array}$

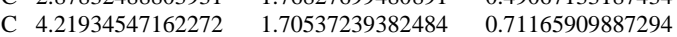

$\begin{array}{llll}\text { C } & 5.15992182764873 & 2.51404147718516 & -0.14241037088841\end{array}$

$\begin{array}{llll}\text { C } & 5.35772714998796 & 4.01120497483244 & 0.24261928757800\end{array}$

$\begin{array}{llll}\text { C } & 4.08254287046897 & 4.68707785375969 & 0.48393001068200\end{array}$

$\begin{array}{llll}\text { C } & 2.97078413174820 & 5.10559770277033 & 0.73685351053970\end{array}$

$\begin{array}{llll}\text { C } & 1.65050416953136 & 5.44318710528582 & 1.20049305591242\end{array}$

C $0.97818599525543 \quad 6.61347750874180 \quad 0.81514582345403$

$\begin{array}{llll}\text { C } & -0.28341482939834 & 6.89622713481496 & 1.33807548990389\end{array}$

$\begin{array}{llll}\text { C } & -0.88012146104004 & 6.02470289842787 & 2.25119575047196\end{array}$

$\begin{array}{llll}\text { C } & -0.22520052692843 & 4.84769916005512 & 2.62004013763089\end{array}$

$\begin{array}{llll}\text { C } & 1.03014264063257 & 4.55427920347525 & 2.09631928524245\end{array}$

H $\quad-3.37424632027204 \quad 3.28621704320431 \quad-0.94161168314766$

$\begin{array}{llll}\mathrm{H} & -1.85662581532330 & 6.26497538494925 & 2.67649003708104\end{array}$

$\begin{array}{llll}\text { H } & 5.92037929294244 & 4.53402454076340 & -0.54780282864958\end{array}$

$\begin{array}{lllr}\mathrm{H} & 4.62920143089515 & 1.11925996618394 & 1.53732707614195\end{array}$

$\begin{array}{llll}\mathrm{H} & 1.45076572240870 & 7.30260389270108 & 0.11328442647829\end{array}$

$\begin{array}{llll}\mathrm{H} & 0.86956148759890 & 3.75742293455143 & -1.54581580009652\end{array}$

$\begin{array}{llll}\mathrm{H} & -0.80385244643067 & 7.80453866260111 & 1.02870503479385\end{array}$

$\begin{array}{llll}\mathrm{H} & -1.45865002134507 & 4.39912665397451 & -2.07191267003124\end{array}$

$\mathrm{H} \quad-0.69583462850166 \quad 4.14832442704989 \quad 3.31296886850076$

$\begin{array}{llll}\mathrm{H} & -2.89383442664443 & 1.50374370720989 & 0.78239654047093\end{array}$

$\begin{array}{llll}\mathrm{H} & -0.57834529599502 & 0.85604797686039 & 1.30618898999711\end{array}$

H $1.54743175040057 \quad 3.62938725848930 \quad 2.35299833680556$

$\begin{array}{llll}\mathrm{H} & 5.96113068816297 & 4.08608189860829 & 1.16132781152646\end{array}$

H $6.16432641120583 \quad 2.06106428246933 \quad-0.13874374413378$

$\begin{array}{llll}\mathrm{H} & 4.80928435978840 & 2.50710075608579 & -1.18924615893258\end{array}$

\section{Allenyne}

35

Free energy $=-732.4505603$ Hartree

C $-2.17317147206613-0.8793380$

.

$\begin{array}{lll}\text { C } & -2.02052095171139 & 3.29826926902255\end{array}$

C $-3.40558208443235 \quad 3.38894998292504$

$\begin{array}{lll}\text { C } & -4.17777224730778 & 2.22858159214627\end{array}$

C $-3.55876640441762 \quad 0.97766349601968$

$\begin{array}{lll}\text { C } & 0.02961146248587 & 1.94180568731707\end{array}$

C $1.22255875045152 \quad 1.86937568543352$

$\begin{array}{lll}\text { C } & 2.66154863289467 & 1.84417464782286\end{array}$

C $2.98325594770235 \quad 1.44758257566606$

$\begin{array}{lll}\text { C } 2.77021673594811 & -0.02095659349925\end{array}$

C $2.14036001966086 \quad-0.84456602491915$

C $1.45405990558488-1.63598644606159$

C $0.00227347655536-1.87426448238316$

C $-0.66324625224848-2.64715434065338$

C $-2.02530925084174 \quad-2.92636458276206$

$\begin{array}{lll}\text { C } & -2.73134201717160 & -2.44344491428093\end{array}$

$\begin{array}{lll}\text { C } & -2.08175808080090 & -1.64763778351167\end{array}$

C $-0.72577781658291 \quad-1.36609906098207$

H $-3.78566142385369-2.69618482377749$

$\begin{array}{lll}\mathrm{H} & -5.26414200424585 & 2.30347743080314\end{array}$

$\begin{array}{lll}\text { H } & 3.07127795804579 & 2.84188089162175\end{array}$

H $3.18008893646976 \quad-0.41227321444174$

H $1.97536796455522-2.17081109428171$

H $-1.41192877692866 \quad 4.19962987459800$

$\begin{array}{lll}\mathrm{H} & -0.20892936626617 & -0.75730677300556\end{array}$

H $\quad-3.89002107902163 \quad 4.36671921870867$

$\mathrm{H} \quad-2.63801243435303 \quad-1.2464722660620$

$\begin{array}{lll}\mathrm{H} & -4.15601947383439 & 0.06755591184485\end{array}$

$\mathrm{H}-2.53051928590110 \quad-3.53514749218573$

H $-0.10147088161741-3.04453386148185$

H $3.16233989093868 \quad 1.14440340392839$

H $-1.68220560601647 \quad-0.09437647832085$

$\begin{array}{lll}\text { H } & 4.02665055307773 & 1.70859698921372\end{array}$

H 2.36523218176091

2.04061557658415
$-0.78036923887719$

$-65140462811714$

$-0.68093557313018$

$-0.79555370409843$

$-0.87710445924064$

$-0.87942965400352$

$-0.44165415072457$

$-0.22694883293209$

0.06631766126023

1.51462446954209

1.80314874272307

1.00402956952545

0.20740336611986

0.33832136265908

$-0.62553705502405$

$-0.49336099856974$

0.61094041283336

1.56160403118653

1.42765464805591

0.73923611479398

0.95234655803576

$-0.14745086350660$

. 74260892120830

0.59377942251630

$-0.59715667434369$

2.1720240481621

$-0.81303671438826$

2.41058751555167

$-0.9577754789703$

$-1.24525679118243$

$-0.78716178344340$

1.74886374701224

2.20549740944127
$-1.47431822055149$
Allenyne_anion

Free energy $=-731.9166458$ Hartree

$\begin{array}{lll}\text { C } & -2.20180847238391 & 0.19440427955187\end{array}$

$\begin{array}{lll}\text { C } & -1.65628214563149 & 1.47971550138723\end{array}$

$\begin{array}{lll}\text { C } & -2.51741998950516 & 2.58564489368374\end{array}$

$\begin{array}{lll}\text { C } & -3.88890849806575 & 2.41381953735268\end{array}$

C $-4.41995358465868 \quad 1.13259261779133$

$\begin{array}{lll}\text { C } & -3.57089607315681 & 0.02336660604821\end{array}$

C $\quad-0.21113668725982 \quad 1.7029011992508$

$\begin{array}{lll}\text { C } & 0.67575245323897 & 0.75406658939651\end{array}$

C $1.52407750468870 \quad-0.20833062082602$

$\begin{array}{lll}\text { C } & 1.79385499554377 & -0.77903195283353\end{array}$

C $1.44645160532997 \quad 0.10279150087020$

$\begin{array}{lll}\text { C } & 0.15974387817560 & 0.36701678471356\end{array}$

$\begin{array}{lll}\text { C } & -1.03143387391838 & 0.58247832606767\end{array}$

$\begin{array}{lll}\text { C } & -2.35001703023063 & 0.88697098993356\end{array}$

$\begin{array}{lll}\text { C } & -2.66025713288157 & 1.83545943432435\end{array}$

$\begin{array}{lll}\text { C } & -3.97179240765508 & 2.12678991212185\end{array}$

C $-5.04939468672062 \quad 1.49556302026036$

$\begin{array}{lll}\text { C } & -4.76720492325857 & 0.56091544227549\end{array}$

C $-3.46324395385190 \quad 0.26201201340757$

H $\quad-5.49213830717016 \quad 0.99395007509852$

H $\quad-6.07405792948668 \quad 1.73182498090044$ H $2.03380673951145 \quad-0.68807420191951$

H 0.12275976265374

H $\quad-3.26683247718013$ 2.74733899651414 $-0.44295886406554$ $-0.66986838513693$ 0.05114295401037

H $\quad-5.58429935904606$

H -3.98709321279331

H $\quad-4.16436559027119$ $-0.97676206120454$ 2.85666256616880 3.28679786255094

$-2.09887933379476$

H -2.098793

H $\quad 2.25260935622142$

H -1.83708754194612 3.58848120043429 0.67648619829581 2.33533051511865 $-1.08095618041764$

H 1.22552729398062

$-1.72694773112520$

$-1.08764675982966$ $-0.93609074401820$ $-0.97543625842007$ 1.14736880077907 $-1.29407845534502$ $-1.26867268134396$ 0.73796686035260 0.52455884907829 $-0.26641603785345$ 1.12973839648692 2.29414252294955 2.64149272687125 2.96087004481277 3.30324983211594 4.3264575224512 4.67553337679779 4.04118998750950 3.03317365833788 2.66255868214609 $-1.44665305678419$ 4.33556409201123 $-1.11348549015498$ $-0.74924250287617$ 1.85112757917353 $-1.07149801973097$ 2.51645003685747 $-1.40604224189629$ 5.46643826901188 $-1.17439234929373$ $-0.86062300715660$ 2.75820156910847 4.8398074479137 1.16694740316284

1.16640796719524

\section{X=-NH-}

Bispropargyl

Free energy $=-748.467639$ Hartree

C $-0.77769109037041 \quad 1.81539924753325$

$\begin{array}{lll}\text { C } & 0.32127962863482 & 2.43527670788216\end{array}$

$\begin{array}{lll}\text { C } & 0.09818566256339 & 3.45801043149717\end{array}$

$\begin{array}{lll}\text { C } & -1.19970473383494 & 3.84720019181918\end{array}$

C $-2.28958874080946 \quad 3.22599684783069$

$\begin{array}{lll}\text { C } & -2.07471140646025 & 2.21257618771011\end{array}$

C 1.67608457225989

C 2.84491635063363

1.89458463779200

1.91732067137620

N $4.70375704301478 \quad 3.10087131109426$

C $4.30306980053037 \quad 4.40252334602842$

C $2.89238311660709 \quad 4.78917818261275$

C $1.74116383547014 \quad 5.07544776984436$

C $0.36527394196794 \quad 5.37253336097333$

$\begin{array}{lll}\text { C } & -0.37333342365034 & 4.5136773525796\end{array}$

C $-1.68771699065098 \quad 4.82447765629690$

C $-2.29009863205225 \quad 5.98201014999225$

C $-1.57535101079006 \quad 6.82162448092857$

$\begin{array}{lll}\text { C } & -0.25761558187745 & 6.52177235145937\end{array}$

H $\quad-3.30930905355754 \quad 6.24042290056654$

H $\quad-3.30252452356942 \quad 3.54153881819033$

H $\quad 4.53521626658390 \quad 1.03707292169269$

H $4.51280830553233 \quad 4.42921601742323$

$\begin{array}{lll}\mathrm{H} & -0.60476582576076 & 1.0300757008203\end{array}$

H $\quad 0.09514196920396 \quad 3.60562734297150$

H $-2.91908346741202 \quad 1.72376704308236$

H -2.24881946644065

$-100587$

H $\quad-1.36584314000721 \quad 4.64437028825364$

H $\quad-2.04774622567983 \quad 7.71748574807564$

$\begin{array}{llll}\mathrm{H} & 0.30803330395464 & 7.18348003571619\end{array}$

H $4.94748439439030 \quad 5.16946448347360$

$\mathrm{H} \quad 0.95401752103322 \quad 3.95222572657348$

$\begin{array}{lll}\mathrm{H} & 4.87479162656543 & 1.83595319749308\end{array}$

H $4.38613508291236 \quad 3.02454139344200$

0.53243384191199 $-0.08423686645485$ $-1.02298328386185$ $-1.34412787803158$ $-0.73032846187618$ 0.20854831857669 0.25243290235263 0.52234285231055 0.88356038258144 1.62651100167512 1.09845099771218 1.34865770464230 61277521897377 93077055110970 2.76550198238975 3.10789434852263 2.61037231865651 1.7527390696939 1.41374832682539 2.90517282755215 $-0.99151893856750$ 1.49122302069457 0.01813635561997 1.2708772986899 3.14706230714884 0.69722585936593 3.76194277698137 2.07054772755213 1.3463203970290 0.75577468348967 1.55258149059059 $-1.48423313721392$ 0.03963246810637 2.59163692656752

Bispropargyl anion

Free energy $=-747.940865$ Hartree

$\begin{array}{llll}\text { C } & -0.75689611857219 & 1.64953623570217 & 0.58931001217465\end{array}$

$\begin{array}{llll}\text { C } & 0.13630998030162 & 3.28961406809616 & -0.95516042553464\end{array}$

$\begin{array}{llll}\text { C } & -1.15533309530082 & 3.69161174547412 & -1.26395264578084\end{array}$

$\begin{array}{llll}\text { C } & -2.26976625209415 & 3.08641590669615 & -0.66695803872457\end{array}$

$\begin{array}{llll}\text { C } & -2.04359577060568 & 2.06695935413132 & 0.26785411867283\end{array}$

$\begin{array}{llll}\text { C } & 1.69582180415103 & 1.83737600905054 & 0.28662929353428\end{array}$ 


$\begin{array}{llll}\text { C } & 2.88045883403150 & 1.63771288157039 & 0.64779623526656 \\ \text { C } & 4.20482989248132 & 1.49085273544701 & 0.93072151948012 \\ \text { N } & 5.20145520063158 & 2.26138149229460 & 0.21871417763414 \\ \text { C } & 5.43592609216178 & 3.63465186010199 & 0.68262184475467 \\ \text { C } & 4.20101253244175 & 4.42219199766456 & 0.87194744903074 \\ \text { C } & 3.09774280188991 & 4.88868222482548 & 1.07643746069140 \\ \text { C } & 1.76572343162492 & 5.30115062305351 & 1.43353649189988 \\ \text { C } & 1.20636106073904 & 6.50811160390299 & 0.98713875406897 \\ \text { C } & -0.07375998437407 & 6.88161530723486 & 1.39401642340592 \\ \text { C } & -0.80883947307063 & 6.05813537071401 & 2.24946825455646 \\ \text { C } & -0.26778214192036 & 4.84379912874904 & 2.67636702683990 \\ \text { C } & 1.00483451150607 & 4.45915664105353 & 2.26525336702498 \\ \text { H } & -3.27799505527284 & 3.41706991361105 & -0.92691212054375 \\ \text { H } & -1.79668402450570 & 6.36946637274545 & 2.59491236143824 \\ \text { H } & 6.10684737606356 & 4.14173243047135 & -0.02806090836885 \\ \text { H } & 4.57475494006332 & 0.75722981534527 & 1.65074673385897 \\ \text { H } & 1.79038116064512 & 7.15811970305382 & 0.33284442783229 \\ \text { H } & 0.98856803157834 & 3.78742124270451 & -1.42085033220041 \\ \text { H } & -0.50182838251924 & 7.82061246345223 & 1.03860433634864 \\ \text { H } & -1.30603488545492 & 4.50319509883306 & -1.98154632516666 \\ \text { H } & -0.84893864614852 & 4.18179466369411 & 3.32113104558177 \\ \text { H } & -2.88700917348105 & 1.57777847926799 & 0.76320730544357 \\ \text { H } & -0.60418028966521 & 0.85227807554330 & 1.32069456416558 \\ \text { H } & 1.42562589756951 & 3.49860839554771 & 2.56369755197824 \\ \text { H } & 5.96527803550745 & 3.61044802966649 & 1.64820666049430 \\ \text { H } & 4.93961546606196 & 2.31807802768334 & -0.76487271629944\end{array}$

\section{Allenyne}

34

Free energy $=-748.4799$ Hartree

C $-2.21612033381527 \quad 1.01642391799824$

C $-1.52823045036534 \quad 2.05016896334360$

$\begin{array}{lll}\text { C } & -2.17920955734372 & 3.27314687910279\end{array}$

C $-3.47977090721962 \quad 3.47442390632869$

$\begin{array}{lll}\text { C } & -4.13996142046008 & 2.46252300384750\end{array}$

$\begin{array}{lll}\text { C } & -3.51306933167614 & 1.23034964145516\end{array}$

C $-0.13536505155239 \quad 1.89918957540925$

$\begin{array}{lll}\text { C } & 1.06397383957609 & 1.81932660821933\end{array}$

$\begin{array}{lll}\text { C } & 2.53689561026449 & 1.76581278771197\end{array}$

N $3.05677091536782 \quad 1.37989761528253$

$\begin{array}{lll}\text { C } 2.71897054263106 & 0.11676975844408\end{array}$

$\begin{array}{lll}\text { C } & 2.08580221691272 & -0.82569792643080\end{array}$

C $1.45491984588382-1.77416126404503$

$\begin{array}{lll}\text { C } & -0.00332485963551 & -2.01839776447235\end{array}$

$\begin{array}{lll}\text { C } & -0.55074745306202 & -3.06543835708909\end{array}$

C $-1.91524424549248 \quad-3.35233927638565$

C $-2.74620695747308 \quad-2.59304771128815$

C $\quad-2.21376755913214 \quad-1.53402351521564$

C $-0.85380557413335-1.24321619291380$

H $-3.80711449386860 \quad-2.84007251214690$

H $\quad-5.14281088225208 \quad 2.63497379949087$

$\begin{array}{lll}\mathrm{H} & 2.94743969866880 & 2.74974260013074\end{array}$

H $3.06716066587631-0.08439959660172$

H $2.03073670756048 \quad-2.45871044044954$

$\begin{array}{lll}\mathrm{H} & -1.64892477007966 & 4.06770034381969\end{array}$

H $-0.42593406435078 \quad-0.42042392674472$

$\begin{array}{lll}\mathrm{H} & -3.97530303620586 & 4.42972224013581\end{array}$

H $-2.86823074087405 \quad-0.93572739927147$

$\begin{array}{lll}\mathrm{H} & -4.03078033582005 & 0.43289982668802\end{array}$

H $\quad-2.32851402647327 \quad-4.17333891406711$

$\begin{array}{lll}\mathrm{H} & 0.10779087643837 & -3.67116487494799\end{array}$

H $2.91322796291766 \quad 1.04977918015206$

$\begin{array}{lll}\mathrm{H} & -1.71593028897137 & 0.05948414096201\end{array}$

H 3.01893445815924

2.10910888354759

Allenyne_anion

33

Free energy $=-747.953909$ Hartree

C $\quad-3.74839357171017 \quad-2.35391183225045$

C $-3.05696488330403 \quad-1.57184936520843$

C $-3.81905948206656 \quad-0.58985213795570$

$\begin{array}{lll}\text { C } & -5.14645113023676 & -0.34452051799894\end{array}$

C $-5.79340431835095 \quad-1.08652458550304$

C $-5.08167434668415 \quad-2.10478673464720$

C $-1.68190563382434 \quad-1.76873770888204$

C $-0.54065000819434 \quad-1.22642781532209$

$\begin{array}{lll}\text { C } & 0.72259647594945 & -0.74948970853789\end{array}$

N $1.54808161764113-1.07816216117096$

$\begin{array}{lll}\text { C } & 1.27651145397479 & -0.51767999968188\end{array}$

$\begin{array}{lll}\text { C } & 0.58007025479344 & 0.58699985755225\end{array}$

$\begin{array}{lll}\text { C } & -0.10721397372061 & 1.70352257272583\end{array}$

$\begin{array}{lll}\text { C } & -1.57968144067055 & 1.79735209026347\end{array}$

$\begin{array}{lll}\text { C } & -2.39236394468652 & 0.65376864526490\end{array}$

$\begin{array}{lll}\text { C } & -3.77565491453376 & 0.77795827346079\end{array}$

$\begin{array}{lll}\text { C } & -4.37502366267013 & 2.04092249269498\end{array}$

C $-3.57892398021429 \quad 3.18456496677260$

$\begin{array}{lll}\text { C } & -2.18949016120490 & 3.06023336801226\end{array}$

$\mathrm{H}-5.46315947736728-2.13299915837821$

$\begin{array}{lll}\mathrm{H} & -5.46315947736728 & 2.1329991583782\end{array}$

$\begin{array}{lll}\text { H } & -6.81746209850010 & -0.84865185889635 \\ \text { H } & 1.71273068472340 & -1.04639946508728\end{array}$ $-0.57282697365358$ $-0.33413822474871$ $-0.79065323279856$ $-1.4901754938816$ $-0.23942304670206$ $-0.06986022165320$ 0.04987893623228 1.34485972362543 1.83357308356208 1.16511792123280 0.50391176304455 0.55325529846197 $-0.20310135740722$ $-0.14462104562724$ 0.67977034668529 1.42227927177816 1.35852976441481 0.75764510466029 $-1.88449399692366$ $-0.21299342862610$ 2.85347495118924 0.19214197661690 1.93479881481724 $-0.60991136674505$ 2.05760624819848 $-2.22752265253058$ $-0.73316883421156$ $-0.82997706452389$ $-0.69664769327899$ $-1.39124372446492$ 2.05126620089789

0.76896235356173 1.73290618506524 2.42024542378831 2.09628721169167 1.1006822830460 0.45775387881908 2.03666264137512 1.97332541191801 1.89200905617396 0.76316286642008 $-0.47720660362733$ $-0.67999397546873$ $-0.80164731513944$ $-0.90776500121494$ $-0.93787439740344$ $-0.9930894229377$ $-0.99251208263512$ $-0.95708639848810$ $-0.92505410274679$ $-1.00875730180439$ $-1.33430862277603$
$-1.23033979583621$ $-1.69290841538576$ $-0.13254483641850$ 0.80555256326496 $\begin{array}{llll}\text { H } & 0.41441926300202 & 2.67078595867955 & -0.77177035831458\end{array}$ $\begin{array}{lllll}\mathrm{H} & -3.20564444544770 & -3.14453177467778 & 0.2471662443795\end{array}$ $\begin{array}{llll}\text { H } & -1.92320950416989 & -0.33193290339553 & -0.88388627618985\end{array}$ $\begin{array}{llll}\mathrm{H} & -5.57167152718433 & -2.70603519882116 & -0.31238833993994\end{array}$ $\begin{array}{llll}\mathrm{H} & -4.39332144649615 & -0.11900702750250 & -1.00439998209625\end{array}$ $\begin{array}{llll}\mathrm{H} & -5.68537774780082 & 0.44655646164908 & 2.62289991670548\end{array}$ $\begin{array}{llll}\mathrm{H} & -4.04299664763043 & 4.17307638025904 & -0.94593874967168\end{array}$ H $-1.56203283690181 \quad 3.95439423553866 \quad-0.89191031063794$ $\begin{array}{llll}\mathrm{H} & 1.13060209879110 & -0.04291725028057 & 2.61971968977200\end{array}$ $\begin{array}{llll}\mathrm{H} & -3.31871425758949 & 0.00675208721407 & 3.18551310441397\end{array}$ $\begin{array}{llll}\mathrm{H} & 1.84904726775763 & -2.04584626236239 & 0.69486140149405\end{array}$

$\mathbf{X}=-\mathbf{O}-$

Bispropargyl

Free energy $=-768.3199554$ Hartree

C $-0.27642006439052 \quad 4.66816251032835 \quad 3.24846154353427$

$\begin{array}{llll}\text { C } & 0.31647222976967 & 5.25139246463887 & 2.11465145193947\end{array}$

$\begin{array}{llll}\text { C } & -0.46057249321292 & 6.06834648222054 & 1.27537816639395\end{array}$

$\begin{array}{llll}\text { C } & -1.78558417674036 & 6.34419883227184 & 1.60437109890610\end{array}$

$\begin{array}{llll}\text { C } & -2.35856878554587 & 5.78973225344879 & 2.75083594053802\end{array}$

$\begin{array}{llll}\text { C } & -1.60603244065738 & 4.93645025818690 & 3.56199299377444\end{array}$

$\begin{array}{llll}\text { C } & 1.70701978298496 & 5.00684237532910 & 1.83321253784679\end{array}$

$\begin{array}{llll}\text { C } & 2.87410145542159 & 4.75972839999984 & 1.61261321549723\end{array}$

$\begin{array}{llll}\text { C } 4.27036799119048 & 4.36465477442116 & 1.33428305923939\end{array}$

$\begin{array}{llll}\text { O } & 4.56236856016639 & 3.05325531290434 & 1.78965190766827\end{array}$

$\begin{array}{llll}\text { C } & 4.25165411935690 & 2.02730211148819 & 0.85808399837697\end{array}$

$\begin{array}{llll}\text { C } & 2.85206196114910 & 2.06377004468998 & 0.38942544529504\end{array}$

$\begin{array}{llll}\text { C } & 1.70493953247648 & 2.26571749995954 & 0.04803562507739\end{array}$

$\begin{array}{llll}\text { C } & 0.35489278303337 & 2.61104861914672 & -0.32071312692065\end{array}$

$\begin{array}{llll}\text { C } & 0.12295814521837 & 3.58316486483907 & -1.3100365555518\end{array}$

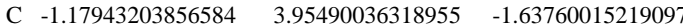

C $\quad-2.26294689941600 \quad 3.36551711293786 \quad-0.98200523836769$

$\begin{array}{llll}\text { C } & -2.03925015379892 & 2.39884385526619 & 0.00307330976737\end{array}$

$\begin{array}{llll}\text { C } & -0.73897352303547 & 2.02050419306074 & 0.33408764649294\end{array}$

$\begin{array}{llll}\mathrm{H} & -3.38493803401314 & 6.04077015553421 & 3.02586288242752\end{array}$

$\begin{array}{llll}\mathrm{H} & -3.27914513310684 & 3.66908557618643 & -1.24547119594455\end{array}$

$\begin{array}{llll}\mathrm{H} & 4.46817372355816 & 1.08053294323881 & 1.37205671010787\end{array}$

$\begin{array}{lllll}\mathrm{H} & 4.47272114367680 & 4.45910175430321 & 0.25257040435754\end{array}$

$\begin{array}{lllr}\mathrm{H} & -0.55724254887131 & 1.27247898057193 & 1.10775836432608\end{array}$

$\mathrm{H} \quad 0.32548180957227 \quad 4.02075655511532 \quad 3.88819816765454$

H $\quad-2.87755948972657 \quad 1.93375077075520 \quad 0.52411323166357$

$\begin{array}{llll}\mathrm{H} & -2.05560444309955 & 4.48416302809873 & 4.44747030239565\end{array}$

$\begin{array}{llll}\mathrm{H} & -1.35529900152219 & 4.71130883097628 & -2.4045907731293\end{array}$

$\begin{array}{llll}\mathrm{H} & -2.37879398591248 & 6.99306063577183 & 0.95766342620736\end{array}$

$\begin{array}{llll}\mathrm{H} & -0.01552663707683 & 6.48901556223996 & 0.37285954694305\end{array}$

$\begin{array}{llll}\mathrm{H} & 4.95876477180393 & 5.04377513631282 & 1.85350527475587\end{array}$

$\begin{array}{llll}\mathrm{H} & 0.97160456290372 & 4.04788490069583 & -1.81304529535714\end{array}$

$\mathrm{H} \quad 4.91659127640992 \quad 2.09744984187183 \quad-0.02208891372454$

Bispropargyl_anion

32

Free energy $=-767.7979357$ Hartree

$\begin{array}{llll}\text { C } & 0.17822036409640 & 9.46783599985170 & 3.53247440937349\end{array}$

C $1.05103151686715 \quad 8.39128899804542$

$\begin{array}{lll}\text { C } & 0.60505648264408 & 7.52489871236708\end{array}$

$\begin{array}{lll}\text { C } & -0.61242275110376 & 7.72534415168306\end{array}$

$\begin{array}{lll}\text { C } & -1.45188302105927 & 8.79184767906358\end{array}$

C $-1.03428342887521 \quad 9.65300969862488$

C $2.27319236676664 \quad 8.18360542753295$

C $3.22292002586583 \quad 7.83066646586134$

C $4.28588822691936 \quad 7.45638329250793$

$\begin{array}{lll}\text { O } & 4.95577029815808 & 6.23476429886493\end{array}$

C $4.41138758303844 \quad 5.13883514964124$

C $2.98535734579391 \quad 4.91492394919525$

C $1.81481368429797 \quad 4.90543145723541$

C $\quad 0.45129365678574 \quad 5.05989071312389$

$\begin{array}{lll}\text { C } & -0.14212688474061 & 4.14485911774939\end{array}$

$\begin{array}{lll}\text { C } & -1.41491166619370 & 4.39294743459015\end{array}$

C $-2.10083595916423 \quad 5.55392440117196$

C $-1.53159980296981 \quad 6.44837712545983$

C $-0.26211313377886 \quad 6.20635199108279$

H $\quad-2.40445463124609 \quad 8.94562256821433$

H $\quad-3.07539785693023 \quad 5.77680386255516$

H $5.00190815762886 \quad 4.25248120408519$

H $4.61084076637806 \quad 7.99094067594868$

$\mathrm{H} \quad 0.41032506344222 \quad 3.25458675350605$

H $\quad 1.23461364083369 \quad 6.67723540895194$

H $-1.86173070159272 \quad 3.68356106383798$

$\begin{array}{lll}\mathrm{H} & -0.92917808349356 & 7.02170814119891\end{array}$

H $\quad-2.06344031725946 \quad 7.36168318834081$

$\begin{array}{lll}\mathrm{H} & -1.67367229211085 & 10.48722769568085\end{array}$

$\begin{array}{lll}\text { H } & 0.47577359585800 & 10.15455733702357\end{array}$

$\begin{array}{lll}\mathrm{H} & 0.22052617785533 & 6.92602946177064\end{array}$

H $\quad 4.51593957728847 \quad 5.29666457523303$ 87498458369431 .92166073541983 5.55760700181079 5.2080188060245 4.18630131240133 3.22400651542199 2.47991521603971 1.72499700537328 1.96589261250929 1.25486333760512 1.57371401133030 1.89945439050867 2.34011922812577 3.22524066114841 3.73702902940976 3.37447500990517 2.46779422589550 1.95016616602306 5.71680740216649 3.81384127439088 1.53218424890057 0.82744200252694 3.52980315777127 5.19979061852072 4.43592218546898 6.33265899314594 2.19764820451882 3.88509259361309 2.73772499867882 1.28771126063053 
33

Free energy $=-768.3295698$ Hartree

C -0.64691061304236

C 2.07091981813662

C 2.53557533610182

$-1.42637213096119$

$-0.11317993030258$
C -0.05844648278630

C -0.86322708133073

C -2.21453638955850

C -2.78534080419845

C -2.00257120830846

C 1.39440303843250

C 2.71983750785811

O 2.97022713673733

C 1.07306206234325

C -0.11935238796556

C -1.51250311336737

C -2.18747972125605

C -3.49977955511675

C -4.14552028719387

C -3.49174937604883

C -2.18088824134168

H -3.83510797928938

H $\quad-5.16022657689764$

H 2.94102233583372

H 3.13640992316010

H 1.93178183094312

H -1.66676435452505

H $\quad-0.40534025124714$

H -4.01679066041934

H -2.83278647258738

H -4.00148624405120

H -2.44811906439559

H -0.0259997033096

H 2.96432557253842

H $\quad-1.65829999384771$
$-2.97230613920576$

$-1.91672515684062$

$-1.13160860695190$

$-2.50064319286020$

$-3.26604097366033$

$-1.67519255602647$

$-0.76811277317649$

0.12333525292615

1.41958281142848

.77509898010640

1.79763469311720

84281649995673

1.96236809335911

3.16824308886283

3.34027447259242

2.31472598202748

10021539928941

0.91660325345347

$-2.75706934276556$

2.46340617841477

2.77515198866382

$-2.32843995763305$

3.9691381396181

$-0.30072095080253$

4.28065111657907

$-0.81975917341492$

0.29445223774999

$-4.09666725831050$

$-3.58501445856086$

.07165032581550

$-0.02529091248797$
$-0.20885262295667$ .50501938367383 1.34651984003474 1.49861195016914 0.80875065940405 $-0.05739848643322$ 0.38163542205408 1.05815702003665 1.77485717055199 1.41833601632985 0.11341441165619 $-0.05167661584218$ $-0.27246427065545$ 0.61605185795700 $-0.35927268024937$ $-0.79246244306662$ $-1.48566140700744$ $-1.7115506381280$ $-1.27566714933043$ 0.96754476103618 $-1.85888350618990$ $-0.08258372045927$ 2.75835931452301 $-0.31715718574824$ 0.16675444355075 1.88674012057170 $-0.59519865252207$ 2.16186759577165 $-0.60759826840416$ $-0.86672263558488$ $-0.61939989452159$

Allenyne_anion Free energy $=-767.8021269$ Hartree

C $-0.93221410181405 \quad-1.98239829977344$ C $0.00505985338541-1.85714187739935$

C $-0.50135667787130 \quad-2.09024122109696$

$\begin{array}{lll}\text { C } & -1.82585271518511 & -2.44280746998019\end{array}$

C $-2.72595036396104 \quad-2.56250025034730$

C $-2.25611804580420 \quad-2.32340923371552$

$\begin{array}{lll}\text { C } & 1.34911591585149 & -1.52704348604294\end{array}$

$\begin{array}{lll}\text { C } & 2.55874377814660 & -1.25036711084778\end{array}$

$\begin{array}{lll}\text { C } 3.86919296797252 & -0.95732707524584\end{array}$

$\begin{array}{lll}\text { O } & 4.82713638050023 & -1.19732260443162\end{array}$

C $5.00112459452721 \quad-2.50287297118927$

$\begin{array}{lll}\text { C } 4.40121558761812 & -3.54613338714824\end{array}$

$\begin{array}{lll}\text { C } & 3.81733482604824 & -4.63253793460434\end{array}$

C $2.13399132632256 \quad-6.47568929416653$

$\begin{array}{lll}\text { C } & 0.97208820239512 & -7.06883617184177\end{array}$

$\begin{array}{lll}\text { C } & 0.22742431654290 & -6.42557662532432\end{array}$

$\begin{array}{lll}\text { C } & 0.64247657334294 & -5.17808454951729\end{array}$

C $1.79140424923358 \quad-4.57993263684494$

H $-0.67293043588176 \quad-6.89218820202542$

H $\quad-3.75867434595022 \quad-2.86695058847437$

H $5.73332853620785-2.63897655669972$

H $4.27545324604760-5.16625122985167$

H $0.18250287654614 \quad-1.98526666336929$

H $2.09799499496796-3.58914632054908$

H $\quad-2.17203962684189-2.64142417798397$

H $0.04529658353960 \quad-4.65252188294319$

H $\quad-2.93705013798942 \quad-2.43068420382106$

H $\quad 0.63786259616598 \quad-8.03052366796860$

H $\quad 2.72152374261760 \quad-6.98847372727123$

H $4.31635168002236-0.70835603005139$

$-1.81705324006522$

.26613831949604

1.70592271227890

0.64731786153846

58044367364570

1.07651106797544

1.60545985991804

55091794002362

2.52271949159816

$-1.6772603706882$

$-0.08354255239356$

$-0.95307476376244$

$-1.54461858874144$

$-2.09182645414703$ $-2.24156345854158$

C $2.56914660863565 \quad-5.23158830940827$

Н -0.58730098533866
C 4.50401439233880

C 3.30709137876207

C 1.86959190669187

C 1.11473292424476

C -0.27677175199766

C -0.92881328347387

C -0.18705463182054

C 1.20493016035196

H -2.01970530947247

H $\quad 0.76846677330378$

H $\quad 6.59827435525860$

H 1.20594474860063

H $\quad-0.06929802268287$

H 2.91618164157442

H 1.79113659658434

H -0.85863981143832

H -0.69924203746737

H 1.62604834173549

H 4.19410795046675

H 5.09600243393479

H 4.10136439088358

Bispropargyl_anion

Free energy $=-1090.744709$ Hartree

C $3.42168223409127 \quad 2.33583807675319$

C 2.80462228692026

C 1.45337154707759

C 0.74245295291665

C 1.36105078808736

C 2.70289135857733

C 3.54506894141996

C 4.18447136047116

C 4.97498019668529

S 6.64179360966905

C 7.42692441898203

C 7.21637155052182

C 7.05710217219406

C 6.89110069401903

C 6.85237206754394

C 6.6537981597544

C 6.49376230196903

C 6.52751955135777

C 6.7176149123959

H 0.79603086062319

H 6.33588639510388

H 5.20062390195079

H 6.71101980252465

H 6.38076486582295

H 6.63983089314552

H 4.46574763488626

H -0.30037119081970

H 3.20368007393600

H 0.96279799987610

H 6.99776711885099

H 7.74981120122924

H 4.41592533821595

2.81575945180415

2.49760482335149

1.71939856592552

1.25396004227379

1.56295107222698

3.61944250991843

4.28841258276476

5.06617653954186

5.60872025060773

4.04147642071633

21452963429699

2.47562646612068

1.81498159364678

2.58787401105802

2.00684121476078

0.62322509190896

$-0.1568728203748$

0.6619376304138

0.16067237129559

4.43908129179550

$-0.21683459777540$

$-1.23757476797560$

2.64281051193023

2.57629913256058

1.46644869221607

1.20313240410390

2.87075113813594

3.66609250121185

3.58510301680741

5.94627916134144

2.15869617813383

Free energy $=-1091.261917$ Hartree

$\begin{array}{ll}\text { C } & -1.98164771826719 \\ \text { C } & -1.28191142008464\end{array}$

C -3.32499918416605

C -3.99601895098232

C -3.32443134593413

C 0.17497972528652

C 0.99242834154658

C 1.82664758488851
0.41533598663746

\section{$\mathbf{X}=-\mathrm{S}-$}

Bispropargyl

33

Free energy $=-1091.252644$ Hartree

$\begin{array}{lll}\text { C } 3.27186089010870 & 2.23801209060656\end{array}$

$\begin{array}{lll}\text { C } & 2.80709181640541 & 2.82808585419648\end{array}$

C $1.59182041471984 \quad 2.38511252227465$

$\begin{array}{lll}\text { C } & 0.86902161769355 & 1.36623961434301\end{array}$

C $\quad 1.34372598222540 \quad 0.77741528481233$

$\begin{array}{lll}\text { C } & 2.54375394444899 & 1.22066147794733\end{array}$

C $3.55844808722795 \quad 3.91515962271450$

$\begin{array}{lll}\text { C } & 4.18210806227301 & 4.87108067739933\end{array}$

$\begin{array}{lll}\text { C } & 4.82232061580332 & 6.13125316842499\end{array}$

S $6.39015320549045 \quad 6.55971698953465$

$\begin{array}{ll}\text { C } 5.90063217422185 & 6.09807482641836\end{array}$

2.64891238127147

1.45976102833441

0.90718702978824

1.53013134255468

2.70437398990920

3.26555295549762

0.87398560705356

0.45374539673565

0.05511107310326

0.92662394247079

2.66007615247428

C -1.97951321963653

S 2.41895766303747

C 1.66923758226739

C 0.21334383703512

C -0.99349690985987

C -2.41716629215381

C -3.24504664770161

C -4.59914417625254

C -5.14774693278546

C -4.34326487903758

C -2.98509143621509

H -5.03497111557786

H -6.20120143422032

H 2.16174793503907

H 2.26051217232965 
$\begin{array}{llll}\mathrm{H} & -5.23408483267018 & -1.01632091013958 & 1.87449566826575\end{array}$ H $-3.85255791009589 \quad 4.01161521644713$

$\mathrm{H}-1.45284009899100-3.6096919961$

H 1.95224313654305

H $-2.81786354967098 \quad-0.48449563418215$

0.43306154208914 0.92986709822442 0.91736061531724

Allenyne_anion

32

Free energy $=-1090.745395$ Hartre

C $-1.65902641298808 \quad 1.49663368352286$

$\begin{array}{lll}\text { C } & -1.00604574638493 & 2.08605127265892\end{array}$

C $-1.71082767969913 \quad 3.09064946355197$

C $-2.96206108934489 \quad 3.52336438129144$

C $-3.56848823030456 \quad 2.96178874308834$

C -2.90910955642233

C 0.32854004873628

C 1.53245500319492

C 2.88869869082761

S 3.82144776087742

C 3.76476685242847

C 3.01072268427927

C 2.22689155118602

C 0.88365569625062

C 0.24464674945585

C -0.97413683680198

C -1.59920098348894

C -1.00134016078015

C 0.22843624502751

H -2.55178387283103

H -4.52891300453722

H 4.43972711140768

H 2.58130488731532

H -1.13950446101673

H $\quad 0.71582035380845$

H -3.37682010555241

H -1.46035307842065

H -3.46820819790794

H -1.49718034432541

H 0.69619247969868

H 3.42891852614160

1.93338952551881

1.68971113973600

1.41751616140370

1.09687145112034

1.24591426897435

3.03441478773666

3.92120183705345

4.82842702442749

5.20273225876022

4.46223203701780

4.87822933564904

6.02884096039205

6.74627895072617

6.33560257604753

6.35745883888396

3.33601188772044

3.35966898736996

5.38918332460521

0.7020052341812

3.54194687290461

1.45933243826429

4.28306605468928

4.32807811268830

7.62872919900787

6.91644670506921

1.41108424071608

3.57699224526669

$-1.13789448783034$ $-0.02813805117924$ 0.67915681563831 0.24696218196009 $-0.88133517276057$ $-1.56099927993295$ 0.27160703325784 0.36245637076350 0.42127762333893 $-1.06535929193742$ $-1.42158320612604$ $-0.81528037647234$ $-0.25665242220316$ $-0.75948569442485$ $-1.76759169514807$ $-2.29453724820139$ $-1.80500792467001$ $-0.76840979772626$ $-0.24955460502759$ $-2.2638725814946$ $-1.24164202704158$ $-2.22313877805120$ 0.61816965999912 $-1.6747221038726$ $-2.11857835435227$ $-2.42693537816243$ $-3.06983819246237$ 0.78583118396206 $-0.35781457215876$ 0.54884401252236 1.32190925311158 1.53260778333712

\section{X=-SO2-}

\section{Bispropargy}

35

Free energy $=-1241.463728$ Hartree

C $-0.51820336357889 \quad 6.06993767909300$

$\begin{array}{lll}\text { C } & 0.24656021126631 & 5.25235425262370\end{array}$

C $-0.33451499731430 \quad 4.72355596545751$

C $\quad-1.64887689204564 \quad 5.04295292659049$

C $-2.39251606214613 \quad 5.89098059689356$

C $-1.82748527726906 \quad 6.39511001751012$

C $1.62736865599823 \quad 4.97348960050588$

$\begin{array}{lll}\text { C } & 2.79897602660349 & 4.73795207589659\end{array}$

$\begin{array}{lll}\text { C } & 4.20521317268862 & 4.42070084039187\end{array}$

$\begin{array}{lll}\text { S } & 4.66899936356298 & 2.81328567874498\end{array}$

C $4.23376755921084 \quad 1.51427512003692$

C $2.84228242437733 \quad 1.64246714588816$

C $\quad 1.69145849313291 \quad 1.92129022643968$

$\begin{array}{lll}\text { C } & 0.34762587896864 & 2.31966298360019\end{array}$

$\begin{array}{llr}\text { C } & -0.74221606534217 & 1.76069929210163\end{array}$

$\begin{array}{lll}\text { C } & -2.03712434695398 & 2.17005044316361\end{array}$

$\begin{array}{lll}\text { C } & -2.25094590708618 & 3.13356634168172\end{array}$

C $-1.16577527305764 \quad 3.69848982049574$

$\begin{array}{lll}\text { C } & 0.13095703977622 & 3.29768142519040\end{array}$

$\begin{array}{lll}\mathrm{H} & -3.40673404827168 & 6.17741934577540\end{array}$

H $\quad-3.26334001737450 \quad 3.45657671381495$

H $4.45585362974189 \quad 0.60321945599748$

H $4.53247573206010 \quad 4.41025012367331$

$\begin{array}{lll}\mathrm{H} & -0.56147457267699 & 1.01370627151284\end{array}$

$\begin{array}{llr}\mathrm{H} & -0.56147457267699 & 1.01370627151284 \\ \mathrm{H} & 0.26711711227034 & 4.08534018477993\end{array}$

H $\quad-2.87788782570859 \quad 1.73002552441152$

H $-2.09184557422076 \quad 4.63594925210563$

$\begin{array}{lll}\mathrm{H} & -1.33508352272724 & 4.45684246982515\end{array}$

$\mathrm{H} \quad-2.41264706478403 \quad 7.04316466657932$

$\begin{array}{lll}\mathrm{H} & -0.07496708586464 & 6.45533411022281\end{array}$

H $4.86311121260766 \quad 5.10237381525329$

H $\quad 0.98763960139877 \quad 3.73673295006552$

H $4.94136818300228 \quad 1.59636357413163$

O $6.12873360197225 \quad 2.80912533699802$

O 3.85577347895031

2.60856693311602

Bispropargyl_anion

34

Free energy $=-1240.978347$ Hartree

$\begin{array}{llll}\text { C } & -0.22162224279939 & 6.38677628945982 & 1.32993542561802\end{array}$

$\begin{array}{llll}\text { C } & 0.32831388298116 & 5.20980935654948 & 1.86318966547227\end{array}$

C -0.44377858183631

C -1.71940482241366

C -2.24398983105920

C -1.49893790659811

C 1.70270538335240

C 2.89655021087732

C 4.33777613181627

S 4.96830080551113

O 4.66920601727667

C 4.17193222310419

C 2.96989062393078

C 1.93760687419287

C 0.76572899475355

C -0.09220928573229

C -1.22886899413886

C -1.55354839747729

C -0.71587031889399

C 0.43209994822566

O 6.38300646259862

H -2.45480792496661

H -3.22045186318539

H 4.87350580911589

H 4.58945998976932

H $\quad-0.02090280215157$

H 1.08095384107617

H $\quad-2.30372942678181$

H -0.97563107150223

H -1.90533313304075

H -1.86898646574964

H $\quad 0.15153002503658$

H 0.39349797377346

H 4.66075287093510

4.41671642826231

4.83190470644597

6.02781006757644

6.79561644794559

4.89976049736458

4.77023429819541

4.85100455458002

6.33865411205401

5.95716087472408

7.76481506136038

8.03639013112309

8.18309778049836

8.23289797220582

9.35355655572373

9.36448051479291

8.25994810813062

7.14027589894016

7.12567422989559

6.39837037190007

8.26369582467890

6.37973555926339

4.01321230154465

8.30479704527319

3.49768583759066

6.24705202089538

4.22389511217278

6.26016624839227

7.72854376080542

10.24935397951030

10.2147124702386

7.00226507206983

4.95286450983605

2.73006913451612

3.10020725452908

2.60336261395955

1.70608811513405

1.58160913269246

1.40401489296064

26139452128853

.18576096027428

3.57896870251014

1.76367689528874

.41184140587267

3.06435842418119

3.87105650451173

3.89089153619443

4.69782941695817

5.48942246026491

5.4704287552717

4.68487481350749

1.75824276924093

6.10397081313690

2.94188242148322

1.72855541080650

0.91622404885654

.65936311340192

3.79363634170452

6.06295882201647

1.31296165294690

4.71375585521790

3.26661464426481

0.67113253201807

0.21620814046882

Allenyne

35

Free energy $=-1241.452329$ Hartree

C $-2.57411722474010 \quad-3.82980821910311$

C -2.15348890019743

C -2.88089640129978

C -4.00522898787146

C -4.41274066727601

C -3.70157143999058

C -0.91820601445734

C -0.26463111873079

C 0.46699639360632

S 0.37535702379121

C 0.03527838437342

C -1.38376735317914

C -2.56244439990378

C -3.95533376695139

C -4.61906813142268

C -5.94124156262023

C -6.61374123181825

C -5.97339691336616

C -4.64951921323908

H -5.28708742962975

H -7.63639831544837

H 0.59672906285682

H 1.23838694035113

H -0.47517887047105

$\mathrm{H}-4.08184946180557$

H -2.5613721170

137211700024

H -6.44930488556236

H $\quad-4.56877711609087$

H -6.50675031596133

H -4.02320753992268

H -2.00488913181455

H $\quad 0.50530209660324$

H -4.13450541710967

O 1.73977873095414

O -0.73645970465564

$-2.72222334782404$

$-2.33791635080676$

$-3.06634663604200$

$-4.17750692148575$

$-4.55296244116114$

$-2.02317729265594$

$-1.14100655128160$

$-0.33095648182193$

1.45953643722485

.91361512268282

2.10648735282705 


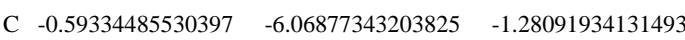

$\begin{array}{llll}\text { C } & -1.82226160977198 & -6.55108292868196 & -0.83068564183577\end{array}$

$\begin{array}{llll}\text { C } & -2.34237400273432 & -6.11460060998720 & 0.38769997955838\end{array}$

$\begin{array}{llll}\text { C } & -1.62921431675790 & -5.18567352673892 & 1.15069241153194\end{array}$

$\begin{array}{llll}\text { C } & -0.40199900039233 & -4.70858504747505 & 0.70503067140498\end{array}$

$\begin{array}{llll}\text { O } & 4.26000737755148 & -0.84151377293597 & -0.50915637731706\end{array}$

$\begin{array}{llll}\mathrm{H} & -3.30337488422365 & -6.48819318170751 & 0.74528132570754\end{array}$

$\begin{array}{llll}\mathrm{H} & -5.49687238387578 & -3.51337885320172 & 0.76948984184606\end{array}$

$\begin{array}{llll}\mathrm{H} & 3.71916830011072 & -3.25794576141194 & 1.06003047714701\end{array}$

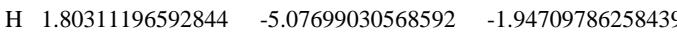

$\begin{array}{llll}\mathrm{H} & -2.10276475687598 & -0.96880441048783 & 1.53648882976248\end{array}$
$\begin{array}{llll}\mathrm{H} & 0.14808666458813 & -3.96760480020652 & 1.28946477783232\end{array}$ $\mathrm{H}-4.30171046729380-1.87622464176708 \quad 2.2256583219672$ $\begin{array}{llll}\mathrm{H} & -2.04889355442542 & -4.80906569462849 & 2.08562743268717\end{array}$

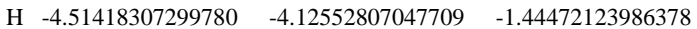

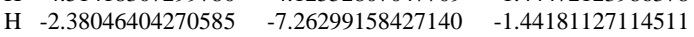
H $\quad-0.18878039841663 \quad-6.42221717866977 \quad-2.23225239790012$ H $2.15976584536772 \quad-1.01259448098172 \quad-2.20235933265483$ $\begin{array}{llll}\text { H } & -2.33235093882097 & -3.19758093113977 & -2.14364398071053\end{array}$

\section{Unsymmetrical Bispropargyl}

\section{$X=-0-$}

\section{$A=4-$ methoxyphenyl, $B=$ phenyl}

\section{Bis
37
Free}

\begin{tabular}{|c|c|c|c|}
\hline \multicolumn{4}{|c|}{ ree energy $=-88$} \\
\hline $\mathrm{C}$ & -0.162708 & 4.688769 & 2.62151 \\
\hline $\mathrm{C}$ & 0.784108 & 5.531896 & \\
\hline $\mathrm{C}$ & 0.310644 & 6.588487 & 615 \\
\hline $\mathrm{C}$ & -1.065456 & 796451 & \\
\hline $\mathrm{C}$ & -1.9935 & & \\
\hline $\mathrm{C}$ & -1.536196 & 04 & \\
\hline $\mathrm{C}$ & 2.1861 & & \\
\hline C & 3. & & \\
\hline C & 4.81 & & \\
\hline $\mathrm{O}$ & 5.509 & 4.2 & 500 \\
\hline $\mathrm{C}$ & 5.3719 & 74 & 367 \\
\hline $\mathrm{C}$ & 4.00 & & \\
\hline C & 2.7 & & \\
\hline C & & & \\
\hline C & & & \\
\hline C & & & \\
\hline $\mathrm{C}$ & & & \\
\hline $\mathrm{C}$ & & & \\
\hline $\mathrm{C}$ & & & \\
\hline 0 & & & \\
\hline $\mathrm{C}$ & -3. & & -0 \\
\hline- & -3 & & 1. \\
\hline $\mathrm{H}$ & & & 2.7 \\
\hline $\mathrm{H}$ & 5.7 & & 257 \\
\hline $\mathrm{H}$ & 1.0 & 02 & \\
\hline $\mathrm{H}$ & 1.38 & 3.8 & -0.9 \\
\hline $\mathrm{H}$ & -1.4 & & 973. \\
\hline $\mathrm{H}$ & -1.0 & 4.0 & -1 . \\
\hline $\mathrm{H}$ & -2.2 & 4.2 & 2.9 \\
\hline $\mathrm{H}$ & -1.4 & & $49-2$ \\
\hline $\mathrm{H}$ & & & 89 \\
\hline $\mathrm{H}$ & -4.5 & & -0.33796 \\
\hline $\mathrm{H}$ & -3.2 & & -1.60049 \\
\hline $\mathrm{H}$ & -3.1 & & \\
\hline $\mathrm{H}$ & $6.0^{7}$ & 2.64 & $0.0074+4$ \\
\hline $\mathrm{H}$ & & & \\
\hline $\mathrm{H}$ & 4.936002 & 4.110208 & 3.37210 \\
\hline
\end{tabular}

Allenyne_A

37

Free energy $=-883.1309908$ Hartree

$\begin{array}{lccc}\mathrm{C} & -0.896367 & -2.842005 & 0.127674 \\ \mathrm{C} & -0.180183 & -1.849866 & 0.846203 \\ \mathrm{C} & -0.902629 & -0.790905 & 1.454609 \\ \mathrm{C} & -2.298432 & -0.733328 & 1.354147 \\ \mathrm{C} & -2.999610 & -1.725397 & 0.645573 \\ \mathrm{C} & -2.293411 & -2.775060 & 0.028887 \\ \mathrm{C} & 1.245960 & -1.894935 & 0.938125 \\ \mathrm{C} & 2.470754 & -1.913966 & 1.031638 \\ \mathrm{C} & 3.927616 & -1.916959 & 1.180404 \\ \mathrm{O} & 4.659488 & -1.690095 & -0.035732 \\ \mathrm{C} & 4.486449 & -0.451023 & -0.610217 \\ \mathrm{C} & 3.734955 & 0.539488 & -0.154178 \\ \mathrm{C} & 2.867873 & 1.473196 & 0.229520 \\ \mathrm{C} & 1.451033 & 1.527191 & -0.171988 \\ \mathrm{C} & 0.914378 & 0.648346 & -1.140201 \\ \mathrm{C} & -0.445173 & 0.659782 & -1.469349 \\ \mathrm{C} & -1.317588 & 1.559571 & -0.816774 \\ \mathrm{C} & -0.795202 & 2.464274 & 0.135196 \\ \mathrm{C} & 0.566479 & 2.446377 & 0.446185 \\ \mathrm{O} & -2.663407 & 1.617875 & -1.021397 \\ \mathrm{C} & -3.242112 & 0.736788 & -1.973532\end{array}$

$\begin{array}{rrrc}\mathrm{H} & -4.097271 & -1.676908 & 0.567160 \\ \mathrm{H} & 4.280452 & -2.906394 & 1.538113 \\ \mathrm{H} & 5.091031 & -0.369265 & -1.530510 \\ \mathrm{H} & 3.197795 & 2.263132 & 0.933715 \\ \mathrm{H} & -0.340441 & -3.661786 & -0.351110 \\ \mathrm{H} & 1.584639 & -0.077756 & -1.625035 \\ \mathrm{H} & -2.837637 & -3.552804 & -0.529702 \\ \mathrm{H} & -0.823668 & -0.063698 & -2.203862 \\ \mathrm{H} & -2.841063 & 0.107650 & 1.811191 \\ \mathrm{H} & -1.488709 & 3.162248 & 0.626605 \\ \mathrm{H} & 0.958215 & 3.150127 & 1.199230 \\ \mathrm{H} & -4.327695 & 0.950674 & -1.974746 \\ \mathrm{H} & -3.081864 & -0.329385 & -1.695873 \\ \mathrm{H} & -2.838377 & 0.911376 & -2.996963 \\ \mathrm{H} & 4.217243 & -1.149651 & 1.939206 \\ \mathrm{H} & -0.347323 & 0.003321 & 1.974286\end{array}$

Allenyne_A2Cyclization_TS

37

Free energy $=-883.1161271$ Hartree

$\begin{array}{llll}\text { C } & -0.793882 & -2.599806 & -0.167298\end{array}$

$\begin{array}{llll}\text { C } & -0.107190 & -1.684876 & 0.682730\end{array}$

$\begin{array}{llll}\text { C } & -0.885068 & -0.816905 & 1.503960\end{array}$

$\begin{array}{llll}\text { C } & -2.283125 & -0.878021 & 1.485816\end{array}$

$\begin{array}{llll}\text { C } & -2.947293 & -1.799140 & 0.654458\end{array}$

$\begin{array}{llll}\text { C } & -2.193357 & -2.651674 & -0.175941\end{array}$

$\begin{array}{llll}\text { C } & 1.307229 & -1.617086 & 0.691904\end{array}$

$\begin{array}{llll}\text { C } & 2.554969 & -1.468614 & 0.701223\end{array}$

$\begin{array}{llll}\text { C } & 3.861279 & -2.032787 & 1.157917\end{array}$

$\begin{array}{llll}\text { O } & 4.916933 & -1.674483 & 0.253748\end{array}$

$\begin{array}{llll}\text { C } & 4.672834 & -0.442800 & -0.275540\end{array}$

$\begin{array}{llll}\text { C } & 3.523735 & 0.205992 & -0.014520\end{array}$

$\begin{array}{llll}\text { C } & 2.827650 & 1.368127 & 0.132090\end{array}$

$\begin{array}{llll}\text { C } & 1.440104 & 1.507055 & -0.259362\end{array}$

$\begin{array}{llll}\text { C } & 0.849614 & 0.626438 & -1.202922\end{array}$

$\begin{array}{llll}\text { C } & -0.522903 & 0.653269 & -1.475824\end{array}$

$\begin{array}{llll}\text { C } & -1.354807 & 1.554462 & -0.778880\end{array}$

$\begin{array}{llll}\text { C } & -0.778058 & 2.477355 & 0.133208\end{array}$

$\begin{array}{llll}\text { C } & 0.591197 & 2.459441 & 0.375041\end{array}$

$\begin{array}{llll}\text { O } & -2.704759 & 1.625948 & -0.915479\end{array}$

$\begin{array}{llll}\text { C } & -3.337794 & 0.763766 & -1.853705\end{array}$

$\begin{array}{lllr}\mathrm{H} & -4.047661 & -1.846036 & 0.645704\end{array}$

$\begin{array}{llll}\mathrm{H} & 3.844416 & -3.139083 & 1.212303\end{array}$

$\begin{array}{llll}\mathrm{H} & 5.436973 & -0.128368 & -1.004193\end{array}$

$\begin{array}{llll}\mathrm{H} & 3.262595 & 2.177226 & 0.749807\end{array}$

$\begin{array}{llll}\mathrm{H} & -0.205967 & -3.267337 & -0.814847\end{array}$

$\begin{array}{llll}\mathrm{H} & 1.494267 & -0.093343 & -1.728362\end{array}$

$\begin{array}{llll}\mathrm{H} & -2.705548 & -3.371564 & -0.834079\end{array}$

$\begin{array}{llll}\mathrm{H} & -0.941664 & -0.067758 & -2.190383\end{array}$

$\begin{array}{llll}\mathrm{H} & -2.863410 & -0.187797 & 2.117278\end{array}$

$\begin{array}{llll}\mathrm{H} & -1.446263 & 3.185087 & 0.645267\end{array}$

$\mathrm{H} \quad 1.025060 \quad 3.167505 \quad 1.100196$

$\begin{array}{llll}\mathrm{H} & -4.420915 & 0.980563 & -1.790890\end{array}$

$\begin{array}{llll}\mathrm{H} & -3.164436 & -0.306536 & -1.606748\end{array}$

$\begin{array}{llll}\mathrm{H} & -2.987022 & 0.962343 & -2.892120\end{array}$

$\mathrm{H} \quad 4.091504 \quad-1.621181 \quad 2.168674$

$\begin{array}{llll}\mathrm{H} & -0.365484 & -0.077647 & 2.130736\end{array}$

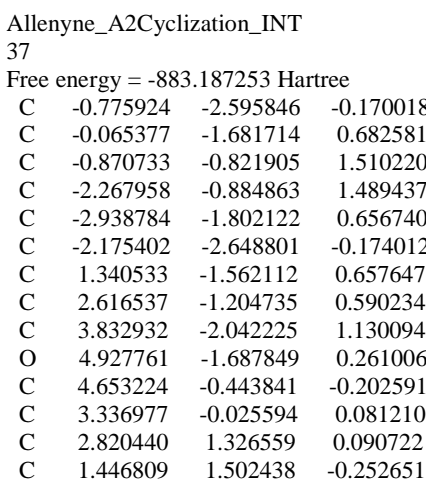




$\begin{array}{crcc}\mathrm{C} & 0.842509 & 0.611015 & -1.190499 \\ \mathrm{C} & -0.527551 & 0.643701 & -1.468345 \\ \mathrm{C} & -1.354492 & 1.546962 & -0.772392 \\ \mathrm{C} & -0.771046 & 2.480692 & 0.135569 \\ \mathrm{C} & 0.594838 & 2.472624 & 0.370047 \\ \mathrm{O} & -2.700118 & 1.626956 & -0.912662 \\ \mathrm{C} & -3.337135 & 0.763761 & -1.852164 \\ \mathrm{H} & -4.038809 & -1.850122 & 0.647110 \\ \mathrm{H} & 3.702568 & -3.146987 & 1.114490 \\ \mathrm{H} & 5.442148 & 0.018494 & -0.835919 \\ \mathrm{H} & 3.390793 & 2.168835 & 0.562424 \\ \mathrm{H} & -0.197043 & -3.263863 & -0.825804 \\ \mathrm{H} & 1.487203 & -0.090695 & -1.739174 \\ \mathrm{H} & -2.683061 & -3.370145 & -0.834910 \\ \mathrm{H} & -0.947187 & -0.075158 & -2.184253 \\ \mathrm{H} & -2.844874 & -0.193027 & 2.123108 \\ \mathrm{H} & -1.441134 & 3.188843 & 0.644745 \\ \mathrm{H} & 1.030660 & 3.187697 & 1.087008 \\ \mathrm{H} & -4.419533 & 0.981400 & -1.784938 \\ \mathrm{H} & -3.161078 & -0.305213 & -1.606117 \\ \mathrm{H} & -2.987393 & 0.967913 & -2.889564 \\ \mathrm{H} & 4.083402 & -1.695407 & 2.161168 \\ \mathrm{H} & -0.361003 & -0.077063 & 2.139168\end{array}$

Allenyne_B

37

Free energy $=-883.1271281$ Hartree

$\begin{array}{cccc}\text { Cree } & 0.659111 & 1.857017 & -0.798974 \\ \mathrm{C} & 0.904356 & 2.403558 & 0.484622 \\ \mathrm{C} & -0.201312 & 2.826815 & 1.259850 \\ \mathrm{C} & -1.511931 & 2.707238 & 0.769658 \\ \mathrm{C} & -1.738803 & 2.164809 & -0.506335 \\ \mathrm{C} & -0.646704 & 1.741362 & -1.287441 \\ \mathrm{C} & 2.271923 & 2.520838 & 1.031351 \\ \mathrm{C} & 3.371669 & 2.066818 & 0.439065 \\ \mathrm{C} & 4.392495 & 1.584586 & -0.255047 \\ \mathrm{O} & 4.918719 & 0.319826 & -0.186463 \\ \mathrm{C} & 4.285596 & -0.569161 & 0.755687 \\ \mathrm{C} & 2.902193 & -0.922540 & 0.441332 \\ \mathrm{C} & 1.730559 & -1.224710 & 0.224451 \\ \mathrm{C} & 0.364733 & -1.572602 & -0.003544 \\ \mathrm{C} & -0.675078 & -0.894088 & 0.677272 \\ \mathrm{C} & -2.020445 & -1.214792 & 0.457483 \\ \mathrm{C} & -2.359432 & -2.231234 & -0.462714 \\ \mathrm{C} & -1.331329 & -2.918086 & -1.151075 \\ \mathrm{C} & 0.006258 & -2.595627 & -0.924493 \\ \mathrm{O} & -3.632808 & -2.615507 & -0.757967 \\ \mathrm{C} & -4.709764 & -1.959619 & -0.107097 \\ \mathrm{H} & -2.765164 & 2.062270 & -0.890902 \\ \mathrm{H} & 4.956246 & 2.201315 & -0.977253 \\ \mathrm{H} & 4.325006 & -0.112097 & 1.774455 \\ \mathrm{H} & 2.350431 & 3.010449 & 2.022241 \\ \mathrm{H} & 1.513657 & 1.507869 & -1.397773 \\ \mathrm{H} & -0.419180 & -0.077339 & 1.368131 \\ \mathrm{H} & -0.820517 & 1.298554 & -2.279602 \\ \mathrm{H} & -2.793637 & -0.649165 & 0.993230 \\ \mathrm{H} & -2.359606 & 3.040101 & 1.388503 \\ \mathrm{H} & -1.618810 & -3.706103 & -1.862992 \\ \mathrm{H} & 0.799745 & -3.132929 & -1.464281 \\ \mathrm{H} & -5.636144 & -2.424034 & -0.492719 \\ \mathrm{H} & -4.731392 & -0.869242 & -0.335243 \\ \mathrm{H} & -4.671374 & -2.092805 & 0.998386 \\ \mathrm{H} & 4.929088 & -1.473112 & 0.757738 \\ \mathrm{H} & -0.025400 & 3.251192 & 2.261507\end{array}$

Allenyne_B2Cyclization_TS

37

Free energy $=-883.1108098$ Hartree

$\begin{array}{lrrr}\mathrm{C} & 0.671361 & 1.733682 & -0.993618 \\ \mathrm{C} & 0.947910 & 2.426843 & 0.215843 \\ \mathrm{C} & -0.147107 & 2.951158 & 0.956446 \\ \mathrm{C} & -1.463141 & 2.736303 & 0.536016 \\ \mathrm{C} & -1.719095 & 2.005591 & -0.643129 \\ \mathrm{C} & -0.648568 & 1.526948 & -1.415137 \\ \mathrm{C} & 2.301007 & 2.507268 & 0.738242 \\ \mathrm{C} & 3.283440 & 1.634132 & 0.376348 \\ \mathrm{C} & 4.587949 & 1.416281 & 0.116939 \\ \mathrm{O} & 5.141292 & 0.186604 & 0.305440 \\ \mathrm{C} & 4.176592 & -0.687606 & 0.908860 \\ \mathrm{C} & 2.794325 & -0.341804 & 0.453428 \\ \mathrm{C} & 1.646698 & -0.809579 & 0.236934 \\ \mathrm{C} & 0.319336 & -1.242013 & 0.011330 \\ \mathrm{C} & -0.709700 & -0.977930 & 0.956302 \\ \mathrm{C} & -2.032710 & -1.374286 & 0.727090 \\ \mathrm{C} & -2.371141 & -2.044936 & -0.469476 \\ \mathrm{C} & -1.360034 & -2.312705 & -1.423658 \\ \mathrm{C} & -0.043432 & -1.924840 & -1.188583 \\ \mathrm{O} & -3.627314 & -2.459526 & -0.800417 \\ \mathrm{C} & -4.684920 & -2.223429 & 0.115639 \\ \mathrm{H} & -2.755804 & 1.816966 & -0.961588\end{array}$

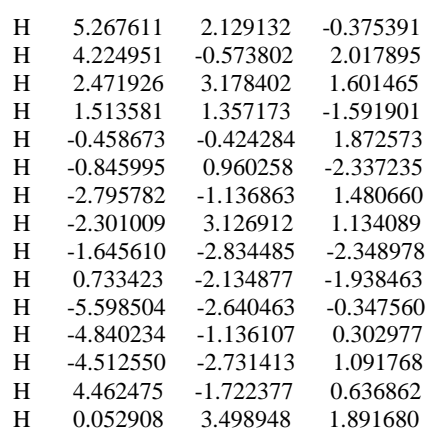

Allenyne_B2Cyclization_INT

37

Free energy $=-883.189253$ Hartree

$\begin{array}{llll}\text { C } & 0.460519 & 1.879348 & -0.456727\end{array}$

$\begin{array}{llll}\text { C } & 1.538356 & 2.909045 & -0.089646\end{array}$

$\begin{array}{llll}\text { C } & 1.211905 & 4.296759 & -0.349324\end{array}$

$\begin{array}{llll}\text { C } & -0.091863 & 4.705862 & -0.481671\end{array}$

$\begin{array}{llll}\text { C } & -1.180482 & 3.759611 & -0.283977\end{array}$

$\begin{array}{llll}\text { C } & -0.938410 & 2.424790 & -0.204545\end{array}$

$\begin{array}{llll}\text { C } & 2.755869 & 2.530290 & 0.425696\end{array}$

$\begin{array}{llll}\text { C } & 2.970100 & 1.165120 & 0.812892\end{array}$

$\begin{array}{llll}\text { C } & 4.074768 & 0.485552 & 1.266343\end{array}$

$\begin{array}{llll}\text { O } & 3.856063 & -0.834072 & 1.515412\end{array}$

$\begin{array}{llll}\text { C } & 2.440468 & -1.091644 & 1.285250\end{array}$

$\begin{array}{llll}\text { C } & 1.882047 & 0.193190 & 0.709520\end{array}$

$\begin{array}{llll}\text { C } & 0.685560 & 0.469660 & 0.110734\end{array}$

$\begin{array}{llll}\text { C } & -0.345628 & -0.562162 & -0.133528\end{array}$

$\begin{array}{llll}\text { C } & -0.622231 & -1.586325 & 0.802392\end{array}$

$\begin{array}{llll}\text { C } & -1.569833 & -2.593211 & 0.555587\end{array}$

$\begin{array}{llll}\text { C } & -2.283652 & -2.600675 & -0.661742\end{array}$

$\begin{array}{llll}\text { C } & -2.035725 & -1.578479 & -1.606909\end{array}$

$\begin{array}{llll}\text { C } & -1.094681 & -0.582537 & -1.343655\end{array}$

$\begin{array}{llll}\text { O } & -3.221946 & -3.526628 & -1.012237\end{array}$

$\begin{array}{llll}\text { C } & -3.509319 & -4.577293 & -0.103801\end{array}$

$\begin{array}{llll}\mathrm{H} & -2.205109 & 4.146187 & -0.161994\end{array}$

$\begin{array}{llll}\mathrm{H} & 5.103383 & 0.830656 & 1.436255\end{array}$

$\begin{array}{llll}\mathrm{H} & 2.001046 & -1.357817 & 2.272069\end{array}$

$\begin{array}{llll}\mathrm{H} & 3.548320 & 3.280604 & 0.580392\end{array}$

$\begin{array}{llll}\mathrm{H} & 0.538704 & 1.794199 & -1.579366\end{array}$

$\begin{array}{llll}\mathrm{H} & -0.118032 & -1.577036 & 1.779391\end{array}$

$\begin{array}{llll}\mathrm{H} & -1.752833 & 1.708907 & -0.017374\end{array}$

$\begin{array}{llll}\mathrm{H} & -1.752924 & -3.355016 & 1.325582\end{array}$

$\begin{array}{llll}\mathrm{H} & -0.325484 & 5.771191 & -0.634642\end{array}$

$\begin{array}{llll}\mathrm{H} & -2.600051 & -1.595178 & -2.550864\end{array}$

$\begin{array}{llll}\mathrm{H} & -0.924381 & 0.194172 & -2.104013\end{array}$

$\begin{array}{llll}\mathrm{H} & -4.279992 & -5.207071 & -0.587263\end{array}$

$\begin{array}{llll}\mathrm{H} & -3.911918 & -4.195612 & 0.862505\end{array}$

$\begin{array}{llll}\mathrm{H} & -2.612019 & -5.204009 & 0.106810\end{array}$

$\begin{array}{llll}\mathrm{H} & 2.333305 & -1.971378 & 0.614997\end{array}$

$\begin{array}{llll}\mathrm{H} & 2.030469 & 5.035827 & -0.344080\end{array}$

\section{Bisallene}

37

Free energy $=-883.1392326$ Hartree

$\begin{array}{llll}\text { Free energy }= & -883.1392326 \text { Hartree } \\ \text { C } & 2.367395 & 7.004657 & -1.374042\end{array}$

$\begin{array}{llll}\text { C } & 3.695426 & 7.163826 & -1.845327\end{array}$

$\begin{array}{llll}\text { C } & 3.957584 & 6.861435 & -3.200917\end{array}$

$\begin{array}{llll}\text { C } & 2.948670 & 6.397260 & -4.055594\end{array}$

$\begin{array}{llll}\text { C } & 1.636005 & 6.223773 & -3.560107\end{array}$

$\begin{array}{llll}\text { C } & 1.354141 & 6.543992 & -2.211839\end{array}$

$\begin{array}{llll}\text { C } & 4.762235 & 7.535993 & -0.909628\end{array}$

$\begin{array}{llll}\text { C } & 6.063910 & 7.604340 & -1.184213\end{array}$

$\begin{array}{llll}\text { C } & 6.754459 & 4.974551 & -1.752400\end{array}$

$\begin{array}{llll}\text { C } & 7.943866 & 5.457931 & -1.432282\end{array}$

$\begin{array}{llll}\text { O } & 8.332186 & 6.783931 & -1.415195\end{array}$

$\begin{array}{llll}\text { C } & 7.350534 & 7.761674 & -1.444349\end{array}$

$\begin{array}{llll}\text { C } & 5.604894 & 4.390205 & -2.084480\end{array}$

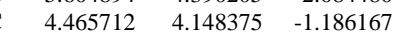

$\begin{array}{llll}\text { C } & 4.508691 & 4.507728 & 0.183642\end{array}$

$\begin{array}{llll}\text { C } & 3.379659 & 4.346945 & 0.996708\end{array}$

$\begin{array}{llll}C & 2.187385 & 3.822469 & 0.461500\end{array}$

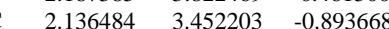

$\begin{array}{llll}\text { C } & 2.136484 & 3.452203 & -0.893668 \\ \text { C } & 3.265034 & 3.612281 & -1.709004\end{array}$

$\begin{array}{llll}\text { O } & 0.584884 & 5.755637 & -4.292173\end{array}$

$\begin{array}{llll}\text { C } & 0.799753 & 5.421351 & -5.653832\end{array}$

$\begin{array}{llll}\mathrm{H} & 1.299198 & 3.702227 & 1.101089\end{array}$

$\begin{array}{llll}\mathrm{H} & 8.795511 & 4.809716 & -1.165182\end{array}$

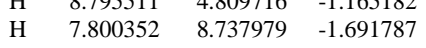

$\begin{array}{llll}\mathrm{H} & 5.440733 & 4.120872 & -3.145798\end{array}$

$\begin{array}{llll}\mathrm{H} & 4.443376 & 7.690645 & 0.139753\end{array}$

$\begin{array}{llll}\mathrm{H} & 5.439300 & 4.932239 & 0.590023\end{array}$

$\begin{array}{llll}\mathrm{H} & 4.984408 & 6.977533 & -3.579701\end{array}$

$\begin{array}{lllr}\mathrm{H} & 3.425302 & 4.637354 & 2.058068\end{array}$ 


$\begin{array}{rrrr}\mathrm{H} & 3.196802 & 6.163786 & -5.099937 \\ \mathrm{H} & 1.205589 & 3.049509 & -1.321696 \\ \mathrm{H} & 0.328026 & 6.395805 & -1.845694 \\ \mathrm{H} & 2.141316 & 7.215782 & -0.317440 \\ \mathrm{H} & -0.173489 & 5.073288 & -6.047638 \\ \mathrm{H} & 1.136671 & 6.299234 & -6.251075 \\ \mathrm{H} & 1.548628 & 4.604025 & -5.769684 \\ \mathrm{H} & 3.214482 & 3.347702 & -2.776807\end{array}$

Bisallene2Cyclization_TS1

37

Free energy $=-883.1347011$ Hartree

$\begin{array}{llll}\text { C } & 2.391751 & 7.068263 & -1.379534\end{array}$

$\begin{array}{llll}\text { C } & 3.730065 & 7.173171 & -1.855885\end{array}$

$\begin{array}{llll}\text { C } & 3.978936 & 6.829992 & -3.209438\end{array}$

$\begin{array}{llll}\text { C } & 2.954285 & 6.368273 & -4.047611\end{array}$

$\begin{array}{llll}\text { C } & 1.643698 & 6.233772 & -3.539975\end{array}$

$\begin{array}{llll}\text { C } & 1.370240 & 6.607957 & -2.199571\end{array}$

$\begin{array}{llll}\text { C } & 4.809185 & 7.479237 & -0.936785\end{array}$

$\begin{array}{llll}\text { C } & 6.128534 & 7.345695 & -1.221940\end{array}$

$\begin{array}{llll}\text { C } & 6.653073 & 5.238933 & -1.718386\end{array}$

$\begin{array}{llll}\text { C } & 7.943637 & 5.499857 & -1.512638\end{array}$

$\begin{array}{llll}\text { O } & 8.427056 & 6.783410 & -1.379737\end{array}$

$\begin{array}{llll}\text { C } & 7.407056 & 7.708369 & -1.315112\end{array}$

$\begin{array}{llll}\text { C } & 5.573324 & 4.501789 & -2.080988\end{array}$

$\begin{array}{llll}\text { C } & 4.452352 & 4.189518 & -1.207117\end{array}$

$\begin{array}{llll}\text { C } & 4.463875 & 4.554027 & 0.166630\end{array}$

$\begin{array}{llll}\text { C } & 3.326508 & 4.365214 & 0.962789\end{array}$

$\begin{array}{llll}\text { C } & 2.157382 & 3.809073 & 0.411700\end{array}$

$\begin{array}{llll}\text { C } & 2.139014 & 3.423351 & -0.943184\end{array}$

$\begin{array}{llll}\text { C } & 3.271108 & 3.608558 & -1.742492\end{array}$

$\begin{array}{llll}\text { O } & 0.579259 & 5.763931 & -4.248763\end{array}$

$\begin{array}{llll}\text { C } & 0.781718 & 5.362245 & -5.594696\end{array}$

$\begin{array}{llll}\mathrm{H} & 1.261857 & 3.670247 & 1.037155\end{array}$

$\begin{array}{llll}\mathrm{H} & 8.735366 & 4.742717 & -1.408680\end{array}$

$\begin{array}{llll}\mathrm{H} & 7.776592 & 8.742420 & -1.388545\end{array}$

$\begin{array}{llll}\mathrm{H} & 5.438155 & 4.265338 & -3.152936\end{array}$

$\begin{array}{llll}\mathrm{H} & 4.520695 & 7.636413 & 0.120144\end{array}$

$\begin{array}{llll}\mathrm{H} & 5.378983 & 4.996080 & 0.587991\end{array}$

$\begin{array}{llll}\mathrm{H} & 5.003265 & 6.924730 & -3.598769\end{array}$

$\begin{array}{llll}\mathrm{H} & 3.347889 & 4.660179 & 2.023719\end{array}$

$\begin{array}{llll}\mathrm{H} & 3.190886 & 6.102203 & -5.086696\end{array}$

$\begin{array}{llll}\mathrm{H} & 1.225673 & 2.990323 & -1.379821\end{array}$

$\begin{array}{llll}\mathrm{H} & 0.341001 & 6.494192 & -1.829510\end{array}$

$\begin{array}{llll}\mathrm{H} & 2.175002 & 7.313916 & -0.328526\end{array}$

$\begin{array}{llll}\mathrm{H} & -0.198984 & 5.012001 & -5.967179\end{array}$

$\begin{array}{llll}\mathrm{H} & 1.129639 & 6.205844 & -6.23343\end{array}$

$\begin{array}{llll}\mathrm{H} & 1.516989 & 4.528523 & -5.673154\end{array}$

$\begin{array}{llll}\mathrm{H} & 3.244579 & 3.335672 & -2.809131\end{array}$

Bisallene2Cyclization_INT1

37

Free energy $=-883.1786541$ Hartree

$\begin{array}{llll}\text { C } & 2.250242 & 6.484346 & -1.122925 \\ \text { C } & 2.967266 & 5.248650 & -1.162414\end{array}$

$\begin{array}{llll}\text { C } & 2.967266 & 5.248650 & -1.162414 \\ \text { C } & 2.261106 & 4.101717 & -1.641631\end{array}$

$\begin{array}{llll}\text { C } & 2.261106 & 4.101717 & -1.641631 \\ \text { C } & 0.293055 & 4.206946 & -2.138027\end{array}$

$\begin{array}{llll}\text { C } & 0.293055 & 5.451433 & -2.137285 \\ \text { C } & 0.937967 & 6.578414 & -1.593261\end{array}$

$\begin{array}{llll}\text { C } & 0.937967 & 6.578414 & -1.593261\end{array}$

$\begin{array}{llll}\text { C } & 4.341335 & 5.122595 & -0.764644\end{array}$

$\begin{array}{llll}\text { C } & 5.371463 & 6.101863 & -0.678200\end{array}$

$\begin{array}{llll}\text { C } & 5.556464 & 7.487020 & -1.214103\end{array}$

$\begin{array}{llll}\text { C } & 6.757916 & 7.931473 & -0.624900\end{array}$

$\begin{array}{llll}\mathrm{O} & 7.330628 & 6.961192 & 0.122483\end{array}$

$\begin{array}{llll}\text { C } & 6.531816 & 5.869172 & 0.086784\end{array}$

$\begin{array}{llll}\text { C } & 4.848384 & 8.309082 & -2.135996\end{array}$

$\begin{array}{llll}\text { C } & 3.850444 & 7.971596 & -3.108965\end{array}$

$\begin{array}{llll}\text { C } & 3.682744 & 6.659197 & -3.641368\end{array}$

$\begin{array}{llll}\text { C } & 2.647383 & 6.340227 & -4.52110\end{array}$

$\begin{array}{llll}\text { C } & 1.706496 & 7.330415 & -4.884173\end{array}$

$\begin{array}{llll}\text { C } & 1.889111 & 8.661717 & -4.429102\end{array}$

$\begin{array}{llll}\text { C } & 2.944398 & 8.974525 & -3.579746\end{array}$

$\begin{array}{llll}\text { O } & 0.615210 & 7.111873 & -5.66724\end{array}$

$\begin{array}{llll}\text { C } & 0.321595 & 5.777950 & -6.057660\end{array}$

$\begin{array}{llll}\mathrm{H} & -0.729927 & 5.535104 & -2.533937\end{array}$

$\begin{array}{llll}\mathrm{H} & 6.854306 & 5.005211 & 0.676813\end{array}$

$\begin{array}{llll}\mathrm{H} & 7.309095 & 8.873397 & -0.714824\end{array}$

$\begin{array}{llll}\mathrm{H} & 4.652109 & 4.108091 & -0.457868\end{array}$

$\begin{array}{llll}\mathrm{H} & 5.131487 & 9.376664 & -2.101678\end{array}$

\begin{tabular}{llll}
$\mathrm{H}$ & 2.744502 & 7.371247 & -0.704644 \\
\hline
\end{tabular}

$\begin{array}{llll}\mathrm{H} & 4.394996 & 5.874794 & -3.352891\end{array}$

$\begin{array}{llll}\mathrm{H} & 0.419256 & 7.548629 & -1.561709\end{array}$

$\begin{array}{llll}\mathrm{H} & 2.552710 & 5.307537 & -4.879143\end{array}$

$\begin{array}{llll}\mathrm{H} & 0.452728 & 3.312014 & -2.532395\end{array}$

$\begin{array}{llll}\mathrm{H} & 1.166195 & 9.424571 & -4.753559\end{array}$

$\begin{array}{llll}\mathrm{H} & 3.060773 & 10.009528 & -3.220736\end{array}$

$\begin{array}{llll}\mathrm{H} & -0.631699 & 5.819472 & -6.617304\end{array}$

$\begin{array}{llll}\mathrm{H} & 1.110199 & 5.354175 & -6.720349\end{array}$

$\begin{array}{llll}\mathrm{H} & 0.201850 & 5.114078 & -5.171264\end{array}$

$\begin{array}{llll}\mathrm{H} & 2.776702 & 3.127585 & -1.655297\end{array}$

\begin{tabular}{llll}
\multicolumn{5}{l}{ Bisallene2Cyclization_TS2_for_A } \\
37 & \multicolumn{3}{l}{ _ } \\
Free energy = -883.1695727 Hartree \\
C & 3.058855 & 8.579036 & -3.445181 \\
C & 4.170787 & 7.864103 & -2.870419 \\
C & 4.170368 & 6.424780 & -3.022866 \\
C & 3.085262 & 5.749716 & -3.616259 \\
C & 1.969267 & 6.475849 & -4.054794 \\
C & 1.987316 & 7.909222 & -4.003641 \\
C & 5.150485 & 8.511883 & -2.089187 \\
C & 5.954163 & 7.776711 & -1.136328 \\
C & 5.505070 & 6.566827 & -0.416261 \\
C & 6.539807 & 6.251566 & 0.436011 \\
O & 7.592701 & 7.124547 & 0.300739 \\
C & 7.222647 & 8.036685 & -0.649947 \\
C & 4.192369 & 5.948568 & -0.577873 \\
C & 3.899941 & 4.546858 & -0.630539 \\
C & 4.908728 & 3.538369 & -0.724071 \\
C & 4.567950 & 2.189602 & -0.870871 \\
C & 3.217135 & 1.789301 & -0.931202 \\
C & 2.205627 & 2.767871 & -0.847808 \\
C & 2.535970 & 4.118458 & -0.703618 \\
O & 0.836545 & 5.928102 & -4.568634 \\
C & 0.724188 & 4.510470 & -4.579399 \\
H & 2.955416 & 0.726091 & -1.044423 \\
H & 6.647057 & 5.486705 & 1.212861 \\
H & 7.975043 & 8.788032 & -0.914106 \\
H & 3.331108 & 6.634524 & -0.519434 \\
H & 5.211910 & 9.612832 & -2.102379 \\
H & 5.967860 & 3.837203 & -0.700709 \\
H & 5.108147 & 5.874906 & -2.870900 \\
H & 5.366392 & 1.434110 & -0.944316 \\
H & 3.131964 & 4.655849 & -3.704490 \\
H & 1.146218 & 2.468410 & -0.894944 \\
H & 1.113089 & 8.446748 & -4.399862 \\
H & 3.044013 & 9.679402 & -3.380924 \\
H & -0.292200 & 4.280107 & -4.949269 \\
H & 1.472338 & 4.042419 & -5.258208 \\
H & 0.856175 & 4.081327 & -3.559878 \\
H & 1.739930 & 4.878244 & -0.646975
\end{tabular}

Bisallene2Cyclization_INT2_for_A

Free energy $=-883.208571$ Hartree

$\begin{array}{llll}\text { C } & 3.472421 & 8.532555 & -3.862570\end{array}$

$\begin{array}{llll}\text { C } & 4.209409 & 7.853593 & -2.817447\end{array}$

$\begin{array}{llll}\text { C } & 3.953740 & 6.353918 & -2.605616\end{array}$

$\begin{array}{llll}\text { C } & 2.691367 & 5.839356 & -3.255353\end{array}$

$\begin{array}{llll}\text { C } & 2.010283 & 6.581049 & -4.17617\end{array}$

$\begin{array}{llll}\text { C } & 2.434489 & 7.935710 & -4.520553\end{array}$

$\begin{array}{llll}\text { C } & 5.160155 & 8.503939 & -2.062741\end{array}$

$\begin{array}{llll}\text { C } & 5.812089 & 7.804314 & -0.981256\end{array}$

$\begin{array}{llll}5.299657 & 6.532611 & -0.507991\end{array}$

$\begin{array}{llll}\text { C } & 6.115987 & 6.151794 & 0.526164\end{array}$

$\begin{array}{llll}\text { O } & 7.102946 & 7.087088 & 0.726020\end{array}$

$\begin{array}{llll}\text { C } & 6.909857 & 8.086257 & -0.185025\end{array}$

$\begin{array}{llll}\text { C } & 4.044269 & 5.938938 & -1.093017\end{array}$

$\begin{array}{llll}\text { C } & 3.934940 & 4.434771 & -0.906118\end{array}$

$\begin{array}{llll}\text { C } & 4.986678 & 3.581904 & -1.307032\end{array}$

$\begin{array}{llll}\text { C } & 4.869566 & 2.188881 & -1.183020\end{array}$

$\begin{array}{llll}\text { C } & 3.696691 & 1.621880 & -0.651730\end{array}$

$\begin{array}{llll}\text { C } & 2.644029 & 2.459684 & -0.247718\end{array}$

$\begin{array}{llll}\text { C } & 2.764496 & 3.854433 & -0.376107\end{array}$

$\begin{array}{llll}\text { O } & 0.890935 & 6.189092 & -4.856894\end{array}$

$\begin{array}{llll}\text { C } & 0.381449 & 4.894579 & -4.584744\end{array}$

$\begin{array}{llll}\mathrm{H} & 3.606048 & 0.528999 & -0.549612\end{array}$

$\begin{array}{llll}\mathrm{H} & 6.135846 & 5.278053 & 1.185355\end{array}$

\begin{tabular}{llll}
$\mathrm{H}$ & 7.630034 & 8.911137 & -0.15869 \\
\hline
\end{tabular}

$\begin{array}{llll}\mathrm{H} & 3.173106 & 6.416474 & -0.587104\end{array}$

$\begin{array}{llll}\mathrm{H} & 5.415672 & 9.553703 & -2.277410\end{array}$

$\begin{array}{llll}\mathrm{H} & 5.910881 & 4.022606 & -1.712923\end{array}$

$\begin{array}{llll}\mathrm{H} & 4.818391 & 5.834999 & -3.098637\end{array}$

$\begin{array}{llll}\mathrm{H} & 5.700733 & 1.540346 & -1.501532\end{array}$

$\begin{array}{llll}\mathrm{H} & 2.388683 & 4.816364 & -2.997926\end{array}$

$\begin{array}{llll}\mathrm{H} & 1.722811 & 2.026277 & 0.172503\end{array}$

$\begin{array}{llll}\mathrm{H} & 1.858996 & 8.461902 & -5.296470\end{array}$

$\begin{array}{llll}\mathrm{H} & 3.743317 & 9.575789 & -4.092106\end{array}$

$\begin{array}{llll}\mathrm{H} & -0.518357 & 4.768658 & -5.215179\end{array}$

\begin{tabular}{llll}
$\mathrm{H}$ & 1.120365 & 4.098979 & -4.836271 \\
\hline
\end{tabular}

$\begin{array}{llll}\mathrm{H} & 0.096941 & 4.780935 & -3.513196 \\ \mathrm{H} & 1.935155 & 4.508961 & -0.063199\end{array}$

Bisallene2Cyclization_TS2_for_B

Free energy $=-883.1659058$ Hartree

$\begin{array}{llll}\text { C } & 3.376495 & 9.252041 & -3.089369\end{array}$

$\begin{array}{llll}\text { C } & 4.102161 & 8.137614 & -2.559290\end{array}$

$\begin{array}{llll}\text { C } & 3.926461 & 6.885159 & -3.216275\end{array}$

C $3.065945 \quad 6.739014 \quad-4.311137$ 


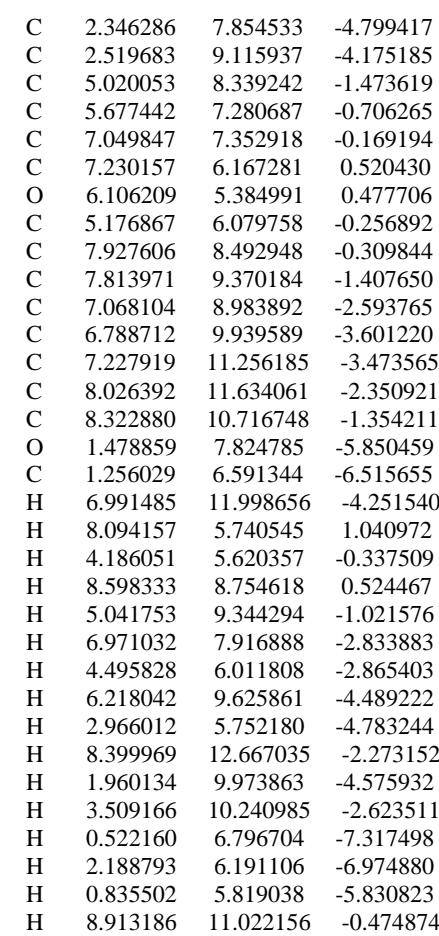

Bisallene2Cyclization_INT2_for_B 37

Free energy $=-883.2062741$ Hartree

\begin{tabular}{cccc} 
Free energy $=-883.2062741$ Hartree \\
$\mathrm{C}$ & 3.122035 & 9.117104 & -2.929490 \\
$\mathrm{C}$ & 4.232616 & 8.267440 & -2.728704 \\
$\mathrm{C}$ & 4.340943 & 7.126373 & -3.547272 \\
$\mathrm{C}$ & 3.383612 & 6.829434 & -4.532350 \\
$\mathrm{C}$ & 2.279643 & 7.691210 & -4.718209 \\
$\mathrm{C}$ & 2.158198 & 8.840020 & -3.904982 \\
$\mathrm{C}$ & 5.302553 & 8.600331 & -1.701561 \\
$\mathrm{C}$ & 5.641655 & 7.452355 & -0.785023 \\
$\mathrm{C}$ & 6.914195 & 7.399778 & -0.090422 \\
$\mathrm{C}$ & 6.856931 & 6.272239 & 0.712326 \\
$\mathrm{O}$ & 5.656554 & 5.639309 & 0.555347 \\
$\mathrm{C}$ & 4.923064 & 6.367787 & -0.350723 \\
$\mathrm{C}$ & 7.913196 & 8.419569 & -0.303305 \\
$\mathrm{C}$ & 7.771547 & 9.304779 & -1.349213 \\
$\mathrm{C}$ & 6.631157 & 9.115295 & -2.363708 \\
$\mathrm{C}$ & 6.404130 & 10.313623 & -3.252651 \\
$\mathrm{C}$ & 7.296558 & 11.334361 & -3.335855 \\
$\mathrm{C}$ & 8.497852 & 11.352856 & -2.512178 \\
$\mathrm{C}$ & 8.708350 & 10.388518 & -1.561387 \\
$\mathrm{O}$ & 1.290763 & 7.502119 & -5.639323 \\
$\mathrm{C}$ & 1.358569 & 6.365464 & -6.484397 \\
$\mathrm{H}$ & 7.113685 & 12.172702 & -4.027403 \\
$\mathrm{H}$ & 7.565334 & 5.801859 & 1.402510 \\
$\mathrm{H}$ & 3.925001 & 5.985711 & -0.585369 \\
$\mathrm{H}$ & 8.782410 & 8.492899 & 0.369766 \\
$\mathrm{H}$ & 4.925283 & 9.437758 & -1.068183 \\
$\mathrm{H}$ & 6.959304 & 8.279531 & -3.038183 \\
$\mathrm{H}$ & 5.192110 & 6.441800 & -3.405660 \\
$\mathrm{H}$ & 5.496651 & 10.306089 & -3.876724 \\
$\mathrm{H}$ & 3.506117 & 5.924551 & -5.143257 \\
$\mathrm{H}$ & 9.216739 & 12.178641 & -2.631452 \\
$\mathrm{H}$ & 1.291421 & 9.499229 & -4.059832 \\
$\mathrm{H}$ & 3.014934 & 10.020359 & -2.307300 \\
$\mathrm{H}$ & 0.469063 & 6.410579 & -7.140936 \\
$\mathrm{H}$ & 2.275018 & 6.369581 & -7.117797 \\
$\mathrm{H}$ & 1.330636 & 5.412904 & -5.907112 \\
$\mathrm{H}$ & 9.587281 & 10.445092 & -0.897731 \\
& & & \\
\hline
\end{tabular}

$\mathrm{A}=$ 2,4-dimethoxyphenyl, $\mathrm{B}=$ phenyl Bispropargyl

41

Free energy $=-997.5247001$ Hartree

$\begin{array}{lrrr}\mathrm{C} & -2.346879 & 1.011717 & -0.082565 \\ \mathrm{C} & -1.938880 & 1.985586 & 0.856936 \\ \mathrm{C} & -2.953847 & 2.761677 & 1.505389 \\ \mathrm{C} & -4.306686 & 2.545967 & 1.202282 \\ \mathrm{C} & -4.680803 & 1.562231 & 0.258087 \\ \mathrm{C} & -3.696186 & 0.786873 & -0.390834 \\ \mathrm{C} & -0.556214 & 2.159292 & 1.148189 \\ \mathrm{C} & 0.659179 & 2.215496 & 1.324051 \\ \mathrm{C} & 2.101997 & 2.046738 & 1.498359\end{array}$

$\begin{array}{lrrr}\mathrm{O} & 2.495411 & 1.204705 & 2.594245 \\ \mathrm{C} & 1.706576 & 0.031697 & 2.789925 \\ \mathrm{C} & 1.425053 & -0.757053 & 1.584422 \\ \mathrm{C} & 1.083403 & -1.325610 & 0.550036 \\ \mathrm{C} & 0.626081 & -1.967804 & -0.642104 \\ \mathrm{C} & 1.513189 & -2.249327 & -1.713904 \\ \mathrm{C} & 1.041178 & -2.859677 & -2.884524 \\ \mathrm{C} & -0.316630 & -3.204831 & -3.009363 \\ \mathrm{C} & -1.204642 & -2.934750 & -1.952130 \\ \mathrm{C} & -0.743815 & -2.321306 & -0.778913 \\ \mathrm{O} & -2.510900 & 3.680803 & 2.400755 \\ \mathrm{C} & -3.459763 & 4.487089 & 3.080654 \\ \mathrm{O} & -6.022278 & 1.444940 & 0.047570 \\ \mathrm{C} & -6.483040 & 0.482019 & -0.887179 \\ \mathrm{H} & -0.682336 & -3.685486 & -3.929811 \\ \mathrm{H} & 0.722530 & 0.292985 & 3.252514 \\ \mathrm{H} & 2.513642 & 1.639455 & 0.541951 \\ \mathrm{H} & -1.435587 & -2.103293 & 0.048149 \\ \mathrm{H} & -1.568361 & 0.413732 & -0.578935 \\ \mathrm{H} & -2.267776 & -3.207219 & -2.042128 \\ \mathrm{H} & -3.960418 & 0.018247 & -1.128859 \\ \mathrm{H} & 1.740605 & -3.071062 & -3.708271 \\ \mathrm{H} & -5.112580 & 3.119969 & 1.676004 \\ \mathrm{H} & -7.586645 & 0.562620 & -0.900065 \\ \mathrm{H} & -6.199844 & -0.554205 & -0.591130 \\ \mathrm{H} & -6.094960 & 0.677964 & -1.912849 \\ \mathrm{H} & 2.608482 & 3.016747 & 1.688393 \\ \mathrm{H} & 2.574476 & -1.977860 & -1.613378 \\ \mathrm{H} & -2.879810 & 5.149657 & 3.749855 \\ \mathrm{H} & -4.053288 & 5.113155 & 2.376150 \\ \mathrm{H} & -4.160682 & 3.875992 & 3.693752 \\ \mathrm{H} & 2.270963 & -0.572809 & 3.532372\end{array}$

Allenyne_A

41

Free energy $=-997.5492781$ Hartree

$\begin{array}{llll}\text { C } & 0.469497 & 2.390894 & 0.512948 \\ \text { C } & 1.391890 & 1.514432 & -0.139734\end{array}$

$\begin{array}{llll}\text { C } & 1.391890 & 1.514432 & -0.139734\end{array}$

$\begin{array}{llll}\text { C } & 0.882940 & 0.672748 & -1.148055\end{array}$

$\begin{array}{llll}\text { C } & -0.468508 & 0.657731 & -1.513593\end{array}$

$\begin{array}{llll}\text { C } & -1.364052 & 1.511317 & -0.838424\end{array}$

$\begin{array}{llll}\text { C } & -0.889862 & 2.387316 & 0.163598\end{array}$

$\begin{array}{llll}\text { C } & 2.803451 & 1.476807 & 0.274182\end{array}$

$\begin{array}{llll}\text { C } & 3.687139 & 0.569742 & -0.136749\end{array}$

$\begin{array}{llll}\text { C } & 4.466790 & -0.388754 & -0.611613\end{array}$

$\begin{array}{llll}\text { O } & 4.665181 & -1.640005 & -0.065847\end{array}$

$\begin{array}{llll}\text { C } & 3.932532 & -1.910038 & 1.139234\end{array}$

$\begin{array}{llll}\text { C } & 2.475942 & -1.921851 & 0.984357\end{array}$

$\begin{array}{llll}\text { C } & 1.251581 & -1.907788 & 0.885174\end{array}$

$\begin{array}{llll}\text { C } & -0.173417 & -1.858989 & 0.781840\end{array}$

$\begin{array}{llll}\text { C } & -0.881891 & -2.805329 & -0.002923\end{array}$

$\begin{array}{llll}\text { C } & -2.277506 & -2.730391 & -0.114444\end{array}$

$\begin{array}{llll}\text { C } & -2.990267 & -1.717273 & 0.553582\end{array}$

$\begin{array}{llll}\text { C } & -2.296612 & -0.770943 & 1.328621\end{array}$

$\begin{array}{llll}\text { C } & -0.902622 & -0.838010 & 1.444431\end{array}$

$\begin{array}{llll}\text { O } & -2.708102 & 1.558615 & -1.062339\end{array}$

$\begin{array}{llll}\text { C } & -3.254989 & 0.708307 & -2.059949\end{array}$

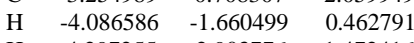

$\begin{array}{llll}\mathrm{H} & 4.297255 & -2.903776 & 1.472416\end{array}$

$\begin{array}{llll}\mathrm{H} & 5.076469 & -0.272150 & -1.524768\end{array}$

$\begin{array}{llll}\mathrm{H} & 3.109082 & 2.248495 & 1.003818\end{array}$

$\begin{array}{llll}\mathrm{H} & -0.320366 & -3.595206 & -0.523531\end{array}$

$\mathrm{H} \quad 1.586290 \quad-0.012688 \quad-1.645329$

$\begin{array}{llll}\mathrm{H} & -2.815750 & -3.472524 & -0.724977\end{array}$

$\begin{array}{llll}\mathrm{H} & -0.813143 & -0.042423 & -2.284938\end{array}$

$\begin{array}{llll}\mathrm{H} & -2.844430 & 0.042829 & 1.826906\end{array}$

$\begin{array}{llll}\mathrm{H} & -1.625682 & 3.033628 & 0.657556\end{array}$

$\begin{array}{llll}\text { O } & 0.991296 & 3.191514 & 1.488710\end{array}$

$\begin{array}{llll}\mathrm{H} & -4.344421 & 0.901892 & -2.069862\end{array}$

$\begin{array}{llll}\mathrm{H} & -3.078086 & -0.365357 & -1.824777\end{array}$

$\begin{array}{llll}\mathrm{H} & -2.838428 & 0.933402 & -3.068107\end{array}$

$\begin{array}{llll}\mathrm{H} & 4.206237 & -1.159458 & 1.920611\end{array}$

$\begin{array}{llll}\mathrm{H} & -0.352055 & -0.080483 & 2.020795\end{array}$

$\begin{array}{llll}\text { C } & 0.131552 & 4.080143 & 2.181252\end{array}$

$\begin{array}{llll}\mathrm{H} & 0.763434 & 4.620809 & 2.910549\end{array}$

$\begin{array}{llll}\mathrm{H} & -0.343117 & 4.819257 & 1.496086\end{array}$

$\begin{array}{llll}\mathrm{H} & -0.671598 & 3.538126 & 2.731039\end{array}$

Allenyne_A2Cyclization_TS

41

Free energy $=-997.5367186$ Hartree

$\begin{array}{lrrr}\mathrm{C} & 0.482768 & 2.433196 & 0.405714 \\ \mathrm{C} & 1.372411 & 1.500457 & -0.230689 \\ \mathrm{C} & 0.813755 & 0.623902 & -1.191648 \\ \mathrm{C} & -0.550249 & 0.613774 & -1.505225 \\ \mathrm{C} & -1.410327 & 1.488813 & -0.813746 \\ \mathrm{C} & -0.886647 & 2.415444 & 0.123394 \\ \mathrm{C} & 2.753739 & 1.390293 & 0.180194 \\ \mathrm{C} & 3.479453 & 0.248723 & 0.013116 \\ \mathrm{C} & 4.634569 & -0.378176 & -0.266800\end{array}$




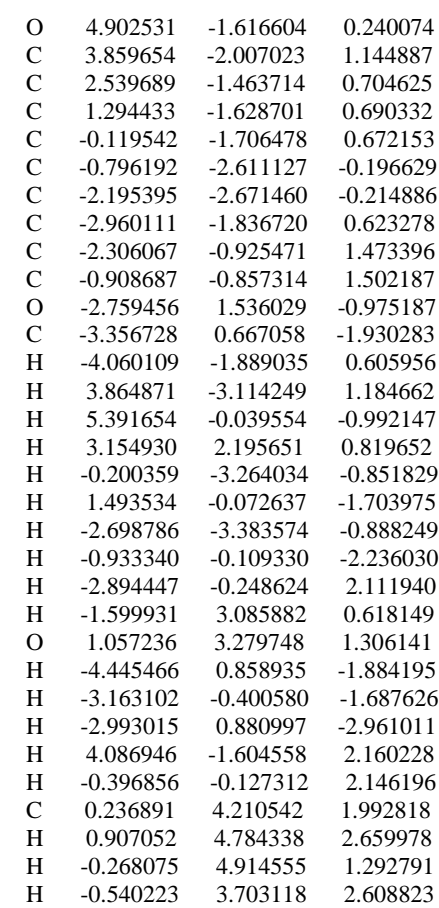

Allenyne_A2Cyclization_INT 41

Free energy $=-997.6116652$ Hartree

$\begin{array}{cccc}\mathrm{C} & 0.876047 & 3.041274 & -0.139670 \\ \mathrm{C} & 1.302963 & 1.683007 & 0.200101 \\ \mathrm{C} & 0.360675 & 0.565550 & -0.250932 \\ \mathrm{C} & -1.105160 & 0.953665 & -0.119436 \\ \mathrm{C} & -1.452115 & 2.266306 & -0.250764 \\ \mathrm{C} & -0.448702 & 3.316439 & -0.385310 \\ \mathrm{C} & 2.492502 & 1.440175 & 0.835528 \\ \mathrm{C} & 2.803749 & 0.101131 & 1.253788 \\ \mathrm{C} & 3.922999 & -0.462758 & 1.814564 \\ \mathrm{O} & 3.816211 & -1.797124 & 2.054763 \\ \mathrm{C} & 2.461731 & -2.195752 & 1.695986 \\ \mathrm{C} & 1.832415 & -0.973143 & 1.059315 \\ \mathrm{C} & 0.674205 & -0.812132 & 0.351905 \\ \mathrm{C} & -0.215612 & -1.945753 & 0.015765 \\ \mathrm{C} & -0.846748 & -2.026276 & -1.255083 \\ \mathrm{C} & -1.647171 & -3.124569 & -1.600766 \\ \mathrm{C} & -1.856536 & -4.171651 & -0.685311 \\ \mathrm{C} & -1.259950 & -4.100697 & 0.585063 \\ \mathrm{C} & -0.454538 & -3.004495 & 0.929471 \\ \mathrm{O} & -2.728181 & 2.765699 & -0.252285 \\ \mathrm{C} & -3.789221 & 1.835048 & -0.137699 \\ \mathrm{H} & -2.486565 & -5.032425 & -0.957696 \\ \mathrm{H} & 2.507171 & -3.082423 & 1.028157 \\ \mathrm{H} & 4.890774 & -0.014432 & 2.076089 \\ \mathrm{H} & 3.181255 & 2.269872 & 1.049248 \\ \mathrm{H} & -0.696691 & -1.220306 & -1.988667 \\ \mathrm{H} & 0.561811 & 0.479809 & -1.358502 \\ \mathrm{H} & -2.111212 & -3.164332 & -2.598612 \\ \mathrm{H} & -1.847786 & 0.155913 & -0.001602 \\ \mathrm{H} & -1.432764 & -4.901141 & 1.321873 \\ \mathrm{H} & -0.817734 & 4.329651 & -0.588421 \\ \mathrm{O} & 1.873664 & 3.968991 & -0.102187 \\ \mathrm{H} & -4.729158 & 2.416643 & -0.185049 \\ \mathrm{H} & -3.752048 & 1.282236 & 0.829642 \\ \mathrm{H} & -3.777084 & 1.088443 & -0.966452 \\ \mathrm{H} & 1.959911 & -2.504179 & 2.639310 \\ \mathrm{H} & -0.039912 & -2.946075 & 1.945861 \\ \mathrm{C} & 1.527969 & 5.334665 & -0.268961 \\ \mathrm{H} & 2.466954 & 5.911026 & -0.173101 \\ \mathrm{H} & 1.086081 & 5.527175 & -1.273257 \\ \mathrm{H} & 0.805337 & 5.674619 & 0.507887\end{array}$

Allenyne_B

41

Free energy $=-997.5467268$ Hartree

$\begin{array}{lrrr}\mathrm{C} & -0.126482 & -1.903532 & -1.073595 \\ \mathrm{C} & 0.305155 & -1.434926 & 0.208986 \\ \mathrm{C} & -0.680582 & -1.183394 & 1.185466 \\ \mathrm{C} & -2.046086 & -1.390342 & 0.941076 \\ \mathrm{C} & -2.448976 & -1.856171 & -0.326258 \\ \mathrm{C} & -1.487324 & -2.117866 & -1.328347 \\ \mathrm{C} & 1.682794 & -1.180449 & 0.464580 \\ \mathrm{C} & 2.860082 & -0.930361 & 0.712107 \\ \mathrm{C} & 4.241002 & -0.579128 & 1.041048\end{array}$

$\begin{array}{lrrr}\mathrm{O} & 4.936272 & 0.173696 & 0.027233 \\ \mathrm{C} & 4.421488 & 1.414759 & -0.255774 \\ \mathrm{C} & 3.369036 & 1.991311 & 0.306757 \\ \mathrm{C} & 2.218430 & 2.480331 & 0.758235 \\ \mathrm{C} & 0.896238 & 2.203008 & 0.158750 \\ \mathrm{C} & 0.767689 & 1.545074 & -1.087577 \\ \mathrm{C} & -0.495769 & 1.234935 & -1.602878 \\ \mathrm{C} & -1.658979 & 1.567202 & -0.885135 \\ \mathrm{C} & -1.546401 & 2.234759 & 0.345902 \\ \mathrm{C} & -0.280942 & 2.555881 & 0.859780 \\ \mathrm{O} & -3.744741 & -2.070227 & -0.697761 \\ \mathrm{C} & -4.770965 & -1.818094 & 0.249452 \\ \mathrm{H} & -2.648341 & 1.291987 & -1.280241 \\ \mathrm{H} & 5.027388 & 1.916354 & -1.030807 \\ \mathrm{H} & 4.251364 & -0.003197 & 1.998790 \\ \mathrm{H} & 2.211050 & 3.104267 & 1.674257 \\ \mathrm{H} & 1.678406 & 1.250854 & -1.630101 \\ \mathrm{H} & -0.356011 & -0.794817 & 2.161355 \\ \mathrm{H} & -0.576406 & 0.706692 & -2.564461 \\ \mathrm{H} & -2.774441 & -1.164995 & 1.729806 \\ \mathrm{H} & -2.451114 & 2.498410 & 0.915244 \\ \mathrm{H} & -1.853115 & -2.466060 & -2.301788 \\ \mathrm{O} & 0.857965 & -2.075034 & -1.994778 \\ \mathrm{H} & -5.726412 & -2.055516 & -0.255156 \\ \mathrm{H} & -4.788091 & -0.751440 & 0.570095 \\ \mathrm{H} & -4.671768 & -2.460675 & 1.153462 \\ \mathrm{H} & 4.861975 & -1.488383 & 1.180953 \\ \mathrm{H} & -0.195816 & 3.070056 & 1.830862 \\ \mathrm{C} & 0.504403 & -2.498239 & -3.301269 \\ \mathrm{H} & 1.448824 & -2.561345 & -3.872912 \\ \mathrm{H} & 0.015623 & -3.498924 & -3.296263 \\ \mathrm{H} & -0.175354 & -1.770932 & -3.802566\end{array}$

Allenyne_B2Cyclization_TS

Free energy $=-997.531394$ Hartree

$\begin{array}{llll}\text { C } & -0.190560 & -1.762186 & -1.097420\end{array}$

$\begin{array}{llll}\text { C } & 0.245262 & -1.265824 & 0.179974\end{array}$

$\begin{array}{llll}\text { C } & -0.726119 & -1.167036 & 1.206841\end{array}$

$\begin{array}{llll}\text { C } & -2.058859 & -1.557294 & 1.017657\end{array}$

$\begin{array}{llll}\text { C } & -2.455071 & -2.063561 & -0.237784\end{array}$

$\begin{array}{llll}\text { C } & -1.517647 & -2.158658 & -1.293505\end{array}$

$\begin{array}{llll}\text { C } & 1.583529 & -0.871876 & 0.380416\end{array}$

$\begin{array}{llll}\text { C } & 2.742133 & -0.431830 & 0.589391\end{array}$

$\begin{array}{llll}\text { C } & 4.108612 & -0.784787 & 1.077155\end{array}$

$\begin{array}{llll}\text { O } & 5.105329 & 0.043489 & 0.461243\end{array}$

$\begin{array}{llll}\text { C } & 4.582493 & 1.271755 & 0.187405\end{array}$

$\begin{array}{llll}\text { C } & 3.280510 & 1.541693 & 0.392059\end{array}$

$\begin{array}{llll}\text { C } & 2.290624 & 2.451230 & 0.615522\end{array}$

$\begin{array}{llll}\text { C } & 0.985147 & 2.348716 & -0.014798 \\ \text { C } & 0.798630 & 1.566391 & -1.187251\end{array}$

$\begin{array}{llll}\text { C } & 0.798630 & 1.566391 & -1.18725\end{array}$

$\begin{array}{llll}\text { C } & -0.484107 & 1.358796 & -1.710393\end{array}$

$\begin{array}{llll}\text { C } & -1.607180 & 1.916905 & -1.079522\end{array}$

$\begin{array}{llll}\text { C } & -1.436996 & 2.726982 & 0.062284\end{array}$

$\begin{array}{llll}\text { C } & -0.157612 & 2.948644 & 0.581702\end{array}$

$\begin{array}{llll}\text { O } & -3.718529 & -2.474915 & -0.546903\end{array}$

$\begin{array}{llll}\text { C } & -4.721066 & -2.397770 & 0.454144\end{array}$

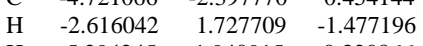

$\begin{array}{llll}\mathrm{H} & 5.294245 & 1.940915 & -0.320866\end{array}$

$\begin{array}{llll}\mathrm{H} & 4.140592 & -0.632193 & 2.182412\end{array}$

$\begin{array}{llll}\mathrm{H} & 2.398577 & 3.175140 & 1.445864\end{array}$

$\begin{array}{llll}\mathrm{H} & 1.675471 & 1.114592 & -1.672063\end{array}$

$\begin{array}{llll}\mathrm{H} & -0.410957 & -0.759532 & 2.178187\end{array}$

$\begin{array}{llll}\mathrm{H} & -0.610452 & 0.735837 & -2.608315\end{array}$

$\begin{array}{llll}\mathrm{H} & -2.771403 & -1.453922 & 1.846332\end{array}$

$\begin{array}{llll}\mathrm{H} & -2.314241 & 3.176278 & 0.553195\end{array}$

$\mathrm{H} \quad-1.878896 \quad-2.539840 \quad-2.256546$

$\begin{array}{llll}\mathrm{O} & 0.762601 & -1.773757 & -2.067022\end{array}$

$\begin{array}{llll}\mathrm{H} & -5.651943 & -2.778648 & -0.006111\end{array}$

$\begin{array}{llll}\mathrm{H} & -4.890181 & -1.350861 & 0.794749\end{array}$

$\begin{array}{llll}\mathrm{H} & -4.474224 & -3.025549 & 1.340389\end{array}$

$\begin{array}{llll}\mathrm{H} & 4.377038 & -1.835540 & 0.849734\end{array}$

$\begin{array}{llll}\mathrm{H} & -0.027876 & 3.562089 & 1.488318\end{array}$

$\begin{array}{llll}\text { C } & 0.407483 & -2.210638 & -3.368907\end{array}$

$\begin{array}{llll}\mathrm{H} & 1.322010 & -2.125552 & -3.985133\end{array}$

$\mathrm{H} \quad 0.063753 \quad-3.269664 \quad-3.371931$

$\begin{array}{llll}\mathrm{H} & -0.392178 & -1.575309 & -3.814919\end{array}$

Allenyne_B2Cyclization_INT

41

Free energy $=-997.6072728$ Hartree

$\begin{array}{lrrr}\mathrm{C} & -0.220657 & -1.699244 & -1.211054 \\ \mathrm{C} & -0.285963 & -0.722004 & -0.171898 \\ \mathrm{C} & -1.318296 & -0.855467 & 0.774042 \\ \mathrm{C} & -2.270179 & -1.890167 & 0.723267 \\ \mathrm{C} & -2.188461 & -2.836415 & -0.316632 \\ \mathrm{C} & -1.160187 & -2.739903 & -1.281499 \\ \mathrm{C} & 0.715398 & 0.375738 & -0.096292 \\ \mathrm{C} & 2.012132 & 0.117764 & 0.206999 \\ \mathrm{C} & 2.703495 & -1.194726 & 0.489856\end{array}$




$\begin{array}{rrrr}\mathrm{O} & 4.104619 & -0.854138 & 0.711448 \\ \mathrm{C} & 4.222682 & 0.498240 & 0.596168 \\ \mathrm{C} & 3.046726 & 1.146353 & 0.302519 \\ \mathrm{C} & 2.674022 & 2.528357 & 0.199282 \\ \mathrm{C} & 1.370110 & 2.869146 & -0.091982 \\ \mathrm{C} & 0.345805 & 1.806778 & -0.531796 \\ \mathrm{C} & -1.086864 & 2.221700 & -0.265234 \\ \mathrm{C} & -1.427967 & 3.523232 & -0.075000 \\ \mathrm{C} & -0.414665 & 4.566196 & -0.022947 \\ \mathrm{C} & 0.918385 & 4.241928 & -0.018083 \\ \mathrm{O} & -3.045816 & -3.885869 & -0.484702 \\ \mathrm{C} & -4.102469 & -4.050804 & 0.446666 \\ \mathrm{H} & -2.486423 & 3.804092 & 0.048182 \\ \mathrm{H} & 5.234184 & 0.893983 & 0.758834 \\ \mathrm{H} & 2.321421 & -1.720558 & 1.391911 \\ \mathrm{H} & 3.409368 & 3.317132 & 0.427616 \\ \mathrm{H} & 0.413946 & 1.779499 & -1.658294 \\ \mathrm{H} & -1.371589 & -0.117481 & 1.589708 \\ \mathrm{H} & -1.861967 & 1.443307 & -0.330094 \\ \mathrm{H} & -3.051282 & -1.946366 & 1.492686 \\ \mathrm{H} & -0.729399 & 5.616195 & 0.081740 \\ \mathrm{H} & -1.135882 & -3.501663 & -2.071192 \\ \mathrm{O} & 0.803241 & -1.546696 & -2.104508 \\ \mathrm{H} & -4.663214 & -4.949468 & 0.127052 \\ \mathrm{H} & -4.793627 & -3.176550 & 0.453011 \\ \mathrm{H} & -3.724962 & -4.212727 & 1.482284 \\ \mathrm{H} & 2.644478 & -1.890569 & -0.375195 \\ \mathrm{H} & 1.679366 & 5.027323 & 0.124458 \\ \mathrm{C} & 0.908966 & -2.457432 & -3.186790 \\ \mathrm{H} & 1.787500 & -2.136165 & -3.777525 \\ \mathrm{H} & 1.071293 & -3.503124 & -2.838224 \\ \mathrm{H} & 0.005242 & -2.434662 & -3.837613\end{array}$

Bisallene

41

Free energy $=-997.5614743$ Hartree

$\begin{array}{llll}\text { C } & 4.665358 & 6.890839 & -3.812754\end{array}$

$\begin{array}{llll}\text { C } & 4.511293 & 6.655947 & -2.425475 \\ \text { C } & 3.201792 & 6.519706 & -1.901873\end{array}$

$\begin{array}{llll}\text { C } & 3.201792 & 6.519706 & -1.901873\end{array}$

$\begin{array}{llll}\text { C } & 2.086399 & 6.619165 & -2.741723\end{array}$

$\begin{array}{llll}\text { C } & 2.253072 & 6.849066 & -4.121034\end{array}$

$\begin{array}{llll}\text { C } & 3.547682 & 6.987351 & -4.652290\end{array}$

$\begin{array}{llll}\text { C } & 5.698578 & 6.623452 & -1.558636\end{array}$

$\begin{array}{llll}\text { C } & 5.709347 & 6.463969 & -0.236186\end{array}$

$\begin{array}{llll}\text { C } & 4.589793 & 8.772252 & 0.807615\end{array}$

$\begin{array}{llll}\text { C } & 5.271700 & 8.364472 & 1.863592\end{array}$

$\begin{array}{llll}\text { O } & 5.726948 & 7.076063 & 2.110253\end{array}$

$\begin{array}{llll}\text { C } & 5.788013 & 6.187937 & 1.055789\end{array}$

$\begin{array}{llll}\text { C } & 3.853533 & 9.225941 & -0.207028\end{array}$

$\begin{array}{llll}\text { C } & 4.358575 & 9.687756 & -1.500060\end{array}$

$\begin{array}{llll}\text { C } & 3.437491 & 9.997842 & -2.551869\end{array}$

$\begin{array}{llll}\text { C } & 3.901955 & 10.313502 & -3.835005\end{array}$

$\begin{array}{llll}\text { C } & 5.287846 & 10.323427 & -4.106733\end{array}$

$\begin{array}{llll}\text { C } & 6.215810 & 10.048877 & -3.079895\end{array}$

$\begin{array}{llll}\text { C } & 5.734483 & 9.737164 & -1.800204\end{array}$

$\begin{array}{llll}\text { O } & 2.116634 & 9.939866 & -2.224557\end{array}$

$\begin{array}{llll}\text { C } & 1.148639 & 10.156885 & -3.237399\end{array}$

$\begin{array}{llll}\text { O } & 5.621354 & 10.608528 & -5.39903\end{array}$

$\begin{array}{llll}\text { C } & 6.993261 & 10.618940 & -5.758972\end{array}$

$\begin{array}{llll}\mathrm{H} & 1.374011 & 6.917236 & -4.780744\end{array}$

$\begin{array}{llll}\mathrm{H} & 5.955176 & 5.164194 & 1.431404\end{array}$

$\begin{array}{llll}\mathrm{H} & 5.536139 & 9.022922 & 2.708128\end{array}$

$\begin{array}{llll}\mathrm{H} & 6.662000 & 6.820003 & -2.068689\end{array}$

$\begin{array}{llll}\mathrm{H} & 2.753068 & 9.177911 & -0.119038\end{array}$

$\begin{array}{llll}\mathrm{H} & 3.078765 & 6.350487 & -0.821138\end{array}$

$\begin{array}{llll}\mathrm{H} & 6.443772 & 9.495399 & -0.994214\end{array}$

$\begin{array}{llll}\mathrm{H} & 1.074775 & 6.520559 & -2.318370\end{array}$

$\begin{array}{llll}\mathrm{H} & 7.297902 & 10.064012 & -3.264133\end{array}$

$\begin{array}{llll}\mathrm{H} & 3.687105 & 7.185378 & -5.726452\end{array}$

$\begin{array}{llll}\mathrm{H} & 3.222008 & 10.528054 & -4.667673\end{array}$

\begin{tabular}{llll}
$\mathrm{H}$ & 7.031435 & 10.857268 & -6.838584 \\
\hline & 7.566450 & 11.391912 & -5.198202
\end{tabular}

H $\quad 7.566450 \quad 11.391912 \quad-5.198202$

$\begin{array}{llll}\mathrm{H} & 7.475362 & 9.628208 & -5.590639\end{array}$

$\begin{array}{llll}\mathrm{H} & 5.677484 & 7.024341 & -4.225976\end{array}$

$\mathrm{H} \quad 0.160921 \quad 10.033516 \quad-2.754909$

\begin{tabular}{llll}
$\mathrm{H}$ & 1.217353 & 11.183946 & -3.662252 \\
\hline
\end{tabular}

$\begin{array}{llll}\mathrm{H} & 1.248361 & 9.413463 & -4.059700\end{array}$

Bisallene2Cyclization_TS1

41

Free energy $=-997.5577286$ Hartree

$\begin{array}{llll}\text { C } & 4.695678 & 6.892870 & -3.807424\end{array}$

$\begin{array}{llll}\text { C } & 4.518816 & 6.698139 & -2.410958\end{array}$

$\begin{array}{llll}\text { C } & 3.195293 & 6.572951 & -1.908670\end{array}$

$\begin{array}{llll}\text { C } & 2.095830 & 6.655500 & -2.771836\end{array}$

$\begin{array}{llll}\text { C } & 2.287120 & 6.856116 & -4.151875\end{array}$

$\begin{array}{lllll}\text { C } & 3.594194 & 6.971394 & -4.665051\end{array}$

$\begin{array}{llll}\text { C } & 5.669962 & 6.726050 & -1.519644\end{array}$

$\begin{array}{llll}\text { C } & 5.616488 & 6.702387 & -0.165964\end{array}$

$\begin{array}{cccc}\text { C } & 4.670352 & 8.561238 & 0.701630 \\ \text { C } & 5.185240 & 8.279198 & 1.895847 \\ \text { O } & 5.742792 & 7.049950 & 2.185999 \\ \text { C } & 5.862314 & 6.258007 & 1.065364 \\ \text { C } & 3.895324 & 9.173815 & -0.225585 \\ \text { C } & 4.376654 & 9.687733 & -1.489403 \\ \text { C } & 3.441351 & 10.023877 & -2.528821 \\ \text { C } & 3.889144 & 10.326935 & -3.816865 \\ \text { C } & 5.273280 & 10.309399 & -4.111794 \\ \text { C } & 6.215260 & 10.027898 & -3.100808 \\ \text { C } & 5.753286 & 9.718732 & -1.813513 \\ \text { O } & 2.128422 & 9.984506 & -2.176748 \\ \text { C } & 1.144033 & 10.166069 & -3.181447 \\ \text { O } & 5.586753 & 10.584109 & -5.409967 \\ \text { C } & 6.951088 & 10.561182 & -5.798561 \\ \text { H } & 1.420410 & 6.913412 & -4.828781 \\ \text { H } & 6.167621 & 5.230447 & 1.314359 \\ \text { H } & 5.257834 & 8.976317 & 2.744813 \\ \text { H } & 6.645253 & 6.939942 & -1.996434 \\ \text { H } & 2.798350 & 9.101377 & -0.120733 \\ \text { H } & 3.051987 & 6.419920 & -0.828524 \\ \text { H } & 6.472324 & 9.475563 & -1.017606 \\ \text { H } & 1.076382 & 6.566927 & -2.364939 \\ \text { H } & 7.293829 & 10.028058 & -3.304953 \\ \text { H } & 3.751280 & 7.140095 & -5.742012 \\ \text { H } & 3.200567 & 10.549162 & -4.640372 \\ \text { H } & 6.971726 & 10.790037 & -6.880644 \\ \text { H } & 7.552233 & 11.325702 & -5.256024 \\ \text { H } & 7.413289 & 9.561061 & -5.631019 \\ \text { H } & 5.715456 & 7.012041 & -4.205981 \\ \text { H } & 0.164337 & 10.051664 & -2.681097 \\ \text { H } & 1.200300 & 11.180346 & -3.637321 \\ \text { H } & 1.239786 & 9.397631 & -3.981293\end{array}$

Bisallene2Cyclization_INT1

Free energy $=-997.5981845$ Hartree

$\begin{array}{lccc}\mathrm{C} & 2.887315 & 8.944085 & -3.619205 \\ \mathrm{C} & 3.816831 & 7.950215 & -3.134770 \\ \mathrm{C} & 3.657674 & 6.633474 & -3.650396 \\ \mathrm{C} & 2.639097 & 6.277196 & -4.534756 \\ \mathrm{C} & 1.694753 & 7.253907 & -4.916532 \\ \mathrm{C} & 1.842981 & 8.593853 & -4.480532 \\ \mathrm{C} & 4.815888 & 8.312855 & -2.179200 \\ \mathrm{C} & 5.528730 & 7.505244 & -1.249118 \\ \mathrm{C} & 5.348452 & 6.130301 & -0.684281 \\ \mathrm{C} & 6.514671 & 5.914860 & 0.077634 \\ \mathrm{O} & 7.312432 & 7.008504 & 0.084757 \\ \mathrm{C} & 6.734285 & 7.962604 & -0.677399 \\ \mathrm{C} & 4.321689 & 5.145947 & -0.748011 \\ \mathrm{C} & 2.949289 & 5.255239 & -1.151448 \\ \mathrm{C} & 2.225719 & 6.488953 & -1.150425 \\ \mathrm{C} & 0.915402 & 6.563458 & -1.630492 \\ \mathrm{C} & 0.279206 & 5.420145 & -2.149404 \\ \mathrm{C} & 0.950230 & 4.177846 & -2.107760 \\ \mathrm{C} & 2.247904 & 4.091916 & -1.599860 \\ \mathrm{O} & 0.611311 & 7.016145 & -5.706111 \\ \mathrm{C} & 0.326634 & 5.672576 & -6.069908 \\ \mathrm{H} & -0.741295 & 5.488276 & -2.555373 \\ \mathrm{H} & 6.843316 & 5.063367 & 0.682159 \\ \mathrm{H} & 7.282051 & 8.904190 & -0.788773 \\ \mathrm{H} & 4.637338 & 4.139611 & -0.420055 \\ \mathrm{H} & 5.088681 & 9.381161 & -2.166031 \\ \mathrm{H} & 2.712098 & 7.388754 & -0.750937 \\ \mathrm{H} & 4.380515 & 5.868267 & -3.338231 \\ \mathrm{H} & 0.391744 & 7.531711 & -1.627579 \\ \mathrm{H} & 2.559905 & 5.237654 & -4.873446 \\ \mathrm{H} & 0.449192 & 3.269976 & -2.479529 \\ \mathrm{H} & 1.092426 & 9.318859 & -4.818644 \\ \mathrm{O} & 3.077802 & 10.205848 & -3.141705 \\ \mathrm{H} & -0.623594 & 5.697827 & -6.635873 \\ \mathrm{H} & 1.121077 & 5.239101 & -6.719036 \\ \mathrm{H} & 0.205499 & 5.027577 & -5.170109 \\ \mathrm{H} & 2.766453 & 3.119391 & -1.580883 \\ \mathrm{C} & 2.220527 & 11.245458 & -3.584676 \\ \mathrm{H} & 2.578019 & 12.172189 & -3.098874 \\ \mathrm{H} & 2.261234 & 11.374615 & -4.690454 \\ \mathrm{H} & 1.163150 & 11.065289 & -3.285304\end{array}$

Bisallene2Cyclization_TS2_for_A

41

Free energy $=-997.5892657$ Hartree

$\begin{array}{llll}\mathrm{C} & 3.126653 & 3.542343 & -0.774237 \\ \mathrm{C} & 3.628124 & 4.883392 & -0.732467 \\ \mathrm{C} & 2.675253 & 5.930995 & -0.530556 \\ \mathrm{C} & 1.312253 & 5.649668 & -0.393216 \\ \mathrm{C} & 0.839681 & 4.321933 & -0.450417 \\ \mathrm{C} & 1.762139 & 3.272299 & -0.640108 \\ \mathrm{C} & 5.040337 & 5.104638 & -0.788932 \\ \mathrm{C} & 5.733299 & 6.387576 & -0.800202\end{array}$




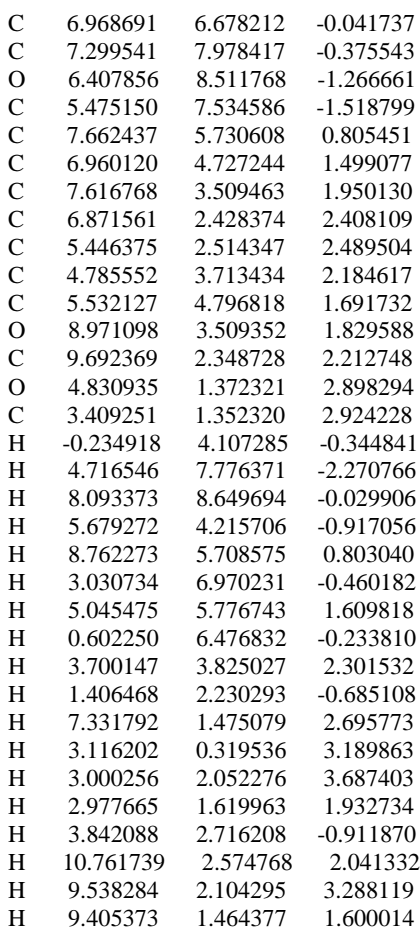

Bisallene2Cyclization_INT2_for_A 41

Free energy $=-997.6280122$ Hartree

$\begin{array}{lcrr}\mathrm{C} & 2.687644 & 3.937887 & -1.040793 \\ \mathrm{C} & 3.446327 & 4.942478 & -0.406076 \\ \mathrm{C} & 2.762168 & 6.007605 & 0.219390 \\ \mathrm{C} & 1.359724 & 6.065872 & 0.209438 \\ \mathrm{C} & 0.614036 & 5.058014 & -0.428376 \\ \mathrm{C} & 1.282947 & 3.992991 & -1.054088 \\ \mathrm{C} & 4.962365 & 4.857993 & -0.352293 \\ \mathrm{C} & 5.652688 & 6.118257 & -0.804304 \\ \mathrm{C} & 7.013705 & 6.402040 & -0.395002 \\ \mathrm{C} & 7.364616 & 7.574720 & -1.042289 \\ \mathrm{O} & 6.323380 & 8.024136 & -1.804559 \\ \mathrm{C} & 5.290575 & 7.128531 & -1.658596 \\ \mathrm{C} & 7.715481 & 5.514653 & 0.502825 \\ \mathrm{C} & 7.013090 & 4.543060 & 1.173518 \\ \mathrm{C} & 7.686940 & 3.584826 & 2.049706 \\ \mathrm{C} & 7.011848 & 2.521166 & 2.597773 \\ \mathrm{C} & 5.597327 & 2.314127 & 2.314504 \\ \mathrm{C} & 4.866616 & 3.202219 & 1.582631 \\ \mathrm{C} & 5.482817 & 4.484780 & 1.083852 \\ \mathrm{O} & 9.017114 & 3.827195 & 2.219162 \\ \mathrm{C} & 9.775520 & 2.918251 & 2.999732 \\ \mathrm{O} & 5.129462 & 1.147872 & 2.859229 \\ \mathrm{C} & 3.763871 & 0.832778 & 2.651212 \\ \mathrm{H} & -0.486481 & 5.105432 & -0.439630 \\ \mathrm{H} & 4.374126 & 7.352705 & -2.213344 \\ \mathrm{H} & 8.268489 & 8.193021 & -1.045246 \\ \mathrm{H} & 5.288905 & 4.030015 & -1.024002 \\ \mathrm{H} & 8.800455 & 5.609550 & 0.651023 \\ \mathrm{H} & 3.342940 & 6.803982 & 0.710686 \\ \mathrm{H} & 5.118921 & 5.314455 & 1.748004 \\ \mathrm{H} & 0.844036 & 6.906269 & 0.700475 \\ \mathrm{H} & 3.797148 & 3.055764 & 1.386257 \\ \mathrm{H} & 0.709171 & 3.199253 & -1.558101 \\ \mathrm{H} & 7.501138 & 1.768420 & 3.228582 \\ \mathrm{H} & 3.581611 & -0.135575 & 3.153952 \\ \mathrm{H} & 3.089644 & 1.604993 & 3.088246 \\ \mathrm{H} & 3.523690 & 0.733731 & 1.567314 \\ \mathrm{H} & 3.209287 & 3.097707 & -1.527518 \\ \mathrm{H} & 10.814505 & 3.298028 & 3.002829 \\ \mathrm{H} & 9.403677 & 2.865917 & 4.048545 \\ \mathrm{H} & 9.761951 & 1.891867 & 2.566790\end{array}$

Bisallene2Cyclization_TS2_for_B

41

Free energy $=-997.585906$ Hartree

$\begin{array}{llll}\mathrm{C} & 2.082717 & 5.199450 & -1.744097 \\ \mathrm{C} & 3.173149 & 5.942091 & -1.165197 \\ \mathrm{C} & 3.163900 & 7.384964 & -1.339592 \\ \mathrm{C} & 2.162409 & 7.999604 & -2.132495 \\ \mathrm{C} & 1.166067 & 7.237649 & -2.738127 \\ \mathrm{C} & 1.116889 & 5.827640 & -2.515476 \\ \mathrm{C} & 4.263500 & 5.297215 & -0.547415 \\ \mathrm{C} & 5.535366 & 5.961740 & -0.374590\end{array}$

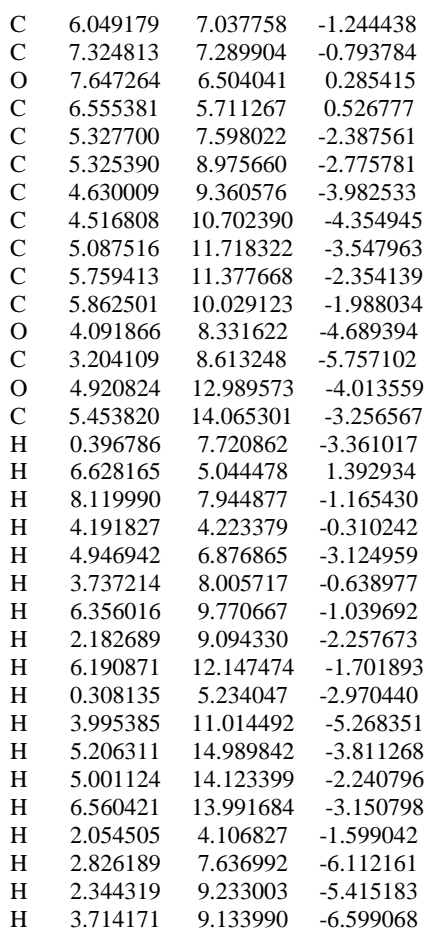

Bisallene2Cyclization_INT2_for_B

Free energy $=-997.624433$ Hartree

$\begin{array}{lccc}\mathrm{C} & 1.933560 & 5.243654 & -1.060901 \\ \mathrm{C} & 3.233683 & 5.879570 & -1.025643 \\ \mathrm{C} & 3.362812 & 7.320005 & -1.547856 \\ \mathrm{C} & 2.170326 & 7.787482 & -2.344915 \\ \mathrm{C} & 1.002036 & 7.096119 & -2.379680 \\ \mathrm{C} & 0.863242 & 5.817859 & -1.695111 \\ \mathrm{C} & 4.336844 & 5.245661 & -0.497952 \\ \mathrm{C} & 5.628263 & 5.887507 & -0.562179 \\ \mathrm{C} & 5.838975 & 7.047336 & -1.408825 \\ \mathrm{C} & 7.161548 & 7.383535 & -1.272575 \\ \mathrm{O} & 7.787607 & 6.527515 & -0.396374 \\ \mathrm{C} & 6.855042 & 5.622252 & 0.024178 \\ \mathrm{C} & 4.730003 & 7.570665 & -2.284803 \\ \mathrm{C} & 4.899247 & 9.018164 & -2.708580 \\ \mathrm{C} & 4.621568 & 9.417251 & -4.048165 \\ \mathrm{C} & 4.727120 & 10.763230 & -4.434778 \\ \mathrm{C} & 5.104680 & 11.745821 & -3.491760 \\ \mathrm{C} & 5.373374 & 11.376232 & -2.159152 \\ \mathrm{C} & 5.262992 & 10.020353 & -1.797481 \\ \mathrm{O} & 4.238616 & 8.418211 & -4.898414 \\ \mathrm{C} & 3.914880 & 8.740515 & -6.240210 \\ \mathrm{O} & 5.176491 & 13.020023 & -3.978089 \\ \mathrm{C} & 5.542729 & 14.062859 & -3.089180 \\ \mathrm{H} & 0.139929 & 7.495423 & -2.937342 \\ \mathrm{H} & 7.200726 & 4.868049 & 0.739151 \\ \mathrm{H} & 7.784232 & 8.173162 & -1.704300 \\ \mathrm{H} & 4.237135 & 4.249087 & -0.039912 \\ \mathrm{H} & 4.705874 & 6.946891 & -3.204203 \\ \mathrm{H} & 3.405811 & 7.977258 & -0.638699 \\ \mathrm{H} & 5.478972 & 9.727483 & -0.758021 \\ \mathrm{H} & 2.264137 & 8.755639 & -2.859933 \\ \mathrm{H} & 5.665463 & 12.117013 & -1.404048 \\ \mathrm{H} & -0.106159 & 5.296265 & -1.726395 \\ \mathrm{H} & 4.521661 & 11.098432 & -5.459003 \\ \mathrm{H} & 5.538254 & 14.996005 & -3.683527 \\ \mathrm{H} & 4.819963 & 14.168037 & -2.247549 \\ \mathrm{H} & 6.562007 & 13.911666 & -2.665723 \\ \mathrm{H} & 1.836663 & 4.249180 & -0.595772 \\ \mathrm{H} & 3.628749 & 7.789861 & -6.727401 \\ \mathrm{H} & 3.058573 & 9.450624 & -6.299851 \\ \mathrm{H} & 4.781998 & 9.180524 & -6.783314\end{array}$

\section{$A=$ 2-naphthyl, $B=$ phenyl}

Bispropargyl

39

Free energy $=-922.1955811$ Hartree

$\begin{array}{llll}\mathrm{C} & -3.349826 & 7.340847 & 2.962534 \\ \mathrm{C} & -3.297100 & 7.502344 & 1.546918 \\ \mathrm{C} & -2.177474 & 8.207477 & 0.965946 \\ \mathrm{C} & -1.166766 & 8.729286 & 1.829645 \\ \mathrm{C} & -1.248958 & 8.558355 & 3.204061 \\ \mathrm{C} & -2.345799 & 7.853471 & 3.775026\end{array}$




$\begin{array}{rrrr}\text { C } & -4.297884 & 6.972915 & 0.675698 \\ \text { C } & -4.196185 & 7.104831 & -0.698304 \\ \text { C } & -3.075994 & 7.784666 & -1.285674 \\ \text { C } & -2.097122 & 8.335685 & -0.446973 \\ \text { C } & -2.894999 & 7.825482 & -2.700505 \\ \text { C } & -2.618080 & 7.803644 & -3.896660 \\ \text { C } & -2.120456 & 7.683814 & -5.272189 \\ \text { O } & -0.706031 & 7.514910 & -5.371726 \\ \text { C } & -0.213933 & 6.235796 & -4.961874 \\ \text { C } & -0.145376 & 6.012530 & -3.511192 \\ \text { C } & -0.121908 & 5.828136 & -2.297091 \\ \text { C } & -0.181085 & 5.637555 & -0.880700 \\ \text { C } & 0.854255 & 6.107950 & -0.031624 \\ \text { C } & 0.754823 & 5.955459 & 1.358187 \\ \text { C } & -0.369316 & 5.331605 & 1.926801 \\ \text { C } & -1.399000 & 4.859292 & 1.094534 \\ \text { C } & -1.309268 & 5.005074 & -0.295326 \\ \text { H } & -2.397421 & 7.716781 & 4.866754 \\ \text { H } & -0.455400 & 5.235727 & 3.019673 \\ \text { H } & 0.806253 & 6.168449 & -5.397268 \\ \text { H } & -2.656224 & 6.846399 & -5.789017 \\ \text { H } & 1.728793 & 6.603115 & -0.479413 \\ \text { H } & -1.227262 & 8.838812 & -0.894567 \\ \text { H } & 1.557742 & 6.338760 & 2.006537 \\ \text { H } & -2.292133 & 4.391121 & 1.535282 \\ \text { H } & -5.155855 & 6.439718 & 1.116014 \\ \text { H } & -4.962684 & 6.676271 & -1.360528 \\ \text { H } & -2.343647 & 8.609231 & -5.846803 \\ \text { H } & -2.125401 & 4.661471 & -0.946877 \\ \text { H } & -4.200891 & 6.797320 & 3.403670 \\ \text { H } & -0.459121 & 8.959966 & 3.857413 \\ \text { H } & -0.309596 & 9.255695 & 1.381420 \\ \text { H } & -0.819482 & 5.418146 & -5.429142\end{array}$

Allenyne_A

39

Free energy $=-922.213213$ Hartree

$\begin{array}{lrrr}\text { C } & -0.665072 & -2.749609 & -0.216201 \\ \mathrm{C} & -0.004663 & -1.807964 & 0.638158 \\ \mathrm{C} & -0.784605 & -0.955935 & 1.426033 \\ \mathrm{C} & -2.203423 & -0.985445 & 1.374842 \\ \mathrm{C} & -2.859281 & -1.936268 & 0.504557 \\ \mathrm{C} & -2.048287 & -2.814082 & -0.276679 \\ \mathrm{C} & 1.470074 & -1.735833 & 0.632468 \\ \mathrm{C} & 2.196304 & -0.842141 & 1.297603 \\ \mathrm{C} & 2.819509 & 0.078944 & 2.016972 \\ \mathrm{O} & 3.157952 & 1.350682 & 1.623162 \\ \mathrm{C} & 2.756575 & 1.715474 & 0.289078 \\ \mathrm{C} & 1.309034 & 1.784632 & 0.085636 \\ \mathrm{C} & 0.097517 & 1.844514 & -0.111127 \\ \mathrm{C} & -1.311245 & 1.908026 & -0.345778 \\ \mathrm{C} & -2.095716 & 2.943391 & 0.225269 \\ \mathrm{C} & -3.480993 & 2.980371 & 0.014928 \\ \mathrm{C} & -4.105587 & 1.992624 & -0.766630 \\ \mathrm{C} & -3.336937 & 0.964303 & -1.339455 \\ \mathrm{C} & -1.953368 & 0.916661 & -1.132786 \\ \mathrm{C} & -4.283891 & -1.941095 & 0.435771 \\ \mathrm{C} & -5.033693 & -1.034571 & 1.173931 \\ \mathrm{H} & -5.195282 & 2.016619 & -0.921058 \\ \mathrm{H} & 3.221407 & 2.708998 & 0.121314 \\ \mathrm{H} & 3.158950 & -0.108914 & 3.050448 \\ \mathrm{H} & 1.984138 & -2.478134 & -0.010556 \\ \mathrm{H} & -1.601731 & 3.712317 & 0.838217 \\ \mathrm{H} & -0.284759 & -0.217958 & 2.072563 \\ \mathrm{H} & -4.079520 & 3.784471 & 0.470348 \\ \mathrm{C} & -3.005364 & -0.067338 & 2.120281 \\ \mathrm{H} & -3.824689 & 0.173697 & -1.929062 \\ \mathrm{H} & -2.541005 & -3.543327 & -0.940249 \\ \mathrm{H} & -0.055351 & -3.426605 & -0.836530 \\ \mathrm{H} & 3.199866 & 0.997362 & -0.442868 \\ \mathrm{H} & -1.355147 & 0.090627 & -1.545253 \\ \mathrm{C} & -4.388229 & -0.086732 & 2.018139 \\ \mathrm{H} & -2.501295 & 0.675733 & 2.757896 \\ \mathrm{H} & -4.992122 & 0.639565 & 2.583493 \\ \mathrm{H} & -6.133079 & -1.041167 & 1.102649 \\ \mathrm{H} & -4.781477 & -2.669367 & -0.224768 \\ & & & \\ & & & \end{array}$

Allenyne_A2Cyclization_TS

39

Free energy $=-922.1985586$ Hartree

$\begin{array}{lrrr}\mathrm{C} & -0.611539 & -2.789558 & -0.067888 \\ \mathrm{C} & 0.000176 & -1.801471 & 0.780224 \\ \mathrm{C} & -0.833448 & -0.945906 & 1.521583 \\ \mathrm{C} & -2.248119 & -0.998046 & 1.401030 \\ \mathrm{C} & -2.844533 & -1.957950 & 0.500731 \\ \mathrm{C} & -1.984295 & -2.861369 & -0.203750 \\ \mathrm{C} & 1.437943 & -1.628034 & 0.771736 \\ \mathrm{C} & 2.056149 & -0.479686 & 1.159233 \\ \mathrm{C} & 3.101522 & 0.162559 & 1.707861 \\ \mathrm{O} & 3.462307 & 1.407876 & 1.290193\end{array}$

$\begin{array}{lrrr}\text { C } & 2.658247 & 1.796440 & 0.165277 \\ \mathrm{C} & 1.268952 & 1.270033 & 0.299720 \\ \mathrm{C} & 0.050162 & 1.455356 & 0.066601 \\ \mathrm{C} & -1.323186 & 1.685726 & -0.199519 \\ \mathrm{C} & -2.034264 & 2.701653 & 0.500580 \\ \mathrm{C} & -3.392013 & 2.927561 & 0.241654 \\ \mathrm{C} & -4.076057 & 2.145272 & -0.705679 \\ \mathrm{C} & -3.388497 & 1.128998 & -1.395673 \\ \mathrm{C} & -2.031307 & 0.898283 & -1.152710 \\ \mathrm{C} & -4.258500 & -1.963333 & 0.335682 \\ \mathrm{C} & -5.057574 & -1.050376 & 1.015891 \\ \mathrm{H} & -5.145838 & 2.317484 & -0.898260 \\ \mathrm{H} & 2.681997 & 2.903150 & 0.123614 \\ \mathrm{H} & 3.664297 & -0.176350 & 2.592168 \\ \mathrm{H} & 2.035146 & -2.400441 & 0.249729 \\ \mathrm{H} & -1.498514 & 3.308445 & 1.245982 \\ \mathrm{H} & -0.378928 & -0.219576 & 2.211506 \\ \mathrm{H} & -3.925726 & 3.717813 & 0.792123 \\ \mathrm{C} & -3.098163 & -0.086355 & 2.096271 \\ \mathrm{H} & -3.924897 & 0.494874 & -2.117788 \\ \mathrm{H} & -2.438287 & -3.611008 & -0.872386 \\ \mathrm{H} & 0.036681 & -3.475431 & -0.637088 \\ \mathrm{H} & 3.109842 & 1.384154 & -0.767336 \\ \mathrm{H} & -1.501093 & 0.082245 & -1.665136 \\ \mathrm{C} & -4.472670 & -0.106585 & 1.903358 \\ \mathrm{H} & -2.638904 & 0.655632 & 2.767669 \\ \mathrm{H} & -5.112730 & 0.618790 & 2.428487 \\ \mathrm{H} & -6.149011 & -1.055800 & 0.866872 \\ \mathrm{H} & -4.710952 & -2.695994 & -0.351919\end{array}$

Allenyne_A2Cyclization_INT

39

Free energy $=-922.2786492$ Hartree

$\begin{array}{llll}\text { C } & -0.410450 & -3.237012 & 0.723296\end{array}$

$\begin{array}{llll}\text { C } & 0.092309 & -1.910167 & 0.982971\end{array}$

$\begin{array}{llll}\text { C } & -0.896579 & -0.776336 & 1.300683\end{array}$

$\begin{array}{llll}\text { C } & -2.371993 & -1.156894 & 1.177348\end{array}$

$\begin{array}{llll}\text { C } & -2.759293 & -2.475281 & 0.800158\end{array}$

$\begin{array}{llll}\text { C } & -1.745746 & -3.501309 & 0.593889\end{array}$

$\begin{array}{llll}\text { C } & 1.441550 & -1.631364 & 0.920020\end{array}$

$\begin{array}{llll}\text { C } & 1.877988 & -0.264249 & 0.839208\end{array}$

$\begin{array}{llll}\text { C } & 3.115333 & 0.307561 & 0.652335\end{array}$

$\begin{array}{llll}\text { O } & 3.081585 & 1.617508 & 0.298407\end{array}$

$\begin{array}{llll}\text { C } & 1.679638 & 1.999479 & 0.149756\end{array}$

$\begin{array}{llll}\text { C } & 0.886522 & 0.781965 & 0.574624\end{array}$

$\begin{array}{llll}\text { C } & -0.455452 & 0.556240 & 0.623100\end{array}$

$\begin{array}{llll}\text { C } & -1.416631 & 1.554473 & 0.095167\end{array}$

$\begin{array}{llll}\text { C } & -1.469914 & 2.869988 & 0.612029\end{array}$

$\begin{array}{llll}\text { C } & -2.366289 & 3.815956 & 0.088210\end{array}$

$\begin{array}{llll}\text { C } & -3.228794 & 3.463442 & -0.963699\end{array}$

$\begin{array}{llll}\text { C } & -3.187918 & 2.158173 & -1.486477\end{array}$

$\begin{array}{llll}\text { C } & -2.294640 & 1.213065 & -0.962502\end{array}$

$\begin{array}{llll}\text { C } & -4.136576 & -2.791628 & 0.696316\end{array}$

$\begin{array}{llll}\text { C } & -5.122766 & -1.844712 & 0.998738\end{array}$

$\mathrm{H} \quad-3.932042 \quad 4.203956 \quad-1.375136$

$\begin{array}{llll}\mathrm{H} & 1.510137 & 2.890732 & 0.786281\end{array}$

$\begin{array}{llll}\mathrm{H} & 4.119183 & -0.134157 & 0.717246\end{array}$

$\begin{array}{llll}\mathrm{H} & 2.167202 & -2.446259 & 0.766036\end{array}$

$\begin{array}{llll}\mathrm{H} & -0.815815 & 3.141474 & 1.455870\end{array}$

$\begin{array}{llll}\mathrm{H} & -0.760118 & -0.570245 & 2.393378\end{array}$

$\begin{array}{llll}\mathrm{H} & -2.396486 & 4.832403 & 0.510819\end{array}$

$\begin{array}{llll}\text { C } & -3.377172 & -0.237299 & 1.530209\end{array}$

$\begin{array}{llll}\mathrm{H} & -3.858751 & 1.873777 & -2.312253\end{array}$

$\begin{array}{llll}\mathrm{H} & -2.086132 & -4.517086 & 0.337061\end{array}$

$\begin{array}{llll}\mathrm{H} & 0.327314 & -4.040579 & 0.563813\end{array}$

$\begin{array}{llll}\mathrm{H} & 1.506986 & 2.296099 & -0.908503\end{array}$

$\begin{array}{llll}\mathrm{H} & -2.269630 & 0.190941 & -1.369252\end{array}$

$\begin{array}{lrrr}\mathrm{C} & -4.740098 & -0.566472 & 1.439011\end{array}$

1.880636

1.712380

$\begin{array}{llll}\mathrm{H} & -6.188217 & -2.108972 & 0.911474 \\ \mathrm{H} & -4.424247 & -3.809343 & 0.387345\end{array}$

Allenyne_B

39

Free energy $=-922.2095565$ Hartree

$\begin{array}{lrrr}\mathrm{C} & 0.726573 & 1.535739 & -0.888137 \\ \mathrm{C} & 0.922057 & 2.139490 & 0.399999 \\ \mathrm{C} & -0.169979 & 2.725224 & 1.051853 \\ \mathrm{C} & -1.455804 & 2.762667 & 0.447792 \\ \mathrm{C} & -1.635293 & 2.185090 & -0.863874 \\ \mathrm{C} & -0.515542 & 1.563991 & -1.496883 \\ \mathrm{C} & 2.226549 & 2.205475 & 0.977517 \\ \mathrm{C} & 3.345345 & 2.321201 & 1.471165 \\ \mathrm{C} & 4.659951 & 2.534405 & 2.078320 \\ \mathrm{O} & 5.614762 & 3.191095 & 1.226099 \\ \mathrm{C} & 5.304660 & 4.479654 & 0.859584 \\ \mathrm{C} & 4.224271 & 5.169888 & 1.193626 \\ \mathrm{C} & 3.085257 & 5.814832 & 1.425970 \\ \mathrm{C} & 1.876021 & 5.739477 & 0.579727\end{array}$




$\begin{array}{rrrr}\text { C } & 1.885741 & 5.093661 & -0.679889 \\ \text { C } & 0.713929 & 4.989385 & -1.438888 \\ \text { C } & -0.494059 & 5.525296 & -0.956988 \\ \text { C } & -0.515637 & 6.183247 & 0.284463 \\ \text { C } & 0.658825 & 6.293021 & 1.043915 \\ \text { C } & -2.913521 & 2.279783 & -1.489728 \\ \text { C } & -3.971474 & 2.917630 & -0.854458 \\ \text { H } & -1.422305 & 5.408932 & -1.536684 \\ \text { H } & 6.110375 & 4.905188 & 0.235889 \\ \text { H } & 4.533602 & 3.123750 & 3.019297 \\ \text { H } & 2.993348 & 6.437679 & 2.338441 \\ \text { H } & 2.827248 & 4.652909 & -1.041800 \\ \text { H } & -0.020308 & 3.208083 & 2.028242 \\ \text { H } & 0.733780 & 4.462146 & -2.404104 \\ \text { C } & -2.566947 & 3.400701 & 1.079095 \\ \text { H } & -1.460132 & 6.597497 & 0.668851 \\ \text { H } & -0.651007 & 1.116380 & -2.493935 \\ \text { H } & 1.588251 & 1.072732 & -1.390606 \\ \text { H } & 5.136618 & 1.567546 & 2.341109 \\ \text { H } & 0.636403 & 6.800362 & 2.022043 \\ \text { C } & -3.796633 & 3.480509 & 0.441758 \\ \text { H } & -3.046813 & 1.843591 & -2.492243 \\ \text { H } & -4.950877 & 2.989849 & -1.352421 \\ \text { H } & -4.643138 & 3.981809 & 0.935781 \\ \text { H } & -2.423690 & 3.844973 & 2.076066\end{array}$

Allenyne_B2Cyclization_TS

39

Free energy $=-922.193504$ Hartree

$\begin{array}{lrrr}\mathrm{C} & 0.716375 & 1.758297 & -0.985645 \\ \mathrm{C} & 0.905757 & 2.418146 & 0.282650 \\ \mathrm{C} & -0.226776 & 2.902780 & 0.963356 \\ \mathrm{C} & -1.532924 & 2.769190 & 0.421422 \\ \mathrm{C} & -1.705631 & 2.119882 & -0.858859 \\ \mathrm{C} & -0.545390 & 1.620538 & -1.530641 \\ \mathrm{C} & 2.208082 & 2.612505 & 0.797539 \\ \mathrm{C} & 3.344975 & 2.906378 & 1.247325 \\ \mathrm{C} & 4.523772 & 2.399672 & 2.015674 \\ \mathrm{O} & 5.721559 & 3.078702 & 1.610253 \\ \mathrm{C} & 5.425443 & 4.357992 & 1.254432 \\ \mathrm{C} & 4.148369 & 4.784689 & 1.203884 \\ \mathrm{C} & 3.249096 & 5.803499 & 1.298647 \\ \mathrm{C} & 2.048235 & 5.863900 & 0.481845 \\ \mathrm{C} & 1.974890 & 5.175204 & -0.758755 \\ \mathrm{C} & 0.767294 & 5.097916 & -1.464674 \\ \mathrm{C} & -0.393904 & 5.686438 & -0.939404 \\ \mathrm{C} & -0.328579 & 6.416186 & 0.266230 \\ \mathrm{C} & 0.880101 & 6.518119 & 0.960928 \\ \mathrm{C} & -3.014584 & 2.017678 & -1.411458 \\ \mathrm{C} & -4.115947 & 2.535512 & -0.738643 \\ \mathrm{H} & -1.354362 & 5.579291 & -1.465837 \\ \mathrm{H} & 6.300257 & 4.923649 & 0.897454 \\ \mathrm{H} & 4.348404 & 2.587102 & 3.101395 \\ \mathrm{H} & 3.317014 & 6.500737 & 2.155598 \\ \mathrm{H} & 2.883280 & 4.693598 & -1.149821 \\ \mathrm{H} & -0.092359 & 3.432212 & 1.917802 \\ \mathrm{H} & 0.720131 & 4.537346 & -2.409694 \\ \mathrm{C} & -2.686078 & 3.285464 & 1.090022 \\ \mathrm{H} & -1.237334 & 6.889266 & 0.668870 \\ \mathrm{H} & -0.671938 & 1.124157 & -2.505859 \\ \mathrm{H} & 1.601220 & 1.380953 & -1.518593 \\ \mathrm{H} & 4.695038 & 1.316659 & 1.856961 \\ \mathrm{H} & 0.925556 & 7.066447 & 1.915748 \\ \mathrm{C} & -3.948086 & 3.173839 & 0.522339 \\ \mathrm{H} & -3.140463 & 1.524161 & -2.388137 \\ \mathrm{H} & -5.121422 & 2.452773 & -1.179501 \\ \mathrm{H} & -4.826047 & 3.580081 & 1.047806 \\ \mathrm{H} & -2.553766 & 3.781976 & 2.064031 \\ & & & \\ & & & \end{array}$

Allenyne_B2Cyclization_INT

39

Free energy $=-922.2685381$ Hartree

$\begin{array}{lrrr}\mathrm{C} & 0.722312 & 2.189885 & -1.178232 \\ \mathrm{C} & 0.544518 & 3.134603 & -0.116163 \\ \mathrm{C} & -0.681695 & 3.168624 & 0.550603 \\ \mathrm{C} & -1.752127 & 2.295274 & 0.196145 \\ \mathrm{C} & -1.558636 & 1.348140 & -0.878719 \\ \mathrm{C} & -0.296807 & 1.323597 & -1.546027 \\ \mathrm{C} & 1.666692 & 4.037899 & 0.264522 \\ \mathrm{C} & 2.753011 & 3.552779 & 0.916991 \\ \mathrm{C} & 3.060235 & 2.155176 & 1.410132 \\ \mathrm{O} & 4.407735 & 2.231625 & 1.962703 \\ \mathrm{C} & 4.803300 & 3.533604 & 1.906331 \\ \mathrm{C} & 3.892865 & 4.377733 & 1.317460 \\ \mathrm{C} & 3.828298 & 5.800049 & 1.137062 \\ \mathrm{C} & 2.738283 & 6.367228 & 0.511790 \\ \mathrm{C} & 1.687980 & 5.503292 & -0.208492 \\ \mathrm{C} & 0.331040 & 6.174083 & -0.298238 \\ \mathrm{C} & 0.199494 & 7.521156 & -0.178452 \\ \mathrm{C} & 1.342315 & 8.362591 & 0.143051\end{array}$

$\begin{array}{lrrr}\text { C } & 2.546577 & 7.801373 & 0.486189 \\ \mathrm{C} & -2.632702 & 0.477121 & -1.234029 \\ \mathrm{C} & -3.845917 & 0.532183 & -0.560572 \\ \mathrm{H} & -0.785349 & 7.993914 & -0.322712 \\ \mathrm{H} & 5.794554 & 3.738580 & 2.332158 \\ \mathrm{H} & 2.370884 & 1.818450 & 2.215703 \\ \mathrm{H} & 4.610979 & 6.446542 & 1.566185 \\ \mathrm{H} & 2.035686 & 5.454412 & -1.281379 \\ \mathrm{H} & -0.828391 & 3.885058 & 1.374492 \\ \mathrm{H} & -0.533016 & 5.547760 & -0.565927 \\ \mathrm{C} & -3.011548 & 2.323405 & 0.870258 \\ \mathrm{H} & 1.211603 & 9.455634 & 0.177557 \\ \mathrm{H} & -0.144001 & 0.605973 & -2.368362 \\ \mathrm{H} & 1.688132 & 2.168800 & -1.707161 \\ \mathrm{H} & 3.054515 & 1.383237 & 0.611691 \\ \mathrm{H} & 3.377192 & 8.438491 & 0.832902 \\ \mathrm{C} & -4.035789 & 1.462076 & 0.500813 \\ \mathrm{H} & -2.482941 & -0.240584 & -2.056538 \\ \mathrm{H} & -4.667244 & -0.143173 & -0.846399 \\ \mathrm{H} & -5.001710 & 1.496437 & 1.028095 \\ \mathrm{H} & -3.157621 & 3.044437 & 1.690589\end{array}$

Bisallene

Free energy $=-922.2223813$ Hartree

$\begin{array}{llll}\text { C } & 5.154883 & 8.914864 & -2.612575\end{array}$

$\begin{array}{llll}\text { C } & 4.006892 & 9.171129 & -1.823497 \\ \text { C } & 2.742754 & 9.216286 & -2.457679\end{array}$

$\begin{array}{llll}\text { C } & 2.742754 & 9.216286 & -2.457679\end{array}$

$\begin{array}{llll}\text { C } & 2.623404 & 8.987598 & -3.834435\end{array}$

$\begin{array}{llll}\text { C } & 3.767896 & 8.718676 & -4.605154\end{array}$

$\begin{array}{llll}\text { C } & 5.034015 & 8.691475 & -3.989844\end{array}$

$\begin{array}{llll}\text { C } & 4.086198 & 9.303715 & -0.360203\end{array}$

$\begin{array}{llll}\text { C } & 5.178743 & 9.153031 & 0.383989\end{array}$

$\begin{array}{llll}\text { C } & 5.831560 & 6.419644 & 0.099004\end{array}$

$\begin{array}{llll}\text { C } & 6.453720 & 6.705006 & 1.230720\end{array}$

$\begin{array}{llll}\text { O } & 6.839173 & 7.953152 & 1.684953\end{array}$

$\begin{array}{llll}\text { C } & 6.279161 & 9.082626 & 1.114019\end{array}$

$\begin{array}{llll}\text { C } & 5.255761 & 6.067203 & -1.048147\end{array}$

$\begin{array}{llll}\text { C } & 3.811129 & 6.026240 & -1.312672\end{array}$

$\begin{array}{llll}\text { C } & 3.351594 & 5.781333 & -2.609597\end{array}$

$\begin{array}{llll}\text { C } & 1.971455 & 5.871437 & -2.940544\end{array}$

$\begin{array}{llll}\text { C } & 1.024261 & 6.213289 & -1.904350\end{array}$

$\begin{array}{llll}\text { C } & 1.509101 & 6.415495 & -0.574445\end{array}$

$\begin{array}{llll}\text { C } & 2.858474 & 6.326890 & -0.284560\end{array}$

$\begin{array}{llll}\text { C } & -0.352950 & 6.354332 & -2.248686\end{array}$

$\begin{array}{llll}\text { C } & -0.782802 & 6.171158 & -3.557560\end{array}$

$\begin{array}{llll}\text { C } & 0.149920 & 5.830158 & -4.577841\end{array}$

$\begin{array}{llll}\text { C } & 1.496028 & 5.681376 & -4.273780\end{array}$

$\begin{array}{lllr}\mathrm{H} & 3.228373 & 6.511993 & 0.735309\end{array}$

$\begin{array}{llll}\mathrm{H} & 3.671945 & 8.523605 & -5.684633\end{array}$

$\begin{array}{llll}\mathrm{H} & 6.864390 & 9.974630 & 1.394217\end{array}$

$\begin{array}{llll}\mathrm{H} & 6.769955 & 5.928625 & 1.947695\end{array}$

$\begin{array}{llll}\mathrm{H} & 3.120528 & 9.470224 & 0.155604\end{array}$

$\begin{array}{llll}\mathrm{H} & 5.908220 & 5.855048 & -1.917659\end{array}$

$\begin{array}{llll}\mathrm{H} & 6.138356 & 8.872879 & -2.119777\end{array}$

$\begin{array}{llll}\mathrm{H} & 4.075529 & 5.565141 & -3.411310\end{array}$

$\begin{array}{llll}\mathrm{H} & 5.933687 & 8.484803 & -4.590456\end{array}$

$\begin{array}{llll}\mathrm{H} & 1.628236 & 8.988074 & -4.304538\end{array}$

$\begin{array}{llll}\mathrm{H} & 0.787098 & 6.661391 & 0.220764\end{array}$

$\begin{array}{llll}\mathrm{H} & 1.841133 & 9.392288 & -1.850749\end{array}$

$\begin{array}{llll}\mathrm{H} & -1.073554 & 6.617730 & -1.457666\end{array}$

$\begin{array}{llll}\mathrm{H} & -1.847853 & 6.290109 & -3.810365\end{array}$

$\begin{array}{llll}\mathrm{H} & -0.201763 & 5.685518 & -5.611030\end{array}$

$\begin{array}{llll}\mathrm{H} & 2.222773 & 5.430072 & -5.062212\end{array}$

Bisallene2Cyclization_TS1

39

Free energy $=-922.2159448$ Hartree

$\begin{array}{lrrr}\mathrm{C} & 5.200603 & 8.880323 & -2.604840 \\ \mathrm{C} & 4.076469 & 9.203250 & -1.796461 \\ \mathrm{C} & 2.801124 & 9.307878 & -2.417454 \\ \mathrm{C} & 2.647578 & 9.044380 & -3.779089 \\ \mathrm{C} & 3.766364 & 8.689700 & -4.561824 \\ \mathrm{C} & 5.041978 & 8.629825 & -3.974592 \\ \mathrm{C} & 4.185686 & 9.274745 & -0.350443 \\ \mathrm{C} & 5.274389 & 8.875246 & 0.356968 \\ \mathrm{C} & 5.743961 & 6.764783 & 0.061781 \\ \mathrm{C} & 6.503634 & 6.747013 & 1.158387 \\ \mathrm{O} & 6.874362 & 7.904436 & 1.806734 \\ \mathrm{C} & 6.238946 & 9.001706 & 1.270231 \\ \mathrm{C} & 5.214337 & 6.234529 & -1.073220 \\ \mathrm{C} & 3.790606 & 6.111408 & -1.337272 \\ \mathrm{C} & 3.332318 & 5.799446 & -2.628100 \\ \mathrm{C} & 1.954459 & 5.853062 & -2.960471 \\ \mathrm{C} & 0.998421 & 6.222435 & -1.938821 \\ \mathrm{C} & 1.475070 & 6.480487 & -0.616745 \\ \mathrm{C} & 2.825089 & 6.428360 & -0.320687 \\ \mathrm{C} & -0.381351 & 6.322896 & -2.289066 \\ \mathrm{C} & -0.807435 & 6.076762 & -3.587997\end{array}$




$\begin{array}{lrrr}\mathrm{C} & 0.133102 & 5.711770 & -4.594831 \\ \mathrm{C} & 1.480506 & 5.600504 & -4.286226 \\ \mathrm{H} & 3.187526 & 6.648789 & 0.694277 \\ \mathrm{H} & 3.637207 & 8.460727 & -5.631032 \\ \mathrm{H} & 6.646950 & 9.952130 & 1.644112 \\ \mathrm{H} & 6.880984 & 5.843636 & 1.659422 \\ \mathrm{H} & 3.244631 & 9.453379 & 0.203641 \\ \mathrm{H} & 5.889639 & 6.057058 & -1.931207 \\ \mathrm{H} & 6.192358 & 8.817484 & -2.132720 \\ \mathrm{H} & 4.061438 & 5.565490 & -3.419887 \\ \mathrm{H} & 5.918745 & 8.368054 & -4.587322 \\ \mathrm{H} & 1.646527 & 9.079363 & -4.235258 \\ \mathrm{H} & 0.745978 & 6.739351 & 0.167851 \\ \mathrm{H} & 1.920939 & 9.540302 & -1.798097 \\ \mathrm{H} & -1.106749 & 6.605059 & -1.508981 \\ \mathrm{H} & -1.874532 & 6.164167 & -3.845044 \\ \mathrm{H} & -0.215919 & 5.518348 & -5.620914 \\ \mathrm{H} & 2.211485 & 5.329961 & -5.064492\end{array}$

Bisallene2Cyclization_INT1

39

Free energy $=-922.2597485$ Hartree

$\begin{array}{llll}\text { C } & 3.183598 & 9.295076 & -3.718873 \\ \text { C } & 3.924978 & 8.214714 & -3.136640\end{array}$

$8.214714-3.136640$

$\begin{array}{llll}C & 3.606553 & 6.891679 & -3.578150\end{array}$

$\begin{array}{llll}\text { C } & 2.584385 & 6.665850 & -4.509976\end{array}$

$\begin{array}{llll}\text { C } & 1.824116 & 7.735802 & -5.004695\end{array}$

$\begin{array}{llll}\text { C } & 2.146160 & 9.058189 & -4.616854\end{array}$

$\begin{array}{llll}\text { C } & 4.920287 & 8.505427 & -2.152966\end{array}$

$\begin{array}{llll}\text { C } & 5.602187 & 7.681514 & -1.212825\end{array}$

$\begin{array}{llll}\text { C } & 5.409318 & 6.314021 & -0.635088\end{array}$

$\begin{array}{llll}\text { C } & 6.577887 & 6.091220 & 0.122507\end{array}$

$\begin{array}{llll}\text { O } & 7.384753 & 7.173074 & 0.118175\end{array}$

$\begin{array}{llll}\text { C } & 6.815497 & 8.127869 & -0.647716\end{array}$

$\begin{array}{llll}\text { C } & 4.379149 & 5.329221 & -0.634587\end{array}$

$\begin{array}{llll}\text { C } & 2.998702 & 5.373079 & -0.987936\end{array}$

$\begin{array}{llll}\text { C } & 2.287358 & 4.122214 & -1.153683\end{array}$

$\begin{array}{llll}\text { C } & 0.975187 & 4.084845 & -1.569596\end{array}$

$\begin{array}{llll}\text { C } & 0.242401 & 5.291436 & -1.837057\end{array}$

$\begin{array}{llll}\text { C } & 0.909204 & 6.554808 & -1.626804\end{array}$

$\begin{array}{llll}\text { C } & 2.267426 & 6.566644 & -1.208539\end{array}$

$\begin{array}{llll}\mathrm{H} & 2.828681 & 3.181787 & -0.962473\end{array}$

$\begin{array}{llll}\mathrm{H} & 0.990611 & 7.550146 & -5.698928\end{array}$

\begin{tabular}{llll}
$\mathrm{H}$ & 7.377514 & 9.059102 & -0.773591 \\
\hline
\end{tabular}

$\begin{array}{llll}\mathrm{H} & 6.897147 & 5.240599 & 0.733491\end{array}$

$\begin{array}{llll}\mathrm{H} & 5.261793 & 9.556085 & -2.143805\end{array}$

$\begin{array}{llll}\mathrm{H} & 4.715170 & 4.353291 & -0.240844\end{array}$

$\begin{array}{llll}\mathrm{H} & 4.208673 & 6.048806 & -3.215451\end{array}$

$\begin{array}{llll}\mathrm{H} & 2.742129 & 7.534814 & -1.007838\end{array}$

$\mathrm{H} \quad 2.357169 \quad 5.635296 \quad-4.821310$

$\begin{array}{llll}\mathrm{H} & 1.568059 & 9.903309 & -5.021058\end{array}$

$\begin{array}{llll}\mathrm{H} & 0.465987 & 3.117441 & -1.707233\end{array}$

$\begin{array}{llll}\mathrm{H} & 3.426137 & 10.327040 & -3.418027\end{array}$

$\begin{array}{llll}\text { C } & -1.103106 & 5.287315 & -2.292953\end{array}$

$\begin{array}{llll}\text { C } & -1.777254 & 6.481966 & -2.543144\end{array}$

$\begin{array}{llll}\text { C } & -1.119538 & 7.725529 & -2.350577\end{array}$

$\begin{array}{llll}\text { C } & 0.197238 & 7.760903 & -1.904170\end{array}$

$\begin{array}{llll}\mathrm{H} & -1.608211 & 4.320661 & -2.449214\end{array}$

$\begin{array}{llll}\mathrm{H} & -2.818537 & 6.462614 & -2.899117\end{array}$

$\begin{array}{llll}\mathrm{H} & -1.652254 & 8.664656 & -2.565394\end{array}$

$\begin{array}{llll}\mathrm{H} & 0.719895 & 8.721333 & -1.776764\end{array}$

Bisallene2Cyclization_TS2_for_A

39

Free energy $=-922.243159$ Hartree

C $\quad 4.780558 \quad 10.169963 \quad-1.610967$

$\begin{array}{llll}\text { C } & 4.523010 & 9.063794 & -2.470581\end{array}$

$\begin{array}{llll}\text { C } & 3.830411 & 9.319456 & -3.689505\end{array}$

$\begin{array}{llll}\text { C } & 3.441178 & 10.616091 & -4.041355\end{array}$

$\begin{array}{llll}\text { C } & 3.719014 & 11.700216 & -3.185423\end{array}$

$\begin{array}{llll}\text { C } & 4.386151 & 11.465290 & -1.967598\end{array}$

$\begin{array}{llll}\text { C } & 4.838848 & 7.698580 & -2.102127\end{array}$

$\begin{array}{llll}\text { C } & 5.819619 & 7.328367 & -1.070520\end{array}$

$\begin{array}{llll}\text { C } & 5.704462 & 6.143900 & -0.206229\end{array}$

$\begin{array}{llll}\text { C } & 6.859854 & 6.139717 & 0.560041\end{array}$

$\begin{array}{llll}\mathrm{O} & 7.667693 & 7.196152 & 0.242920\end{array}$

$\begin{array}{llll}\text { C } & 7.029147 & 7.898551 & -0.750803\end{array}$

$\begin{array}{llll}\text { C } & 4.615619 & 5.203236 & -0.276130\end{array}$

$\begin{array}{llll}\text { C } & 3.367603 & 5.587969 & -0.813019\end{array}$

$\begin{array}{llll}\text { C } & 2.439786 & 4.636101 & -1.318157\end{array}$

$\begin{array}{llll}\text { C } & 1.286548 & 5.019091 & -2.033305\end{array}$

$\begin{array}{llll}\text { C } & 1.006907 & 6.439746 & -2.232183 \\ \text { C } & 1.866291 & 7.393658 & -1.646103\end{array}$

$\begin{array}{llll}\text { C } & 1.866291 & 7.393658 & -1.646103\end{array}$

$\begin{array}{llll}\text { C } & 3.044801 & 7.016163 & -0.965589\end{array}$

$\begin{array}{llll}\text { C } & -0.161348 & 6.811725 & -2.974200\end{array}$

$\begin{array}{llll}\text { C } & -1.012347 & 5.851800 & -3.497312\end{array}$

$\begin{array}{llll}\text { C } & -0.739504 & 4.460613 & -3.303422\end{array}$

$\begin{array}{llll}\text { C } & 0.378105 & 4.058661 & -2.590748\end{array}$

$\begin{array}{llll}\mathrm{H} & 3.445837 & 7.712164 & -0.213432\end{array}$

$\begin{array}{llll}\mathrm{H} & 3.411270 & 12.719851 & -3.462828\end{array}$

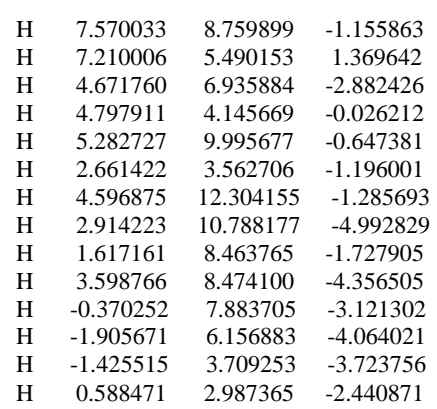

Bisallene2Cyclization_INT2_for_A

39

Free energy $=-922.2744115$ Hartre

$\begin{array}{llll}\text { C } & 4.309454 & 10.077392 & -1.317771\end{array}$

$\begin{array}{llll}\text { C } & 4.401391 & 9.143200 & -2.372303\end{array}$

$\begin{array}{llll}\text { C } & 4.281084 & 9.610082 & -3.696833\end{array}$

$\begin{array}{llll}\text { C } & 4.068297 & 10.973446 & -3.964770\end{array}$

$\begin{array}{llll}\text { C } & 3.972771 & 11.892361 & -2.907089\end{array}$

$\begin{array}{llll}\text { C } & 4.095768 & 11.439272 & -1.581061\end{array}$

$\begin{array}{llll}\text { C } & 4.570472 & 7.661192 & -2.081076\end{array}$

$\begin{array}{llll}\text { C } & 5.721704 & 7.348484 & -1.160632\end{array}$

$\begin{array}{llll}\text { C } & 5.751182 & 6.118692 & -0.389452\end{array}$

$\begin{array}{llll}\text { C } & 6.973541 & 6.120400 & 0.267895\end{array}$

$\begin{array}{llll}\text { O } & 7.677670 & 7.247514 & -0.043813\end{array}$

$\begin{array}{llll}\text { C } & 6.912695 & 7.983055 & -0.918165\end{array}$

$\begin{array}{llll}\text { C } & 4.656433 & 5.187235 & -0.440330\end{array}$

$\begin{array}{llll}\text { C } & 3.461345 & 5.567020 & -1.029423\end{array}$

$\begin{array}{llll}\text { C } & 2.367512 & 4.655166 & -1.174842\end{array}$

$\begin{array}{llll}\text { C } & 1.202946 & 4.970678 & -1.865626\end{array}$

$\begin{array}{llll}\text { C } & 1.075870 & 6.297282 & -2.513523\end{array}$

$\begin{array}{llll}\text { C } & 2.068151 & 7.234553 & -2.359778\end{array}$

$\begin{array}{llll}\text { C } & 3.265961 & 7.025122 & -1.477841 \\ \text { C } & -0.118107 & 6.558648 & -3.302298\end{array}$

$\begin{array}{llll}\text { C } & -0.118107 & 6.558648 & -3.302298\end{array}$

$\begin{array}{llll}\text { C } & -1.109358 & 5.618824 & -3.423183\end{array}$

$\begin{array}{llll}\text { C } & -0.988884 & 4.330851 & -2.774011\end{array}$

$\begin{array}{llll}\text { C } & 0.124930 & 4.023538 & -2.028277\end{array}$

$\begin{array}{llll}\mathrm{H} & 3.072348 & 7.605430 & -0.534412\end{array}$

$\begin{array}{llll}\mathrm{H} & 3.802504 & 12.960277 & -3.114763\end{array}$

$\begin{array}{llll}\mathrm{H} & 7.345802 & 8.926571 & -1.264213\end{array}$

$\begin{array}{llll}\mathrm{H} & 7.451448 & 5.429856 & 0.970852\end{array}$

$\begin{array}{llll}\mathrm{H} & 4.754118 & 7.136043 & -3.047975\end{array}$

$\begin{array}{llll}\mathrm{H} & 4.764879 & 4.177917 & -0.013482\end{array}$

$\begin{array}{llll}\mathrm{H} & 4.415370 & 9.728293 & -0.278162\end{array}$

$\begin{array}{llll}\mathrm{H} & 2.484047 & 3.639106 & -0.762209\end{array}$

$\begin{array}{llll}\mathrm{H} & 4.024336 & 12.152949 & -0.745446\end{array}$

$\begin{array}{llll}\mathrm{H} & 3.975382 & 11.318556 & -5.006363\end{array}$

$\begin{array}{llll}\mathrm{H} & 1.970966 & 8.216377 & -2.849149\end{array}$

$\begin{array}{llll}\mathrm{H} & 4.350649 & 8.891009 & -4.529108\end{array}$

$\begin{array}{llll}\mathrm{H} & -0.210607 & 7.541335 & -3.791413\end{array}$

$\begin{array}{llll}\mathrm{H} & -2.011225 & 5.838373 & -4.015608\end{array}$

$\begin{array}{llll}\mathrm{H} & -1.799388 & 3.594095 & -2.888135\end{array}$

$\begin{array}{llll}\mathrm{H} & 0.218352 & 3.038885 & -1.542965\end{array}$

Bisallene2Cyclization_TS2_for_B

Free energy $=-922.2489829$ Hartree

$\begin{array}{llll}\text { C } & 0.562820 & 7.377027 & -1.155917\end{array}$

$\begin{array}{llll}\text { C } & 1.424243 & 6.235088 & -1.17839 \\ \text { C } & 0.828716 & 4.923583 & -1.320484\end{array}$

$\begin{array}{llll}\text { C } & 0.828716 & 4.923583 & -1.320484\end{array}$

$\begin{array}{llll}\text { C } & -0.584159 & 4.813587 & -1.440371\end{array}$

$\begin{array}{llll}\text { C } & -1.394419 & 5.945538 & -1.419080\end{array}$

$\begin{array}{llll}\text { C } & -0.813859 & 7.235777 & -1.273734\end{array}$

$\begin{array}{llll}\text { C } & 2.835063 & 6.350544 & -1.064199\end{array}$

$\begin{array}{llll}\text { C } & 3.681976 & 5.219510 & -1.088873\end{array}$

$\begin{array}{llll}\text { C } & 3.062962 & 3.920405 & -1.212201\end{array}$

$\begin{array}{llll}\text { C } & 1.695773 & 3.782167 & -1.327335\end{array}$

$\begin{array}{llll}\text { C } & 5.103563 & 5.297948 & -0.912713\end{array}$

$\begin{array}{llll}\text { C } & 5.895868 & 6.528953 & -0.973473\end{array}$

$\begin{array}{llll}\text { C } & 7.050368 & 6.844370 & -0.112357\end{array}$

$\begin{array}{llll}\text { C } & 7.515283 & 8.066983 & -0.567201\end{array}$

$\begin{array}{llll}\text { O } & 6.776382 & 8.522239 & -1.624314\end{array}$

$\begin{array}{llll}\text { C } & 5.810525 & 7.575408 & -1.862651\end{array}$

$\begin{array}{llll}\text { C } & 7.578161 & 5.974583 & 0.911271\end{array}$

$\begin{array}{llll}\text { C } & 6.762601 & 5.023806 & 1.558958\end{array}$

$\begin{array}{llll}\text { C } & 7.324965 & 3.870641 & 2.212970\end{array}$

$\begin{array}{llll}\text { C } & 6.514245 & 2.843967 & 2.672126\end{array}$

$\begin{array}{llll}\text { C } & 5.095080 & 2.920218 & 2.532010\end{array}$

$\begin{array}{llll}\text { C } & 4.510208 & 4.057222 & 1.977878\end{array}$

$\begin{array}{llll}\text { C } & 5.314501 & 5.116843 & 1.490045\end{array}$

$\begin{array}{llll}\mathrm{H} & 3.709896 & 3.028858 & -1.210867\end{array}$

$\begin{array}{llll}\mathrm{H} & 4.465904 & 2.093165 & 2.895857\end{array}$

$\begin{array}{llll}\mathrm{H} & 8.314584 & 8.729563 & -0.217304\end{array}$

$\begin{array}{llll}\mathrm{H} & 5.159959 & 7.755861 & -2.724879\end{array}$

$\begin{array}{llll}\mathrm{H} & 8.665653 & 5.965097 & 1.091136\end{array}$

$\begin{array}{llll}\mathrm{H} & 5.670037 & 4.354867 & -0.982456\end{array}$ 


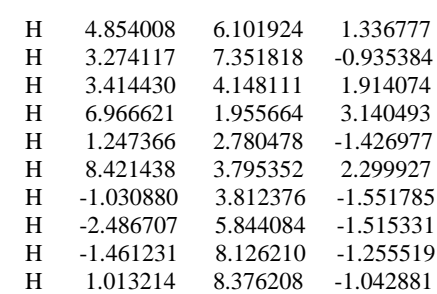

Bisallene2Cyclization_INT2_for_B 39

Free energy $=-922.2863679$ Hartree

$\begin{array}{lrrr}\mathrm{C} & 0.571437 & 7.316530 & -0.585740 \\ \mathrm{C} & 1.418025 & 6.204735 & -0.884425 \\ \mathrm{C} & 0.839715 & 5.039548 & -1.515525 \\ \mathrm{C} & -0.554982 & 5.039369 & -1.821917 \\ \mathrm{C} & -1.349627 & 6.136996 & -1.519288 \\ \mathrm{C} & -0.781416 & 7.284000 & -0.894723 \\ \mathrm{C} & 2.810879 & 6.207393 & -0.581478 \\ \mathrm{C} & 3.626957 & 5.116708 & -0.875514 \\ \mathrm{C} & 3.043452 & 3.971756 & -1.497282 \\ \mathrm{C} & 1.691903 & 3.933560 & -1.809526 \\ \mathrm{C} & 5.098924 & 5.117867 & -0.495680 \\ \mathrm{C} & 5.846882 & 6.343764 & -0.952654 \\ \mathrm{C} & 7.080924 & 6.746602 & -0.305825 \\ \mathrm{C} & 7.539110 & 7.841319 & -1.020779 \\ \mathrm{O} & 6.680287 & 8.132765 & -2.042287 \\ \mathrm{C} & 5.659663 & 7.213637 & -1.996717 \\ \mathrm{C} & 7.590093 & 6.018625 & 0.831285 \\ \mathrm{C} & 6.786152 & 5.098779 & 1.467850 \\ \mathrm{C} & 7.273024 & 4.293732 & 2.567870 \\ \mathrm{C} & 6.523973 & 3.285012 & 3.114723 \\ \mathrm{C} & 5.205235 & 2.967206 & 2.584484 \\ \mathrm{C} & 4.643808 & 3.718403 & 1.602308 \\ \mathrm{C} & 5.312532 & 4.955523 & 1.052625 \\ \mathrm{H} & 3.685689 & 3.106789 & -1.727384 \\ \mathrm{H} & 4.666401 & 2.095593 & 2.988525 \\ \mathrm{H} & 8.409946 & 8.497409 & -0.918656 \\ \mathrm{H} & 4.881668 & 7.316725 & -2.760216 \\ \mathrm{H} & 8.618634 & 6.193575 & 1.184359 \\ \mathrm{H} & 5.580603 & 4.232391 & -0.972122 \\ \mathrm{H} & 4.779373 & 5.831742 & 1.509111 \\ \mathrm{H} & 3.248179 & 7.102765 & -0.110721 \\ \mathrm{H} & 3.645214 & 3.472614 & 1.209352 \\ \mathrm{H} & 6.931998 & 2.676400 & 3.936521 \\ \mathrm{H} & 1.259959 & 3.042229 & -2.292186 \\ \mathrm{H} & 8.295490 & 4.487071 & 2.931119 \\ \mathrm{H} & -0.991465 & 4.150006 & -2.304403 \\ \mathrm{H} & -2.423190 & 6.124509 & -1.763159 \\ \mathrm{H} & -1.421265 & 8.147909 & -0.656588 \\ \mathrm{H} & 1.012156 & 8.203700 & -0.103326\end{array}$

\section{A = 6-methoxynaphthyl, $B=$ phenyl}

Bispropargyl

43

Free energy $=-1036.614737$ Hartree

$\begin{array}{rrrr}\text { C } & 1.508084 & 4.933030 & -0.234434 \\ \mathrm{C} & 0.489459 & 4.917164 & -1.219405 \\ \mathrm{C} & -0.809646 & 5.469170 & -0.903675 \\ \mathrm{C} & -1.010890 & 6.027188 & 0.391502 \\ \mathrm{C} & -0.001700 & 6.036042 & 1.344004 \\ \mathrm{C} & 1.272637 & 5.470074 & 1.034545 \\ \mathrm{C} & -1.849911 & 5.402764 & -1.867725 \\ \mathrm{C} & -1.646929 & 4.805696 & -3.119229 \\ \mathrm{C} & -0.344648 & 4.286063 & -3.436910 \\ \mathrm{C} & 0.685296 & 4.343447 & -2.516159 \\ \mathrm{C} & -2.747704 & 4.626691 & -4.008239 \\ \mathrm{C} & -3.750497 & 4.401683 & -4.681893 \\ \mathrm{C} & -5.015565 & 4.008610 & -5.311497 \\ \mathrm{O} & -5.590767 & 2.806700 & -4.788453 \\ \mathrm{C} & -6.063510 & 2.894711 & -3.444177 \\ \mathrm{C} & -5.031368 & 2.768644 & -2.405182 \\ \mathrm{C} & -4.151889 & 2.669944 & -1.553461 \\ \mathrm{C} & -3.062248 & 2.594333 & -0.630893 \\ \mathrm{C} & -3.183976 & 3.078587 & 0.697282 \\ \mathrm{C} & -2.083063 & 3.050791 & 1.564976 \\ \mathrm{C} & -0.846638 & 2.542197 & 1.131331 \\ \mathrm{C} & -0.718657 & 2.054734 & -0.181248 \\ \mathrm{C} & -1.811917 & 2.072544 & -1.055571 \\ \mathrm{O} & 2.310971 & 5.410493 & 1.918420 \\ \mathrm{C} & 2.137622 & 5.937382 & 3.224488 \\ \mathrm{H} & 0.022540 & 2.544875 & 1.805949 \\ \mathrm{H} & -6.795832 & 2.063678 & -3.337859 \\ \mathrm{H} & -5.745651 & 4.855021 & -5.230471 \\ \mathrm{H} & -1.706907 & 1.717484 & -2.090326\end{array}$

$\begin{array}{rrrr}\mathrm{H} & -2.848579 & 5.783784 & -1.608680 \\ \mathrm{H} & 0.251368 & 1.674493 & -0.534890 \\ \mathrm{H} & -2.186765 & 3.445877 & 2.587037 \\ \mathrm{H} & 1.673322 & 3.926314 & -2.766794 \\ \mathrm{H} & -0.188910 & 3.821579 & -4.421594 \\ \mathrm{H} & -4.868797 & 3.810240 & -6.394624 \\ \mathrm{H} & -4.148335 & 3.489038 & 1.031756 \\ \mathrm{H} & 2.498885 & 4.505776 & -0.448004 \\ \mathrm{H} & -0.199510 & 6.464576 & 2.335278 \\ \mathrm{H} & -1.999113 & 6.443049 & 0.641057 \\ \mathrm{H} & 3.096855 & 5.778231 & 3.752896 \\ \mathrm{H} & 1.913018 & 7.028212 & 3.210658 \\ \mathrm{H} & 1.329824 & 5.412853 & 3.784353 \\ \mathrm{H} & -6.634163 & 3.845694 & -3.285911\end{array}$

Allenyne_A

Free energy $=-1036.630925$ Hartre

$\begin{array}{llll}\text { C } & -4.273027 & -0.119908 & 2.131478\end{array}$

$\begin{array}{llll}\text { C } & -4.953152 & -1.017664 & 1.251694\end{array}$

$\begin{array}{llll}\text { C } & -4.224460 & -1.935887 & 0.492201\end{array}$

$\begin{array}{llll}\text { C } & -2.807291 & -1.966365 & 0.549352\end{array}$

$\begin{array}{llll}\text { C } & -2.113699 & -1.054408 & 1.433984\end{array}$

$\begin{array}{llll}\text { C } & -2.889638 & -0.150097 & 2.216882\end{array}$

$\begin{array}{llll}\text { C } & -2.021448 & -2.832951 & -0.274070\end{array}$

$\begin{array}{llll}\text { C } & -0.637358 & -2.776099 & -0.250108\end{array}$

$\begin{array}{llll}\text { C } & 0.056840 & -1.859672 & 0.606346\end{array}$

$\begin{array}{llll}\text { C } & -0.695530 & -1.033597 & 1.446675\end{array}$

$\begin{array}{llll}\text { C } & 1.527935 & -1.773003 & 0.542421\end{array}$

$\begin{array}{llll}\text { C } & 2.271370 & -0.884662 & 1.197171\end{array}$

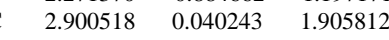

$\begin{array}{llll}\text { O } & 3.189572 & 1.327966 & 1.518395\end{array}$

$\begin{array}{llll}\text { C } & 2.708054 & 1.706732 & 0.216443\end{array}$

$\begin{array}{llll}\text { C } & 1.250115 & 1.770228 & 0.097673\end{array}$

$\begin{array}{llll}\text { C } & 0.030451 & 1.837529 & -0.038473\end{array}$

$\begin{array}{llll}\text { C } & -1.387111 & 1.919560 & -0.206252\end{array}$

$\begin{array}{llll}\text { C } & -2.131369 & 2.961283 & 0.406091\end{array}$

$\begin{array}{llll}\text { C } & -3.523515 & 3.023222 & 0.252267\end{array}$

$\begin{array}{llll}\text { C } & -4.195418 & 2.053877 & -0.513130\end{array}$

$\begin{array}{llll}\text { C } & -3.467688 & 1.018251 & -1.125541\end{array}$

$\begin{array}{llll}\text { C } & -2.078014 & 0.945169 & -0.973762\end{array}$

$\begin{array}{llll}\mathrm{H} & -5.289977 & 2.097970 & -0.627325\end{array}$

$\begin{array}{llll}\mathrm{H} & 3.154265 & 2.706542 & 0.035431\end{array}$

$\begin{array}{llll}\mathrm{H} & 3.290722 & -0.151763 & 2.920475\end{array}$

$\begin{array}{llll}\mathrm{H} & 2.022920 & -2.493698 & -0.139090\end{array}$

$\begin{array}{llll}\mathrm{H} & -1.600057 & 3.717320 & 1.003661\end{array}$

$\begin{array}{llll}\mathrm{H} & -0.174049 & -0.316756 & 2.099905\end{array}$

$\begin{array}{llll}\mathrm{H} & -4.089045 & 3.834410 & 0.736727\end{array}$

$\begin{array}{llll}\mathrm{H} & -3.992143 & 0.241111 & -1.701100\end{array}$

$\begin{array}{llll}\mathrm{H} & -2.536423 & -3.538133 & -0.946146\end{array}$

$\begin{array}{llll}\mathrm{H} & -0.050594 & -3.437511 & -0.908508\end{array}$

$\begin{array}{llll}\mathrm{H} & 3.111263 & 1.003066 & -0.551749\end{array}$

$\begin{array}{llll}\mathrm{H} & -1.511003 & 0.113739 & -1.418168\end{array}$

$\begin{array}{llll}\mathrm{H} & -2.367699 & 0.562183 & 2.874728\end{array}$

$\begin{array}{llll}\mathrm{H} & -4.832392 & 0.613934 & 2.726603\end{array}$

$\begin{array}{llll}\text { O } & -6.308347 & -1.029905 & 1.083335\end{array}$

$\begin{array}{llll}\mathrm{H} & -4.772115 & -2.612466 & -0.180847\end{array}$

$\begin{array}{llll}\text { C } & -7.097601 & -0.090817 & 1.794741\end{array}$

$\begin{array}{llll}\mathrm{H} & -8.145301 & -0.269034 & 1.486424\end{array}$

$\begin{array}{llll}H & -7.021306 & -0.226305 & 2.898438\end{array}$

$\begin{array}{llll}\text { H } & -6.823386 & 0.960040 & 1.544770\end{array}$

Allenyne_A2Cyclization_TS

Free energy $=-1036.618501$ Hartree

$\begin{array}{lrrr}\mathrm{C} & -4.364128 & -0.119674 & 2.106748 \\ \mathrm{C} & -4.994517 & -1.034959 & 1.212105 \\ \mathrm{C} & -4.222881 & -1.961696 & 0.502227 \\ \mathrm{C} & -2.812338 & -1.972326 & 0.618530 \\ \mathrm{C} & -2.165758 & -1.031179 & 1.506500 \\ \mathrm{C} & -2.983635 & -0.130573 & 2.246947 \\ \mathrm{C} & -1.984701 & -2.868539 & -0.137862 \\ \mathrm{C} & -0.607917 & -2.792511 & -0.070458 \\ \mathrm{C} & 0.048073 & -1.812217 & 0.755664 \\ \mathrm{C} & -0.749132 & -0.975193 & 1.556132 \\ \mathrm{C} & 1.479976 & -1.625624 & 0.665681 \\ \mathrm{C} & 2.114706 & -0.480591 & 1.037532 \\ \mathrm{C} & 3.178728 & 0.163605 & 1.544127 \\ \mathrm{O} & 3.507582 & 1.420131 & 1.130017 \\ \mathrm{C} & 2.646012 & 1.820330 & 0.053186 \\ \mathrm{C} & 1.267336 & 1.285659 & 0.247948 \\ \mathrm{C} & 0.036647 & 1.467936 & 0.084862 \\ \mathrm{C} & -1.352767 & 1.685384 & -0.092892 \\ \mathrm{C} & -2.030653 & 2.689858 & 0.656029 \\ \mathrm{C} & -3.403872 & 2.904100 & 0.482248 \\ \mathrm{C} & -4.138634 & 2.119234 & -0.424516 \\ \mathrm{C} & -3.484784 & 1.113570 & -1.161817 \\ \mathrm{C} & -2.112735 & 0.896366 & -1.004697 \\ \mathrm{H} & -5.219754 & 2.283035 & -0.552598 \\ \mathrm{H} & 2.662869 & 2.927786 & 0.027006\end{array}$




$\begin{array}{rrrr}\mathrm{H} & 3.792125 & -0.183457 & 2.391175 \\ \mathrm{H} & 2.050749 & -2.383716 & 0.094847 \\ \mathrm{H} & -1.456124 & 3.298795 & 1.370178 \\ \mathrm{H} & -0.261750 & -0.254310 & 2.229255 \\ \mathrm{H} & -3.909457 & 3.687496 & 1.068333 \\ \mathrm{H} & -4.058898 & 0.475381 & -1.850486 \\ \mathrm{H} & -2.469666 & -3.609312 & -0.794002 \\ \mathrm{H} & 0.011222 & -3.468854 & -0.682276 \\ \mathrm{H} & 3.051857 & 1.424564 & -0.907368 \\ \mathrm{H} & -1.608054 & 0.087316 & -1.552858 \\ \mathrm{H} & -2.498203 & 0.597339 & 2.915066 \\ \mathrm{H} & -4.955740 & 0.614746 & 2.668756 \\ \mathrm{O} & -6.340038 & -1.069861 & 0.987074 \\ \mathrm{H} & -4.734737 & -2.660755 & -0.175979 \\ \mathrm{C} & -7.167183 & -0.115375 & 1.631923 \\ \mathrm{H} & -8.197907 & -0.307775 & 1.278875 \\ \mathrm{H} & -7.143743 & -0.218499 & 2.741343 \\ \mathrm{H} & -6.883438 & 0.928556 & 1.364567\end{array}$

Allenyne_A2Cyclization_INT

43

Free energy $=-1036.697676$ Hartree

$\begin{array}{llll}\text { C } & -4.676256 & -0.382554 & 1.659356\end{array}$

$\begin{array}{llll}\text { C } & -5.135091 & -1.623392 & 1.170286\end{array}$

$\begin{array}{llll}\text { C } & -4.194036 & -2.594197 & 0.776187\end{array}$

$\begin{array}{llll}\text { C } & -2.808773 & -2.335622 & 0.830891\end{array}$

$\begin{array}{llll}\text { C } & -2.341046 & -1.055556 & 1.253934\end{array}$

$\begin{array}{llll}\text { C } & -3.293852 & -0.125430 & 1.698349\end{array}$

$\begin{array}{llll}\text { C } & -1.852652 & -3.392643 & 0.525402\end{array}$

$\begin{array}{llll}\text { C } & -0.502685 & -3.189596 & 0.600865\end{array}$

$\begin{array}{llll}\text { C } & 0.071903 & -1.900259 & 0.899491\end{array}$

$\begin{array}{llll}\text { C } & -0.847012 & -0.743805 & 1.326325\end{array}$

$\begin{array}{llll}\text { C } & 1.427600 & -1.677861 & 0.778273\end{array}$

$\begin{array}{llll}\text { C } & 1.922655 & -0.328845 & 0.743462\end{array}$

$\begin{array}{llll}\text { C } & 3.173858 & 0.196553 & 0.516548\end{array}$

$\begin{array}{llll}\text { O } & 3.183243 & 1.523844 & 0.232039\end{array}$

$\begin{array}{llll}\text { C } & 1.795430 & 1.976110 & 0.186683\end{array}$

$\begin{array}{llll}\text { C } & 0.969031 & 0.772882 & 0.588653\end{array}$

$\begin{array}{llll}\text { C } & -0.377748 & 0.602671 & 0.696875\end{array}$

$\begin{array}{llll}\text { C } & -1.316591 & 1.669792 & 0.274434\end{array}$

$\begin{array}{llll}\text { C } & -1.272564 & 2.960121 & 0.852413\end{array}$

$\begin{array}{llll}\text { C } & -2.146066 & 3.973940 & 0.425173\end{array}$

$\begin{array}{llll}\text { C } & -3.084514 & 3.715690 & -0.588613\end{array}$

$\begin{array}{llll}\text { C } & -3.142079 & 2.436070 & -1.170455\end{array}$

$\begin{array}{llll}\text { C } & -2.270576 & 1.423754 & -0.743676\end{array}$

$\begin{array}{llll}\mathrm{H} & -3.769008 & 4.509754 & -0.925075\end{array}$

$\begin{array}{llll}\mathrm{H} & 1.705244 & 2.835766 & 0.880817\end{array}$

$\begin{array}{llll}\mathrm{H} & 4.157437 & -0.292726 & 0.500018\end{array}$

$\begin{array}{llll}\mathrm{H} & 2.105923 & -2.514057 & 0.544531\end{array}$

$\begin{array}{llll}\mathrm{H} & -0.558241 & 3.158929 & 1.667130\end{array}$

$\begin{array}{llll}\mathrm{H} & -0.644082 & -0.602887 & 2.418849\end{array}$

$\begin{array}{llll}\mathrm{H} & -2.097607 & 4.969818 & 0.892830\end{array}$

$\begin{array}{llll}\mathrm{H} & -3.872539 & 2.224603 & -1.967067\end{array}$

$\begin{array}{llll}\mathrm{H} & -2.248704 & -4.379230 & 0.237367\end{array}$

$\begin{array}{llll}\mathrm{H} & 0.190247 & -4.014065 & 0.36528\end{array}$

$\begin{array}{llll}\mathrm{H} & 1.578302 & 2.342071 & -0.84128\end{array}$

$\begin{array}{llll}\mathrm{H} & -2.321135 & 0.422424 & -1.196532\end{array}$

$\begin{array}{llll}\mathrm{H} & -2.959740 & 0.847659 & 2.086510\end{array}$

$\begin{array}{llll}\mathrm{H} & -5.373168 & 0.390489 & 2.007905\end{array}$

$\begin{array}{llll}\text { O } & -6.449057 & -1.982365 & 1.062767\end{array}$

$\begin{array}{llll}\mathrm{H} & -4.565599 & -3.574217 & 0.441094\end{array}$

$\begin{array}{llll}\text { C } & -7.437597 & -1.050256 & 1.468276\end{array}$

$\begin{array}{llll}\mathrm{H} & -8.416369 & -1.535325 & 1.291266\end{array}$

$\begin{array}{llll}\mathrm{H} & -7.353846 & -0.794935 & 2.549843\end{array}$

$\begin{array}{llll}\mathrm{H} & -7.391963 & -0.107295 & 0.876673\end{array}$

Allenyne_B

43

Free energy $=-1036.628234$ Hartree

$\begin{array}{llll}\text { C } & -3.778791 & 3.284991 & 0.641591\end{array}$

$\begin{array}{llll}\text { C } & -3.984607 & 2.713922 & -0.652910\end{array}$

$\begin{array}{llll}\text { C } & -2.916840 & 2.111579 & -1.324429\end{array}$

$\begin{array}{llll}\text { C } & -1.623570 & 2.061449 & -0.744034\end{array}$

$\begin{array}{llll}\text { C } & -1.410195 & 2.642255 & 0.562542\end{array}$

$\begin{array}{llll}\text { C } & -2.519980 & 3.241439 & 1.225085\end{array}$

$\begin{array}{llll}\text { C } & -0.504348 & 1.474214 & -1.415627\end{array}$

$\begin{array}{llll}\text { C } & 0.756449 & 1.485154 & -0.848492\end{array}$

$\begin{array}{llll}\text { C } & 0.982194 & 2.095193 & 0.433302\end{array}$

$\begin{array}{llll}\text { C } & -0.104554 & 2.647367 & 1.121666\end{array}$

$\begin{array}{llll}\text { C } & 2.303765 & 2.203061 & 0.962180\end{array}$

$\begin{array}{llll}\text { C } & 3.436515 & 2.357100 & 1.412517\end{array}$

$\begin{array}{llll}\text { C } & 4.764834 & 2.612510 & 1.970127\end{array}$

$\begin{array}{llll}\text { O } & 5.666279 & 3.302691 & 1.084830\end{array}$

$\begin{array}{llll}\text { C } & 5.301202 & 4.579854 & 0.731752\end{array}$

$\begin{array}{llll}\text { C } & 4.214842 & 5.238153 & 1.108745\end{array}$

$\begin{array}{llll}\text { C } & 3.066716 & 5.848752 & 1.385180 \\ \text { C } & 1.827733 & 5.735694 & 0.587178\end{array}$

$\begin{array}{llll}\text { C } & 1.827733 & 5.735694 & 0.587178\end{array}$

$\begin{array}{llll}\text { C } & 1.806415 & 5.085884 & -0.670144\end{array}$

$\begin{array}{llll}\text { C } & 0.609109 & 4.944222 & -1.381629\end{array}$

$\begin{array}{llll}\text { C } & -0.594092 & 5.446578 & -0.853816\end{array}$

$\begin{array}{lrrr}\mathrm{C} & -0.585697 & 6.108572 & 0.385512 \\ \mathrm{C} & 0.614211 & 6.255447 & 1.097592 \\ \mathrm{H} & -1.540371 & 5.298897 & -1.395985 \\ \mathrm{H} & 6.067411 & 5.030143 & 0.076061 \\ \mathrm{H} & 4.655124 & 3.195966 & 2.916850 \\ \mathrm{H} & 2.991929 & 6.470763 & 2.299837 \\ \mathrm{H} & 2.745141 & 4.670430 & -1.067168 \\ \mathrm{H} & 0.062630 & 3.136154 & 2.092419 \\ \mathrm{H} & 0.605800 & 4.412807 & -2.344640 \\ \mathrm{H} & -1.525797 & 6.497056 & 0.806234 \\ \mathrm{H} & -0.659340 & 1.022278 & -2.407627 \\ \mathrm{H} & 1.613177 & 1.047901 & -1.381782 \\ \mathrm{H} & 5.284754 & 1.662450 & 2.211717 \\ \mathrm{H} & 0.615673 & 6.765314 & 2.074696 \\ \mathrm{H} & -3.099563 & 1.679267 & -2.319185 \\ \mathrm{O} & -5.185803 & 2.713759 & -1.300291 \\ \mathrm{H} & -4.607982 & 3.759941 & 1.182814 \\ \mathrm{H} & -2.363452 & 3.692841 & 2.216833 \\ \mathrm{C} & -6.311637 & 3.304238 & -0.669132 \\ \mathrm{H} & -7.159374 & 3.181262 & -1.369590 \\ \mathrm{H} & -6.566420 & 2.800806 & 0.291303 \\ \mathrm{H} & -6.161989 & 4.391024 & -0.474992\end{array}$

Allenyne_B2Cyclization_TS

Free energy $=-1036.61174$ Hartree

$\begin{array}{llll}\text { C } & -3.922467 & 3.166471 & 0.675100\end{array}$

$\begin{array}{llll}\text { C } & -4.121758 & 2.522775 & -0.584564\end{array}$

$\begin{array}{llll}\text { C } & -3.024747 & 2.003192 & -1.280094\end{array}$

$\begin{array}{llll}\text { C } & -1.712221 & 2.107037 & -0.756990\end{array}$

$\begin{array}{llll}\text { C } & -1.503622 & 2.759127 & 0.517622\end{array}$

$\begin{array}{llll}\text { C } & -2.642333 & 3.273862 & 1.204035\end{array}$

$\begin{array}{llll}\text { C } & -0.565150 & 1.604502 & -1.454037\end{array}$

$\begin{array}{llll}\text { C } & 0.707363 & 1.745892 & -0.938178\end{array}$

$\begin{array}{llll}\text { C } & 0.930762 & 2.412073 & 0.322676\end{array}$

$\begin{array}{llll}\text { C } & -0.185908 & 2.897874 & 1.027654\end{array}$

$\begin{array}{llll}\text { C } & 2.244800 & 2.613651 & 0.802225\end{array}$

$\begin{array}{llll}\text { C } & 3.390535 & 2.917714 & 1.223993\end{array}$

$\begin{array}{llll}\text { C } & 4.584762 & 2.415331 & 1.970985\end{array}$

$\begin{array}{llll}\text { O } & 5.772735 & 3.100887 & 1.547747\end{array}$

$\begin{array}{llll}\text { C } & 5.463065 & 4.377741 & 1.19213\end{array}$

$\begin{array}{llll}\text { C } & 4.182546 & 4.795690 & 1.158484\end{array}$

$\begin{array}{llll}\text { C } & 3.282898 & 5.814496 & 1.258003\end{array}$

$\begin{array}{llll}\text { C } & 2.071201 & 5.864529 & 0.456267\end{array}$

$\begin{array}{llll}\text { C } & 1.984189 & 5.164549 & -0.777133\end{array}$

$\begin{array}{llll}\text { C } & 0.767163 & 5.073400 & -1.464986\end{array}$

$\begin{array}{llll}\text { C } & -0.389899 & 5.661071 & -0.929736\end{array}$

$\begin{array}{llll}\text { C } & -0.311510 & 6.402425 & 0.267777\end{array}$

$\begin{array}{llll}\text { C } & 0.906161 & 6.517057 & 0.944864\end{array}$

$\begin{array}{llll}\mathrm{H} & -1.356783 & 5.543133 & -1.442012\end{array}$

$\begin{array}{llll}\mathrm{H} & 6.328934 & 4.948310 & 0.821818\end{array}$

$\begin{array}{llll}\mathrm{H} & 4.426683 & 2.599936 & 3.060013\end{array}$

\begin{tabular}{llll}
$\mathrm{H}$ & 3.358498 & 6.516264 & 2.110585 \\
\hline
\end{tabular}

$\mathrm{H} \quad 2.889427 \quad 4.683252 \quad-1.175823$

$\begin{array}{lllr}\mathrm{H} & -0.030141 & 3.432197 & 1.976243\end{array}$

$\begin{array}{llll}\mathrm{H} & 0.709835 & 4.503571 & -2.403941\end{array}$

$\begin{array}{llll}\mathrm{H} & -1.216959 & 6.874784 & 0.678890\end{array}$

$\begin{array}{llll}\mathrm{H} & -0.713770 & 1.104782 & -2.424167\end{array}$

$\begin{array}{llll}\mathrm{H} & 1.578860 & 1.366695 & -1.491681\end{array}$

$\begin{array}{llll}\mathrm{H} & 4.759257 & 1.333442 & 1.807852\end{array}$

$\begin{array}{llll}\mathrm{H} & 0.961583 & 7.073755 & 1.894395\end{array}$

$\begin{array}{llll}\mathrm{H} & -3.202456 & 1.513787 & -2.249130\end{array}$

$\begin{array}{llll}\text { O } & -5.342157 & 2.372784 & -1.178227\end{array}$

$\begin{array}{llll}\mathrm{H} & -4.772819 & 3.578719 & 1.234307\end{array}$

$\begin{array}{llll}\mathrm{H} & -2.494664 & 3.774701 & 2.173524\end{array}$

$\begin{array}{llll}\text { C } & -6.494170 & 2.881170 & -0.524935\end{array}$

$\mathrm{H} \quad-7.352965 \quad 2.644143 \quad-1.180934$

$\begin{array}{llll}\mathrm{H} & -6.660532 & 2.401919 & 0.466834 \\ \mathrm{H} & -6.444238 & 3.985468 & -0.384557\end{array}$

Allenyne_B2Cyclization_INT

43

Free energy $=-1036.689526$ Hartree

$\begin{array}{lrrr}\mathrm{C} & -3.957073 & 1.363614 & 0.964331 \\ \mathrm{C} & -4.247273 & 1.249740 & -0.430296 \\ \mathrm{C} & -3.352198 & 1.770473 & -1.369279 \\ \mathrm{C} & -2.154014 & 2.409891 & -0.959851 \\ \mathrm{C} & -1.851406 & 2.521323 & 0.449277 \\ \mathrm{C} & -2.787005 & 1.986361 & 1.381856 \\ \mathrm{C} & -1.218961 & 2.965327 & -1.889478 \\ \mathrm{C} & -0.058989 & 3.584648 & -1.458969 \\ \mathrm{C} & 0.268199 & 3.690486 & -0.060785 \\ \mathrm{C} & -0.646598 & 3.161864 & 0.857450 \\ \mathrm{C} & 1.543320 & 4.304988 & 0.365500 \\ \mathrm{C} & 2.300041 & 3.797064 & 1.383940 \\ \mathrm{C} & 2.069694 & 2.624221 & 2.312451 \\ \mathrm{O} & 3.348400 & 2.391955 & 2.971894 \\ \mathrm{C} & 4.178573 & 3.409072 & 2.616437 \\ \mathrm{C} & 3.637600 & 4.289076 & 1.709848 \\ \mathrm{C} & 4.146143 & 5.469014 & 1.069798\end{array}$




$\begin{array}{crrr}\text { C } & 3.391300 & 6.102385 & 0.110338 \\ \text { C } & 2.111667 & 5.471783 & -0.456564 \\ \text { C } & 1.076383 & 6.529238 & -0.812973 \\ \text { C } & 1.464634 & 7.782635 & -1.167303 \\ \text { C } & 2.863532 & 8.180467 & -1.092033 \\ \text { C } & 3.775877 & 7.383280 & -0.446437 \\ \text { H } & 0.716599 & 8.525726 & -1.486759 \\ \text { H } & 5.176665 & 3.383212 & 3.073663 \\ \text { H } & 1.332204 & 2.841582 & 3.115992 \\ \text { H } & 5.117210 & 5.889700 & 1.375379 \\ \text { H } & 2.440005 & 5.043907 & -1.447776 \\ \text { H } & -0.467045 & 3.276916 & 1.937182 \\ \text { H } & 0.016420 & 6.234165 & -0.838229 \\ \text { H } & 3.162167 & 9.172602 & -1.464198 \\ \text { H } & -1.434840 & 2.888734 & -2.967196 \\ \text { H } & 0.638316 & 3.989995 & -2.207054 \\ \text { H } & 1.747856 & 1.693452 & 1.796976 \\ \text { H } & 4.798038 & 7.748711 & -0.253755 \\ \text { H } & -3.597426 & 1.674880 & -2.437903 \\ \text { O } & -5.370406 & 0.653194 & -0.926667 \\ \text { H } & -4.651002 & 0.963158 & 1.715739 \\ \text { H } & -2.571554 & 2.070543 & 2.459291 \\ \text { C } & -6.316812 & 0.105013 & -0.023674 \\ \text { H } & -7.128494 & -0.319147 & -0.644100 \\ \text { H } & -5.880704 & -0.709881 & 0.598819 \\ \text { H } & -6.750313 & 0.878288 & 0.651093\end{array}$

Bisallene

43

Free energy $=-1036.639086$ Hartree

$\begin{array}{llll}\text { C } & 4.071894 & 6.695597 & -4.097399 \\ \text { C } & 3.609998 & 7.470109 & -3.004719\end{array}$

$-3.004719$

$\begin{array}{llll}\text { C } & 2.218532 & 7.688449 & -2.866832\end{array}$

$\begin{array}{llll}\text { C } & 1.312587 & 7.132587 & -3.779648\end{array}$

$\begin{array}{llll}\text { C } & 1.781197 & 6.353101 & -4.851647\end{array}$

$\begin{array}{llll}\text { C } & 3.164700 & 6.143313 & -5.010192\end{array}$

$\begin{array}{llll}\text { C } & 4.528393 & 7.975379 & -1.971927\end{array}$

$\begin{array}{llll}\text { C } & 5.836251 & 7.741041 & -1.902640\end{array}$

$\begin{array}{llll}\text { C } \quad 7.149006 & 7.588302 & -1.846254\end{array}$

$\begin{array}{llll}\text { O } & 7.850076 & 6.556426 & -1.24856\end{array}$

$\begin{array}{llll}\text { C } & 7.184152 & 5.385633 & -0.929155\end{array}$

$\begin{array}{llll}\text { C } & 6.011606 & 4.980411 & -1.387191\end{array}$

$\begin{array}{llll}\text { C } & 4.847110 & 4.502599 & -1.821802\end{array}$

$\begin{array}{llll}\text { C } & 3.527143 & 4.803511 & -1.254149\end{array}$

$\begin{array}{llll}\text { C } & 2.372713 & 4.366292 & -1.908487\end{array}$

$\begin{array}{llll}\text { C } & 1.077484 & 4.754608 & -1.471024\end{array}$

$\begin{array}{llll}\text { C } & 0.948736 & 5.612979 & -0.314477\end{array}$

$\begin{array}{llll}\text { C } & 2.142834 & 6.015586 & 0.366465\end{array}$

$\begin{array}{llll}\text { C } & 3.388720 & 5.627164 & -0.087464\end{array}$

$\begin{array}{llll}\text { C } & -0.340811 & 6.036382 & 0.09706\end{array}$

$\begin{array}{llll}\text { C } & -1.485712 & 5.642476 & -0.601442\end{array}$

$\begin{array}{llll}\text { C } & -1.363142 & 4.796999 & -1.747504\end{array}$

$\begin{array}{llll}\text { C } & -0.108108 & 4.368827 & -2.159853\end{array}$

$\begin{array}{llll}\text { O } & -2.677449 & 6.106803 & -0.124402\end{array}$

$\begin{array}{llll}\text { C } & -3.877277 & 5.739181 & -0.786353\end{array}$

$\begin{array}{llll}\mathrm{H} & 4.301965 & 5.964030 & 0.425376\end{array}$

$\begin{array}{llll}\mathrm{H} & 1.069313 & 5.904287 & -5.561501\end{array}$

$\begin{array}{llll}\mathrm{H} & 7.851775 & 8.329595 & -2.262937\end{array}$

$\begin{array}{llll}\mathrm{H} & 7.797458 & 4.780563 & -0.239778\end{array}$

$\begin{array}{llll}\mathrm{H} & 4.052873 & 8.534875 & -1.142559\end{array}$

$\begin{array}{llll}\mathrm{H} & 4.842971 & 3.889269 & -2.743778\end{array}$

$\mathrm{H} \quad 5.153485 \quad 6.517216 \quad-4.200961$

$\begin{array}{llll}\mathrm{H} & 2.461612 & 3.753369 & -2.819683\end{array}$

$\begin{array}{llll}\mathrm{H} & 3.537097 & 5.536428 & -5.850620\end{array}$

$\begin{array}{llll}\mathrm{H} & 0.231572 & 7.283687 & -3.636453\end{array}$

$\begin{array}{llll}\mathrm{H} & 2.052643 & 6.656427 & 1.258100\end{array}$

$\begin{array}{llll}\mathrm{H} & 1.845138 & 8.265950 & -2.006784\end{array}$

$\begin{array}{llll}\mathrm{H} & -0.461348 & 6.685170 & 0.97764\end{array}$

$\begin{array}{llll}\mathrm{H} & -2.252476 & 4.481611 & -2.308974\end{array}$

$\begin{array}{llll}\mathrm{H} & -0.016978 & 3.728730 & -3.051687\end{array}$

$\begin{array}{llll}\mathrm{H} & -4.700198 & 6.221198 & -0.226703\end{array}$

$\begin{array}{llll}\mathrm{H} & -3.902882 & 6.100340 & -1.839659\end{array}$

$\mathrm{H} \quad-4.038486 \quad 4.636644 \quad-0.781613$

Bisallene2Cyclization_TS1

43

Free energy $=-1036.63473$ Hartree

$\begin{array}{llll}\mathrm{C} & 4.087630 & 6.557830 & -4.133394 \\ \mathrm{C} & 3.672571 & 7.437793 & -3.096270 \\ \mathrm{C} & 2.285840 & 7.727259 & -2.967168 \\ \mathrm{C} & 1.349504 & 7.122162 & -3.807462 \\ \mathrm{C} & 1.772853 & 6.225541 & -4.810592 \\ \mathrm{C} & 3.143656 & 5.962117 & -4.980381 \\ \mathrm{C} & 4.611685 & 7.918876 & -2.097731 \\ \mathrm{C} & 5.879482 & 7.451455 & -1.954185 \\ \mathrm{C} & 7.205071 & 7.559094 & -1.845728 \\ \mathrm{O} & 7.972517 & 6.548994 & -1.309702 \\ \mathrm{C} & 7.220041 & 5.416968 & -1.085120 \\ \mathrm{C} & 5.954134 & 5.320782 & -1.495682\end{array}$

$\begin{array}{llll}\text { C } & 4.815797 & 4.653005 & -1.826020\end{array}$

$\begin{array}{llll}\text { C } & 3.512638 & 4.909663 & -1.240065\end{array}$

$\begin{array}{llll}\text { C } & 2.351546 & 4.384313 & -1.830632\end{array}$

$\begin{array}{llll}\text { C } & 1.059148 & 4.770666 & -1.396910\end{array}$

$\begin{array}{llll}\text { C } & 0.927323 & 5.715306 & -0.306883\end{array}$

$\begin{array}{llll}2.120009 & 6.196256 & 0.321187\end{array}$

$\begin{array}{lll}3.368778 & 5.810939 & -0.128002\end{array}$

$\begin{array}{llll}\text { C } & -0.366378 & 6.135954 & 0.095449\end{array}$

$\begin{array}{llll}\text { C } & -1.511834 & 5.661013 & -0.547975\end{array}$

$\begin{array}{lll}-1.387452 & 4.730267 & -1.627856\end{array}$

$\begin{array}{llll}-0.130730 & 4.302130 & -2.029503\end{array}$

$\begin{array}{llll}-2.706885 & 6.133435 & -0.088586\end{array}$

$\begin{array}{llll}-3.908427 & 5.687431 & -0.697405\end{array}$

$\begin{array}{llll}\text { H } & 4.280334 & 6.202534 & 0.346371\end{array}$

$\begin{array}{llll}1.030955 & 5.734831 & -5.45930\end{array}$

$\begin{array}{llll}\mathrm{H} & 7.808542 & 8.410255 & -2.194221\end{array}$

$\begin{array}{llll}\mathrm{H} & 7.771149 & 4.650011 & -0.520784\end{array}$

$\mathrm{H} \quad 4.187051 \quad 8.529693 \quad-1.278433$

$\begin{array}{llll}\mathrm{H} & 4.828959 & 4.017013 & -2.731078\end{array}$

H $\quad 5.160566 \quad 6.342199 \quad-4.247331$

$\begin{array}{llll}\mathrm{H} & 2.441733 & 3.707495 & -2.695380\end{array}$

$\begin{array}{llll}\mathrm{H} & 3.478380 & 5.273557 & -5.772544\end{array}$

$\begin{array}{llll}\mathrm{H} & 0.276312 & 7.320773 & -3.663786\end{array}$

$\begin{array}{llll}\mathrm{H} & 2.026354 & 6.896735 & 1.166530\end{array}$

$\begin{array}{llll}\mathrm{H} & 1.949585 & 8.389734 & -2.154228\end{array}$

$\begin{array}{llll}\mathrm{H} & -0.488793 & 6.849828 & 0.924074\end{array}$

$\begin{array}{llll}\mathrm{H} & -2.278276 & 4.351068 & -2.145785\end{array}$

$\begin{array}{llll}\mathrm{H} & -0.038719 & 3.595436 & -2.869761\end{array}$

$\begin{array}{llll}-4.733084 & 6.193413 & -0.162266\end{array}$

$\begin{array}{llll}\mathrm{H} & -3.960127 & 5.963821 & -1.775303\end{array}$

$\begin{array}{llll}\mathrm{H} & -4.043272 & 4.585498 & -0.602327\end{array}$

Bisallene2Cyclization_INT1

43

Free energy $=-1036.677077$ Hartree

C $\quad 0.263873 \quad 7.860659 \quad-1.929593$

$\begin{array}{llll}\text { C } & 0.939691 & 6.643062 & -1.629325\end{array}$

$\begin{array}{llll}\text { C } & 0.215041 & 5.403049 & -1.790161\end{array}$

$\begin{array}{llll}\text { C } & -1.133047 & 5.433574 & -2.209157\end{array}$

$\begin{array}{llll}\text { C } & -1.778861 & 6.650226 & -2.478791\end{array}$

$\begin{array}{llll}\text { C } & -1.065942 & 7.876087 & -2.339945\end{array}$

$\begin{array}{llll}\text { C } & 2.304941 & 6.616757 & -1.239814\end{array}$

$\begin{array}{llll}\text { C } & 2.995767 & 5.401455 & -0.997375\end{array}$

$\begin{array}{llll}\text { C } & 2.230677 & 4.175408 & -1.120134\end{array}$

$\begin{array}{llll}\text { C } & 0.910284 & 4.174778 & -1.505801\end{array}$

$\begin{array}{llll}\text { C } & 4.374085 & 5.308365 & -0.655874\end{array}$

$\begin{array}{llll}\text { C } & 5.445872 & 6.247802 & -0.658445\end{array}$

$\begin{array}{llll}\text { C } & 5.692761 & 7.615508 & -1.215136\end{array}$

$\begin{array}{llll}\text { C } & 6.926518 & 8.000923 & -0.649906\end{array}$

$\begin{array}{llll}\text { O } & 7.459531 & 7.009253 & 0.096037\end{array}$

$\begin{array}{llll}\text { C } & 6.609695 & 5.962604 & 0.087159\end{array}$

$\begin{array}{llll}\text { C } & 5.037213 & 8.482888 & -2.135256\end{array}$

$\begin{array}{llll}\text { C } & 4.033735 & 8.245102 & -3.121784\end{array}$

$\begin{array}{llll}\text { C } & 3.337807 & 9.361543 & -3.694802\end{array}$

$\begin{array}{llll}\text { C } & 2.288875 & 9.177462 & -4.590666\end{array}$

$\begin{array}{llll}\text { C } & 1.906340 & 7.872731 & -4.987539\end{array}$

$\begin{array}{llll}\text { C } & 2.621860 & 6.767696 & -4.505482\end{array}$

$\begin{array}{llll}\text { C } & 3.655565 & 6.940255 & -3.574202\end{array}$

$\begin{array}{llll}\mathrm{H} & 2.739293 & 3.219841 & -0.914268\end{array}$

$\begin{array}{llll}\mathrm{H} & 1.063734 & 7.729344 & -5.680821\end{array}$

$\begin{array}{llll}\mathrm{H} & 7.528004 & 8.908436 & -0.765055\end{array}$

$\begin{array}{llll}\mathrm{H} & 6.896973 & 5.088555 & 0.680757\end{array}$

$\begin{array}{llll}\mathrm{H} & 5.416373 & 9.520082 & -2.107219\end{array}$

H $4.673719 \quad 4.319558 \quad-0.264136$

$\begin{array}{llll}\mathrm{H} & 4.224354 & 6.069188 & -3.224703\end{array}$

$\begin{array}{llll}\mathrm{H} & 2.813585 & 7.571572 & -1.062174\end{array}$

$\mathrm{H} \quad 2.349263 \quad 5.750585 \quad-4.824612$

$\begin{array}{llll}\mathrm{H} & 1.749017 & 10.050879 & -4.988112 \\ \mathrm{H} & 0.362757 & 3.223988 & -1.605158\end{array}$

$\begin{array}{cccc}\mathrm{H} & 0.362757 & 3.223988 & -1.605158 \\ \mathrm{H} & 3.627102 & 10.379613 & -3.387829\end{array}$

$\begin{array}{llll}\mathrm{H} & 3.627102 & 10.379613 & -3.387829 \\ \mathrm{H} & -1.702078 & 4.500150 & -2.333899\end{array}$

$\begin{array}{llll}\mathrm{O} & -3.080993 & 6.564181 & -2.876717\end{array}$

$\begin{array}{llll}\mathrm{H} & -1.547128 & 8.836981 & -2.566754\end{array}$

$\begin{array}{llll}\mathrm{H} & 0.821471 & 8.806429 & -1.849851\end{array}$

$\begin{array}{llll}\text { C } & -3.795283 & 7.757227 & -3.155620\end{array}$

$\mathrm{H} \quad-4.817142 \quad 7.447933 \quad-3.444470$

\begin{tabular}{llll}
$\mathrm{H}$ & -3.859601 & 8.424398 & -2.265439 \\
\hline
\end{tabular}

$\begin{array}{llll}\mathrm{H} & -3.343115 & 8.329028 & -3.998378\end{array}$

Bisallene2Cyclization_TS2_for_A 43

Free energy $=-1036.659798$ Hartree

$\begin{array}{lrrr}\mathrm{C} & 0.460929 & 3.833152 & -2.346067 \\ \mathrm{C} & 1.354189 & 4.833702 & -1.847097 \\ \mathrm{C} & 1.001762 & 6.236783 & -2.060056 \\ \mathrm{C} & -0.205110 & 6.547184 & -2.750394 \\ \mathrm{C} & -1.048856 & 5.542628 & -3.222656 \\ \mathrm{C} & -0.707265 & 4.163651 & -3.014920 \\ \mathrm{C} & 1.848236 & 7.235491 & -1.524958 \\ \mathrm{C} & 3.072784 & 6.918220 & -0.896542\end{array}$




\begin{tabular}{cccc}
$\mathrm{C}$ & 3.462485 & 5.505606 & -0.732959 \\
$\mathrm{C}$ & 2.552443 & 4.509059 & -1.179160 \\
$\mathrm{C}$ & 4.746176 & 5.180975 & -0.244653 \\
$\mathrm{C}$ & 5.796004 & 6.167312 & -0.232296 \\
$\mathrm{C}$ & 5.820186 & 7.341566 & -1.116770 \\
$\mathrm{C}$ & 7.014457 & 7.971654 & -0.856976 \\
$\mathrm{O}$ & 7.726284 & 7.313496 & 0.117566 \\
$\mathrm{C}$ & 6.981891 & 6.226089 & 0.483242 \\
$\mathrm{C}$ & 4.776129 & 7.645705 & -2.108034 \\
$\mathrm{C}$ & 4.388324 & 8.988721 & -2.493315 \\
$\mathrm{C}$ & 4.637297 & 10.125704 & -1.672576 \\
$\mathrm{C}$ & 4.171898 & 11.394376 & -2.040009 \\
$\mathrm{C}$ & 3.439673 & 11.571261 & -3.229868 \\
$\mathrm{C}$ & 3.168877 & 10.455391 & -4.046154 \\
$\mathrm{C}$ & 3.629601 & 9.185300 & -3.683316 \\
$\mathrm{H}$ & 3.469790 & 7.639399 & -0.165789 \\
$\mathrm{H}$ & 3.076603 & 12.570109 & -3.515747 \\
$\mathrm{H}$ & 7.497276 & 8.851163 & -1.294719 \\
$\mathrm{H}$ & 7.397094 & 5.605096 & 1.284404 \\
$\mathrm{H}$ & 4.616698 & 6.861940 & -2.869129 \\
$\mathrm{H}$ & 4.982445 & 4.135995 & 0.012508 \\
$\mathrm{H}$ & 5.190060 & 9.996621 & -0.729688 \\
$\mathrm{H}$ & 2.821299 & 3.447561 & -1.048792 \\
$\mathrm{H}$ & 4.377940 & 12.258109 & -1.388258 \\
$\mathrm{H}$ & 2.591120 & 10.581058 & -4.975265 \\
$\mathrm{H}$ & 1.549303 & 8.292367 & -1.607143 \\
$\mathrm{H}$ & 3.403247 & 8.314655 & -4.318897 \\
$\mathrm{H}$ & -0.489358 & 7.596258 & -2.921153 \\
$\mathrm{O}$ & -2.178800 & 5.949383 & -3.872171 \\
$\mathrm{H}$ & -1.363918 & 3.362929 & -3.380886 \\
$\mathrm{H}$ & 0.716995 & 2.772781 & -2.190903 \\
$\mathrm{C}$ & -3.081742 & 4.976435 & -4.371399 \\
$\mathrm{H}$ & -3.909329 & 5.536231 & -4.846337 \\
$\mathrm{H}$ & -2.610686 & 4.318606 & -5.137793 \\
$\mathrm{H}$ & -3.502514 & 4.338214 & -3.561306 \\
& & & \\
\hline & & &
\end{tabular}

Bisallene2Cyclization_INT2_for_A

43

Free energy $=-1036.690099$ Hartree

$\begin{array}{lccc}\mathrm{C} & 0.235919 & 3.838127 & -1.918259 \\ \mathrm{C} & 1.278942 & 4.823011 & -1.781671 \\ \mathrm{C} & 1.083651 & 6.136943 & -2.437460 \\ \mathrm{C} & -0.129440 & 6.346617 & -3.197834 \\ \mathrm{C} & -1.096387 & 5.368667 & -3.297334 \\ \mathrm{C} & -0.907725 & 4.086970 & -2.640304 \\ \mathrm{C} & 2.050104 & 7.108418 & -2.309004 \\ \mathrm{C} & 3.269754 & 6.946923 & -1.446431 \\ \mathrm{C} & 3.527793 & 5.501604 & -0.990558 \\ \mathrm{C} & 2.465802 & 4.550850 & -1.110357 \\ \mathrm{C} & 4.746130 & 5.167902 & -0.420065 \\ \mathrm{C} & 5.807118 & 6.137853 & -0.395106 \\ \mathrm{C} & 5.719037 & 7.360051 & -1.174224 \\ \mathrm{C} & 6.890133 & 8.039213 & -0.956777 \\ \mathrm{O} & 7.696768 & 7.338794 & -0.090620 \\ \mathrm{C} & 7.039848 & 6.189049 & 0.240958 \\ \mathrm{C} & 4.541035 & 7.623356 & -2.076386 \\ \mathrm{C} & 4.314528 & 9.095640 & -2.376626 \\ \mathrm{C} & 4.214377 & 10.036560 & -1.328874 \\ \mathrm{C} & 3.946844 & 11.387434 & -1.599329 \\ \mathrm{C} & 3.776691 & 11.822815 & -2.926019 \\ \mathrm{C} & 3.879415 & 10.897200 & -3.977204 \\ \mathrm{C} & 4.146627 & 9.544991 & -3.702025 \\ \mathrm{H} & 3.076298 & 7.524602 & -0.500927 \\ \mathrm{H} & 3.563350 & 12.881820 & -3.139202 \\ \mathrm{H} & 7.282199 & 8.995516 & -1.316246 \\ \mathrm{H} & 7.554765 & 5.522071 & 0.940552 \\ \mathrm{H} & 4.726060 & 7.097123 & -3.042407 \\ \mathrm{H} & 4.897499 & 4.166367 & 0.012264 \\ \mathrm{H} & 4.355983 & 9.701461 & -0.288907 \\ \mathrm{H} & 2.624512 & 3.541698 & -0.694457 \\ \mathrm{H} & 3.869455 & 12.106402 & -0.768731 \\ \mathrm{H} & 3.748278 & 11.228213 & -5.019280 \\ \mathrm{H} & 1.908844 & 8.083641 & -2.799746 \\ \mathrm{H} & 4.220117 & 8.820104 & -4.528868 \\ \mathrm{H} & -0.298025 & 7.311430 & -3.698396 \\ \mathrm{O} & -2.214099 & 5.664939 & -4.027987 \\ \mathrm{H} & -1.675505 & 3.305133 & -2.721065 \\ \mathrm{H} & 0.372009 & 2.859454 & -1.431061 \\ \mathrm{C} & -3.249628 & 4.704408 & -4.148821 \\ \mathrm{H} & -4.036285 & 5.175125 & -4.769053 \\ \mathrm{H} & -2.908521 & 3.773320 & -4.656959 \\ \mathrm{H} & -3.692947 & 4.431470 & -3.163714\end{array}$

Bisallene2Cyclization_TS2_for_B 43

Free energy $=-1036.66595$ Hartree

$\begin{array}{llll}\text { C } & 4.321878 & 6.088095 & -2.784736\end{array}$

$\begin{array}{llll}\text { C } & 4.340619 & 7.531100 & -2.955239\end{array}$

$\begin{array}{llll}\text { C } & 3.258222 & 8.127103 & -3.695570\end{array}$

$\begin{array}{llll}\text { C } & 2.190815 & 7.362607 & -4.140039\end{array}$

\begin{tabular}{lrrr} 
C & 2.153123 & 5.955269 & -3.898061 \\
$\mathrm{C}$ & 3.221939 & 5.330531 & -3.258910 \\
$\mathrm{C}$ & 5.324361 & 8.318813 & -2.323713 \\
$\mathrm{C}$ & 6.115917 & 7.801182 & -1.233131 \\
$\mathrm{C}$ & 5.686333 & 6.733425 & -0.311118 \\
$\mathrm{C}$ & 6.689643 & 6.638349 & 0.625538 \\
$\mathrm{O}$ & 7.712532 & 7.516790 & 0.364002 \\
$\mathrm{C}$ & 7.351236 & 8.210086 & -0.759417 \\
$\mathrm{C}$ & 4.405819 & 6.022847 & -0.371449 \\
$\mathrm{C}$ & 4.223298 & 4.625754 & -0.108396 \\
$\mathrm{C}$ & 5.291049 & 3.703705 & -0.028610 \\
$\mathrm{C}$ & 5.069607 & 2.314546 & 0.160372 \\
$\mathrm{C}$ & 3.715187 & 1.812925 & 0.273322 \\
$\mathrm{C}$ & 2.637801 & 2.757501 & 0.176678 \\
$\mathrm{C}$ & 2.879798 & 4.100963 & -0.010647 \\
$\mathrm{C}$ & 3.496569 & 0.428580 & 0.461760 \\
$\mathrm{C}$ & 4.569334 & -0.469231 & 0.536382 \\
$\mathrm{C}$ & 5.907027 & 0.015544 & 0.422612 \\
$\mathrm{C}$ & 6.139527 & 1.373560 & 0.239934 \\
$\mathrm{O}$ & 4.247407 & -1.782942 & 0.716758 \\
$\mathrm{C}$ & 5.287715 & -2.744148 & 0.786491 \\
$\mathrm{H}$ & 2.036992 & 4.805226 & -0.095464 \\
$\mathrm{H}$ & 1.293813 & 5.363900 & -4.250652 \\
$\mathrm{H}$ & 8.082895 & 8.934536 & -1.133881 \\
$\mathrm{H}$ & 6.789261 & 6.039656 & 1.537082 \\
$\mathrm{H}$ & 5.400462 & 9.389927 & -2.572147 \\
$\mathrm{H}$ & 3.502494 & 6.654933 & -0.374119 \\
$\mathrm{H}$ & 5.264474 & 5.569610 & -2.564627 \\
$\mathrm{H}$ & 6.325752 & 4.064171 & -0.135229 \\
$\mathrm{H}$ & 3.226735 & 4.238596 & -3.114918 \\
$\mathrm{H}$ & 1.357264 & 7.843467 & -4.676147 \\
$\mathrm{H}$ & 1.603364 & 2.386119 & 0.252513 \\
$\mathrm{H}$ & 3.270524 & 9.216601 & -3.862770 \\
$\mathrm{H}$ & 2.474340 & 0.030855 & 0.550249 \\
$\mathrm{H}$ & 6.760609 & -0.673700 & 0.477868 \\
$\mathrm{H}$ & 7.174755 & 1.740154 & 0.152032 \\
$\mathrm{H}$ & 4.795890 & -3.724682 & 0.928263 \\
$\mathrm{H}$ & 5.890371 & -2.778673 & -0.150324 \\
$\mathrm{H}$ & 5.972474 & -2.560183 & 1.645907 \\
& & & \\
\hline
\end{tabular}

Bisallene2Cyclization_INT2_for_B

Free energy $=-1036.702768$ Hartree

$\begin{array}{cccc}\text { Free energy }= & -1036.702768 \text { Hartree } \\ \text { C } & 4.152386 & 6.136367 & -2.369790\end{array}$

$\begin{array}{llll}\mathrm{C} & 4.152386 & 6.136367 & -2.369790 \\ \mathrm{C} & 4.424303 & 7.570716 & -2.85327 \\ \mathrm{C} & 3.695508 & 8.060560 & -4.003955\end{array}$

$\begin{array}{llll}\text { C } & 3.695508 & 8.060560 & -4.003955\end{array}$

$\begin{array}{llll}\text { C } & 2.651696 & 7.362101 & -4.551952\end{array}$

$\begin{array}{llll}\text { C } & 2.218056 & 6.097659 & -3.973055\end{array}$

$\begin{array}{llll}\text { C } & 2.218056 & 6.097659 & -3.973055 \\ \text { C } & 2.890092 & 5.532763 & -2.936894\end{array}$

$\begin{array}{llll}\text { C } & 5.382221 & 8.334417 & -2.223157\end{array}$

$\begin{array}{llll}\text { C } & 6.028843 & 7.831128 & -1.035306\end{array}$

$\begin{array}{llll}\text { C } & 5.504914 & 6.669856 & -0.341475\end{array}$

$\begin{array}{llll}\text { C } & 5.504914 & 6.669856 & -0.341475 \\ \text { O } & 6.320641 & 6.472059 & 0.743402\end{array}$

$\begin{array}{llll}\text { O } & 7.318109 & 7.417223 & 0.771401\end{array}$

$\begin{array}{llll}\text { C } & 7.132286 & 8.239201 & -0.303820\end{array}$

$\begin{array}{llll}\text { C } & 4.239330 & 5.996425 & -0.806492\end{array}$

$\begin{array}{llll}\text { C } & 4.102119 & 4.554205 & -0.346518\end{array}$

$\begin{array}{llll}\text { C } & 5.134691 & 3.638317 & -0.532504\end{array}$

$\begin{array}{llll}\text { C } & 5.001921 & 2.271705 & -0.150052\end{array}$

$\begin{array}{llll}\text { C } & 3.763684 & 1.819434 & 0.446432\end{array}$

$\begin{array}{llll}\text { C } & 2.716303 & 2.775380 & 0.627780\end{array}$

$\begin{array}{llll}\text { C } & 2.716303 & 2.775380 & 0.627780 \\ \text { C } & 2.883160 & 4.096986 & 0.242595\end{array}$

$\begin{array}{llll}\text { C } & 3.630376 & 0.458553 & 0.829963\end{array}$

$\begin{array}{llll}\text { C } & 4.675247 & -0.448790 & 0.639163\end{array}$

$\begin{array}{llll}\text { C } & 5.899836 & -0.006379 & 0.048786\end{array}$

$\begin{array}{llll}\text { C } & 6.048258 & 1.321408 & -0.331148\end{array}$

$\begin{array}{lllr}\text { O } & 4.446675 & -1.733231 & 1.040892\end{array}$

$\begin{array}{llll}\text { C } & 5.470308 & -2.700955 & 0.875506\end{array}$

$\begin{array}{llll}\mathrm{H} & 2.062380 & 4.817380 & 0.388665\end{array}$

$\begin{array}{llll}\mathrm{H} & 1.323836 & 5.604188 & -4.385112\end{array}$

$\begin{array}{llll}\mathrm{H} & 7.860865 & 9.047643 & -0.426709\end{array}$

$\begin{array}{llll}\mathrm{H} & 6.332781 & 5.732139 & 1.550409\end{array}$

$\begin{array}{llll}\mathrm{H} & 5.647754 & 9.327232 & -2.619130\end{array}$

$\begin{array}{llll}\mathrm{H} & 3.379710 & 6.574763 & -0.393889\end{array}$

$\begin{array}{llll}\mathrm{H} & 5.002288 & 5.514385 & -2.759695\end{array}$

$\begin{array}{llll}\mathrm{H} & 6.085775 & 3.973178 & -0.977153\end{array}$

$\begin{array}{llll}\mathrm{H} & 2.558050 & 4.574794 & -2.508027\end{array}$

$\begin{array}{llll}\mathrm{H} & 2.103784 & 7.772854 & -5.413910\end{array}$

$\begin{array}{llll}\mathrm{H} & 1.769252 & 2.445817 & 1.083303\end{array}$

$\begin{array}{llll}\mathrm{H} & 3.977284 & 9.046909 & -4.406729\end{array}$

$\begin{array}{llll}\mathrm{H} & 2.696744 & 0.096242 & 1.285477\end{array}$

$\begin{array}{llll}\mathrm{H} & 6.730662 & -0.707075 & -0.111714\end{array}$

$\begin{array}{llll}\mathrm{H} & 6.994964 & 1.654094 & -0.785650\end{array}$

$\begin{array}{llll}\mathrm{H} & 5.063403 & -3.652835 & 1.265364\end{array}$

$\begin{array}{llll}\mathrm{H} & 5.746505 & -2.842683 & -0.194307\end{array}$

$\begin{array}{lllr}\mathrm{H} & 6.388908 & -2.443786 & 1.450739\end{array}$ 


\section{$A=2$-pyridyl, $B=$ phenyl}

\section{Bispropargyl}

32

Free energy $=-784.7269148$ Hartree

$\begin{array}{lllr}\text { C } & 2.567065 & 1.057030 & -1.15342 \\ \text { C } & 2.781810 & 0.696767 & 0.20363\end{array}$

$\begin{array}{llll}\text { C } & 2.781810 & 0.696767 & 0.203631 \\ \text { C } & 1.811407 & -0.099876 & 0.864264\end{array}$

$\begin{array}{llll}\text { C } & 0.654122 & -0.509564 & 0.186767\end{array}$

$\begin{array}{llll}\text { C } & 0.443134 & -0.135743 & -1.152303\end{array}$

$\begin{array}{llll}\text { C } & 1.404920 & 0.645841 & -1.817861\end{array}$

$\begin{array}{llll}\text { C } & 3.948276 & 1.167050 & 0.881828\end{array}$

$\begin{array}{llll}\text { C } & 4.948191 & 1.640692 & 1.413687\end{array}$

$\begin{array}{llll}\text { C } & 6.123912 & 2.291007 & 2.003932\end{array}$

$\begin{array}{llll}\text { O } & 6.713184 & 3.308818 & 1.192333\end{array}$

$\begin{array}{llll}\text { C } & 6.004157 & 4.548251 & 1.184613\end{array}$

$\begin{array}{llll}\text { C } & 4.758574 & 4.549398 & 0.404619\end{array}$

$\begin{array}{llll}\text { C } & 3.713585 & 4.491733 & -0.235874\end{array}$

$\begin{array}{llll}\text { C } & 2.456908 & 4.310850 & -0.906435\end{array}$

$\begin{array}{llll}\mathrm{N} & 2.459305 & 4.269465 & -2.263135\end{array}$

$\begin{array}{llll}\text { C } & 1.290821 & 4.040384 & -2.878186\end{array}$

$\begin{array}{llll}\text { C } & 0.071058 & 3.836580 & -2.205763\end{array}$

$\begin{array}{llll}\text { C } & 0.070441 & 3.885682 & -0.802582\end{array}$

$\begin{array}{llll}\text { C } & 1.276818 & 4.131377 & -0.138115\end{array}$

$\begin{array}{llll}\mathrm{H} & -0.468815 & -0.458335 & -1.677972\end{array}$

H $\quad-0.850920 \quad 3.635626 \quad-2.771889$

$\begin{array}{llll}\mathrm{H} & 6.711764 & 5.292698 & 0.760600\end{array}$

$\mathrm{H} \quad 5.853154 \quad 2.701455 \quad 3.010564$

$\begin{array}{llll}\mathrm{H} & 1.333428 & 4.160986 & 0.959540\end{array}$

$\begin{array}{llll}\mathrm{H} & 3.312938 & 1.678184 & -1.669459\end{array}$

$\begin{array}{llll}\mathrm{H} & -0.857028 & 3.722679 & -0.23243\end{array}$

$\begin{array}{llll}\mathrm{H} & 1.246188 & 0.948410 & -2.863658\end{array}$

$\begin{array}{llll}\mathrm{H} & 1.324432 & 4.008491 & -3.983119\end{array}$

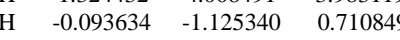

$\begin{array}{llll}\mathrm{H} & 1.976422 & -0.387655 & 1.912999\end{array}$

$\begin{array}{llll}\mathrm{H} & 6.928756 & 1.542269 & 2.171324\end{array}$

$\begin{array}{llll}\mathrm{H} & 5.776898 & 4.872995 & 2.231962\end{array}$

Allenyne_A

32

Free energy $=-784.7496997$ Hartree

$\begin{array}{lrrr}\mathrm{C} & 0.034528 & 0.769027 & -1.392990 \\ \mathrm{C} & 0.529732 & 1.456383 & -0.257919 \\ \mathrm{~N} & -0.232330 & 2.320231 & 0.455073 \\ \mathrm{C} & -1.501387 & 2.509136 & 0.063498 \\ \mathrm{C} & -2.084685 & 1.864689 & -1.042201 \\ \mathrm{C} & -1.290458 & 0.977167 & -1.787006 \\ \mathrm{C} & 1.911413 & 1.257512 & 0.236208 \\ \mathrm{C} & 2.752390 & 0.340105 & -0.227819 \\ \mathrm{C} & 3.493805 & -0.602528 & -0.791744 \\ \mathrm{O} & 3.577862 & -1.923002 & -0.423203 \\ \mathrm{C} & 2.782749 & -2.306909 & 0.712836 \\ \mathrm{C} & 1.337663 & -2.172272 & 0.523294 \\ \mathrm{C} & 0.127764 & -2.002292 & 0.398913 \\ \mathrm{C} & -1.272237 & -1.746930 & 0.265304 \\ \mathrm{C} & -1.913825 & -0.836032 & 1.143890 \\ \mathrm{C} & -3.276843 & -0.550365 & 0.992530 \\ \mathrm{C} & -4.021773 & -1.158917 & -0.033195 \\ \mathrm{C} & -3.393944 & -2.061005 & -0.911093 \\ \mathrm{C} & -2.032331 & -2.359519 & -0.764069 \\ \mathrm{H} & -5.091181 & -0.925345 & -0.152865 \\ \mathrm{H} & -3.136926 & 2.048107 & -1.304466 \\ \mathrm{H} & 4.160953 & -0.394998 & -1.646760 \\ \mathrm{H} & 3.089188 & -1.701677 & 1.600688 \\ \mathrm{H} & 2.185922 & 1.922034 & 1.077491 \\ \mathrm{H} & 0.691727 & 0.069534 & -1.930538 \\ \mathrm{H} & -1.320350 & -0.336209 & 1.922824 \\ \mathrm{H} & -1.707554 & 0.440430 & -2.652257 \\ \mathrm{H} & -3.759440 & 0.166927 & 1.674134 \\ \mathrm{H} & -2.098164 & 3.215033 & 0.670203 \\ \mathrm{H} & -3.971934 & -2.538784 & -1.717425 \\ \mathrm{H} & -1.535055 & -3.061309 & -1.450285 \\ \mathrm{H} & 3.060113 & -3.363739 & 0.904791\end{array}$

Allenyne_A2Cyclization_TS

Free energy $=-784.7325804$ Hartree

$\begin{array}{lrrr}\mathrm{C} & -0.681306 & -0.981670 & 0.936898 \\ \mathrm{C} & 0.336080 & -1.256357 & -0.017297 \\ \mathrm{~N} & 0.072369 & -1.891853 & -1.194166 \\ \mathrm{C} & -1.199020 & -2.194140 & -1.466716 \\ \mathrm{C} & -2.283092 & -1.899689 & -0.609278 \\ \mathrm{C} & -2.008049 & -1.306425 & 0.630027 \\ \mathrm{C} & 1.698203 & -0.790038 & 0.183359 \\ \mathrm{C} & 2.002341 & 0.233235 & 1.029483 \\ \mathrm{C} & 2.871134 & 0.783499 & 1.901633 \\ \mathrm{O} & 2.926812 & 2.129926 & 2.077075 \\ \mathrm{C} & 2.071011 & 2.774421 & 1.122804 \\ \mathrm{C} & 0.881764 & 1.917785 & 0.818959 \\ \mathrm{C} & -0.320499 & 1.940197 & 0.450429 \\ \mathrm{C} & -1.646177 & 1.851213 & -0.038208\end{array}$

$\begin{array}{llll}\text { C } & -1.892908 & 1.459265 & -1.386499\end{array}$

$\begin{array}{llll}\text { C } & -3.203914 & 1.341933 & -1.862914\end{array}$

$\begin{array}{llll}\text { C } & -4.297871 & 1.606968 & -1.018818\end{array}$

$\begin{array}{llll}\text { C } & -4.068318 & 1.991331 & 0.316323\end{array}$

$\begin{array}{llll}\text { C } & -2.763238 & 2.117365 & 0.805724\end{array}$

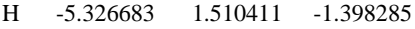

$\begin{array}{llll}\mathrm{H} & -3.312201 & -2.146762 & -0.909451\end{array}$

$\begin{array}{llll}\mathrm{H} & 3.487213 & 0.221611 & 2.620782\end{array}$

$\begin{array}{llll}\text { H } & 2.639009 & 2.941183 & 0.177802\end{array}$

H $\quad 2.442666 \quad-1.163788 \quad-0.542601$

\begin{tabular}{llll}
\hline & -0.411697 & -0.515988 & 1.895260
\end{tabular}

$\begin{array}{llll}\mathrm{H} & -1.037436 & 1.223117 & -2.036033\end{array}$

\begin{tabular}{llll}
\hline & -2.819087 & -1.068189 & 1.334436
\end{tabular}

$\begin{array}{llll}\mathrm{H} & -3.374890 & 1.028686 & -2.904582\end{array}$

$\begin{array}{llll}\mathrm{H} & -1.385550 & -2.697415 & -2.433478\end{array}$

$\begin{array}{llll}H & -4.920001 & 2.201195 & 0.982051\end{array}$

$\begin{array}{llll}-2.583218 & 2.417214 & 1.84872\end{array}$

$\begin{array}{llll}\mathrm{H} & 1.783906 & 3.753820 & 1.551631\end{array}$

Allenyne_A2Cyclization_INT

32

Free energy $=-784.8147732$ Hartree

$\begin{array}{llll}\text { C } & -0.761657 & -0.817143 & 0.441619 \\ \text { C } & 0.544264 & -1.611190 & 0.579701\end{array}$

$\begin{array}{llll}\text { C } & 0.544264 & -1.611190 & 0.579701\end{array}$

$\begin{array}{llll}\mathrm{N} & 0.495189 & -2.996502 & 0.555950\end{array}$

$\begin{array}{llll}\text { C } & -0.521182 & -3.567795 & -0.037321\end{array}$

$\begin{array}{llll}\text { C } & -1.535138 & -2.828900 & -0.787323\end{array}$

$\begin{array}{llll}\text { C } & -1.631048 & -1.485476 & -0.615212\end{array}$

$\begin{array}{llll}\text { C } & 1.759350 & -0.993382 & 0.757147\end{array}$

$\begin{array}{llll}\text { C } & 1.845471 & 0.432520 & 0.638566\end{array}$

$\begin{array}{llll}\text { C } & 2.881480 & 1.316196 & 0.833075\end{array}$

$\begin{array}{llll}\text { O } & 2.548826 & 2.617470 & 0.641234\end{array}$

$\begin{array}{llll}\text { C } & 1.161207 & 2.652350 & 0.199435\end{array}$

$\begin{array}{llll}\text { C } & 0.663710 & 1.225785 & 0.304473\end{array}$

$\begin{array}{llll}\text { C } & -0.593268 & 0.692125 & 0.217678\end{array}$

$\begin{array}{llll}\text { C } & -1.813499 & 1.506182 & 0.031191\end{array}$

$\begin{array}{llll}\text { C } & -1.811094 & 2.704800 & -0.729440\end{array}$

$\begin{array}{llll}\text { C } & -2.965556 & 3.489937 & -0.862806\end{array}$

$\begin{array}{llll}\text { C } & -4.165987 & 3.099484 & -0.244960\end{array}$

$\begin{array}{llll}\text { C } & -4.197137 & 1.906527 & 0.497620\end{array}$

$\begin{array}{llll}\text { C } & -3.044061 & 1.119635 & 0.628791\end{array}$

$\begin{array}{llll}\mathrm{H} & -5.072734 & 3.714879 & -0.349085\end{array}$

$\begin{array}{llll}\mathrm{H} & -2.176290 & -3.368306 & -1.502747\end{array}$

$\begin{array}{llll}\mathrm{H} & 3.919540 & 1.135966 & 1.143200\end{array}$

$\begin{array}{llll}\mathrm{H} & 1.170771 & 3.035455 & -0.844494\end{array}$

$\begin{array}{llll}\mathrm{H} & 2.646092 & -1.615335 & 0.948953\end{array}$

$\begin{array}{llll}\mathrm{H} & -1.296048 & -0.958586 & 1.423227\end{array}$

$\begin{array}{llll}\mathrm{H} & -0.902131 & 3.006221 & -1.268194\end{array}$

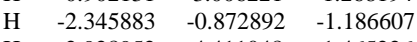

$\begin{array}{llll}\mathrm{H} & -2.928952 & 4.411048 & -1.465226\end{array}$

$\begin{array}{llll}\mathrm{H} & -0.548089 & -4.675053 & -0.036584\end{array}$

$\begin{array}{llll}\mathrm{H} & -5.131258 & 1.583964 & 0.983046\end{array}$

$\begin{array}{llll}\mathrm{H} & -3.097161 & 0.193821 & 1.219955\end{array}$

$\begin{array}{llll}\mathrm{H} & 0.599405 & 3.377650 & 0.825894\end{array}$

Allenyne_B

32

Free energy $=-784.7443417$ Hartree

$\begin{array}{llll}\text { C } & 0.916977 & 0.735750 & -1.023139\end{array}$

$\begin{array}{llll}\mathrm{C} & 1.337558 & 1.375877 & 0.170909\end{array}$

$\begin{array}{llll}\mathrm{N} & 0.484116 & 2.049767 & 0.980793\end{array}$

$\begin{array}{llll}\text { C } & -0.801509 & 2.111457 & 0.611208\end{array}$

$\begin{array}{llll}\text { C } & -1.317543 & 1.516770 & -0.555988\end{array}$

$\begin{array}{llll}\text { C } & -0.432601 & 0.812467 & -1.387495\end{array}$

$\begin{array}{llll}\text { C } & 2.720583 & 1.348670 & 0.557490\end{array}$

$\begin{array}{llll}\text { C } & 3.905107 & 1.358537 & 0.877375\end{array}$

$\begin{array}{llll}\text { C } & 5.302081 & 1.435251 & 1.310138\end{array}$

$\begin{array}{llll}\text { O } & 6.210385 & 1.939440 & 0.317836\end{array}$

$\begin{array}{llll}\text { C } & 5.993264 & 3.239705 & -0.078835\end{array}$

$\begin{array}{llll}\text { C } & 5.039126 & 4.051352 & 0.351743\end{array}$

$\begin{array}{llll}\text { C } & 3.998419 & 4.805318 & 0.692045\end{array}$

$\begin{array}{llll}\text { C } & 2.690030 & 4.802190 & 0.006370\end{array}$

$\begin{array}{llll}\text { C } & 2.489764 & 4.111506 & -1.212481\end{array}$

$\begin{array}{llll}\text { C } & 1.229465 & 4.088679 & -1.821566\end{array}$

$\begin{array}{llll}\text { C } & 0.142669 & 4.756665 & -1.227814\end{array}$

$\begin{array}{llll}\text { C } & 0.329343 & 5.453307 & -0.021902\end{array}$

$\begin{array}{llll}\text { C } & 1.591536 & 5.476317 & 0.588609\end{array}$

$\begin{array}{llll}\mathrm{H} & -0.849660 & 4.727264 & -1.703344\end{array}$

$\begin{array}{llll}\mathrm{H} & -2.385971 & 1.609546 & -0.803388\end{array}$

$\begin{array}{llll}\mathrm{H} & 5.689835 & 0.427233 & 1.564046\end{array}$

$\begin{array}{llll}\mathrm{H} & 6.753557 & 3.548626 & -0.817432\end{array}$

$\begin{array}{llll}\mathrm{H} & 4.074866 & 5.467730 & 1.577001\end{array}$

$\begin{array}{llll}\mathrm{H} & 1.651319 & 0.202115 & -1.644017\end{array}$

$\begin{array}{llll}\mathrm{H} & 3.339976 & 3.578808 & -1.665021\end{array}$

$\begin{array}{llll}\mathrm{H} & -0.788788 & 0.331343 & -2.311591\end{array}$

$\begin{array}{llll}\mathrm{H} & 1.087875 & 3.537123 & -2.763018\end{array}$

$\begin{array}{llll}\mathrm{H} & -1.470491 & 2.677033 & 1.284854\end{array}$

$\begin{array}{llll}\mathrm{H} & -0.517792 & 5.973175 & 0.451580\end{array}$

$\begin{array}{llll}\mathrm{H} & 1.731230 & 6.010165 & 1.542072\end{array}$

$\begin{array}{llll}\mathrm{H} & 5.358182 & 2.070275 & 2.227708\end{array}$ 


\begin{tabular}{|c|c|c|c|}
\hline \multicolumn{4}{|c|}{ Allenyne_B2Cyclization_TS } \\
\hline \multicolumn{4}{|c|}{ Free energy $=-784.7289391$ Hartree } \\
\hline $\mathrm{C}$ & 0.864348 & 0.534565 & -1.219176 \\
\hline $\mathrm{C}$ & 1.361770 & 1.398313 & \\
\hline $\mathrm{N}$ & 0.549132 & 2.239362 & \\
\hline $\mathrm{C}$ & -0.75 & 2.246964 & \\
\hline $\mathrm{C}$ & -1 . & 8 & \\
\hline $\mathrm{C}$ & -0 & 0.3 & \\
\hline $\mathrm{C}$ & 3 & 1.4 & \\
\hline $\mathrm{C}$ & 4 & 1. & \\
\hline $\mathrm{C}$ & 7 & 0. & \\
\hline $\mathrm{O}$ & 6.36 & 1.1 & \\
\hline $\mathrm{C}$ & 7 & 2.3 & -0 \\
\hline $\mathrm{C}$ & 7 & & \\
\hline $\mathrm{C}$ & & & \\
\hline $\mathrm{C}$ & & & \\
\hline $\mathrm{C}$ & & & \\
\hline $\mathrm{C}$ & & & \\
\hline $\mathrm{C}$ & & & \\
\hline $\mathrm{C}$ & & & \\
\hline $\mathrm{C}$ & & & \\
\hline $\mathrm{H}$ & & & \\
\hline $\mathrm{H}$ & & & \\
\hline $\mathrm{H}$ & & & \\
\hline $\mathrm{H}$ & & & \\
\hline $\mathrm{H}$ & & & \\
\hline $\mathrm{H}$ & 155 & -0.1 & -1. \\
\hline $\mathrm{H}$ & 3.398 & 2.6 & -2.329851 \\
\hline $\mathrm{H}$ & -0.90 & -0.0 & -2.315307 \\
\hline $\mathrm{H}$ & 1.081451 & 2.875082 & -3.266369 \\
\hline $\mathrm{H}$ & -1.3 & 52 & \\
\hline $\mathrm{H}$ & & 6.490766 & 3524 \\
\hline $\mathrm{H}$ & & 6.286274 & 0.005973 \\
\hline$\Pi$ & & & 2.000 \\
\hline
\end{tabular}

Allenyne_B2Cyclization_INT

32

Free energy $=-784.8083764$ Hartree

$\begin{array}{lrrr}\text { C } & 1.372776 & 0.240172 & -1.209990 \\ \mathrm{C} & 1.229549 & 1.533333 & -0.643910 \\ \mathrm{~N} & 0.057872 & 1.931211 & -0.091102 \\ \mathrm{C} & -0.965891 & 1.064286 & -0.057623 \\ \mathrm{C} & -0.901346 & -0.246186 & -0.562477 \\ \mathrm{C} & 0.299833 & -0.659438 & -1.161633 \\ \mathrm{C} & 2.381455 & 2.479495 & -0.661094 \\ \mathrm{C} & 3.591132 & 2.100461 & -0.160779 \\ \mathrm{C} & 4.011817 & 0.850351 & 0.590924 \\ \mathrm{O} & 5.427230 & 1.027593 & 0.884443 \\ \mathrm{C} & 5.796959 & 2.244507 & 0.398642 \\ \mathrm{C} & 4.785899 & 2.940153 & -0.217567 \\ \mathrm{C} & 4.711698 & 4.247572 & -0.806775 \\ \mathrm{C} & 3.513764 & 4.717003 & -1.291149 \\ \mathrm{C} & 2.262958 & 3.830850 & -1.385692 \\ \mathrm{C} & 0.990757 & 4.622090 & -1.113410 \\ \mathrm{C} & 0.939174 & 5.950107 & -1.395261 \\ \mathrm{C} & 2.125455 & 6.670176 & -1.836848 \\ \mathrm{C} & 3.362596 & 6.083748 & -1.753244 \\ \mathrm{H} & -0.003094 & 6.506013 & -1.263204 \\ \mathrm{H} & -1.773115 & -0.914727 & -0.497790 \\ \mathrm{H} & 3.908310 & -0.091538 & 0.014796 \\ \mathrm{H} & 6.849634 & 2.512214 & 0.561104 \\ \mathrm{H} & 5.601670 & 4.897808 & -0.819427 \\ \mathrm{H} & 2.317155 & -0.037364 & -1.701770 \\ \mathrm{H} & 2.183631 & 3.572355 & -2.481574 \\ \mathrm{H} & 0.395526 & -1.665833 & -1.598313 \\ \mathrm{H} & 0.119112 & 4.065527 & -0.740259 \\ \mathrm{H} & -1.899916 & 1.435737 & 0.401722 \\ \mathrm{H} & 2.033215 & 7.722828 & -2.146825 \\ \mathrm{H} & 4.274359 & 6.673025 & -1.945153 \\ \mathrm{H} & 3.467578 & 0.724922 & 1.551894\end{array}$

Bisallene

32

Free energy $=-784.7604359$ Hartree

$\begin{array}{llll}\mathrm{N} & 1.947760 & 4.905609 & -2.344901 \\ \mathrm{C} & 2.805342 & 5.360574 & -1.399846 \\ \mathrm{C} & 2.396642 & 6.223352 & -0.352382 \\ \mathrm{C} & 1.063210 & 6.644134 & -0.307418 \\ \mathrm{C} & 0.178450 & 6.194468 & -1.302279 \\ \mathrm{C} & 0.676107 & 5.321450 & -2.288689 \\ \mathrm{C} & 4.212684 & 4.957159 & -1.569490 \\ \mathrm{C} & 5.232412 & 5.371978 & -0.822694 \\ \mathrm{C} & 6.256459 & 5.691986 & -0.048082 \\ \mathrm{O} & 7.071288 & 6.802237 & -0.155869 \\ \mathrm{C} & 6.646577 & 7.864714 & -0.934724 \\ \mathrm{C} & 5.417124 & 8.098645 & -1.362580 \\ \mathrm{C} & 4.207507 & 8.422161 & -1.815921\end{array}$

$\begin{array}{lrrr}\mathrm{C} & 3.593863 & 7.941971 & -3.062956 \\ \mathrm{C} & 2.251706 & 8.282426 & -3.356202 \\ \mathrm{C} & 1.615646 & 7.770713 & -4.495317 \\ \mathrm{C} & 2.308307 & 6.905416 & -5.359519 \\ \mathrm{C} & 3.644964 & 6.564932 & -5.082427 \\ \mathrm{C} & 4.284881 & 7.078706 & -3.948494 \\ \mathrm{H} & 1.806143 & 6.491798 & -6.247265 \\ \mathrm{H} & -0.876048 & 6.508966 & -1.316259 \\ \mathrm{H} & 6.593358 & 5.049803 & 0.783143 \\ \mathrm{H} & 7.489654 & 8.546514 & -1.137466 \\ \mathrm{H} & 4.390291 & 4.321530 & -2.456940 \\ \mathrm{H} & 3.557939 & 9.047886 & -1.172748 \\ \mathrm{H} & 3.135608 & 6.558212 & 0.390005 \\ \mathrm{H} & 5.325103 & 6.804975 & -3.717608 \\ \mathrm{H} & 0.717778 & 7.321890 & 0.489253 \\ \mathrm{H} & 4.189235 & 5.884664 & -5.755834 \\ \mathrm{H} & 0.005393 & 4.943669 & -3.081668 \\ \mathrm{H} & 0.568240 & 8.037518 & -4.703074 \\ \mathrm{H} & 1.698799 & 8.937417 & -2.664011\end{array}$

Bisallene2Cyclization_TS1

32

Free energy $=-784.7552169$ Hartree

$\begin{array}{lrrr}\mathrm{N} & 1.897439 & 4.907987 & -2.206063 \\ \mathrm{C} & 2.785900 & 5.435417 & -1.318745 \\ \mathrm{C} & 2.403239 & 6.395997 & -0.342269 \\ \mathrm{C} & 1.081190 & 6.856478 & -0.328903 \\ \mathrm{C} & 0.180424 & 6.349341 & -1.277869 \\ \mathrm{C} & 0.643295 & 5.361718 & -2.175607 \\ \mathrm{C} & 4.178812 & 5.051573 & -1.495866 \\ \mathrm{C} & 5.217119 & 5.653432 & -0.863584 \\ \mathrm{C} & 6.315179 & 5.718505 & -0.109509 \\ \mathrm{O} & 7.164578 & 6.801211 & -0.142955 \\ \mathrm{C} & 6.643615 & 7.828438 & -0.897670 \\ \mathrm{C} & 5.398684 & 7.794863 & -1.373781 \\ \mathrm{C} & 4.250325 & 8.306648 & -1.885672 \\ \mathrm{C} & 3.623849 & 7.850304 & -3.115116 \\ \mathrm{C} & 2.304331 & 8.268104 & -3.440512 \\ \mathrm{C} & 1.642051 & 7.740323 & -4.551997 \\ \mathrm{C} & 2.276772 & 6.778263 & -5.362663 \\ \mathrm{C} & 3.585741 & 6.364716 & -5.062852 \\ \mathrm{C} & 4.258033 & 6.894023 & -3.953990 \\ \mathrm{H} & 1.747449 & 6.350292 & -6.227528 \\ \mathrm{H} & -0.863446 & 6.695017 & -1.320644 \\ \mathrm{H} & 6.632371 & 4.954156 & 0.615434 \\ \mathrm{H} & 7.374681 & 8.630861 & -1.077882 \\ \mathrm{H} & 4.363928 & 4.370537 & -2.346046 \\ \mathrm{H} & 3.647393 & 8.979862 & -1.247196 \\ \mathrm{H} & 3.150850 & 6.763447 & 0.374839 \\ \mathrm{H} & 5.281936 & 6.574956 & -3.712651 \\ \mathrm{H} & 0.759776 & 7.610979 & 0.406713 \\ \mathrm{H} & 4.083439 & 5.613997 & -5.696056 \\ \mathrm{H} & -0.051452 & 4.925006 & -2.916648 \\ \mathrm{H} & 0.615984 & 8.064550 & -4.782931 \\ \mathrm{H} & 1.795068 & 8.993835 & -2.786649\end{array}$

Bisallene2Cyclization_INT1

32

Free energy $=-784.7978413$ Hartree

$\begin{array}{lrrr}\mathrm{N} & 2.207026 & 4.181609 & -1.550810 \\ \mathrm{C} & 2.868736 & 5.306898 & -1.139650 \\ \mathrm{C} & 2.200290 & 6.563813 & -1.045269 \\ \mathrm{C} & 0.870149 & 6.668601 & -1.454739 \\ \mathrm{C} & 0.225102 & 5.525409 & -1.957312 \\ \mathrm{C} & 0.942143 & 4.308362 & -1.956606 \\ \mathrm{C} & 4.257374 & 5.121161 & -0.805745 \\ \mathrm{C} & 5.324017 & 6.060637 & -0.745461 \\ \mathrm{C} & 6.499320 & 5.784478 & -0.022578 \\ \mathrm{O} & 7.330487 & 6.853349 & 0.000288 \\ \mathrm{C} & 6.759257 & 7.848141 & -0.719665 \\ \mathrm{C} & 5.528996 & 7.443689 & -1.271486 \\ \mathrm{C} & 4.808612 & 8.283816 & -2.168062 \\ \mathrm{C} & 3.786075 & 7.957982 & -3.127942 \\ \mathrm{C} & 2.886590 & 8.975093 & -3.568540 \\ \mathrm{C} & 1.829938 & 8.681002 & -4.436603 \\ \mathrm{C} & 1.655038 & 7.371028 & -4.925881 \\ \mathrm{C} & 2.568795 & 6.365228 & -4.554479 \\ \mathrm{C} & 3.611893 & 6.649358 & -3.670298 \\ \mathrm{H} & 0.819229 & 7.138588 & -5.603703 \\ \mathrm{H} & -0.813130 & 5.562086 & -2.318440 \\ \mathrm{H} & 6.814576 & 4.903532 & 0.545913 \\ \mathrm{H} & 7.335339 & 8.773949 & -0.821007 \\ \mathrm{H} & 4.514265 & 4.078762 & -0.552476 \\ \mathrm{H} & 5.109026 & 9.346508 & -2.139963 \\ \mathrm{H} & 2.737868 & 7.437356 & -0.652632 \\ \mathrm{H} & 4.315573 & 5.854911 & -3.386895 \\ \mathrm{H} & 0.347671 & 7.635895 & -1.401206 \\ \mathrm{H} & 2.454687 & 5.342961 & -4.945110 \\ \mathrm{H} & 0.446881 & 3.381524 & -2.302219 \\ \mathrm{H} & 1.133387 & 9.478666 & -4.738588\end{array}$


H $\quad 3.013341 \quad 10.000446 \quad-3.185699$

Bisallene2Cyclization_TS2_for_A

32

Free energy $=-784.7882554$ Hartree

$\begin{array}{lccc}\mathrm{C} & 4.061235 & 7.016592 & -3.558182 \\ \mathrm{C} & 4.183955 & 8.243169 & -2.836981 \\ \mathrm{C} & 3.493229 & 9.383608 & -3.351199 \\ \mathrm{C} & 2.711401 & 9.296091 & -4.505739 \\ \mathrm{C} & 2.593266 & 8.071873 & -5.196036 \\ \mathrm{C} & 3.276952 & 6.938100 & -4.714092 \\ \mathrm{C} & 5.027061 & 8.388942 & -1.681205 \\ \mathrm{C} & 5.623118 & 7.294925 & -0.915948 \\ \mathrm{C} & 6.957347 & 7.328307 & -0.288760 \\ \mathrm{C} & 7.071130 & 6.121344 & 0.379781 \\ \mathrm{O} & 5.941953 & 5.361864 & 0.238744 \\ \mathrm{C} & 5.074634 & 6.090377 & -0.536550 \\ \mathrm{C} & 7.872488 & 8.442824 & -0.347215 \\ \mathrm{C} & 7.854285 & 9.350561 & -1.426253 \\ \mathrm{~N} & 8.439489 & 10.586863 & -1.235454 \\ \mathrm{C} & 8.236718 & 11.518907 & -2.151518 \\ \mathrm{C} & 7.454640 & 11.334452 & -3.336338 \\ \mathrm{C} & 6.959863 & 10.061808 & -3.608541 \\ \mathrm{C} & 7.162840 & 9.029223 & -2.663783 \\ \mathrm{H} & 1.979716 & 8.004829 & -6.107232 \\ \mathrm{H} & 7.302941 & 12.172681 & -4.033510 \\ \mathrm{H} & 7.892818 & 5.665102 & 0.942337 \\ \mathrm{H} & 4.084584 & 5.650583 & -0.696164 \\ \mathrm{H} & 8.512377 & 8.685595 & 0.514823 \\ \mathrm{H} & 5.040194 & 9.378150 & -1.194540 \\ \mathrm{H} & 7.045118 & 7.981303 & -2.969113 \\ \mathrm{H} & 4.608672 & 6.130029 & -3.205483 \\ \mathrm{H} & 6.418129 & 9.847400 & -4.543041 \\ \mathrm{H} & 3.200724 & 5.981191 & -5.254036 \\ \mathrm{H} & 8.716087 & 12.499603 & -1.969720 \\ \mathrm{H} & 2.187293 & 10.190030 & -4.878237 \\ \mathrm{H} & 3.589610 & 10.344788 & -2.821681\end{array}$

Bisallene2Cyclization_INT2_for_A 32

Free energy $=-784.8285613$ Hartree

$\begin{array}{llll}\text { C } & 4.532740 & 7.190090 & -3.845915\end{array}$

$\begin{array}{llll}\text { C } & 4.374021 & 8.314812 & -3.006231\end{array}$

$\begin{array}{llll}\text { C } & 3.288207 & 9.182784 & -3.243061\end{array}$

$\begin{array}{llll}\text { C } & 2.381962 & 8.937410 & -4.289656\end{array}$

$\begin{array}{llll}\text { C } & 2.551046 & 7.815700 & -5.118266\end{array}$

$\begin{array}{llll}\text { C } & 3.630369 & 6.941932 & -4.892226\end{array}$

$\begin{array}{llll}\text { C } & 5.380607 & 8.609013 & -1.905485\end{array}$

$\begin{array}{llll}\text { C } & 5.646249 & 7.441566 & -0.990378\end{array}$

$\begin{array}{llll}\text { C } & 6.869955 & 7.367080 & -0.214666\end{array}$

$\begin{array}{llll}\text { C } & 6.746437 & 6.232548 & 0.571039\end{array}$

$\begin{array}{llll}\text { O } & 5.552620 & 5.616991 & 0.328798\end{array}$

$\begin{array}{llll}\text { C } & 4.887489 & 6.362529 & -0.615503 \\ \text { C } & 7.897353 & 8.369878 & -0.350754\end{array}$

$\begin{array}{llll}\text { C } & 7.897353 & 8.369878 & -0.350754\end{array}$

$\begin{array}{llll}\text { C } & 7.836456 & 9.268209 & -1.391246\end{array}$

$\begin{array}{llll}\text { N } & 8.808711 & 10.257468 & -1.460191\end{array}$

$\begin{array}{llll}\text { C } & 8.680776 & 11.207873 & -2.345401\end{array}$

$\begin{array}{llll}\text { C } & 7.542545 & 11.346188 & -3.252974\end{array}$

$\begin{array}{llll}\text { C } & 6.603091 & 10.369266 & -3.300688\end{array}$

$\begin{array}{llll}\text { C } & 6.752190 & 9.115106 & -2.478049\end{array}$

$\begin{array}{llll}\mathrm{H} & 1.842135 & 7.620708 & -5.938012\end{array}$

$\begin{array}{llll}\mathrm{H} & 7.461204 & 12.251945 & -3.874631\end{array}$

$\begin{array}{llll}\mathrm{H} & 7.403034 & 5.746531 & 1.300463\end{array}$

$\begin{array}{llll}\mathrm{H} & 3.901892 & 5.994956 & -0.916407\end{array}$

$\begin{array}{llll}\mathrm{H} & 8.735634 & 8.442747 & 0.357784\end{array}$

$\begin{array}{llll}\mathrm{H} & 4.977634 & 9.440017 & -1.279454\end{array}$

$\begin{array}{llll}\mathrm{H} & 7.100255 & 8.304995 & -3.172207\end{array}$

$\begin{array}{llll}\mathrm{H} & 5.369812 & 6.497434 & -3.665213\end{array}$

$\begin{array}{llll}\mathrm{H} & 5.727249 & 10.445229 & -3.966171\end{array}$

$\begin{array}{llll}\mathrm{H} & 3.767548 & 6.058134 & -5.534810\end{array}$

$\begin{array}{llll}\mathrm{H} & 9.476484 & 11.978121 & -2.371356\end{array}$

$\begin{array}{llll}\mathrm{H} & 1.538698 & 9.626037 & -4.455549\end{array}$

$\begin{array}{llll}\mathrm{H} & 3.153735 & 10.065043 & -2.596152\end{array}$

Bisallene2Cyclization_TS2_for_B

32

Free energy $=-784.787966$ Hartree

$\begin{array}{llll}\mathrm{C} & 4.020970 & 6.241150 & -3.090378 \\ \mathrm{C} & 4.002986 & 7.683915 & -2.931417 \\ \mathrm{C} & 2.859365 & 8.395930 & -3.436331 \\ \mathrm{C} & 1.775923 & 7.715572 & -3.972451 \\ \mathrm{C} & 1.782986 & 6.291825 & -4.057909 \\ \mathrm{C} & 2.905022 & 5.573333 & -3.647597 \\ \mathrm{C} & 5.020769 & 8.344269 & -2.209526 \\ \mathrm{C} & 5.893527 & 7.625521 & -1.312392 \\ \mathrm{C} & 5.537236 & 6.378070 & -0.610564 \\ \mathrm{C} & 6.614604 & 6.100968 & 0.200413 \\ \mathrm{O} & 7.612888 & 7.033641 & 0.053004 \\ \mathrm{C} & 7.162744 & 7.945410 & -0.860561 \\ \mathrm{C} & 4.256048 & 5.683173 & -0.727078\end{array}$

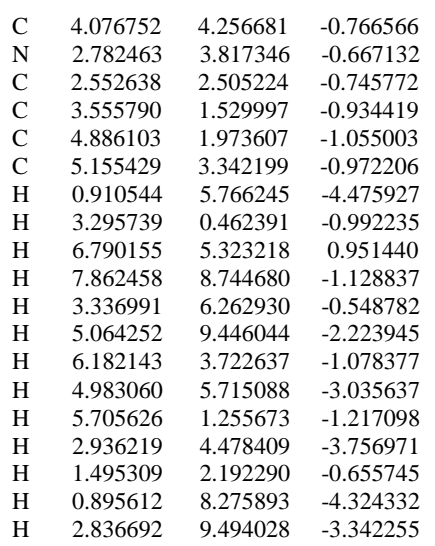

Bisallene2Cyclization_INT2 for_B

32

Free energy $=-784.8277137$ Hartree

$\begin{array}{llll}\mathrm{C} & 3.790200 & 6.195581 & -2.601150 \\ \mathrm{C} & 4.037138 & 7.694821 & -2.839018 \\ \mathrm{C} & 3.205313 & 8.377544 & -3.807823 \\ \mathrm{C} & 2.087446 & 7.792141 & -4.342563 \\ \mathrm{C} & 1.670865 & 6.462101 & -3.920242 \\ \mathrm{C} & 2.436877 & 5.721847 & -3.078143 \\ \mathrm{C} & 5.069984 & 8.335801 & -2.192018 \\ \mathrm{C} & 5.830566 & 7.635908 & -1.185349 \\ \mathrm{C} & 5.386387 & 6.354476 & -0.672247 \\ \mathrm{C} & 6.305555 & 5.984340 & 0.277303 \\ \mathrm{O} & 7.292081 & 6.933664 & 0.385954 \\ \mathrm{C} & 6.996114 & 7.932645 & -0.497727 \\ \mathrm{C} & 4.091277 & 5.735730 & -1.129295 \\ \mathrm{C} & 4.062906 & 4.220287 & -1.006763 \\ \mathrm{~N} & 2.956176 & 3.675838 & -0.459596 \\ \mathrm{C} & 2.882524 & 2.336225 & -0.370952 \\ \mathrm{C} & 3.891405 & 1.468153 & -0.821603 \\ \mathrm{C} & 5.040719 & 2.034789 & -1.398129 \\ \mathrm{C} & 5.129677 & 3.430160 & -1.491746 \\ \mathrm{H} & 0.707027 & 6.068865 & -4.279266 \\ \mathrm{H} & 3.777481 & 0.377856 & -0.720901 \\ \mathrm{H} & 6.402331 & 5.111833 & 0.931693 \\ \mathrm{H} & 7.701708 & 8.769340 & -0.537213 \\ \mathrm{H} & 3.263387 & 6.113810 & -0.489010 \\ \mathrm{H} & 5.311434 & 9.382070 & -2.437809 \\ \mathrm{H} & 6.017565 & 3.915542 & -1.924349 \\ \mathrm{H} & 4.551935 & 5.667824 & -3.236015 \\ \mathrm{H} & 5.858444 & 1.397031 & -1.768970 \\ \mathrm{H} & 2.102449 & 4.730191 & -2.739303 \\ \mathrm{H} & 1.962248 & 1.929604 & 0.086354 \\ \mathrm{H} & 1.461644 & 8.347977 & -5.058170 \\ \mathrm{H} & 3.471285 & 9.413777 & -4.072480\end{array}$

\section{$A$ = 2-pyridyl, B= 4-methoxyphenyl}

Bispropargyl

Free energy $=-899.1525682$ Hartree

$\begin{array}{lrrr}\mathrm{C} & -0.352717 & -2.393240 & -0.051753 \\ \mathrm{C} & 0.613912 & -1.590960 & 0.613223 \\ \mathrm{C} & 0.152922 & -0.514319 & 1.411832 \\ \mathrm{C} & -1.210790 & -0.220677 & 1.523819 \\ \mathrm{C} & -2.154635 & -1.004912 & 0.826011 \\ \mathrm{C} & -1.713294 & -2.105736 & 0.054163 \\ \mathrm{C} & 2.014496 & -1.765436 & 0.411323 \\ \mathrm{C} & 3.226088 & -1.776004 & 0.204664 \\ \mathrm{C} & 4.634382 & -1.551684 & -0.133325 \\ \mathrm{O} & 4.853248 & -0.498354 & -1.080362 \\ \mathrm{C} & 4.496239 & 0.805595 & -0.624916 \\ \mathrm{C} & 3.070807 & 1.143682 & -0.744198 \\ \mathrm{C} & 1.880542 & 1.427309 & -0.843282 \\ \mathrm{C} & 0.463850 & 1.624807 & -0.970171 \\ \mathrm{C} & -0.294089 & 0.705338 & -1.743701 \\ \mathrm{C} & -1.678964 & 0.875751 & -1.824580 \\ \mathrm{C} & -2.267658 & 1.960929 & -1.153142 \\ \mathrm{C} & -1.429864 & 2.818174 & -0.416869 \\ \mathrm{~N} & -0.100931 & 2.668925 & -0.313969 \\ \mathrm{O} & -3.495759 & -0.770644 & 0.816290 \\ \mathrm{C} & -4.005397 & 0.288721 & 1.614745 \\ \mathrm{H} & -3.353394 & 2.137460 & -1.194639 \\ \mathrm{H} & 5.087162 & 1.510644 & -1.249801 \\ \mathrm{H} & 5.213560 & -1.353286 & 0.804998 \\ \mathrm{H} & 0.212094 & -0.138155 & -2.233519 \\ \mathrm{H} & 0.888485 & 0.129724 & 1.914539 \\ \mathrm{H} & -2.294816 & 0.162382 & -2.393120\end{array}$




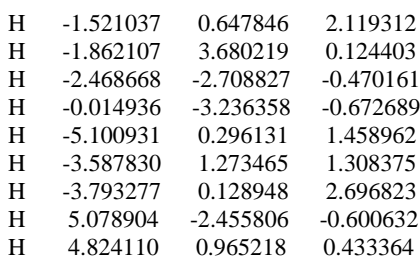

Allenyne_A

36

Free energy $=-899.1667747$ Hartree

$\begin{array}{llll}\text { C } & 0.021253 & 0.759299 & -1.383303 \\ \text { C } & 0.504457 & 1.451707 & -0.246514\end{array}$

$\begin{array}{llll}\text { C } & 0.504457 & 1.451707 & -0.246514\end{array}$

$\begin{array}{llll}\text { C } & -0.378282 & 2.341943 & 0.422484\end{array}$

$\begin{array}{llll}\text { C } & -1.689701 & 2.514129 & -0.019407\end{array}$

$\begin{array}{llll}\text { C } & -2.161322 & 1.811299 & -1.152708\end{array}$

$\begin{array}{llll}\text { C } & -1.290861 & 0.936604 & -1.839389\end{array}$

$\begin{array}{llll}\text { C } & 1.825912 & 1.206920 & 0.233672\end{array}$

$\begin{array}{llll}\text { C } & 2.957020 & 0.930933 & 0.626343\end{array}$

$\begin{array}{llll}\text { C } & 4.295914 & 0.526839 & 1.053784\end{array}$

$\begin{array}{llll}\text { O } & 4.359795 & -0.081954 & 2.359006\end{array}$

$\begin{array}{llll}\text { C } & 3.682306 & -1.266370 & 2.504253\end{array}$

$\begin{array}{llll}\text { C } & 2.960376 & -1.900178 & 1.591019\end{array}$

$\begin{array}{llll}\text { C } & 2.121906 & -2.472380 & 0.734383\end{array}$

$\begin{array}{llll}\text { C } & 0.680541 & -2.148780 & 0.624473\end{array}$

$\begin{array}{llll}\mathrm{N} & 0.021906 & -2.731115 & -0.406563\end{array}$

$\begin{array}{llll}\text { C } & -1.278372 & -2.442193 & -0.565330\end{array}$

$\begin{array}{llll}\text { C } & -1.995920 & -1.570602 & 0.273303\end{array}$

$\begin{array}{llll}\text { C } & -1.314113 & -0.973619 & 1.347155\end{array}$

$\begin{array}{llll}\text { C } & 0.040484 & -1.266583 & 1.529011\end{array}$

$\begin{array}{llll}\mathrm{O} & -3.461410 & 2.035528 & -1.492905\end{array}$

$\begin{array}{llll}\text { C } & -3.999383 & 1.352026 & -2.615013\end{array}$

$\begin{array}{llll}\mathrm{H} & -3.057949 & -1.355382 & 0.081362\end{array}$

$\begin{array}{llll}\mathrm{H} & 3.825228 & -1.671772 & 3.521366\end{array}$

$\begin{array}{llll}\mathrm{H} & 4.717577 & -0.180064 & 0.297884\end{array}$

$\begin{array}{llll}\mathrm{H} & 2.468471 & -3.224537 & 0.000216\end{array}$

$\begin{array}{llll}\mathrm{H} & 0.624723 & -0.808491 & 2.340292\end{array}$

$\mathrm{H} \quad 0.681646 \quad 0.044329 \quad-1.895124$

$\begin{array}{llll}\mathrm{H} & -1.829761 & -0.271643 & 2.019994\end{array}$

$\begin{array}{llll}\mathrm{H} & -1.630469 & 0.367591 & -2.714810\end{array}$

$\begin{array}{llll}\mathrm{H} & -1.783985 & -2.928251 & -1.420163\end{array}$

$\begin{array}{llll}\mathrm{H} & -2.384254 & 3.192237 & 0.49818\end{array}$

$\begin{array}{llll}\mathrm{H} & -0.020841 & 2.886176 & 1.309177\end{array}$

$\begin{array}{llll}\mathrm{H} & -5.050866 & 1.681936 & -2.706230\end{array}$

$\begin{array}{llll}\mathrm{H} & -3.977294 & 0.246371 & -2.477890\end{array}$

$\begin{array}{llll}\mathrm{H} & -3.459403 & 1.606127 & -3.555379\end{array}$

$\mathrm{H} \quad 4.974668 \quad 1.401914 \quad 1.123380$

Allenyne_A2Cyclization_TS

Free energy $=-899.1510255$ Hartree

$\begin{array}{llll}\text { C } & -0.678798 & -0.982143 & 0.944292\end{array}$

$\begin{array}{llll}\text { C } & 0.331829 & -1.259355 & -0.018002\end{array}$

$\begin{array}{llll}\text { C } & -0.057055 & -1.938658 & -1.211809\end{array}$

$\begin{array}{llll}\text { C } & -1.381970 & -2.308836 & -1.426175\end{array}$

$\begin{array}{llll}\text { C } & -2.375435 & -2.026617 & -0.456680\end{array}$

$\begin{array}{llll}\text { C } & -2.010486 & -1.360342 & 0.734645\end{array}$

$\begin{array}{llll}\text { C } & 1.664083 & -0.836137 & 0.192114\end{array}$

$\begin{array}{llll}\text { C } & 2.799380 & -0.343985 & 0.424095\end{array}$

$\begin{array}{llll}\text { C } & 4.179973 & -0.678796 & 0.895004\end{array}$

$\begin{array}{llll}\text { O } & 5.136462 & 0.240134 & 0.348226\end{array}$

$\begin{array}{llll}\text { C } & 4.556297 & 1.460352 & 0.192405\end{array}$

$\begin{array}{llll}\text { C } & 3.237157 & 1.634229 & 0.413601\end{array}$

$\begin{array}{llll}\text { C } & 2.227516 & 2.477490 & 0.772922\end{array}$

$\begin{array}{llll}\text { C } & 0.896394 & 2.385033 & 0.196591\end{array}$

$\begin{array}{llll}\mathrm{N} & -0.131332 & 2.896314 & 0.932529\end{array}$

$\begin{array}{llll}\text { C } & -1.375426 & 2.687073 & 0.494366\end{array}$

$\begin{array}{llll}\text { C } & -1.689846 & 1.972668 & -0.683070\end{array}$

$\begin{array}{llll}\text { C } & -0.633771 & 1.504105 & -1.476669\end{array}$

$\begin{array}{llll}\text { C } & 0.678513 & 1.715857 & -1.039066\end{array}$

$\begin{array}{llll}\text { O } & -3.640574 & -2.425523 & -0.768203\end{array}$

$\begin{array}{llll}\text { C } & -4.682141 & -2.177641 & 0.164085\end{array}$

$\begin{array}{llll}\mathrm{H} & -2.738912 & 1.805690 & -0.970250\end{array}$

$\begin{array}{llll}\mathrm{H} & 5.229575 & 2.213201 & -0.245699\end{array}$

$\begin{array}{llll}\mathrm{H} & 4.202113 & -0.607829 & 2.008158\end{array}$

$\begin{array}{llll}\mathrm{H} & 2.318370 & 3.119066 & 1.667945\end{array}$

$\begin{array}{llll}\mathrm{H} & 1.540912 & 1.368694 & -1.625054\end{array}$

$\begin{array}{llll}\mathrm{H} & -0.405563 & -0.433835 & 1.857636\end{array}$

$\begin{array}{llll}\mathrm{H} & -0.831061 & 0.955065 & -2.409527\end{array}$

$\begin{array}{llll}\mathrm{H} & -2.758589 & -1.114028 & 1.500195\end{array}$

$\begin{array}{llll}\mathrm{H} & -2.188838 & 3.104713 & 1.116646\end{array}$

$\begin{array}{llll}\mathrm{H} & -1.688650 & -2.829196 & -2.345543\end{array}$

$\begin{array}{llll}\mathrm{H} & 0.705738 & -2.160096 & -1.972745\end{array}$

$\begin{array}{llll}\mathrm{H} & -5.606781 & -2.584586 & -0.28571\end{array}$

$\begin{array}{llll}\mathrm{H} & -4.822174 & -1.088709 & 0.35316\end{array}$

$\begin{array}{llll}\mathrm{H} & -4.500285 & -2.687838 & 1.13712\end{array}$

$\begin{array}{llll}\mathrm{H} & 4.493372 & -1.695538 & 0.588467\end{array}$

\begin{tabular}{lccc}
\multicolumn{5}{l}{ Allenyne_A2Cyclization_INT } \\
36 \\
Free & \multicolumn{4}{l}{} \\
C & $-0.576 y=-899.2343715$ & Hartree \\
C & -0.310823 & -1.537978 & 0.777717 \\
C & -1.109161 & -0.561947 & -1.366198 \\
C & -2.082323 & -1.539692 & -1.574554 \\
C & -2.316345 & -2.539435 & -0.602470 \\
C & -1.555524 & -2.525920 & 0.586268 \\
C & 0.745021 & 0.480526 & -0.001666 \\
C & 1.929473 & 0.232684 & 0.635252 \\
C & 2.465781 & -1.014287 & 1.306194 \\
O & 3.874901 & -0.746004 & 1.562281 \\
C & 4.108878 & 0.550508 & 1.232121 \\
C & 3.021365 & 1.203571 & 0.701451 \\
C & 2.841760 & 2.536896 & 0.207602 \\
C & 1.639657 & 2.889092 & -0.360395 \\
N & 1.427265 & 4.214401 & -0.707036 \\
C & 0.193052 & 4.634003 & -0.816337 \\
C & -0.968163 & 3.818727 & -0.465014 \\
C & -0.810964 & 2.477888 & -0.321085 \\
C & 0.540362 & 1.857032 & -0.649382 \\
O & -3.287688 & -3.449056 & -0.898386 \\
C & -3.561179 & -4.478988 & 0.038420 \\
H & -1.937842 & 4.310087 & -0.285874 \\
H & 5.133959 & 0.903103 & 1.407680 \\
H & 1.998066 & -1.217900 & 2.294915 \\
H & 3.625643 & 3.302901 & 0.303606 \\
H & 0.556973 & 1.713568 & -1.766851 \\
H & -0.040133 & -1.524656 & 1.736952 \\
H & -1.640535 & 1.822204 & -0.014118 \\
H & -1.726411 & -3.268468 & 1.377656 \\
H & 0.043714 & 5.692027 & -1.105350 \\
H & -2.683403 & -1.558571 & -2.495610 \\
H & -0.952755 & 0.195491 & -2.148591 \\
H & -4.363383 & -5.099169 & -0.404165 \\
H & -3.917850 & -4.073261 & 1.012817 \\
H & -2.669525 & -5.121316 & 0.223920 \\
H & 2.377050 & -1.934354 & 0.689529
\end{tabular}

Allenyne_B

Free energy $=-899.1659414$ Hartree

$\begin{array}{llll}\text { C } & 0.896403 & 0.746021 & -1.024143\end{array}$

$\begin{array}{llll}\text { C } & 1.348771 & 1.388572 & 0.14994\end{array}$

$\begin{array}{llll}\text { C } & 0.396046 & 2.094914 & 0.924572\end{array}$

$\begin{array}{llll}C & -0.948760 & 2.140003 & 0.554708\end{array}$

$\begin{array}{llll}\text { C } & -1.386702 & 1.475426 & -0.612334\end{array}$

$\begin{array}{llll}\text { C } & -0.447967 & 0.785504 & -1.411475\end{array}$

$\begin{array}{llll}\text { C } & 2.750724 & 1.300474 & 0.594646\end{array}$

$\begin{array}{llll}\text { C } & 3.669819 & 0.496053 & 0.066368\end{array}$

$\begin{array}{llll}\text { C } & 4.477623 & -0.361817 & -0.537431\end{array}$

$\begin{array}{llll}4.675974 & -1.685307 & -0.204413\end{array}$

$\begin{array}{lll}3.940385 & -2.152330 & 0.936791\end{array}$

$\begin{array}{llll}\text { C } & 2.483673 & -2.118351 & 0.787265\end{array}$

$\begin{array}{llll}\text { C } & 1.261411 & -2.035224 & 0.713955\end{array}$

$\begin{array}{llll}\text { C } & -0.164511 & -1.891089 & 0.627552\end{array}$

$\begin{array}{llll}\mathrm{N} & -0.762323 & -1.118406 & 1.568359\end{array}$

$\begin{array}{llll}\text { C } & -2.084819 & -0.933908 & 1.472939\end{array}$

$\begin{array}{llll}\mathrm{C} & -2.891361 & -1.500224 & 0.467849\end{array}$

$\begin{array}{llll}\text { C } & -2.272546 & -2.313357 & -0.495477\end{array}$

$\begin{array}{llll}\text { C } & -0.888559 & -2.514616 & -0.421232\end{array}$

$\begin{array}{llll}\text { O } & -2.724862 & 1.542520 & -0.874829\end{array}$

$\begin{array}{llll}\text { C } & -3.204919 & 0.981190 & -2.086979\end{array}$

$\begin{array}{llll}\mathrm{H} & -3.972327 & -1.297118 & 0.442140\end{array}$

$\begin{array}{llll}\mathrm{H} & 4.294353 & -3.191742 & 1.096313\end{array}$

$\begin{array}{llll}\mathrm{H} & 5.115122 & -0.086724 & -1.396101\end{array}$

$\begin{array}{llll}\mathrm{H} & 3.019954 & 1.934231 & 1.463108\end{array}$

$\begin{array}{llll}\mathrm{H} & -0.356182 & -3.133622 & -1.158277\end{array}$

$\begin{array}{llll}\mathrm{H} & 1.622069 & 0.187848 & -1.635395\end{array}$

$\begin{array}{llll}\mathrm{H} & -2.860447 & -2.786047 & -1.298169\end{array}$

$\begin{array}{llll}\mathrm{H} & -0.759797 & 0.258792 & -2.322782\end{array}$

$\begin{array}{llll}\mathrm{H} & -2.538877 & -0.282448 & 2.241271\end{array}$

$\begin{array}{llll}\mathrm{H} & -1.695331 & 2.669971 & 1.164040\end{array}$

$\begin{array}{llll}\mathrm{H} & 0.719758 & 2.600516 & 1.848217\end{array}$

$\mathrm{H} \quad-4.294154 \quad 1.174618 \quad-2.110320$

$\begin{array}{llll}\mathrm{H} & -3.031109 & -0.117812 & -2.132419 \\ \mathrm{H} & -2.733593 & 1.455667 & -2.977226\end{array}$

$\begin{array}{llll}\mathrm{H} & 4.219077 & -1.548861 & 1.835037\end{array}$

Allenyne_B2Cyclization_TS

Free energy $=-899.1520996$ Hartree

$\begin{array}{llll}\text { C } & 0.826412 & 0.592256 & -1.212041\end{array}$

$\begin{array}{llll}\text { C } & 1.380794 & 1.455099 & -0.232226\end{array}$

$\begin{array}{llll}\text { C } & 0.501842 & 2.364168 & 0.422119\end{array}$

$\begin{array}{llll}\text { C } & -0.861949 & 2.362703 & 0.156345\end{array}$

$\begin{array}{llll}\text { C } & -1.401481 & 1.465852 & -0.801490\end{array}$

$\begin{array}{llll}\text { C } & -0.540646 & 0.604314 & -1.513123\end{array}$

$\begin{array}{llll}\text { C } & 2.769202 & 1.345079 & 0.166004\end{array}$ 


$\begin{array}{rrrr}\text { C } & 3.499187 & 0.207740 & 0.000663 \\ \text { C } & 4.655399 & -0.422595 & -0.270219 \\ \text { O } & 4.921661 & -1.654670 & 0.246712 \\ \text { C } & 3.877771 & -2.038518 & 1.154173 \\ \text { C } & 2.558903 & -1.495194 & 0.712589 \\ \text { C } & 1.311973 & -1.634277 & 0.717745 \\ \text { C } & -0.108711 & -1.657645 & 0.706961 \\ \text { N } & -0.760508 & -0.852298 & 1.594386 \\ \text { C } & -2.098272 & -0.853953 & 1.572635 \\ \text { C } & -2.880340 & -1.635855 & 0.700658 \\ \text { C } & -2.209654 & -2.469488 & -0.213406 \\ \text { C } & -0.811936 & -2.485647 & -0.217014 \\ \text { O } & -2.751248 & 1.511754 & -0.958913 \\ \text { C } & -3.344480 & 0.717401 & -1.977598 \\ \text { H } & -3.979325 & -1.586121 & 0.738024 \\ \text { H } & 3.880726 & -3.145290 & 1.200861 \\ \text { H } & 5.412661 & -0.090024 & -0.998296 \\ \text { H } & 3.178123 & 2.153658 & 0.801857 \\ \text { H } & -0.246497 & -3.119496 & -0.916002 \\ \text { H } & 1.495831 & -0.096881 & -1.748149 \\ \text { H } & -2.773530 & -3.104234 & -0.915659 \\ \text { H } & -0.931407 & -0.092395 & -2.266426 \\ \text { H } & -2.592754 & -0.180056 & 2.296448 \\ \text { H } & -1.554481 & 3.032267 & 0.686646 \\ \text { H } & 0.907056 & 3.049948 & 1.183789 \\ \text { H } & -4.431287 & 0.920168 & -1.935500 \\ \text { H } & -3.169869 & -0.367832 & -1.807015 \\ \text { H } & -2.961216 & 0.993188 & -2.986241 \\ \text { H } & 4.104070 & -1.629807 & 2.166767\end{array}$

Allenyne_B2Cyclization_INT 36

Free energy $=-899.2272953$ Hartree

$\begin{array}{llll}\text { C } & 0.457408 & 0.550811 & -0.797135\end{array}$

$\begin{array}{llll}\text { C } & 1.572067 & 1.602796 & -0.742880\end{array}$

$\begin{array}{llll}\text { C } & 1.223876 & 2.918030 & -1.247561\end{array}$

$\begin{array}{llll}\text { C } & -0.080409 & 3.319520 & -1.335364\end{array}$

$\begin{array}{llll}\text { C } & -1.155012 & 2.451876 & -0.860545\end{array}$

$\begin{array}{llll}\text { C } & -0.915872 & 1.150714 & -0.529268\end{array}$

$\begin{array}{llll}\text { C } & 2.827155 & 1.327144 & -0.256137\end{array}$

$\begin{array}{llll}\text { C } & 3.087137 & 0.065634 & 0.379500\end{array}$

$\begin{array}{llll}\text { C } & 4.188757 & -0.458543 & 1.009864\end{array}$

$\begin{array}{llll}\text { O } & 3.994999 & -1.695596 & 1.544176\end{array}$

$\begin{array}{llll}\text { C } & 2.617701 & -2.080671 & 1.269516\end{array}$

$\begin{array}{llll}\text { C } & 2.021175 & -0.929368 & 0.479360\end{array}$

$\begin{array}{llll}\text { C } & 0.767729 & -0.740450 & -0.021293 \\ \text { C } & -0.238547 & -1.839025 & 0.037979\end{array}$

$\begin{array}{llll}\text { C } & -0.238547 & -1.839025 & 0.037979\end{array}$

$\begin{array}{llll}\mathrm{N} & -1.455087 & -1.594209 & 0.584028\end{array}$

$\begin{array}{llll}\text { C } & -2.343392 & -2.597188 & 0.654462\end{array}$

$\begin{array}{llll}\text { C } & -2.093244 & -3.901661 & 0.194066\end{array}$

$\begin{array}{llll}\text { C } & -0.846498 & -4.158816 & -0.398991 \\ \text { C } & 0.086563 & -3.117529 & -0.484348\end{array}$

$\begin{array}{llll}\text { C } & 0.086563 & -3.117529 & -0.484348\end{array}$

$\begin{array}{llll}\text { O } & -2.359156 & 3.098576 & -0.763909\end{array}$

$\begin{array}{llll}\text { C } & -3.458282 & 2.344253 & -0.284212\end{array}$

$\begin{array}{llll}\mathrm{H} & -2.860219 & -4.685426 & 0.287187\end{array}$

$\begin{array}{llll}\mathrm{H} & 2.645916 & -3.047554 & 0.727160\end{array}$

$\begin{array}{llll}\mathrm{H} & 5.194687 & -0.040907 & 1.150300\end{array}$

$\begin{array}{llll}\mathrm{H} & 3.616196 & 2.095841 & -0.299940\end{array}$

$\begin{array}{llll}\mathrm{H} & 1.060370 & -3.273597 & -0.971812\end{array}$

$\begin{array}{llll}\mathrm{H} & 0.429401 & 0.236016 & -1.880489\end{array}$

$\begin{array}{llll}\mathrm{H} & -0.608729 & -5.155325 & -0.803010\end{array}$

$\begin{array}{llll}\mathrm{H} & -1.684287 & 0.476656 & -0.131407\end{array}$

$\begin{array}{llll}\mathrm{H} & -3.320006 & -2.348236 & 1.108273\end{array}$

$\begin{array}{llll}\mathrm{H} & -0.354312 & 4.332577 & -1.665648\end{array}$

$\begin{array}{llll}\mathrm{H} & 2.037355 & 3.625481 & -1.477620\end{array}$

$\begin{array}{llll}\mathrm{H} & -4.333973 & 3.021006 & -0.286813\end{array}$

$\begin{array}{llll}\mathrm{H} & -3.283686 & 1.972986 & 0.751815\end{array}$

$\begin{array}{llll}\mathrm{H} & -3.670852 & 1.464203 & -0.935389\end{array}$

$\begin{array}{llll}\mathrm{H} & 2.100952 & -2.248603 & 2.239184\end{array}$

\section{Bisallene}

36

Free energy $=-899.1800083$ Hartree

$\begin{array}{llll}\mathrm{C} & 2.007140 & 4.752815 & -2.328622 \\ \mathrm{C} & 2.950120 & 5.201430 & -1.368127 \\ \mathrm{C} & 2.495823 & 6.072464 & -0.350690 \\ \mathrm{C} & 1.171325 & 6.523684 & -0.314065 \\ \mathrm{C} & 0.260024 & 6.103261 & -1.308680 \\ \mathrm{C} & 0.684168 & 5.187889 & -2.299373 \\ \mathrm{C} & 4.366050 & 4.856638 & -1.524159 \\ \mathrm{C} & 5.380317 & 5.329803 & -0.800375 \\ \mathrm{C} & 6.400490 & 5.723710 & -0.058365 \\ \mathrm{O} & 7.142815 & 6.884865 & -0.217714 \\ \mathrm{C} & 6.604059 & 7.915545 & -0.960844 \\ \mathrm{C} & 5.345557 & 8.060720 & -1.345212 \\ \mathrm{C} & 4.103940 & 8.331967 & -1.740719 \\ \mathrm{C} & 3.474804 & 7.876448 & -2.993084 \\ \mathrm{~N} & 2.168456 & 8.202511 & -3.147157 \\ \mathrm{C} & 1.535757 & 7.755094 & -4.240926\end{array}$

$\begin{array}{cccc}\mathrm{C} & 2.150842 & 6.979921 & -5.240781 \\ \mathrm{C} & 3.510094 & 6.655802 & -5.086626 \\ \mathrm{C} & 4.184783 & 7.105483 & -3.947474 \\ \mathrm{H} & 1.575681 & 6.639658 & -6.115027 \\ \mathrm{O} & -1.029963 & 6.527383 & -1.407436 \\ \mathrm{H} & 6.804909 & 5.130941 & 0.779618 \\ \mathrm{H} & 7.377436 & 8.670156 & -1.183016 \\ \mathrm{H} & 4.607209 & 4.211236 & -2.392280 \\ \mathrm{H} & 3.417402 & 8.889367 & -1.076397 \\ \mathrm{H} & 3.214781 & 6.424317 & 0.404181 \\ \mathrm{H} & 5.242773 & 6.865245 & -3.766350 \\ \mathrm{H} & 0.865471 & 7.224642 & 0.474065 \\ \mathrm{H} & 4.034022 & 6.053357 & -5.845610 \\ \mathrm{H} & -0.043475 & 4.869386 & -3.059404 \\ \mathrm{H} & 0.466548 & 8.020482 & -4.325517 \\ \mathrm{H} & 2.337561 & 4.078695 & -3.134987 \\ \mathrm{C} & -1.481101 & 7.538531 & -0.518263 \\ \mathrm{H} & -2.522334 & 7.769364 & -0.811213 \\ \mathrm{H} & -1.474206 & 7.195652 & 0.541495 \\ \mathrm{H} & -0.864959 & 8.462052 & -0.602689\end{array}$

Bisallene2Cyclization_TS1

Free energy $=-899.1754169$ Hartree

$\begin{array}{llll}\text { C } & 2.009455 & 4.714019 & -2.199752\end{array}$

$\begin{array}{llll}\text { C } & 2.969636 & 5.238673 & -1.286723\end{array}$

$\begin{array}{llll}\text { C } & 2.518902 & 6.170901 & -0.316108\end{array}$

$\begin{array}{llll}\text { C } & 1.190496 & 6.612902 & -0.290485\end{array}$

$\begin{array}{llll}\text { C } & 0.274851 & 6.129049 & -1.248569\end{array}$

$\begin{array}{llll}\text { C } & 0.689532 & 5.143435 & -2.180378\end{array}$

$\begin{array}{llll}\text { C } & 4.377560 & 4.952160 & -1.470194\end{array}$

$\begin{array}{llll}\text { C } & 5.389147 & 5.621961 & -0.864826\end{array}$

$\begin{array}{llll}\text { C } & 6.534578 & 5.789484 & -0.206181\end{array}$

$\begin{array}{llll}\text { O } & 7.269833 & 6.951200 & -0.303787\end{array}$

$\begin{array}{llll}\text { C } & 6.588564 & 7.935257 & -0.986331\end{array}$

$\begin{array}{llll}\text { C } & 5.314514 & 7.808878 & -1.355992\end{array}$

$\begin{array}{llll}\text { C } & 4.091416 & 8.221306 & -1.770619\end{array}$

$\begin{array}{llll}\text { C } & 3.449277 & 7.802055 & -3.010434\end{array}$

$\begin{array}{llll}\mathrm{N} & 2.146069 & 8.163846 & -3.169182\end{array}$

$\begin{array}{llll}\text { C } & 1.497869 & 7.703915 & -4.245078\end{array}$

$\begin{array}{llll}\text { C } & 2.081040 & 6.876100 & -5.225250\end{array}$

$\begin{array}{llll}\text { C } & 3.433630 & 6.524546 & -5.076300\end{array}$

$\begin{array}{llll}\text { C } & 4.131399 & 6.989024 & -3.957388\end{array}$

\begin{tabular}{llll}
$\mathrm{H}$ & 1.487316 & 6.524697 & -6.082485 \\
\hline
\end{tabular}

$\begin{array}{llll}\mathrm{O} & -1.013784 & 6.545587 & -1.369242\end{array}$

$\begin{array}{llll}\mathrm{H} & 6.981997 & 5.071900 & 0.498767\end{array}$

$\begin{array}{llll}\mathrm{H} & 7.218207 & 8.814738 & -1.188703\end{array}$

$\begin{array}{llll}\mathrm{H} & 4.633988 & 4.287383 & -2.318110\end{array}$

$\begin{array}{llll}\mathrm{H} & 3.434908 & 8.782660 & -1.082235\end{array}$

$\begin{array}{llll}\mathrm{H} & 3.240571 & 6.561763 & 0.415584\end{array}$

$\begin{array}{llll}\mathrm{H} & 5.187212 & 6.734177 & -3.785660\end{array}$

$\begin{array}{llll}\mathrm{H} & 0.888521 & 7.360826 & 0.454770\end{array}$

$\begin{array}{llll}\mathrm{H} & 3.934816 & 5.889028 & -5.823787\end{array}$

$\begin{array}{llll}\mathrm{H} & -0.047539 & 4.775375 & -2.908268\end{array}$

$\begin{array}{llll}\mathrm{H} & 0.436730 & 7.999831 & -4.335612\end{array}$

$\begin{array}{llll}\mathrm{H} & 2.335259 & 3.992567 & -2.965991\end{array}$

$\begin{array}{llll}\text { C } & -1.451759 & 7.633540 & -0.566778\end{array}$

$\begin{array}{llll}\mathrm{H} & -2.490791 & 7.850616 & -0.876960\end{array}$

$\begin{array}{llll}\mathrm{H} & -1.446000 & 7.379848 & 0.517685\end{array}$

$\begin{array}{llll}\mathrm{H} & -0.823102 & 8.537261 & -0.731223\end{array}$

Bisallene2Cyclization_INT1

36

Free energy $=-899.2196237$ Hartree

$\begin{array}{lrrr}\text { C } & 3.689052 & 6.653241 & -3.648846 \\ \mathrm{C} & 3.845245 & 7.964915 & -3.112272 \\ \mathrm{C} & 2.929930 & 8.960733 & -3.576818 \\ \mathrm{C} & 1.875640 & 8.641566 & -4.426931 \\ \mathrm{C} & 1.703629 & 7.310953 & -4.885553 \\ \mathrm{C} & 2.653348 & 6.326388 & -4.524107 \\ \mathrm{C} & 4.842665 & 8.304535 & -2.137006 \\ \mathrm{C} & 5.545700 & 7.474971 & -1.217814 \\ \mathrm{C} & 5.345945 & 6.087417 & -0.696074 \\ \mathrm{C} & 6.504210 & 5.831836 & 0.061834 \\ \mathrm{O} & 7.315964 & 6.914708 & 0.108550 \\ \mathrm{C} & 6.752583 & 7.900247 & -0.627204 \\ \mathrm{C} & 4.299759 & 5.127353 & -0.793565 \\ \mathrm{C} & 2.924396 & 5.291478 & -1.177027 \\ \mathrm{~N} & 2.290930 & 4.162083 & -1.626003 \\ \mathrm{C} & 1.047534 & 4.281319 & -2.093458 \\ \mathrm{C} & 0.320254 & 5.492942 & -2.127856 \\ \mathrm{C} & 0.925230 & 6.636289 & -1.575436 \\ \mathrm{C} & 2.233345 & 6.540431 & -1.100549 \\ \mathrm{O} & 0.613891 & 7.084216 & -5.666457 \\ \mathrm{H} & -0.699000 & 5.523876 & -2.540185 \\ \mathrm{H} & 6.817623 & 4.956849 & 0.640256 \\ \mathrm{H} & 7.315347 & 8.836045 & -0.709216 \\ \mathrm{H} & 4.568945 & 4.088805 & -0.537665 \\ \mathrm{H} & 5.127887 & 9.371414 & -2.101209 \\ \mathrm{H} & 2.739299 & 7.413609 & -0.667429\end{array}$




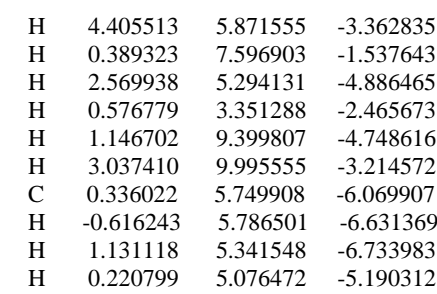

Bisallene2Cyclization_TS2_for_A 36

Free energy $=-899.2069515$ Hartree

$\begin{array}{cccc}\mathrm{C} & 2.908154 & 7.164697 & -1.050423 \\ \mathrm{C} & 3.098522 & 5.724774 & -1.125460 \\ \mathrm{~N} & 2.162525 & 4.913563 & -1.738470 \\ \mathrm{C} & 1.161214 & 5.493030 & -2.379046 \\ \mathrm{C} & 0.971252 & 6.908466 & -2.477900 \\ \mathrm{C} & 1.829854 & 7.738823 & -1.761981 \\ \mathrm{C} & 4.281343 & 5.122531 & -0.652184 \\ \mathrm{C} & 5.465728 & 5.902164 & -0.380033 \\ \mathrm{C} & 5.814541 & 7.172103 & -1.043588 \\ \mathrm{C} & 7.054106 & 7.511665 & -0.548923 \\ \mathrm{O} & 7.499593 & 6.597452 & 0.372227 \\ \mathrm{C} & 6.530057 & 5.633495 & 0.463323 \\ \mathrm{C} & 5.019360 & 7.828734 & -2.080672 \\ \mathrm{C} & 4.876472 & 9.246014 & -2.251476 \\ \mathrm{C} & 5.245803 & 10.195396 & -1.253907 \\ \mathrm{C} & 5.041479 & 11.568862 & -1.429532 \\ \mathrm{C} & 4.448472 & 12.049253 & -2.620832 \\ \mathrm{C} & 4.055876 & 11.122532 & -3.620867 \\ \mathrm{C} & 4.260632 & 9.760551 & -3.437420 \\ \mathrm{O} & 4.209160 & 13.360400 & -2.898995 \\ \mathrm{C} & 4.579690 & 14.341115 & -1.941046 \\ \mathrm{H} & 0.128659 & 7.313817 & -3.058736 \\ \mathrm{H} & 6.710529 & 4.836261 & 1.192803 \\ \mathrm{H} & 7.745030 & 8.325638 & -0.793117 \\ \mathrm{H} & 4.318772 & 4.022643 & -0.621399 \\ \mathrm{H} & 4.711194 & 7.190569 & -2.925301 \\ \mathrm{H} & 3.410653 & 7.742785 & -0.264018 \\ \mathrm{H} & 5.686610 & 9.837672 & -0.311226 \\ \mathrm{H} & 1.679471 & 8.830247 & -1.738351 \\ \mathrm{H} & 5.342321 & 12.259138 & -0.629684 \\ \mathrm{H} & 0.429581 & 4.816583 & -2.861063 \\ \mathrm{H} & 3.589806 & 11.515542 & -4.536524 \\ \mathrm{H} & 3.941838 & 9.053457 & -4.219162 \\ \mathrm{H} & 4.301224 & 15.320100 & -2.373832 \\ \mathrm{H} & 4.037853 & 14.207187 & -0.977525 \\ \mathrm{H} & 5.675282 & 14.332726 & -1.740300 \\ & & & \\ & & & \end{array}$

Bisallene2Cyclization_INT2_for_A 36

Free energy $=-899.2477211$ Hartree

$\begin{array}{llll}\text { C } & 3.198915 & 7.251612 & -1.408208\end{array}$

$\begin{array}{llll}\text { C } & 3.186547 & 5.749096 & -1.058870\end{array}$

$\begin{array}{llll}\mathrm{N} & 2.037453 & 4.977543 & -1.179332\end{array}$

C $\quad 1.002642 \quad 5.479576 \quad-1.796869$

$\begin{array}{llll}\text { C } & 0.974392 & 6.797509 & -2.430925\end{array}$

$\begin{array}{llll}\text { C } & 2.028887 & 7.636353 & -2.276297\end{array}$

$\begin{array}{llll}\text { C } & 4.318256 & 5.153698 & -0.550538\end{array}$

$\begin{array}{llll}\text { C } & 5.543739 & 5.909791 & -0.468521\end{array}$

$\begin{array}{llll}\text { C } & 5.679032 & 7.188599 & -1.14046\end{array}$

$\begin{array}{llll}\text { C } & 6.952448 & 7.625564 & -0.879750\end{array}$

$\begin{array}{llll}\text { O } & 7.616340 & 6.718540 & -0.087745\end{array}$

$\begin{array}{llll}\text { C } & 6.761647 & 5.681477 & 0.151448\end{array}$

$\begin{array}{llll}\text { C } & 4.570277 & 7.731330 & -2.004711\end{array}$

$\begin{array}{llll}\text { C } & 4.618563 & 9.236493 & -2.205365\end{array}$

$\begin{array}{llll}\text { C } & 4.704716 & 10.113954 & -1.107069\end{array}$

$\begin{array}{llll}\text { C } & 4.700909 & 11.509024 & -1.273278\end{array}$

$\begin{array}{llll}\text { C } & 4.607415 & 12.059088 & -2.570951\end{array}$

$\begin{array}{llll}\text { C } & 4.520801 & 11.191270 & -3.682155\end{array}$

$\begin{array}{llll}\text { C } & 4.526476 & 9.805069 & -3.494834\end{array}$

$\begin{array}{llll}\text { O } & 4.596148 & 13.393881 & -2.851721\end{array}$

$\begin{array}{llll}\text { C } & 4.674088 & 14.315138 & -1.776549\end{array}$

$\begin{array}{llll}\mathrm{H} & 0.097700 & 7.081936 & -3.033998\end{array}$

$\begin{array}{llll}\mathrm{H} & 7.144971 & 4.872662 & 0.782389\end{array}$

$\begin{array}{llll}\mathrm{H} & 7.512828 & 8.521844 & -1.163608\end{array}$

$\mathrm{H} \quad 4.258876 \quad 4.106920 \quad-0.219190$

$\begin{array}{llll}\mathrm{H} & 4.660388 & 7.246034 & -3.005175\end{array}$

$\begin{array}{llll}\mathrm{H} & 3.088518 & 7.810227 & -0.441160\end{array}$

$\begin{array}{llll}\mathrm{H} & 4.788814 & 9.697441 & -0.090586\end{array}$

$\begin{array}{llll}\mathrm{H} & 2.045788 & 8.633403 & -2.744701\end{array}$

$\begin{array}{llll}\mathrm{H} & 4.774432 & 12.154129 & -0.387151\end{array}$

$\begin{array}{llll}\mathrm{H} & 0.104707 & 4.835863 & -1.872863\end{array}$

$\mathrm{H} \quad 4.451276 \quad 11.635621 \quad-4.685968$

$\begin{array}{llll}\mathrm{H} & 4.456767 & 9.143323 & -4.373489\end{array}$

$\begin{array}{llll}\mathrm{H} & 4.644904 & 15.324863 & -2.227238\end{array}$

$\begin{array}{llll}\mathrm{H} & 3.815863 & 14.210178 & -1.073170\end{array}$

$\begin{array}{llll}\mathrm{H} & 5.622233 & 14.206791 & -1.200779\end{array}$

\begin{tabular}{lccc}
\multicolumn{5}{l}{ Bisallene2Cyclization_TS2_for_B } \\
36 & \multicolumn{4}{l}{} \\
Free & energy $=-899.2099522$ & Hartree \\
C & 2.644930 & 5.996296 & -0.549694 \\
C & 3.511965 & 4.869502 & -0.720719 \\
N & 3.007496 & 3.591882 & -0.772760 \\
C & 1.685236 & 3.430963 & -0.687410 \\
C & 0.758005 & 4.483608 & -0.532705 \\
C & 1.266952 & 5.795210 & -0.455742 \\
C & 4.938896 & 4.979302 & -0.762728 \\
C & 5.719116 & 6.211594 & -0.783508 \\
C & 6.952422 & 6.446337 & -0.003395 \\
C & 7.377137 & 7.711335 & -0.371348 \\
O & 6.544152 & 8.270132 & -1.300159 \\
C & 5.555241 & 7.348251 & -1.544738 \\
C & 7.566825 & 5.489520 & 0.888848 \\
C & 6.805611 & 4.513839 & 1.569598 \\
C & 5.374630 & 4.648289 & 1.719281 \\
C & 4.583278 & 3.585404 & 2.185441 \\
C & 5.185234 & 2.359167 & 2.510745 \\
C & 6.611827 & 2.238261 & 2.482182 \\
C & 7.396053 & 3.286597 & 2.038474 \\
O & 4.512721 & 1.240724 & 2.882524 \\
C & 3.092935 & 1.250505 & 2.783646 \\
H & -0.320976 & 4.276918 & -0.469412 \\
H & 4.833157 & 7.613322 & -2.324300 \\
H & 8.206063 & 8.339869 & -0.027731 \\
H & 5.478267 & 4.026972 & -0.878932 \\
H & 8.667488 & 5.437124 & 0.931588 \\
H & 3.073583 & 7.006840 & -0.476773 \\
H & 4.923355 & 5.645286 & 1.635136 \\
H & 0.589826 & 6.653371 & -0.319743 \\
H & 3.498128 & 3.731491 & 2.266995 \\
H & 1.320643 & 2.387097 & -0.742447 \\
H & 7.051653 & 1.278054 & 2.789417 \\
H & 8.489798 & 3.164720 & 1.977422 \\
H & 2.757340 & 0.221560 & 3.010658 \\
H & 2.632370 & 1.949682 & 3.517635 \\
H & 2.763419 & 1.536307 & 1.759730 \\
& & & \\
\hline
\end{tabular}

Bisallene2Cyclization_INT2_for_B

36

Free energy $=-899.2476232$ Hartree

$\begin{array}{llll}\text { C } & 2.702514 & 6.083990 & -0.007887\end{array}$

$\begin{array}{llll}\text { C } & 3.392772 & 4.930982 & -0.446135\end{array}$

$\begin{array}{llll}\mathrm{N} & 2.752680 & 3.874909 & -0.990906\end{array}$

$\begin{array}{llll}\text { C } & 1.416297 & 3.940144 & -1.124705\end{array}$

$\begin{array}{llll}\text { C } & 0.644223 & 5.043685 & -0.724228\end{array}$

$\begin{array}{llll}\text { C } & 1.309465 & 6.139390 & -0.148582\end{array}$

$\begin{array}{llll}\text { C } & 4.897400 & 4.805168 & -0.269814\end{array}$

$\begin{array}{llll}\text { C } & 5.663560 & 6.021679 & -0.719060\end{array}$

$\begin{array}{lllll}\text { C } & 6.969590 & 6.330540 & -0.169679\end{array}$

$\begin{array}{llll}\text { C } & 7.412831 & 7.448630 & -0.856907\end{array}$

$\begin{array}{llll}\text { O } & 6.479260 & 7.841719 & -1.773587\end{array}$

$\begin{array}{llll}\text { C } & 5.425103 & 6.964956 & -1.687231\end{array}$

$\begin{array}{llll}\text { C } & 7.548730 & 5.512254 & 0.867755\end{array}$

$\begin{array}{llll}\text { C } & 6.775843 & 4.567041 & 1.502982\end{array}$

$\begin{array}{llll}\text { C } & 5.268376 & 4.480952 & 1.222530\end{array}$

$\begin{array}{llll}\text { C } & 4.625064 & 3.194276 & 1.686154\end{array}$

$\begin{array}{llll}\text { C } & 5.255868 & 2.363680 & 2.565230\end{array}$

$\begin{array}{llll}\text { C } & 6.611899 & 2.643998 & 3.029678\end{array}$

$\begin{array}{llll}\text { C } & 7.329962 & 3.681164 & 2.505095\end{array}$

$\begin{array}{llll}\text { O } & 4.747647 & 1.204874 & 3.084241\end{array}$

$\begin{array}{llll}\text { C } & 3.445276 & 0.822203 & 2.673966\end{array}$

$\begin{array}{llll}\mathrm{H} & -0.447776 & 5.042806 & -0.862934\end{array}$

$\begin{array}{llll}\mathrm{H} & 4.589376 & 7.148815 & -2.370222\end{array}$

$\begin{array}{llll}\mathrm{H} & 8.320404 & 8.058514 & -0.798332\end{array}$

$\begin{array}{llll}\mathrm{H} & 5.210180 & 3.930916 & -0.88328\end{array}$

$\begin{array}{llll}\mathrm{H} & 8.606851 & 5.641266 & 1.146020\end{array}$

$\begin{array}{llll}\mathrm{H} & 3.264226 & 6.924615 & 0.426515\end{array}$

$\begin{array}{llll}\mathrm{H} & 4.817795 & 5.317768 & 1.822011\end{array}$

$\begin{array}{llll}\mathrm{H} & 0.749495 & 7.027167 & 0.185250\end{array}$

$\begin{array}{llll}\mathrm{H} & 3.617732 & 2.977160 & 1.310110\end{array}$

$\begin{array}{llll}\mathrm{H} & 0.930378 & 3.057614 & -1.579733\end{array}$

$\begin{array}{llll}\mathrm{H} & 7.043840 & 1.955243 & 3.771352\end{array}$

$\begin{array}{llll}\mathrm{H} & 8.377174 & 3.837165 & 2.810760\end{array}$

H $\quad 3.216767 \quad-0.127990 \quad 3.191513$

$\begin{array}{llll}\mathrm{H} & 2.683620 & 1.585784 & 2.955927\end{array}$

$\begin{array}{llll}\mathrm{H} & 3.390431 & 0.663099 & 1.572456\end{array}$

\section{$A=1$-benzyl-3-indolyl,$B=$ phenyl}

Bispropargyl

50

Free energy $=-1170.246349$ Hartree

$\begin{array}{llll}\text { C } & 10.453043 & 3.911078 & -2.903257\end{array}$

$\begin{array}{llll}\text { C } & 10.065018 & 5.263570 & -2.841667\end{array}$ 


\begin{tabular}{|c|c|c|c|}
\hline $\mathrm{C}$ & 10.957044 & 6.253676 & -3.300969 \\
\hline $\mathrm{C}$ & 12.211076 & 5.898916 & -3.824401 \\
\hline $\mathrm{C}$ & 12.590618 & 4.546792 & -3.886017 \\
\hline $\mathrm{C}$ & 11.708950 & 3.555536 & -3.422116 \\
\hline $\mathrm{C}$ & 8.697659 & 5.668254 & -2.310247 \\
\hline $\mathrm{N}$ & 8.121954 & 4.714378 & -1.380152 \\
\hline $\mathrm{C}$ & 8.614429 & 4.456090 & -0.104121 \\
\hline $\mathrm{C}$ & 7.776010 & 3.468021 & 0.496069 \\
\hline $\mathrm{C}$ & 6.759883 & 3.123113 & -0.484412 \\
\hline $\mathrm{C}$ & 7.022603 & 3.914361 & -1.607517 \\
\hline $\mathrm{C}$ & 9.704732 & 5.029605 & 0.573407 \\
\hline $\mathrm{C}$ & 9.924978 & 4.619064 & 1.894838 \\
\hline $\mathrm{C}$ & 9.087637 & 3.656669 & 2.517484 \\
\hline $\mathrm{C}$ & 8.017347 & 3.072987 & 1.827742 \\
\hline $\mathrm{C}$ & 5.646574 & 2.276717 & -0.290185 \\
\hline $\mathrm{C}$ & 4.634001 & 1.616582 & -0.059142 \\
\hline $\mathrm{C}$ & 3.302752 & 1.140360 & 0.322309 \\
\hline $\mathrm{O}$ & 2.314181 & 2.173477 & 0.433434 \\
\hline $\mathrm{C}$ & 2.583824 & 3.162627 & 1.427507 \\
\hline $\mathrm{C}$ & 3.497905 & 4.240284 & 1.018901 \\
\hline C & 4.266428 & 5.139744 & 0.688468 \\
\hline $\mathrm{C}$ & 5.190940 & 6.156139 & 0.294577 \\
\hline C & 4.982166 & 6.905342 & -0.895093 \\
\hline $\mathrm{C}$ & 5.888614 & 7.903393 & -1.275784 \\
\hline $\mathrm{C}$ & 7.014768 & 8.183190 & -0.477570 \\
\hline $\mathrm{C}$ & 7.236374 & 7.441014 & 0.695800 \\
\hline $\mathrm{C}$ & 6.340483 & 6.433715 & 1.079473 \\
\hline $\mathrm{H}$ & 7.717711 & 8.978862 & -0.770350 \\
\hline $\mathrm{H}$ & 2.893592 & 0.437598 & -0.434931 \\
\hline $\mathrm{H}$ & 1.593533 & 3.602363 & 1.678355 \\
\hline $\mathrm{H}$ & 4.096927 & 6.689948 & -1.511627 \\
\hline $\mathrm{H}$ & 5.711962 & 8.476636 & -2.199359 \\
\hline $\mathrm{H}$ & 8.125530 & 7.632575 & 1.315283 \\
\hline $\mathrm{H}$ & 6.531203 & 5.836020 & 1.982100 \\
\hline $\mathrm{H}$ & 7.978031 & 5.791396 & -3.146378 \\
\hline $\mathrm{H}$ & 8.753657 & 6.659038 & -1.810503 \\
\hline $\mathrm{H}$ & 10.666518 & 7.316075 & -3.245374 \\
\hline $\mathrm{H}$ & 12.898700 & 6.682572 & -4.178113 \\
\hline $\mathrm{H}$ & 13.575874 & 4.266193 & -4.288537 \\
\hline $\mathrm{H}$ & 12.002505 & 2.494863 & -3.459542 \\
\hline $\mathrm{H}$ & 9.768868 & 3.133526 & -2.529858 \\
\hline $\mathrm{H}$ & 6.465702 & 3.980279 & -2.548666 \\
\hline $\mathrm{H}$ & 2.972405 & 2.690682 & 2.364946 \\
\hline $\mathrm{H}$ & 7.365979 & 2.324860 & 2.304744 \\
\hline $\mathrm{H}$ & 9.291802 & 3.360211 & 3.557552 \\
\hline $\mathrm{H}$ & 10.767126 & 5.048172 & 2.459012 \\
\hline $\mathrm{H}$ & 10.353668 & 5.770699 & 0.084687 \\
\hline $\mathrm{H}$ & 3.377953 & 0.571969 & 1.285092 \\
\hline
\end{tabular}

Allenyne_A

Free energy $=-1170.262295$ Hartree

\begin{tabular}{cccc}
$\mathrm{C}$ & 5.120552 & 7.194518 & -1.053969 \\
$\mathrm{C}$ & 5.255678 & 6.264386 & -0.014177 \\
$\mathrm{C}$ & 6.343611 & 6.429402 & 0.861774 \\
$\mathrm{C}$ & 7.298232 & 7.488242 & 0.715840 \\
$\mathrm{C}$ & 7.135192 & 8.409080 & -0.343813 \\
$\mathrm{C}$ & 6.049788 & 8.254896 & -1.216000 \\
$\mathrm{~N}$ & 6.697573 & 5.693671 & 1.987596 \\
$\mathrm{C}$ & 7.825272 & 6.252136 & 2.550713 \\
$\mathrm{C}$ & 8.242031 & 7.359970 & 1.810217 \\
$\mathrm{C}$ & 5.933623 & 4.579905 & 2.524361 \\
$\mathrm{C}$ & 5.883445 & 3.372497 & 1.602271 \\
$\mathrm{C}$ & 4.727725 & 2.568379 & 1.555561 \\
$\mathrm{C}$ & 4.681711 & 1.430489 & 0.732546 \\
$\mathrm{C}$ & 5.790909 & 1.090156 & -0.060440 \\
$\mathrm{C}$ & 6.943455 & 1.895374 & -0.027084 \\
$\mathrm{C}$ & 6.989122 & 3.029545 & 0.798861 \\
$\mathrm{C}$ & 9.350392 & 8.250796 & 2.131407 \\
$\mathrm{C}$ & 10.083128 & 8.180507 & 3.243065 \\
$\mathrm{C}$ & 10.721167 & 7.996409 & 4.387649 \\
$\mathrm{O}$ & 10.392168 & 8.537152 & 5.613783 \\
$\mathrm{C}$ & 9.230481 & 9.380364 & 5.621597 \\
$\mathrm{C}$ & 7.972980 & 8.709888 & 5.279832 \\
$\mathrm{C}$ & 6.898562 & 8.194798 & 4.978448 \\
$\mathrm{C}$ & 5.633096 & 7.639789 & 4.608824 \\
$\mathrm{C}$ & 5.035531 & 7.989508 & 3.369594 \\
$\mathrm{C}$ & 3.791626 & 7.458513 & 3.005199 \\
$\mathrm{C}$ & 3.118127 & 6.571202 & 3.865401 \\
$\mathrm{C}$ & 3.704256 & 6.210157 & 5.093274 \\
$\mathrm{C}$ & 4.950062 & 6.735321 & 5.464653 \\
$\mathrm{H}$ & 2.139912 & 6.157557 & 3.576988 \\
$\mathrm{H}$ & 11.632979 & 7.380415 & 4.479897 \\
$\mathrm{H}$ & 9.184950 & 9.785143 & 6.653753 \\
$\mathrm{H}$ & 9.550542 & 9.056603 & 1.398838 \\
$\mathrm{H}$ & 5.409093 & 6.457373 & 6.424804 \\
$\mathrm{H}$ & 3.184293 & 5.513277 & 5.768985 \\
$\mathrm{H}$ & 3.358575 & 7.726437 & 2.030132 \\
$\mathrm{H}$ & 5.575361 & 8.659747 & 2.685701 \\
$\mathrm{H}$ & 6.392980 & 4.306524 & 3.496384 \\
& & & \\
\hline
\end{tabular}

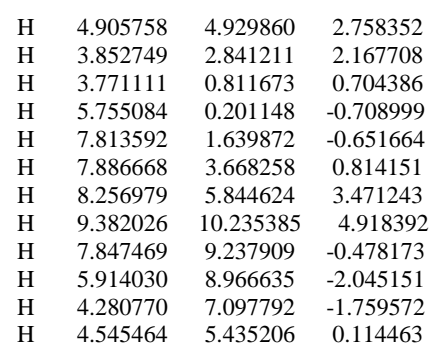

Allenyne_A2Cyclization_TS

50

Free energy $=-1170.252388$ Hartree

\begin{tabular}{|c|c|c|c|}
\hline $\mathrm{C}$ & 5.318144 & 6.981741 & -1.271865 \\
\hline $\mathrm{C}$ & 5.303852 & 6.174361 & -0.125479 \\
\hline C & 6.351042 & 6.347168 & 0.795493 \\
\hline C & 7.406059 & 7.290415 & 0.594834 \\
\hline $\mathrm{C}$ & 7.391005 & 8.092955 & -0.565362 \\
\hline $\mathrm{C}$ & 6.348712 & 7.930465 & -1.488925 \\
\hline $\mathrm{N}$ & 6.576224 & 5.705002 & 2.013997 \\
\hline C & 7.714574 & 6.217122 & 2.586543 \\
\hline $\mathrm{C}$ & 8.276097 & 7.206251 & 1.759411 \\
\hline $\mathrm{C}$ & 5.715165 & 4.678896 & 2.575053 \\
\hline $\mathrm{C}$ & 5.691967 & 70 & 9263 \\
\hline C & 4. & 63 & 207 \\
\hline $\mathrm{C}$ & 4.5 & 1.371800 & 1.060159 \\
\hline $\mathrm{C}$ & 5.638331 & 0.9 & 0.333942 \\
\hline $\mathrm{C}$ & 6.791459 & 0085 & 0.321075 \\
\hline C & 6.818242 & 2.9 & 1.034484 \\
\hline $\mathrm{C}$ & 9.386283 & 8.045917 & 2.100121 \\
\hline $\mathrm{C}$ & 9.832942 & 8.200084 & 3.376971 \\
\hline $\mathrm{C}$ & 10.787797 & 8.349033 & 4.304974 \\
\hline $\mathrm{O}$ & 10.539244 & 8.997940 & 5.481960 \\
\hline $\mathrm{C}$ & 9.209325 & 9.539364 & 5.478000 \\
\hline $\mathrm{C}$ & 8.263706 & 8.652553 & 4.743966 \\
\hline $\mathrm{C}$ & 7.097992 & 8.215218 & 4.597651 \\
\hline C & 5.775985 & 7.736810 & 4.405489 \\
\hline $\mathrm{C}$ & 5.018092 & 8.115406 & 3.258847 \\
\hline C & 3.716665 & 7.635955 & 3.072427 \\
\hline C & 3.132183 & 64 & 2692 \\
\hline C & 3.8 & & 45 \\
\hline $\mathrm{C}$ & & 6.8 & 5.344944 \\
\hline $\mathrm{H}$ & 2.108790 & 322 & 3.860901 \\
\hline $\mathrm{H}$ & 11.789483 & 0666 & 4.279474 \\
\hline $\mathrm{H}$ & 8.918934 & 9.673074 & 6.538861 \\
\hline $\mathrm{H}$ & 9.762410 & 8.728024 & 1.314782 \\
\hline $\mathrm{H}$ & 5.747923 & 6.546236 & 6.233051 \\
\hline $\mathrm{H}$ & 3.423320 & 5.694527 & 5.888549 \\
\hline $\mathrm{H}$ & 3.158672 & 7.931367 & 2.170502 \\
\hline $\mathrm{H}$ & 5.484916 & 8.765821 & 2.504781 \\
\hline $\mathrm{H}$ & 6.064533 & 4.487819 & 3.610305 \\
\hline $\mathrm{H}$ & 4.688055 & 5.090510 & 2.677122 \\
\hline $\mathrm{H}$ & 3.645151 & 2.914438 & 2.328549 \\
\hline $\mathrm{H}$ & 3.598128 & 0.752816 & 1.068507 \\
\hline $\mathrm{H}$ & 5.617869 & 0.008125 & -0.225808 \\
\hline $\mathrm{H}$ & 7.677746 & 1.445523 & -0.251502 \\
\hline $\mathrm{H}$ & 7.718273 & 3.604068 & 1.013041 \\
\hline $\mathrm{H}$ & 8.084428 & 5.841351 & .545859 \\
\hline $\mathrm{H}$ & 0045 & 10.535785 & 4.976361 \\
\hline $\mathrm{H}$ & 8.185157 & 8.835178 & -0.741381 \\
\hline $\mathrm{H}$ & 6.328923 & 8.547123 & -2.400718 \\
\hline $\mathrm{H}$ & 4.515188 & 6.873722 & -2.017707 \\
\hline 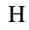 & 4.512665 & 5.430697 & 0.046650 \\
\hline
\end{tabular}

Allenyne_A2Cyclization_INT

50

$\begin{array}{cccc}\text { Free energy }=-1170.345595 & \text { Hartree } \\ \mathrm{C} & 6.875705 & 6.634017 & -2.572976 \\ \mathrm{C} & 6.126373 & 6.405547 & -1.404435 \\ \mathrm{C} & 6.719639 & 6.704107 & -0.160477 \\ \mathrm{C} & 8.060720 & 7.197584 & -0.100683 \\ \mathrm{C} & 8.784737 & 7.431300 & -1.280916 \\ \mathrm{C} & 8.189616 & 7.144861 & -2.522690 \\ \mathrm{~N} & 6.172255 & 6.606883 & 1.114009 \\ \mathrm{C} & 7.280072 & 6.718484 & 2.104801 \\ \mathrm{C} & 8.355636 & 7.455147 & 1.301235 \\ \mathrm{C} & 4.940767 & 5.891332 & 1.373609 \\ \mathrm{C} & 5.060406 & 4.379181 & 1.537351 \\ \mathrm{C} & 4.065783 & 3.678920 & 2.250909 \\ \mathrm{C} & 4.150072 & 2.289269 & 2.426749 \\ \mathrm{C} & 5.241376 & 1.576545 & 1.897853 \\ \mathrm{C} & 6.236979 & 2.264995 & 1.185024 \\ \mathrm{C} & 6.145233 & 3.656447 & 1.002229 \\ \mathrm{C} & 9.264398 & 8.304310 & 1.868882 \\ \mathrm{C} & 9.094429 & 8.651151 & 3.257800 \\ \mathrm{C} & 9.834436 & 9.389631 & 4.147433 \\ \mathrm{O} & 9.266687 & 9.529048 & 5.376978\end{array}$




$\begin{array}{cccc}\text { C } & 7.975301 & 8.856896 & 5.338356 \\ \text { C } & 7.890827 & 8.217976 & 3.968112 \\ \text { C } & 6.979279 & 7.335077 & 3.464399 \\ \text { C } & 5.880249 & 6.802401 & 4.309112 \\ \text { C } & 4.952003 & 7.670647 & 4.932110 \\ \text { C } & 3.933578 & 7.167465 & 5.757349 \\ \text { C } & 3.820957 & 5.782899 & 5.976188 \\ \text { C } & 4.736807 & 4.908688 & 5.365183 \\ \text { C } & 5.755111 & 5.410937 & 4.541903 \\ \text { H } & 3.019706 & 5.386030 & 6.619048 \\ \text { H } & 10.814731 & 9.870265 & 4.029053 \\ \text { H } & 7.926065 & 8.124042 & 6.172249 \\ \text { H } & 10.032703 & 8.800189 & 1.256083 \\ \text { H } & 6.457481 & 4.712957 & 4.062591 \\ \text { H } & 4.656667 & 3.821828 & 5.522270 \\ \text { H } & 3.216084 & 7.860152 & 6.224545 \\ \text { H } & 5.017594 & 8.752356 & 4.735292 \\ \text { H } & 4.462769 & 6.316753 & 2.278693 \\ \text { H } & 4.240555 & 6.119837 & 0.539936 \\ \text { H } & 3.225780 & 4.237616 & 2.694515 \\ \text { H } & 3.364757 & 1.759755 & 2.989423 \\ \text { H } & 5.316035 & 0.487613 & 2.044684 \\ \text { H } & 7.094771 & 1.717002 & 0.764792 \\ \text { H } & 6.926892 & 4.184739 & 0.435202 \\ \text { H } & 7.657730 & 5.671648 & 2.286392 \\ \text { H } & 7.195319 & 9.627573 & 5.515065 \\ \text { H } & 9.813176 & 7.820181 & -1.227546 \\ \text { H } & 8.751267 & 7.306957 & -3.454604 \\ \text { H } & 6.424629 & 6.395035 & -3.548760 \\ \text { H } & 5.111958 & 5.986994 & -1.467819\end{array}$

Allenyne_B

50

Free energy $=-1170.262105$ Hartree

$\begin{array}{llll}\text { C } & 5.671662 & 6.622948 & -0.324051\end{array}$

$\begin{array}{llll}\text { C } & 4.478924 & 6.497981 & 0.402005\end{array}$

$\begin{array}{llll}\text { C } & 4.071896 & 7.607037 & 1.165307\end{array}$

$\begin{array}{llll}\text { C } & 4.837124 & 8.813208 & 1.228518\end{array}$

$\begin{array}{llll}\text { C } & 6.031951 & 8.914144 & 0.486835\end{array}$

$\begin{array}{llll}\text { C } & 6.437509 & 7.817121 & -0.284681 \\ \text { N } & 2.931942 & 7.785107 & 1.941877\end{array}$

$\begin{array}{llll}\mathrm{N} & 2.931942 & 7.785107 & 1.941877\end{array}$

$\begin{array}{llll}\text { C } & 2.949146 & 9.054607 & 2.481458\end{array}$

$\begin{array}{llll}\text { C } & 4.101810 & 9.733458 & 2.078875\end{array}$

$\begin{array}{llll}\text { C } & 1.883818 & 6.797065 & 2.115371\end{array}$

$\begin{array}{llll}\text { C } & 2.319531 & 5.568238 & 2.900651\end{array}$

$\begin{array}{llll}\text { C } & 3.345357 & 5.647946 & 3.862344\end{array}$

$\begin{array}{llll}\text { C } & 3.720201 & 4.511299 & 4.597089\end{array}$

$\begin{array}{llll}\text { C } & 3.073927 & 3.282193 & 4.379595\end{array}$

$\begin{array}{llll}\text { C } & 2.051649 & 3.195072 & 3.419092\end{array}$

$\begin{array}{llll}\text { C } & 1.680802 & 4.331523 & 2.681645\end{array}$

$\begin{array}{llll}\text { C } & 4.410145 & 11.087087 & 2.350537\end{array}$

$\begin{array}{llll}\text { C } & 4.643464 & 12.276943 & 2.555815\end{array}$

$\begin{array}{llll}\text { C } & 4.807755 & 13.711658 & 2.776241\end{array}$

$\begin{array}{llll}\text { O } & 4.895041 & 14.507556 & 1.575769\end{array}$

$\begin{array}{llll}\text { C } & 3.798668 & 14.491563 & 0.752448\end{array}$

$\begin{array}{llll}\text { C } & 2.663346 & 13.827911 & 0.920466\end{array}$

$\begin{array}{llll}\text { C } & 1.582775 & 13.056153 & 0.980427\end{array}$

$\begin{array}{llll}\text { C } & 1.487467 & 11.690392 & 0.420402\end{array}$

$\begin{array}{llll}\text { C } & 0.351413 & 10.893334 & 0.697138\end{array}$

$\begin{array}{llll}\text { C } & 0.261606 & 9.577461 & 0.215036\end{array}$

$\begin{array}{llll}\text { C } & 1.303428 & 9.038192 & -0.559554\end{array}$

$\begin{array}{llll}\text { C } & 2.435176 & 9.822876 & -0.848699\end{array}$

$\begin{array}{llll}\text { C } & 2.525200 & 11.133102 & -0.365256\end{array}$

$\begin{array}{llll}\mathrm{H} & 1.240851 & 8.005878 & -0.936489\end{array}$

$\begin{array}{llll}\mathrm{H} & 3.955947 & 14.080347 & 3.399256\end{array}$

$\begin{array}{llll}\mathrm{H} & 3.963250 & 15.150421 & -0.119033\end{array}$

$\begin{array}{llll}\mathrm{H} & 0.680477 & 13.420514 & 1.510915\end{array}$

$\begin{array}{llll}\mathrm{H} & 3.420852 & 11.740187 & -0.566724\end{array}$

$\begin{array}{llll}\mathrm{H} & 3.265848 & 9.397396 & -1.431844\end{array}$

$\begin{array}{llll}\mathrm{H} & -0.628401 & 8.969762 & 0.444023\end{array}$

$\begin{array}{llll}\mathrm{H} & -0.466565 & 11.312349 & 1.305913\end{array}$

$\begin{array}{llll}\mathrm{H} & 1.036466 & 7.301438 & 2.625115\end{array}$

$\begin{array}{llll}\mathrm{H} & 1.504750 & 6.494756 & 1.114705\end{array}$

$\begin{array}{llll}\mathrm{H} & 3.862807 & 6.606322 & 4.022940\end{array}$

$\begin{array}{llll}\mathrm{H} & 4.529040 & 4.586105 & 5.340611\end{array}$

$\begin{array}{llll}\mathrm{H} & 3.372447 & 2.390497 & 4.951881\end{array}$

$\begin{array}{llll}\mathrm{H} & 1.546271 & 2.234401 & 3.235294\end{array}$

$\begin{array}{llll}\mathrm{H} & 0.886107 & 4.254873 & 1.920618\end{array}$

$\begin{array}{llll}\mathrm{H} & 2.112739 & 9.418341 & 3.088013\end{array}$

$\begin{array}{llll}\mathrm{H} & 5.750049 & 13.934985 & 3.318924\end{array}$

$\begin{array}{llll}\mathrm{H} & 6.621811 & 9.842771 & 0.519970\end{array}$

$\begin{array}{llll}\mathrm{H} & 7.369860 & 7.876118 & -0.866696\end{array}$

$\begin{array}{llll}\mathrm{H} & 6.022733 & 5.774693 & -0.93148\end{array}$

$\begin{array}{llll}\mathrm{H} & 3.890336 & 5.569028 & 0.385480\end{array}$

Allenyne_B2Cyclization_TS

Free energy $=-1170.244548$ Hartree

$\begin{array}{llll}\text { C } & 5.713819 & 6.563973 & -0.643667\end{array}$

$\begin{array}{llll}\text { C } & 4.615482 & 6.538358 & 0.227912\end{array}$

$\begin{array}{cccc}\mathrm{C} & 4.328049 & 7.719897 & 0.934805 \\ \mathrm{C} & 5.119044 & 8.901416 & 0.796677 \\ \mathrm{C} & 6.216082 & 8.902098 & -0.086061 \\ \mathrm{C} & 6.503651 & 7.731394 & -0.801400 \\ \mathrm{~N} & 3.293710 & 8.000172 & 1.823206 \\ \mathrm{C} & 3.393197 & 9.316128 & 2.230134 \\ \mathrm{C} & 4.510403 & 9.924566 & 1.638132 \\ \mathrm{C} & 2.201204 & 7.097581 & 2.135187 \\ \mathrm{C} & 2.624614 & 5.850193 & 2.897130 \\ \mathrm{C} & 3.769873 & 5.853189 & 3.716416 \\ \mathrm{C} & 4.127864 & 4.704772 & 4.441547 \\ \mathrm{C} & 3.343175 & 3.541614 & 4.358092 \\ \mathrm{C} & 2.200265 & 3.531325 & 3.539822 \\ \mathrm{C} & 1.846987 & 4.678053 & 2.810098 \\ \mathrm{C} & 4.898579 & 11.267300 & 1.736018 \\ \mathrm{C} & 5.141961 & 12.503584 & 1.737191 \\ \mathrm{C} & 6.008283 & 13.558773 & 2.345874 \\ \mathrm{O} & 6.145923 & 14.676405 & 1.456533 \\ \mathrm{C} & 5.017793 & 14.804499 & 0.702428 \\ \mathrm{C} & 4.029232 & 13.891190 & 0.770836 \\ \mathrm{C} & 2.703057 & 13.605359 & 0.617340 \\ \mathrm{C} & 2.240298 & 12.324433 & 0.109126 \\ \mathrm{C} & 0.966950 & 11.811826 & 0.482190 \\ \mathrm{C} & 0.597180 & 10.503673 & 0.152856 \\ \mathrm{C} & 1.478162 & 9.681769 & -0.582139 \\ \mathrm{C} & 2.711941 & 10.194282 & -1.017780 \\ \mathrm{C} & 3.090452 & 11.498881 & -0.676484 \\ \mathrm{H} & 1.192720 & 8.647611 & -0.830398 \\ \mathrm{H} & 5.533599 & 13.904244 & 3.294420 \\ \mathrm{H} & 5.073599 & 15.627929 & -0.026524 \\ \mathrm{H} & 1.952071 & 14.267396 & 1.088397 \\ \mathrm{H} & 4.064831 & 11.895892 & -0.996481 \\ \mathrm{H} & 3.400645 & 9.558611 & -1.594663 \\ \mathrm{H} & -0.381267 & 10.113342 & 0.474349 \\ \mathrm{H} & 0.287344 & 12.445916 & 1.074901 \\ \mathrm{H} & 1.459520 & 7.671068 & 2.729730 \\ \mathrm{H} & 1.686442 & 6.811264 & 1.190773 \\ \mathrm{H} & 4.390948 & 6.760595 & 3.772174 \\ \mathrm{H} & 5.030589 & 4.718195 & 5.071793 \\ \mathrm{H} & 3.626046 & 2.640539 & 4.923509 \\ \mathrm{H} & 1.584574 & 2.621580 & 3.462071 \\ \mathrm{H} & 0.954435 & 4.661654 & 2.162767 \\ \mathrm{H} & 2.637670 & 9.760094 & 2.887175 \\ \mathrm{H} & 7.029474 & 13.192114 & 2.567856 \\ \mathrm{H} & 6.822102 & 9.813057 & -0.207401 \\ & 7.358268 & 7.713185 & -1.495334 \\ & 4.008875 & 5.657481 & -1.213652 \\ 5.631116 & 0.362200\end{array}$

Bisallene

Free energy $=-1170.276216$ Hartree

$\begin{array}{llll}\text { C } & 4.171426 & 5.316534 & 3.522940 \\ \text { C } & 3.012153 & 4.649118 & 3.968028\end{array}$

$\begin{array}{llll}\text { C } & 3.012153 & 4.649118 & 3.968028\end{array}$

$\begin{array}{llll}\text { C } & 1.941962 & 5.411466 & 4.478314\end{array}$

$\begin{array}{llll}\text { C } & 2.021032 & 6.813292 & 4.525531\end{array}$

$\begin{array}{llll}\text { C } & 3.174535 & 7.470244 & 4.062834\end{array}$

$\begin{array}{llll}\text { C } & 4.253008 & 6.718192 & 3.565931\end{array}$

$\begin{array}{llll}\text { C } & 2.891952 & 3.143597 & 3.808117\end{array}$

$\begin{array}{llll}\mathrm{N} & 2.357976 & 2.762238 & 2.502871\end{array}$

$\begin{array}{llll}\text { C } & 3.109736 & 2.616439 & 1.351664\end{array}$

$\begin{array}{llll}\text { C } & 2.279264 & 2.346878 & 0.263277\end{array}$

$\begin{array}{llll}\text { C } & 0.924377 & 2.342851 & 0.781121\end{array}$

$\begin{array}{llll}\text { C } & 1.009147 & 2.625123 & 2.182365\end{array}$

$\begin{array}{llll}\text { C } & 2.665888 & 2.230189 & -1.131568\end{array}$

$\begin{array}{llll}\text { C } & 3.895379 & 2.388251 & -1.625112\end{array}$

$\begin{array}{llll}\text { C } & 5.113902 & 2.460087 & -2.131218\end{array}$

$\begin{array}{llll}\text { O } & 5.780816 & 3.609486 & -2.531499\end{array}$

$\begin{array}{llll}\text { C } & 5.289838 & 4.831254 & -2.110026\end{array}$

$\begin{array}{llll}\text { C } & 4.411756 & 5.055129 & -1.145768\end{array}$

$\begin{array}{llll}\text { C } & 3.578570 & 5.406776 & -0.167439\end{array}$

$\begin{array}{llll}\text { C } & 2.112673 & 5.489683 & -0.257412\end{array}$

$\begin{array}{llll}\text { C } & 1.417171 & 5.200948 & -1.456801\end{array}$

$\begin{array}{llll}\text { C } & 0.016324 & 5.204843 & -1.482775\end{array}$

$\begin{array}{llll}\text { C } & -0.716642 & 5.489315 & -0.315164\end{array}$

$\begin{array}{llll}\text { C } & -0.036367 & 5.785023 & 0.878165\end{array}$

$\begin{array}{llll}\text { C } & 1.364468 & 5.792714 & 0.904586\end{array}$

$\begin{array}{llll}\mathrm{H} & -1.816998 & 5.462967 & -0.332345\end{array}$

$\begin{array}{llll}\mathrm{H} & 5.745147 & 1.576771 & -2.327523\end{array}$

$\begin{array}{llll}\mathrm{H} & 5.759170 & 5.645937 & -2.686969\end{array}$

$\begin{array}{llll}\mathrm{H} & 1.833932 & 2.082882 & -1.846988\end{array}$

H $\quad 3.998052 \quad 5.602926 \quad 0.838416$

$\begin{array}{llll}\mathrm{H} & 1.996292 & 4.957155 & -2.360785\end{array}$

$\begin{array}{llll}\mathrm{H} & -0.512521 & 4.968539 & -2.419677\end{array}$

$\begin{array}{llll}\mathrm{H} & -0.599185 & 5.992376 & 1.800768\end{array}$

$\begin{array}{llll}\mathrm{H} & 1.893491 & 6.012349 & 1.842733\end{array}$

$\begin{array}{llll}\mathrm{H} & 2.232027 & 2.713811 & 4.587704\end{array}$

$\begin{array}{llll}\mathrm{H} & 3.883935 & 2.661345 & 3.920196\end{array}$

$\begin{array}{llll}\mathrm{H} & 1.028693 & 4.904997 & 4.827436\end{array}$ 


$\begin{array}{lrrr}\mathrm{H} & 1.173944 & 7.395729 & 4.919307 \\ \mathrm{H} & 3.234273 & 8.569185 & 4.092036 \\ \mathrm{H} & 5.161783 & 7.224859 & 3.206434 \\ \mathrm{H} & 5.018235 & 4.730688 & 3.130074 \\ \mathrm{H} & 4.199815 & 2.726779 & 1.370027 \\ \mathrm{C} & -0.135433 & 2.722677 & 2.994817 \\ \mathrm{C} & -0.344130 & 2.152207 & 0.195096 \\ \mathrm{C} & -1.485780 & 2.245298 & 0.999584 \\ \mathrm{C} & -1.382187 & 2.529467 & 2.383800 \\ \mathrm{H} & -0.434409 & 1.946101 & -0.881936 \\ \mathrm{H} & -2.481067 & 2.104437 & 0.551340 \\ \mathrm{H} & -2.296869 & 2.601089 & 2.992126 \\ \mathrm{H} & -0.063708 & 2.945327 & 4.069081\end{array}$

Bisallene2Cyclization_TS 1

50

Free energy $=-1170.274596$ Hartree

$\begin{array}{llll}\text { C } & 4.262911 & 5.356797 & 3.494196\end{array}$

$\begin{array}{llll}\text { C } & 3.106782 & 4.703141 & 3.966770\end{array}$

$\begin{array}{llll}\text { C } & 2.048992 & 5.478801 & 4.482721\end{array}$

$\begin{array}{llll}\text { C } & 2.137216 & 6.880552 & 4.509059\end{array}$

$\begin{array}{llll}\text { C } & 3.286860 & 7.523799 & 4.017964\end{array}$

$\begin{array}{llll}\text { C } & 4.352850 & 6.758523 & 3.514660\end{array}$

$\begin{array}{llll}\text { C } & 2.974954 & 3.196659 & 3.829494\end{array}$

$\begin{array}{llll}\mathrm{N} & 2.403974 & 2.800760 & 2.544240\end{array}$

$\begin{array}{llll}\text { C } & 3.120434 & 2.647577 & 1.374286\end{array}$

$\begin{array}{llll}\text { C } & 2.256140 & 2.341861 & 0.313702\end{array}$

$\begin{array}{llll}\text { C } & 0.916692 & 2.332590 & 0.878451 \\ \text { C } & 1.044492 & 2.642088 & 2.268487\end{array}$

$\begin{array}{llll}\text { C } & 1.044492 & 2.642088 & 2.268487\end{array}$

$\begin{array}{llll}\text { C } & 2.607628 & 2.251315 & -1.076839\end{array}$

$\begin{array}{llll}\text { C } & 3.830020 & 2.554839 & -1.575693\end{array}$

$\begin{array}{llll}\text { C } & 4.976683 & 2.419117 & -2.234999\end{array}$

$\begin{array}{llll}\text { O } & 5.746538 & 3.503184 & -2.60926\end{array}$

$\begin{array}{llll}\text { C } & 5.285139 & 4.683213 & -2.063710\end{array}$

$\begin{array}{llll}\text { C } & 4.321755 & 4.732478 & -1.146289\end{array}$

$\begin{array}{llll}\text { C } & 3.549325 & 5.248015 & -0.161172\end{array}$

$\begin{array}{llll}\text { C } & 2.103145 & 5.428416 & -0.234173\end{array}$

$\begin{array}{llll}\text { C } & 1.373767 & 5.130575 & -1.415064\end{array}$

$\begin{array}{llll}\text { C } & -0.027172 & 5.195460 & -1.420552\end{array}$

$\begin{array}{llll}\text { C } & -0.728135 & 5.551652 & -0.254823\end{array}$

$\begin{array}{llll}\text { C } & -0.015070 & 5.864312 & 0.918177\end{array}$

$\begin{array}{llll}\text { C } & 1.382183 & 5.811725 & 0.926830\end{array}$

$\begin{array}{llll}\mathrm{C} & 1.82183 & 5.811725 & 0.926830 \\ \mathrm{H} & -1.828789 & 5.573770 & -0.255105\end{array}$

$\begin{array}{llll}\mathrm{H} & 5.444808 & 1.465352 & -2.523098\end{array}$

$\begin{array}{llll}\mathrm{H} & 5.794496 & 5.560026 & -2.491549\end{array}$

$\begin{array}{llll}\mathrm{H} & 1.769961 & 2.119455 & -1.787081\end{array}$

$\begin{array}{llll}\mathrm{H} & 4.005916 & 5.430804 & 0.829646\end{array}$

$\begin{array}{llll}\mathrm{H} & 1.926820 & 4.840971 & -2.321249\end{array}$

$\begin{array}{llll}\mathrm{H} & -0.580055 & 4.952247 & -2.341829\end{array}$

$\begin{array}{lllr}\mathrm{H} & -0.554992 & 6.134413 & 1.838591\end{array}$

$\begin{array}{llll}\mathrm{H} & 1.934817 & 6.040298 & 1.849063\end{array}$

$\begin{array}{llll}\mathrm{H} & 2.333875 & 2.779711 & 4.631429\end{array}$

$\begin{array}{llll}\mathrm{H} & 3.967352 & 2.710913 & 3.921267\end{array}$

$\mathrm{H} \quad 1.137850 \quad 4.982731 \quad 4.851800$

$\begin{array}{llll}\mathrm{H} & 1.300090 & 7.473686 & 4.908065\end{array}$

$\begin{array}{llll}\mathrm{H} & 3.353117 & 8.622723 & 4.029838\end{array}$

$\begin{array}{llll}\mathrm{H} & 5.258305 & 7.254561 & 3.132783\end{array}$

$\begin{array}{llll}\mathrm{H} & 5.101090 & 4.760001 & 3.099217\end{array}$

$\begin{array}{llll}\mathrm{H} & 4.210334 & 2.755196 & 1.357141\end{array}$

$\begin{array}{llll}\text { C } & -0.073546 & 2.746086 & 3.11522\end{array}$

$\begin{array}{llll}\text { C } & -0.366580 & 2.129182 & 0.333910\end{array}$

$\begin{array}{llll}\text { C } & -1.484247 & 2.229045 & 1.172012\end{array}$

$\begin{array}{llll}\text { C } & -1.338214 & 2.534842 & 2.546705\end{array}$

$\begin{array}{llll}\mathrm{H} & -0.487159 & 1.906138 & -0.736697\end{array}$

$\begin{array}{llll}\mathrm{H} & -2.492172 & 2.077435 & 0.756956\end{array}$

$\begin{array}{llll}\mathrm{H} & -2.233361 & 2.611616 & 3.182847\end{array}$

$\begin{array}{llll}\mathrm{H} & 0.030043 & 2.989903 & 4.182216\end{array}$

Bisallene2Cyclization_INT1

50

Free energy $=-1170.31365$ Hartree

$\begin{array}{llll}\text { C } & 4.059843 & 5.518456 & -0.006217 \\ \text { C } & 4.265479 & 5.051275 & -1.348452\end{array}$

$\begin{array}{llll}\text { C } & 4.265479 & 5.051275 & -1.348452 \\ \text { C } & 3.880913 & 5.942958 & -2.403068\end{array}$

$\begin{array}{llll}\text { C } & 3.880913 & 5.942958 & -2.403068 \\ \text { C } & 3.400929 & 7.230520 & -2.125486\end{array}$

$\begin{array}{llll}\text { C } & 3.223342 & 7.665189 & -0.800678 \\ \text { C } & 3.54637 & 6.786699 & 0.258500\end{array}$

$\begin{array}{llll}\text { C } & 3.544637 & 6.786699 & 0.258500\end{array}$

$\begin{array}{llll}\text { C } & 4.867086 & 3.771043 & -1.55744\end{array}$

$\begin{array}{llll}\text { C } & 5.156528 & 3.065067 & -2.763563\end{array}$

$\begin{array}{llll}\text { C } & 4.565043 & 3.057155 & -4.131614\end{array}$

$\begin{array}{llll}\text { C } & 5.454250 & 2.276567 & -4.901613\end{array}$

$\begin{array}{llll}\text { O } & 6.431668 & 1.759501 & -4.133050\end{array}$

$\begin{array}{llll}\text { C } & 6.259778 & 2.197873 & -2.861467\end{array}$

$\begin{array}{llll}\text { C } & 3.325852 & 3.486371 & -4.681416\end{array}$

$\begin{array}{llll}\text { C } & 2.114469 & 3.891714 & -4.077097\end{array}$

$\begin{array}{llll}\text { C } & 0.893424 & 4.251352 & -4.806676\end{array}$

$\begin{array}{llll}\text { C } & -0.143113 & 4.425776 & -3.842432\end{array}$

$\begin{array}{llll}\mathrm{N} & 0.406262 & 4.185640 & -2.579477\end{array}$

$\begin{array}{llll}\text { C } & 1.745303 & 3.914704 & -2.706859\end{array}$

$\begin{array}{llll}\text { C } & 0.592864 & 4.433921 & -6.169776\end{array}$

$\begin{array}{lrrr}\mathrm{C} & -0.715042 & 4.787965 & -6.536039 \\ \mathrm{C} & -1.724408 & 4.964633 & -5.560323 \\ \mathrm{C} & -1.453378 & 4.788647 & -4.194832 \\ \mathrm{C} & -0.324815 & 4.274880 & -1.322592 \\ \mathrm{C} & -0.857727 & 5.668913 & -1.033089 \\ \mathrm{C} & -0.063730 & 6.796767 & -1.313631 \\ \mathrm{C} & -0.538190 & 8.085235 & -1.026991 \\ \mathrm{C} & -1.811971 & 8.260766 & -0.457467 \\ \mathrm{C} & -2.610194 & 7.138358 & -0.178277 \\ \mathrm{C} & -2.135477 & 5.847684 & -0.468967 \\ \mathrm{H} & 2.825836 & 8.668861 & -0.588792 \\ \mathrm{H} & 5.436514 & 1.971324 & -5.953037 \\ \mathrm{H} & 7.020948 & 1.899052 & -2.134046 \\ \mathrm{H} & 3.261773 & 3.364019 & -5.776923 \\ \mathrm{H} & 5.334269 & 3.331984 & -0.658649 \\ \mathrm{H} & 4.040824 & 5.642986 & -3.446522 \\ \mathrm{H} & 3.150834 & 7.901315 & -2.962508 \\ \mathrm{H} & 3.399681 & 7.109733 & 1.301523 \\ \mathrm{H} & 4.334790 & 4.855465 & 0.830394 \\ \mathrm{H} & -1.160430 & 3.541549 & -1.337487 \\ \mathrm{H} & 0.372866 & 3.957776 & -0.520017 \\ \mathrm{H} & -2.766968 & 4.969571 & -0.255185 \\ \mathrm{H} & -3.610824 & 7.267232 & 0.262019 \\ \mathrm{H} & -2.184637 & 9.272184 & -0.233612 \\ \mathrm{H} & 0.095400 & 8.957527 & -1.250283 \\ \mathrm{H} & 0.933178 & 6.661993 & -1.756526 \\ \mathrm{H} & 2.345386 & 3.652883 & -1.830397 \\ \mathrm{H} & 1.372745 & 4.310343 & -6.937001 \\ \mathrm{H} & -0.959591 & 4.941116 & -7.598428 \\ \mathrm{H} & -2.738186 & 5.254775 & -5.875727 \\ \mathrm{H} & -2.227916 & 4.946798 & -3.430952 \\ & & & \end{array}$

Bisallene2Cyclization_TS2_for_A

Free energy $=-1170.310148$ Hartree

$\begin{array}{llll}\text { C } & 0.403913 & 5.599114 & 0.639417\end{array}$

$\begin{array}{llll}\text { C } & 0.613076 & 5.363277 & -0.734602\end{array}$

$\begin{array}{llll}\text { C } & 0.909437 & 6.450876 & -1.575730\end{array}$

$\begin{array}{llll}\text { C } & 0.990378 & 7.753442 & -1.052817\end{array}$

$\begin{array}{llll}\text { C } & 0.781296 & 7.981598 & 0.316488\end{array}$

$\begin{array}{llll}\text { C } & 0.488584 & 6.897369 & 1.163224\end{array}$

$\begin{array}{llll}\text { C } & 0.501187 & 3.935718 & -1.259297\end{array}$

$\begin{array}{llll}\mathrm{N} & 0.925619 & 3.753460 & -2.633600\end{array}$

$\begin{array}{llll}\text { C } & 0.188284 & 4.103508 & -3.767138\end{array}$

$\begin{array}{llll}\text { C } & 1.009992 & 3.878977 & -4.914974\end{array}$

$\begin{array}{llll}\text { C } & 2.307878 & 3.400303 & -4.436152\end{array}$

$\begin{array}{llll}\text { C } & 2.182972 & 3.335335 & -3.020269\end{array}$

$\begin{array}{llll}\text { C } & -1.116465 & 4.615239 & -3.864516\end{array}$

$\begin{array}{llll}\text { C } & -1.610884 & 4.880996 & -5.151194\end{array}$

$\begin{array}{llll}\text { C } & -0.818823 & 4.655666 & -6.302355\end{array}$

$\begin{array}{llll}\text { C } & 0.491214 & 4.163365 & -6.192622\end{array}$

$\begin{array}{llll}\text { C } & 3.459557 & 3.098347 & -5.194565\end{array}$

$\begin{array}{llll}\text { C } & 4.762923 & 2.917034 & -4.59468\end{array}$

$\begin{array}{llll}\text { C } & 5.279333 & 3.590336 & -3.387816\end{array}$

$\begin{array}{llll}\text { C } & 6.546486 & 3.067251 & -3.197643\end{array}$

$\begin{array}{llll}\text { O } & 6.861650 & 2.146413 & -4.160230\end{array}$

$\begin{array}{llll}\text { C } & 5.779677 & 2.065250 & -4.995783\end{array}$

$\begin{array}{llll}\text { C } & 4.627044 & 4.662674 & -2.655380\end{array}$

$\begin{array}{llll}\text { C } & 4.609492 & 4.832307 & -1.233877\end{array}$

$\begin{array}{llll}\text { C } & 4.078932 & 6.034383 & -0.665240\end{array}$

$\begin{array}{llll}\text { C } & 3.976550 & 6.204063 & 0.715728\end{array}$

$\begin{array}{llll}\text { C } & 4.398069 & 5.183781 & 1.594228\end{array}$

$\begin{array}{llll}\text { C } & 4.926776 & 3.990537 & 1.060663\end{array}$

$\begin{array}{llll}\text { C } & 5.035322 & 3.812305 & -0.322490\end{array}$

$\begin{array}{llll}\mathrm{H} & 4.311325 & 5.316355 & 2.683832\end{array}$

$\begin{array}{llll}\mathrm{H} & 5.850951 & 1.331209 & -5.806034\end{array}$

$\begin{array}{llll}\mathrm{H} & 7.337407 & 3.306513 & -2.478815\end{array}$

$\begin{array}{llll}\mathrm{H} & 3.360429 & 2.990222 & -6.286240\end{array}$

$\mathrm{H} \quad 4.102779 \quad 5.418485 \quad-3.262735$

$\begin{array}{llll}\mathrm{H} & 5.427544 & 2.865556 & -0.724559\end{array}$

$\begin{array}{llll}\mathrm{H} & 5.253175 & 3.184484 & 1.737104\end{array}$

$\begin{array}{llll}\mathrm{H} & 3.548237 & 7.136281 & 1.114108\end{array}$

$\begin{array}{llll}\mathrm{H} & 3.738326 & 6.834456 & -1.339125\end{array}$

$\begin{array}{llll}\mathrm{H} & -0.551145 & 3.586166 & -1.166718\end{array}$

$\begin{array}{llll}\mathrm{H} & 1.112569 & 3.261193 & -0.625211\end{array}$

$\begin{array}{llll}\mathrm{H} & 0.182617 & 4.752956 & 1.310314\end{array}$

$\begin{array}{llll}\mathrm{H} & 0.330357 & 7.064691 & 2.239980\end{array}$

$\begin{array}{llll}\mathrm{H} & 0.848914 & 9.001395 & 0.725916\end{array}$

$\begin{array}{llll}\mathrm{H} & 1.225380 & 8.594268 & -1.723520\end{array}$

$\begin{array}{llll}\mathrm{H} & 1.091096 & 6.279759 & -2.647358\end{array}$

$\begin{array}{llll}\mathrm{H} & 2.864695 & 2.904364 & -2.282502\end{array}$

$\begin{array}{llll}\mathrm{H} & 1.107877 & 4.006583 & -7.090853\end{array}$

$\begin{array}{llll}\mathrm{H} & -1.234794 & 4.881217 & -7.295971\end{array}$

$\begin{array}{llll}\mathrm{H} & -2.630207 & 5.280171 & -5.263930\end{array}$

$\mathrm{H} \quad-1.720965 \quad 4.816396 \quad-2.968239$

Bisallene2Cyclization_INT2 for_A

Free energy $=-1170.36421$ Hartree 


$\begin{array}{rrrr}\mathrm{C} & 0.680453 & 5.860220 & 0.638778 \\ \mathrm{C} & 0.842648 & 5.707462 & -0.752295 \\ \mathrm{C} & 1.016517 & 6.853345 & -1.549166 \\ \mathrm{C} & 1.044541 & 8.131391 & -0.964303 \\ \mathrm{C} & 0.897142 & 8.275499 & 0.425210 \\ \mathrm{C} & 0.710399 & 7.133646 & 1.225793 \\ \mathrm{C} & 0.851202 & 4.310093 & -1.356519 \\ \mathrm{~N} & 1.315495 & 4.236648 & -2.728090 \\ \mathrm{C} & 0.451140 & 4.248275 & -3.813832 \\ \mathrm{C} & 1.096274 & 3.743605 & -4.984279 \\ \mathrm{C} & 2.463633 & 3.409230 & -4.617024 \\ \mathrm{C} & 2.575302 & 3.582402 & -3.097639 \\ \mathrm{C} & -0.883819 & 4.693949 & -3.858193 \\ \mathrm{C} & -1.563814 & 4.621642 & -5.088651 \\ \mathrm{C} & -0.936744 & 4.126645 & -6.251430 \\ \mathrm{C} & 0.399700 & 3.690104 & -6.202868 \\ \mathrm{C} & 3.554392 & 3.065125 & -5.367016 \\ \mathrm{C} & 4.836042 & 2.991643 & -4.690770 \\ \mathrm{C} & 5.009887 & 3.587063 & -3.375988 \\ \mathrm{C} & 6.321768 & 3.382952 & -3.032541 \\ \mathrm{O} & 6.977471 & 2.704995 & -4.029704 \\ \mathrm{C} & 6.073642 & 2.476359 & -5.031643 \\ \mathrm{C} & 3.881760 & 4.329728 & -2.696900 \\ \mathrm{C} & 4.063238 & 4.495784 & -1.198615 \\ \mathrm{C} & 4.031208 & 5.774211 & -0.609222 \\ \mathrm{C} & 4.135846 & 5.929931 & 0.782742 \\ \mathrm{C} & 4.275251 & 4.803137 & 1.608265 \\ \mathrm{C} & 4.323285 & 3.520563 & 1.031384 \\ \mathrm{C} & 4.223493 & 3.369746 & -0.360780 \\ \mathrm{H} & 4.351058 & 4.922417 & 2.700497 \\ \mathrm{H} & 6.448844 & 1.931859 & -5.904300 \\ \mathrm{H} & 6.915575 & 3.646126 & -2.151448 \\ \mathrm{H} & 3.476299 & 2.899023 & -6.452140 \\ \mathrm{H} & 3.806948 & 5.342079 & -3.156206 \\ \mathrm{H} & 4.274297 & 2.365006 & -0.809952 \\ \mathrm{H} & 4.444095 & 2.631724 & 1.670122 \\ \mathrm{H} & -1.372970 & 5.107171 & -2.964308 \\ \mathrm{H} & 4.085941 & 6.937864 & 1.221595 \\ \mathrm{H} & 3.893490 & 6.660378 & -1.246773 \\ \mathrm{H} & -0.175639 & 3.877011 & -1.293698 \\ \mathrm{H} & 1.497070 & 3.658393 & -0.731613 \\ \mathrm{H} & 0.559735 & 4.966786 & 1.272690 \\ \mathrm{H} & 0.597032 & 7.237636 & 2.316423 \\ \mathrm{H} & 0.925848 & 9.275456 & 0.885597 \\ \mathrm{H} & 1.188475 & 9.019658 & -1.599043 \\ \mathrm{H} & 1.157509 & 6.731164 & -2.634251 \\ \mathrm{H} & 0.821906 & 2.565934 & -2.626054 \\ & -1.495065 & 3.301725 & -7.105631 \\ & & & \\ \mathrm{H} & & \end{array}$

Bisallene2Cyclization_TS2_for_B

50

Free energy $=-1170.298623$ Hartree

$\begin{array}{lrrr}\text { Free energy }=-1170.298623 & \text { Hartree } \\ \mathrm{C} & -1.896604 & 5.475989 & -0.713871 \\ \mathrm{C} & -0.700193 & 5.286791 & -1.433618 \\ \mathrm{C} & 0.083642 & 6.408510 & -1.767965 \\ \mathrm{C} & -0.318643 & 7.696669 & -1.379941 \\ \mathrm{C} & -1.508407 & 7.878253 & -0.653859 \\ \mathrm{C} & -2.297568 & 6.763773 & -0.321927 \\ \mathrm{C} & -0.258709 & 3.880285 & -1.819946 \\ \mathrm{~N} & 0.569174 & 3.834239 & -3.011178 \\ \mathrm{C} & 0.121945 & 4.129026 & -4.297788 \\ \mathrm{C} & 1.249358 & 4.084657 & -5.173111 \\ \mathrm{C} & 2.421571 & 3.776733 & -4.359169 \\ \mathrm{C} & 1.938171 & 3.632684 & -3.042430 \\ \mathrm{C} & -1.175245 & 4.424106 & -4.753850 \\ \mathrm{C} & -1.335012 & 4.646404 & -6.129331 \\ \mathrm{C} & -0.234969 & 4.575947 & -7.019535 \\ \mathrm{C} & 1.057662 & 4.295720 & -6.552588 \\ \mathrm{C} & 3.743705 & 3.577036 & -4.844838 \\ \mathrm{C} & 4.905040 & 3.342722 & -3.985664 \\ \mathrm{C} & 6.045109 & 2.468604 & -4.318851 \\ \mathrm{C} & 6.905485 & 2.575799 & -3.240843 \\ \mathrm{O} & 6.420086 & 3.439820 & -2.291657 \\ \mathrm{C} & 5.216258 & 3.898509 & -2.765573 \\ \mathrm{C} & 6.193200 & 1.754671 & -5.567584 \\ \mathrm{C} & 5.072078 & 1.350780 & -6.320266 \\ \mathrm{C} & 5.171809 & 1.060339 & -7.727714 \\ \mathrm{C} & 4.038134 & 0.865788 & -8.502072 \\ \mathrm{C} & 2.737237 & 0.923475 & -7.916421 \\ \mathrm{C} & 2.604539 & 1.116503 & -6.542487 \\ \mathrm{C} & 3.744633 & 1.323495 & -5.726681 \\ \mathrm{H} & 1.845064 & 0.781490 & -8.545104 \\ \mathrm{H} & 4.718711 & 4.662340 & -2.157935 \\ \mathrm{H} & 7.856730 & 2.087778 & -3.003829 \\ \mathrm{H} & 3.950403 & 3.868222 & -5.886053 \\ \mathrm{H} & 7.200740 & 1.646663 & -6.000793 \\ \mathrm{H} & 3.652895 & 1.179990 & -4.642059\end{array}$

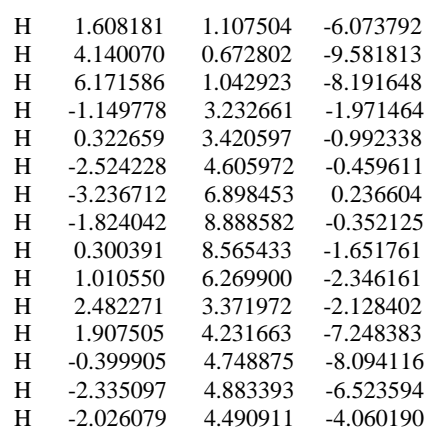

Bisallene2Cyclization_INT2_for_B

50

Free energy $=-1170.334569$ Hartree

\begin{tabular}{|c|c|c|c|}
\hline C & -1.156560 & 5.157015 & -0.235717 \\
\hline $\mathrm{C}$ & -0.563981 & 5.033373 & -1.509161 \\
\hline $\mathrm{C}$ & 0.151642 & 6.124462 & -2.039238 \\
\hline $\mathrm{C}$ & 0.269760 & 7.318993 & -1.306998 \\
\hline$C$ & -0.323561 & 7.436132 & -0.039544 \\
\hline$C$ & -1.038500 & 6.349518 & 0.495534 \\
\hline$c$ & -0.716837 & 3.725661 & -2.280885 \\
\hline $\mathbf{N}$ & 0.100697 & 3.636194 & -3.474812 \\
\hline & -0.234799 & 4.136989 & -4.727111 \\
\hline$c$ & 0.941021 & 4.074591 & -5.547563 \\
\hline$c$ & 2.007136 & 3.546752 & -4.725811 \\
\hline$c$ & 1.449465 & 02025 & -3.478730 \\
\hline$c$ & -1.454555 & 981 & -5.206876 \\
\hline & -1.496891 & 755 & -6 \\
\hline $\mathrm{C}$ & -0.352883 & 4.997391 & -7.377433 \\
\hline $\mathrm{C}$ & 0.863451 & 4.502299 & -6.893376 \\
\hline C & 3.426092 & 3.272279 & -5.149237 \\
\hline $\mathrm{C}$ & 4.444103 & 3.591866 & -4.083134 \\
\hline $\mathrm{C}$ & 5.765091 & 2.992654 & -4.111478 \\
\hline $\mathrm{C}$ & 6.457028 & 3.559393 & -3.053333 \\
\hline 0 & 5.660758 & 4.447940 & -2.387870 \\
\hline $\mathrm{C}$ & 4.443200 & 4.467054 & -3.026451 \\
\hline $\mathrm{C}$ & 6.118751 & 2.035133 & -5.132248 \\
\hline $\mathrm{C}$ & 5.133676 & 1.486329 & -5.923332 \\
\hline C & 5.446094 & 0.582505 & -7.010448 \\
\hline $\mathrm{C}$ & 4.486702 & 0.140200 & -7.883394 \\
\hline $\mathrm{C}$ & 3.105006 & 0.583762 & -7.7 \\
\hline$c$ & 2.713033 & 31 & 00 \\
\hline $\mathrm{C}$ & 3.656510 & 3787 & -5.620972 \\
\hline $\mathrm{H}$ & 2.373956 & 0.271562 & -8.519292 \\
\hline $\mathrm{H}$ & 3.689845 & 5.144619 & -2.611876 \\
\hline $\mathrm{H}$ & 7.468524 & 3.424483 & -2.656511 \\
\hline $\mathrm{H}$ & 3.650686 & 3.917446 & -6.031572 \\
\hline $\mathrm{H}$ & 7.172374 & 1.750247 & -5.281765 \\
\hline $\mathrm{H}$ & 3.388192 & 1.166517 & -4.725695 \\
\hline $\mathrm{H}$ & 1.665924 & 1.703057 & -6.635144 \\
\hline $\mathrm{H}$ & 4.761941 & -0.525563 & -8.716283 \\
\hline $\mathrm{H}$ & 6.499939 & 0.288469 & -7.146730 \\
\hline $\mathrm{H}$ & -1.778655 & 3.580123 & -2.573429 \\
\hline $\mathrm{H}$ & -0.463927 & 2.869146 & -1.620898 \\
\hline $\mathrm{H}$ & -1.712689 & 4.305698 & 0.190792 \\
\hline $\mathrm{H}$ & -1.502017 & 6.429042 & 1.490755 \\
\hline $\mathrm{H}$ & -0.227072 & 8.371269 & 0.533519 \\
\hline $\mathrm{H}$ & 0.830422 & 8.165050 & -1.733783 \\
\hline $\mathrm{H}$ & 0.617358 & 6.042172 & -3.032726 \\
\hline $\mathrm{H}$ & 1.925449 & 2.919211 & -2.568452 \\
\hline $\mathrm{H}$ & 1.747719 & 4.439674 & -7.545895 \\
\hline $\mathrm{H}$ & -0.425550 & 5.338452 & -8.421297 \\
\hline & -2.436163 & 5.481972 & -6.946813 \\
\hline & & 4.728402 & \\
\hline
\end{tabular}

A = 1-benzyl-3-indolyl, $B=4$-methoxyphenyl Bispropargyl

54

Free energy $=-1284.663911$ Hartree

C $\quad 6.473861 \quad 7.007174 \quad-1.711475$

$\begin{array}{llll}\text { C } & 7.748451 & 6.925965 & -1.100931\end{array}$

$\begin{array}{llll}\text { C } & 7.803383 & 6.670473 & 0.297180\end{array}$

$\begin{array}{llll}\text { C } & 6.636651 & 6.518543 & 1.045388\end{array}$

$\begin{array}{llll}C & 5.370448 & 6.609050 & 0.425098\end{array}$

$\begin{array}{llll}\text { C } & 5.294662 & 6.858057 & -0.963722\end{array}$

$\begin{array}{llll}\text { C } & 8.952798 & 7.054335 & -1.863554\end{array}$

$\begin{array}{llll}\text { C } & 10.022155 & 7.103227 & -2.468583\end{array}$

$\begin{array}{llll}\text { C } & 11.281678 & 7.148613 & -3.232932\end{array}$

$\begin{array}{llll}\text { O } & 12.468499 & 6.975975 & -2.451734\end{array}$

$\begin{array}{llll}\text { C } & 12.816029 & 5.599945 & -2.210271\end{array}$

$\begin{array}{llll}\text { C } & 11.728413 & 4.830034 & -1.594689\end{array}$

$\begin{array}{llll}\text { C } & 10.682436 & 4.394709 & -1.115368\end{array}$ 


$\begin{array}{cccc}\mathrm{C} & 9.428988 & 3.957459 & -0.632103 \\ \mathrm{C} & 8.247084 & 3.730541 & -1.448169 \\ \mathrm{C} & 7.195085 & 3.355000 & -0.559266 \\ \mathrm{~N} & 7.690633 & 3.427514 & 0.740072 \\ \mathrm{C} & 9.022000 & 3.789114 & 0.694667 \\ \mathrm{C} & 7.978946 & 3.884207 & -2.821988 \\ \mathrm{C} & 6.677999 & 3.640523 & -3.277002 \\ \mathrm{C} & 5.646504 & 3.249206 & -2.384038 \\ \mathrm{C} & 5.886945 & 3.100977 & -1.011359 \\ \mathrm{C} & 6.920836 & 3.100530 & 1.928132 \\ \mathrm{C} & 6.506425 & 1.636280 & 2.003463 \\ \mathrm{C} & 5.357389 & 1.271546 & 2.731859 \\ \mathrm{C} & 4.974792 & -0.076786 & 2.830840 \\ \mathrm{C} & 5.736780 & -1.073877 & 2.199311 \\ \mathrm{C} & 6.882404 & -0.715473 & 1.467404 \\ \mathrm{C} & 7.264993 & 0.631406 & 1.369780 \\ \mathrm{O} & 4.287963 & 6.437559 & 1.244437 \\ \mathrm{C} & 2.993126 & 6.540878 & 0.678216 \\ \mathrm{H} & 13.108995 & 5.113165 & -3.173431 \\ \mathrm{H} & 11.378563 & 8.130998 & -3.744261 \\ \mathrm{H} & 6.410194 & 7.178638 & -2.796796 \\ \mathrm{H} & 4.324987 & 6.921891 & -1.476832 \\ \mathrm{H} & 6.682990 & 6.302716 & 2.123149 \\ \mathrm{H} & 8.780834 & 6.610032 & 0.794180 \\ \mathrm{H} & 7.536584 & 3.372361 & 2.810705 \\ \mathrm{H} & 6.020421 & 3.752410 & 1.964476 \\ \mathrm{H} & 4.750669 & 2.053306 & 3.223242 \\ \mathrm{H} & 4.072612 & -0.348338 & 3.398654 \\ \mathrm{H} & 5.429064 & -2.129722 & 2.268370 \\ \mathrm{H} & 7.481678 & -1.487546 & 0.966113 \\ \mathrm{H} & 8.155766 & 0.914046 & 0.786975 \\ \mathrm{H} & 9.609510 & 3.896446 & 1.616655 \\ \mathrm{H} & 2.277145 & 6.404420 & 1.509552 \\ \mathrm{H} & 2.820728 & 7.539396 & 0.214398 \\ \mathrm{H} & 2.807143 & 5.754719 & -0.091082 \\ \mathrm{H} & 11.232575 & 6.378752 & -4.045628 \\ \mathrm{H} & 8.782803 & 4.192140 & -3.506557 \\ \mathrm{H} & 6.445998 & 3.756693 & -4.348502 \\ \mathrm{H} & 4.633524 & 3.071331 & -2.774812 \\ \mathrm{H} & 5.089039 & 2.804975 & -0.315348 \\ \mathrm{H} & 13.717334 & 5.600791 & -1.559677\end{array}$

Allenyne_A

54

Free energy $=-1284.68113$ Hartree

$\begin{array}{llll}\text { C } & 4.512043 & 6.696157 & 0.709884\end{array}$

$\begin{array}{llll}\text { C } & 5.036716 & 5.537044 & 0.103903\end{array}$

$\begin{array}{llll}\text { C } & 6.343070 & 5.570926 & -0.421318\end{array}$

$\begin{array}{llll}\text { C } & 7.107892 & 6.747045 & -0.349554 \\ \text { C } & 6.574832 & 7.902857 & 0.246883\end{array}$

$\begin{array}{llll}\text { C } & 6.574832 & 7.902857 & 0.246883\end{array}$

$\begin{array}{llll}\text { C } & 5.273221 & 7.874571 & 0.777216\end{array}$

$\begin{array}{llll}\text { C } & 4.179892 & 4.284517 & 0.008436\end{array}$

$\begin{array}{llll}\mathrm{N} & 4.938069 & 3.049670 & -0.058046\end{array}$

$\begin{array}{llll}\text { C } & 5.002490 & 2.191323 & -1.135874\end{array}$

$\begin{array}{llll}\text { C } & 5.770587 & 1.069734 & -0.819009\end{array}$

$\begin{array}{llll}\text { C } & 6.208101 & 1.257629 & 0.551919\end{array}$

$\begin{array}{llll}\text { C } & 5.667106 & 2.508454 & 0.995027\end{array}$

$\begin{array}{llll}\text { C } & 6.009112 & -0.088112 & -1.673598\end{array}$

$\begin{array}{llll}\text { C } & 5.461682 & -0.268810 & -2.875476\end{array}$

$\begin{array}{llll}\text { C } & 4.858232 & -0.301484 & -4.052869\end{array}$

$\begin{array}{llll}\text { O } & 3.605871 & -0.807552 & -4.330201\end{array}$

$\begin{array}{llll}\text { C } & 2.893462 & -1.344280 & -3.202632\end{array}$

$\begin{array}{llll}\text { C } & 2.563868 & -0.375560 & -2.155035\end{array}$

$\begin{array}{llll}\text { C } & 2.285822 & 0.398878 & -1.241641\end{array}$

$\begin{array}{llll}\text { C } & 1.989570 & 1.276340 & -0.154100\end{array}$

$\begin{array}{llll}\text { C } & 2.696716 & 1.165280 & 1.075776\end{array}$

$\begin{array}{llll}\text { C } & 2.431724 & 2.027996 & 2.136883\end{array}$

$\begin{array}{llll}\text { C } & 1.451534 & 3.040332 & 2.008952 \\ \text { C } & 0.735156 & 3.16493 & 0.797388\end{array}$

$\begin{array}{llll}\text { C } & 0.735156 & 3.164493 & 0.797388\end{array}$

$\begin{array}{llll}\text { C } & 1.006335 & 2.289193 & -0.265383\end{array}$

$\begin{array}{llll}\text { O } & 1.295976 & 3.856634 & 3.089915\end{array}$

$\begin{array}{llll}\text { C } & 0.335652 & 4.898770 & 3.023055\end{array}$

$\begin{array}{llll}\mathrm{H} & 5.326568 & 0.074698 & -4.979707\end{array}$

$\begin{array}{llll}\mathrm{H} & 1.969085 & -1.776898 & -3.638312\end{array}$

$\begin{array}{llll}\mathrm{H} & 6.666836 & -0.873603 & -1.252387\end{array}$

$\begin{array}{llll}\mathrm{H} & 0.449088 & 2.391544 & -1.208648\end{array}$

$\begin{array}{llll}\mathrm{H} & -0.030832 & 3.941368 & 0.666387\end{array}$

$\begin{array}{llll}\mathrm{H} & 3.001152 & 1.962772 & 3.074890\end{array}$

$\begin{array}{llll}\mathrm{H} & 3.488795 & 0.408734 & 1.172290\end{array}$

$\begin{array}{llll}\mathrm{H} & 3.532397 & 4.323920 & -0.892704\end{array}$

$\begin{array}{llll}\mathrm{H} & 3.488474 & 4.230201 & 0.876679\end{array}$

$\begin{array}{llll}\mathrm{H} & 3.496332 & 6.672738 & 1.137641\end{array}$

$\begin{array}{llll}\mathrm{H} & 4.852119 & 8.773097 & 1.254473\end{array}$

$\begin{array}{llll}\mathrm{H} & 7.175958 & 8.823419 & 0.306629\end{array}$

$\begin{array}{llll}\mathrm{H} & 8.129539 & 6.758110 & -0.758824\end{array}$

$\begin{array}{llll}\mathrm{H} & 6.764084 & 4.661819 & -0.877809\end{array}$

$\begin{array}{llll}\mathrm{H} & 4.466438 & 2.411366 & -2.06539\end{array}$

$\begin{array}{llll}\text { C } & 5.868814 & 3.005291 & 2.294706\end{array}$

$\begin{array}{llll}\mathrm{H} & 0.373960 & 5.421683 & 3.997080\end{array}$

$\begin{array}{llll}\mathrm{H} & 0.567832 & 5.627588 & 2.212305\end{array}$

$\begin{array}{rrrc}\mathrm{H} & -0.694900 & 4.507178 & 2.864304 \\ \mathrm{H} & 3.485765 & -2.176014 & -2.748692 \\ \mathrm{C} & 6.965080 & 0.485987 & 1.461767 \\ \mathrm{C} & 7.163995 & 0.970881 & 2.760905 \\ \mathrm{C} & 6.623775 & 2.216437 & 3.172978 \\ \mathrm{H} & 7.386927 & -0.483515 & 1.156259 \\ \mathrm{H} & 7.750756 & 0.376478 & 3.478305 \\ \mathrm{H} & 6.803628 & 2.570724 & 4.199316 \\ \mathrm{H} & 5.452154 & 3.974352 & 2.604431\end{array}$

Allenyne_A2Cyclization_TS

Free energy $=-1284.670613$ Hartree

$\begin{array}{llll}\text { C } & 4.726314 & 6.624633 & 1.351480 \\ \text { C } & 5.245812 & 5.753771 & 0.363319\end{array}$

$\begin{array}{llll}\text { C } & 5.245812 & 5.753771 & 0.36331\end{array}$

$\begin{array}{llll}\text { C } & 6.415712 & 6.119694 & -0.328656\end{array}$

$\begin{array}{llll}\text { C } & 7.032961 & 7.350849 & -0.050871\end{array}$

$\begin{array}{llll}\text { C } & 6.484328 & 8.229203 & 0.910145\end{array}$

$\begin{array}{llll}\text { C } & 5.329802 & 7.862336 & 1.614261\end{array}$

$\begin{array}{llll}C & 4.491502 & 4.467277 & 0.053411\end{array}$

$\begin{array}{llll}\mathrm{N} & 5.307028 & 3.273904 & -0.154578\end{array}$

$\begin{array}{llll}\text { C } & 5.141687 & 2.369467 & -1.196353\end{array}$

$\begin{array}{llll}\text { C } & 5.945213 & 1.244815 & -1.010584\end{array}$

$\begin{array}{llll}\text { C } & 6.617329 & 1.455728 & 0.256349\end{array}$

$\begin{array}{llll}\text { C } & 6.173940 & 2.715925 & 0.776095\end{array}$

$\begin{array}{llll}\text { C } & 6.076610 & 0.067494 & -1.846098\end{array}$

$\begin{array}{llll}\text { C } & 5.323263 & -0.347013 & -2.855815\end{array}$

$\begin{array}{llll}\text { C } & 4.603577 & -0.701937 & -3.886605\end{array}$

$\begin{array}{llll}\text { O } & 3.478505 & -1.515994 & -3.882294\end{array}$

$\begin{array}{llll}\text { C } & 3.019094 & -1.982102 & -2.610546\end{array}$

$\begin{array}{llll}\text { C } & 2.555646 & -0.889573 & -1.756494\end{array}$

$\begin{array}{llll}\text { C } & 2.186855 & -0.035153 & -0.968958\end{array}$

$\begin{array}{llll}\text { C } & 1.733000 & 0.970631 & -0.062440\end{array}$

$\begin{array}{llll}\text { C } & 2.550625 & 1.445025 & 0.973708\end{array}$

$\begin{array}{llll}\text { C } & 2.152087 & 2.502393 & 1.809059\end{array}$

$\begin{array}{llll}\text { C } & 0.927200 & 3.109116 & 1.627428\end{array}$

$\begin{array}{llll}\text { C } & 0.070552 & 2.613432 & 0.627717\end{array}$

$\begin{array}{llll}\text { C } & 0.481489 & 1.559135 & -0.202762\end{array}$

$\begin{array}{llll}\text { O } & 0.695477 & 4.238379 & 2.360861\end{array}$

$\begin{array}{llll}\text { C } & -0.601171 & 4.756182 & 2.419943\end{array}$

$\begin{array}{llll}\mathrm{H} & 4.833419 & -0.367405 & -4.898688\end{array}$

$\mathrm{H} \quad 2.151860 \quad-2.684252 \quad-2.801017$

$\mathrm{H} \quad \begin{array}{llll}6.923211 & -0.592083 & -1.536171\end{array}$

$\begin{array}{llll}\mathrm{H} & -0.197693 & 1.173942 & -0.978342\end{array}$

$\begin{array}{lllr}\mathrm{H} & -0.197693 & 1.173942 & -0.978342 \\ \mathrm{H} & -0.894597 & 3.062778 & 0.465697\end{array}$

$\begin{array}{llll}\mathrm{H} & 2.836768 & 2.934704 & 2.539774\end{array}$

$\begin{array}{llll}\mathrm{H} & 3.523398 & 1.040044 & 1.063065\end{array}$

$\begin{array}{llll}\mathrm{H} & 3.922341 & 4.600510 & -0.896624\end{array}$

$\begin{array}{llll}\mathrm{H} & 3.753183 & 4.266086 & 0.861636\end{array}$

$\begin{array}{llll}\mathrm{H} & 3.843966 & 6.308412 & 1.927253\end{array}$

$\begin{array}{llll}\mathrm{H} & 4.904844 & 8.539511 & 2.376911\end{array}$

$\begin{array}{llll}\mathrm{H} & 6.963746 & 9.195663 & 1.117353\end{array}$

$\begin{array}{llll}\mathrm{H} & 7.949242 & 7.629824 & -0.587290\end{array}$

$\begin{array}{llll}\mathrm{H} & 6.850818 & 5.427174 & -1.075447\end{array}$

$\begin{array}{llll}\mathrm{H} & 4.463299 & 2.583698 & -2.021411\end{array}$

$\begin{array}{llll}\text { C } & 6.567812 & 3.187677 & 2.039467\end{array}$

$\begin{array}{llll}\mathrm{H} & -0.609983 & 5.459850 & 3.217703\end{array}$

$\begin{array}{llll}\mathrm{H} & -0.913108 & 5.244289 & 1.507987\end{array}$

$\begin{array}{llll}\mathrm{H} & -1.310642 & 3.990848 & 2.626779\end{array}$

$\begin{array}{llll}\mathrm{H} & 3.867464 & -2.583791 & -2.095152\end{array}$

$\begin{array}{llll}\text { C } & 7.481812 & 0.661460 & 1.033531\end{array}$

$\begin{array}{llll}\text { C } & 7.885722 & 1.129804 & 2.284449\end{array}$

$\begin{array}{llll}\text { C } & 7.430854 & 2.376540 & 2.784396\end{array}$

$\begin{array}{llll}\mathrm{H} & 7.827439 & -0.318450 & 0.665432\end{array}$

$\begin{array}{llll}H & 8.562197 & 0.519405 & 2.899226\end{array}$

$\begin{array}{llll}H & 7.768330 & 2.711079 & 3.774621\end{array}$

$\begin{array}{llll}\mathrm{H} & 6.208414 & 4.147467 & 2.433511\end{array}$

Allenyne_B

Free energy $=-1284.68838$ Hartree

$\begin{array}{llll}\text { C } & 5.680195 & 6.540216 & -0.307690\end{array}$

$\begin{array}{llll}\text { C } & 4.508955 & 6.445719 & 0.469092\end{array}$

$\begin{array}{llll}\text { C } & 4.074801 & 7.574483 & 1.193117\end{array}$

$\begin{array}{llll}\text { C } & 4.790836 & 8.781468 & 1.132060\end{array}$

$\begin{array}{llll}\text { C } & 5.958095 & 8.869051 & 0.353722\end{array}$

$\begin{array}{llll}\text { C } & 6.401441 & 7.744335 & -0.363178\end{array}$

$\begin{array}{llll}C & 3.696929 & 5.161070 & 0.524040\end{array}$

$\begin{array}{lll}4.468508 & 3.959069 & 0.254597\end{array}$

$\begin{array}{llll}\text { C } & 4.240797 & 3.062880 & -0.768456\end{array}$

$\begin{array}{llll}\text { C } & 5.096490 & 1.964386 & -0.652002\end{array}$

$\begin{array}{llll}\text { C } & 5.897900 & 2.203835 & 0.535854\end{array}$

$\begin{array}{llll}\text { C } & 5.877694 & 3.462169 & 1.072562\end{array}$

$\begin{array}{llll}\text { C } & 5.086635 & 0.820736 & -1.485526\end{array}$

$\begin{array}{llll}\text { C } & 5.020550 & -0.169399 & -2.212400\end{array}$

$\begin{array}{llll}\text { C } & 4.848883 & -1.310790 & -3.108803\end{array}$

$\begin{array}{llll}\text { C } & 4.420440 & -2.535902 & -2.478976\end{array}$

$\begin{array}{llll}\text { C } & 3.201247 & -2.500563 & -1.847828\end{array}$

$\begin{array}{llll}\text { C } & 2.368403 & -1.474406 & -1.747160\end{array}$ 


$\begin{array}{lrrr}\mathrm{C} & 1.608423 & -0.419579 & -1.464575 \\ \mathrm{C} & 1.805648 & 0.464536 & -0.298458 \\ \mathrm{C} & 1.077745 & 1.674643 & -0.181531 \\ \mathrm{C} & 1.305219 & 2.559427 & 0.877894 \\ \mathrm{C} & 2.267771 & 2.250414 & 1.865180 \\ \mathrm{C} & 2.986100 & 1.037679 & 1.779061 \\ \mathrm{C} & 2.752231 & 0.168076 & 0.708222 \\ \mathrm{O} & 2.445508 & 3.183869 & 2.848575 \\ \mathrm{C} & 3.263483 & 2.842575 & 3.963994 \\ \mathrm{H} & 4.113214 & -1.031489 & -3.902363 \\ \mathrm{H} & 2.964769 & -3.491981 & -1.422067 \\ \mathrm{H} & 0.789239 & -0.132854 & -2.153735 \\ \mathrm{H} & 3.339632 & -0.758212 & 0.624599 \\ \mathrm{H} & 3.756443 & 0.783006 & 2.518946 \\ \mathrm{H} & 0.743979 & 3.500902 & 0.969959 \\ \mathrm{H} & 0.325682 & 1.928998 & -0.946726 \\ \mathrm{H} & 2.875213 & 5.195286 & -0.221813 \\ \mathrm{H} & 3.208899 & 5.048465 & 1.517151 \\ \mathrm{H} & 6.035107 & 5.656026 & -0.859088 \\ \mathrm{H} & 7.320495 & 7.801612 & -0.966498 \\ \mathrm{H} & 6.525152 & 9.811836 & 0.311727 \\ \mathrm{H} & 4.439594 & 9.656113 & 1.701192 \\ \mathrm{H} & 3.164828 & 7.506595 & 1.811771 \\ \mathrm{H} & 3.447770 & 3.239201 & -1.502585 \\ \mathrm{C} & 6.047514 & 4.010522 & 2.236299 \\ \mathrm{H} & 3.216923 & 3.705002 & 4.655973 \\ \mathrm{H} & 2.878993 & 1.936064 & 4.483391 \\ \mathrm{H} & 4.322312 & 2.677304 & 3.669206 \\ \mathrm{H} & 5.803542 & -1.581354 & -3.605804 \\ \mathrm{C} & 6.892678 & 1.464439 & 1.208430 \\ \mathrm{C} & 7.449317 & 1.995501 & 2.379533 \\ \mathrm{C} & 7.036034 & 3.255746 & 2.884153 \\ \mathrm{H} & 7.214343 & 0.489573 & 0.812677 \\ \mathrm{H} & 8.227721 & 1.431403 & 2.916034 \\ \mathrm{H} & 7.500565 & 3.650473 & 3.800351 \\ \mathrm{H} & 5.726174 & 4.989669 & 2.617478 \\ & & & \\ & & & \end{array}$

Allenyne_B2Cyclization_TS

54

Free energy $=-1284.671913$ Hartree

$\begin{array}{llll}\text { C } & 5.697653 & 6.482873 & -0.623512 \\ \text { C } & 4.609262 & 6.506515 & 0.270071\end{array}$

$\begin{array}{llll}\text { C } & 4.609262 & 6.506515 & 0.270071\end{array}$

$\begin{array}{llll}\text { C } & 4.304080 & 7.705330 & 0.945475\end{array}$

$\begin{array}{llll}\text { C } & 5.064164 & 8.865129 & 0.722047\end{array}$

$\begin{array}{llll}\text { C } & 6.147376 & 8.835238 & -0.173035\end{array}$

$\begin{array}{llll}\text { C } & 6.463118 & 7.640079 & -0.841899\end{array}$

$\begin{array}{llll}\text { C } & 3.745871 & 5.275567 & 0.496063\end{array}$

$\begin{array}{llll}\mathrm{N} & 4.425136 & 4.016825 & 0.238781\end{array}$

$\begin{array}{lllr}\mathrm{C} & 4.013448 & 3.051851 & -0.658522 \\ \mathrm{C} & 4.793865 & 1.82146 & -0.526864\end{array}$

$\begin{array}{llll}\text { C } & 4.793865 & 1.892146 & -0.526864\end{array}$

$\begin{array}{llll}\text { C } & 5.736233 & 2.175806 & 0.548328\end{array}$

$\begin{array}{llll}\text { C } & 5.476791 & 3.510204 & 0.994348\end{array}$

$\begin{array}{llll}\text { C } & 4.619507 & 0.690266 & -1.228964\end{array}$

$\begin{array}{llll}\text { C } & 4.294604 & -0.376975 & -1.818220\end{array}$

$\begin{array}{llll}\text { C } & 4.661740 & -1.279196 & -2.95233\end{array}$

$\begin{array}{llll}\text { O } & 4.181254 & -2.612061 & -2.720282\end{array}$

$\begin{array}{llll}\text { C } & 3.042530 & -2.565695 & -1.969896\end{array}$

$\begin{array}{llll}\text { C } & 2.590545 & -1.398925 & -1.473034 \\ \text { C } & 1.544813 & -0.658601 & -0.996725\end{array}$

$\begin{array}{llll}\text { C } & 1.544813 & -0.658601 & -0.996725\end{array}$

$\begin{array}{llll}\text { C } & 1.709706 & 0.256925 & 0.116738\end{array}$

$\begin{array}{llll}\text { C } & 1.709786 & 0.256925 & 0.116738 \\ \text { C } & 1.228250 & 1.435931 & 0.232344\end{array}$

$\begin{array}{llll}\text { C } & 1.228250 & 2.421028 & 1.167582\end{array}$

$\begin{array}{llll}\text { C } & 2.320155 & 2.244601 & 2.056242\end{array}$

$\begin{array}{llll}\text { C } & 3.059595 & 1.045046 & 2.022589\end{array}$

$\begin{array}{llll}\text { C } & 2.760886 & 0.079263 & 1.054493\end{array}$

$\begin{array}{llll}\text { O } & 2.577242 & 3.284579 & 2.904137\end{array}$

$\begin{array}{llll}\text { C } & 3.499419 & 3.073497 & 3.970200\end{array}$

H $\quad 4.193717 \quad-0.883294 \quad-3.884386$

$\begin{array}{llll}\mathrm{H} & 2.641012 & -3.562321 & -1.730249\end{array}$

$\begin{array}{llll}\mathrm{H} & 0.624394 & -0.566919 & -1.603614\end{array}$

$\begin{array}{llll}\mathrm{H} & 3.365600 & -0.837528 & 0.998433\end{array}$

$\begin{array}{llll}\mathrm{H} & 3.906449 & 0.882171 & 2.702101\end{array}$

$\begin{array}{llll}\mathrm{H} & 0.643489 & 3.350244 & 1.241107\end{array}$

$\begin{array}{llll}\mathrm{H} & 0.079333 & 1.591530 & -0.464707\end{array}$

\begin{tabular}{llll}
$\mathrm{H}$ & 2.857851 & 5.307106 & -0.169015 \\
\hline
\end{tabular}

$\begin{array}{llll}\mathrm{H} & 3.349487 & 5.255153 & 1.534783\end{array}$

$\begin{array}{llll}\mathrm{H} & 5.951690 & 5.544685 & -1.140396\end{array}$

$\begin{array}{llll}\mathrm{H} & 7.316012 & 7.605990 & -1.537743\end{array}$

$\begin{array}{llll}\mathrm{H} & 6.748456 & 9.741528 & -0.344650\end{array}$

$\begin{array}{llll}\mathrm{H} & 4.813222 & 9.795212 & 1.255080\end{array}$

$\begin{array}{llll}\mathrm{H} & 3.459547 & 7.730451 & 1.654221\end{array}$

H $\quad 3.156936 \quad 3.226954 \quad-1.317496$

$\begin{array}{llll}\text { C } & 6.206455 & 4.106668 & 2.038654\end{array}$

$\begin{array}{llll}\mathrm{H} & 3.512366 & 4.011441 & 4.557171\end{array}$

$\begin{array}{llll}\mathrm{H} & 3.170650 & 2.235474 & 4.624980\end{array}$

$\begin{array}{llll}\mathrm{H} & 4.526892 & 2.870124 & 3.600178\end{array}$

$\begin{array}{llll}\mathrm{H} & 5.756997 & -1.349950 & -3.10198\end{array}$

$\begin{array}{llll}\text { C } & 6.736484 & 1.416233 & 1.186916\end{array}$

$\begin{array}{llll}\text { C } & 7.455576 & 1.997718 & 2.240483\end{array}$

$\begin{array}{llll}\text { C } & 7.196091 & 3.328476 & 2.657647\end{array}$

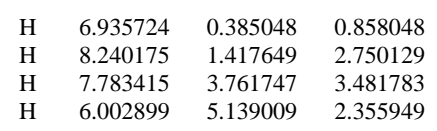

Allenyne_B2Cyclization_INT

54

Free energy $=-1284.737664$ Hartree

$\begin{array}{llll}\text { C } & 5.784030 & 6.060855 & -0.92986\end{array}$

$\begin{array}{llll}\text { C } & 5.586318 & 6.635241 & 0.340518\end{array}$

$\begin{array}{llll}\text { C } & 5.998447 & 7.964185 & 0.562852\end{array}$

$\begin{array}{llll}\text { C } & 6.587803 & 8.712009 & -0.469461\end{array}$

$\begin{array}{llll}\text { C } & 6.778943 & 8.134079 & -1.736645\end{array}$

$\begin{array}{llll}\text { C } & 6.377793 & 6.806601 & -1.961834\end{array}$

$\begin{array}{llll}\mathrm{C} & 4.914841 & 5.853111 & 1.463584\end{array}$

$\begin{array}{llll}\mathrm{N} & 4.969584 & 4.413613 & 1.297267\end{array}$

$\begin{array}{llll}\text { C } & 3.938113 & 3.607921 & 0.829456\end{array}$

$\begin{array}{llll}\text { C } & 4.384287 & 2.302452 & 0.640996\end{array}$

$\begin{array}{llll}\text { C } & 5.788126 & 2.309437 & 1.006526\end{array}$

$\begin{array}{llll}\text { C } & 6.122552 & 3.645873 & 1.396724\end{array}$

$\begin{array}{llll}\text { C } & 3.636889 & 1.135459 & 0.131893\end{array}$

$\begin{array}{llll}\text { C } & 4.001357 & 0.548024 & -1.039203\end{array}$

$\begin{array}{llll}\text { C } & 5.070888 & 0.933176 & -2.037571\end{array}$

$\begin{array}{llll}\text { O } & 5.013359 & -0.089337 & -3.077598\end{array}$

$\begin{array}{llll}\text { C } & 3.985933 & -0.929745 & -2.772686\end{array}$

$\begin{array}{llll}\text { C } & 3.336283 & -0.627285 & -1.600260\end{array}$

$\begin{array}{llll}\text { C } & 2.158374 & -1.140245 & -0.959997\end{array}$

$\begin{array}{llll}\text { C } & 1.714053 & -0.575095 & 0.215574\end{array}$

$\begin{array}{llll}\text { C } & 0.430900 & -0.930137 & 0.782381\end{array}$

$\begin{array}{llll}\text { C } & -0.158914 & -0.177493 & 1.760224\end{array}$

$\begin{array}{llll}\text { C } & 0.483923 & 1.036814 & 2.252400\end{array}$

$\begin{array}{llll}\text { C } & 1.746303 & 1.376742 & 1.860721\end{array}$

$\begin{array}{llll}\text { C } & 2.566245 & 0.438555 & 0.994749\end{array}$

$\begin{array}{llll}\text { O } & -0.314552 & 1.751973 & 3.105754\end{array}$

$\begin{array}{llll}\text { C } & 0.211880 & 2.952633 & 3.638740\end{array}$

\begin{tabular}{llll}
$\mathrm{H}$ & 4.888061 & 1.924080 & -2.510501 \\
\hline & 3.807717 & -1.733288 & -3.500230
\end{tabular}

$\begin{array}{llll}\mathrm{H} & 3.807717 & -1.733288 & -3.500230\end{array}$

$\begin{array}{llll}\mathrm{H} & 1.564081 & -1.929620 & -1.448476\end{array}$

$\begin{array}{llll}\mathrm{H} & 3.156025 & -0.187440 & 1.724768\end{array}$

$\begin{array}{llll}\mathrm{H} & 2.259680 & 2.257956 & 2.264284\end{array}$

$\begin{array}{llll}\mathrm{H} & -1.157055 & -0.416983 & 2.154920\end{array}$

$\mathrm{H} \quad-0.107165 \quad-1.790170 \quad 0.351388$

$\begin{array}{llll}\mathrm{H} & 3.844682 & 6.141003 & 1.538354\end{array}$

\begin{tabular}{llll}
$\mathrm{H}$ & 5.375591 & 6.124375 & 2.438274 \\
\hline
\end{tabular}

$\mathrm{H} \quad 5.478256 \quad 5.018272 \quad-1.108066$

$\begin{array}{llll}\mathrm{H} & 6.532318 & 6.343877 & -2.949004\end{array}$

$\begin{array}{llll}\mathrm{H} & 7.245430 & 8.716428 & -2.546029\end{array}$

$\begin{array}{llll}\mathrm{H} & 6.906564 & 9.748613 & -0.280706\end{array}$

$\begin{array}{llll}\mathrm{H} & 5.858882 & 8.419577 & 1.557646\end{array}$

$\begin{array}{llll}\mathrm{H} & 2.946487 & 4.035439 & 0.640495\end{array}$

$\begin{array}{llll}\text { C } & 7.424431 & 4.009868 & 1.788165\end{array}$

$\begin{array}{llll}\mathrm{H} & -0.571873 & 3.382194 & 4.291555\end{array}$

$\begin{array}{llll}\mathrm{H} & 1.131573 & 2.770784 & 4.242901\end{array}$

$\begin{array}{llll}\mathrm{H} & 0.461677 & 3.685847 & 2.836312\end{array}$

$\begin{array}{llll}\mathrm{H} & 6.098411 & 0.942673 & -1.616907\end{array}$

$\begin{array}{llll}\text { C } & 6.786021 & 1.309892 & 1.047414\end{array}$

$\begin{array}{llll}\text { C } & 8.080387 & 1.664845 & 1.445643\end{array}$

$\begin{array}{llll}\text { C } & 8.396098 & 3.000439 & 1.807019\end{array}$

$\begin{array}{llll}\mathrm{H} & 6.535431 & 0.273353 & 0.771654\end{array}$

$\begin{array}{llll}\mathrm{H} & 8.869863 & 0.898205 & 1.479629\end{array}$

$\mathrm{H} \quad 9.425117 \quad 3.249883 \quad 2.107954$

$\begin{array}{llll}\mathrm{H} & 7.672904 & 5.047113 & 2.056744\end{array}$

Bisallene

54

Free energy $=-1284.697162$ Hartree

$\begin{array}{llll}\text { C } & 4.138666 & 5.421389 & 3.360663\end{array}$

$\begin{array}{llll}\text { C } & 3.010447 & 4.710901 & 3.818822\end{array}$

$\begin{array}{llll}\text { C } & 1.906798 & 5.433754 & 4.314981\end{array}$

$\begin{array}{llll}\text { C } & 1.922837 & 6.838434 & 4.336775\end{array}$

$\begin{array}{llll}\text { C } & 3.046220 & 7.537954 & 3.862239\end{array}$

$\begin{array}{llll}\text { C } & 4.157361 & 6.825869 & 3.378162\end{array}$

$\begin{array}{llll}C & 2.960437 & 3.198180 & 3.691521\end{array}$

$\begin{array}{llll}\mathrm{N} & 2.458034 & 2.762531 & 2.390935\end{array}$

$\begin{array}{llll}\mathrm{C} & 3.228115 & 2.626528 & 1.249851\end{array}$

$\begin{array}{llll}\text { C } & 2.421094 & 2.296002 & 0.160993\end{array}$

$\begin{array}{llll}\text { C } & 1.063422 & 2.236405 & 0.667822\end{array}$

$\begin{array}{llll}\text { C } & 1.121037 & 2.554971 & 2.062324\end{array}$

$\begin{array}{llll}\text { C } & 2.820722 & 2.172489 & -1.230009\end{array}$

$\begin{array}{llll}\text { C } & 4.038972 & 2.391019 & -1.728281\end{array}$

$\begin{array}{llll}\text { C } & 5.249323 & 2.513331 & -2.244836\end{array}$

$\begin{array}{llll}\text { O } & 5.850559 & 3.681951 & -2.687774\end{array}$

$\begin{array}{llll}\text { C } & 5.293924 & 4.890729 & -2.304960\end{array}$

$\begin{array}{llll}\text { C } & 4.423941 & 5.100397 & -1.330858\end{array}$

$\begin{array}{llll}\text { C } & 3.582597 & 5.445076 & -0.35580\end{array}$

$\begin{array}{llll}\text { C } & 2.116211 & 5.433061 & -0.413475\end{array}$

$\begin{array}{llll}\text { C } & 1.406239 & 5.036874 & -1.569219\end{array}$

$\begin{array}{llll}\text { C } & 0.010028 & 4.915424 & -1.559812\end{array}$

$\begin{array}{llll}\text { C } & -0.711503 & 5.185853 & -0.374595\end{array}$ 


$\begin{array}{crrr}\mathrm{C} & -0.017613 & 5.614733 & 0.778377 \\ \mathrm{C} & 1.370121 & 5.738917 & 0.752676 \\ \mathrm{O} & -2.061662 & 5.046610 & -0.240823 \\ \mathrm{C} & -2.800007 & 4.540608 & -1.339500 \\ \mathrm{H} & 5.925789 & 1.658455 & -2.415899 \\ \mathrm{H} & 5.707033 & 5.705657 & -2.923386 \\ \mathrm{H} & 2.000816 & 1.958827 & -1.942698 \\ \mathrm{H} & 4.007397 & 5.706908 & 0.633238 \\ \mathrm{H} & 1.971311 & 4.796105 & -2.482668 \\ \mathrm{H} & -0.504562 & 4.585663 & -2.472402 \\ \mathrm{H} & -0.593220 & 5.813570 & 1.693363 \\ \mathrm{H} & 1.898870 & 6.054102 & 1.663364 \\ \mathrm{H} & 2.313290 & 2.756531 & 4.475030 \\ \mathrm{H} & 3.972521 & 2.765097 & 3.823823 \\ \mathrm{H} & 1.016799 & 4.893370 & 4.673263 \\ \mathrm{H} & 1.049888 & 7.389249 & 4.719466 \\ \mathrm{H} & 3.056437 & 8.638859 & 3.871009 \\ \mathrm{H} & 5.042440 & 7.366099 & 3.008501 \\ \mathrm{H} & 5.010313 & 4.867172 & 2.976536 \\ \mathrm{H} & 4.310655 & 2.793581 & 1.274333 \\ \mathrm{C} & -0.037077 & 2.628074 & 2.858893 \\ \mathrm{H} & -3.851555 & 4.467280 & -1.003188 \\ \mathrm{H} & -2.750749 & 5.216012 & -2.224714 \\ \mathrm{H} & -2.447151 & 3.528569 & -1.641946 \\ \mathrm{C} & -0.190430 & 1.975247 & 0.074790 \\ \mathrm{C} & -1.344024 & 2.043604 & 0.864040 \\ \mathrm{C} & -1.267880 & 2.370846 & 2.240393 \\ \mathrm{H} & -0.257948 & 1.734309 & -0.996654 \\ \mathrm{H} & -2.327985 & 1.850771 & 0.411095 \\ \mathrm{H} & -2.192422 & 2.428650 & 2.834742 \\ \mathrm{H} & 0.012013 & 2.884798 & 3.926698 \\ & & & \end{array}$

Bisallene2Cyclization_TS

54

Free energy $=-1284.695553$ Hartree

$\begin{array}{llll}\text { C } & 4.138550 & 5.534378 & 3.318734\end{array}$

$\begin{array}{llll}\text { C } & 3.039679 & 4.788140 & 3.791132 \\ \text { C } & 1.914813 & 5.474979 & 4.290759\end{array}$

$\begin{array}{llll}\text { C } & 1.914813 & 5.474979 & 4.290759\end{array}$

$\begin{array}{llll}\text { C } & 1.881080 & 6.879410 & 4.301859\end{array}$

$\begin{array}{llll}\text { C } & 2.974739 & 7.614531 & 3.811581\end{array}$

$\begin{array}{llll}\text { C } & 4.106715 & 6.938776 & 3.323957\end{array}$

$\begin{array}{llll}\text { C } & 3.041203 & 3.274148 & 3.672420\end{array}$

$\begin{array}{llll}\mathrm{N} & 2.533105 & 2.814505 & 2.382384\end{array}$

$\begin{array}{llll}\text { C } & 3.285557 & 2.713743 & 1.226948\end{array}$

$\begin{array}{llll}\text { C } & 2.470410 & 2.334594 & 0.152868\end{array}$

$\begin{array}{llll}\text { C } & 1.125799 & 2.213037 & 0.692172\end{array}$

$\begin{array}{llll}\text { C } & 1.198968 & 2.542818 & 2.081689\end{array}$

$\begin{array}{llll}\text { C } & 2.847201 & 2.275434 & -1.234488\end{array}$

$\begin{array}{llll}\text { C } & 4.042193 & 2.683872 & -1.724544\end{array}$

$\begin{array}{llll}\text { C } & 5.204696 & 2.637864 & -2.368614\end{array}$

$\begin{array}{lllll}\text { O } & 5.882223 & 3.775841 & -2.76109\end{array}$

$\begin{array}{llll}\text { C } & 5.311586 & 4.925326 & -2.251627\end{array}$

$\begin{array}{llll}\text { C } & 4.337886 & 4.910924 & -1.344483\end{array}$

$\begin{array}{llll}\text { C } & 3.519638 & 5.377544 & -0.371532\end{array}$

$\begin{array}{llll}\text { C } & 2.065407 & 5.401590 & -0.42702\end{array}$

$\begin{array}{llll}\text { C } & 1.348180 & 4.980744 & -1.574025\end{array}$

$\begin{array}{llll}\text { C } & -0.047316 & 4.839728 & -1.549435\end{array}$

$\begin{array}{llll}\text { C } & -0.759975 & 5.113244 & -0.361768\end{array}$

$\begin{array}{llll}\text { C } & -0.063464 & 5.584024 & 0.77652\end{array}$

$\begin{array}{llll}\text { C } & 1.318960 & 5.729148 & 0.738565\end{array}$

$\begin{array}{llll}\text { O } & -2.103530 & 4.938544 & -0.204923\end{array}$

$\begin{array}{llll}\text { C } & -2.842357 & 4.387939 & -1.281897\end{array}$

$\begin{array}{llll}\mathrm{H} & 5.756295 & 1.722446 & -2.632794\end{array}$

$\begin{array}{llll}\mathrm{H} & 5.743762 & 5.830819 & -2.704350\end{array}$

$\begin{array}{llll}\mathrm{H} & 2.033779 & 2.062082 & -1.952707\end{array}$

$\begin{array}{llll}\mathrm{H} & 3.962953 & 5.626925 & 0.611387\end{array}$

$\begin{array}{llll}\mathrm{H} & 1.906926 & 4.743815 & -2.491680\end{array}$

$\begin{array}{llll}\mathrm{H} & -0.565212 & 4.488375 & -2.452112\end{array}$

$\begin{array}{llll}\mathrm{H} & -0.636505 & 5.792881 & 1.691076\end{array}$

$\begin{array}{llll}\mathrm{H} & 1.850808 & 6.068014 & 1.638819\end{array}$

$\begin{array}{llll}\mathrm{H} & 2.423147 & 2.813401 & 4.468205\end{array}$

\begin{tabular}{llll}
$\mathrm{H}$ & 4.070232 & 2.877912 & 3.789954 \\
\hline
\end{tabular}

$\begin{array}{llll}\mathrm{H} & 1.047257 & 4.905740 & 4.659185\end{array}$

$\begin{array}{llll}\mathrm{H} & 0.992398 & 7.402028 & 4.687910\end{array}$

$\begin{array}{llll}\mathrm{H} & 2.945170 & 8.715159 & 3.810722\end{array}$

$\begin{array}{llll}\mathrm{H} & 4.968555 & 7.507175 & 2.941915\end{array}$

$\begin{array}{llll}\mathrm{H} & 5.027202 & 5.008064 & 2.934416\end{array}$

$\begin{array}{llll}\mathrm{H} & 4.360586 & 2.921869 & 1.229012\end{array}$

$\begin{array}{llll}\text { C } & 0.055093 & 2.572513 & 2.900775\end{array}$

$\mathrm{H} \quad-3.885178 \quad 4.285732 \quad-0.926733$

$\begin{array}{llll}\mathrm{H} & -2.829866 & 5.048423 & -2.179477\end{array}$

$\begin{array}{llll}\mathrm{H} & -2.460241 & 3.383188 & -1.57271\end{array}$

$\begin{array}{llll}\text { C } & -0.126278 & 1.901310 & 0.123939\end{array}$

$\begin{array}{llll}\text { C } & -1.267891 & 1.928124 & 0.934582\end{array}$

$\begin{array}{llll}\text { C } & -1.176804 & 2.263277 & 2.307353\end{array}$

$\mathrm{H} \quad-0.202764 \quad 1.654491 \quad-0.945525$

$\begin{array}{llll}\mathrm{H} & -2.252012 & 1.695208 & 0.501249\end{array}$

$\begin{array}{llll}\mathrm{H} & -2.090590 & 2.286258 & 2.920526\end{array}$

$\begin{array}{llll}\mathrm{H} & 0.114115 & 2.837346 & 3.966137\end{array}$
Bisallene2Cyclization_INT1

54

Free energy $=-1284.735338$ Hartree

$\begin{array}{llll}\text { C } & -2.192399 & 5.890055 & -0.817252\end{array}$

$\begin{array}{llll}\text { C } & -0.844917 & 5.507918 & -0.954885\end{array}$

$\begin{array}{llll}\text { C } & 0.169273 & 6.426132 & -0.619870\end{array}$

$\begin{array}{llll}\text { C } & -0.157992 & 7.706803 & -0.148975\end{array}$

$\begin{array}{llll}\text { C } & -1.507500 & 8.082999 & -0.014321\end{array}$

$\begin{array}{llll}\text { C } & -2.524436 & 7.173627 & -0.348863\end{array}$

$\begin{array}{llll}\text { C } & -0.466106 & 4.120774 & -1.443973\end{array}$

$\begin{array}{llll}\mathrm{N} & 0.334182 & 4.124362 & -2.663862\end{array}$

$\begin{array}{llll}\text { C } & -0.152814 & 4.425417 & -3.935906\end{array}$

$\begin{array}{llll}\text { C } & 0.914887 & 4.240159 & -4.865964\end{array}$

$\begin{array}{llll}\text { C } & 2.090301 & 3.812983 & -4.103349\end{array}$

$\begin{array}{llll}\text { C } & 1.671134 & 3.808420 & -2.750148\end{array}$

$\begin{array}{lllll}\text { C } & -1.435236 & 4.841560 & -4.332241\end{array}$

$\begin{array}{llll}\text { C } & -1.644153 & 5.067026 & -5.701311\end{array}$

$\begin{array}{llll}\text { C } & -0.601701 & 4.885811 & -6.641422\end{array}$

$\begin{array}{llll}\text { C } & 0.676987 & 4.475352 & -6.233517\end{array}$

$\begin{array}{llll}\text { C } & 3.317946 & 3.389573 & -4.668547\end{array}$

$\begin{array}{llll}\text { C } & 4.522726 & 2.944005 & -4.054339\end{array}$

$\begin{array}{llll}\text { C } & 5.085545 & 3.049499 & -2.679098\end{array}$

$\begin{array}{llll}\text { C } & 6.167269 & 2.147210 & -2.678740\end{array}$

$\begin{array}{llll}\text { O } & 6.353908 & 1.600092 & -3.903123\end{array}$

$\begin{array}{llll}\text { C } & 5.404014 & 2.079286 & -4.734762\end{array}$

$\begin{array}{llll}\text { C } & 4.827264 & 3.880723 & -1.548501\end{array}$

$\begin{array}{llll}\text { C } & 4.220659 & 5.170249 & -1.435882\end{array}$

$\begin{array}{llll}\text { C } & 4.147464 & 5.795223 & -0.142386\end{array}$

$\begin{array}{llll}\text { C } & 3.670360 & 7.087408 & 0.029022\end{array}$

$\begin{array}{llll}\text { C } & 3.223328 & 7.840593 & -1.088697\end{array}$

$\begin{array}{llll}\text { C } & 3.262469 & 7.256871 & -2.369887\end{array}$

$\begin{array}{llll}\text { C } & 3.736732 & 5.947457 & -2.53531\end{array}$

$\begin{array}{llll}\mathrm{O} & 2.752020 & 9.094119 & -0.812091\end{array}$

$\begin{array}{llll}\mathrm{H} & 5.395896 & 1.683461 & -5.755459\end{array}$

$\begin{array}{llll}\mathrm{H} & 6.904003 & 1.891916 & -1.910286\end{array}$

$\begin{array}{llll}\mathrm{H} & 3.290511 & 3.248904 & -5.763170\end{array}$

$\begin{array}{llll}\mathrm{H} & 5.334637 & 3.539940 & -0.628805\end{array}$

$\mathrm{H} \quad 3.807738 \quad 5.545914 \quad-3.552493$

$\begin{array}{llll}\mathrm{H} & 2.915501 & 7.805487 & -3.255597\end{array}$

$\begin{array}{llll}\mathrm{H} & 3.626205 & 7.554944 & 1.023863\end{array}$

$\begin{array}{llll}\mathrm{H} & 4.504920 & 5.235504 & 0.736850\end{array}$

$\begin{array}{llll}\mathrm{H} & -1.378720 & 3.513462 & -1.624210\end{array}$

$\begin{array}{llll}\mathrm{H} & 0.126558 & 3.591562 & -0.669882\end{array}$

$\begin{array}{llll}\mathrm{H} & -2.991268 & 5.175280 & -1.075705\end{array}$

$\begin{array}{llll}\mathrm{H} & -3.581852 & 7.462851 & -0.244614\end{array}$

$\begin{array}{llll}\mathrm{H} & -1.766730 & 9.086716 & 0.356702\end{array}$

$\begin{array}{llll}\mathrm{H} & 0.649055 & 8.406598 & 0.115211\end{array}$

$\begin{array}{llll}\mathrm{H} & 1.225084 & 6.133176 & -0.727627\end{array}$

H $\quad 2.232446 \quad 3.508099 \quad-1.859326$

$\begin{array}{llll}\mathrm{H} & 1.484210 & 4.347150 & -6.971177\end{array}$

$\begin{array}{llll}\mathrm{H} & -0.797040 & 5.079571 & -7.707419\end{array}$

$\begin{array}{llll}\mathrm{H} & -2.634538 & 5.399751 & -6.047825\end{array}$

$\begin{array}{llll}\mathrm{H} & -2.237002 & 5.003767 & -3.598342\end{array}$

$\begin{array}{llll}\text { C } & 2.229962 & 9.867902 & -1.884292\end{array}$

$\begin{array}{llll}\mathrm{H} & 1.899020 & 10.826613 & -1.443720\end{array}$

$\mathrm{H} \quad 3.001793 \quad 10.073055 \quad-2.659705$

$\begin{array}{llll}\mathrm{H} & 1.357671 & 9.367324 & -2.362283\end{array}$

Bisallene2Cyclization_TS2_for_A

Free energy $=-1284.729833$ Hartree

C $\quad 4.985888 \quad 5.465796 \quad-1.639136$

$\begin{array}{llll}\text { C } & 4.913925 & 4.301433 & -0.815503\end{array}$

$\begin{array}{llll}\text { C } & 4.709902 & 4.519267 & 0.586705\end{array}$

$\begin{array}{llll}\mathrm{C} & 4.571472 & 5.794053 & 1.115424\end{array}$

$\begin{array}{llll}\text { C } & 4.677022 & 6.934125 & 0.281254\end{array}$

$\begin{array}{llll}\text { C } & 4.885147 & 6.757930 & -1.105024\end{array}$

$\begin{array}{llll}\text { C } & 5.099157 & 2.973900 & -1.315373\end{array}$

$\begin{array}{llll}\text { C } & 5.052772 & 2.557267 & -2.706515\end{array}$

$\begin{array}{llll}\text { C } & 5.880969 & 1.499417 & -3.316045\end{array}$

$\begin{array}{llll}\text { C } & 5.409616 & 1.370098 & -4.610853\end{array}$

$\begin{array}{llll}\text { O } & 4.379447 & 2.239731 & -4.858839\end{array}$

\begin{tabular}{llll}
\hline & 4.175855 & 2.947107 & -3.702959
\end{tabular}

$\begin{array}{llll}\text { C } & 6.975289 & 0.808362 & -2.665071\end{array}$

\begin{tabular}{llll}
\hline & 7.803433 & 1.471027 & -1.735728
\end{tabular}

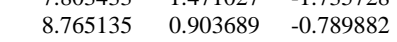

$\begin{array}{llll}\text { C } & 9.312107 & 1.988806 & -0.035103\end{array}$

$\begin{array}{llll}\text { C } & 8.734568 & 3.172618 & -0.499370\end{array}$

$\begin{array}{llll}\text { C } & 7.837282 & 2.876788 & -1.505450\end{array}$

$\begin{array}{llll}\text { C } & 9.187317 & -0.408517 & -0.505601\end{array}$

$\begin{array}{llll}\text { C } & 10.141591 & -0.615333 & 0.503277\end{array}$

$\begin{array}{llll}\text { C } & 10.669091 & 0.472050 & 1.238946\end{array}$

$\begin{array}{llll}\text { C } & 10.257168 & 1.790533 & 0.985772\end{array}$

$\begin{array}{llll}\text { C } & 9.006418 & 4.510591 & -0.006388\end{array}$

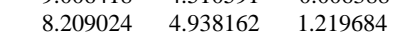

$\begin{array}{llll}\text { C } & 7.991707 & 6.311330 & 1.449246\end{array}$

$\begin{array}{llll}\text { C } & 7.334278 & 6.749515 & 2.608987\end{array}$

$\begin{array}{llll}\text { C } & 6.884615 & 5.815124 & 3.557334\end{array}$

$\begin{array}{lll}7.076671 & 4.443386 & 3.324955\end{array}$

$\begin{array}{llll}\text { C } & 7.730806 & 4.005820 & 2.160852\end{array}$ 


$\begin{array}{cccc}\mathrm{O} & 4.585983 & 8.142889 & 0.914519 \\ \mathrm{C} & 4.694756 & 9.322846 & 0.137467 \\ \mathrm{H} & 5.733397 & 0.754932 & -5.457286 \\ \mathrm{H} & 3.330384 & 3.643712 & -3.705463 \\ \mathrm{H} & 7.163574 & -0.251016 & -2.897810 \\ \mathrm{H} & 5.238831 & 2.169360 & -0.574951 \\ \mathrm{H} & 5.159344 & 5.344494 & -2.720109 \\ \mathrm{H} & 4.975499 & 7.622042 & -1.777668 \\ \mathrm{H} & 4.410017 & 5.951007 & 2.190572 \\ \mathrm{H} & 4.660414 & 3.648691 & 1.258664 \\ \mathrm{H} & 10.094371 & 4.575580 & 0.220552 \\ \mathrm{H} & 8.819178 & 5.222519 & -0.836877 \\ \mathrm{H} & 8.331307 & 7.047863 & 0.703111 \\ \mathrm{H} & 7.144410 & 7.823208 & 2.758008 \\ \mathrm{H} & 6.365785 & 6.157128 & 4.466064 \\ \mathrm{H} & 6.709225 & 3.703280 & 4.052472 \\ \mathrm{H} & 7.868491 & 2.929040 & 1.981985 \\ \mathrm{H} & 7.404334 & 3.684508 & -2.101155 \\ \mathrm{H} & 8.771166 & -1.259274 & -1.066356 \\ \mathrm{H} & 10.480848 & -1.637006 & 0.731623 \\ \mathrm{H} & 11.410978 & 0.282065 & 2.030070 \\ \mathrm{H} & 10.650575 & 2.632259 & 1.574760 \\ \mathrm{H} & 4.592386 & 10.171935 & 0.839071 \\ \mathrm{H} & 5.682438 & 9.396065 & -0.374025 \\ \mathrm{H} & 3.891489 & 9.393120 & -0.632169\end{array}$

Bisallene2Cyclization_INT2_for_A

54

Free energy $=-1284.783564$ Hartree

$\begin{array}{llll}\mathrm{C} & 5.820628 & 5.537824 & -1.60626 \\ \mathrm{C} & 5.481218 & 4.415104 & -0.829123\end{array}$

$\begin{array}{lllr}\text { C } & 5.481218 & 4.415104 & -0.829123 \\ \text { C } & 4.786162 & 4.636280 & 0.380127\end{array}$

$\begin{array}{llll}\text { C } & 4.466442 & 5.924286 & 0.812701\end{array}$

$\begin{array}{llll}\text { C } & 4.859503 & 7.043754 & 0.049106\end{array}$

$\begin{array}{llll}\text { C } & 5.528792 & 6.845333 & -1.177875\end{array}$

$\begin{array}{llll}\text { C } & 5.875693 & 3.010652 & -1.243509\end{array}$

$\begin{array}{llll}\text { C } & 5.245629 & 2.532744 & -2.531860\end{array}$

$\begin{array}{llll}\text { C } & 5.771162 & 1.373023 & -3.23527\end{array}$

$\begin{array}{llll}\text { C } & 4.921484 & 1.175054 & -4.308646\end{array}$

$\begin{array}{llll}\text { O } & 3.932883 & 2.121702 & -4.316072\end{array}$

$\begin{array}{llll}\text { C } & 4.137894 & 2.937240 & -3.230085\end{array}$

$\begin{array}{llll}\text { C } & 6.940157 & 0.677662 & -2.726709\end{array}$

$\begin{array}{llll}\text { C } & 7.708000 & 1.332141 & -1.802759\end{array}$

$\begin{array}{llll}\text { C } & 8.815643 & 0.895655 & -0.966398\end{array}$

$\begin{array}{llll}\text { C } & 9.093665 & 1.947851 & -0.039848\end{array}$

$\begin{array}{llll}\mathrm{N} & 8.244955 & 3.027673 & -0.244125\end{array}$

$\begin{array}{llll}\text { C } & 7.405757 & 2.786148 & -1.422786\end{array}$

$\begin{array}{llll}\text { C } & 9.549152 & -0.301189 & -0.911831\end{array}$

$\begin{array}{llll}\text { C } & 10.564307 & -0.444186 & 0.051566\end{array}$

$\begin{array}{llll}\text { C } & 10.833632 & 0.602976 & 0.957682\end{array}$

$\begin{array}{llll}\text { C } & 10.104511 & 1.806927 & 0.93038\end{array}$

$\begin{array}{llll}\text { C } & 8.571455 & 4.374764 & 0.189543\end{array}$

$\begin{array}{llll}\text { C } & 7.845556 & 4.850525 & 1.438546\end{array}$

$\begin{array}{llll}\text { C } & 7.688300 & 6.230220 & 1.672203\end{array}$

$\begin{array}{llll}\text { C } & 7.009614 & 6.690150 & 2.811185\end{array}$

$\begin{array}{llll}\text { C } & 6.486087 & 5.771289 & 3.737150\end{array}$

$\begin{array}{llll}\text { C } & 6.649427 & 4.392760 & 3.516645\end{array}$

$\begin{array}{llll}\text { C } & 7.323981 & 3.935506 & 2.372325\end{array}$

$\begin{array}{llll}\text { O } & 4.564619 & 8.264783 & 0.586278\end{array}$

$\begin{array}{llll}\text { C } & 4.966340 & 9.428315 & -0.116849\end{array}$

$\begin{array}{llll}\mathrm{H} & 4.898416 & 0.449040 & -5.128557\end{array}$

$\begin{array}{llll}\mathrm{H} & 3.420004 & 3.751697 & -3.089815\end{array}$

$\begin{array}{llll}\mathrm{H} & 7.165878 & -0.353997 & -3.035656\end{array}$

$\begin{array}{llll}\mathrm{H} & 5.562068 & 2.311572 & -0.433500\end{array}$

$\begin{array}{llll}\mathrm{H} & 6.332842 & 5.396011 & -2.571445\end{array}$

$\begin{array}{llll}\mathrm{H} & 5.829016 & 7.695140 & -1.805054\end{array}$

$\begin{array}{lllr}\mathrm{H} & 3.953421 & 6.096275 & 1.768887\end{array}$

$\begin{array}{llll}\mathrm{H} & 4.514002 & 3.776543 & 1.010797\end{array}$

$\begin{array}{llll}\mathrm{H} & 9.672107 & 4.438102 & 0.358177\end{array}$

$\begin{array}{llll}\mathrm{H} & 8.346634 & 5.078491 & -0.640506\end{array}$

$\begin{array}{llll}\mathrm{H} & 8.068250 & 6.951853 & 0.931375\end{array}$

$\begin{array}{llll}\mathrm{H} & 6.860435 & 7.770748 & 2.960366\end{array}$

$\begin{array}{llll}\mathrm{H} & 5.944977 & 6.130787 & 4.626124\end{array}$

$\begin{array}{llll}\mathrm{H} & 6.239465 & 3.666866 & 4.236069\end{array}$

\begin{tabular}{llll}
$\mathrm{H}$ & 7.430871 & 2.856991 & 2.178522 \\
\hline & 7.741966 & 3.453307 & -2.259410
\end{tabular}

$\begin{array}{llll}\mathrm{H} & 7.741966 & 3.453307 & -2.259410\end{array}$

$\mathrm{H} \quad 9.331746 \quad-1.114546 \quad-1.621858$

$\begin{array}{llll}\mathrm{H} & 11.147140 & -1.376137 & 0.102143\end{array}$

$\begin{array}{llll}\mathrm{H} & 11.626413 & 0.476261 & 1.711911\end{array}$

$\begin{array}{llll}\mathrm{H} & 10.305027 & 2.605042 & 1.660876\end{array}$

$\begin{array}{llll}\mathrm{H} & 4.645147 & 10.291543 & 0.495849\end{array}$

$\begin{array}{llll}\mathrm{H} & 6.072100 & 9.473109 & -0.251829\end{array}$

$\begin{array}{llll}\mathrm{H} & 4.483377 & 9.500978 & -1.117953\end{array}$

Bisallene2Cyclization_TS2_for_B

54

Free energy $=-1284.719569$ Hartree

$\begin{array}{llll}\text { C } & 4.311905 & 5.110532 & -3.557322\end{array}$

$\begin{array}{llll}\text { C } & 4.807854 & 5.091875 & -2.193090\end{array}$

\begin{tabular}{lrrl} 
& & & \\
$\mathrm{C}$ & 4.268216 & 6.081350 & -1.294277 \\
$\mathrm{C}$ & 3.252797 & 6.932077 & -1.686341 \\
$\mathrm{C}$ & 2.727869 & 6.880951 & -3.020651 \\
$\mathrm{C}$ & 3.297659 & 6.011185 & -3.956565 \\
$\mathrm{C}$ & 5.673319 & 4.071457 & -1.750062 \\
$\mathrm{C}$ & 5.825189 & 2.847448 & -2.510353 \\
$\mathrm{C}$ & 4.753475 & 2.256163 & -3.335131 \\
$\mathrm{C}$ & 5.274104 & 1.076556 & -3.817486 \\
$\mathrm{O}$ & 6.567858 & 0.884500 & -3.394739 \\
$\mathrm{C}$ & 6.883418 & 1.964010 & -2.608088 \\
$\mathrm{C}$ & 3.431448 & 2.850534 & -3.505215 \\
$\mathrm{C}$ & 2.647260 & 2.792006 & -4.690770 \\
$\mathrm{C}$ & 1.319532 & 3.378917 & -4.848674 \\
$\mathrm{C}$ & 0.935072 & 3.206415 & -6.214229 \\
$\mathrm{~N}$ & 1.978404 & 2.562902 & -6.872799 \\
$\mathrm{C}$ & 2.996626 & 2.303940 & -5.965147 \\
$\mathrm{C}$ & 0.448379 & 4.050272 & -3.968056 \\
$\mathrm{C}$ & -0.785924 & 4.507084 & -4.453569 \\
$\mathrm{C}$ & -1.157327 & 4.308093 & -5.807526 \\
$\mathrm{C}$ & -0.304469 & 3.653353 & -6.708538 \\
$\mathrm{C}$ & 1.928584 & 2.104563 & -8.247666 \\
$\mathrm{C}$ & 0.982805 & 0.931342 & -8.477580 \\
$\mathrm{C}$ & 0.465504 & 0.691564 & -9.766223 \\
$\mathrm{C}$ & -0.380165 & -0.404358 & -10.003498 \\
$\mathrm{C}$ & -0.725029 & -1.268611 & -8.949809 \\
$\mathrm{C}$ & -0.219752 & -1.029022 & -7.660562 \\
$\mathrm{C}$ & 0.630236 & 0.064410 & -7.425009 \\
$\mathrm{O}$ & 1.697348 & 7.735895 & -3.270375 \\
$\mathrm{C}$ & 1.146372 & 7.756764 & -4.582701 \\
$\mathrm{H}$ & 1.896824 & 8.092619 & -5.335044 \\
$\mathrm{H}$ & 4.833224 & 0.261315 & -4.401294 \\
$\mathrm{H}$ & 7.905236 & 1.987862 & -2.214962 \\
$\mathrm{H}$ & 2.923098 & 3.176470 & -2.584778 \\
$\mathrm{H}$ & 6.129896 & 4.142694 & -0.749916 \\
$\mathrm{H}$ & 4.903106 & 4.615974 & -4.338968 \\
$\mathrm{H}$ & 2.956711 & 5.990752 & -4.999639 \\
$\mathrm{H}$ & 2.808670 & 7.658222 & -0.988995 \\
$\mathrm{H}$ & 4.643548 & 6.116616 & -0.258941 \\
$\mathrm{H}$ & 1.639476 & 2.954603 & -8.904038 \\
$\mathrm{H}$ & 2.961841 & 1.823822 & -8.543592 \\
$\mathrm{H}$ & 0.725564 & 1.372412 & -10.593648 \\
$\mathrm{H}$ & -0.778693 & -0.579209 & -11.014759 \\
$\mathrm{H}$ & -1.392120 & -2.125076 & -9.133462 \\
$\mathrm{H}$ & -0.492549 & -1.695974 & -6.828132 \\
$\mathrm{H}$ & 1.017151 & 0.254693 & -6.411974 \\
$\mathrm{H}$ & 0.738302 & 1.814580 & -6.292580 \\
$\mathrm{H}$ & 4.221643 & -2.920740 \\
\hline & & & \\
$\mathrm{H}$ & & &
\end{tabular}

Bisallene2Cyclization_INT2_for_B

Free energy $=-1284.757111$ Hartree

$\begin{array}{lrrr}\text { C } & 4.009269 & 4.788183 & -3.547455 \\ \mathrm{C} & 4.941067 & 4.886469 & -2.331402 \\ \mathrm{C} & 5.028502 & 6.149547 & -1.628375 \\ \mathrm{C} & 4.181930 & 7.187847 & -1.897363 \\ \mathrm{C} & 3.142832 & 7.059642 & -2.915487 \\ \mathrm{C} & 3.042300 & 5.937453 & -3.683964 \\ \mathrm{C} & 5.692864 & 3.795320 & -1.957021 \\ \mathrm{C} & 5.491484 & 2.529643 & -2.622454 \\ \mathrm{C} & 4.328370 & 2.313080 & -3.463196 \\ \mathrm{C} & 4.409512 & 1.014654 & -3.899145 \\ \mathrm{O} & 5.542040 & 0.412073 & -3.403920 \\ \mathrm{C} & 6.187889 & 1.332513 & -2.628095 \\ \mathrm{C} & 3.285495 & 3.390243 & -3.622367 \\ \mathrm{C} & 2.428428 & 3.240399 & -4.851074 \\ \mathrm{C} & 1.032549 & 3.598922 & -4.977744 \\ \mathrm{C} & 0.653674 & 3.353705 & -6.338823 \\ \mathrm{~N} & 1.777120 & 2.890945 & -7.010733 \\ \mathrm{C} & 2.832177 & 2.811991 & -6.108702 \\ \mathrm{C} & 0.067382 & 4.111641 & -4.079479 \\ \mathrm{C} & -1.233100 & 4.344754 & -4.543816 \\ \mathrm{C} & -1.592366 & 4.071142 & -5.889791 \\ \mathrm{C} & -0.657850 & 3.567854 & -6.804404 \\ \mathrm{C} & 1.782829 & 2.358994 & -8.358957 \\ \mathrm{C} & 1.189055 & 0.959817 & -8.487954 \\ \mathrm{C} & 0.944018 & 0.427858 & -9.770703 \\ \mathrm{C} & 0.417732 & -0.864851 & -9.919565 \\ \mathrm{C} & 0.125181 & -1.641651 & -8.784455 \\ \mathrm{C} & 0.363130 & -1.115581 & -7.504075 \\ \mathrm{C} & 0.892633 & 0.177968 & -7.355451 \\ \mathrm{O} & 2.324505 & 8.152751 & -2.984175 \\ \mathrm{C} & 1.260720 & 8.108818 & -3.921807 \\ \mathrm{H} & 3.770880 & 0.392426 & -4.533934 \\ \mathrm{H} & 7.124014 & 1.003027 & -2.165712 \\ \mathrm{H} & 2.615537 & 3.342674 & -2.730520\end{array}$




$\begin{array}{rrrr}\mathrm{H} & 6.428494 & 3.880921 & -1.141012 \\ \mathrm{H} & 4.676848 & 4.801192 & -4.450059 \\ \mathrm{H} & 2.280207 & 5.845454 & -4.468245 \\ \mathrm{H} & 4.227376 & 8.134917 & -1.339481 \\ \mathrm{H} & 5.776645 & 6.243270 & -0.823781 \\ \mathrm{H} & 1.235594 & 3.061599 & -9.023701 \\ \mathrm{H} & 2.834304 & 2.355093 & -8.717073 \\ \mathrm{H} & 1.167465 & 1.034042 & -10.664262 \\ \mathrm{H} & 0.229429 & -1.266661 & -10.927250 \\ \mathrm{H} & -0.291532 & -2.653755 & -8.899275 \\ \mathrm{H} & 0.130739 & -1.713632 & -6.609570 \\ \mathrm{H} & 1.068898 & 0.588313 & -6.349305 \\ \mathrm{H} & 3.811849 & 2.445806 & -6.437044 \\ \mathrm{H} & 0.341790 & 4.328017 & -3.035898 \\ \mathrm{H} & -1.994840 & 4.738606 & -3.853468 \\ \mathrm{H} & -2.626134 & 4.253533 & -6.220732 \\ \mathrm{H} & -0.942410 & 3.338229 & -7.841700 \\ \mathrm{H} & 0.696191 & 9.053234 & -3.804636 \\ \mathrm{H} & 1.636341 & 8.034648 & -4.968653 \\ \mathrm{H} & 0.581651 & 7.244304 & -3.738292\end{array}$

\section{$\mathrm{X}=-\mathrm{SO} 2-$}

\section{$A=4$-methoxyphenyl, $B$ = phenyl} Bisallene

39

Free energy $=-1356.370908$ Hartree

$\begin{array}{lccc}\mathrm{C} & -0.074178 & 3.171462 & 0.824025 \\ \mathrm{C} & 0.830819 & 2.443258 & 0.018436 \\ \mathrm{C} & 0.325309 & 1.627863 & -1.023930 \\ \mathrm{C} & -1.056197 & 1.528456 & -1.230096 \\ \mathrm{C} & -1.952937 & 2.242081 & -0.410192 \\ \mathrm{C} & -1.457041 & 3.069132 & 0.612973 \\ \mathrm{C} & 2.278227 & 2.626506 & 0.206946 \\ \mathrm{C} & 3.232769 & 1.873310 & -0.325767 \\ \mathrm{C} & 4.134422 & 1.195313 & -0.994248 \\ \mathrm{~S} & 4.367588 & 1.547234 & -2.778956 \\ \mathrm{O} & 5.335097 & 0.559332 & -3.310960 \\ \mathrm{H} & -3.038557 & 2.160817 & -0.578215 \\ \mathrm{C} & 5.103597 & 3.229032 & -2.927731 \\ \mathrm{C} & 4.800391 & 4.177446 & -2.078909 \\ \mathrm{C} & 4.280973 & 5.010352 & -1.179661 \\ \mathrm{C} & 2.871722 & 5.424445 & -1.186623 \\ \mathrm{C} & 2.385697 & 6.344984 & -0.223693 \\ \mathrm{C} & 1.023054 & 6.635725 & -0.128024 \\ \mathrm{C} & 0.098158 & 6.013624 & -0.999745 \\ \mathrm{C} & 0.568806 & 5.104257 & -1.974778 \\ \mathrm{C} & 1.932711 & 4.811747 & -2.054498 \\ \mathrm{O} & -1.209237 & 6.331992 & -0.810929 \\ \mathrm{C} & -2.177404 & 5.785140 & -1.699628 \\ \mathrm{O} & 3.010637 & 1.678985 & -3.384392 \\ \mathrm{H} & 4.728315 & 0.328649 & -0.658532 \\ \mathrm{H} & 5.615415 & 3.326828 & -3.901438 \\ \mathrm{H} & 2.592261 & 3.506951 & 0.796427 \\ \mathrm{H} & 4.909996 & 5.344960 & -0.333922 \\ \mathrm{H} & 1.032753 & 1.124037 & -1.701817 \\ \mathrm{H} & 2.269008 & 4.034956 & -2.760370 \\ \mathrm{H} & -1.440469 & 0.899961 & -2.048780 \\ \mathrm{H} & -0.126935 & 4.568673 & -2.633423 \\ \mathrm{H} & -2.149339 & 3.648476 & 1.242814 \\ \mathrm{H} & 0.637497 & 7.340496 & 0.623742 \\ \mathrm{H} & 0.315928 & 3.836819 & 1.610112 \\ \mathrm{H} & 3.092010 & 6.830778 & 0.468642 \\ \mathrm{H} & -3.154027 & 6.193990 & -1.380768 \\ \mathrm{H} & -2.208404 & 4.674562 & -1.637452 \\ \mathrm{H} & -1.983270 & 6.086955 & -2.753405 \\ & & & \\ & & \end{array}$

Bisallene2Cyclization_TS_for_A

39

Free energy $=-1356.347844$ Hartree

$\begin{array}{lcrr}\mathrm{C} & -0.081100 & 3.437733 & 0.675185 \\ \mathrm{C} & 0.906255 & 2.636248 & 0.042060 \\ \mathrm{C} & 0.510951 & 1.387724 & -0.507938 \\ \mathrm{C} & -0.817455 & 0.957469 & -0.413022 \\ \mathrm{C} & -1.783342 & 1.752698 & 0.236738 \\ \mathrm{C} & -1.406961 & 2.993456 & 0.781389 \\ \mathrm{C} & 2.272751 & 3.138539 & -0.048656 \\ \mathrm{C} & 3.329558 & 2.488017 & -0.615915 \\ \mathrm{C} & 4.057794 & 1.376144 & -0.670998 \\ \mathrm{~S} & 5.126325 & 1.093401 & -2.090619 \\ \mathrm{O} & 6.506577 & 0.850395 & -1.604602 \\ \mathrm{H} & -2.824221 & 1.404003 & 0.318254 \\ \mathrm{C} & 4.975481 & 2.745925 & -2.801193 \\ \mathrm{C} & 4.324203 & 3.687709 & -2.131013 \\ \mathrm{C} & 4.003748 & 5.007788 & -1.961568 \\ \mathrm{C} & 2.652248 & 5.494065 & -1.889593\end{array}$

$\begin{array}{rrrr}\mathrm{C} & 2.352152 & 6.759963 & -1.300752 \\ \mathrm{C} & 1.038851 & 7.157720 & -1.089415 \\ \mathrm{C} & -0.039341 & 6.309825 & -1.469630 \\ \mathrm{C} & 0.230333 & 5.079935 & -2.106764 \\ \mathrm{C} & 1.557077 & 4.681470 & -2.300667 \\ \mathrm{O} & -1.279031 & 6.772379 & -1.177990 \\ \mathrm{C} & -2.405158 & 5.959612 & -1.500401 \\ \mathrm{O} & 4.508645 & 0.130931 & -3.037248 \\ \mathrm{H} & 4.158225 & 0.624166 & 0.129140 \\ \mathrm{H} & 5.375680 & 2.830720 & -3.824080 \\ \mathrm{H} & 2.445571 & 4.149267 & 0.359516 \\ \mathrm{H} & 4.823584 & 5.696023 & -1.685432 \\ \mathrm{H} & 1.260745 & 0.766070 & -1.021843 \\ \mathrm{H} & 1.761448 & 3.719022 & -2.791291 \\ \mathrm{H} & -1.106943 & -0.012125 & -0.846441 \\ \mathrm{H} & -0.579570 & 4.407660 & -2.415012 \\ \mathrm{H} & -2.152271 & 3.621059 & 1.294833 \\ \mathrm{H} & 0.796655 & 8.119236 & -0.614137 \\ \mathrm{H} & 0.207375 & 4.417796 & 1.085830 \\ \mathrm{H} & 3.178064 & 7.415209 & -0.981959 \\ \mathrm{H} & -3.294176 & 6.508486 & -1.139370 \\ \mathrm{H} & -2.349980 & 4.971438 & -0.993689 \\ \mathrm{H} & -2.495586 & 5.802408 & -2.598472\end{array}$

Bisallene2Cyclization_TS_for_B

Free energy $=-1356.34497$ Hartree

$\begin{array}{llll}\text { C } & 1.890838 & 2.279832 & 1.032206\end{array}$

$\begin{array}{llll}\text { C } & 2.989716 & 1.955905 & 0.203679\end{array}$

$\begin{array}{llll}\text { C } & 2.786259 & 1.172775 & -0.939662\end{array}$

$\begin{array}{llll}\text { C } & 1.503404 & 0.679147 & -1.285992\end{array}$

$\begin{array}{llll}\text { C } & 0.408544 & 1.024553 & -0.443678\end{array}$

$\begin{array}{llll}\text { C } & 0.597948 & 1.807377 & 0.690683\end{array}$

$\begin{array}{llll}\text { C } & 1.348044 & -0.122986 & -2.489597\end{array}$

$\begin{array}{llll}\text { C } & 0.168456 & -0.637526 & -2.945258\end{array}$

$\begin{array}{llll}\text { C } & -0.917353 & -1.343109 & -2.638086\end{array}$

$\begin{array}{llll}\text { S } & -2.385682 & -1.161129 & -3.660925\end{array}$

$\begin{array}{llll}\text { O } & -3.351340 & -0.229059 & -3.025046\end{array}$

$\begin{array}{llll}\text { O } & 1.965900 & 3.035100 & 2.159836\end{array}$

$\begin{array}{llll}\text { C } & 3.227782 & 3.555172 & 2.554994\end{array}$

$\begin{array}{llll}\text { C } & -1.581006 & -0.292926 & -5.020255\end{array}$

$\begin{array}{llll}\text { C } & -0.264190 & -0.119282 & -5.001026\end{array}$

$\begin{array}{llll}\text { C } & 0.884205 & 0.196407 & -5.672021\end{array}$

$\begin{array}{llll}\text { C } & 1.821351 & 1.201869 & -5.229909\end{array}$

$\begin{array}{llll}\text { C } & 3.154292 & 1.227295 & -5.731319\end{array}$

$\begin{array}{llll}\text { C } & 4.093840 & 2.121914 & -5.212935\end{array}$

$\begin{array}{llll}\text { C } & 3.729638 & 3.019329 & -4.184614\end{array}$

$\begin{array}{llll}\text { C } & 2.411754 & 3.031766 & -3.700674\end{array}$

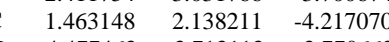

$\mathrm{H} \quad 4.477463 \quad 3.713113 \quad-3.770662$

$\begin{array}{llll}\mathrm{O} & -2.845879 & -2.503218 & -4.093229\end{array}$

$\begin{array}{llll}\mathrm{H} & -1.002380 & -2.103298 & -1.843624\end{array}$

$\begin{array}{llll}\mathrm{H} & 3.444175 & 0.514317 & -6.519883\end{array}$

$\begin{array}{llll}\mathrm{H} & 5.123335 & 2.121523 & -5.602899\end{array}$

$\begin{array}{llll}\mathrm{H} & 2.121398 & 3.730601 & -2.901842\end{array}$

$\begin{array}{llll}\mathrm{H} & -0.603382 & 0.680548 & -0.707275\end{array}$

$\begin{array}{llll}\mathrm{H} & 4.001760 & 2.312350 & 0.437327\end{array}$

$\begin{array}{llll}\mathrm{H} & -0.243214 & 2.083055 & 1.343349\end{array}$

$\begin{array}{llll}\mathrm{H} & 3.642680 & 0.939935 & -1.591763\end{array}$

$\mathrm{H} \quad 0.427244 \quad 2.161010 \quad-3.850464$

$\begin{array}{llll}\mathrm{H} & -2.277990 & 0.129760 & -5.761012\end{array}$

\begin{tabular}{llll}
$\mathrm{H}$ & 1.178705 & -0.462964 & -6.50884 \\
\hline
\end{tabular}

H $\quad 2.259111 \quad-0.287960 \quad-3.089874$

$\begin{array}{llll}\mathrm{H} & 3.960411 & 2.744896 & 2.770546\end{array}$

$\begin{array}{llll}\mathrm{H} & 3.657379 & 4.231805 & 1.781490\end{array}$

$\begin{array}{llll}\mathrm{H} & 3.049378 & 4.134036 & 3.480147\end{array}$

A = 2,4-dimethoxyphenyl, $B$ = phenyl Bisallene

Free energy $=-1470.792057$ Hartree

$\begin{array}{lccc}\mathrm{C} & 1.877367 & 4.788517 & -2.102683 \\ \mathrm{C} & 2.796393 & 5.414149 & -1.229879 \\ \mathrm{C} & 2.278335 & 6.338321 & -0.269559 \\ \mathrm{C} & 0.901500 & 6.602238 & -0.206272 \\ \mathrm{C} & 0.011861 & 5.957993 & -1.097199 \\ \mathrm{C} & 0.505721 & 5.053017 & -2.060599 \\ \mathrm{C} & 4.207802 & 5.024796 & -1.189912 \\ \mathrm{C} & 4.761437 & 4.201548 & -2.077312 \\ \mathrm{C} & 5.098452 & 3.245576 & -2.905210 \\ \mathrm{~S} & 4.372228 & 1.561274 & -2.740426 \\ \mathrm{O} & 3.009225 & 1.670979 & -3.338156 \\ \mathrm{O} & -1.302348 & 6.263565 & -0.928692 \\ \mathrm{C} & -2.249502 & 5.699531 & -1.829096 \\ \mathrm{C} & 4.148365 & 1.222825 & -0.949382 \\ \mathrm{C} & 3.259421 & 1.911965 & -0.275743 \\ \mathrm{C} & 2.308969 & 2.670453 & 0.257844 \\ \mathrm{C} & 0.861338 & 2.488706 & 0.070617 \\ \mathrm{C} & -0.043191 & 3.239684 & 0.855213\end{array}$




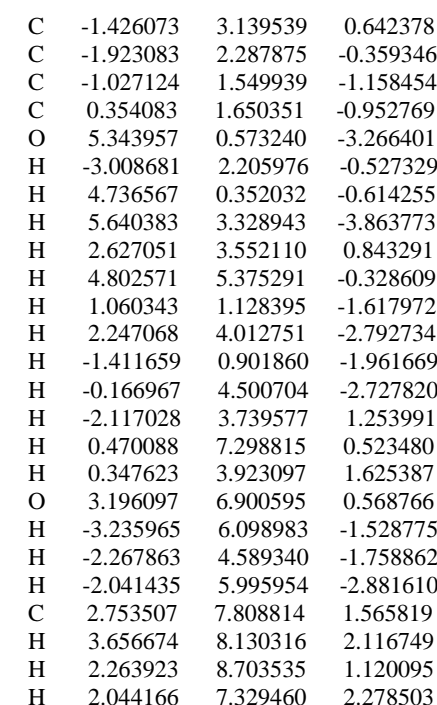

Bisallene2Cyclization_TS_for_A
43

Free energy $=-1470.770102$ Hartree

$\begin{array}{cccc}\text { C } & 1.548367 & 4.646974 & -2.330382 \\ \mathrm{C} & 2.610758 & 5.481679 & -1.888036 \\ \mathrm{C} & 2.253770 & 6.736437 & -1.274273 \\ \mathrm{C} & 0.910310 & 7.073271 & -1.084272 \\ \mathrm{C} & -0.117431 & 6.191950 & -1.513411 \\ \mathrm{C} & 0.202845 & 4.984703 & -2.169018 \\ \mathrm{C} & 3.975751 & 5.045093 & -1.942086 \\ \mathrm{C} & 4.326411 & 3.734184 & -2.128127 \\ \mathrm{C} & 4.966603 & 2.802763 & -2.821478 \\ \mathrm{~S} & 5.114464 & 1.133508 & -2.146309 \\ \mathrm{O} & 4.478908 & 0.196566 & -3.107213 \\ \mathrm{O} & -1.378449 & 6.609885 & -1.243955 \\ \mathrm{C} & -2.470580 & 5.776899 & -1.628387 \\ \mathrm{C} & 4.065924 & 1.389726 & -0.705822 \\ \mathrm{C} & 3.347257 & 2.505107 & -0.613591 \\ \mathrm{C} & 2.309454 & 3.163826 & -0.022293 \\ \mathrm{C} & 0.932848 & 2.695321 & 0.080003 \\ \mathrm{C} & -0.032343 & 3.528053 & 0.706934 \\ \mathrm{C} & -1.369561 & 3.120547 & 0.819647 \\ \mathrm{C} & -1.780718 & 1.886587 & 0.285396 \\ \mathrm{C} & -0.837282 & 1.061391 & -0.360812 \\ \mathrm{C} & 0.502199 & 1.454125 & -0.460268 \\ \mathrm{O} & 6.498685 & 0.866838 & -1.683045 \\ \mathrm{H} & -2.830348 & 1.566434 & 0.371471 \\ \mathrm{H} & 4.177304 & 0.620192 & 0.075757 \\ \mathrm{H} & 5.361209 & 2.903561 & -3.845192 \\ \mathrm{H} & 2.507752 & 4.168983 & 0.388548 \\ \mathrm{H} & 4.761142 & 5.754621 & -1.631160 \\ \mathrm{H} & 1.234506 & 0.808935 & -0.970134 \\ \mathrm{H} & 1.807924 & 3.697362 & -2.820323 \\ \mathrm{H} & -1.153587 & 0.096851 & -0.786933 \\ \mathrm{H} & -0.573352 & 4.286831 & -2.503067 \\ \mathrm{H} & -2.096539 & 3.774423 & 1.326833 \\ \mathrm{H} & 0.601286 & 8.005602 & -0.596618 \\ \mathrm{H} & 0.281837 & 4.505123 & 1.105563 \\ \mathrm{O} & 3.294236 & 7.514358 & -0.881637 \\ \mathrm{H} & -3.385796 & 6.293876 & -1.285872 \\ \mathrm{H} & -2.408482 & 4.778288 & -1.144492 \\ \mathrm{H} & -2.516727 & 5.647573 & -2.732707 \\ \mathrm{C} & 3.027124 & 8.754352 & -0.238949 \\ \mathrm{H} & 4.011175 & 9.202187 & -0.009545 \\ \mathrm{H} & 2.455927 & 9.443968 & -0.899896 \\ \mathrm{H} & 2.462620 & 8.611590 & 0.709799 \\ & & & \end{array}$

Bisallene2Cyclization_TS_for_B

43

Free energy $=-1470.766791$ Hartree

$\begin{array}{llll}\text { C } & 1.526546 & 2.126708 & -4.102938\end{array}$

$\begin{array}{llll}\text { C } & 1.906428 & 1.190241 & -5.107257\end{array}$

$\begin{array}{llll}\text { C } & 3.233605 & 1.257735 & -5.619595\end{array}$

$\begin{array}{llll}\text { C } & 4.134848 & 2.217521 & -5.150822\end{array}$

$\begin{array}{llll}\text { C } & 3.741493 & 3.134029 & -4.15219\end{array}$

$\begin{array}{llll}\text { C } & 2.439926 & 3.078260 & -3.62795\end{array}$

$\begin{array}{llll}\text { C } & 0.987077 & 0.173524 & -5.565499\end{array}$

$\begin{array}{llll}\text { C } & -0.192681 & -0.134349 & -4.947468\end{array}$

$\begin{array}{llll}\text { C } & -1.509028 & -0.292723 & -5.039202\end{array}$

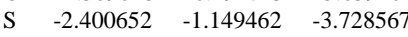

$\begin{array}{llll}\text { O } & -2.855061 & -2.485749 & -4.18593\end{array}$

$\begin{array}{llll}\text { C } & -0.994724 & -1.356307 & -2.625323\end{array}$

$\begin{array}{llll}\text { C } & 0.116713 & -0.667821 & -2.871583 \\ \text { C } & 1.298559 & -0.192393 & -2.384336\end{array}$

$\begin{array}{llll}\text { C } & 1.298559 & -0.192393 & -2.384336\end{array}$

$\begin{array}{lrrr}\text { C } & 1.453349 & 0.620459 & -1.195263 \\ \mathrm{C} & 2.749881 & 1.133459 & -0.871475 \\ \mathrm{C} & 2.940954 & 1.970575 & 0.244569 \\ \mathrm{C} & 1.836541 & 2.309152 & 1.058644 \\ \mathrm{C} & 0.546124 & 1.814053 & 0.752322 \\ \mathrm{C} & 0.370910 & 0.989508 & -0.354714 \\ \mathrm{O} & 1.918496 & 3.110332 & 2.154346 \\ \mathrm{C} & 3.179634 & 3.643503 & 2.529648 \\ \mathrm{O} & -3.389387 & -0.205631 & -3.147904 \\ \mathrm{H} & 4.452861 & 3.893190 & -3.791124 \\ \mathrm{H} & -1.133474 & -2.125000 & -1.846711 \\ \mathrm{H} & 3.545115 & 0.538390 & -6.394097 \\ \mathrm{H} & 5.154133 & 2.258616 & -5.564938 \\ \mathrm{H} & 2.128416 & 3.782739 & -2.841542 \\ \mathrm{H} & -0.635398 & 0.623350 & -0.609195 \\ \mathrm{H} & 3.940533 & 2.352903 & 0.477338 \\ \mathrm{H} & -0.294339 & 2.103369 & 1.398282 \\ \mathrm{O} & 3.754234 & 0.761812 & -1.708434 \\ \mathrm{H} & 0.496941 & 2.113380 & -3.719022 \\ \mathrm{H} & -2.158182 & 0.140447 & -5.816508 \\ \mathrm{H} & 1.309524 & -0.485269 & -6.392472 \\ \mathrm{H} & 2.215826 & -0.374115 & -2.966098 \\ \mathrm{H} & 3.915156 & 2.841538 & 2.766166 \\ \mathrm{H} & 3.605024 & 4.300380 & 1.736652 \\ \mathrm{H} & 3.003585 & 4.248028 & 3.438763 \\ \mathrm{C} & 5.044867 & 1.323254 & -1.551435 \\ \mathrm{H} & 5.656338 & 0.927599 & -2.383107 \\ \mathrm{H} & 5.016313 & 2.433464 & -1.628220 \\ \mathrm{H} & 5.510075 & 1.031128 & -0.582735\end{array}$

\section{$A$ = 2-naphthyl, $B$ = phenyl}

\section{Bisallene}

Free energy $=-1395.446204$ Hartree

$\begin{array}{cccc}\text { Free energy }=-1395.446204 & \text { Hartree } \\ \mathrm{C} & 1.929637 & 4.858893 & -1.949705 \\ \mathrm{C} & 2.944028 & 5.501999 & -1.192546 \\ \mathrm{C} & 2.578134 & 6.540700 & -0.303970 \\ \mathrm{C} & 1.235439 & 6.930359 & -0.177488 \\ \mathrm{C} & 0.239030 & 6.284004 & -0.929577 \\ \mathrm{C} & 0.592363 & 5.248719 & -1.815023 \\ \mathrm{C} & 4.336895 & 5.023065 & -1.234540 \\ \mathrm{C} & 4.773625 & 4.140788 & -2.127448 \\ \mathrm{C} & 5.026929 & 3.169038 & -2.965862 \\ \mathrm{~S} & 4.299765 & 1.496155 & -2.736597 \\ \mathrm{O} & 2.931370 & 1.606742 & -3.319940 \\ \mathrm{H} & -0.816205 & 6.575485 & -0.816768 \\ \mathrm{C} & 4.113603 & 1.219257 & -0.935543 \\ \mathrm{C} & 3.198849 & 1.899608 & -0.286462 \\ \mathrm{C} & 2.221718 & 2.650742 & 0.206987 \\ \mathrm{C} & 0.782176 & 2.407140 & 0.019437 \\ \mathrm{C} & -0.160591 & 3.074192 & 0.863844 \\ \mathrm{C} & -1.522237 & 2.922777 & 0.660989 \\ \mathrm{C} & -2.021468 & 2.107945 & -0.400466 \\ \mathrm{C} & -1.072938 & 1.445103 & -1.269201 \\ \mathrm{C} & 0.319245 & 1.614392 & -1.035966 \\ \mathrm{O} & 5.260241 & 0.494063 & -3.253011 \\ \mathrm{C} & -3.416744 & 1.937939 & -0.645815 \\ \mathrm{H} & 4.744085 & 0.391516 & -0.569749 \\ \mathrm{H} & 5.491502 & 3.246129 & -3.964408 \\ \mathrm{H} & 2.505831 & 3.567125 & 0.756359 \\ \mathrm{H} & 5.023794 & 5.368520 & -0.439948 \\ \mathrm{H} & 1.043231 & 1.165029 & -1.735059 \\ \mathrm{H} & 2.189936 & 4.006050 & -2.597451 \\ \mathrm{C} & -1.564927 & 0.651433 & -2.352075 \\ \mathrm{H} & -0.186396 & 4.713356 & -2.378506 \\ \mathrm{H} & -2.240044 & 3.441764 & 1.315859 \\ \mathrm{H} & 0.964891 & 7.740616 & 0.517529 \\ \mathrm{H} & 0.210142 & 3.721498 & 1.673923 \\ \mathrm{H} & 3.355894 & 7.041044 & 0.294834 \\ \mathrm{C} & -2.929034 & 0.508304 & -2.563117 \\ \mathrm{C} & -3.861934 & 1.154117 & -1.702607 \\ \mathrm{H} & -0.839034 & 0.155922 & -3.015671 \\ \mathrm{H} & -3.296403 & -0.105505 & -3.400244 \\ \mathrm{H} & -4.941760 & 1.030180 & -1.879977 \\ \mathrm{H} & -4.137859 & 2.440586 & 0.018523\end{array}$

Bisallene2Cyclization_TS_for_A

Free energy $=-1395.425178$ Hartree

$\begin{array}{llll}\mathrm{C} & 1.733740 & 5.257835 & -1.756995 \\ \mathrm{C} & 2.980490 & 5.722623 & -1.245219 \\ \mathrm{C} & 2.980261 & 6.876138 & -0.409023 \\ \mathrm{C} & 1.783273 & 7.514671 & -0.078353 \\ \mathrm{C} & 0.554235 & 7.023687 & -0.572591 \\ \mathrm{C} & 0.535383 & 5.903110 & -1.417190 \\ \mathrm{C} & 4.201318 & 4.998882 & -1.503392 \\ \mathrm{C} & 4.242956 & 3.725096 & -1.999744 \\ \mathrm{C} & 4.695592 & 2.877811 & -2.917273 \\ \mathrm{~S} & 4.543141 & 1.103803 & -2.633239\end{array}$




$\begin{array}{rrrr}\mathrm{O} & 3.628221 & 0.542875 & -3.662157 \\ \mathrm{H} & -0.386682 & 7.525598 & -0.300078 \\ \mathrm{C} & 3.664679 & 1.226912 & -1.074012 \\ \mathrm{C} & 3.104502 & 2.392710 & -0.763014 \\ \mathrm{C} & 2.073112 & 2.993034 & -0.101223 \\ \mathrm{C} & 0.680414 & 2.579379 & -0.251563 \\ \mathrm{C} & -0.327505 & 3.239018 & 0.530706 \\ \mathrm{C} & -1.660281 & 2.886501 & 0.417539 \\ \mathrm{C} & -2.079982 & 1.866815 & -0.491987 \\ \mathrm{C} & -1.076759 & 1.211340 & -1.303343 \\ \mathrm{C} & 0.285161 & 1.587998 & -1.163581 \\ \mathrm{O} & 5.876424 & 0.489313 & -2.432514 \\ \mathrm{C} & -3.444492 & 1.481239 & -0.636944 \\ \mathrm{H} & 3.630843 & 0.301589 & -0.476323 \\ \mathrm{H} & 5.089598 & 3.148159 & -3.909537 \\ \mathrm{H} & 2.272189 & 3.860185 & 0.549731 \\ \mathrm{H} & 5.151736 & 5.430910 & -1.140270 \\ \mathrm{H} & 1.038824 & 1.108452 & -1.808736 \\ \mathrm{H} & 1.720793 & 4.399115 & -2.442765 \\ \mathrm{C} & -1.489464 & 0.202512 & -2.229596 \\ \mathrm{H} & -0.417672 & 5.518254 & -1.808212 \\ \mathrm{H} & -2.420489 & 3.396443 & 1.030329 \\ \mathrm{H} & 1.797555 & 8.401398 & 0.574208 \\ \mathrm{H} & -0.022109 & 4.037714 & 1.224930 \\ \mathrm{H} & 3.936364 & 7.249188 & -0.008925 \\ \mathrm{C} & -2.826954 & -0.148946 & -2.345437 \\ \mathrm{C} & -3.811918 & 0.493363 & -1.542823 \\ \mathrm{H} & -0.724089 & -0.292306 & -2.847831 \\ \mathrm{H} & -3.131787 & -0.929371 & -3.060004 \\ \mathrm{H} & -4.869403 & 0.203521 & -1.642910 \\ \mathrm{H} & -4.206443 & 1.980218 & -0.017096 \\ & & & \end{array}$

Bisallene2Cyclization_TS_for_B

41

Free energy $=-1395.432615$ Hartree

$\begin{array}{llll}\mathrm{C} & 1.688606 & 2.501402 & 1.144820 \\ \mathrm{C} & 2.869249 & 1.891917 & 0.667140\end{array}$

$\begin{array}{llll}\mathrm{C} & 2.869249 & 1.891917 & 0.667140 \\ \mathrm{C} & 2.748404 & 0.710934 & -0.148236\end{array}$

$\begin{array}{llll}\text { C } & 2.748404 & 0.710934 & -0.148236 \\ \text { C } & 1.507688 & 0.216496 & -0.496976\end{array}$

$\begin{array}{llll}\text { C } & 0.298938 & 0.851984 & -0.053731\end{array}$

$\begin{array}{llll}\text { C } & 0.401224 & 2.003601 & 0.811047\end{array}$

$\begin{array}{llll}\text { C } & 4.145750 & 2.517314 & 0.886101\end{array}$

$\begin{array}{llll}\text { C } & 4.265676 & 3.802652 & 1.340311\end{array}$

$\begin{array}{llll}\text { C } & 4.703910 & 4.677178 & 2.233529\end{array}$

$\begin{array}{llll}\mathrm{S} & 4.555381 & 6.448786 & 1.906259\end{array}$

$\begin{array}{llll}\text { O } & 5.888096 & 7.056674 & 1.680763\end{array}$

$\begin{array}{llll}\text { C } & 3.684265 & 6.309080 & 0.338113\end{array}$

$\begin{array}{llll}\text { C } & 3.134310 & 5.144634 & 0.013504\end{array}$

$\begin{array}{llll}\text { C } & 2.184611 & 4.483376 & -0.708705\end{array}$

$\begin{array}{llll}\text { C } & 0.771166 & 4.847034 & -0.769772\end{array}$

$\begin{array}{llll}\text { C } & -0.090951 & 4.115551 & -1.626949\end{array}$

$\begin{array}{llll}\text { C } & -1.453468 & 4.429760 & -1.712042\end{array}$

$\begin{array}{llll}\text { C } & -1.988089 & 5.469262 & -0.931749\end{array}$

$\begin{array}{llll}\text { C } & -1.148410 & 6.186060 & -0.05660\end{array}$

$\begin{array}{llll}\text { C } & 0.215615 & 5.881249 & 0.028594\end{array}$

$\begin{array}{llll}\mathrm{H} & -3.059268 & 5.715105 & -0.994674\end{array}$

$\begin{array}{llll}\text { O } & 3.652489 & 7.024009 & 2.935769\end{array}$

$\begin{array}{llll}\text { C } & -0.993165 & 0.396066 & -0.436326\end{array}$

$\begin{array}{llll}\mathrm{H} & 3.704519 & 7.222107 & -0.279371\end{array}$

$\begin{array}{llll}\mathrm{H} & 3.664003 & 0.221389 & -0.514996\end{array}$

$\begin{array}{llll}\mathrm{H} & 1.428768 & -0.677121 & -1.136490\end{array}$

$\begin{array}{llll}\text { C } & -0.789821 & 2.649866 & 1.255143\end{array}$

$\begin{array}{llll}\mathrm{H} & 0.866087 & 6.432361 & 0.726229\end{array}$

\begin{tabular}{llll}
$\mathrm{H}$ & -2.105231 & 3.847172 & -2.380645 \\
\hline
\end{tabular}

$\begin{array}{llll}\mathrm{H} & -1.564796 & 6.991669 & 0.568131\end{array}$

$\begin{array}{llll}\mathrm{H} & 0.321159 & 3.284052 & -2.220076\end{array}$

$\begin{array}{llll}\mathrm{H} & 1.761580 & 3.374875 & 1.809026\end{array}$

$\begin{array}{llll}\mathrm{H} & 5.080604 & 4.441550 & 3.241736\end{array}$

$\begin{array}{llll}\mathrm{H} & 5.054202 & 2.016972 & 0.504720\end{array}$

$\begin{array}{llll}\mathrm{H} & 2.490352 & 3.607858 & -1.306923\end{array}$

$\begin{array}{llll}\text { C } & -2.036807 & 2.184497 & 0.858111\end{array}$

$\begin{array}{llll}\text { C } & -2.138776 & 1.050835 & 0.008972\end{array}$

$\begin{array}{llll}\mathrm{H} & -0.702012 & 3.544658 & 1.889749\end{array}$

$\begin{array}{llll}\mathrm{H} & -2.948163 & 2.702799 & 1.190810\end{array}$

$\begin{array}{llll}\mathrm{H} & -3.131399 & 0.689533 & -0.300449\end{array}$

$\begin{array}{llll}\mathrm{H} & -1.075645 & -0.484473 & -1.093184\end{array}$

\section{A = 6-methoxynaphthyl, $B$ = phenyl}

$$
\text { Bisallene }
$$

45

Free energy $=-1509.865279$ Hartree

$\begin{array}{llll}\mathrm{C} & -3.825726 & 1.083854 & -1.771442 \\ \mathrm{C} & -3.390635 & 1.882954 & -0.710083 \\ \mathrm{C} & -2.007853 & 2.067789 & -0.454515 \\ \mathrm{C} & -1.035140 & 1.413581 & -1.305406 \\ \mathrm{C} & -1.511218 & 0.612184 & -2.385288 \\ \mathrm{C} & -2.868923 & 0.443696 & -2.619962 \\ \mathrm{C} & 0.351351 & 1.598401 & -1.057714 \\ \mathrm{C} & 0.794122 & 2.401304 & -0.001323\end{array}$

$\begin{array}{lrrr}\mathrm{C} & -0.170684 & 3.063015 & 0.824976 \\ \mathrm{C} & -1.527370 & 2.896854 & 0.608705 \\ \mathrm{C} & 2.226629 & 2.659659 & 0.205431 \\ \mathrm{C} & 3.220363 & 1.918524 & -0.271917 \\ \mathrm{C} & 4.148685 & 1.247193 & -0.911481 \\ \mathrm{~S} & 4.349805 & 1.526067 & -2.710202 \\ \mathrm{O} & 5.316733 & 0.527168 & -3.222105 \\ \mathrm{C} & 5.075954 & 3.201134 & -2.931564 \\ \mathrm{C} & 4.818385 & 4.170424 & -2.091636 \\ \mathrm{C} & 4.374607 & 5.050856 & -1.200322 \\ \mathrm{C} & 2.980793 & 5.528015 & -1.167243 \\ \mathrm{C} & 1.971909 & 4.881952 & -1.929157 \\ \mathrm{C} & 0.633011 & 5.268917 & -1.801883 \\ \mathrm{C} & 0.272868 & 6.305174 & -0.920505 \\ \mathrm{C} & 1.264086 & 6.955285 & -0.164683 \\ \mathrm{C} & 2.608164 & 6.567828 & -0.282814 \\ \mathrm{O} & 2.987049 & 1.636070 & -3.307439 \\ \mathrm{H} & -0.783499 & 6.594591 & -0.812835 \\ \mathrm{H} & 4.787165 & 0.428071 & -0.539819 \\ \mathrm{H} & 5.545218 & 3.281633 & -3.927638 \\ \mathrm{H} & 2.493872 & 3.578786 & 0.758924 \\ \mathrm{H} & 5.055538 & 5.396478 & -0.400681 \\ \mathrm{H} & 1.087766 & 1.152448 & -1.745904 \\ \mathrm{H} & 2.237385 & 4.028562 & -2.574022 \\ \mathrm{H} & -0.141482 & 4.729823 & -2.367641 \\ \mathrm{H} & -2.257540 & 3.413182 & 1.251423 \\ \mathrm{H} & 0.987801 & 7.766329 & 0.527184 \\ \mathrm{H} & 0.181810 & 3.720342 & 1.635370 \\ \mathrm{H} & 3.381714 & 7.070284 & 0.319719 \\ \mathrm{H} & -0.777585 & 0.119624 & -3.042399 \\ \mathrm{H} & -3.196584 & -0.181422 & -3.461896 \\ \mathrm{O} & -5.175050 & 0.970180 & -1.929931 \\ \mathrm{H} & -4.146252 & 2.368757 & -0.074993 \\ \mathrm{C} & -5.683415 & 0.168907 & -2.985508 \\ \mathrm{H} & -6.786403 & 0.223005 & -2.912009 \\ \mathrm{H} & -5.372173 & 0.546965 & -3.986093 \\ \mathrm{H} & -5.369655 & -0.895928 & -2.892229\end{array}$

Bisallene2Cyclization_TS_for_A

Free energy $=-1509.844666$ Hartree

$\begin{array}{llll}\text { C } & -3.750933 & 0.414971 & -1.583411\end{array}$

$\begin{array}{llll}\text { C } & -3.396500 & 1.420274 & -0.676832\end{array}$

$\begin{array}{llll}\text { C } & -2.046132 & 1.818761 & -0.521660\end{array}$

$\begin{array}{llll}\mathrm{C} & -1.019113 & 1.172700 & -1.313214\end{array}$

$\begin{array}{llll}\text { C } & -1.413624 & 0.154915 & -2.232861\end{array}$

$\begin{array}{llll}\text { C } & -2.741892 & -0.222708 & -2.370803\end{array}$

$\begin{array}{llll}\text { C } & 0.335194 & 1.565409 & -1.161458\end{array}$

$\begin{array}{llll}\text { C } & 0.708774 & 2.570227 & -0.254415\end{array}$

$\begin{array}{llll}\text { C } & -0.320881 & 3.223788 & 0.507166\end{array}$

$\begin{array}{llll}\text { C } & -1.647243 & 2.855023 & 0.383643\end{array}$

$\begin{array}{llll}\text { C } & 2.092821 & 3.003785 & -0.095236\end{array}$

$\begin{array}{llll}\text { C } & 3.136062 & 2.415763 & -0.749725\end{array}$

$\begin{array}{llll}\text { C } & 3.716298 & 1.258546 & -1.054420\end{array}$

$\begin{array}{llll}\text { S } & 4.590413 & 1.139567 & -2.617445\end{array}$

$\begin{array}{llll}\text { O } & 5.931269 & 0.540094 & -2.419373\end{array}$

$\begin{array}{llll}\text { C } & 4.719847 & 2.913752 & -2.914382\end{array}$

$\begin{array}{llll}\text { C } & 4.263805 & 3.764178 & -2.001216\end{array}$

$\begin{array}{llll}\text { C } & 4.212544 & 5.038359 & -1.508692\end{array}$

$\begin{array}{llll}\text { C } & 2.987145 & 5.755341 & -1.247318\end{array}$

$\begin{array}{llll}\text { C } & 1.741068 & 5.281474 & -1.751333\end{array}$

$\begin{array}{llll}\text { C } & 0.540420 & 5.920838 & -1.408963\end{array}$

$\begin{array}{llll}\text { C } & 0.555715 & 7.047093 & -0.571422\end{array}$

$\begin{array}{llll}\text { C } & 1.783965 & 7.548167 & -0.086745\end{array}$

$\begin{array}{llll}\text { C } & 2.983325 & 6.913910 & -0.418568\end{array}$

$\begin{array}{llll}\text { O } & 3.678154 & 0.561492 & -3.640205\end{array}$

$\begin{array}{llll}\mathrm{H} & -0.386998 & 7.545232 & -0.298448\end{array}$

$\begin{array}{llll}\mathrm{H} & 3.701423 & 0.335326 & -0.452360\end{array}$

$\begin{array}{llll}\mathrm{H} & 5.102331 & 3.180220 & -3.912246\end{array}$

$\begin{array}{llll}\mathrm{H} & 2.275182 & 3.878500 & 0.550604\end{array}$

$\begin{array}{llll}\mathrm{H} & 5.161511 & 5.480643 & -1.154677\end{array}$

$\begin{array}{llll}\mathrm{H} & 1.101211 & 1.089669 & -1.794929\end{array}$

$\begin{array}{llll}\mathrm{H} & 1.729910 & 4.417330 & -2.430337\end{array}$

$\begin{array}{llll}\mathrm{H} & -0.411997 & 5.527269 & -1.793111\end{array}$

$\begin{array}{llll}\mathrm{H} & -2.420272 & 3.362149 & 0.981971\end{array}$

$\begin{array}{llll}\mathrm{H} & 1.796472 & 8.439563 & 0.559386\end{array}$

$\begin{array}{llll}\mathrm{H} & -0.035133 & 4.034492 & 1.195982\end{array}$

$\begin{array}{llll}\mathrm{H} & 3.938837 & 7.295213 & -0.025080\end{array}$

$\begin{array}{llll}\mathrm{H} & -0.639179 & -0.339417 & -2.839963\end{array}$

$\begin{array}{llll}\mathrm{H} & -3.005832 & -1.014238 & -3.085409\end{array}$

$\begin{array}{llll}\mathrm{O} & -5.074848 & 0.102932 & -1.652127\end{array}$

$\begin{array}{llll}\mathrm{H} & -4.192594 & 1.896898 & -0.085965\end{array}$

$\begin{array}{llll}\text { C } & -5.509530 & -0.880301 & -2.579843\end{array}$

$\begin{array}{llll}\mathrm{H} & -6.608948 & -0.942568 & -2.472575\end{array}$

$\begin{array}{llll}\mathrm{H} & -5.265951 & -0.600422 & -3.630327\end{array}$

$\begin{array}{llll}\mathrm{H} & -5.071975 & -1.881701 & -2.364491\end{array}$

Bisallene2Cyclization_TS_for_B

45

Free energy $=-1509.851499$ Hartree 


$\begin{array}{crrr}\mathrm{C} & -2.139273 & 1.174244 & 0.079853 \\ \mathrm{C} & -1.003447 & 0.498124 & -0.389004 \\ \mathrm{C} & 0.293968 & 0.913609 & -0.011951 \\ \mathrm{C} & 0.440837 & 2.042569 & 0.878892 \\ \mathrm{C} & -0.730825 & 2.693354 & 1.358191 \\ \mathrm{C} & -1.997291 & 2.279007 & 0.971251 \\ \mathrm{C} & 1.738634 & 2.520072 & 1.185072 \\ \mathrm{C} & 2.900412 & 1.908425 & 0.664288 \\ \mathrm{C} & 2.740293 & 0.739078 & -0.163578 \\ \mathrm{C} & 1.486333 & 0.266796 & -0.491895 \\ \mathrm{C} & 4.184531 & 2.525608 & 0.849380 \\ \mathrm{C} & 4.316942 & 3.810344 & 1.305045 \\ \mathrm{C} & 4.759017 & 4.682145 & 2.198414 \\ \mathrm{~S} & 4.579200 & 6.455274 & 1.890566 \\ \mathrm{O} & 3.675150 & 7.003605 & 2.934265 \\ \mathrm{O} & 5.900278 & 7.088560 & 1.664210 \\ \mathrm{C} & 3.696787 & 6.321467 & 0.328093 \\ \mathrm{C} & 3.154472 & 5.154801 & -0.001635 \\ \mathrm{C} & 2.208931 & 4.479331 & -0.715409 \\ \mathrm{C} & 0.787366 & 4.810172 & -0.766248 \\ \mathrm{C} & -0.066746 & 4.048502 & -1.605640 \\ \mathrm{C} & -1.439139 & 4.321481 & -1.672074 \\ \mathrm{C} & -1.992981 & 5.351848 & -0.892326 \\ \mathrm{C} & -1.161010 & 6.099864 & -0.036181 \\ \mathrm{C} & 0.212561 & 5.834548 & 0.031318 \\ \mathrm{H} & -3.071565 & 5.567373 & -0.942238 \\ \mathrm{H} & 3.708701 & 7.238879 & -0.282968 \\ \mathrm{H} & 3.639380 & 0.242330 & -0.560592 \\ \mathrm{H} & 1.380384 & -0.613400 & -1.145620 \\ \mathrm{H} & 0.857181 & 6.409322 & 0.715201 \\ \mathrm{H} & -2.082920 & 3.713777 & -2.326533 \\ \mathrm{H} & -1.590562 & 6.899068 & 0.587997 \\ \mathrm{H} & 0.359847 & 3.224267 & -2.198428 \\ \mathrm{H} & 1.839226 & 3.387696 & 1.853737 \\ \mathrm{H} & 5.153936 & 4.445790 & 3.199623 \\ \mathrm{H} & 5.079664 & 2.026521 & 0.436100 \\ \mathrm{H} & 2.525231 & 3.605142 & -1.310040 \\ \mathrm{H} & -0.620034 & 3.566785 & 2.018259 \\ \mathrm{H} & -2.876476 & 2.823277 & 1.337823 \\ \mathrm{O} & -3.338814 & 0.714533 & -0.369516 \\ \mathrm{H} & -1.145768 & -0.354427 & -1.069825 \\ \mathrm{C} & -4.528697 & 1.378082 & 0.035294 \\ \mathrm{H} & -5.362032 & 0.848519 & -0.461548 \\ \mathrm{H} & -4.678169 & 1.330717 & 1.137747 \\ \mathrm{H} & -4.536848 & 2.444670 & -0.284079\end{array}$

\section{A = 2-pyridyl, $B$ = phenyl}

Bisallene

34

Free energy $=-1257.983951$ Hartree

$\begin{array}{llll}\mathrm{N} & 0.131294 & 3.202567 & 0.648452 \\ \mathrm{C} & 1.022514 & 2.621159 & -0.188820\end{array}$

$\begin{array}{llll}\text { C } & 1.022514 & 2.621159 & -0.188820\end{array}$

$\begin{array}{llll}\text { C } & 0.798536 & 1.353550 & -0.776354\end{array}$

$\begin{array}{llll}\text { C } & -0.386968 & 0.669880 & -0.477069\end{array}$

$\begin{array}{llll}\text { C } & -1.308985 & 1.267784 & 0.395111\end{array}$

$\begin{array}{llll}\text { C } & -0.994816 & 2.536227 & 0.924921\end{array}$

$\begin{array}{llll}\text { C } & 2.274182 & 3.342223 & -0.472318\end{array}$

$\begin{array}{llll}\text { C } & 2.574217 & 4.570109 & -0.065021\end{array}$

$\begin{array}{llll}\text { C } & 2.901220 & 5.799091 & 0.244731\end{array}$

$\begin{array}{llll}\text { S } & 3.756654 & 6.397603 & 1.739716\end{array}$

$\begin{array}{llll}\text { O } & 4.857736 & 7.261442 & 1.250533\end{array}$

$\begin{array}{llll}\text { C } & 4.481893 & 4.953423 & 2.588950\end{array}$

$\begin{array}{llll}\text { C } & 4.717246 & 3.773882 & 2.072541\end{array}$

$\begin{array}{llll}\text { C } & 4.906407 & 2.552441 & 1.586191\end{array}$

$\begin{array}{llll}\text { C } & 3.914371 & 1.467441 & 1.634173\end{array}$

$\begin{array}{llll}\text { C } & 2.681934 & 1.614818 & 2.319855\end{array}$

$\begin{array}{llll}\text { C } & 1.756729 & 0.567123 & 2.352627\end{array}$

$\begin{array}{llll}\text { C } & 2.030709 & -0.642599 & 1.687549\end{array}$

$\begin{array}{llll}\text { C } & 3.243037 & -0.798978 & 0.994109\end{array}$

$\begin{array}{llll}\text { C } & 4.179834 & 0.245602 & 0.970741\end{array}$

$\begin{array}{llll}\mathrm{H} & 1.293534 & -1.460222 & 1.704202\end{array}$

$\begin{array}{llll}\mathrm{O} & 2.749773 & 6.943231 & 2.679678\end{array}$

$\begin{array}{llll}\mathrm{H} & -2.251333 & 0.768384 & 0.665233\end{array}$

$\begin{array}{llll}\mathrm{H} & 2.671252 & 6.679813 & -0.382340\end{array}$

$\begin{array}{llll}\mathrm{H} & 4.714325 & 5.249802 & 3.627903\end{array}$

$\begin{array}{llll}\mathrm{H} & 3.035752 & 2.801152 & -1.063025\end{array}$

$\begin{array}{llll}\mathrm{H} & 5.869487 & 2.336698 & 1.087476\end{array}$

$\begin{array}{llll}\mathrm{H} & 2.447760 & 2.572875 & 2.808398\end{array}$

$\begin{array}{llll}\mathrm{H} & -1.699455 & 3.036956 & 1.614226\end{array}$

$\begin{array}{llll}\mathrm{H} & 0.798056 & 0.703762 & 2.875060\end{array}$

$\begin{array}{llll}\mathrm{H} & -0.582115 & -0.322467 & -0.911138\end{array}$

$\begin{array}{llll}\mathrm{H} & 3.462522 & -1.742088 & 0.470539\end{array}$

$\begin{array}{llll}\mathrm{H} & 1.557847 & 0.908661 & -1.436793\end{array}$

$\begin{array}{llll}\mathrm{H} & 5.133567 & 0.118001 & 0.434114\end{array}$

Bisallene2Cyclization_TS_for_A

34

Free energy $=-1257.964346$ Hartree

$\begin{array}{llll}\text { C } & 1.915207 & 2.273759 & 1.081474\end{array}$

$\begin{array}{lrrr}\mathrm{C} & 2.978947 & 1.940253 & 0.200602 \\ \mathrm{C} & 2.683076 & 1.192125 & -0.969797 \\ \mathrm{C} & 1.366283 & 0.815032 & -1.263164 \\ \mathrm{C} & 0.319888 & 1.162553 & -0.389240 \\ \mathrm{C} & 0.602861 & 1.888722 & 0.784597 \\ \mathrm{C} & 4.364307 & 2.316532 & 0.470167 \\ \mathrm{C} & 4.762105 & 3.083161 & 1.527209 \\ \mathrm{C} & 4.527686 & 4.199949 & 2.213743 \\ \mathrm{~S} & 5.022691 & 4.249274 & 3.939026 \\ \mathrm{O} & 3.863383 & 3.897192 & 4.797867 \\ \mathrm{H} & -0.713984 & 0.861782 & -0.617557 \\ \mathrm{C} & 6.125598 & 2.824019 & 3.862719 \\ \mathrm{C} & 6.311051 & 2.205699 & 2.701266 \\ \mathrm{C} & 7.034199 & 1.314471 & 1.956930 \\ \mathrm{C} & 6.439181 & 0.200206 & 1.256604 \\ \mathrm{C} & 5.094870 & -0.195345 & 1.517623 \\ \mathrm{C} & 4.510953 & -1.188829 & 0.717960 \\ \mathrm{C} & 5.280267 & -1.768729 & -0.298123 \\ \mathrm{C} & 6.623450 & -1.345365 & -0.451259 \\ \mathrm{~N} & 7.195512 & -0.393937 & 0.287486 \\ \mathrm{O} & 5.789313 & 5.486597 & 4.214931 \\ \mathrm{H} & 4.866175 & -2.547304 & -0.956700 \\ \mathrm{H} & 4.077216 & 5.126500 & 1.823369 \\ \mathrm{H} & 7.259979 & -1.811059 & -1.226164 \\ \mathrm{H} & 3.467156 & -1.493411 & 0.884516 \\ \mathrm{H} & 2.136360 & 2.818317 & 2.012893 \\ \mathrm{H} & 1.152074 & 0.244394 & -2.179989 \\ \mathrm{H} & -0.209955 & 2.151855 & 1.478845 \\ \mathrm{H} & 3.503808 & 0.909642 & -1.647516 \\ \mathrm{H} & 4.539515 & 0.259595 & 2.349284 \\ \mathrm{H} & 6.518771 & 2.493844 & 4.836875 \\ \mathrm{H} & 8.091732 & 1.532813 & 1.728238 \\ \mathrm{H} & 5.127786 & 1.932943 & -0.227260\end{array}$

Bisallene2Cyclization_TS_for_B

34

Free energy $=-1257.963633$ Hartree

$\begin{array}{lrrr}\mathrm{C} & 4.773342 & 0.165061 & 1.131279 \\ \mathrm{C} & 4.223978 & 1.364770 & 1.669835 \\ \mathrm{C} & 2.879139 & 1.341839 & 2.144883 \\ \mathrm{C} & 2.108424 & 0.173608 & 2.052433 \\ \mathrm{C} & 2.658869 & -0.988579 & 1.492714 \\ \mathrm{C} & 3.998200 & -0.992305 & 1.042562 \\ \mathrm{C} & 4.976463 & 2.593350 & 1.646223 \\ \mathrm{C} & 4.413454 & 3.832831 & 1.791550 \\ \mathrm{C} & 4.389557 & 5.000477 & 2.424943 \\ \mathrm{~S} & 3.460423 & 6.369810 & 1.703739 \\ \mathrm{O} & 2.346599 & 6.715636 & 2.625130 \\ \mathrm{C} & 2.808221 & 5.447441 & 0.309945 \\ \mathrm{C} & 2.870168 & 4.118055 & 0.350861 \\ \mathrm{C} & 2.284766 & 2.957864 & -0.063142 \\ \mathrm{C} & 0.846746 & 2.695545 & 0.045156 \\ \mathrm{~N} & 0.427634 & 1.525163 & -0.501698 \\ \mathrm{C} & -0.877123 & 1.229860 & -0.429581 \\ \mathrm{C} & -1.835918 & 2.053661 & 0.190440 \\ \mathrm{C} & -1.397583 & 3.256430 & 0.774177 \\ \mathrm{C} & -0.040705 & 3.587439 & 0.706217 \\ \mathrm{O} & 4.377169 & 7.435250 & 1.234795 \\ \mathrm{H} & 2.048949 & -1.900625 & 1.403956 \\ \mathrm{H} & -2.894074 & 1.753085 & 0.222106 \\ \mathrm{H} & 2.388666 & 6.057962 & -0.505273 \\ \mathrm{H} & 4.817419 & 5.209362 & 3.417938 \\ \mathrm{H} & 2.876536 & 2.132213 & -0.488163 \\ \mathrm{H} & 6.033626 & 2.553224 & 1.326875 \\ \mathrm{H} & 2.460725 & 2.243644 & 2.613500 \\ \mathrm{H} & -1.184343 & 0.272452 & -0.888414 \\ \mathrm{H} & 1.067906 & 0.178790 & 2.407915 \\ \mathrm{H} & -2.107134 & 3.927316 & 1.282885 \\ \mathrm{H} & 4.427816 & -1.908160 & 0.608671 \\ \mathrm{H} & 0.346554 & 4.507755 & 1.169453 \\ \mathrm{H} & 5.810352 & 0.168280 & 0.760692\end{array}$

$A$ = 2-pyridyl, B = 4-methoxyphenyl Bisallene

38

Free energy $=-1372.407335$ Hartree

$\begin{array}{lccc}\mathrm{C} & 2.020429 & 2.587176 & 1.404592 \\ \mathrm{C} & 2.834293 & 2.150138 & 0.327239 \\ \mathrm{~N} & 2.329896 & 1.540445 & -0.770563 \\ \mathrm{C} & 1.004339 & 1.332438 & -0.816703 \\ \mathrm{C} & 0.115561 & 1.721101 & 0.202096 \\ \mathrm{C} & 0.641855 & 2.367060 & 1.333770 \\ \mathrm{C} & 4.279949 & 2.456579 & 0.285701 \\ \mathrm{C} & 4.920239 & 3.016782 & 1.304409 \\ \mathrm{C} & 5.360412 & 3.694593 & 2.333658 \\ \mathrm{~S} & 4.779913 & 5.409534 & 2.652766 \\ \mathrm{O} & 5.872596 & 6.155242 & 3.318537 \\ \mathrm{C} & 4.460223 & 6.202589 & 1.033525 \\ \mathrm{C} & 3.453688 & 5.782936 & 0.303835 \\ \mathrm{C} & 2.394507 & 5.256653 & -0.300498\end{array}$




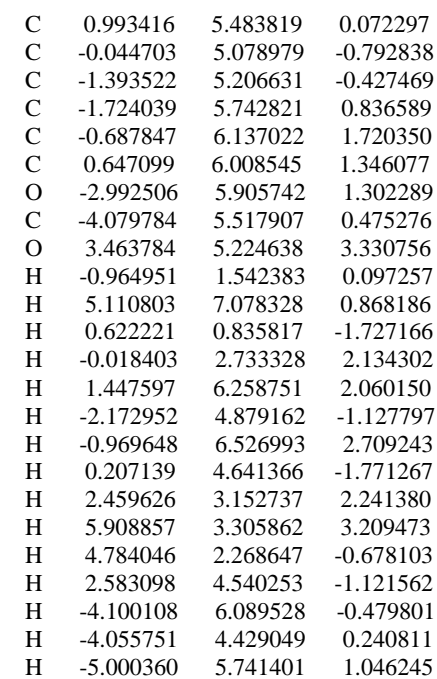

Bisallene2Cyclization_TS_for_A 38

Free energy $=-1372.383668$ Hartree

$\begin{array}{cccc}\mathrm{C} & 1.893212 & 2.249717 & 1.047405 \\ \mathrm{C} & 2.989895 & 1.928770 & 0.195816 \\ \mathrm{~N} & 2.839192 & 1.153628 & -0.917487 \\ \mathrm{C} & 1.611161 & 0.729766 & -1.219582 \\ \mathrm{C} & 0.455605 & 1.041873 & -0.463672 \\ \mathrm{C} & 0.609874 & 1.805609 & 0.700629 \\ \mathrm{C} & 4.302649 & 2.485278 & 0.432994 \\ \mathrm{C} & 4.537180 & 3.521428 & 1.292709 \\ \mathrm{C} & 5.215203 & 4.009224 & 2.326210 \\ \mathrm{~S} & 5.170618 & 5.779470 & 2.666197 \\ \mathrm{O} & 6.485665 & 6.401642 & 2.380326 \\ \mathrm{C} & 4.012772 & 6.202342 & 1.356825 \\ \mathrm{C} & 3.318354 & 5.221570 & 0.784105 \\ \mathrm{C} & 2.197744 & 4.906230 & 0.069764 \\ \mathrm{C} & 0.844924 & 5.321116 & 0.406643 \\ \mathrm{C} & -0.231880 & 4.925652 & -0.426830 \\ \mathrm{C} & -1.553674 & 5.293291 & -0.146076 \\ \mathrm{C} & -1.833516 & 6.066177 & 1.005071 \\ \mathrm{C} & -0.769215 & 6.455044 & 1.858609 \\ \mathrm{C} & 0.540990 & 6.091061 & 1.565797 \\ \mathrm{O} & -3.072891 & 6.474924 & 1.380947 \\ \mathrm{C} & -4.190336 & 6.111058 & 0.581011 \\ \mathrm{O} & 4.540534 & 5.982297 & 3.995353 \\ \mathrm{H} & -0.531430 & 0.674991 & -0.782580 \\ \mathrm{H} & 4.006893 & 7.266581 & 1.069019 \\ \mathrm{H} & 1.520433 & 0.103845 & -2.126615 \\ \mathrm{H} & -0.255631 & 2.065350 & 1.328407 \\ \mathrm{H} & 1.354578 & 6.382685 & 2.247633 \\ \mathrm{H} & -2.356225 & 4.973962 & -0.824033 \\ \mathrm{H} & -1.013341 & 7.042976 & 2.755529 \\ \mathrm{H} & -0.021651 & 4.311548 & -1.316794 \\ \mathrm{H} & 2.063528 & 2.825446 & 1.967415 \\ \mathrm{H} & 5.753429 & 3.413259 & 3.080271 \\ \mathrm{H} & 5.109225 & 2.151989 & -0.242811 \\ \mathrm{H} & 2.307756 & 4.263178 & -0.820458 \\ \mathrm{H} & -4.112830 & 6.523193 & -0.449899 \\ \mathrm{H} & -4.312955 & 5.005802 & 0.519194 \\ \mathrm{H} & -5.079400 & 6.544551 & 1.074519 \\ & & & \\ & & & \end{array}$

Bisallene2Cyclization_TS_for_B

38

Free energy $=-1372.386009$ Hartree

$\begin{array}{lrrr}\mathrm{C} & 0.530000 & 1.462182 & -0.509942 \\ \mathrm{C} & 0.963282 & 2.687558 & 0.070330 \\ \mathrm{C} & -0.019713 & 3.494278 & 0.723886 \\ \mathrm{C} & -1.357817 & 3.119233 & 0.758568 \\ \mathrm{C} & -1.778635 & 1.942450 & 0.099364 \\ \mathrm{C} & -0.815544 & 1.095922 & -0.512668 \\ \mathrm{C} & 2.308775 & 3.162516 & -0.105305 \\ \mathrm{C} & 2.638387 & 4.491224 & -0.013539 \\ \mathrm{C} & 3.373909 & 5.443080 & 0.549063 \\ \mathrm{~S} & 3.334881 & 7.108388 & -0.147188 \\ \mathrm{O} & 2.810554 & 8.025213 & 0.897254 \\ \mathrm{O} & -3.111931 & 1.705921 & 0.105245 \\ \mathrm{C} & -3.622412 & 0.597869 & -0.628160 \\ \mathrm{C} & 2.072089 & 6.781267 & -1.382040 \\ \mathrm{C} & 1.396978 & 5.634941 & -1.322867 \\ \mathrm{C} & 0.278026 & 4.956622 & -1.713383 \\ \mathrm{C} & -1.100436 & 5.384802 & -1.499310 \\ \mathrm{~N} & -2.053081 & 4.512477 & -1.928605 \\ \mathrm{C} & -3.337102 & 4.822080 & -1.701850 \\ \mathrm{C} & -3.759555 & 5.990454 & -1.041532\end{array}$

$\begin{array}{rrrr}\mathrm{C} & -2.773845 & 6.893952 & -0.600177 \\ \mathrm{C} & -1.428708 & 6.594793 & -0.830508 \\ \mathrm{O} & 4.612117 & 7.434339 & -0.826821 \\ \mathrm{H} & -4.830133 & 6.183491 & -0.875230 \\ \mathrm{H} & 1.988428 & 7.545527 & -2.171135 \\ \mathrm{H} & 3.941793 & 5.360355 & 1.489414 \\ \mathrm{H} & 0.368373 & 3.954133 & -2.163264 \\ \mathrm{H} & 3.060792 & 2.474580 & -0.532152 \\ \mathrm{H} & 0.294955 & 4.432929 & 1.203423 \\ \mathrm{H} & -4.082468 & 4.087938 & -2.057397 \\ \mathrm{H} & -2.119481 & 3.753761 & 1.233254 \\ \mathrm{H} & -3.055658 & 7.820194 & -0.075647 \\ \mathrm{H} & -1.118254 & 0.162066 & -1.004486 \\ \mathrm{H} & -0.625758 & 7.261814 & -0.482365 \\ \mathrm{H} & 1.267896 & 0.810760 & -1.004554 \\ \mathrm{H} & -4.723090 & 0.641933 & -0.527456 \\ \mathrm{H} & -3.262080 & -0.371673 & -0.217623 \\ \mathrm{H} & -3.350298 & 0.661708 & -1.705254\end{array}$

A = 1-benzyl-3-indolyl, $B=$ phenyl Bisallene

Free energy $=-1643.497814$ Hartree

C $\quad 0.073386 \quad 3.568784 \quad 0.363466$

$\begin{array}{llll}\text { C } & 1.040812 & 2.669715 & -0.145745 \\ \text { C } & 0.629614 & 1.363779 & -0.521058\end{array}$

$\begin{array}{llll}\text { C } & 0.629614 & 1.363779 & -0.521058\end{array}$

$\begin{array}{llll}\text { C } & -0.707644 & 0.975884 & -0.381166\end{array}$

$\begin{array}{llll}\text { C } & -1.660857 & 1.875074 & 0.132518\end{array}$

$\begin{array}{llll}\text { C } & -1.266914 & 3.173562 & 0.499304\end{array}$

$\begin{array}{llll}\text { C } & 2.463299 & 3.053972 & -0.168513\end{array}$

$\begin{array}{llll}\text { C } & 3.417787 & 2.283199 & -0.679730\end{array}$

$\begin{array}{llll}\text { C } & 4.264846 & 1.379794 & -1.101261\end{array}$

$\begin{array}{llll}\mathrm{S} & 4.625483 & -0.143183 & -0.135034\end{array}$

$\begin{array}{llll}\text { O } & 3.570291 & -1.107636 & -0.567417\end{array}$

$\begin{array}{llll}\text { C } & 4.338375 & 0.242961 & 1.631739\end{array}$

$\begin{array}{llll}\text { C } & 3.101301 & 0.415272 & 2.035114\end{array}$

$\begin{array}{llll}\text { C } & 1.783924 & 0.498351 & 2.205706\end{array}$

$\begin{array}{llll}\text { C } & 0.872254 & -0.632576 & 2.183136\end{array}$

$\begin{array}{llll}\text { C } & 1.133597 & -1.877238 & 1.605590\end{array}$

$\begin{array}{llll}\mathrm{N} & -0.005698 & -2.654627 & 1.632185\end{array}$

$\begin{array}{llll}\text { C } & -1.036197 & -1.932555 & 2.230901\end{array}$

$\begin{array}{llll}\text { C } & -0.519486 & -0.644747 & 2.582500\end{array}$

$\begin{array}{llll}\text { O } & 6.060202 & -0.474840 & -0.308852\end{array}$

$\begin{array}{llll}\mathrm{H} & -2.703155 & 1.551927 & 0.271817\end{array}$

$\begin{array}{llll}\mathrm{H} & 5.265284 & 0.186360 & 2.227218\end{array}$

$\begin{array}{llll}\mathrm{H} & 4.713450 & 1.303676 & -2.107298\end{array}$

$\begin{array}{llll}\mathrm{H} & 1.335018 & 1.502995 & 2.307116\end{array}$

$\begin{array}{llll}\mathrm{H} & 2.745376 & 4.001521 & 0.325856\end{array}$

$\begin{array}{llll}\mathrm{H} & 1.382897 & 0.634632 & -0.858607\end{array}$

$\begin{array}{llll}\mathrm{H} & -1.003590 & -0.052425 & -0.636437\end{array}$

$\begin{array}{llll}\mathrm{H} & -2.006431 & 3.880857 & 0.905377\end{array}$

$\begin{array}{llll}\mathrm{H} & 0.382276 & 4.582054 & 0.666446\end{array}$

$\begin{array}{llll}\text { C } & -2.372446 & -2.300470 & 2.470855\end{array}$

$\begin{array}{llll}\text { C } & -3.208845 & -1.336804 & 3.051426\end{array}$

$\begin{array}{llll}\text { C } & -2.722303 & -0.047149 & 3.388019\end{array}$

$\begin{array}{llll}\text { C } & -1.385212 & 0.305291 & 3.165021\end{array}$

$\begin{array}{llll}\mathrm{H} & -2.741511 & -3.305830 & 2.221216\end{array}$

$\begin{array}{llll}\mathrm{H} & -4.260900 & -1.588219 & 3.254570\end{array}$

$\begin{array}{llll}\mathrm{H} & -3.409686 & 0.686201 & 3.836919\end{array}$

$\begin{array}{llll}\mathrm{H} & -1.017344 & 1.308348 & 3.427754\end{array}$

$\begin{array}{llll}\text { C } & -0.115644 & -4.027249 & 1.167486\end{array}$

$\begin{array}{llll}\mathrm{H} & 0.808845 & -4.256415 & 0.596577\end{array}$

$\begin{array}{llll}\text { C } & -0.304600 & -5.038868 & 2.290259\end{array}$

$\begin{array}{llll}\mathrm{C} & -0.304600 & -5.038868 & 2.290259 \\ \mathrm{H} & -0.958411 & -4.101855 & 0.445532\end{array}$

$\begin{array}{llll}\text { C } & -0.995019 & -6.241783 & 2.043343\end{array}$

$\begin{array}{llll}\text { C } & -1.145204 & -7.200107 & 3.059253\end{array}$

$\begin{array}{llll}\text { C } & -0.612751 & -6.960111 & 4.337510\end{array}$

$\begin{array}{llll}\text { C } & 0.069847 & -5.757984 & 4.592085\end{array}$

$\begin{array}{llll}\text { C } & 0.223438 & -4.802576 & 3.574532\end{array}$

$\begin{array}{llll}\mathrm{H} & -1.422694 & -6.430125 & 1.044918\end{array}$

$\begin{array}{llll}\mathrm{H} & -1.687414 & -8.135825 & 2.852984\end{array}$

$\begin{array}{llll}\mathrm{H} & -0.736042 & -7.707254 & 5.136945\end{array}$

$\begin{array}{llll}\mathrm{H} & 0.483861 & -5.559799 & 5.592588\end{array}$

$\begin{array}{llll}\mathrm{H} & 0.747842 & -3.856369 & 3.779856\end{array}$

$\begin{array}{llll}\mathrm{H} & 2.048836 & -2.210706 & 1.099605\end{array}$

Bisallene2Cyclization_TS_for_A

52

Free energy $=-1643.487284$ Hartree

$\begin{array}{rrrr}\mathrm{C} & 2.308268 & 2.035831 & 1.777036 \\ \mathrm{C} & 3.546712 & 1.801657 & 1.044584 \\ \mathrm{C} & 3.154838 & 1.469835 & -0.272318 \\ \mathrm{~N} & 1.792909 & 1.486656 & -0.384573 \\ \mathrm{C} & 1.236653 & 1.830506 & 0.852989 \\ \mathrm{C} & 4.887605 & 1.758974 & 1.520966 \\ \mathrm{C} & 5.899702 & 1.201300 & 0.786502 \\ \mathrm{C} & 7.047763 & 1.285374 & 0.134869 \\ \mathrm{~S} & 7.813248 & -0.209898 & -0.537893 \\ \mathrm{O} & 9.019563 & -0.587390 & 0.237655 \\ \mathrm{C} & 6.482209 & -1.357836 & -0.140080\end{array}$




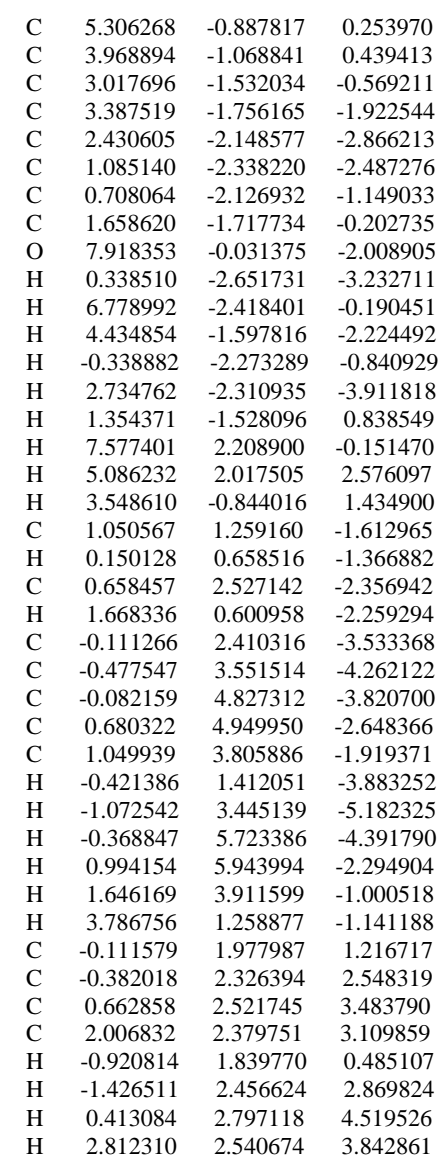

Bisallene2Cyclization_TS_for_B

52

Free energy $=-1643.477688$ Hartree

$\begin{array}{llll}\text { C } & 0.194366 & 3.933769 & 0.620020\end{array}$

$\begin{array}{llll}\text { C } & 0.985428 & 2.883455 & 0.073797\end{array}$

$\begin{array}{llll}\text { C } & 0.319617 & 1.716765 & -0.402485\end{array}$

$\begin{array}{llll}\text { C } & -1.074743 & 1.602039 & -0.312678\end{array}$

$\begin{array}{llll}\text { C } & -1.837433 & 2.645957 & 0.236417\end{array}$

$\begin{array}{llll}\text { C } & -1.196917 & 3.815325 & 0.697157\end{array}$

$\begin{array}{llll}\text { C } & 2.428259 & 2.983319 & 0.052155\end{array}$

$\begin{array}{llll}\text { C } & 3.277470 & 1.943948 & -0.195421\end{array}$

$\begin{array}{llll}\text { C } & 4.291493 & 1.436345 & -0.885954\end{array}$

$\begin{array}{llll}\text { S } & 5.177404 & -0.003298 & -0.259186\end{array}$

$\begin{array}{llll}\text { O } & 5.040536 & -1.102058 & -1.249607\end{array}$

$\begin{array}{llll}\text { C } & 4.118594 & -0.298932 & 1.166818\end{array}$

$\begin{array}{llll}\text { C } & 2.938150 & 0.311979 & 1.220119\end{array}$

$\begin{array}{llll}\text { C } & 1.680837 & 0.310274 & 1.750376\end{array}$

$\begin{array}{llll}\text { C } & 0.806967 & -0.835360 & 1.763855\end{array}$

$\begin{array}{llll}\text { C } & 1.102819 & -2.140614 & 1.344885\end{array}$

$\begin{array}{llll}\mathrm{N} & -0.005878 & -2.946162 & 1.48688\end{array}$

$\begin{array}{llll}\text { C } & -1.054095 & -2.191709 & 2.014687\end{array}$

$\begin{array}{llll}\text { C } & -0.582884 & -0.854341 & 2.190510\end{array}$

$\begin{array}{llll}\text { O } & 6.529544 & 0.382666 & 0.214651\end{array}$

$\begin{array}{llll}\mathrm{H} & -2.931150 & 2.547134 & 0.314897\end{array}$

$\begin{array}{llll}\mathrm{H} & 4.579138 & -0.916262 & 1.956600\end{array}$

$\mathrm{H} \quad 4.620470 \quad 1.765706 \quad-1.883927$

$\begin{array}{llll}\mathrm{H} & 1.272755 & 1.250927 & 2.154129\end{array}$

$\begin{array}{llll}\mathrm{H} & 2.885882 & 3.933887 & 0.382100\end{array}$

$\begin{array}{llll}\mathrm{H} & 0.911874 & 0.911257 & -0.859597\end{array}$

$\begin{array}{llll}\mathrm{H} & -1.568727 & 0.684642 & -0.666063\end{array}$

$\begin{array}{llll}\mathrm{H} & -1.792434 & 4.637041 & 1.124679\end{array}$

$\begin{array}{llll}\mathrm{H} & 0.692883 & 4.843212 & 0.992255\end{array}$

$\begin{array}{llll}\text { C } & -2.365220 & -2.580324 & 2.344155\end{array}$

$\begin{array}{llll}\text { C } & -3.220818 & -1.586946 & 2.840293 \\ \text { C } & -2.778671 & -0.249328 & 3.004894\end{array}$

$\begin{array}{llll}\text { C } & -2.778671 & -0.249328 & 3.004894\end{array}$

$\begin{array}{llll}\text { C } & -1.467204 & 0.125902 & 2.686624\end{array}$

$\begin{array}{llll}\mathrm{H} & -2.698904 & -3.621873 & 2.230945\end{array}$

$\begin{array}{llll}\mathrm{H} & -4.253645 & -1.852817 & 3.111575\end{array}$

$\begin{array}{llll}\mathrm{H} & -3.480533 & 0.505685 & 3.390368\end{array}$

$\begin{array}{llll}\mathrm{H} & -1.137031 & 1.168543 & 2.804867\end{array}$

$\begin{array}{llll}\text { C } & -0.074876 & -4.371785 & 1.204810\end{array}$

$\begin{array}{llll}\mathrm{H} & 0.865471 & -4.651326 & 0.684297\end{array}$

$\begin{array}{llll}\text { C } & -0.264908 & -5.228224 & 2.449355\end{array}$

$\begin{array}{llll}\mathrm{H} & -0.901783 & -4.559897 & 0.485978\end{array}$

$\begin{array}{llll}\text { C } & -0.976494 & -6.441213 & 2.367260\end{array}$

$\begin{array}{llll}\text { C } & -1.130850 & -7.256022 & 3.501321\end{array}$

$\begin{array}{llll}\text { C } & -0.582262 & -6.858987 & 4.732585 \\ \text { C } & 0.121128 & -5.645233 & 4.822478\end{array}$

$\begin{array}{llll}\text { C } & 0.121128 & -5.645233 & 4.822478\end{array}$

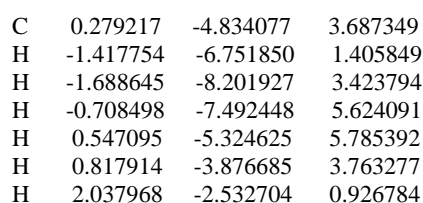

\section{A = 1-benzyl-3-indolyl, B = 4-methoxyphenyl}

Bisallene

56

Free energy $=-1757.922577$ Hartree

$\begin{array}{llll}\text { C } & -0.115510 & -4.382689 & 3.282868\end{array}$

$\begin{array}{llll}\text { C } & -0.849615 & -4.098865 & 2.114516\end{array}$

$\begin{array}{llll}\text { C } & -1.923665 & -4.938272 & 1.759427\end{array}$

$\begin{array}{llll}\text { C } & -2.252585 & -6.050691 & 2.552005\end{array}$

$\begin{array}{llll}\text { C } & -1.514339 & -6.329380 & 3.714474\end{array}$

$\begin{array}{llll}\text { C } & -0.446145 & -5.490771 & 4.078381\end{array}$

$\begin{array}{llll}\text { C } & -0.485050 & -2.915305 & 1.233719\end{array}$

$\begin{array}{llll}\mathrm{N} & -0.012053 & -1.757109 & 1.978188\end{array}$

$\begin{array}{llll}\text { C } & 1.188524 & -1.108191 & 1.783561\end{array}$

$\begin{array}{llll}\text { C } & 1.210302 & 0.084321 & 2.509401\end{array}$

$\begin{array}{llll}\text { C } & -0.065787 & 0.179062 & 3.186130\end{array}$

$\begin{array}{llll}\text { C } & -0.804391 & -0.997080 & 2.835900\end{array}$

$\begin{array}{llll}\text { C } & -0.663663 & 1.137232 & 4.031719\end{array}$

$\begin{array}{llll}\text { C } & -1.965016 & 0.910437 & 4.498906\end{array}$

$\begin{array}{llll}\text { C } & -2.674236 & -0.267021 & 4.150014\end{array}$

$\begin{array}{llll}\text { C } & -2.102602 & -1.240048 & 3.316073\end{array}$

$\begin{array}{llll}\text { C } & 2.250381 & 1.096457 & 2.426905\end{array}$

$\begin{array}{llll}\text { C } & 3.515881 & 0.867223 & 2.086298\end{array}$

$\begin{array}{llll}\text { C } & 4.690080 & 0.581201 & 1.574184\end{array}$

$\begin{array}{llll}\text { S } & 4.837261 & 0.112019 & -0.193356\end{array}$

$\begin{array}{llll}\text { O } & 6.259310 & -0.221170 & -0.452608\end{array}$

$\begin{array}{llll}\text { C } & 4.402576 & 1.567983 & -1.237507\end{array}$

$\begin{array}{llll}\text { C } & 3.352300 & 2.288511 & -0.930728\end{array}$

$\begin{array}{llll}\text { C } & 2.214700 & 2.755855 & -0.424055\end{array}$

$\begin{array}{llll}\text { C } & 0.952019 & 2.005326 & -0.453211\end{array}$

$\begin{array}{llll}\text { C } & -0.176597 & 2.486072 & 0.246025\end{array}$

$\begin{array}{llll}\text { C } & -1.338640 & 1.711495 & 0.388085\end{array}$

$\begin{array}{llll}\text { C } & -1.393361 & 0.428701 & -0.194932\end{array}$

$\begin{array}{llll}\text { C } & -0.279201 & -0.053081 & -0.924583\end{array}$

$\begin{array}{llll}\text { C } & 0.873298 & 0.715079 & -1.043337\end{array}$

$\begin{array}{llll}\text { O } & 3.762879 & -0.889964 & -0.466848\end{array}$

$\begin{array}{llll}\text { O } & -2.451309 & -0.425281 & -0.097078\end{array}$

$\begin{array}{llll}\mathrm{H} & 5.649057 & 0.434822 & 2.101278\end{array}$

$\begin{array}{llll}\mathrm{H} & 4.994439 & 1.572108 & -2.169466\end{array}$

$\begin{array}{llll}\mathrm{H} & 1.938408 & 2.146192 & 2.582591\end{array}$

$\begin{array}{llll}\mathrm{H} & 2.232795 & 3.700711 & 0.14846\end{array}$

$\begin{array}{llll}\mathrm{H} & 1.757059 & 0.291307 & -1.542322\end{array}$

$\mathrm{H} \quad-0.338111 \quad-1.057846 \quad-1.367070$

$\begin{array}{llll}\mathrm{H} & -2.175894 & 2.100113 & 0.981969\end{array}$

$\begin{array}{llll}\mathrm{H} & -0.136095 & 3.476982 & 0.725142\end{array}$

$\begin{array}{llll}\mathrm{H} & -2.645021 & -2.157589 & 3.047434\end{array}$

$\begin{array}{llll}\mathrm{H} & -3.689306 & -0.424791 & 4.544579\end{array}$

$\begin{array}{llll}\mathrm{H} & -2.442706 & 1.651526 & 5.158494\end{array}$

$\begin{array}{llll}\mathrm{H} & -0.113556 & 2.046326 & 4.319636\end{array}$

$\begin{array}{llll}\mathrm{H} & 0.321176 & -3.197711 & 0.524928\end{array}$

$\begin{array}{llll}\mathrm{H} & -1.359875 & -2.603274 & 0.621764\end{array}$

$\begin{array}{llll}\mathrm{H} & -2.510639 & -4.716426 & 0.853327\end{array}$

$\begin{array}{llll}\mathrm{H} & -3.095689 & -6.698278 & 2.264889\end{array}$

$\begin{array}{llll}\mathrm{H} & -1.773915 & -7.197088 & 4.340397\end{array}$

$\begin{array}{llll}\mathrm{H} & 0.132395 & -5.698263 & 4.991748\end{array}$

$\begin{array}{llll}\mathrm{H} & 0.713081 & -3.717546 & 3.573356\end{array}$

$\begin{array}{llll}\mathrm{H} & 1.942253 & -1.477087 & 1.074562\end{array}$

$\begin{array}{llll}\text { C } & -3.643421 & 0.039961 & 0.529919\end{array}$

$\begin{array}{llll}\mathrm{H} & -4.368375 & -0.793438 & 0.469792\end{array}$

$\begin{array}{llll}\mathrm{H} & -3.472643 & 0.291186 & 1.599176\end{array}$

$\begin{array}{lllr}\mathrm{H} & -3.472643 & 0.291186 & 1.599176 \\ \mathrm{H} & -4.057934 & 0.926081 & -0.000821\end{array}$

Bisallene2Cyclization_TS_for_A

56

Free energy $=-1757.905714$ Hartree

$\begin{array}{lrrr}\mathrm{C} & 2.037736 & 2.375629 & 3.123107 \\ \mathrm{C} & 2.343049 & 2.046157 & 1.787377 \\ \mathrm{C} & 1.272728 & 1.834300 & 0.862381 \\ \mathrm{C} & -0.076923 & 1.962419 & 1.228595 \\ \mathrm{C} & -0.350883 & 2.296804 & 2.563131 \\ \mathrm{C} & 0.692369 & 2.497629 & 3.499340 \\ \mathrm{~N} & 1.832098 & 1.504862 & -0.376880 \\ \mathrm{C} & 3.195324 & 1.502485 & -0.263930 \\ \mathrm{C} & 3.583730 & 1.829184 & 1.053662 \\ \mathrm{C} & 4.926474 & 1.786603 & 1.529375 \\ \mathrm{C} & 5.931024 & 1.221585 & 0.790202 \\ \mathrm{C} & 7.075825 & 1.300388 & 0.131074 \\ \mathrm{~S} & 7.829384 & -0.196480 & -0.549799 \\ \mathrm{O} & 7.928296 & -0.012069 & -2.020893 \\ \mathrm{C} & 1.092654 & 1.262026 & -1.602851 \\ \mathrm{C} & 0.673302 & 2.517804 & -2.351605 \\ \mathrm{C} & -0.088460 & 2.378648 & -3.530849\end{array}$




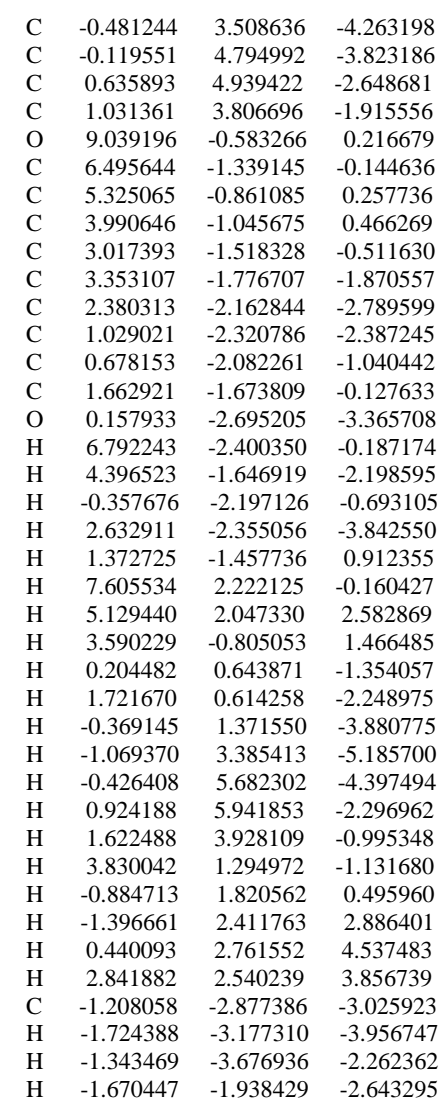

Bisallene2Cyclization_TS_for_B

56

Free energy $=-1757.899183$ Hartree

$\begin{array}{lrrr}\text { C } & 0.169210 & -4.739106 & 3.621026 \\ \text { C } & -0.420699 & -5.061640 & 2.383287 \\ \text { C } & -1.192221 & -6.235271 & 2.275777 \\ \text { C } & -1.360640 & -7.082342 & 3.383777 \\ \text { C } & -0.765590 & -6.757462 & 4.614728 \\ \text { C } & -0.002390 & -5.582696 & 4.730294 \\ \text { C } & -0.214115 & -4.173107 & 1.164152 \\ \text { N } & -0.049381 & -2.765724 & 1.489495\end{array}$

$\begin{array}{llll}\text { C } & 1.107602 & -2.026546 & 1.348382\end{array}$

$\begin{array}{llll}\text { C } & 0.901656 & -0.718187 & 1.805660\end{array}$

$\begin{array}{llll}\text { C } & -0.478330 & -0.663907 & 2.261794\end{array}$

$\begin{array}{llll}\text { C } & -1.037747 & -1.962825 & 2.056463\end{array}$

$\begin{array}{lll}-1.290773 & 0.355079 & 2.800606\end{array}$

$\begin{array}{lll}-2.620726 & 0.058866 & 3.121772\end{array}$

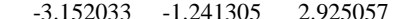

$\begin{array}{llll}-2.368275 & -2.273525 & 2.392204\end{array}$

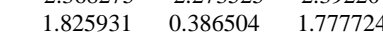

$\begin{array}{llll}\text { C } & 3.068732 & 0.363240 & 1.214445\end{array}$

$\begin{array}{lll}4.241864 & -0.260162 & 1.132450\end{array}$

$\begin{array}{llll}5.295843 & 0.068731 & -0.288906\end{array}$

$\begin{array}{lll}6.652673 & 0.428793 & 0.193333\end{array}$

$\begin{array}{llll}\text { C } & 4.411217 & 1.525631 & -0.879310\end{array}$

$\begin{array}{lll}3.389718 & 2.006246 & -0.180479\end{array}$

$\begin{array}{lll}2.488547 & 3.008179 & 0.050055\end{array}$

$\begin{array}{lll}1.058503 & 2.810934 & 0.050126\end{array}$

$\begin{array}{lll}0.181891 & 3.760427 & 0.641686 \\ -1.193454 & 3.527398 & 0.738917\end{array}$

$\begin{array}{llll}C & -1.193454 & 3.527398 & 0.738917\end{array}$

$\begin{array}{lll}-1.740024 & 2.320446 & 0.233607\end{array}$

$\begin{array}{lll}-0.891374 & 1.379542 & -0.391706\end{array}$

$\begin{array}{lll}0.477336 & 1.617917 & -0.478542\end{array}$

$\begin{array}{lll}5.150465 & -0.996425 & -1.314793\end{array}$

$\begin{array}{lll}-3.048263 & 1.971207 & 0.322645\end{array}$

$\begin{array}{lll}4.708425 & -0.892059 & 1.906638\end{array}$

$\begin{array}{lll}4.733438 & 1.868335 & -1.875175\end{array}$

$\begin{array}{lll}1.459530 & 1.340236 & 2.191915\end{array}$

$\begin{array}{lll}2.883298 & 3.976592 & 0.407320\end{array}$

$\begin{array}{lll}1.128924 & 0.876686 & -0.963198\end{array}$

$\begin{array}{llll}-1.336596 & 0.448563 & -0.769141\end{array}$

$\begin{array}{lll}-1.834345 & 4.280858 & 1.215746\end{array}$

$\begin{array}{lll}0.599220 & 4.694867 & 1.049824\end{array}$

$\begin{array}{lll}-2.771445 & -3.287335 & 2.254021\end{array}$

$\begin{array}{lll}-4.197702 & -1.446563 & 3.199692\end{array}$

$\begin{array}{lll}-3.266979 & 0.845659 & 3.539369\end{array}$

$\begin{array}{llll}-0.890378 & 1.370020 & 2.942673\end{array}$

$\begin{array}{llll}0.691717 & -4.491680 & 0.606098\end{array}$

$\begin{array}{lll}-1.071128 & -4.286266 & 0.464630\end{array}$

$\begin{array}{llll}-1.669406 & -6.489055 & 1.314834\end{array}$

$\begin{array}{llll}\mathrm{H} & -1.965587 & -7.997134 & 3.286407\end{array}$

$\begin{array}{llll}\mathrm{H} & -0.902426 & -7.416551 & 5.485998\end{array}$

$\begin{array}{llll} & 0.460112 & -5.317867 & 5.693355\end{array}$

$\begin{array}{llll}\mathrm{H} & 0.755320 & -3.811706 & 3.717071\end{array}$

$\begin{array}{llll}\text { H } & 2.007903 & -2.465069 & 0.901127\end{array}$

$\begin{array}{llll}\text { C } & -3.942730 & 2.824683 & 1.020816\end{array}$

$\begin{array}{llll}\mathrm{H} & -4.925304 & 2.317526 & 1.012372\end{array}$

$\begin{array}{llll}\mathrm{H} & -3.625123 & 2.978029 & 2.076852\end{array}$

$\begin{array}{llll}\mathrm{H} & -4.042397 & 3.817014 & 0.525979\end{array}$

\section{Coordinates from the geometry optimized in solvent (THF)}

$\mathrm{X}=-\mathrm{SO} 2-$

\begin{tabular}{|c|c|c|c|}
\hline \multicolumn{4}{|c|}{$A=4-$ methoxyphenyl, $B=$ phenyl } \\
\hline \multicolumn{4}{|c|}{ Bispropargyl } \\
\hline \multicolumn{4}{|l|}{39} \\
\hline \multicolumn{4}{|c|}{ Free energy $=-1356.382145$ Hartree } \\
\hline $\mathrm{C}$ & -0.469143 & 6.099183 & 0.794403 \\
\hline $\mathrm{C}$ & 0.734775 & 5.923647 & 0.057281 \\
\hline $\mathrm{C}$ & 0.686220 & 5.153031 & -1.130841 \\
\hline $\mathrm{C}$ & -0.500823 & 4.549568 & -1.558899 \\
\hline $\mathrm{C}$ & -1.678783 & 4.702234 & -0.795339 \\
\hline $\mathrm{C}$ & -1.654787 & 5.500965 & 0.373013 \\
\hline $\mathrm{C}$ & 1.979853 & 6.421807 & 0.534156 \\
\hline $\mathrm{C}$ & 3.084632 & 6.767712 & 0.948596 \\
\hline $\mathrm{C}$ & 4.410110 & 6.980162 & 1.469471 \\
\hline $\mathrm{S}$ & 5.157894 & 5.422606 & 2.224753 \\
\hline $\mathrm{O}$ & 4.249181 & 4.918193 & 3.276867 \\
\hline $\mathrm{O}$ & -2.869351 & 4.114926 & -1.089157 \\
\hline $\mathrm{C}$ & -2.960764 & 3.309465 & -2.257295 \\
\hline $\mathrm{C}$ & 5.188485 & 4.185993 & 0.797607 \\
\hline $\mathrm{C}$ & 3.932144 & 3.513237 & 0.577752 \\
\hline $\mathrm{C}$ & 2.843937 & 2.955995 & 0.461139 \\
\hline $\mathrm{C}$ & 1.536264 & 2.388062 & 0.367463 \\
\hline $\mathrm{C}$ & 0.542137 & 2.778617 & 1.302567 \\
\hline $\mathrm{C}$ & -0.758955 & 2.272793 & 1.200794 \\
\hline $\mathrm{C}$ & -1.091448 & 1.364284 & 0.179592 \\
\hline $\mathrm{C}$ & -0.110937 & 0.965991 & -0.746744 \\
\hline $\mathrm{C}$ & 1.194529 & 1.471472 & -0.658814 \\
\hline $\mathrm{O}$ & 6.580920 & 5.748024 & 2.498728 \\
\hline
\end{tabular}

$\begin{array}{rrrr}\mathrm{H} & -2.116731 & 0.971122 & 0.105715 \\ \mathrm{H} & 5.157510 & 7.306557 & 0.716113 \\ \mathrm{H} & 1.962839 & 1.166851 & -1.384748 \\ \mathrm{H} & -0.364530 & 0.251186 & -1.545624 \\ \mathrm{H} & -1.526385 & 2.601969 & 1.917237 \\ \mathrm{H} & -0.450994 & 6.695752 & 1.718316 \\ \mathrm{H} & -0.487940 & 3.929255 & -2.464011 \\ \mathrm{H} & -2.587208 & 5.612209 & 0.945356 \\ \mathrm{H} & 1.612995 & 4.994466 & -1.700715 \\ \mathrm{H} & 0.806988 & 3.503139 & 2.085743 \\ \mathrm{H} & 6.000484 & 3.506938 & 1.137220 \\ \mathrm{H} & -2.756377 & 3.897385 & -3.180616 \\ \mathrm{H} & -2.260721 & 2.445299 & -2.214809 \\ \mathrm{H} & -3.999526 & 2.932698 & -2.293497 \\ \mathrm{H} & 4.451855 & 7.677716 & 2.333875 \\ \mathrm{H} & 5.562858 & 4.758666 & -0.077580\end{array}$

Alkyne anionA

Free energy $=-1355.888883$ Hartree

$\begin{array}{lrrr}\mathrm{C} & -0.693094 & 3.307309 & 0.936554 \\ \mathrm{C} & 0.269425 & 2.657612 & 0.108241 \\ \mathrm{C} & -0.229854 & 1.785422 & -0.904299 \\ \mathrm{C} & -1.604632 & 1.626349 & -1.106817 \\ \mathrm{C} & -2.539053 & 2.318537 & -0.305001 \\ \mathrm{C} & -2.069165 & 3.144339 & 0.738535 \\ \mathrm{C} & 1.642481 & 2.954174 & 0.291960 \\ \mathrm{C} & 2.759240 & 3.485514 & 0.448931 \\ \mathrm{C} & 3.952348 & 4.143459 & 0.727421 \\ \mathrm{~S} & 3.881733 & 5.521003 & 1.748487 \\ \mathrm{O} & 2.907578 & 5.332053 & 2.870934 \\ \mathrm{O} & -3.864777 & 2.147699 & -0.626647 \\ \mathrm{C} & -4.835339 & 2.805669 & 0.176619 \\ \mathrm{C} & 3.164144 & 7.026941 & 0.798761 \\ \mathrm{C} & 1.829561 & 6.806805 & 0.298767\end{array}$




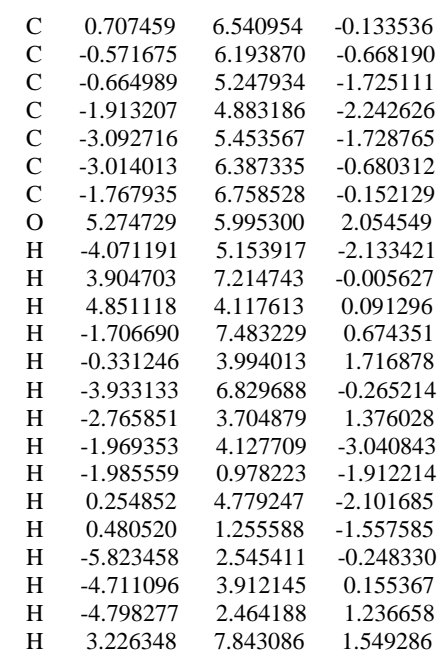

Alkyne_anionB

38

Free energy $=-1355.895457$ Hartree

$\begin{array}{llll}\text { C } & 0.392807 & 2.800433 & 1.363990\end{array}$

$\begin{array}{llll}\text { C } & 1.483898 & 2.372367 & 0.541836\end{array}$

$\begin{array}{llll}\text { C } & 1.215154 & 1.348308 & -0.420252\end{array}$

$\begin{array}{llll}\text { C } & -0.065767 & 0.793025 & -0.544977\end{array}$

$\begin{array}{llll}\text { C } & -1.127091 & 1.226590 & 0.274954\end{array}$

$\begin{array}{llll}\text { C } & -0.880802 & 2.240869 & 1.223473\end{array}$

$\begin{array}{llll}\text { C } & 2.758166 & 2.964837 & 0.675388\end{array}$

$\begin{array}{llll}\text { C } & 3.845155 & 3.566813 & 0.800645\end{array}$

$\begin{array}{llll}\text { C } & 5.051180 & 4.225845 & 0.978508\end{array}$

$\begin{array}{llll}\text { S } & 5.167100 & 5.574763 & 2.039997\end{array}$

$\begin{array}{llll}\text { O } & 6.614576 & 5.923376 & 2.246886\end{array}$

$\begin{array}{llll}\text { C } & 4.490584 & 7.145019 & 1.177136\end{array}$

$\begin{array}{llll}\text { C } & 3.139018 & 6.938039 & 0.720993\end{array}$

$\begin{array}{llll}\text { C } & 2.027513 & 6.550860 & 0.357823\end{array}$

$\begin{array}{llll}\text { C } & 0.790104 & 5.989233 & -0.071683\end{array}$

$\begin{array}{llll}\text { C } & 0.772444 & 5.064775 & -1.147127\end{array}$

$\begin{array}{llll}\text { C } & -0.407489 & 4.421372 & -1.537621\end{array}$

$\begin{array}{llll}\text { C } & -1.610868 & 4.685669 & -0.848821\end{array}$

$\begin{array}{llll}\text { C } & -1.616518 & 5.624343 & 0.210341\end{array}$

$\begin{array}{llll}\text { C } & -0.437074 & 6.263104 & 0.594116\end{array}$

$\begin{array}{llll}\text { O } & -2.803340 & 4.085944 & -1.121862\end{array}$

$\begin{array}{llll}\text { C } & -2.858999 & 3.142930 & -2.190103\end{array}$

$\begin{array}{llll}\text { O } & 4.271957 & 5.419466 & 3.230872\end{array}$

$\begin{array}{llll}\mathrm{H} & -2.131955 & 0.789432 & 0.170934\end{array}$

$\begin{array}{llll}\mathrm{H} & 5.210278 & 7.342835 & 0.356187\end{array}$

\begin{tabular}{llll}
$\mathrm{H}$ & 2.032658 & 1.003621 & -1.072593 \\
\hline
\end{tabular}

$\begin{array}{llll}\mathrm{H} & -0.240468 & 0.006229 & -1.297758\end{array}$

$\begin{array}{llll}\mathrm{H} & -1.703985 & 2.614767 & 1.853066\end{array}$

$\begin{array}{llll}\mathrm{H} & -0.447940 & 6.973562 & 1.434898\end{array}$

$\begin{array}{llll}\mathrm{H} & -0.366646 & 3.676566 & -2.343208\end{array}$

$\begin{array}{llll}\mathrm{H} & -2.564248 & 5.816443 & 0.735156\end{array}$

$\begin{array}{llll}\mathrm{H} & 1.717229 & 4.816932 & -1.651036\end{array}$

$\begin{array}{llll}\mathrm{H} & 0.567888 & 3.611558 & 2.086547\end{array}$

$\begin{array}{llll}\mathrm{H} & 5.917065 & 4.133562 & 0.303895\end{array}$

$\begin{array}{llll}\mathrm{H} & -2.600161 & 3.613133 & -3.165327\end{array}$

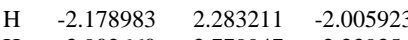

$\begin{array}{llll}\mathrm{H} & -3.902660 & 2.779947 & -2.229356\end{array}$

$\begin{array}{llll}\mathrm{H} & 4.597261 & 7.929872 & 1.955438\end{array}$

\section{$A$ = 2,4-dimethoxyphenyl, $B$ = phenyl}

Bispropargyl

43

Free energy $=-1470.795479$ Hartree

\begin{tabular}{|c|c|c|c|}
\hline & 4.043222 & -0.066150 & -5.190974 \\
\hline & 3.059681 & 0.237928 & -6.165859 \\
\hline & 3.347510 & 1.195034 & -7.166803 \\
\hline & 4.597304 & 1.824605 & -7.193319 \\
\hline & 5.571314 & 1.521057 & -6.225654 \\
\hline & 5.287109 & 0.575049 & -5.225356 \\
\hline & 1.777065 & -0.408139 & -6.154183 \\
\hline & 0.688082 & -0.979922 & -6.217212 \\
\hline & -0.593448 & -1.622533 & -6.331344 \\
\hline & -1.737544 & -0.781539 & -7.556708 \\
\hline & -2.812970 & -1.767149 & -7.859309 \\
\hline & -2.544483 & 0.618465 & -6.598969 \\
\hline & -1.575465 & 1.456094 & \\
\hline & -0.677250 & 2.093497 & -5.394862 \\
\hline & 0.488944 & 2.795260 & -4.984662 \\
\hline & 0.950885 & 2.786305 & -3.642434 \\
\hline c & 2.149000 & 3.398327 & -3.277482 \\
\hline & 2.932021 & 4.038009 & -4.266496 \\
\hline & 2.487867 & 4.094798 & -5.603377 \\
\hline
\end{tabular}

$\begin{array}{cccc}\mathrm{C} & 1.278267 & 3.475002 & -5.965551 \\ \mathrm{O} & 0.797498 & 3.460764 & -7.231298 \\ \mathrm{C} & 1.534811 & 4.123331 & -8.256728 \\ \mathrm{O} & 4.111492 & 4.564721 & -3.847478 \\ \mathrm{C} & 4.976972 & 5.161394 & -4.814340 \\ \mathrm{O} & -0.920021 & -0.205433 & -8.654313 \\ \mathrm{H} & 6.551742 & 2.025277 & -6.249589 \\ \mathrm{H} & -3.267828 & 0.119960 & -5.920758 \\ \mathrm{H} & 3.814765 & -0.806158 & -4.407563 \\ \mathrm{H} & 6.043569 & 0.337718 & -4.459913 \\ \mathrm{H} & 4.814876 & 2.569371 & -7.977861 \\ \mathrm{H} & 0.350033 & 2.261554 & -2.884446 \\ \mathrm{H} & 3.096443 & 4.587850 & -6.368763 \\ \mathrm{H} & 2.518558 & 3.368858 & -2.242233 \\ \mathrm{H} & 2.571312 & 1.440718 & -7.907551 \\ \mathrm{H} & -0.554822 & -2.623949 & -6.814308 \\ \mathrm{H} & 5.282592 & 4.425211 & -5.592325 \\ \mathrm{H} & 4.502696 & 6.041524 & -5.302590 \\ \mathrm{H} & 5.873978 & 5.493446 & -4.260714 \\ \mathrm{H} & 0.975683 & 3.952532 & -9.194986 \\ \mathrm{H} & 1.604267 & 5.217967 & -8.068838 \\ \mathrm{H} & 2.556974 & 3.703145 & -8.359957 \\ \mathrm{H} & -3.103638 & 1.122299 & -7.417883 \\ \mathrm{H} & -1.158147 & -1.707203 & -5.378709\end{array}$

Alkyne_anionA

Free energy $=-1470.308496$ Hartree

$\begin{array}{lccc}\mathrm{C} & 1.152617 & 4.953085 & -2.014437 \\ \mathrm{C} & 2.544123 & 5.027359 & -1.738658 \\ \mathrm{C} & 2.995482 & 5.960393 & -0.765897 \\ \mathrm{C} & 2.084228 & 6.787060 & -0.098604 \\ \mathrm{C} & 0.706173 & 6.700348 & -0.373043 \\ \mathrm{C} & 0.247723 & 5.784160 & -1.336530 \\ \mathrm{C} & 3.484233 & 4.197416 & -2.424571 \\ \mathrm{C} & 4.324863 & 3.495348 & -2.988540 \\ \mathrm{C} & 5.351064 & 2.724817 & -3.645868 \\ \mathrm{~S} & 6.442541 & 3.742053 & -4.859946 \\ \mathrm{O} & 7.423853 & 2.701967 & -5.323840 \\ \mathrm{C} & 7.272635 & 4.991676 & -4.027766 \\ \mathrm{C} & 6.513316 & 6.081256 & -3.611330 \\ \mathrm{C} & 5.732353 & 7.010560 & -3.330366 \\ \mathrm{C} & 4.608389 & 7.807399 & -3.005111 \\ \mathrm{C} & 3.370112 & 7.577825 & -3.658978 \\ \mathrm{C} & 2.190814 & 8.249218 & -3.300307 \\ \mathrm{C} & 2.229029 & 9.190605 & -2.256351 \\ \mathrm{C} & 3.454978 & 9.482615 & -1.611476 \\ \mathrm{C} & 4.626020 & 8.802812 & -1.973305 \\ \mathrm{O} & 5.838984 & 8.990102 & -1.365966 \\ \mathrm{C} & 5.925233 & 9.928945 & -0.303991 \\ \mathrm{O} & 1.132561 & 9.859856 & -1.767405 \\ \mathrm{C} & -0.122961 & 9.620763 & -2.389916 \\ \mathrm{O} & 5.443453 & 4.287742 & -5.834673 \\ \mathrm{H} & -0.003195 & 7.361147 & 0.148442 \\ \mathrm{H} & 6.082520 & 2.282497 & -2.938285 \\ \mathrm{H} & 8.206435 & 4.653577 & -3.552328 \\ \mathrm{H} & 0.792917 & 4.240996 & -2.772997 \\ \mathrm{H} & 3.342021 & 6.797777 & -4.434748 \\ \mathrm{H} & -0.827715 & 5.718059 & -1.567350 \\ \mathrm{H} & 1.251784 & 7.989827 & -3.804969 \\ \mathrm{H} & 2.456126 & 7.524327 & 0.629171 \\ \mathrm{H} & 3.439021 & 10.218538 & -0.796521 \\ \mathrm{H} & 4.074774 & 6.050431 & -0.575279 \\ \mathrm{H} & -0.864534 & 10.238100 & -1.849099 \\ \mathrm{H} & -0.421405 & 8.550867 & -2.318953 \\ \mathrm{H} & -0.116549 & 9.919015 & -3.463353 \\ \mathrm{H} & 6.977244 & 9.920941 & 0.037832 \\ \mathrm{H} & 5.660467 & 10.959075 & -0.635969 \\ \mathrm{H} & 5.265423 & 9.652458 & 0.549988 \\ \mathrm{H} & 4.947195 & 1.922539 & -4.298818\end{array}$

Alkyne_anionB

Free energy $=-1470.315081$ Hartree

$\begin{array}{llll}\text { C } & 1.114726 & 2.835740 & -3.486171\end{array}$

$\begin{array}{llll}\text { C } & 1.177670 & 1.613981 & -4.203389\end{array}$

$\begin{array}{llll}\text { C } & 2.426847 & 1.253702 & -4.801748\end{array}$

$\begin{array}{lll}3.544562 & 2.103640 & -4.700238\end{array}$

$\begin{array}{llll}\text { C } & 3.434638 & 3.323834 & -4.003270\end{array}$

$\begin{array}{lll}2.218978 & 3.682861 & -3.377929\end{array}$

$\begin{array}{llll}\text { C } & 0.039104 & 0.789348 & -4.415026\end{array}$

$\begin{array}{llll}\text { C } & -0.927966 & 0.077419 & -4.688767\end{array}$

$\begin{array}{lll}-2.002615 & -0.701814 & -5.251646\end{array}$

$\begin{array}{lll}-2.356847 & -0.192005 & -7.060093\end{array}$

$\begin{array}{llll}-2.830307 & 1.224307 & -6.941488\end{array}$

$\begin{array}{llll}2.444852 & 0.069924 & -5.460571\end{array}$

$\begin{array}{lll}3.589733 & -0.274498 & -6.233711\end{array}$

$\begin{array}{lll}4.453486 & 4.223323 & -3.890668\end{array}$

$\begin{array}{lll}5.703107 & 3.919457 & -4.506696\end{array}$

$\begin{array}{rrrr}\text { C } & -0.918508 & -0.302934 & -7.999140\end{array}$ 


$\begin{array}{lrrr}\mathrm{C} & 0.036282 & 0.697765 & -7.960625 \\ \mathrm{C} & 0.872422 & 1.625452 & -7.951332 \\ \mathrm{C} & 1.829458 & 2.646952 & -7.785547 \\ \mathrm{C} & 1.561919 & 3.769027 & -6.936389 \\ \mathrm{C} & 2.514030 & 4.773782 & -6.744597 \\ \mathrm{C} & 3.769096 & 4.719429 & -7.386922 \\ \mathrm{C} & 4.051128 & 3.625770 & -8.230117 \\ \mathrm{C} & 3.105243 & 2.611609 & -8.435895 \\ \mathrm{O} & -3.322232 & -1.254161 & -7.508727 \\ \mathrm{H} & 4.515003 & 5.513168 & -7.226736 \\ \mathrm{H} & -1.777580 & -1.787070 & -5.31109 \\ \mathrm{H} & 3.338039 & 1.767170 & -9.103842 \\ \mathrm{H} & 5.026773 & 3.562350 & -8.740082 \\ \mathrm{H} & 2.284767 & 5.605971 & -6.059960 \\ \mathrm{H} & 0.157191 & 3.123774 & -3.027120 \\ \mathrm{H} & 4.476178 & 1.838179 & -5.209408 \\ \mathrm{H} & 2.159886 & 4.641412 & -2.842799 \\ \mathrm{H} & 0.601320 & 3.804026 & -6.402301 \\ \mathrm{H} & -0.746578 & -1.313311 & -8.402227 \\ \mathrm{H} & 6.163882 & 3.005315 & -4.069607 \\ \mathrm{H} & 5.590253 & 3.784444 & -5.604817 \\ \mathrm{H} & 6.363650 & 4.785403 & -4.311249 \\ \mathrm{H} & 3.337153 & -1.217155 & -6.752937 \\ \mathrm{H} & 3.815589 & 0.510241 & -6.986558 \\ \mathrm{H} & 4.485046 & -0.441691 & -5.593323 \\ \mathrm{H} & -2.983918 & -0.560446 & -4.750632\end{array}$

\section{A = 2-naphthyl, $B$ = phenyl}

Bispropargyl

41

Free energy $=-1395.461439$ Hartree

$\begin{array}{llll}\text { C } & -0.781102 & -0.556351 & -0.230452\end{array}$

$\begin{array}{llll}\text { C } & -0.300085 & -1.758399 & 0.351860\end{array}$

$\begin{array}{llll}\text { C } & 0.203161 & -1.728737 & 1.677224\end{array}$

$\begin{array}{llll}\text { C } & 0.233131 & -0.524173 & 2.393611\end{array}$

$\begin{array}{llll}\text { C } & -0.238257 & 0.662691 & 1.807476\end{array}$

$\begin{array}{llll}\text { C } & -0.744289 & 0.639744 & 0.495484\end{array}$

$\begin{array}{llll}\text { C } & -0.300862 & -2.962242 & -0.417445\end{array}$

$\begin{array}{llll}\text { C } & -0.294142 & -3.959521 & -1.134000\end{array}$

$\begin{array}{llll}\text { C } & -0.317837 & -5.093237 & -2.025020\end{array}$

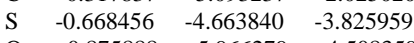

$\begin{array}{llll}\mathrm{O} & -0.875888 & -5.966279 & -4.508359\end{array}$

$\begin{array}{llll}\text { C } & 0.971701 & -4.004163 & -4.479669\end{array}$

$\begin{array}{llll}\text { C } & 1.416215 & -2.817083 & -3.795251\end{array}$

$\begin{array}{llll}\text { C } & 1.700643 & -1.838869 & -3.108212\end{array}$

$\begin{array}{llll}\text { C } & 1.963387 & -0.728874 & -2.254430\end{array}$

$\begin{array}{llll}\text { C } & 2.564314 & -0.928544 & -1.004037\end{array}$

$\begin{array}{llll}\text { C } & 2.748579 & 0.147002 & -0.094367\end{array}$

$\begin{array}{llll}\text { C } & 2.310171 & 1.471126 & -0.472006\end{array}$

$\begin{array}{llll}\text { C } & 1.719014 & 1.655849 & -1.759249\end{array}$

$\begin{array}{llll}\text { C } & 1.547114 & 0.594031 & -2.628839\end{array}$

$\begin{array}{llll}\text { C } & 3.328537 & -0.047052 & 1.196028\end{array}$

$\begin{array}{llll}\text { C } & 3.467193 & 1.013703 & 2.079220\end{array}$

$\begin{array}{llll}\text { C } & 3.027114 & 2.315425 & 1.710319\end{array}$

$\begin{array}{llll}\text { C } & 2.463115 & 2.538164 & 0.460116\end{array}$

$\begin{array}{llll}\text { O } & -1.670169 & -3.577581 & -3.880355\end{array}$

$\begin{array}{llll}\mathrm{H} & -0.194183 & 1.609525 & 2.365725\end{array}$

$\begin{array}{llll}\mathrm{H} & 0.625410 & -5.679717 & -2.048117\end{array}$

$\begin{array}{llll}\mathrm{H} & 0.625410 & -5.679717 & -2.048117 \\ \mathrm{H} & 1.074945 & 0.742118 & -3.610609\end{array}$

\begin{tabular}{llll}
$\mathrm{H}$ & 1.384411 & 2.664424 & -2.049084 \\
\hline & -1.154446 & -0.578161 & -1.263875
\end{tabular}

$\begin{array}{llll}\mathrm{H} & -1.154446 & -0.578161 & -1.263875\end{array}$

$\begin{array}{llll}\mathrm{H} & 0.641535 & -0.508422 & 3.414892\end{array}$

$\begin{array}{llll}\mathrm{H} & -1.099280 & 1.569293 & 0.025608\end{array}$

$\begin{array}{llll}\mathrm{H} & 0.581206 & -2.657725 & 2.128800\end{array}$

$\begin{array}{llll}\mathrm{H} & 2.865129 & -1.943511 & -0.705912\end{array}$

$\mathrm{H} \quad 0.721883 \quad-3.843094 \quad-5.550581$

$\begin{array}{llll}\mathrm{H} & 3.653762 & -1.059156 & 1.482041\end{array}$

$\begin{array}{llll}\mathrm{H} & 3.911301 & 0.849718 & 3.072820\end{array}$

$\begin{array}{llll}\mathrm{H} & 3.136656 & 3.149182 & 2.421145\end{array}$

$\begin{array}{llll}\mathrm{H} & 2.120261 & 3.545223 & 0.174493\end{array}$

$\begin{array}{llll}\mathrm{H} & 1.669109 & -4.864962 & -4.405713\end{array}$

$\begin{array}{llll}\mathrm{H} & -1.155015 & -5.797619 & -1.823913\end{array}$

Alkyne_anionA

40

Free energy $=-1394.974448$ Hartree

$\begin{array}{llll}\text { C } & 3.113726 & 4.539605 & -0.580587\end{array}$

$\begin{array}{llll}\text { C } & 3.743218 & 5.760915 & -0.350119\end{array}$

$\begin{array}{llll}\text { C } & 3.009771 & 6.889975 & 0.113585\end{array}$

$\begin{array}{llll}\text { C } & 1.586160 & 6.762762 & 0.350035\end{array}$

$\begin{array}{llll}\text { C } & 0.967298 & 5.497263 & 0.087643\end{array}$

$\begin{array}{llll}\text { C } & 1.711287 & 4.414277 & -0.362303\end{array}$

$\begin{array}{llll}\text { C } & 0.859780 & 7.864356 & 0.866824\end{array}$

$\begin{array}{llll}\text { C } & 1.489059 & 9.091809 & 1.181434\end{array}$

$\begin{array}{llll}\text { C } & 2.900907 & 9.214020 & 0.896693\end{array}$

$\begin{array}{llll}\text { C } & 3.625963 & 8.152162 & 0.387589\end{array}$

$\begin{array}{llll}\text { C } & 0.775714 & 10.138926 & 1.797735\end{array}$

$\begin{array}{llll}\text { C } & 0.125183 & 10.942010 & 2.500284\end{array}$

$\begin{array}{llll}\text { C } & -0.651145 & 11.799166 & 3.257866\end{array}$

$\begin{array}{cccc}\text { S } & -2.200587 & 11.280455 & 3.805796 \\ \mathrm{O} & -2.934089 & 12.445794 & 4.404462 \\ \mathrm{C} & -1.990745 & 10.120580 & 5.306977 \\ \mathrm{C} & -1.132422 & 8.999546 & 5.011951 \\ \mathrm{C} & -0.338821 & 8.128956 & 4.655134 \\ \mathrm{C} & 0.622705 & 7.173019 & 4.204902 \\ \mathrm{C} & 0.250111 & 5.839376 & 3.888679 \\ \mathrm{C} & 1.202728 & 4.935320 & 3.397979 \\ \mathrm{C} & 2.537646 & 5.338247 & 3.214708 \\ \mathrm{C} & 2.918300 & 6.654860 & 3.529771 \\ \mathrm{C} & 1.975943 & 7.566027 & 4.023485 \\ \mathrm{O} & -2.886022 & 10.422819 & 2.788187 \\ \mathrm{H} & 3.272470 & 4.637415 & 2.790577 \\ \mathrm{H} & -0.280053 & 12.695703 & 3.777956 \\ \mathrm{H} & -1.590733 & 10.776968 & 6.107216 \\ \mathrm{H} & -0.211296 & 7.743592 & 1.089750 \\ \mathrm{H} & -0.798304 & 5.530583 & 4.016341 \\ \mathrm{H} & 0.896031 & 3.912582 & 3.131314 \\ \mathrm{H} & 4.706711 & 8.266378 & 0.199917 \\ \mathrm{H} & 3.953416 & 6.985186 & 3.356261 \\ \mathrm{H} & 3.401538 & 10.169320 & 1.120135 \\ \mathrm{H} & 2.259343 & 8.607152 & 4.233019 \\ \mathrm{H} & -0.112692 & 5.389842 & 0.279036 \\ \mathrm{H} & 1.216905 & 3.446457 & -0.544929 \\ \mathrm{H} & 3.694476 & 3.672500 & -0.932547 \\ \mathrm{H} & 4.827987 & 5.868330 & -0.518540 \\ \mathrm{H} & -3.035227 & 9.837735 & 5.555659\end{array}$

Alkyne_anionB

40

Free energy $=-1394.974631$ Hartree

$\begin{array}{llll}\text { C } & 3.062012 & 2.835314 & 1.033435 \\ \text { C } & 3.187668 & 2.903450 & -0.350881\end{array}$

$\begin{array}{llll}\text { C } & 3.187668 & 2.903450 & -0.350881\end{array}$

$\begin{array}{llll}\text { C } & 2.824859 & 1.792074 & -1.171604\end{array}$

$\begin{array}{llll}\text { C } & 2.332118 & 0.587472 & -0.539653\end{array}$

$\begin{array}{llll}\text { C } & 2.212687 & 0.549467 & 0.884891\end{array}$

$\begin{array}{llll}\text { C } & 2.567541 & 1.650995 & 1.653566\end{array}$

$\begin{array}{llll}\text { C } & 1.958447 & -0.521178 & -1.349219\end{array}$

$\begin{array}{llll}\text { C } & 2.048563 & -0.461879 & -2.747940\end{array}$

$\begin{array}{llll}\text { C } & 2.540107 & 0.738576 & -3.369357\end{array}$

$\begin{array}{llll}\text { C } & 2.918156 & 1.826568 & -2.599419\end{array}$

$\begin{array}{llll}\text { C } & 1.646670 & -1.591073 & -3.525062\end{array}$

$\begin{array}{llll}\text { C } & 1.269005 & -2.593363 & -4.131194\end{array}$

$\begin{array}{llll}\text { C } & 0.772747 & -3.817082 & -4.710971\end{array}$

$\begin{array}{llll}\text { S } & -0.568209 & -4.631043 & -3.627558\end{array}$

$\begin{array}{llll}\text { O } & -1.676088 & -3.626092 & -3.601934\end{array}$

$\begin{array}{llll}\text { O } & -0.771519 & -5.939939 & -4.337546\end{array}$

$\begin{array}{llll}\text { C } & 0.063896 & -4.927171 & -2.055892\end{array}$

$\begin{array}{llll}\text { C } & -0.025347 & -3.987406 & -1.045459\end{array}$

$\begin{array}{llll}\text { C } & -0.131863 & -3.163224 & -0.110194\end{array}$

$\begin{array}{llll}\text { C } & -0.264768 & -2.210654 & 0.926534\end{array}$

$\begin{array}{llll}\text { C } & 0.323026 & -2.412775 & 2.216016\end{array}$

$\begin{array}{llll}\text { C } & 0.179298 & -1.458512 & 3.234438\end{array}$

$\begin{array}{llll}\text { C } & -0.553757 & -0.274226 & 3.020709\end{array}$

$\begin{array}{llll}\text { C } & -1.138291 & -0.058493 & 1.756501\end{array}$

$\begin{array}{llll}\text { C } & -1.000852 & -0.998517 & 0.727547\end{array}$

$\begin{array}{llll}\mathrm{H} & -0.658268 & 0.474208 & 3.822774\end{array}$

$\begin{array}{llll}\mathrm{H} & 0.655263 & -5.851058 & -1.995597\end{array}$

$\begin{array}{llll}\mathrm{H} & 2.609388 & 0.780586 & -4.467668\end{array}$

$\begin{array}{llll}\mathrm{H} & 3.292835 & 2.742550 & -3.084499\end{array}$

$\begin{array}{llll}\mathrm{H} & -1.448736 & -0.811963 & -0.258352\end{array}$

$\begin{array}{llll}\mathrm{H} & 0.647653 & -1.643362 & 4.213781\end{array}$

$\begin{array}{llll}\mathrm{H} & -1.700383 & 0.869303 & 1.564941\end{array}$

$\begin{array}{llll}\mathrm{H} & 0.895618 & -3.334987 & 2.398789\end{array}$

$\begin{array}{llll}\mathrm{H} & 1.564526 & -1.433221 & -0.874413\end{array}$

$\begin{array}{llll}\mathrm{H} & 0.267929 & -3.677787 & -5.690279\end{array}$

$\mathrm{H} \quad 1.810962-0.360123 \quad 1.360120$

$\begin{array}{llll}\mathrm{H} & 2.454971 & 1.612794 & 2.748357\end{array}$

$\begin{array}{llll}\mathrm{H} & 3.340275 & 3.702777 & 1.655181\end{array}$

$\begin{array}{llll}\mathrm{H} & 3.564194 & 3.821023 & -0.832552\end{array}$

$\begin{array}{llll}\mathrm{H} & 1.551484 & -4.596502 & -4.817770\end{array}$

A = 6-methoxynaphthyl, $B$ = phenyl Bispropargyl

45

Free energy $=-1509.883907$ Hartree

$\begin{array}{lrrr}\mathrm{C} & 2.291893 & 3.759826 & -1.947786 \\ \mathrm{C} & 1.262660 & 3.134900 & -2.693887 \\ \mathrm{C} & -0.099816 & 3.598701 & -2.547834 \\ \mathrm{C} & -0.353592 & 4.685257 & -1.661558 \\ \mathrm{C} & 0.667498 & 5.289178 & -0.941921 \\ \mathrm{C} & 2.008737 & 4.815044 & -1.074766 \\ \mathrm{C} & 1.514437 & 2.035591 & -3.575454 \\ \mathrm{C} & 0.486475 & 1.420564 & -4.265206 \\ \mathrm{C} & -0.870535 & 1.871924 & -4.118782 \\ \mathrm{C} & -1.139246 & 2.953257 & -3.267623 \\ \mathrm{C} & -1.940779 & 1.200080 & -4.774643 \\ \mathrm{C} & -2.903594 & 0.617711 & -5.268741 \\ \mathrm{C} & -4.069663 & -0.136573 & -5.650026\end{array}$




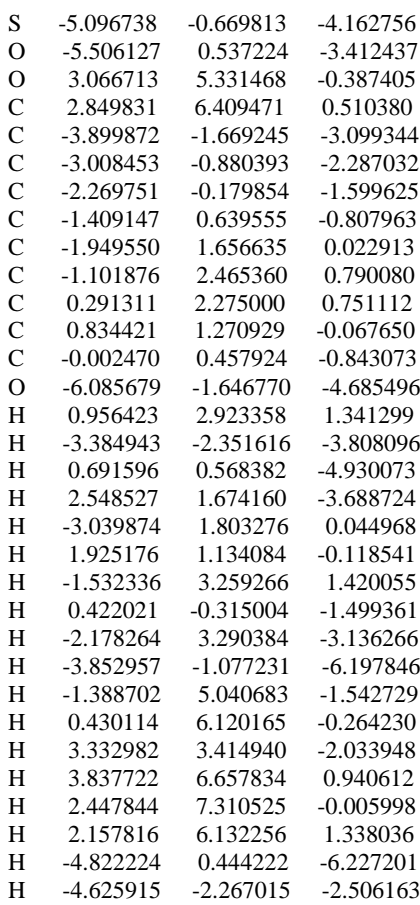

Alkyne_anionA

$$
44
$$

Free energy $=-1509.388365$ Hartree

$\begin{array}{llll}\text { C } & -2.229873 & 2.622955 & -2.090180\end{array}$

$\begin{array}{llll}\text { C } & -2.437889 & 2.064885 & -0.800963\end{array}$

$\begin{array}{llll}\text { C } & -1.624797 & 0.976321 & -0.384871\end{array}$

$\begin{array}{llll}\text { C } & -0.615664 & 0.482989 & -1.223632\end{array}$

$\begin{array}{llll}\text { C } & -0.413333 & 1.045643 & -2.498030\end{array}$

$\begin{array}{llll}\text { C } & -1.229054 & 2.109522 & -2.929767\end{array}$

$\begin{array}{llll}\text { C } & -3.415460 & 2.589967 & 0.101527\end{array}$

$\begin{array}{llll}\text { C } & -4.206485 & 3.008847 & 0.946232\end{array}$

$\begin{array}{llll}\text { C } & -5.053118 & 3.476341 & 2.016396\end{array}$

$\begin{array}{llll}\text { S } & -4.213884 & 3.356446 & 3.724138\end{array}$

$\begin{array}{llll}\text { O } & -2.948229 & 4.137982 & 3.568672\end{array}$

$\begin{array}{llll}\text { C } & -3.904179 & 1.708752 & 4.108538\end{array}$

$\begin{array}{llll}\text { C } & -2.754578 & 1.067425 & 3.684563\end{array}$

$\begin{array}{llll}\text { C } & -1.698985 & 0.508768 & 3.315614\end{array}$

$\begin{array}{llll}\text { C } & -0.532803 & -0.131678 & 2.845343\end{array}$

$\begin{array}{llll}\text { C } & 0.479227 & 0.564672 & 2.145709\end{array}$

$\begin{array}{llll}\text { C } & 1.606334 & -0.102526 & 1.596049\end{array}$

$\begin{array}{llll}\text { C } & 1.758433 & -1.532219 & 1.775075\end{array}$

$\begin{array}{llll}\text { C } & 0.743076 & -2.223263 & 2.515161\end{array}$

$\begin{array}{llll}\text { C } & -0.355849 & -1.557733 & 3.025876\end{array}$

$\begin{array}{llll}\text { C } & 2.606004 & 0.580984 & 0.837664\end{array}$

$\begin{array}{llll}\text { C } & 3.699619 & -0.086321 & 0.293916\end{array}$

$\begin{array}{llll}\text { C } & 3.848280 & -1.493131 & 0.487673\end{array}$

$\begin{array}{llll}\text { C } & 2.883528 & -2.195270 & 1.218448\end{array}$

$\begin{array}{llll}\text { O } & 4.896505 & -2.227070 & -0.008190\end{array}$

$\begin{array}{llll}\text { C } & 5.915495 & -1.555608 & -0.740448\end{array}$

$\begin{array}{llll}\text { O } & -5.299601 & 3.874236 & 4.624858\end{array}$

\begin{tabular}{llll}
$\mathrm{H}$ & 0.382281 & 0.658396 & -3.153883 \\
\hline
\end{tabular}

$\begin{array}{llll}\mathrm{H} & -4.780411 & 1.195017 & 4.535175\end{array}$

$\begin{array}{llll}\mathrm{H} & -5.309369 & 4.553709 & 1.937429\end{array}$

$\begin{array}{rrrr}\mathrm{H} & -5.309369 & 4.553709 & 1.937429 \\ \mathrm{H} & 0.368268 & 1.647700 & 1.981529\end{array}$

$\begin{array}{llll}\mathrm{H} & -1.783868 & 0.547033 & 0.613836\end{array}$

$\begin{array}{llll}\mathrm{H} & 0.030063 & -0.334492 & -0.865803\end{array}$

$\begin{array}{llll}\mathrm{H} & 0.845602 & -3.310162 & 2.668968\end{array}$

$\begin{array}{llll}\mathrm{H} & -1.078309 & 2.549711 & -3.927367\end{array}$

$\begin{array}{llll}\mathrm{H} & -1.130008 & -2.114991 & 3.576538\end{array}$

$\begin{array}{llll}\mathrm{H} & -2.856942 & 3.464607 & -2.421672\end{array}$

$\begin{array}{llll}\mathrm{H} & 2.498282 & 1.665592 & 0.675037\end{array}$

$\begin{array}{llll}\mathrm{H} & 4.441214 & 0.481499 & -0.284991\end{array}$

$\begin{array}{lllr}\mathrm{H} & 3.010219 & -3.280997 & 1.355530\end{array}$

$\begin{array}{llll}\mathrm{H} & 6.653347 & -2.328304 & -1.027274\end{array}$

$\begin{array}{llll}\mathrm{H} & 5.520664 & -1.077811 & -1.665322\end{array}$

$\begin{array}{llll}\mathrm{H} & 6.426964 & -0.779423 & -0.127765\end{array}$

$\begin{array}{llll}\mathrm{H} & -5.989477 & 2.892455 & 2.128977\end{array}$

Alkyne_anionB

44

Free energy $=-1509.393243$ Hartree

C $\quad-2.621773 \quad 2.541253 \quad-0.094555$

$\begin{array}{llll}\text { C } & -2.192061 & 1.173035 & -0.07622 \\ \text { C } & -1.149033 & 0.826056 & 0.844555\end{array}$

$\begin{array}{llll}\mathrm{C} & -1.149033 & 0.826056 & 0.844555\end{array}$

$\begin{array}{llll}\text { C } & -0.575262 & 1.790952 & 1.682589\end{array}$

$\begin{array}{llll}\text { C } & -1.016785 & 3.130565 & 1.655214\end{array}$

$\begin{array}{llll}\text { C } & -2.039618 & 3.489378 & 0.753364\end{array}$

$\begin{array}{lrrr}\text { C } & -2.743548 & 0.226749 & -0.963687 \\ \text { C } & -3.258678 & -0.592671 & -1.753853 \\ \text { C } & -3.849092 & -1.509244 & -2.599206 \\ \text { S } & -4.919262 & -1.002657 & -3.850360 \\ \text { O } & -5.791373 & -2.151646 & -4.266630 \\ \text { C } & -3.923567 & -0.673065 & -5.444805 \\ \text { C } & -2.853093 & 0.271869 & -5.231708 \\ \text { C } & -1.934309 & 1.029799 & -4.921609 \\ \text { C } & -0.871822 & 1.894913 & -4.514696 \\ \text { C } & -0.511876 & 1.961528 & -3.162999 \\ \text { C } & 0.530439 & 2.818873 & -2.718062 \\ \text { C } & 1.242911 & 3.634282 & -3.677348 \\ \text { C } & 0.860759 & 3.549488 & -5.056432 \\ \text { C } & -0.163456 & 2.713261 & -5.465470 \\ \text { C } & 0.899895 & 2.905938 & -1.341000 \\ \text { C } & 1.916742 & 3.749329 & -0.919370 \\ \text { C } & 2.624705 & 4.548956 & -1.871099 \\ \text { C } & 2.285879 & 4.484968 & -3.225940 \\ \text { O } & 3.639794 & 5.401581 & -1.538546 \\ \text { C } & 4.013602 & 5.530677 & -0.168863 \\ \text { O } & -5.545589 & 0.314264 & -3.530456 \\ \text { H } & -0.558554 & 3.889323 & 2.306722 \\ \text { H } & -3.562874 & -2.571472 & -2.667956 \\ \text { H } & -0.449202 & 2.662593 & -6.527804 \\ \text { H } & 1.396038 & 4.166883 & -5.794612 \\ \text { H } & -3.401931 & 2.840430 & -0.811920 \\ \text { H } & 0.231688 & 1.491431 & 2.372867 \\ \text { H } & -2.387553 & 4.532884 & 0.703917 \\ \text { H } & -0.785685 & -0.213890 & 0.869266 \\ \text { H } & -1.060130 & 1.347682 & -2.430701 \\ \text { H } & -3.566859 & -1.672923 & -5.763482 \\ \text { H } & 0.353883 & 2.301365 & -0.602177 \\ \text { H } & 2.166079 & 3.792740 & 0.147585 \\ \text { H } & 2.840335 & 5.112790 & -3.941873 \\ \text { H } & 4.835206 & 6.272787 & -0.141664 \\ \text { H } & 3.172907 & 5.903396 & 0.458300 \\ \text { H } & 4.380173 & 4.567621 & 0.251423 \\ \text { H } & -4.698734 & -0.324951 & -6.161017\end{array}$

\section{A = 2-pyridyl, $B$ = phenyl}

\begin{tabular}{lrrr} 
Bispropargyl & \multicolumn{3}{l}{} \\
34 & \multicolumn{4}{l}{} \\
Free & energy $=-1257.99674$ Hartree \\
C & 1.483049 & 3.444406 & 0.058105 \\
C & 2.513234 & 3.824143 & -0.841722 \\
N & 2.324570 & 3.912937 & -2.181314 \\
C & 1.105906 & 3.616075 & -2.653158 \\
C & 0.023688 & 3.216485 & -1.846308 \\
C & 0.221813 & 3.130981 & -0.459139 \\
C & 3.827484 & 4.088930 & -0.329852 \\
C & 4.935026 & 4.256873 & 0.171759 \\
C & 6.235227 & 4.379430 & 0.781175 \\
S & 7.048448 & 2.736852 & 1.212525 \\
C & 5.893279 & 1.950899 & 2.475549 \\
C & 4.716236 & 1.374080 & 1.874978 \\
C & 3.762968 & 0.908906 & 1.256695 \\
C & 2.682892 & 0.450976 & 0.442714 \\
C & 2.787872 & 0.572396 & -0.968921 \\
C & 1.718457 & 0.191034 & -1.788019 \\
C & 0.536431 & -0.320623 & -1.221252 \\
C & 0.428231 & -0.451104 & 0.174340 \\
C & 1.491414 & -0.070160 & 1.005785 \\
H & -0.303544 & -0.615311 & -1.868871 \\
H & -0.947615 & 2.969401 & -2.300333 \\
H & 7.001673 & 4.816829 & 0.105341 \\
H & 5.693345 & 2.745629 & 3.225117 \\
H & 1.700178 & 3.368820 & 1.132966 \\
H & 3.708620 & 0.991768 & -1.400184 \\
H & -0.589487 & 2.806793 & 0.209482 \\
H & 1.804864 & 0.306143 & -2.879288 \\
H & 0.978396 & 3.695946 & -3.748259 \\
H & -0.494384 & -0.853830 & 0.620542 \\
H & 1.409292 & -0.164802 & 2.098968 \\
H & 6.573794 & 1.202516 & 2.937778 \\
H & 6.238264 & 4.949326 & 1.733519 \\
O & 7.079244 & 1.891311 & 0.001659 \\
O & 8.277462 & 3.093301 & 1.964422 \\
& & & \\
& & &
\end{tabular}

Alkyne_anionA

Free energy $=-1257.513194$ Hartree

$\begin{array}{llll}\mathrm{C} & 1.222377 & 5.277422 & -1.121386 \\ \mathrm{C} & 2.498553 & 4.662226 & -1.352803 \\ \mathrm{~N} & 2.767206 & 4.023647 & -2.541090 \\ \mathrm{C} & 1.797014 & 3.977173 & -3.467048 \\ \mathrm{C} & 0.517414 & 4.541548 & -3.320250 \\ \mathrm{C} & 0.239438 & 5.211028 & -2.107593 \\ \mathrm{C} & 3.501907 & 4.683971 & -0.363436 \\ \mathrm{C} & 4.361043 & 4.664149 & 0.545595\end{array}$




$\begin{array}{cccc}\text { C } & 5.319694 & 4.681507 & 1.535520 \\ \text { S } & 6.754008 & 3.732789 & 1.404396 \\ \text { C } & 6.430498 & 1.998400 & 2.118084 \\ \text { C } & 5.277192 & 1.375763 & 1.515869 \\ \text { C } & 4.239266 & 0.984539 & 0.983735 \\ \text { C } & 3.022299 & 0.607059 & 0.335428 \\ \text { C } & 2.647205 & 1.247272 & -0.876081 \\ \text { C } & 1.429398 & 0.929574 & -1.493207 \\ \text { C } & 0.574476 & -0.036633 & -0.930760 \\ \text { C } & 0.944324 & -0.685899 & 0.262390 \\ \text { C } & 2.154732 & -0.367865 & 0.896400 \\ \text { H } & -0.381660 & -0.281594 & -1.418839 \\ \text { H } & -0.229974 & 4.461155 & -4.123934 \\ \text { H } & 5.175778 & 5.120813 & 2.535038 \\ \text { H } & 6.321993 & 2.152767 & 3.211502 \\ \text { H } & 1.033855 & 5.784905 & -0.163284 \\ \text { H } & 3.300789 & 2.013727 & -1.318629 \\ \text { H } & -0.745666 & 5.674439 & -1.936216 \\ \text { H } & 1.145436 & 1.455007 & -2.418019 \\ \text { H } & 2.054398 & 3.446147 & -4.403484 \\ \text { H } & 0.279383 & -1.443009 & 0.707501 \\ \text { H } & 2.438106 & -0.864349 & 1.836730 \\ \text { H } & 7.386812 & 1.468888 & 1.918956 \\ \text { O } & 7.109737 & 3.478750 & -0.025536 \\ \text { O } & 7.795591 & 4.266642 & 2.343975\end{array}$

Alkyne_anionB

33

Free energy $=-1257.502442$ Hartree

C $\quad 0.386128 \quad 0.871454 \quad 0.383278$

$\begin{array}{llll}\text { C } & 0.446484 & 1.069599 & 1.801575\end{array}$

$\begin{array}{llll}\text { C } & -0.400907 & 0.245890 & 2.611630\end{array}$

$\begin{array}{llll}\text { C } & -1.251114 & -0.706245 & 2.03380\end{array}$

$\begin{array}{llll}\text { C } & -1.306903 & -0.875267 & 0.634674\end{array}$

$\begin{array}{llll}\text { C } & -0.479173 & -0.073863 & -0.178846\end{array}$

$\begin{array}{llll}\text { C } & 1.308166 & 2.033888 & 2.368740\end{array}$

$\begin{array}{llll}\text { C } & 2.168387 & 2.815276 & 2.835401\end{array}$

$\begin{array}{llll}\text { C } & 3.149542 & 3.654970 & 3.315525\end{array}$

$\begin{array}{llll}\text { S } & 3.942569 & 4.817248 & 2.308283\end{array}$

$\begin{array}{llll}\text { C } & 4.179060 & 3.983196 & 0.618384\end{array}$

$\begin{array}{llll}\text { C } & 2.968865 & 4.002911 & -0.164790\end{array}$

$\begin{array}{llll}\text { C } & 1.891549 & 4.080322 & -0.752313\end{array}$

$\begin{array}{llll}\text { C } & 0.555578 & 4.129981 & -1.272952\end{array}$

$\begin{array}{llll}\text { C } & -0.528909 & 3.917675 & -0.379623\end{array}$

$\begin{array}{llll}\text { C } & -1.833230 & 3.954453 & -0.885623\end{array}$

$\begin{array}{llll}\text { C } & -2.020778 & 4.202363 & -2.25642\end{array}$

$\begin{array}{llll}\text { C } & -0.882915 & 4.403545 & -3.059739\end{array}$

$\begin{array}{llll}\mathrm{N} & 0.379021 & 4.371338 & -2.598956\end{array}$

$\begin{array}{llll}\mathrm{H} & -1.980497 & -1.622052 & 0.185598\end{array}$

$\begin{array}{llll}\mathrm{H} & -3.027225 & 4.239548 & -2.700125\end{array}$

$\begin{array}{llll}\mathrm{H} & 4.992918 & 4.596823 & 0.178648\end{array}$

$\begin{array}{llll}\mathrm{H} & 3.528618 & 3.675633 & 4.349118\end{array}$

$\begin{array}{llll}\mathrm{H} & -0.321674 & 3.716631 & 0.682012\end{array}$

$\begin{array}{llll}\mathrm{H} & -0.377133 & 0.373246 & 3.706016\end{array}$

$\begin{array}{llll}\mathrm{H} & -2.692660 & 3.786105 & -0.218632\end{array}$

$\begin{array}{llll}\mathrm{H} & -1.887333 & -1.325967 & 2.687464\end{array}$

$\begin{array}{llll}\mathrm{H} & -1.000671 & 4.603952 & -4.140352\end{array}$

$\begin{array}{llll}\mathrm{H} & -0.501937 & -0.194103 & -1.274967\end{array}$

$\begin{array}{llll}\mathrm{H} & 1.039093 & 1.478352 & -0.260630\end{array}$

$\begin{array}{llll}\mathrm{H} & 4.558081 & 2.962674 & 0.828158\end{array}$

$\begin{array}{llll}\mathrm{O} & 3.140520 & 6.044764 & 1.991672\end{array}$

$\begin{array}{llll}\text { O } & 5.323534 & 5.049221 & 2.851127\end{array}$

\section{A = 2-pyridyl, $B$ = 4-methoxyphenyl}

Bispropargyl

38

Free energy $=-1372.42044$ Hartree

$\begin{array}{cccc}\mathrm{C} & 1.683273 & 0.009332 & 1.197664 \\ \mathrm{C} & 2.933453 & 0.388164 & 0.657219 \\ \mathrm{C} & 3.070568 & 0.457982 & -0.759194 \\ \mathrm{C} & 1.988196 & 0.186694 & -1.590472 \\ \mathrm{C} & 0.733022 & -0.158173 & -1.038509 \\ \mathrm{C} & 0.589541 & -0.263106 & 0.363181 \\ \mathrm{C} & 4.046507 & 0.770637 & 1.459461 \\ \mathrm{C} & 5.060211 & 1.189295 & 2.013294 \\ \mathrm{C} & 6.287647 & 1.803697 & 2.450591 \\ \mathrm{~S} & 7.049969 & 2.840502 & 1.076270 \\ \mathrm{O} & 8.300551 & 3.407295 & 1.640550 \\ \mathrm{C} & 5.887079 & 4.314381 & 0.886092 \\ \mathrm{C} & 4.617894 & 4.047689 & 0.256788 \\ \mathrm{C} & 3.541424 & 3.833356 & -0.293648 \\ \mathrm{C} & 2.255708 & 3.559020 & -0.871077 \\ \mathrm{C} & 1.161682 & 3.249085 & -0.022253 \\ \mathrm{C} & -0.076413 & 2.953880 & -0.603624 \\ \mathrm{C} & -0.191264 & 2.980441 & -2.002548 \\ \mathrm{C} & 0.951810 & 3.308459 & -2.755891 \\ \mathrm{~N} & 2.147759 & 3.593953 & -2.222795 \\ \mathrm{O} & 7.038307 & 2.051852 & -0.173901 \\ \mathrm{O} & -0.275147 & -0.340323 & -1.935683\end{array}$

$\begin{array}{rrrr}\mathrm{H} & -1.139960 & 2.740382 & -2.504567 \\ \mathrm{H} & 6.534323 & 4.989614 & 0.285404 \\ \mathrm{H} & 6.193030 & 2.486652 & 3.321049 \\ \mathrm{H} & 1.308549 & 3.215783 & 1.066443 \\ \mathrm{H} & 4.035449 & 0.771260 & -1.183851 \\ \mathrm{H} & -0.937858 & 2.694197 & 0.030817 \\ \mathrm{H} & 2.073048 & 0.272443 & -2.683122 \\ \mathrm{H} & 0.894445 & 3.338704 & -3.859468 \\ \mathrm{H} & -0.372219 & -0.539273 & 0.815328 \\ \mathrm{H} & 1.564226 & -0.054278 & 2.289714 \\ \mathrm{H} & 7.113829 & 1.087751 & 2.655023 \\ \mathrm{H} & 5.803948 & 4.733696 & 1.910322 \\ \mathrm{C} & -1.580787 & -0.605828 & -1.448346 \\ \mathrm{H} & -2.235048 & -0.688390 & -2.335983 \\ \mathrm{H} & -1.627782 & -1.560136 & -0.876231 \\ \mathrm{H} & -1.953710 & 0.220158 & -0.799273\end{array}$

Alkyne_anionA

Free energy $=-1371.934352$ Hartree

$\begin{array}{llll}\text { C } & 2.809081 & -0.739316 & 0.579352\end{array}$

$\begin{array}{llll}\text { C } & 3.514294 & 0.414116 & 0.155645\end{array}$

$\begin{array}{llll}\text { C } & 3.084071 & 1.066577 & -1.034469\end{array}$

$\begin{array}{llll}\text { C } & 1.986817 & 0.590105 & -1.750882\end{array}$

$\begin{array}{llll}\text { C } & 1.289501 & -0.562426 & -1.317803\end{array}$

$\begin{array}{llll}\text { C } & 1.712320 & -1.230823 & -0.145938\end{array}$

$\begin{array}{llll}\text { C } & 4.603798 & 0.952615 & 0.906619\end{array}$

$\begin{array}{llll}\text { C } & 5.527049 & 1.479747 & 1.526439\end{array}$

$\begin{array}{llll}\text { C } & 6.521752 & 2.242211 & 2.241581\end{array}$

$\begin{array}{llll}\text { S } & 6.621099 & 4.040040 & 1.633369\end{array}$

$\begin{array}{llll}\text { O } & 7.516079 & 4.668262 & 2.661983\end{array}$

$\begin{array}{llll}\text { C } & 5.052872 & 4.756181 & 1.718795\end{array}$

$\begin{array}{llll}\text { C } & 4.160471 & 4.645955 & 0.674446\end{array}$

$\begin{array}{llll}\text { C } & 3.355264 & 4.581998 & -0.281110\end{array}$

$\begin{array}{llll}\text { C } & 2.417903 & 4.453416 & -1.325761\end{array}$

$\begin{array}{llll}\text { C } & 1.050878 & 4.852420 & -1.143971\end{array}$

$\begin{array}{llll}\text { C } & 0.140359 & 4.678932 & -2.185155\end{array}$

$\begin{array}{llll}\text { C } & 0.579132 & 4.116093 & -3.404532\end{array}$

$\begin{array}{llll}\text { C } & 1.937731 & 3.766656 & -3.501264\end{array}$

$\begin{array}{llll}\mathrm{N} & 2.841736 & 3.921427 & -2.521516\end{array}$

$\begin{array}{llll}\text { O } & 7.092140 & 3.928545 & 0.218576\end{array}$

$\begin{array}{llll}\mathrm{O} & 0.232242 & -0.947882 & -2.088743\end{array}$

$\begin{array}{llll}\mathrm{H} & -0.105947 & 3.955292 & -4.250291\end{array}$

$\begin{array}{llll}\mathrm{H} & 4.789757 & 5.113120 & 2.726870\end{array}$

$\begin{array}{llll}\mathrm{H} & 6.312485 & 2.322418 & 3.328673\end{array}$

$\begin{array}{llll}\mathrm{H} & 0.737417 & 5.282306 & -0.180657\end{array}$

$\begin{array}{llll}\mathrm{H} & 3.602350 & 1.970919 & -1.386117\end{array}$

$\begin{array}{llll}\mathrm{H} & -0.912276 & 4.977164 & -2.051888\end{array}$

$\mathrm{H} \quad 1.639622 \quad 1.111320 \quad-2.655596$

\begin{tabular}{llll}
$\mathrm{H}$ & 2.322170 & 3.326676 & -4.441485 \\
\hline
\end{tabular}

$\begin{array}{llll}\mathrm{H} & 1.194597 & -2.130092 & 0.214236\end{array}$

$\begin{array}{llll}\mathrm{H} & 3.123159 & -1.256846 & 1.498467\end{array}$

\begin{tabular}{llll}
\hline & 7.562739 & 1.878952 & 2.100464 \\
\hline & -0.515474 & -2.096633 & -1.698426
\end{tabular}

$\begin{array}{llll}\text { C } & -0.515474 & -2.096633 & -1.698426\end{array}$

$\begin{array}{llll}\mathrm{H} & -1.315512 & -2.217996 & -2.452148\end{array}$

$\mathrm{H} \quad 0.114025 \quad-3.014643 \quad-1.686824$

$\begin{array}{llll}\mathrm{H} & -0.979808 & -1.965724 & -0.695525\end{array}$

Alkyne_anionB

Free energy $=-1371.92684$ Hartree

$\begin{array}{llll}\mathrm{N} & 0.633931 & 1.786605 & -1.208675\end{array}$

$\begin{array}{llll}\text { C } & 0.534814 & 3.110058 & -0.907552\end{array}$

$\begin{array}{llll}\text { C } & -0.641108 & 3.861739 & -1.179262\end{array}$

$\begin{array}{llll}\text { C } & -1.739024 & 3.215705 & -1.759617\end{array}$

$\begin{array}{llll}\text { C } & -1.644893 & 1.845352 & -2.052842\end{array}$

$\begin{array}{llll}\text { C } & -0.436483 & 1.188851 & -1.751292\end{array}$

$\begin{array}{llll}\text { C } & 1.671658 & 3.741186 & -0.304055\end{array}$

$\begin{array}{llll}\text { C } & 2.643525 & 4.300118 & 0.207011\end{array}$

$\begin{array}{llll}\text { C } & 3.781896 & 4.926761 & 0.826073\end{array}$

$\begin{array}{lll}3.845852 & 4.774622 & 2.751624\end{array}$

$\begin{array}{llll}\text { O } & 5.101521 & 5.536245 & 3.067966\end{array}$

C $\quad 4.029886 \quad 3.131226 \quad 3.213686$

$\begin{array}{llll}\text { C } & 2.929453 & 2.310028 & 2.991346\end{array}$

$\begin{array}{llll}\text { C } & 1.860718 & 1.708659 & 2.769949\end{array}$

$\begin{array}{llll}\text { C } & 0.628500 & 1.234423 & 2.259761\end{array}$

$\begin{array}{llll}\text { C } & -0.425667 & 2.176253 & 2.036818\end{array}$

$\begin{array}{llll}\text { C } & -1.625650 & 1.791851 & 1.44225\end{array}$

$\begin{array}{llll}\text { C } & -1.833135 & 0.452791 & 1.042277\end{array}$

$\begin{array}{llll}\text { C } & -0.824705 & -0.503794 & 1.286619\end{array}$

$\begin{array}{llll}\text { C } & 0.382043 & -0.116238 & 1.891552\end{array}$

$\begin{array}{llll}\mathrm{O} & 2.524545 & 5.335427 & 3.179447\end{array}$

$\begin{array}{llll}\text { O } & -3.021960 & 0.194303 & 0.404166\end{array}$

$\begin{array}{llll}\mathrm{H} & -2.487735 & 1.290243 & -2.489484\end{array}$

$\begin{array}{llll}\mathrm{H} & 3.799739 & 6.027579 & 0.684985\end{array}$

$\begin{array}{llll}\mathrm{H} & 5.078175 & 2.792410 & 3.196922\end{array}$

$\begin{array}{llll}\mathrm{H} & -0.677215 & 4.929978 & -0.920111\end{array}$

$\begin{array}{llll}\mathrm{H} & 1.168963 & -0.869031 & 2.053973\end{array}$

$\begin{array}{llll}\mathrm{H} & -2.663461 & 3.775157 & -1.972611\end{array}$

$\begin{array}{lllr}\mathrm{H} & -0.956380 & -1.553367 & 0.988116\end{array}$ 
$\begin{array}{crrr}\mathrm{H} & -0.330843 & 0.109374 & -1.963256 \\ \mathrm{H} & -2.415123 & 2.528589 & 1.232741 \\ \mathrm{H} & -0.251999 & 3.229161 & 2.304076 \\ \mathrm{H} & 4.749366 & 4.498767 & 0.493764 \\ \mathrm{C} & -3.212371 & -1.097095 & -0.155585 \\ \mathrm{H} & -4.194887 & -1.078455 & -0.663865 \\ \mathrm{H} & -2.421921 & -1.343234 & -0.900642 \\ \mathrm{H} & -3.226341 & -1.894510 & 0.622286\end{array}$

\section{A = 1-benzyl-3-indolyl, $B$ = phenyl}

Bispropargyl

Free energy $=-1643.520487$ Hartree

$\begin{array}{llll}\text { C } & 6.616810 & 6.246193 & 1.394820\end{array}$

$\begin{array}{llll}\text { C } & 5.598724 & 5.679086 & 0.587134\end{array}$

$\begin{array}{llll}\text { C } & 5.482276 & 6.088206 & -0.767049\end{array}$

$\begin{array}{llll}\text { C } & 6.366208 & 7.039251 & -1.292708\end{array}$

$\begin{array}{llll}\text { C } & 7.375732 & 7.594953 & -0.485325\end{array}$

$\begin{array}{llll}\text { C } & 7.497658 & 7.192674 & 0.856678\end{array}$

$\begin{array}{llll}\text { C } & 4.706854 & 4.689636 & 1.100932\end{array}$

$\begin{array}{llll}\text { C } & 3.936071 & 3.811023 & 1.477389\end{array}$

$\begin{array}{llll}\text { C } & 2.999201 & 2.779560 & 1.848076\end{array}$

$\begin{array}{llll}\mathrm{S} & 2.078032 & 2.010736 & 0.389778\end{array}$

$\begin{array}{llll}\text { C } & 3.299041 & 0.755761 & -0.317571\end{array}$

$\begin{array}{llll}\text { C } & 4.547708 & 1.354487 & -0.709260\end{array}$

$\begin{array}{llll}\text { C } & 5.545322 & 2.032894 & -0.953748\end{array}$

$\begin{array}{llll}\text { C } & 6.678475 & 2.853701 & -1.131139\end{array}$

$\begin{array}{llll}\text { C } & 7.625020 & 3.238442 & -0.095977\end{array}$

$\begin{array}{llll}\text { C } & 8.548907 & 4.146129 & -0.695954\end{array}$

$\begin{array}{llll}\text { N } & 8.180131 & 4.310279 & -2.028241\end{array}$

$\begin{array}{llll}\text { C } & 7.068409 & 3.538193 & -2.287855\end{array}$

$\begin{array}{llll}\text { C } & 7.743798 & 2.933565 & 1.274436\end{array}$

$\begin{array}{llll}\text { C } & 8.783385 & 3.524266 & 2.003062\end{array}$

$\begin{array}{llll}\text { C } & 9.706887 & 4.405785 & 1.383848\end{array}$

$\begin{array}{llll}\text { C } & 9.605977 & 4.729160 & 0.024948\end{array}$

$\begin{array}{llll}\text { C } & 8.866544 & 5.178933 & -2.968475\end{array}$

$\begin{array}{llll}\text { C } & 10.282544 & 4.728749 & -3.297108\end{array}$

$\begin{array}{llll}\text { C } & 10.631269 & 3.364052 & -3.282210\end{array}$

$\begin{array}{llll}\text { C } & 11.934634 & 2.960682 & -3.614700\end{array}$

C $12.903696 \quad 3.916232 \quad-3.966220$

$\begin{array}{llll}\text { C } & 12.563923 & 5.279944 & -3.977800\end{array}$

$\begin{array}{llll}\text { C } & 11.261053 & 5.682719 & -3.640271\end{array}$

$\begin{array}{llll}\mathrm{H} & 8.067204 & 8.344436 & -0.901668\end{array}$

$\begin{array}{llll}\mathrm{H} & 2.708339 & 0.337850 & -1.161084\end{array}$

$\begin{array}{llll}\mathrm{H} & 2.141526 & 3.153153 & 2.449277\end{array}$

$\begin{array}{llll}\mathrm{H} & 4.697495 & 5.636553 & -1.390729\end{array}$

$\begin{array}{llll}\mathrm{H} & 6.266198 & 7.350748 & -2.344147\end{array}$

$\begin{array}{llll}\mathrm{H} & 8.295263 & 7.611967 & 1.488068\end{array}$

$\begin{array}{llll}\mathrm{H} & 6.722296 & 5.913713 & 2.436825\end{array}$

$\begin{array}{llll}\mathrm{H} & 8.251629 & 5.220644 & -3.891807\end{array}$

$\begin{array}{llll}\mathrm{H} & 8.879869 & 6.209341 & -2.551606\end{array}$

$\begin{array}{llll}\mathrm{H} & 11.000984 & 6.754277 & -3.64029\end{array}$

$\begin{array}{llll}\mathrm{H} & 13.319447 & 6.035631 & -4.243006\end{array}$

$\begin{array}{llll}\mathrm{H} & 13.925267 & 3.598453 & -4.225006\end{array}$

$\mathrm{H} \quad 12.196480 \quad 1.891392 \quad-3.593376$

$\begin{array}{llll}\mathrm{H} & 9.877153 & 2.615566 & -2.992743\end{array}$

$\begin{array}{llll}\mathrm{H} & 6.603890 & 3.537683 & -3.280255\end{array}$

$\begin{array}{llll}\mathrm{H} & 3.451595 & 1.920960 & 2.388360\end{array}$

$\begin{array}{llll}\mathrm{H} & 7.018670 & 2.256757 & 1.750264\end{array}$

$\begin{array}{llll}\mathrm{H} & 8.892308 & 3.298563 & 3.074983\end{array}$

$\begin{array}{llll}\mathrm{H} & 10.518098 & 4.846692 & 1.983054\end{array}$

$\begin{array}{llll}\mathrm{H} & 10.319480 & 5.410682 & -0.459582\end{array}$

$\begin{array}{llll}\mathrm{H} & 3.384726 & -0.020998 & 0.471467\end{array}$

\begin{tabular}{lllr}
$\mathrm{O}$ & 1.832901 & 3.056979 & -0.626713 \\
\hline & 0.972900 & 1.218682 & 0.989772
\end{tabular}

Alkyne_anionA

51

Free energy $=-1643.024441$ Hartree

$\begin{array}{lccc}\mathrm{C} & 10.390623 & 3.939775 & -2.845946 \\ \mathrm{C} & 10.225374 & 5.338910 & -2.865002 \\ \mathrm{C} & 11.302209 & 6.148856 & -3.282925 \\ \mathrm{C} & 12.518948 & 5.571087 & -3.684502 \\ \mathrm{C} & 12.675918 & 4.172618 & -3.666548 \\ \mathrm{C} & 11.608427 & 3.359964 & -3.244212 \\ \mathrm{C} & 8.903777 & 5.984641 & -2.464308 \\ \mathrm{~N} & 8.035513 & 5.133012 & -1.682461 \\ \mathrm{C} & 8.237790 & 4.803658 & -0.354397 \\ \mathrm{C} & 7.179079 & 3.928691 & 0.044564 \\ \mathrm{C} & 6.315088 & 3.732145 & -1.114844 \\ \mathrm{C} & 6.893429 & 4.474993 & -2.149121 \\ \mathrm{C} & 9.248997 & 5.199794 & 0.542691 \\ \mathrm{C} & 9.163369 & 4.723728 & 1.859034 \\ \mathrm{C} & 8.100710 & 3.875991 & 2.273603 \\ \mathrm{C} & 7.103547 & 3.468628 & 1.374673 \\ \mathrm{C} & 5.122641 & 2.982214 & -1.031036 \\ \mathrm{C} & 4.078679 & 2.425758 & -0.643809 \\ \mathrm{C} & 2.984357 & 1.788217 & -0.056870 \\ \mathrm{~S} & 2.930804 & 1.761140 & 1.654165\end{array}$

$\begin{array}{cccc}\text { C } & 2.486241 & 3.491869 & 2.365472 \\ \mathrm{C} & 3.434209 & 4.518135 & 2.009174 \\ \mathrm{C} & 4.272850 & 5.342962 & 1.644773 \\ \mathrm{C} & 5.243326 & 6.278647 & 1.173181 \\ \mathrm{C} & 5.236924 & 6.686651 & -0.187722 \\ \mathrm{C} & 6.206669 & 7.578332 & -0.665231 \\ \mathrm{C} & 7.194625 & 8.084817 & 0.199642 \\ \mathrm{C} & 7.212195 & 7.684566 & 1.548160 \\ \mathrm{C} & 6.247376 & 6.792557 & 2.035884 \\ \mathrm{H} & 7.959955 & 8.780287 & -0.180456 \\ \mathrm{H} & 1.990679 & 1.687333 & -0.521222 \\ \mathrm{H} & 1.464820 & 3.683942 & 1.976986 \\ \mathrm{H} & 4.482195 & 6.262821 & -0.865392 \\ \mathrm{H} & 6.200945 & 7.868782 & -1.727132 \\ \mathrm{H} & 7.997111 & 8.057976 & 2.223674 \\ \mathrm{H} & 6.275246 & 6.459234 & 3.083219 \\ \mathrm{H} & 8.347361 & 6.294484 & -3.373904 \\ \mathrm{H} & 9.098610 & 6.919173 & -1.892954 \\ \mathrm{H} & 11.185863 & 7.245697 & -3.291821 \\ \mathrm{H} & 13.351712 & 6.215909 & -4.007194 \\ \mathrm{H} & 13.630167 & 3.718549 & -3.976523 \\ \mathrm{H} & 11.724097 & 2.264579 & -3.219936 \\ \mathrm{H} & 9.557763 & 3.302465 & -2.509528 \\ \mathrm{H} & 6.555994 & 4.627688 & -3.180169 \\ \mathrm{H} & 2.431013 & 3.300168 & 3.457661 \\ \mathrm{H} & 6.256795 & 2.832570 & 1.683584 \\ \mathrm{H} & 8.060976 & 3.536984 & 3.320874 \\ \mathrm{H} & 9.931312 & 5.024618 & 2.589703 \\ \mathrm{H} & 10.067053 & 5.863387 & 0.225278 \\ \mathrm{O} & 1.756367 & 0.940643 & 2.109842 \\ \mathrm{O} & 4.280188 & 1.491496 & 2.256853 \\ & & & \end{array}$

\begin{tabular}{|c|c|c|c|}
\hline $\begin{array}{l}51 \\
\text { Free }\end{array}$ & $\mathrm{nB}$ & & \\
\hline $\mathrm{C}$ & 5.295731 & 12.646629 & -0.997780 \\
\hline $\mathrm{C}$ & 5.238913 & 11.402111 & -1.657010 \\
\hline $\mathrm{C}$ & 4.091615 & 11.073053 & 2005 \\
\hline $\mathrm{C}$ & 3.009253 & 11.967111 & 4173 \\
\hline $\mathrm{C}$ & 3.063260 & 13.200676 & 00941 \\
\hline $\mathrm{C}$ & 4.213595 & & 63703 \\
\hline $\mathrm{C}$ & 6.38 & & 5144 \\
\hline $\mathrm{N}$ & & & \\
\hline $\mathrm{C}$ & & & \\
\hline $\mathrm{C}$ & 6.3 & & \\
\hline $\mathrm{C}$ & $5.8 \mathrm{c}$ & & \\
\hline $\mathrm{C}$ & 5.90 & & \\
\hline $\mathrm{C}$ & 7.12 & 10. & \\
\hline $\mathrm{C}$ & 7.3 & 10. & -5 \\
\hline $\mathrm{C}$ & 7.081400 & & -6 \\
\hline $\mathrm{C}$ & 6.600906 & & 331 \\
\hline $\mathrm{C}$ & 5.4 & & 373 \\
\hline $\mathrm{C}$ & 5.0 & & \\
\hline C & 4.4 & & \\
\hline $\mathrm{S}$ & 3.11 & & \\
\hline C & & & \\
\hline $\mathrm{C}$ & 3.8 & & \\
\hline $\mathrm{C}$ & & & \\
\hline C & & & \\
\hline $\mathrm{C}$ & & & \\
\hline $\mathrm{C}$ & & & \\
\hline $\mathrm{C}$ & 4.0 & & \\
\hline $\mathrm{C}$ & 3.2 & & \\
\hline C & 3.1 & & \\
\hline $\mathrm{H}$ & 4.10 & 11. & 3272 \\
\hline $\mathrm{H}$ & 3.9 & & 2779 \\
\hline $\mathrm{H}$ & 4.3 & & 92 \\
\hline $\mathrm{H}$ & 5.4 & & \\
\hline $\mathrm{H}$ & & & \\
\hline $\mathrm{H}$ & 2.5 & & \\
\hline $\mathrm{H}$ & 2.48 & & \\
\hline $\mathrm{H}$ & 6.3 & & \\
\hline $\mathrm{H}$ & & & \\
\hline $\mathrm{H}$ & & & \\
\hline $\mathrm{H}$ & & & \\
\hline $\mathrm{H}$ & & & \\
\hline $\mathrm{H}$ & & & \\
\hline $\mathrm{H}$ & & & 927 \\
\hline $\mathrm{H}$ & & & \\
\hline $\mathrm{H}$ & & & \\
\hline $\mathrm{H}$ & & & \\
\hline $\mathrm{H}$ & & & -7.627004 \\
\hline $\mathrm{H}$ & & & -6.486460 \\
\hline $\mathrm{H}$ & & & -4.016517 \\
\hline $\mathrm{O}$ & & & \\
\hline $\mathrm{O}$ & 2.784860 & 1.995436 & -1.969879 \\
\hline
\end{tabular}

A = 1-benzyl-3-indolyl, $\mathrm{B}=$ 4-methoxyphenyl

Bispropargyl 
56

Free energy $=-1757.943403$ Hartree

\begin{tabular}{|c|c|c|c|}
\hline $\mathrm{C}$ & 11.292981 & 5.837008 & -3.641656 \\
\hline 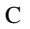 & 10.347684 & 4.833206 & -3.352240 \\
\hline & 10.760326 & 3.486211 & -3.341817 \\
\hline $\mathrm{C}$ & 12.093255 & 3.149324 & -3.626177 \\
\hline C & 13.029460 & 4.154831 & -3.924495 \\
\hline $\mathrm{C}$ & 12.626198 & 5.501054 & -3.931488 \\
\hline 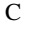 & 8.903624 & 5.212931 & -3.064699 \\
\hline $\mathrm{N}$ & 8.260410 & & -2.081538 \\
\hline$C$ & 8.648135 & & \\
\hline $\mathrm{C}$ & 7.7 & 2954 & 71 \\
\hline $\mathrm{C}$ & 6.727243 & 3.005862 & -1.067789 \\
\hline $\mathrm{C}$ & 7.118499 & 3.602747 & -2.271348 \\
\hline $\mathrm{C}$ & 9.740941 & 4.842894 & -0.083911 \\
\hline C & 9.860315 & 4.597835 & 1.290513 \\
\hline $\mathrm{C}$ & 8.921328 & 3.787882 & 1.979313 \\
\hline $\mathrm{C}$ & 7.845467 & 3.194855 & 1.307295 \\
\hline $\mathrm{C}$ & 5.577420 & 2.221494 & -0.828225 \\
\hline $\mathrm{C}$ & 4.577866 & 1.563178 & -0.541930 \\
\hline C & 3.363895 & 0.883861 & -0.171913 \\
\hline & 1.922816 & 2.013069 & 0.290589 \\
\hline $\mathrm{O}$ & 0.820038 & 1.1 & 171 \\
\hline $\mathrm{C}$ & 2.473831 & 2.882079 & 1.869683 \\
\hline $\mathrm{C}$ & 3.464683 & 3.90 & 392 \\
\hline $\mathrm{C}$ & 4.305478 & 4.73 & 1.2 \\
\hline $\mathrm{C}$ & 5.28 & & 31 \\
\hline $\mathrm{C}$ & 6.3 & 6.1 & 38 \\
\hline $\mathrm{C}$ & 7.333567 & 20 & 80 \\
\hline $\mathrm{C}$ & 7.332879 & 7.221723 & -0.350220 \\
\hline $\mathrm{C}$ & 6.283778 & 6.731831 & -1.159180 \\
\hline $\mathrm{C}$ & 5.275812 & 5.945979 & -0.602941 \\
\hline $\mathrm{O}$ & 1.747552 & 3.025522 & -0.773854 \\
\hline $\mathrm{O}$ & 8.317877 & 7.910602 & -1.006608 \\
\hline $\mathrm{H}$ & 2.900449 & 0.309517 & -1.003933 \\
\hline $\mathrm{H}$ & 1.498210 & 3.270910 & 2.235919 \\
\hline $\mathrm{H}$ & 4.477677 & 5.530906 & -1.234978 \\
\hline $\mathrm{H}$ & 6.295001 & 6.965628 & -2.233809 \\
\hline $\mathrm{H}$ & 8.141931 & 7.317540 & 1.682351 \\
\hline $\mathrm{H}$ & 6.349261 & 5.916345 & 2.659421 \\
\hline $\mathrm{H}$ & 8.299710 & 5.160055 & -3.994642 \\
\hline $\mathrm{H}$ & 8.852553 & 6.262858 & -2.703695 \\
\hline $\mathrm{H}$ & 10.980512 & 259 & -3.636405 \\
\hline $\mathrm{H}$ & 13.355070 & 5104 & -4.156431 \\
\hline $\mathrm{H}$ & 14.074421 & 3.888978 & -4.146333 \\
\hline $\mathrm{H}$ & 12.404868 & 2.093404 & -3.609521 \\
\hline $\mathrm{H}$ & 10.030913 & 2.700258 & -3.090936 \\
\hline $\mathrm{H}$ & 6.630724 & 3.561943 & -3.251653 \\
\hline $\mathrm{H}$ & 2.797422 & 2.067116 & 2.551306 \\
\hline $\mathrm{H}$ & 7.107116 & 2.574403 & 1.837245 \\
\hline $\mathrm{H}$ & 9.045608 & 3.621593 & 3.060429 \\
\hline $\mathrm{H}$ & 10.703097 & 5.039260 & 1.844488 \\
\hline $\mathrm{H}$ & 10.472645 & 5.456572 & -0.628259 \\
\hline $\mathrm{H}$ & 3.468361 & 0.204215 & 0.700183 \\
\hline $\mathrm{C}$ & 9.370642 & 8.482192 & -0.240961 \\
\hline $\mathrm{H}$ & 10.040409 & 8.987712 & -0.962009 \\
\hline $\mathrm{H}$ & 8.987247 & 9.232967 & 0.486185 \\
\hline & & & \\
\hline
\end{tabular}

Alkyne_anionA

55

Free energy $=-1757.448262$ Hartree

$\begin{array}{llll}\mathrm{C} & 6.207061 & 6.789018 & 2.100289 \\ \mathrm{C} & 5.106975 & 6.282758 & 1.364423 \\ \mathrm{C} & 4.999192 & 6.640039 & -0.008088 \\ \mathrm{C} & 5.964408 & 7.442792 & -0.616645 \\ \mathrm{C} & 7.073878 & 7.907268 & 0.123557 \\ \mathrm{C} & 7.180737 & 7.594729 & 1.494995 \\ \mathrm{C} & 4.181105 & 5.370930 & 1.952063 \\ \mathrm{C} & 3.408784 & 4.530634 & 2.415244 \\ \mathrm{C} & 2.576999 & 3.439650 & 2.859624 \\ \mathrm{~S} & 3.145140 & 1.748711 & 2.145817 \\ \mathrm{O} & 4.554561 & 1.623935 & 2.651593 \\ \mathrm{C} & 3.076467 & 1.748801 & 0.435163 \\ \mathrm{C} & 4.079643 & 2.432088 & -0.254039 \\ \mathrm{C} & 5.046346 & 3.032280 & -0.758674 \\ \mathrm{C} & 6.209257 & 3.791102 & -1.009895 \\ \mathrm{C} & 7.199640 & 4.047009 & 0.029954 \\ \mathrm{C} & 8.206142 & 4.892945 & -0.534887 \\ \mathrm{~N} & 7.854775 & 5.150132 & -1.846678 \\ \mathrm{C} & 6.662782 & 4.480342 & -2.140165 \\ \mathrm{C} & 7.278070 & 3.659204 & 1.382370 \\ \mathrm{C} & 8.372368 & 4.105394 & 2.138792 \\ \mathrm{C} & 9.379147 & 4.924658 & 1.561045 \\ \mathrm{C} & 9.312801 & 5.330245 & 0.219749 \\ \mathrm{C} & 8.594503 & 6.019809 & -2.743111 \\ \mathrm{C} & 9.977433 & 5.488193 & -3.090023 \\ \mathrm{C} & 10.175076 & 4.113017 & -3.332320 \\ \mathrm{C} & 11.447811 & 3.623258 & -3.667904 \\ \mathrm{C} & 12.542733 & 4.503573 & -3.762048\end{array}$

$\begin{array}{cccc}\text { C } & 12.355832 & 5.874609 & -3.513040 \\ \mathrm{C} & 11.079864 & 6.361655 & -3.175017 \\ \mathrm{O} & 2.094804 & 0.822180 & 2.694437 \\ \mathrm{O} & 8.006119 & 8.623699 & -0.580347 \\ \mathrm{H} & 2.067791 & 1.545288 & 0.042878 \\ \mathrm{H} & 1.518994 & 3.525913 & 2.535479 \\ \mathrm{H} & 4.167730 & 6.234680 & -0.601871 \\ \mathrm{H} & 5.903620 & 7.688356 & -1.686935 \\ \mathrm{H} & 8.040300 & 7.930472 & 2.089083 \\ \mathrm{H} & 6.320651 & 6.508287 & 3.157472 \\ \mathrm{H} & 7.991212 & 6.137128 & -3.667094 \\ \mathrm{H} & 8.675021 & 7.029784 & -2.286172 \\ \mathrm{H} & 10.938487 & 7.434971 & -2.965258 \\ \mathrm{H} & 13.209384 & 6.568295 & -3.572995 \\ \mathrm{H} & 13.540918 & 4.118751 & -4.023290 \\ \mathrm{H} & 11.587873 & 2.546919 & -3.855876 \\ \mathrm{H} & 9.320093 & 3.423536 & -3.243045 \\ \mathrm{H} & 6.206171 & 4.582779 & -3.131056 \\ \mathrm{H} & 2.608534 & 3.273385 & 3.957438 \\ \mathrm{H} & 6.471988 & 3.043718 & 1.817277 \\ \mathrm{H} & 8.453531 & 3.820902 & 3.199704 \\ \mathrm{H} & 10.227948 & 5.256759 & 2.180331 \\ \mathrm{H} & 10.092854 & 5.962998 & -0.228735 \\ \mathrm{C} & 9.162604 & 9.093633 & 0.108553 \\ \mathrm{H} & 9.783407 & 9.612402 & -0.646076 \\ \mathrm{H} & 8.895320 & 9.810635 & 0.916263 \\ \mathrm{H} & 9.745308 & 8.255265 & 0.548281\end{array}$

Alkyne_anionB

55

Free energy $=-1757.44882$ Hartree

$\begin{array}{llll}\text { C } & 2.810793 & 8.349595 & -0.031740\end{array}$

$\begin{array}{llll}\text { C } & 3.724615 & 7.487274 & 0.659334\end{array}$

$\begin{array}{llll}\text { C } & 4.565534 & 8.099957 & 1.634188\end{array}$

$\begin{array}{llll}\text { C } & 4.513252 & 9.481970 & 1.897097\end{array}$

$\begin{array}{llll}\text { C } & 3.610521 & 10.307575 & 1.192820\end{array}$

$\begin{array}{llll}\text { C } & 2.757604 & 9.717060 & 0.230374\end{array}$

$\begin{array}{llll}\text { C } & 3.797983 & 6.113092 & 0.327270\end{array}$

$\begin{array}{llll}\text { C } & 3.891775 & 4.940540 & -0.091762\end{array}$

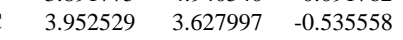

$\begin{array}{llll}\text { S } & 3.182701 & 3.151537 & -1.995110\end{array}$

$\begin{array}{llll}\mathrm{O} & 3.044010 & 1.654958 & -2.024287\end{array}$

$\begin{array}{llll}\text { C } & 4.337703 & 3.449924 & -3.485793\end{array}$

$\begin{array}{llll}\text { C } & 4.820395 & 4.808513 & -3.542818\end{array}$

$\begin{array}{llll}\text { C } & 5.235216 & 5.964653 & -3.449376\end{array}$

$\begin{array}{llll}\text { C } & 5.714521 & 7.286805 & -3.266578\end{array}$

$\begin{array}{llll}\text { C } & 6.252071 & 8.208265 & -4.254552\end{array}$

$\begin{array}{llll}\text { C } & 6.618573 & 9.402764 & -3.550542\end{array}$

$\begin{array}{llll}\mathrm{N} & 6.315174 & 9.214434 & -2.206482\end{array}$

$\begin{array}{llll}\text { C } & 5.776250 & 7.955736 & -2.039496\end{array}$

$\begin{array}{llll}\text { C } & 6.461307 & 8.137282 & -5.649078\end{array}$

$\begin{array}{llll}\text { C } & 7.025308 & 9.241120 & -6.304557\end{array}$

$\begin{array}{llll}\text { C } & 7.381178 & 10.416173 & -5.590589\end{array}$

$\begin{array}{llll}\text { C } & 7.180428 & 10.515220 & -4.205509\end{array}$

$\begin{array}{llll}\text { C } & 6.516922 & 10.188941 & -1.144528\end{array}$

$\begin{array}{llll}\text { C } & 5.684045 & 11.451588 & -1.288382\end{array}$

$\begin{array}{llll}\text { C } & 6.131133 & 12.649838 & -0.698038\end{array}$

$\begin{array}{llll}\text { C } & 5.334476 & 13.807068 & -0.739442\end{array}$

$\begin{array}{llll}\text { C } & 4.084193 & 13.777544 & -1.380624\end{array}$

$\begin{array}{llll}\text { C } & 3.642919 & 12.589323 & -1.989782\end{array}$

$\begin{array}{llll}\text { C } & 4.439007 & 11.434384 & -1.945086\end{array}$

$\begin{array}{llll}\text { O } & 1.988066 & 4.005748 & -2.288780\end{array}$

$\begin{array}{llll}\text { O } & 3.488644 & 11.662921 & 1.352797\end{array}$

H $\quad 3.707163 \quad 3.155578 \quad-4.351376$

$\mathrm{H} \quad 4.664754 \quad 2.877821 \quad-0.157023$

$\begin{array}{llll}\mathrm{H} & 5.285431 & 7.477205 & 2.187333\end{array}$

$\begin{array}{llll}\mathrm{H} & 5.191738 & 9.903495 & 2.653250\end{array}$

$\begin{array}{llll}\mathrm{H} & 2.064495 & 10.370433 & -0.319702\end{array}$

$\begin{array}{llll}\mathrm{H} & 2.152794 & 7.913157 & -0.798613\end{array}$

$\begin{array}{llll}\mathrm{H} & 6.245751 & 9.688850 & -0.189915\end{array}$

$\begin{array}{cccc}\mathrm{H} & 6.245751 & 9.688850 & -0.189915 \\ \mathrm{H} & 7.595905 & 10.447073 & -1.080817\end{array}$

$\begin{array}{llll}\mathrm{H} & 4.085942 & 10.501142 & -2.408373\end{array}$

$\begin{array}{llll}\mathrm{H} & 2.665626 & 12.557713 & -2.495937\end{array}$

$\begin{array}{llll}\mathrm{H} & 3.455132 & 14.681216 & -1.408768\end{array}$

$\begin{array}{llll}\mathrm{H} & 5.691354 & 14.734451 & -0.264586\end{array}$

$\begin{array}{llll}\mathrm{H} & 7.111209 & 12.675922 & -0.194105\end{array}$

$\begin{array}{llll}\mathrm{H} & 5.447668 & 7.606162 & -1.055086\end{array}$

$\begin{array}{llll}\mathrm{H} & 5.146856 & 2.700784 & -3.357347\end{array}$

$\begin{array}{llll}\mathrm{H} & 6.182245 & 7.226207 & -6.201675\end{array}$

$\begin{array}{llll}\mathrm{H} & 6.182245 & 7.226207 & -6.201675 \\ \mathrm{H} & 7.196329 & 9.198877 & -7.391793\end{array}$

$\begin{array}{llll}\mathrm{H} & 7.819099 & 11.268463 & -6.133641\end{array}$

$\begin{array}{llll}\mathrm{H} & 7.440408 & 11.429171 & -3.651795\end{array}$

$\begin{array}{llll}\text { C } & 4.355842 & 12.313245 & 2.267212\end{array}$

$\begin{array}{llll}\mathrm{H} & 4.126993 & 13.393870 & 2.200631\end{array}$

$\begin{array}{llll}\mathrm{H} & 4.194463 & 11.967006 & 3.314652\end{array}$

$\begin{array}{llll}\mathrm{H} & 5.425937 & 12.166081 & 1.996996\end{array}$ 


\begin{tabular}{|c|c|c|c|c|c|c|c|}
\hline \multicolumn{4}{|c|}{ A = 4-methoxyphenyl, $B=$ phenyl } & $\mathrm{C}$ & 0.135770 & 0.312265 & -0.558826 \\
\hline \multirow{2}{*}{\multicolumn{4}{|c|}{ Bispropargyl }} & $\mathrm{C}$ & 0.341257 & 1.266536 & 0.465532 \\
\hline \multirow{2}{*}{\multicolumn{4}{|c|}{37}} & $\mathrm{C}$ & 3.962095 & 0.734671 & $\begin{array}{l}1.347119 \\
1570115\end{array}$ \\
\hline \multirow{2}{*}{\multicolumn{4}{|c|}{ Free energy $=-883.1257596$ Hartree }} & $\mathrm{C}$ & 5.155168 & 1.116649 & 1.570115 \\
\hline & -0140968 & & & $\mathrm{C}$ & 6.435615 & 1.463936 & 1.869582 \\
\hline & 0.00580 & 4.110400 & 2.642405 & $\mathrm{O}$ & 6.730509 & 2.602940 & 2.654362 \\
\hline & 0.800588 & 5.536912 & 1.974149 & $\mathrm{C}$ & 7.169982 & 3.743134 & 1.882700 \\
\hline $\mathrm{C}$ & 0.322636 & 6.564460 & 1.118215 & $\mathrm{C}$ & 6.137527 & 4.209759 & 0.951915 \\
\hline $\mathrm{C}$ & -1.055305 & 6.759154 & 0.945535 & $\mathrm{C}$ & 5.178742 & 4.371768 & 0.196195 \\
\hline $\mathrm{C}$ & -1.978928 & 5.941425 & 1.622670 & $\mathrm{C}$ & 4.005351 & 4.373666 & -0.619681 \\
\hline $\mathrm{C}$ & -1.516106 & 4.914193 & 2.465406 & $\mathrm{C}$ & 3.258001 & 5.542042 & -0.926685 \\
\hline $\mathrm{C}$ & 2.204978 & 5.321978 & 2.150132 & $\mathrm{C}$ & 2.077314 & 5.455736 & -1.669617 \\
\hline $\mathrm{C}$ & 3.403327 & 5.092413 & 2.300915 & $\mathrm{C}$ & 1.597882 & 4.200182 & -2.116557 \\
\hline $\mathrm{C}$ & 4.836331 & 4.813872 & 2.485456 & $\mathrm{C}$ & 2.340702 & 3.031795 & -1.836494 \\
\hline $\mathrm{O}$ & 5.507310 & 4.283023 & 1.335055 & $\mathrm{C}$ & 3.531580 & 3.127914 & -1.108047 \\
\hline $\mathrm{C}$ & 5.365618 & 2.866527 & 1.151400 & $\mathrm{O}$ & 0.420023 & 4.220281 & -2.807214 \\
\hline $\mathrm{C}$ & 3.995702 & 2.449505 & 0.830036 & $\mathrm{C}$ & -0.116224 & 2.977270 & -3.263516 \\
\hline $\mathrm{C}$ & 2.784915 & 2.301446 & 0.670304 & $\mathrm{H}$ & -0.847794 & 0.199772 & -1.040546 \\
\hline C & 1.367632 & 2.271655 & 0.512016 & $\mathrm{H}$ & 8.097554 & 3.485296 & 1.311982 \\
\hline C & 0.540656 & 1.443458 & 1.319829 & $\mathrm{H}$ & 3.319986 & -1.008022 & -0.650840 \\
\hline C & -0.849215 & 1.521263 & 1.231800 & $\mathrm{H}$ & 4.087089 & 2.214424 & -0.855202 \\
\hline C & -1.460402 & 2.433740 & 0.338449 & $\mathrm{H}$ & 1.104129 & -1.243130 & -1.744016 \\
\hline C & -0.653551 & 3.242347 & -0.492818 & $\mathrm{H}$ & 1.985989 & 2.034006 & -2.129946 \\
\hline $\mathrm{C}$ & 0.740420 & 3.158119 & -0.400225 & $\mathrm{H}$ & -0.491078 & 1.920205 & 0.776935 \\
\hline $\mathrm{O}$ & -2.819362 & 2.472239 & 0.364718 & $\mathrm{H}$ & 1.489308 & 6.355517 & -1.906754 \\
\hline C & -3.496080 & 3.345434 & -0.539109 & $\mathrm{H}$ & 3.606591 & 6.521946 & -0.566641 \\
\hline $\mathrm{H}$ & -3.060249 & 6.103898 & 1.491989 & $\mathrm{H}$ & 7.445633 & 4.533525 & 2.614680 \\
\hline $\mathrm{H}$ & 5.364563 & 5.762127 & 2.723569 & $\mathrm{H}$ & 7.312192 & 1.006765 & 1.370151 \\
\hline $\mathrm{H}$ & 5.717700 & 2.317466 & 2.059716 & $\mathrm{H}$ & -1.087722 & 3.213494 & -3.736035 \\
\hline $\mathrm{H}$ & 1.045675 & 7.203815 & 0.589953 & $\mathrm{H}$ & 0.549432 & 2.497386 & -4.016307 \\
\hline $\mathrm{H}$ & 1.364649 & 3.816617 & -1.021808 & $\mathrm{H}$ & -0.271850 & 2.263482 & -2.425597 \\
\hline $\mathrm{H}$ & -1.413198 & 7.560201 & 0.279677 & $\mathrm{H}$ & 1.715623 & 2.163283 & 1.881737 \\
\hline
\end{tabular}

A = 2,4-dimethoxyphenyl, $B$ = phenyl

$\begin{array}{llll}\mathrm{H} & -2.233447 & 4.253333 & 2.975109\end{array}$

$\begin{array}{llll}\mathrm{H} & -1.495449 & 0.896946 & 1.866606\end{array}$

$\begin{array}{llll}\mathrm{H} & 1.006356 & 0.748475 & 2.035057\end{array}$

$\begin{array}{llll}\mathrm{H} & -4.576505 & 3.225517 & -0.334848\end{array}$

$\begin{array}{llll}\mathrm{H} & -3.290933 & 3.074085 & -1.598727\end{array}$

$\begin{array}{llll}\mathrm{H} & -3.207649 & 4.406349 & -0.374408\end{array}$

$\begin{array}{llll}\mathrm{H} & 6.065044 & 2.610640 & 0.32725\end{array}$

$\begin{array}{llll}\mathrm{H} & 0.222186 & 3.892889 & 3.282187\end{array}$

$\mathrm{H} \quad 4.970290 \quad 4.138976 \quad 3.365803$

Alkyne_anionA

36

Free energy $=-882.6017937$ Hartree

$\begin{array}{lrrr}\mathrm{C} & 2.012748 & -0.437890 & 0.967722 \\ \mathrm{C} & 2.961554 & 0.511982 & 0.496337 \\ \mathrm{C} & 2.725945 & 1.150984 & -0.750326 \\ \mathrm{C} & 1.582498 & 0.841058 & -1.500687 \\ \mathrm{C} & 0.651042 & -0.102132 & -1.026842 \\ \mathrm{C} & 0.870972 & -0.737825 & 0.210109 \\ \mathrm{C} & 4.137188 & 0.809755 & 1.257043 \\ \mathrm{C} & 5.169117 & 1.034697 & 1.889131 \\ \mathrm{C} & 6.408674 & 1.363010 & 2.607045 \\ \mathrm{O} & 6.732003 & 2.761297 & 2.622937 \\ \mathrm{C} & 6.975685 & 3.295496 & 1.329266 \\ \mathrm{C} & 6.021156 & 4.000897 & 0.681284 \\ \mathrm{C} & 5.122643 & 4.751484 & 0.149308 \\ \mathrm{C} & 3.961087 & 4.572006 & -0.669382 \\ \mathrm{C} & 4.031565 & 4.396702 & -2.090233 \\ \mathrm{C} & 2.888612 & 4.156395 & -2.861383 \\ \mathrm{C} & 1.611258 & 4.089972 & -2.262606 \\ \mathrm{C} & 1.501562 & 4.310754 & -0.873360 \\ \mathrm{C} & 2.648780 & 4.556850 & -0.103456 \\ \mathrm{O} & 0.555086 & 3.775657 & -3.101004 \\ \mathrm{C} & -0.723785 & 3.605920 & -2.510597 \\ \mathrm{H} & -0.247815 & -0.337121 & -1.618342 \\ \mathrm{H} & 6.324274 & 1.044532 & 3.670504 \\ \mathrm{H} & 7.924384 & 2.961674 & 0.864262 \\ \mathrm{H} & 3.435280 & 1.910841 & -1.109573 \\ \mathrm{H} & 2.542790 & 4.701380 & 0.983386 \\ \mathrm{H} & 1.411664 & 1.359227 & -2.456482 \\ \mathrm{H} & 0.525583 & 4.263398 & -0.368865 \\ \mathrm{H} & 0.144322 & -1.474473 & 0.588655 \\ \mathrm{H} & 2.968670 & 3.981723 & -3.946832 \\ \mathrm{H} & 5.019252 & 4.414199 & -2.578732 \\ \mathrm{H} & 7.248438 & 0.773202 & 2.158840 \\ \mathrm{H} & -1.416233 & 3.327860 & -3.328184 \\ \mathrm{H} & -0.722114 & 2.795453 & -1.744354 \\ \mathrm{H} & -1.093643 & 4.542186 & -2.031462 \\ \mathrm{H} & 2.184199 & -0.934507 & 1.934725\end{array}$

Alkyne_anionB

36

Free energy $=-882.6077922$ Hartree

C $\quad 1.580036 \quad 1.404920 \quad 1.094580$

\begin{tabular}{|c|c|c|c|}
\hline $\begin{array}{l}\text { Bis } \\
41 \\
\text { Fre }\end{array}$ & energy $=-99$ & $.5410978 \mathrm{H}$ & Irtree \\
\hline $\mathrm{C}$ & -3.926616 & 1.271634 & $0.40^{\circ}$ \\
\hline $\mathrm{C}$ & -3.0360 & & \\
\hline $\mathrm{C}$ & -3.5 & 3.2 & \\
\hline $\mathrm{C}$ & -4.990 & 3.409 & \\
\hline $\mathrm{C}$ & -5.852127 & 2.497 & \\
\hline $\mathrm{C}$ & -5.318 & & \\
\hline $\mathrm{C}$ & -1.623139 & & \\
\hline $\mathrm{C}$ & -0.40 & & \\
\hline $\mathrm{C}$ & & & \\
\hline $\mathrm{O}$ & & & \\
\hline $\mathrm{C}$ & & & \\
\hline $\mathrm{C}$ & & & \\
\hline $\mathrm{C}$ & & & \\
\hline $\mathrm{C}$ & & & \\
\hline $\mathrm{C}$ & & & \\
\hline $\mathrm{C}$ & & & \\
\hline $\mathrm{C}$ & & & \\
\hline $\mathrm{C}$ & & & \\
\hline C & & & \\
\hline $\mathrm{O}$ & & & \\
\hline $\mathrm{C}$ & -3.19 & & \\
\hline $\mathrm{O}$ & -7.180 & & \\
\hline $\mathrm{C}$ & -8.1 & & \\
\hline $\mathrm{H}$ & & & \\
\hline $\mathrm{H}$ & -0. & & \\
\hline $\mathrm{H}$ & & & -0 . \\
\hline $\mathrm{H}$ & & & \\
\hline $\mathrm{H}$ & & & \\
\hline $\mathrm{H}$ & & & \\
\hline $\mathrm{H}$ & & & 1767 \\
\hline & & & \\
\hline $\mathrm{H}$ & & & \\
\hline $\mathrm{H}$ & -9 . & & \\
\hline 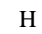 & -8 & & \\
\hline $\mathrm{H}$ & -7.9 & & 717 \\
\hline 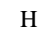 & & & \\
\hline $\mathrm{H}$ & & & -0 . \\
\hline $\mathrm{H}$ & -2.30 & & 49800 \\
\hline $\mathrm{H}$ & -3.7 & & 2.532939 \\
\hline $\mathrm{H}$ & -3 & & \\
\hline $\mathrm{H}$ & 1.538999 & -0.840902 & 2.925348 \\
\hline
\end{tabular}

Alkyne_anionA

40

Free energy $=-997.0199306$ Hartree

$\begin{array}{llll}\text { C } & 2.693772 & 5.645637 & -2.033989 \\ \text { C } & 3.032699 & 5.283207 & -0.695487\end{array}$

$\begin{array}{llll}\text { C } & 3.032699 & 5.283207 & -0.695487\end{array}$

$\begin{array}{llll}\text { C } & 1.980769 & 4.632236 & 0.054779\end{array}$

$\begin{array}{llll}\text { C } & 0.721683 & 4.385325 & -0.503204\end{array}$ 


$\begin{array}{rrrr}\mathrm{C} & 0.431992 & 4.764519 & -1.837488 \\ \mathrm{C} & 1.425321 & 5.400420 & -2.602408 \\ \mathrm{C} & 4.286099 & 5.580131 & -0.094371 \\ \mathrm{C} & 5.512160 & 5.206030 & 0.009866 \\ \mathrm{C} & 6.813998 & 4.884708 & 0.171096 \\ \mathrm{O} & 7.349630 & 4.042467 & 1.192266 \\ \mathrm{C} & 6.424135 & 3.639759 & 2.189784 \\ \mathrm{C} & 5.420807 & 2.674212 & 1.719885 \\ \mathrm{C} & 4.544897 & 1.943278 & 1.262113 \\ \mathrm{C} & 3.443326 & 1.249442 & 0.675117 \\ \mathrm{C} & 2.913887 & 1.708242 & -0.562228 \\ \mathrm{C} & 1.770963 & 1.109570 & -1.110161 \\ \mathrm{C} & 1.141647 & 0.037322 & -0.450407 \\ \mathrm{C} & 1.666897 & -0.435719 & 0.768686 \\ \mathrm{C} & 2.805930 & 0.160862 & 1.329457 \\ \mathrm{O} & 2.333654 & 4.254153 & 1.324094 \\ \mathrm{C} & 1.405522 & 3.506857 & 2.089469 \\ \mathrm{O} & -0.844659 & 4.453714 & -2.280778 \\ \mathrm{C} & -1.167985 & 4.777557 & -3.620341 \\ \mathrm{H} & 0.238481 & -0.423659 & -0.879198 \\ \mathrm{H} & 7.029742 & 3.201207 & 3.012580 \\ \mathrm{H} & 7.615326 & 5.149296 & -0.536969 \\ \mathrm{H} & 3.204154 & -0.195739 & 2.291191 \\ \mathrm{H} & 3.468388 & 6.132762 & -2.648221 \\ \mathrm{H} & 1.178924 & -1.273830 & 1.290742 \\ \mathrm{H} & 1.240511 & 5.702942 & -3.642938 \\ \mathrm{H} & 1.355702 & 1.505147 & -2.049082 \\ \mathrm{H} & -0.060386 & 3.858036 & 0.059194 \\ \mathrm{H} & -2.210786 & 4.444840 & -3.787308 \\ \mathrm{H} & -0.505373 & 4.258173 & -4.351972 \\ \mathrm{H} & -1.105744 & 5.874323 & -3.817082 \\ \mathrm{H} & 5.880629 & 4.527102 & 2.605801 \\ \mathrm{H} & 3.386513 & 2.567147 & -1.057962 \\ \mathrm{H} & 1.915728 & 3.252041 & 3.037181 \\ \mathrm{H} & 0.482160 & 4.089916 & 2.316069 \\ \mathrm{H} & 1.114094 & 2.561323 & 1.578188 \\ & & & \\ & & & \end{array}$

Alkyne_anionB

40

Free energy $=-997.0225749$ Hartree

$\begin{array}{lrrr}\text { C } & 0.137451 & -1.400426 & -1.709506 \\ \text { C } & -0.177355 & -1.665500 & -0.321786\end{array}$

$\begin{array}{llll}\text { C } & -1.345672 & -2.487267 & -0.090023\end{array}$

$\begin{array}{llll}\text { C } & -2.146600 & -2.935794 & -1.142648\end{array}$

$\begin{array}{llll}\text { C } & -1.848933 & -2.617006 & -2.489785\end{array}$

$\begin{array}{llll}\text { C } & -0.686951 & -1.850087 & -2.744623\end{array}$

$\begin{array}{llll}\text { C } & 0.567378 & -1.135669 & 0.732279\end{array}$

$\begin{array}{llll}\text { C } & 1.028285 & -0.480193 & 1.719709\end{array}$

$\begin{array}{llll}\text { C } & 1.440486 & 0.178343 & 2.834690\end{array}$

$\begin{array}{llll}\text { O } & 2.348334 & 1.248558 & 2.903640\end{array}$

$\begin{array}{llll}\text { C } & 2.462957 & 2.138139 & 1.773225\end{array}$

$\begin{array}{llll}\text { C } & 1.177716 & 2.679569 & 1.309013\end{array}$

$\begin{array}{llll}\text { C } & -0.036572 & 2.604087 & 1.078447\end{array}$

$\begin{array}{llll}\text { C } & -1.339009 & 2.263679 & 0.625038\end{array}$

$\begin{array}{llll}\text { C } & -2.549979 & 2.740508 & 1.221502\end{array}$

$\begin{array}{llll}\text { C } & -3.787304 & 2.208207 & 0.824070\end{array}$

$\begin{array}{llll}\text { C } & -3.844879 & 1.189746 & -0.161873\end{array}$

$\begin{array}{llll}\text { C } & -2.667930 & 0.734138 & -0.788872\end{array}$

$\begin{array}{llll}\text { C } & -1.443726 & 1.283275 & -0.391618\end{array}$

$\begin{array}{llll}\text { O } & -2.402793 & 3.696147 & 2.181292\end{array}$

$\begin{array}{llll}\text { C } & -3.564840 & 4.180843 & 2.847010\end{array}$

$\begin{array}{llll}\text { O } & -5.092797 & 0.706598 & -0.433957\end{array}$

$\begin{array}{llll}\text { C } & -5.201611 & -0.368550 & -1.366588\end{array}$

\begin{tabular}{llll}
$\mathrm{H}$ & -2.493541 & -2.963612 & -3.31145 \\
\hline & 0.983489 & -0.012471 & 3.820527
\end{tabular}

$\begin{array}{llll}\mathrm{H} & 0.983489 & -0.012471 & 3.820527\end{array}$

$\begin{array}{llll}\mathrm{H} & 2.927495 & 1.601209 & 0.909048\end{array}$

$\begin{array}{llll}\mathrm{H} & -1.620333 & -2.726696 & 0.949667\end{array}$

$\begin{array}{llll}\mathrm{H} & -0.520447 & 0.914652 & -0.852648\end{array}$

$\begin{array}{llll}\mathrm{H} & -3.039909 & -3.540811 & -0.908875\end{array}$

$\begin{array}{llll}\mathrm{H} & -2.669139 & -0.070653 & -1.537338\end{array}$

$\begin{array}{llll}\mathrm{H} & -0.418742 & -1.595610 & -3.784772\end{array}$

$\begin{array}{llll}\mathrm{H} & -4.732493 & 2.535783 & 1.275082\end{array}$

H $\quad-6.271216 \quad-0.647421 \quad-1.39700$

$\begin{array}{llll}\mathrm{H} & -4.596506 & -1.247836 & -1.051573\end{array}$

$\begin{array}{llll}\mathrm{H} & -4.874507 & -0.064218 & -2.386218\end{array}$

$\begin{array}{llll}\mathrm{H} & 3.177084 & 2.922507 & 2.099951\end{array}$

$\begin{array}{llll}\mathrm{H} & 1.032144 & -0.800353 & -1.941383\end{array}$

$\begin{array}{llll}\mathrm{H} & -3.213010 & 4.931779 & 3.578842\end{array}$

$\begin{array}{llll}\mathrm{H} & -4.272923 & 4.667933 & 2.139254\end{array}$

$\begin{array}{llll}\mathrm{H} & -4.098586 & 3.367671 & 3.387923\end{array}$

\section{A = 2-naphthyl, $B$ = phenyl}

Bispropargyl

39

Free energy $=-922.2066553$ Hartree

$\begin{array}{llll}\mathrm{C} & -3.343943 & 7.316492 & 2.954467 \\ \mathrm{C} & -3.288670 & 7.489925 & 1.539717 \\ \mathrm{C} & -2.177341 & 8.215859 & 0.967564 \\ \mathrm{C} & -1.175983 & 8.747201 & 1.837305\end{array}$ $\begin{array}{llll}\text { C } & -1.260253 & 8.564242 & 3.210767\end{array}$

$\begin{array}{llll}\text { C } & -2.348811 & 7.838301 & 3.773288\end{array}$

$\begin{array}{llll}\text { C } & -4.279581 & 6.950508 & 0.662375\end{array}$

$\begin{array}{llll}\text { C } & -4.175576 & 7.092321 & -0.711023\end{array}$

$\begin{array}{llll}\text { C } & -3.062895 & 7.793694 & -1.289216\end{array}$

$\begin{array}{llll}\mathrm{C} & -2.094154 & 8.354958 & -0.444785\end{array}$

$\begin{array}{llll}\text { C } & -2.879389 & 7.844523 & -2.704614\end{array}$

$\begin{array}{llll}\text { C } & -2.608814 & 7.829076 & -3.903442\end{array}$

$\begin{array}{llll}\text { C } & -2.110486 & 7.703569 & -5.279329\end{array}$

$\begin{array}{llll}C & -0.693509 & 7.508184 & -5.362978\end{array}$

$\begin{array}{lll}-0.233923 & 6.208857 & -4.963959\end{array}$

$\begin{array}{llll}-0.162870 & 5.988661 & -3.511758\end{array}$

$\begin{array}{llll}-0.139498 & 5.817695 & -2.294603\end{array}$

$\begin{array}{llll}\text { C } & -0.192922 & 5.637949 & -0.875331\end{array}$

$\begin{array}{lll}0.838010 & 6.132619 & -0.033576\end{array}$

$\begin{array}{lll}0.746099 & 5.986867 & 1.358058\end{array}$

$\begin{array}{llll}\text { C } & -0.364619 & 5.344765 & 1.934326\end{array}$

$\begin{array}{llll}\text { C } & -1.389867 & 4.849258 & 1.109037\end{array}$

$\begin{array}{llll}\text { C } & -1.308698 & 4.989144 & -0.282715\end{array}$

$\begin{array}{llll}\text { H } & -2.399224 & 7.690055 & 4.863656\end{array}$

$\begin{array}{llll}H & -0.443788 & 5.252487 & 3.028162\end{array}$

$\begin{array}{llll}\mathrm{H} & 0.780487 & 6.110373 & -5.405746\end{array}$

$\begin{array}{llll}\mathrm{H} & -2.654301 & 6.876194 & -5.798885\end{array}$

$\begin{array}{llll}\mathrm{H} & 1.702425 & 6.642867 & -0.484682\end{array}$

$\begin{array}{llll}\mathrm{H} & -1.229845 & 8.876185 & -0.882946\end{array}$

$\begin{array}{llll}\mathrm{H} & 1.543776 & 6.390169 & 2.000637\end{array}$

$\begin{array}{llll}\mathrm{H} & -2.272518 & 4.366972 & 1.555944\end{array}$

$\begin{array}{llll}\mathrm{H} & -5.129140 & 6.399036 & 1.096150\end{array}$

$\begin{array}{llll}\mathrm{H} & -4.933407 & 6.653272 & -1.376612\end{array}$

$\begin{array}{llll}\mathrm{H} & -2.314103 & 8.633048 & -5.853321\end{array}$

$\begin{array}{llll}\mathrm{H} & -2.122194 & 4.625349 & -0.927030\end{array}$

$\begin{array}{llll}\mathrm{H} & -4.186583 & 6.754695 & 3.388697\end{array}$

$\begin{array}{llll}\mathrm{H} & -0.477298 & 8.970261 & 3.869783\end{array}$

$\begin{array}{llll}\mathrm{H} & -0.324680 & 9.289032 & 1.395993\end{array}$

$\begin{array}{llll}\text { H } & -0.867724 & 5.413445 & -5.426715\end{array}$

Alkyne_anionA

Free energy $=-921.692334$ Hartree

$\begin{array}{llll}\text { C } & -0.190928 & -3.066335 & 3.053093\end{array}$

$\begin{array}{llll}\text { C } & 0.293509 & -1.739736 & 2.874756 \\ \text { C } & -0.514993 & -0.806305 & 2.212572\end{array}$

$\begin{array}{lll}-0.514993 & -0.806305 & 2.212572\end{array}$

$\begin{array}{llll}\mathrm{C} & -1.796949 & -1.153182 & 1.716617 \\ \mathrm{C} & -2.301668 & -2.508937 & 1.891963\end{array}$

$\begin{array}{llll}\mathrm{C} & -2.301668 & -2.508937 & 1.891963 \\ \mathrm{C} & -1.445611 & -3.441327 & 2.580710\end{array}$

$\begin{array}{llll}\text { C } & -2.645302 & -0.215383 & 1.030801\end{array}$

$\begin{array}{llll}\text { C } & -3.885449 & -0.571936 & 0.549525\end{array}$

$\begin{array}{llll}\text { C } & -4.411077 & -1.926468 & 0.693982\end{array}$

$\begin{array}{llll}\text { C } & -3.574085 & -2.862911 & 1.391243\end{array}$

$\begin{array}{llll}\text { C } & -5.652759 & -2.221129 & 0.152918\end{array}$

$\begin{array}{llll}\text { C } & -6.736497 & -2.446158 & -0.471653\end{array}$

$\begin{array}{llll}\text { C } & -7.877888 & -2.636769 & -1.174373\end{array}$

$\begin{array}{llll}\text { O } & -8.541088 & -1.639572 & -1.927779\end{array}$

$\begin{array}{llll}\text { C } & -8.009163 & -0.323134 & -1.812443\end{array}$

$\begin{array}{llll}\text { C } & -6.689622 & -0.150026 & -2.437339\end{array}$

$\begin{array}{llll}\text { C } & -5.566269 & -0.083244 & -2.932630\end{array}$

$\begin{array}{llll}\text { C } & -4.213562 & -0.067625 & -3.398636\end{array}$

$\begin{array}{llll}\text { C } & -3.806023 & 0.753183 & -4.483380\end{array}$

$\begin{array}{llll}\text { C } & -2.468344 & 0.755056 & -4.906669\end{array}$

$\begin{array}{llll}\text { C } & -1.517176 & -0.057588 & -4.260417\end{array}$

$\begin{array}{llll}\text { C } & -1.912112 & -0.876710 & -3.185849\end{array}$

$\begin{array}{llll}\text { C } & -3.246263 & -0.887336 & -2.754351\end{array}$

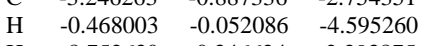

$\begin{array}{llll}\mathrm{H} & -8.752630 & 0.346624 & -2.293875\end{array}$

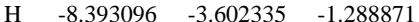

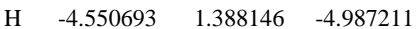

$\begin{array}{llll}\mathrm{H} & -3.930408 & -3.896832 & 1.529159\end{array}$

$\begin{array}{llll}\mathrm{H} & -2.164593 & 1.394853 & -5.750341\end{array}$

$\begin{array}{llll}\mathrm{H} & -1.172590 & -1.510219 & -2.671928\end{array}$

$\begin{array}{llll}\mathrm{H} & -2.280565 & 0.815371 & 0.883948\end{array}$

$\begin{array}{llll}\mathrm{H} & -4.505573 & 0.164862 & 0.014148\end{array}$

$\begin{array}{llll}\mathrm{H} & -7.931547 & -0.027545 & -0.735605\end{array}$

$\begin{array}{llll}\mathrm{H} & -3.559736 & -1.519218 & -1.910715\end{array}$

$\begin{array}{llll}\mathrm{H} & -1.804184 & -4.473863 & 2.728052\end{array}$

$\begin{array}{llll}\mathrm{H} & 0.437913 & -3.809786 & 3.571482\end{array}$

$\mathrm{H} \quad 1.288259 \quad-1.454260 \quad 3.250158$

$\begin{array}{lrrr}\mathrm{H} & -0.158055 & 0.227594 & 2.062968\end{array}$

Alkyne_anionB

42

Free energy $=-921.6922116$ Hartree

$\begin{array}{lccc}\mathrm{C} & 0.412215 & 3.508328 & 0.005637 \\ \mathrm{C} & 0.539995 & 2.473618 & 0.974849 \\ \mathrm{C} & -0.553876 & 1.672748 & 1.298954 \\ \mathrm{C} & -1.819154 & 1.878669 & 0.673819 \\ \mathrm{C} & -1.950453 & 2.940864 & -0.300534 \\ \mathrm{C} & -0.806840 & 3.739130 & -0.623289 \\ \mathrm{C} & -2.968450 & 1.073810 & 0.965524 \\ \mathrm{C} & -4.182555 & 1.294704 & 0.332210 \\ \mathrm{C} & -4.321800 & 2.347679 & -0.634354\end{array}$




$\begin{array}{lrrr}\text { C } & -3.207258 & 3.156903 & -0.927584 \\ \text { C } & -5.539453 & 2.604376 & -1.335583 \\ \text { C } & -6.475735 & 2.939095 & -2.062291 \\ \text { C } & -7.503768 & 3.545295 & -2.917788 \\ \text { O } & -7.901095 & 4.849540 & -2.494663 \\ \text { C } & -6.931268 & 5.870515 & -2.613419 \\ \text { C } & -5.666684 & 5.712471 & -3.075174 \\ \text { C } & -4.475243 & 5.559833 & -3.492287 \\ \text { C } & -3.127533 & 5.289490 & -3.711732 \\ \text { C } & -2.091432 & 6.286534 & -3.545270 \\ \text { C } & -0.742460 & 5.972769 & -3.727377 \\ \text { C } & -0.326130 & 4.667181 & -4.084121 \\ \text { C } & -1.321006 & 3.678531 & -4.262038 \\ \text { C } & -2.676705 & 3.964942 & -4.091939 \\ \text { H } & 0.740200 & 4.428519 & -4.215714 \\ \text { H } & -7.307916 & 6.799711 & -2.158922 \\ \text { H } & -7.118129 & 3.581951 & -3.968545 \\ \text { H } & -2.384753 & 7.307104 & -3.250689 \\ \text { H } & -3.313593 & 3.973985 & -1.653629 \\ \text { H } & 0.011776 & 6.764190 & -3.575865 \\ \text { H } & -1.025490 & 2.650755 & -4.533467 \\ \text { H } & -2.873390 & 0.263638 & 1.707121 \\ \text { H } & -5.053585 & 0.662172 & 0.563325 \\ \text { H } & -8.431866 & 2.935515 & -2.921932 \\ \text { H } & -3.431052 & 3.172599 & -4.217198 \\ \text { H } & -0.451758 & 0.864893 & 2.042492 \\ \text { H } & 1.511395 & 2.302126 & 1.464816 \\ \text { H } & 1.288148 & 4.123856 & -0.253705 \\ \text { H } & -0.908995 & 4.521667 & -1.392557 \\ & & & \end{array}$

\section{$A=$ 6-methoxynaphthyl, $B$ = phenyl}

Bispropargyl

43

Free energy $=-1036.627816$ Hartree

$\begin{array}{rrrr}\text { Free } & \text { energy }=-1036.627816 \text { Hartree } \\ \text { C } & 1.496841 & 4.929067 & -0.235021 \\ \text { C } & 0.476017 & 4.924157 & -1.218371 \\ \text { C } & -0.817899 & 5.487264 & -0.899098 \\ \text { C } & -1.013372 & 6.046256 & 0.397762 \\ \text { C } & -0.003195 & 6.043224 & 1.349101 \\ \text { C } & 1.265743 & 5.464213 & 1.037052 \\ \text { C } & -1.861268 & 5.429916 & -1.860456 \\ \text { C } & -1.664387 & 4.831636 & -3.113460 \\ \text { C } & -0.366963 & 4.300932 & -3.435696 \\ \text { C } & 0.665591 & 4.349339 & -2.516303 \\ \text { C } & -2.769810 & 4.662849 & -4.000280 \\ \text { C } & -3.775262 & 4.444929 & -4.674488 \\ \text { C } & -5.042305 & 4.046082 & -5.299100 \\ \text { O } & -5.572248 & 2.807398 & -4.801521 \\ \text { C } & -6.050453 & 2.851732 & -3.452213 \\ \text { C } & -5.007641 & 2.732881 & -2.421527 \\ \text { C } & -4.118481 & 2.642891 & -1.577442 \\ \text { C } & -3.029359 & 2.582243 & -0.651189 \\ \text { C } & -3.158900 & 3.088163 & 0.669019 \\ \text { C } & -2.062620 & 3.071787 & 1.543617 \\ \text { C } & -0.824657 & 2.552691 & 1.124654 \\ \text { C } & -0.688858 & 2.045196 & -0.180072 \\ \text { C } & -1.777367 & 2.052056 & -1.061762 \\ \text { O } & 2.300428 & 5.392248 & 1.921386 \\ \text { C } & 2.123318 & 5.903419 & 3.242183 \\ \text { H } & 0.040020 & 2.564243 & 1.805103 \\ \text { H } & -6.758234 & 1.998694 & -3.361397 \\ \text { H } & -5.791905 & 4.866303 & -5.172487 \\ \text { H } & -1.663815 & 1.679788 & -2.089874 \\ \text { H } & -2.854776 & 5.821741 & -1.596924 \\ \text { H } & 0.282567 & 1.657751 & -0.522105 \\ \text { H } & -2.170840 & 3.485464 & 2.557671 \\ \text { H } & 1.648545 & 3.921470 & -2.768596 \\ \text { H } & -0.214404 & 3.833781 & -4.419874 \\ \text { H } & -4.910302 & 3.890046 & -6.390569 \\ \text { H } & -4.122401 & 3.509476 & 0.992809 \\ \text { H } & 2.481314 & 4.488882 & -0.453160 \\ \text { H } & -0.196020 & 6.469944 & 2.341996 \\ \text { H } & -1.996955 & 6.471504 & 0.650049 \\ \text { H } & 3.079092 & 5.728005 & 3.770429 \\ \text { H } & 1.907096 & 6.994384 & 3.238236 \\ \text { H } & 1.308997 & 5.373730 & 3.784075 \\ \text { H } & -6.645076 & 3.781682 & -3.273928 \\ & & & \end{array}$

Alkyne_anionA

42

Free energy $=-1036.107249$ Hartree

$\begin{array}{lrrr}\mathrm{C} & -4.994476 & -1.352297 & 0.711606 \\ \mathrm{C} & -5.800703 & -0.410117 & 0.014876 \\ \mathrm{C} & -5.250134 & 0.272678 & -1.102122 \\ \mathrm{C} & -3.930072 & 0.021364 & -1.505474 \\ \mathrm{C} & -3.138933 & -0.911956 & -0.807991 \\ \mathrm{C} & -3.677189 & -1.596190 & 0.298451 \\ \mathrm{C} & -7.136112 & -0.167002 & 0.466667 \\ \mathrm{C} & -8.252979 & -0.018258 & 0.958899\end{array}$

$\begin{array}{cccc}\text { C } & -9.573059 & 0.063608 & 1.602251 \\ \text { O } & -10.291803 & -1.164707 & 1.614157 \\ \text { C } & -9.771522 & -2.171462 & 2.466608 \\ \text { C } & -8.592556 & -2.094112 & 3.130396 \\ \text { C } & -7.473641 & -1.982626 & 3.721976 \\ \text { C } & -6.185797 & -1.838626 & 4.222168 \\ \text { C } & -5.461821 & -0.602396 & 3.942669 \\ \text { C } & -4.173873 & -0.390363 & 4.381865 \\ \text { C } & -3.463890 & -1.375817 & 5.153520 \\ \text { C } & -4.162255 & -2.615838 & 5.457533 \\ \text { C } & -5.486399 & -2.820447 & 4.998363 \\ \text { C } & -3.438666 & -3.589382 & 6.224317 \\ \text { C } & -2.130817 & -3.371848 & 6.662520 \\ \text { C } & -1.464851 & -2.151775 & 6.357100 \\ \mathrm{C} & -2.139585 & -1.174290 & 5.609379 \\ \text { O } & -0.174257 & -1.848366 & 6.748686 \\ \mathrm{C} & 0.537354 & -2.809670 & 7.510665 \\ \text { H } & -2.102874 & -1.105163 & -1.126656 \\ \text { H } & -10.219301 & 0.788418 & 1.062871 \\ \text { H } & -10.429633 & -3.053443 & 2.453178 \\ \text { H } & -5.869720 & 1.001962 & -1.646329 \\ \text { H } & -5.988889 & -3.772274 & 5.236184 \\ \text { H } & -3.514231 & 0.556868 & -2.373268 \\ \text { H } & -3.062564 & -2.322506 & 0.852760 \\ \text { H } & -3.661792 & 0.554583 & 4.133475 \\ \text { H } & -5.971630 & 0.165281 & 3.338534 \\ \text { H } & -9.433342 & 0.444843 & 2.645834 \\ \text { H } & -5.418898 & -1.875640 & 1.580427 \\ \text { H } & -3.935222 & -4.542195 & 6.472571 \\ \text { H } & -1.626437 & -4.157662 & 7.243735 \\ \text { H } & -1.615023 & -0.232952 & 5.376978 \\ \text { H } & 1.530957 & -2.371494 & 7.724611 \\ \text { H } & 0.033732 & -3.039894 & 8.478194 \\ \text { H } & 0.680828 & -3.766129 & 6.956108\end{array}$

\begin{tabular}{lrrr} 
Alkyne_anionB & & \\
42 & & & \\
Free & energy $=-1036.109406$ & Hartree \\
C & -3.938410 & 3.945512 & 0.074172 \\
C & -3.836360 & 2.567316 & -0.366606 \\
C & -3.203173 & 1.653278 & 0.561045 \\
C & -2.707990 & 2.090875 & 1.791967 \\
C & -2.802497 & 3.446435 & 2.191148 \\
C & -3.427553 & 4.356147 & 1.306675 \\
C & -4.297974 & 2.173700 & -1.619690 \\
C & -4.480830 & 1.911102 & -2.851154 \\
C & -4.662457 & 1.641452 & -4.166542 \\
O & -4.998957 & 2.587095 & -5.164116 \\
C & -5.187449 & 3.929927 & -4.718841 \\
C & -3.955438 & 4.575791 & -4.247528 \\
C & -2.888473 & 4.971216 & -3.776949 \\
C & -1.709318 & 5.316766 & -3.048363 \\
C & -0.842445 & 6.396551 & -3.431554 \\
C & 0.272282 & 6.716847 & -2.672163 \\
C & 0.594025 & 5.989201 & -1.478371 \\
C & -0.275902 & 4.899973 & -1.086619 \\
C & -1.404287 & 4.581110 & -1.888503 \\
C & 0.016522 & 4.187576 & 0.115218 \\
C & 1.124225 & 4.509660 & 0.892619 \\
C & 1.990433 & 5.573248 & 0.495272 \\
C & 1.719969 & 6.299845 & -0.671655 \\
O & 3.099626 & 5.951706 & 1.200742 \\
C & 3.429663 & 5.248930 & 2.395305 \\
H & -2.397540 & 3.782755 & 3.157294 \\
H & -4.443926 & 0.667867 & -4.631686 \\
H & -5.944018 & 3.959741 & -3.893278 \\
H & -3.099379 & 0.595281 & 0.270674 \\
H & -2.052935 & 3.744090 & -1.597629 \\
H & -2.223091 & 1.358315 & 2.460084 \\
H & -3.507097 & 5.420866 & 1.584952 \\
H & 0.928057 & 7.548069 & -2.978442 \\
H & -1.076525 & 6.971952 & -4.340470 \\
H & -5.614862 & 4.476401 & -5.586081 \\
H & -4.399584 & 4.677119 & -0.607437 \\
H & 2.396491 & 7.122306 & -0.951552 \\
H & 1.312021 & 3.946143 & 1.816352 \\
H & -0.667707 & 3.384890 & 0.433716 \\
H & 4.353427 & 5.716546 & 2.783304 \\
H & 2.629484 & 5.337474 & 3.164398 \\
H & 3.626104 & 4.170657 & 2.202053 \\
& & & \\
\hline
\end{tabular}

\section{$A$ = 2-pyridyl, B = phenyl}

Bispropargyl

Free energy $=-784.7416599$ Hartree

$\begin{array}{llll}\text { C } & 2.730950 & 0.912747 & -1.230825\end{array}$

$\begin{array}{llll}\text { C } & 2.801248 & 0.771124 & 0.181766\end{array}$

$\begin{array}{llll}\text { C } & 1.671385 & 0.271789 & 0.879832\end{array}$

$\begin{array}{llll}\text { C } & 0.503362 & -0.067624 & 0.180892\end{array}$ 


$\begin{array}{rrrr}\text { C } & 0.440969 & 0.083160 & -1.216277 \\ \mathrm{C} & 1.558199 & 0.573729 & -1.917755 \\ \mathrm{C} & 3.979989 & 1.189028 & 0.875275 \\ \mathrm{C} & 4.987096 & 1.637439 & 1.417975 \\ \mathrm{C} & 6.168682 & 2.291642 & 1.996546 \\ \mathrm{O} & 6.720380 & 3.329704 & 1.176147 \\ \mathrm{C} & 5.989939 & 4.562114 & 1.183797 \\ \mathrm{C} & 4.736217 & 4.533161 & 0.416351 \\ \mathrm{C} & 3.684461 & 4.435945 & -0.209942 \\ \mathrm{C} & 2.424166 & 4.209981 & -0.862601 \\ \mathrm{~N} & 2.358636 & 4.404289 & -2.204974 \\ \mathrm{C} & 1.189241 & 4.142571 & -2.812187 \\ \mathrm{C} & 0.040494 & 3.683581 & -2.141998 \\ \mathrm{C} & 0.110872 & 3.489279 & -0.752696 \\ \mathrm{C} & 1.316990 & 3.759733 & -0.097111 \\ \mathrm{H} & -0.482058 & -0.174622 & -1.758082 \\ \mathrm{H} & -0.883359 & 3.475881 & -2.702347 \\ \mathrm{H} & 6.678620 & 5.317630 & 0.750054 \\ \mathrm{H} & 5.918628 & 2.682768 & 3.013156 \\ \mathrm{H} & 1.429472 & 3.603089 & 0.984958 \\ \mathrm{H} & 3.597935 & 1.317971 & -1.772909 \\ \mathrm{H} & -0.758269 & 3.117974 & -0.189418 \\ \mathrm{H} & 1.509991 & 0.708670 & -3.009152 \\ \mathrm{H} & 1.160981 & 4.306041 & -3.904660 \\ \mathrm{H} & -0.369738 & -0.448802 & 0.733232 \\ \mathrm{H} & 1.717671 & 0.163866 & 1.974001 \\ \mathrm{H} & 6.987927 & 1.552417 & 2.128475 \\ \mathrm{H} & 5.770192 & 4.881931 & 2.231776\end{array}$

Alkyne_anionA

31

Free energy $=-784.235177$ Hartree

N $\quad 1.252383 \quad 3.972700 \quad-0.437613$

$\begin{array}{llll}\text { C } & 2.392391 & 4.694878 & -0.798911\end{array}$

$\begin{array}{llll}\text { C } & 2.316103 & 5.646027 & -1.898094\end{array}$

$\begin{array}{llll}\text { C } & 1.118891 & 5.831764 & -2.573149\end{array}$

$\begin{array}{llll}\text { C } & -0.035923 & 5.092863 & -2.189516\end{array}$

$\begin{array}{llll}\text { C } & 0.120861 & 4.189918 & -1.121754\end{array}$

$\begin{array}{llll}\text { C } & 3.578200 & 4.497887 & -0.108018\end{array}$

$\begin{array}{llll}\text { C } & 4.701752 & 4.300044 & 0.455414\end{array}$

$\begin{array}{llll}\text { C } & 5.910475 & 4.098963 & 1.029322\end{array}$

$\begin{array}{llll}\text { O } & 6.194162 & 3.211357 & 2.093442\end{array}$

$\begin{array}{llll}\text { C } & 5.062073 & 2.662703 & 2.759895\end{array}$

$\begin{array}{llll}\text { C } & 4.308956 & 1.671825 & 1.977939\end{array}$

$\begin{array}{llll}\text { C } & 3.651974 & 0.895031 & 1.288728\end{array}$

$\begin{array}{llll}\text { C } & 2.811938 & 0.111524 & 0.434327\end{array}$

$\begin{array}{llll}\text { C } & 3.012737 & -1.282964 & 0.257201\end{array}$

$\begin{array}{llll}\text { C } & 2.173026 & -2.015154 & -0.596502\end{array}$

$\begin{array}{llll}\text { C } & 1.124089 & -1.373116 & -1.282355 \\ \text { C } & 0.920956 & 0.010121 & -1.111477\end{array}$

$\begin{array}{llll}\text { C } & 0.920956 & 0.010121 & -1.111477\end{array}$

$\begin{array}{llll}\text { C } & 1.752524 & 0.756213 & -0.262640\end{array}$

\begin{tabular}{llll}
$\mathrm{H}$ & 0.467127 & -1.951778 & -1.951165 \\
\hline
\end{tabular}

$\begin{array}{llll}\mathrm{H} & -1.003739 & 5.211644 & -2.698716\end{array}$

$\begin{array}{llll}\mathrm{H} & 6.834493 & 4.602015 & 0.704764\end{array}$

$\begin{array}{llll}\mathrm{H} & 4.355968 & 3.477656 & 3.060069\end{array}$

$\begin{array}{llll}\mathrm{H} & 3.220207 & 6.208036 & -2.181373\end{array}$

$\begin{array}{llll}\mathrm{H} & 1.600109 & 1.848110 & -0.137580\end{array}$

$\begin{array}{llll}\mathrm{H} & 1.068182 & 6.551007 & -3.408817\end{array}$

$\begin{array}{llll}\mathrm{H} & 0.105025 & 0.519560 & -1.648958\end{array}$

$\begin{array}{llll}\mathrm{H} & -0.748464 & 3.582697 & -0.796236\end{array}$

$\begin{array}{llll}\mathrm{H} & 2.339970 & -3.095909 & -0.730702\end{array}$

\begin{tabular}{llll}
$\mathrm{H}$ & 3.835203 & -1.782624 & 0.791755 \\
\hline
\end{tabular}

$\begin{array}{llll}\mathrm{H} & 5.455716 & 2.197228 & 3.688664\end{array}$

Alkyne_anionB

31

Free energy $=-784.2261876$ Hartree

$\begin{array}{llll}\text { C } & -0.090210 & 0.172295 & 2.129324 \\ \text { C } & 0.792393 & 0.974194 & 1.307349\end{array}$

$\begin{array}{llll}\text { C } & 0.792393 & 0.974194 & 1.307349\end{array}$

$\begin{array}{llll}\text { C } & 0.679136 & 0.772957 & -0.124483\end{array}$

$\begin{array}{llll}\text { C } & -0.236165 & -0.133761 & -0.662845 \\ \text { C } & -1.090171 & -0.90458 & 0.160763\end{array}$

$\begin{array}{llll}\text { C } & -1.090171 & -0.904158 & 0.160763\end{array}$

$\begin{array}{llll}\text { C } & -0.994643 & -0.729191 & 1.562505\end{array}$

$\begin{array}{llll}\text { C } & 1.690748 & 1.894632 & 1.835761\end{array}$

$\begin{array}{llll}\text { C } & 2.474364 & 2.800481 & 2.259173\end{array}$

$\begin{array}{llll}\text { C } & 3.316980 & 3.778227 & 2.676730\end{array}$

$\begin{array}{llll}\text { O } & 4.098754 & 4.601561 & 1.822593\end{array}$

$\begin{array}{llll}\text { C } & 4.215368 & 4.156065 & 0.479385\end{array}$

$\begin{array}{llll}\text { C } & 2.988105 & 4.333764 & -0.309562\end{array}$

$\begin{array}{llll}\text { C } & 1.909244 & 4.466044 & -0.885273\end{array}$

$\begin{array}{llll}\text { C } & 0.579636 & 4.443834 & -1.421957\end{array}$

$\begin{array}{llll}\mathrm{N} & 0.343202 & 5.031700 & -2.627144\end{array}$

$\begin{array}{llll}\text { C } & -0.904670 & 4.956199 & -3.117190\end{array}$

$\begin{array}{llll}\text { C } & -1.974093 & 4.314256 & -2.461213\end{array}$

$\begin{array}{llll}\text { C } & -1.726275 & 3.716197 & -1.213438\end{array}$

$\begin{array}{llll}\text { C } & -0.433961 & 3.776044 & -0.679555\end{array}$

$\begin{array}{llll}\mathrm{H} & -1.808109 & -1.616500 & -0.275373\end{array}$

$\begin{array}{llll}\mathrm{H} & -2.972154 & 4.284267 & -2.924264\end{array}$

$\begin{array}{llll}\mathrm{H} & 5.047103 & 4.740182 & 0.030941\end{array}$

$\begin{array}{llll}\mathrm{H} & 3.398965 & 4.132647 & 3.715432\end{array}$

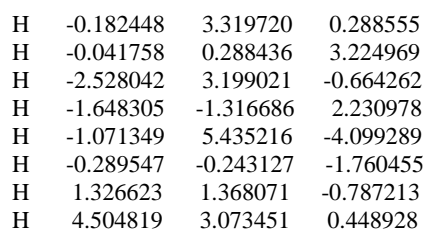

\section{$A$ = 2-pyridyl, $B$ = 4-methoxyphenyl} Bispropargyl

Free energy $=-899.1670172$ Hartree

$\begin{array}{llll}\text { C } & -0.319434 & -2.372961 & -0.215694\end{array}$

$\begin{array}{llll}\text { C } & 0.617549 & -1.611904 & 0.535906\end{array}$

$\begin{array}{llll}\text { C } & 0.120674 & -0.601807 & 1.399117\end{array}$

$\begin{array}{llll}\text { C } & -1.249376 & -0.331436 & 1.493141\end{array}$

$\begin{array}{llll}\text { C } & -2.163625 & -1.071453 & 0.710310\end{array}$

$\begin{array}{llll}\text { C } & -1.686168 & -2.107771 & -0.128475\end{array}$

$\begin{array}{llll}\text { C } & 2.026099 & -1.759964 & 0.364464\end{array}$

$\begin{array}{llll}\text { C } & 3.244273 & -1.752066 & 0.192143\end{array}$

$\begin{array}{llll}\text { C } & 4.658603 & -1.499526 & -0.102319\end{array}$

$\begin{array}{llll}\text { O } & 4.876205 & -0.406866 & -1.012149\end{array}$

$\begin{array}{llll}\text { C } & 4.509278 & 0.879369 & -0.504405\end{array}$

$\begin{array}{llll}\text { C } & 3.082101 & 1.208427 & -0.639512\end{array}$

$\begin{array}{llll}\text { C } & 1.888453 & 1.475329 & -0.754363\end{array}$

$\begin{array}{llll}\text { C } & 0.470649 & 1.667828 & -0.888166\end{array}$

$\begin{array}{llll}\text { C } & -0.275634 & 0.793101 & -1.720197\end{array}$

$\begin{array}{llll}\text { C } & -1.661047 & 0.965155 & -1.805548\end{array}$

$\begin{array}{llll}\text { C } & -2.260604 & 2.006486 & -1.076836\end{array}$

$\begin{array}{llll}\text { C } & -1.435337 & 2.824096 & -0.283859\end{array}$

$\begin{array}{lll}-0.103953 & 2.674322 & -0.179721\end{array}$

\begin{tabular}{llll}
\hline & -3.504347 & -0.851201 & 0.681686
\end{tabular}

$\begin{array}{llll}-4.052425 & 0.152587 & 1.536860\end{array}$

$\begin{array}{llll}\mathrm{H} & -3.346299 & 2.181466 & -1.116722\end{array}$

$\begin{array}{llll}\text { H } & 5.107223 & 1.615122 & -1.084535\end{array}$

$\begin{array}{llll}\mathrm{H} & 5.212569 & -1.321007 & 0.852393\end{array}$

$\begin{array}{llll}\mathrm{H} & 0.235488 & -0.018259 & -2.256867\end{array}$

$\begin{array}{llll}\mathrm{H} & 0.832084 & 0.007535 & 1.975281\end{array}$

$\begin{array}{llll}\mathrm{H} & -2.268141 & 0.287143 & -2.424397\end{array}$

$\begin{array}{llll}\mathrm{H} & -1.589446 & 0.482152 & 2.147395\end{array}$

$\begin{array}{llll}\mathrm{H} & -1.878692 & 3.649476 & 0.302211\end{array}$

$\begin{array}{llll}\mathrm{H} & -2.415077 & -2.678364 & -0.722787\end{array}$

$\begin{array}{llll}\mathrm{H} & 0.043004 & -3.163538 & -0.890196\end{array}$

$\begin{array}{llll}\mathrm{H} & -5.141435 & 0.160861 & 1.345091\end{array}$

$\begin{array}{llll}\mathrm{H} & -3.634256 & 1.156490 & 1.307750\end{array}$

$\begin{array}{llll}\mathrm{H} & -3.870073 & -0.081423 & 2.609318\end{array}$

$\begin{array}{llll}\mathrm{H} & 5.126236 & -2.381006 & -0.588941\end{array}$

$\begin{array}{llll}\mathrm{H} & 4.814587 & 0.990479 & 0.564641\end{array}$

Alkyne_anionA

Free energy $=-898.6569028$ Hartree

$\begin{array}{llll}\text { C } & -0.477634 & 0.741787 & -1.684174\end{array}$

$\begin{array}{llll}\text { C } & 0.397743 & 1.684917 & -1.018667\end{array}$

$\begin{array}{llll}\mathrm{N} & -0.122836 & 2.851500 & -0.474501\end{array}$

$\begin{array}{llll}\text { C } & -1.439536 & 3.070454 & -0.604386\end{array}$

$\begin{array}{llll}\text { C } & -2.354724 & 2.217000 & -1.247512\end{array}$

$\begin{array}{llll}\text { C } & -1.834608 & 1.009880 & -1.785903\end{array}$

$\begin{array}{llll}\text { C } & 1.764218 & 1.425247 & -0.911684\end{array}$

$\begin{array}{llll}\text { C } & 2.964880 & 1.064911 & -0.727028\end{array}$

$\begin{array}{llll}\text { C } & 4.261570 & 0.679300 & -0.570389\end{array}$

$\begin{array}{llll}\text { O } & 4.729478 & -0.549323 & -1.075429\end{array}$

$\begin{array}{llll}\text { C } & 4.704970 & -1.653371 & -0.129380\end{array}$

$\begin{array}{llll}\text { C } & 3.340131 & -1.983282 & 0.288741\end{array}$

$\begin{array}{llll}\text { C } & 2.122083 & -1.936098 & 0.475626\end{array}$

$\begin{array}{llll}\text { C } & 0.727021 & -1.724430 & 0.676130\end{array}$

$\begin{array}{llll}\text { C } & 0.296732 & -0.530484 & 1.313134\end{array}$

$\begin{array}{llll}\text { C } & -1.061902 & -0.210843 & 1.417156\end{array}$

$\begin{array}{lll}-2.030841 & -1.087599 & 0.883597\end{array}$

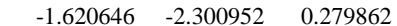

$\begin{array}{lll}-0.264434 & -2.610537 & 0.171128\end{array}$

$\begin{array}{llll}\text { O } & -3.373376 & -0.851893 & 0.894593\end{array}$

$\begin{array}{lll}-3.842784 & 0.363137 & 1.481370\end{array}$

$\begin{array}{llll}\mathrm{H} & -3.421580 & 2.476645 & -1.318781\end{array}$

$\begin{array}{llll}\mathrm{H} & 4.983393 & 1.237326 & 0.057645\end{array}$

$\begin{array}{llll}\mathrm{H} & 5.328094 & -1.406367 & 0.765078\end{array}$

$\begin{array}{llll}\mathrm{H} & -0.054568 & -0.201319 & -2.061804\end{array}$

$\begin{array}{llll}\mathrm{H} & 1.051454 & 0.179918 & 1.676766\end{array}$

$\begin{array}{llll}\mathrm{H} & -2.503873 & 0.276141 & -2.264928\end{array}$

$\begin{array}{llll}\mathrm{H} & -1.349527 & 0.752595 & 1.856966\end{array}$

$\begin{array}{llll}\mathrm{H} & -1.810758 & 4.014502 & -0.155508\end{array}$

$\mathrm{H} \quad-2.392762 \quad-2.972666 \quad-0.124016$

$\begin{array}{llll}\mathrm{H} & 0.047135 & -3.541552 & -0.326450\end{array}$

$\begin{array}{llll}\mathrm{H} & -4.943388 & 0.355598 & 1.367061\end{array}$

$\begin{array}{llll}\mathrm{H} & -3.423730 & 1.249622 & 0.957214\end{array}$

$\begin{array}{llll}\mathrm{H} & -3.588447 & 0.419272 & 2.563539\end{array}$

$\begin{array}{llll}\mathrm{H} & 5.193048 & -2.509033 & -0.645067\end{array}$ 


\begin{tabular}{lrrr}
\multicolumn{5}{c}{ Alkyne_anionB } & & \\
35 & & & \\
Free & energy $=-898.6437165$ & Hartree \\
C & 0.242205 & 0.536312 & -1.538258 \\
C & 1.374241 & 1.216556 & -0.983057 \\
C & 1.090235 & 2.416673 & -0.252634 \\
C & -0.221751 & 2.852962 & -0.043022 \\
C & -1.320961 & 2.136083 & -0.569305 \\
C & -1.071530 & 0.984376 & -1.344423 \\
C & 2.686151 & 0.697773 & -1.177050 \\
C & 3.487088 & -0.215299 & -0.775095 \\
C & 4.341579 & -1.231192 & -0.492072 \\
O & 4.101715 & -2.516531 & -1.039440 \\
C & 3.341977 & -3.395329 & -0.180639 \\
C & 2.013044 & -2.883345 & 0.168748 \\
C & 0.949946 & -2.337218 & 0.462972 \\
C & -0.223633 & -1.623040 & 0.880762 \\
N & -1.443703 & -2.167853 & 0.621951 \\
C & -2.525733 & -1.479941 & 1.033482 \\
C & -2.472206 & -0.251766 & 1.711409 \\
C & -1.206862 & 0.313299 & 1.965336 \\
C & -0.066912 & -0.382887 & 1.555941 \\
O & -2.578888 & 2.619735 & -0.260113 \\
C & -3.693933 & 1.990180 & -0.870717 \\
H & -3.397586 & 0.260949 & 2.012541 \\
H & 3.256172 & -4.364285 & -0.720409 \\
H & 5.119061 & -1.194549 & 0.296264 \\
H & 0.941364 & 0.027250 & 1.703501 \\
H & 0.415212 & -0.395877 & -2.097567 \\
H & -1.112925 & 1.288680 & 2.464058 \\
H & -1.898935 & 0.390155 & -1.756007 \\
H & -3.505550 & -1.940032 & 0.807629 \\
H & -0.426297 & 3.757682 & 0.551768 \\
H & 1.926147 & 2.987571 & 0.181859 \\
H & -4.593818 & 2.532625 & -0.522565 \\
H & -3.786843 & 0.920614 & -0.575607 \\
H & -3.645348 & 2.044713 & -1.984051 \\
H & 3.907277 & -3.595041 & 0.763809 \\
& & & \\
\hline
\end{tabular}

\section{A = 1-benzyl-3-indolyl, $B$ = phenyl}

Bispropargyl
50

Free energy $=-1170.262861$ Hartree

$\begin{array}{cccc}\text { Free energy }=-1170.262861 \text { Hartree } \\ \mathrm{C} & 10.477319 & 3.839316 & -3.101026 \\ \mathrm{C} & 10.135719 & 5.206474 & -3.067359 \\ \mathrm{C} & 11.122490 & 6.167232 & -3.367515 \\ \mathrm{C} & 12.426507 & 5.768796 & -3.710719 \\ \mathrm{C} & 12.759174 & 4.402777 & -3.748088 \\ \mathrm{C} & 11.781216 & 3.439617 & -3.439528 \\ \mathrm{C} & 8.718598 & 5.649449 & -2.734278 \\ \mathrm{~N} & 8.048937 & 4.778698 & -1.781208 \\ \mathrm{C} & 8.415375 & 4.648180 & -0.445643 \\ \mathrm{C} & 7.523753 & 3.713136 & 0.162501 \\ \mathrm{C} & 6.599216 & 3.277921 & -0.873368 \\ \mathrm{C} & 6.970699 & 3.961065 & -2.038257 \\ \mathrm{C} & 9.441237 & 5.286087 & 0.274750 \\ \mathrm{C} & 9.547678 & 4.985246 & 1.639375 \\ \mathrm{C} & 8.656739 & 4.074316 & 2.265498 \\ \mathrm{C} & 7.644463 & 3.433458 & 1.538749 \\ \mathrm{C} & 5.483932 & 2.435071 & -0.666617 \\ \mathrm{C} & 4.508449 & 1.754949 & -0.344083 \\ \mathrm{C} & 3.254929 & 1.210733 & 0.185833 \\ \mathrm{O} & 2.330431 & 2.207530 & 0.657764 \\ \mathrm{C} & 2.775970 & 2.950445 & 1.798335 \\ \mathrm{C} & 3.659932 & 4.084106 & 1.485979 \\ \mathrm{C} & 4.407444 & 5.015933 & 1.196738 \\ \mathrm{C} & 5.317471 & 6.042007 & 0.790937 \\ \mathrm{C} & 5.325255 & 6.490027 & -0.556445 \\ \mathrm{C} & 6.247697 & 7.458761 & -0.974981 \\ \mathrm{C} & 7.173891 & 7.999231 & -0.064117 \\ \mathrm{C} & 7.170713 & 7.564237 & 1.273964 \\ \mathrm{C} & 6.252028 & 6.597341 & 1.703581 \\ \mathrm{H} & 7.901963 & 8.755108 & -0.397889 \\ \mathrm{H} & 2.701233 & 0.650952 & -0.597218 \\ \mathrm{H} & 1.851867 & 3.326734 & 2.288877 \\ \mathrm{H} & 4.614025 & 6.050605 & -1.270873 \\ \mathrm{H} & 6.247035 & 7.790618 & -2.024878 \\ \mathrm{H} & 7.903348 & 7.970662 & 1.987383 \\ \mathrm{H} & 6.265381 & 6.240458 & 2.743284 \\ \mathrm{H} & 8.097550 & 5.678415 & -3.652881 \\ \mathrm{H} & 8.726639 & 6.681863 & -2.324884 \\ \mathrm{H} & 10.868472 & 7.239419 & -3.329229 \\ \mathrm{H} & 13.188207 & 6.529655 & -3.942277 \\ \mathrm{H} & 13.780667 & 4.088595 & -4.012566 \\ \mathrm{H} & 12.036135 & 2.368301 & -3.459386 \\ \mathrm{H} & 9.716670 & 3.083155 & -2.849889 \\ \mathrm{H} & 6.516159 & 3.933352 & -3.035090 \\ \mathrm{H} & 3.279591 & 2.279039 & 2.537087 \\ \mathrm{H} & 6.941816 & 2.737578 & 2.022014\end{array}$

$\begin{array}{cccc}\mathrm{H} & 8.765605 & 3.870556 & 3.342217 \\ \mathrm{H} & 10.333247 & 5.471584 & 2.238036 \\ \mathrm{H} & 10.125343 & 5.996055 & -0.211934 \\ \mathrm{H} & 3.483020 & 0.484387 & 1.005216\end{array}$

$\begin{array}{cccc}\text { Alkyne_anionA } & & \\ \text { 49 } & & & \\ \text { Free } & \text { energy }=-1169.729454 \text { Hartree } \\ \text { C } & 5.021421 & 7.049968 & -1.031615 \\ \text { C } & 5.224152 & 6.223756 & 0.108572 \\ \text { C } & 6.338610 & 6.492670 & 0.948068 \\ \text { C } & 7.203318 & 7.559249 & 0.666306 \\ \text { C } & 6.986992 & 8.376500 & -0.459413 \\ \text { C } & 5.896044 & 8.110260 & -1.310129 \\ \text { C } & 4.331648 & 5.148900 & 0.410543 \\ \text { C } & 3.578749 & 4.223059 & 0.711405 \\ \text { C } & 2.702107 & 3.087731 & 1.050915 \\ \text { O } & 2.437460 & 2.199366 & -0.022215 \\ \text { C } & 3.606564 & 1.542443 & -0.543120 \\ \text { C } & 4.847239 & 1.688195 & -0.055048 \\ \text { C } & 6.019347 & 1.811425 & 0.527019 \\ \text { C } & 6.950603 & 2.876610 & 0.168749 \\ \text { C } & 8.031642 & 3.388807 & 0.999757 \\ \text { C } & 8.690696 & 4.439436 & 0.277912 \\ \text { N } & 8.053496 & 4.565583 & -0.946650 \\ \text { C } & 7.018033 & 3.638240 & -1.006036 \\ \text { C } & 8.477983 & 3.072713 & 2.301179 \\ \text { C } & 9.553076 & 3.788659 & 2.849111 \\ \text { C } & 10.195689 & 4.820987 & 2.112021 \\ \text { C } & 9.776501 & 5.156404 & 0.814903 \\ \text { C } & 8.449502 & 5.475283 & -2.000365 \\ \text { C } & 9.740920 & 5.087475 & -2.708054 \\ \text { C } & 10.465124 & 6.060332 & -3.428546 \\ \text { C } & 11.648224 & 5.717727 & -4.105280 \\ \text { C } & 12.126708 & 4.394923 & -4.063890 \\ \text { C } & 11.411461 & 3.420827 & -3.344238 \\ \text { C } & 10.226462 & 3.764991 & -2.670706 \\ \text { H } & 7.672641 & 9.209578 & -0.679678 \\ \text { H } & 3.326760 & 0.986864 & -1.453637 \\ \text { H } & 1.709345 & 3.462884 & 1.383271 \\ \text { H } & 4.167882 & 6.845532 & -1.695737 \\ \text { H } & 5.725889 & 8.736420 & -2.200369 \\ \text { H } & 8.070413 & 7.734917 & 1.321669 \\ \text { H } & 6.531216 & 5.833874 & 1.806624 \\ \text { H } & 7.615647 & 5.527603 & -2.732229 \\ \text { H } & 8.538724 & 6.499745 & -1.579570 \\ \text { H } & 10.098660 & 7.099898 & -3.455558 \\ \text { H } & 12.204830 & 6.489729 & -4.659779 \\ \text { H } & 13.059223 & 4.126074 & -4.584128 \\ \text { H } & 11.781396 & 2.384163 & -3.300761 \\ \text { H } & 9.671526 & 3.002933 & -2.101562 \\ \text { H } & 6.371076 & 3.589684 & -1.890855 \\ \text { H } & 3.165322 & 2.550435 & 1.916577 \\ \text { H } & 7.970952 & 2.266363 & 2.855729 \\ \text { H } & 9.912526 & 1.547819 & 3.862354 \\ & 10.274619 & 5.952419 & 0.241100\end{array}$

Alkyne_anionB

Free energy $=-1169.748063$ Hartree

$\begin{array}{llll}\text { C } & 4.335499 & 7.789933 & 1.328219\end{array}$

$\begin{array}{llll}\text { C } & 3.500407 & 7.094339 & 0.380237\end{array}$

$\begin{array}{llll}\text { C } & 2.693626 & 7.927889 & -0.478259\end{array}$

$\begin{array}{llll}\text { C } & 2.750989 & 9.322344 & -0.412433\end{array}$

$\begin{array}{llll}\text { C } & 3.594484 & 9.984013 & 0.511008\end{array}$

$\begin{array}{lll}4.378535 & 9.190177 & 1.378345\end{array}$

\begin{tabular}{lll}
3.446558 & 5.685723 & 0.289518 \\
\hline
\end{tabular}

$\begin{array}{lll}4.010646 & 4.648435 & -0.221995\end{array}$

$\begin{array}{lll}4.553666 & 3.553656 & -0.789436\end{array}$

$\begin{array}{lll}4.252545 & 3.076146 & -2.096333\end{array}$

$\begin{array}{lll}3.500180 & 3.976433 & -2.909644\end{array}$

$\begin{array}{lll}4.173172 & 5.255975 & -3.179172\end{array}$

$\begin{array}{lll}4.755160 & 6.336595 & -3.275014\end{array}$

$\begin{array}{lll}5.378073 & 7.610937 & -3.278819\end{array}$

$\begin{array}{lll}5.929361 & 8.358504 & -4.397639\end{array}$

$\begin{array}{lll}6.401057 & 9.605600 & -3.869839\end{array}$

$\begin{array}{llll}6.166794 & 9.602237 & -2.498741\end{array}$

$\begin{array}{lll}5.547872 & 8.420582 & -2.149377\end{array}$

$\begin{array}{lll}6.057647 & 8.104179 & -5.780572\end{array}$

$\begin{array}{llll}6.636230 & 9.086442 & -6.597720\end{array}$

$\begin{array}{lll}7.083565 & 10.321554 & -6.058121\end{array}$

$\begin{array}{llll}6.969948 & 10.599848 & -4.687231\end{array}$

$\begin{array}{llll}6.374470 & 10.728239 & -1.603934\end{array}$

$\begin{array}{llll}5.392505 & 11.871710 & -1.810397\end{array}$

$\begin{array}{lll}4.171607 & 11.680339 & -2.485030\end{array}$

$\begin{array}{llll}3.250660 & 12.734754 & -2.604889\end{array}$

$\begin{array}{llll}\text { C } & 3.539837 & 13.994474 & -2.050534\end{array}$

\begin{tabular}{llll}
\hline & .761121 & 14.194280 & -1.380688
\end{tabular}

$\begin{array}{llll}\text { C } & 5.682677 & 13.139339 & -1.266307\end{array}$ 


$\begin{array}{cccc}\mathrm{H} & 3.651644 & 11.083037 & 0.533727 \\ \mathrm{H} & 2.509753 & 4.195847 & -2.438059 \\ \mathrm{H} & 5.367649 & 2.956460 & -0.348030 \\ \mathrm{H} & 4.973659 & 7.195720 & 2.002358 \\ \mathrm{H} & 5.053377 & 9.678249 & 2.103131 \\ \mathrm{H} & 2.142196 & 9.918180 & -1.112799 \\ \mathrm{H} & 2.055202 & 7.435737 & -1.229174 \\ \mathrm{H} & 6.262809 & 10.344048 & -0.568122 \\ \mathrm{H} & 7.418613 & 11.093018 & -1.707660 \\ \mathrm{H} & 3.935321 & 10.693321 & -2.908913 \\ \mathrm{H} & 2.297987 & 12.568021 & -3.131430 \\ \mathrm{H} & 2.816858 & 14.820431 & -2.142990 \\ \mathrm{H} & 4.998963 & 15.178252 & -0.947009 \\ \mathrm{H} & 6.638563 & 13.299902 & -0.741397 \\ \mathrm{H} & 5.254597 & 8.225795 & -1.112138 \\ \mathrm{H} & 3.300612 & 3.431323 & -3.856999 \\ \mathrm{H} & 5.703300 & 7.149729 & -6.200246 \\ \mathrm{H} & 6.743474 & 8.901121 & -7.678285 \\ \mathrm{H} & 7.522668 & 11.078175 & -6.727067 \\ \mathrm{H} & 7.301219 & 11.561696 & -4.268911\end{array}$

\section{A = 1-benzyl-3-indolyl, $B$ = 4-methoxyphenyl} Bispropargyl

54

Free energy $=-1284.688929$ Hartree

$\begin{array}{llll}\text { C } & 6.714336 & 6.973339 & -1.809593\end{array}$

$\begin{array}{lllr}\text { C } & 7.959290 & 7.170319 & -1.164421 \\ \text { C } & 8.028429 & 6.957877 & 0.240105\end{array}$

0.240105

$\begin{array}{llll}\text { C } & 6.911763 & 6.523307 & 0.952864 \\ \text { C } & 5.687631 & 6.287425 & 0.286202\end{array}$

$\begin{array}{llll}\text { C } & 5.585273 & 6.545589 & -1.097378\end{array}$

$\begin{array}{llll}\text { C } & 9.150585 & 7.475321 & -1.891189\end{array}$

$\begin{array}{llll}\text { C } & 10.228088 & 7.627600 & -2.463623\end{array}$

$\begin{array}{llll}\text { C } & 11.561773 & 7.689786 & -3.079650\end{array}$

$\begin{array}{llll}\text { O } & 12.590344 & 7.042908 & -2.319174\end{array}$

$\begin{array}{llll}\text { C } & 12.621184 & 5.609981 & -2.425638\end{array}$

$\begin{array}{llll}\text { C } & 11.529083 & 4.908298 & -1.743037\end{array}$

$\begin{array}{llll}\text { C } & 10.536148 & 4.446913 & -1.179321\end{array}$

$\begin{array}{llll}\text { C } & 9.306366 & 3.985008 & -0.652916\end{array}$

$\begin{array}{llll}\text { C } & 8.087068 & 3.782529 & -1.419583\end{array}$

$\begin{array}{llll}\text { C } & 7.069213 & 3.412730 & -0.488158\end{array}$

$\begin{array}{llll}\mathrm{N} & 7.635828 & 3.403010 & 0.780876\end{array}$

$\begin{array}{llll}\text { C } & 8.969124 & 3.737742 & 0.683884\end{array}$

$\begin{array}{llll}\text { C } & 7.761232 & 3.918397 & -2.784420\end{array}$

$\begin{array}{llll}\text { C } & 6.442554 & 3.667731 & -3.187378\end{array}$

$\begin{array}{llll}\text { C } & 5.449417 & 3.279127 & -2.250677\end{array}$

$\begin{array}{llll}\text { C } & 5.747681 & 3.142984 & -0.887442\end{array}$

$\begin{array}{llll}\text { C } & 6.886270 & 3.159465 & 2.006842\end{array}$

$\begin{array}{llll}\text { C } & 6.886270 & 3.159465 & 2.006842 \\ \text { C } & 6.356305 & 1.737901 & 2.111789\end{array}$

$\begin{array}{llll}\text { C } & 5.069385 & 1.498847 & 2.633343\end{array}$

$\begin{array}{llll}\text { C } & 4.579551 & 0.185931 & 2.752859\end{array}$

$\begin{array}{llll}\text { C } & 5.371608 & -0.901236 & 2.343332\end{array}$

$\begin{array}{llll}\text { C } & 6.654705 & -0.668492 & 1.813659\end{array}$

$\begin{array}{llll}\text { C } & 7.143360 & 0.643411 & 1.698993\end{array}$

$\begin{array}{llll}\text { O } & 4.678919 & 5.783057 & 1.057419\end{array}$

$\begin{array}{llll}\text { C } & 3.406055 & 5.555095 & 0.452468\end{array}$

$\begin{array}{llll}\mathrm{H} & 12.635767 & 5.306614 & -3.501548\end{array}$

$\mathrm{H} \quad 11.890900 \quad 8.747235 \quad-3.178056$

$\begin{array}{llll}\mathrm{H} & 6.639454 & 7.125606 & -2.896087\end{array}$

$\begin{array}{llll}\mathrm{H} & 4.647771 & 6.368355 & -1.639355\end{array}$

$\begin{array}{llll}\mathrm{H} & 6.971868 & 6.328827 & 2.034095\end{array}$

$\begin{array}{llll}\mathrm{H} & 8.987477 & 7.102163 & 0.758541\end{array}$

$\begin{array}{llll}\mathrm{H} & 7.560214 & 3.386188 & 2.858080\end{array}$

$\begin{array}{llll}\mathrm{H} & 6.044745 & 3.883958 & 2.053357\end{array}$

$\begin{array}{llll}\mathrm{H} & 4.441885 & 2.349496 & 2.945294\end{array}$

$\begin{array}{llll}\mathrm{H} & 3.571200 & 0.012772 & 3.159527\end{array}$

$\begin{array}{llll}\mathrm{H} & 4.987271 & -1.929497 & 2.431139\end{array}$

$\begin{array}{llll}\mathrm{H} & 7.278200 & -1.514509 & 1.485482\end{array}$

$\begin{array}{llll}\mathrm{H} & 8.143631 & 0.824521 & 1.274843\end{array}$

$\begin{array}{llll}\mathrm{H} & 9.591954 & 3.810096 & 1.582363\end{array}$

$\begin{array}{llll}\mathrm{H} & 2.756540 & 5.140295 & 1.245365\end{array}$

$\begin{array}{llll}\mathrm{H} & 2.962006 & 6.501131 & 0.072508\end{array}$

$\begin{array}{llll}\mathrm{H} & 3.475817 & 4.826707 & -0.384429\end{array}$

H $\quad 11.514528 \quad 7.270636 \quad-4.114854$

$\begin{array}{llll}\mathrm{H} & 8.532387 & 4.231556 & -3.504790\end{array}$

$\begin{array}{llll}\mathrm{H} & 6.167379 & 3.776702 & -4.24798\end{array}$

$\begin{array}{llll}\mathrm{H} & 4.424055 & 3.082124 & -2.599404\end{array}$

$\begin{array}{llll}\mathrm{H} & 4.983193 & 2.842087 & -0.157507\end{array}$

$\begin{array}{llll}\mathrm{H} & 13.600702 & 5.311361 & -1.995414\end{array}$

Alkyne_anionA

53

Free energy $=-1284.15485$ Hartree

$\begin{array}{llll}\mathrm{C} & 4.701369 & 7.131447 & 0.614504 \\ \mathrm{C} & 4.910854 & 5.799274 & 0.224333 \\ \mathrm{C} & 6.129466 & 5.484852 & -0.410939 \\ \mathrm{C} & 7.123934 & 6.488638 & -0.681003\end{array}$

$\begin{array}{llll}\text { C } & 6.129466 & 5.484852 & -0.410939 \\ \text { C } & 7.123934 & 6.488638 & -0.681003\end{array}$

\begin{tabular}{|c|c|c|c|}
\hline $\mathrm{C}$ & 6.884684 & 7.818306 & -0.276360 \\
\hline $\mathrm{C}$ & 5.678930 & 8.134249 & 0.370563 \\
\hline $\mathrm{N}$ & 6.622746 & 4.278056 & -0.856338 \\
\hline $\mathrm{C}$ & 7.883611 & 4.489145 & -1.435887 \\
\hline $\mathrm{C}$ & 8.256296 & 5.838941 & -1.335305 \\
\hline $\mathrm{C}$ & 5.929547 & 3.013692 & -0.822996 \\
\hline $\mathrm{C}$ & 5.054055 & 2.721721 & -2.034557 \\
\hline $\mathrm{C}$ & 4.286680 & 1.537504 & -2.062490 \\
\hline $\mathrm{C}$ & 3.471946 & 1.236497 & -3.167012 \\
\hline $\mathrm{C}$ & 3.413097 & 2.121021 & -4.261096 \\
\hline $\mathrm{C}$ & 4.172469 & 3.304572 & -4.237770 \\
\hline $\mathrm{C}$ & 4.988095 & 3.603701 & -3.130816 \\
\hline $\mathrm{C}$ & 9.454733 & 6.470229 & 3121 \\
\hline$=$ & 10.574115 & 85 & 6679 \\
\hline $\mathrm{C}$ & 11.8 & 48 & 5245 \\
\hline $\mathrm{O}$ & 12.9 & & 4683 \\
\hline $\mathrm{C}$ & 13.1 & 80 & 05311 \\
\hline $\mathrm{C}$ & 12. & 2236 & 0.786471 \\
\hline $\mathrm{C}$ & 11.014610 & 8658 & 1.076480 \\
\hline $\mathrm{C}$ & 9.783521 & 3.752919 & 1.425126 \\
\hline $\mathrm{C}$ & 8.657115 & 4.545458 & 1.786984 \\
\hline $\mathrm{C}$ & 7.437619 & 3.953719 & 2.107435 \\
\hline $\mathrm{C}$ & 7.292441 & 2.547152 & 2.059523 \\
\hline $\mathrm{C}$ & 8.394461 & 1.742408 & 1.702561 \\
\hline $\mathrm{C}$ & 9.628619 & 2.345447 & 1.400380 \\
\hline $\mathrm{O}$ & 6.039102 & 2.070587 & 2.336187 \\
\hline $\mathrm{C}$ & 5.796233 & 0.675858 & 2.184402 \\
\hline I & 12.027935 & 46 & -0.540173 \\
\hline $\mathrm{H}$ & 14.083119 & 17 & 8006 \\
\hline H & 10.484085 & 593 & 009 \\
\hline I & 8.30 & & 431 \\
\hline $\mathrm{H}$ & & 4.5 & 2.353091 \\
\hline $\mathrm{H}$ & 8.745270 & 5.64 & 1.768488 \\
\hline $\mathrm{H}$ & 6.689319 & 2.209780 & -0.703738 \\
\hline $\mathrm{H}$ & 5.318766 & 2.969021 & 0.102774 \\
\hline $\mathrm{H}$ & 4.330473 & 0.843041 & -1.206288 \\
\hline $\mathrm{H}$ & 2.880673 & 0.306989 & -3.174899 \\
\hline $\mathrm{H}$ & 2.776915 & 1.887174 & -5.129337 \\
\hline $\mathrm{H}$ & 4.131467 & 4.003168 & -5.088829 \\
\hline $\mathrm{H}$ & 5.584222 & 4.529228 & -3.112758 \\
\hline $\mathrm{H}$ & 8.452395 & 3.636059 & -1.821220 \\
\hline $\mathrm{H}$ & 4.727861 & 0.517357 & 2.421104 \\
\hline $\mathrm{H}$ & 5.993107 & 0.338399 & 1.141433 \\
\hline $\mathrm{H}$ & 6.415831 & 0.067437 & 2.879794 \\
\hline $\mathrm{H}$ & 13.375477 & 6.615336 & 1.064377 \\
\hline $\mathrm{H}$ & 7.648544 & 8.588487 & -0.472117 \\
\hline $\mathrm{H}$ & 18 & 249 & 0.693869 \\
\hline $\mathrm{H}$ & .759282 & 7.403658 & 1.116957 \\
\hline $\mathrm{H}$ & 4.148249 & 5.027403 & 0.411356 \\
\hline
\end{tabular}

\begin{tabular}{lccc}
\multicolumn{5}{l}{ Alkyne_anionB } & \multicolumn{3}{l}{} \\
53 & & & \\
Free energy $=-1284.163849$ & Hartree \\
C & 6.299996 & 6.297130 & 0.924120 \\
C & 4.915888 & 6.409133 & 0.721798 \\
C & 4.340096 & 7.683108 & 0.882106 \\
C & 5.114361 & 8.833088 & 1.248761 \\
C & 6.505285 & 8.691099 & 1.445889 \\
C & 7.085581 & 7.424980 & 1.281141 \\
N & 3.017528 & 8.088946 & 0.743723 \\
C & 2.924773 & 9.434758 & 1.027648 \\
C & 4.188819 & 9.950816 & 1.339103 \\
C & 1.894546 & 7.214276 & 0.442123 \\
C & 1.597069 & 6.208143 & 1.543030 \\
C & 1.787113 & 6.548256 & 2.895568 \\
C & 1.444373 & 5.641822 & 3.910531 \\
C & 0.905270 & 4.384284 & 3.585377 \\
C & 0.719974 & 4.034758 & 2.235399 \\
C & 1.069621 & 4.941840 & 1.220324 \\
C & 4.449523 & 11.307665 & 1.656878 \\
C & 4.571607 & 12.501949 & 1.929911 \\
C & 4.562105 & 13.959576 & 2.125903 \\
O & 4.270485 & 14.703199 & 0.943948 \\
C & 2.993411 & 14.440331 & 0.362378 \\
C & 1.993452 & 13.775004 & 0.972555 \\
C & 1.053811 & 13.178909 & 1.624288 \\
C & 0.592215 & 11.872785 & 1.953164 \\
C & 0.999161 & 11.242134 & 3.182084 \\
C & 0.536210 & 9.981541 & 3.558610 \\
C & -0.374466 & 9.262948 & 2.745589 \\
C & -0.786063 & 9.841798 & 1.526174 \\
C & -0.314653 & 11.113307 & 1.142289 \\
O & -0.798389 & 8.042718 & 3.236484 \\
C & -1.583557 & 7.221609 & 2.394151 \\
H & 3.824694 & 14.198228 & 2.932882 \\
H & 2.981949 & 14.775039 & -0.686960 \\
H & -0.642668 & 11.533205 & 0.178186 \\
H & -1.477416 & 9.308368 & 0.856184 \\
H & 0.874531 & 9.517050 & 4.498406 \\
H & 1.720984 & 11.769402 & 3.825455
\end{tabular}




$\begin{array}{crrr}\mathrm{H} & 1.011007 & 7.870985 & 0.290640 \\ \mathrm{H} & 2.080385 & 6.690324 & -0.519394 \\ \mathrm{H} & 2.185808 & 7.541228 & 3.151549 \\ \mathrm{H} & 1.588251 & 5.925872 & 4.964697 \\ \mathrm{H} & 0.632009 & 3.675353 & 4.382608 \\ \mathrm{H} & 0.305467 & 3.049607 & 1.970111 \\ \mathrm{H} & 0.925522 & 4.666060 & 0.162770 \\ \mathrm{H} & 1.960394 & 9.955250 & 0.990505 \\ \mathrm{H} & -1.723090 & 6.262647 & 2.928707 \\ \mathrm{H} & -2.581126 & 7.670410 & 2.175907 \\ \mathrm{H} & -1.075554 & 7.006280 & 1.426077 \\ \mathrm{H} & 5.555545 & 14.318568 & 2.470203 \\ \mathrm{H} & 7.113965 & 9.564551 & 1.728024 \\ \mathrm{H} & 8.168713 & 7.298061 & 1.436780 \\ \mathrm{H} & 6.784367 & 5.314905 & 0.806773 \\ \mathrm{H} & 4.301267 & 5.535929 & 0.458756\end{array}$

$X=-S-$

\section{A = 4-methoxyphenyl, $B$ = phenyl}

Bispropargyl

37

Free energy $=-1206.091162$ Hartree

\begin{tabular}{|c|c|c|c|}
\hline $\mathrm{C}$ & 0.168535 & 6.774548 & 1.309743 \\
\hline $\mathrm{C}$ & 0.864193 & 5.821538 & 2.099658 \\
\hline C & 0.113064 & 4.894368 & 2.870813 \\
\hline $\mathrm{C}$ & -1.288571 & 4.917608 & 2.840888 \\
\hline $\mathrm{C}$ & -1.966678 & 5.857822 & 2.042324 \\
\hline $\mathrm{C}$ & -1.232577 & 6.788696 & 1.284506 \\
\hline C & 2.294678 & 5.797281 & 2.118802 \\
\hline $\mathrm{C}$ & 3.524157 & 5.787770 & 2.126512 \\
\hline $\mathrm{C}$ & 4.980151 & 5.772808 & 2.130534 \\
\hline & 5.778078 & 6.013437 & 3.792749 \\
\hline $\mathrm{C}$ & 5.687965 & 7.865320 & 3.950124 \\
\hline C & 4.327927 & 8.375654 & 3.950175 \\
\hline $\mathrm{C}$ & 3.136532 & 8.667375 & 3.859373 \\
\hline C & 1.736117 & 8.910271 & 3.737268 \\
\hline C & 1.221347 & 9.850815 & 2.803170 \\
\hline $\mathrm{C}$ & -0.154398 & 10.014365 & 2.639922 \\
\hline $\mathrm{C}$ & -1.063357 & 9.242274 & 3.401676 \\
\hline $\mathrm{C}$ & -0.569586 & 8.320594 & 4.351257 \\
\hline $\mathrm{C}$ & 0.812122 & 8.159405 & 4.507504 \\
\hline $\mathrm{O}$ & -2.382703 & 851 & 3.137630 \\
\hline $\mathrm{C}$ & -3.350019 & 8.728849 & 3.905423 \\
\hline $\mathrm{H}$ & -3.067843 & 5.864841 & 2.010824 \\
\hline $\mathrm{H}$ & 5.363972 & 4.787179 & 1.789607 \\
\hline $\mathrm{H}$ & 6.291363 & 8.3 & 3.136023 \\
\hline $\mathrm{H}$ & 0.644888 & 4.157655 & 3.491785 \\
\hline $\mathrm{H}$ & 1.192471 & 7.414615 & 5.222662 \\
\hline $\mathrm{H}$ & -1.858100 & 4.191673 & 3.442203 \\
\hline $\mathrm{H}$ & -1.250647 & 7.697133 & 4.944616 \\
\hline $\mathrm{H}$ & -1.758247 & 7.545521 & 0.682524 \\
\hline $\mathrm{H}$ & -0.559634 & 10.728091 & 1.906574 \\
\hline $\mathrm{H}$ & 1.918806 & 10.444117 & 2.193076 \\
\hline $\mathrm{H}$ & -4.339498 & 9.037534 & 3.521132 \\
\hline $\mathrm{H}$ & -3.282045 & 8.983616 & 4.986378 \\
\hline $\mathrm{H}$ & -3.236495 & 7.629368 & 3.783670 \\
\hline 11 & 6.218577 & 8.075084 & 4.904641 \\
\hline $\mathrm{H}$ & 0.742520 & 7.514551 & 0.733642 \\
\hline & & 840 & $1.435 / 20$ \\
\hline
\end{tabular}

Alkyne_anionA

36

Free energy $=-1205.58045$ Hartree

$\begin{array}{llll}\text { C } & 3.872969 & 5.268890 & -0.003698 \\ \text { C } & 5.129221 & 4.719136 & -0.415845\end{array}$

$\begin{array}{llll}\text { C } & 5.129221 & 4.719136 & -0.415845\end{array}$

$\begin{array}{llll}\text { C } & 5.155646 & 4.077936 & -1.693437\end{array}$

$\begin{array}{llll}\text { C } & 4.043034 & 4.072023 & -2.544594\end{array}$

$\begin{array}{llll}\text { C } & 2.836381 & 4.681372 & -2.137804\end{array}$

$\begin{array}{llll}\text { C } & 2.759372 & 5.253005 & -0.848657\end{array}$

$\begin{array}{llll}\text { C } & 6.302040 & 4.803999 & 0.371638\end{array}$

$\begin{array}{llll}\text { C } & 7.423583 & 5.155191 & 0.812164\end{array}$

$\begin{array}{llll}\text { C } & 8.690628 & 5.477565 & 1.275286\end{array}$

$\begin{array}{llll}\mathrm{S} & 10.100852 & 5.058123 & 0.341783\end{array}$

$\begin{array}{llll}\text { C } & 10.264261 & 6.399554 & -1.075185\end{array}$

$\begin{array}{llll}\text { C } & 9.100209 & 6.438977 & -1.925580\end{array}$

$\begin{array}{llll}\text { C } & 8.029726 & 6.468724 & -2.538756\end{array}$

$\begin{array}{llll}\text { C } & 6.765435 & 6.539157 & -3.199567\end{array}$

$\begin{array}{llll}\text { C } & 5.663188 & 7.166555 & -2.55493\end{array}$

$\begin{array}{llll}\text { C } & 4.423668 & 7.265386 & -3.198021\end{array}$

$\begin{array}{llll}\text { C } & 4.247755 & 6.744895 & -4.494464\end{array}$

$\begin{array}{llll}\text { C } & 5.325374 & 6.111712 & -5.138985 \\ \text { C } & 6.573619 & 6.011225 & -4.505421\end{array}$

$\begin{array}{llll}\text { C } & 6.573619 & 6.011225 & -4.50542\end{array}$

$\begin{array}{llll}\text { O } & 1.715583 & 4.805419 & -2.931388\end{array}$

$\begin{array}{cccc}\mathrm{C} & 1.763527 & 4.239891 & -4.232288 \\ \mathrm{H} & 3.267444 & 6.818428 & -4.991234 \\ \mathrm{H} & 11.190996 & 6.115354 & -1.619036 \\ \mathrm{H} & 8.812870 & 6.179078 & 2.122535 \\ \mathrm{H} & 5.793944 & 7.543533 & -1.531302 \\ \mathrm{H} & 6.100738 & 3.632605 & -2.039076 \\ \mathrm{H} & 3.578023 & 7.731120 & -2.671296 \\ \mathrm{H} & 4.143799 & 3.627841 & -3.544923 \\ \mathrm{H} & 5.193865 & 5.689467 & -6.147900 \\ \mathrm{H} & 1.809713 & 5.716905 & -0.538212 \\ \mathrm{H} & 3.795925 & 5.744498 & 0.986428 \\ \mathrm{H} & 10.434230 & 7.372320 & -0.568007 \\ \mathrm{H} & 0.779584 & 4.439462 & -4.699361 \\ \mathrm{H} & 2.560888 & 4.703431 & -4.858017 \\ \mathrm{H} & 1.932916 & 3.138314 & -4.204253 \\ \mathrm{H} & 7.416154 & 5.516773 & -5.013114\end{array}$

\begin{tabular}{lrrr}
\multicolumn{5}{l}{ Alkyne_anionB } & & \\
36 & & & \\
Free & energy $=-1205.590253$ & Hartree \\
C & 3.532952 & 3.229368 & -2.023648 \\
C & 4.158677 & 4.459388 & -1.673976 \\
C & 3.463199 & 5.663110 & -1.963965 \\
C & 2.211002 & 5.650796 & -2.591162 \\
C & 1.619799 & 4.422772 & -2.954338 \\
C & 2.285784 & 3.211546 & -2.648983 \\
C & 5.470658 & 4.520456 & -1.124881 \\
C & 6.630991 & 4.664547 & -0.728026 \\
C & 8.014616 & 5.002089 & -0.520038 \\
S & 8.795436 & 5.698127 & -2.186603 \\
C & 7.771279 & 7.002870 & -2.719216 \\
C & 6.643473 & 6.790888 & -3.493047 \\
C & 5.635430 & 6.604008 & -4.217653 \\
C & 4.479491 & 6.334465 & -4.972867 \\
C & 3.615030 & 7.377982 & -5.446511 \\
C & 2.459634 & 7.087875 & -6.183351 \\
C & 2.105007 & 5.756594 & -6.489323 \\
C & 2.935826 & 4.715420 & -6.021867 \\
C & 4.094141 & 4.987852 & -5.289027 \\
O & 0.423018 & 4.301485 & -3.599385 \\
C & -0.301817 & 5.491005 & -3.903290 \\
H & 1.191579 & 5.533571 & -7.061964 \\
H & 8.148774 & 5.785806 & 0.255240 \\
H & 3.871560 & 8.423896 & -5.213369 \\
H & 3.945204 & 6.622632 & -1.731650 \\
H & 1.819181 & 7.917718 & -6.526134 \\
H & 1.735196 & 6.605909 & -2.847965 \\
H & 2.661486 & 3.666115 & -6.219080 \\
H & 1.807298 & 2.263748 & -2.938105 \\
H & 4.051288 & 2.281897 & -1.811131 \\
H & 8.654968 & 4.131480 & -0.260240 \\
H & 7.975351 & 8.016046 & -2.323596 \\
H & -1.225744 & 5.168287 & -4.418613 \\
H & -0.577751 & 6.048939 & -2.980018 \\
H & 0.280112 & 6.157129 & -4.576671 \\
H & 4.711567 & 4.163217 & -4.902282 \\
& & & \\
\hline
\end{tabular}

$A=$ 2,4-dimethoxyphenyl, $B=$ phenyl

Bispropargyl

Free energy $=-1320.513149$ Hartree

$\begin{array}{lrrr}\mathrm{C} & 0.017634 & 1.023407 & 1.769911 \\ \mathrm{C} & 1.049758 & 1.519999 & 0.941860 \\ \mathrm{C} & 0.675338 & 2.210236 & -0.260676 \\ \mathrm{C} & -0.676293 & 2.352596 & -0.599503 \\ \mathrm{C} & -1.680350 & 1.806780 & 0.230655 \\ \mathrm{C} & -1.337493 & 1.155581 & 1.435585 \\ \mathrm{C} & 2.425887 & 1.294023 & 1.226153 \\ \mathrm{C} & 3.621684 & 1.067032 & 1.405661 \\ \mathrm{C} & 5.031469 & 0.724015 & 1.459946 \\ \mathrm{~S} & 5.486932 & -0.803190 & 0.490386 \\ \mathrm{C} & 5.034295 & -0.261578 & -1.228799 \\ \mathrm{C} & 3.622032 & -0.386792 & -1.558206 \\ \mathrm{C} & 2.422240 & -0.470014 & -1.809466 \\ \mathrm{C} & 1.009925 & -0.520873 & -2.027262 \\ \mathrm{C} & 0.432058 & -0.018628 & -3.222732 \\ \mathrm{C} & -0.961286 & -0.009332 & -3.388484 \\ \mathrm{C} & -1.801548 & -0.510839 & -2.378176 \\ \mathrm{C} & -1.236946 & -1.029707 & -1.198614 \\ \mathrm{C} & 0.152683 & -1.036907 & -1.019651 \\ \mathrm{O} & 1.699865 & 2.666713 & -1.021607 \\ \mathrm{C} & 1.401298 & 3.333451 & -2.245334 \\ \mathrm{O} & -2.952341 & 1.946399 & -0.235073 \\ \mathrm{C} & -4.015096 & 1.326768 & 0.486298 \\ \mathrm{H} & -2.894818 & -0.488276 & -2.504697 \\ \mathrm{H} & 5.658651 & -0.896429 & -1.893515 \\ \mathrm{H} & 5.663267 & 1.562993 & 1.095177 \\ \mathrm{H} & 0.592331 & -1.411483 & -0.084268 \\ \mathrm{H} & 0.297999 & 0.495124 & 2.693370\end{array}$




$\begin{array}{rrrr}\mathrm{H} & -1.886382 & -1.412456 & -0.396969 \\ \mathrm{H} & -2.102716 & 0.731812 & 2.097220 \\ \mathrm{H} & -1.394901 & 0.397042 & -4.315588 \\ \mathrm{H} & -0.993098 & 2.830260 & -1.534171 \\ \mathrm{H} & -4.938579 & 1.531939 & -0.086024 \\ \mathrm{H} & -4.122594 & 1.749648 & 1.509311 \\ \mathrm{H} & -3.868394 & 0.226492 & 0.562050 \\ \mathrm{H} & 5.366566 & 0.488964 & 2.493115 \\ \mathrm{H} & 1.089090 & 0.372804 & -4.014075 \\ \mathrm{H} & 5.385765 & 0.784817 & -1.364707 \\ \mathrm{H} & 2.374654 & 3.589994 & -2.701242 \\ \mathrm{H} & 0.834232 & 2.674289 & -2.937546 \\ \mathrm{H} & 0.820948 & 4.266873 & -2.071964\end{array}$

Alkyne_anionA

40

Free energy $=-1320.002523$ Hartree

$\begin{array}{llll}\text { C } & 2.630890 & -0.063029 & -0.866065\end{array}$

$\begin{array}{llll}\text { C } & 3.280623 & -0.998271 & -0.013702\end{array}$

$\begin{array}{llll}\text { C } & 2.659113 & -2.258608 & 0.210396\end{array}$

$\begin{array}{llll}\text { C } & 1.444021 & -2.571288 & -0.414421\end{array}$

$\begin{array}{llll}\text { C } & 0.824578 & -1.648033 & -1.273311\end{array}$

$\begin{array}{llll}\text { C } & 1.418611 & -0.389457 & -1.487565 \\ \text { C } & 4.558373 & -0.740096 & 0.559198\end{array}$

$\begin{array}{llll}\text { C } & 4.558373 & -0.740096 & 0.559198\end{array}$

$\begin{array}{llll}\text { C } & 5.700803 & -0.643172 & 1.020554\end{array}$

$\begin{array}{llll}\text { C } & 7.073481 & -0.799812 & 1.405110\end{array}$

$\begin{array}{llll}\text { S } & 7.937144 & -2.168344 & 0.216486\end{array}$

$\begin{array}{llll}\text { C } & 6.908635 & -3.560832 & 0.178380\end{array}$

$\begin{array}{llll}\text { C } & 5.834777 & -3.669506 & -0.700369\end{array}$

$\begin{array}{llll}\text { C } & 4.883722 & -3.784657 & -1.506113\end{array}$

$\begin{array}{llll}\text { C } & 3.781908 & -3.768366 & -2.389428\end{array}$

$\begin{array}{llll}\text { C } & 2.752173 & -4.772578 & -2.326804\end{array}$

$\begin{array}{llll}\text { C } & 1.641524 & -4.734118 & -3.178639\end{array}$

$\begin{array}{llll}\text { C } & 1.491041 & -3.683709 & -4.115166\end{array}$

$\begin{array}{llll}\text { C } & 2.472516 & -2.680987 & -4.197977\end{array}$

$\begin{array}{llll}\text { C } & 3.593076 & -2.738148 & -3.349236\end{array}$

$\begin{array}{llll}\text { O } & 2.931127 & -5.721490 & -1.354587\end{array}$

$\begin{array}{llll}\text { C } & 1.928968 & -6.710670 & -1.182462\end{array}$

$\begin{array}{llll}\text { O } & 0.339492 & -3.728964 & -4.874289\end{array}$

$\begin{array}{llll}\text { C } & 0.114611 & -2.673373 & -5.794797\end{array}$

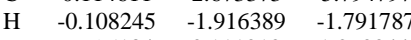

$\begin{array}{llll}\mathrm{H} & 7.695184 & 0.111010 & 1.270044\end{array}$

$\begin{array}{llll}\mathrm{H} & 7.008236 & -4.305920 & 0.992152\end{array}$

$\begin{array}{llll}\mathrm{H} & 3.171588 & -3.001055 & 0.837360\end{array}$

$\begin{array}{llll}\mathrm{H} & 4.344558 & -1.935002 & -3.394941\end{array}$

$\begin{array}{llll}\mathrm{H} & 0.995751 & -3.563809 & -0.257231\end{array}$

$\begin{array}{llll}\mathrm{H} & 2.373955 & -1.838060 & -4.895650\end{array}$

$\begin{array}{llll}\mathrm{H} & 0.940692 & 0.337532 & -2.163398\end{array}$

$\begin{array}{llll}\mathrm{H} & 0.844937 & -5.488430 & -3.124508\end{array}$

$\begin{array}{llll}\mathrm{H} & 7.188630 & -1.167982 & 2.445948\end{array}$

$\begin{array}{llll}\mathrm{H} & -0.854160 & -2.883304 & -6.287270\end{array}$

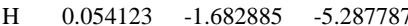

$\begin{array}{llll}\mathrm{H} & 0.910229 & -2.617987 & -6.573958\end{array}$

$\begin{array}{llll}\mathrm{H} & 3.105202 & 0.913514 & -1.049567\end{array}$

$\begin{array}{llll}\mathrm{H} & 2.259508 & -7.350587 & -0.342146\end{array}$

$\begin{array}{llll}\mathrm{H} & 0.941192 & -6.262689 & -0.926253\end{array}$

$\begin{array}{llll}\mathrm{H} & 1.803623 & -7.344055 & -2.091038\end{array}$

Alkyne_anionB

40

Free energy $=-1320.009096$ Hartree

$\begin{array}{llll}\text { C } & 2.955466 & -0.595427 & -0.574786\end{array}$

$\begin{array}{llll}\text { C } & 2.995395 & -0.236444 & 0.815241\end{array}$

$\begin{array}{llll}\text { C } & 1.729357 & -0.065774 & 1.470649\end{array}$

$\begin{array}{llll}\text { C } & 0.523220 & -0.236289 & 0.779663\end{array}$

$\begin{array}{llll}\text { C } & 0.508549 & -0.580715 & -0.588883\end{array}$

$\begin{array}{llll}\text { C } & 1.743118 & -0.765770 & -1.248365\end{array}$

$\begin{array}{llll}\text { C } & 4.217978 & -0.081391 & 1.493209\end{array}$

$\begin{array}{llll}\text { C } & 5.332714 & 0.000252 & 2.065376\end{array}$

$\begin{array}{llll}\text { C } & 5.332714 & 0.000252 & 2.065376 \\ \text { C } & 6.559814 & 0.117629 & 2.693599\end{array}$

$\begin{array}{llll}\mathrm{S} & 8.068704 & -0.081019 & 1.843039\end{array}$

$\begin{array}{llll}\text { C } & 8.448027 & -2.007848 & 1.807103\end{array}$

$\begin{array}{llll}\text { C } & 7.335413 & -2.722210 & 1.237679\end{array}$

$\begin{array}{llll}\text { C } & 6.251445 & -3.108284 & 0.790323\end{array}$

$\begin{array}{llll}\text { C } & 4.938543 & -3.449471 & 0.368530\end{array}$

$\begin{array}{llll}\text { C } & 4.646606 & -3.805453 & -0.988644 \\ \text { C } & 3.326836 & -4.048597 & -1.391556\end{array}$

$\begin{array}{llll}\text { C } & 3.326836 & -4.048597 & -1.391556\end{array}$

$\begin{array}{llll}\text { C } & 2.264879 & -3.919324 & -0.464869\end{array}$

$\begin{array}{llll}\text { C } & 2.527948 & -3.587385 & 0.877573\end{array}$

$\begin{array}{llll}\text { C } & 3.852765 & -3.354274 & 1.271514\end{array}$

$\begin{array}{llll}\text { O } & 5.721799 & -3.867677 & -1.826349 \\ \text { C } & 5.495687 & -4.144904 & -3.203582\end{array}$

$\begin{array}{llll}\text { C } & 5.495687 & -4.144904 & -3.203582\end{array}$

$\begin{array}{llll}\text { O } & 1.017170 & -4.129760 & -0.978156\end{array}$

$\begin{array}{llll}\text { C } & -0.098001 & -4.023609 & -0.096339\end{array}$

$\begin{array}{llll}\mathrm{H} & -0.442967 & -0.720099 & -1.124965\end{array}$

$\begin{array}{llll}\mathrm{H} & 8.661909 & -2.318748 & 2.851203\end{array}$

$\begin{array}{llll}\mathrm{H} & 1.719205 & 0.201587 & 2.539155\end{array}$

$\begin{array}{llll}\mathrm{H} & 4.066285 & -3.037595 & 2.301932\end{array}$

$\begin{array}{llll}\mathrm{H} & -0.428731 & -0.097425 & 1.319542\end{array}$

$\begin{array}{llll}\mathrm{H} & 1.716789 & -3.438809 & 1.600205\end{array}$

$\begin{array}{crcc}\mathrm{H} & 1.758687 & -1.074181 & -2.306171 \\ \mathrm{H} & 3.071350 & -4.305530 & -2.427159 \\ \mathrm{H} & 9.384294 & -2.091363 & 1.214056 \\ \mathrm{H} & 6.598849 & 0.232185 & 3.793848 \\ \mathrm{H} & -0.997774 & -4.217157 & -0.709965 \\ \mathrm{H} & -0.046191 & -4.776882 & 0.722288 \\ \mathrm{H} & -0.168764 & -3.005268 & 0.342601 \\ \mathrm{H} & 3.907654 & -0.781321 & -1.094089 \\ \mathrm{H} & 6.486739 & -4.118716 & -3.693505 \\ \mathrm{H} & 5.044131 & -5.151444 & -3.355347 \\ \mathrm{H} & 4.836901 & -3.381471 & -3.675765\end{array}$

\section{A = 2-naphthyl, B = phenyl}

\begin{tabular}{llll}
\multicolumn{4}{l}{ Bispropargyl } \\
39 & & & \\
Free & energy $=-1245.172003$ & Hartree \\
C & -2.204265 & 3.992307 & -4.682830 \\
C & -2.722012 & 5.168183 & -4.069459 \\
C & -1.950349 & 5.814097 & -3.062931 \\
C & -0.699846 & 5.299066 & -2.688382 \\
C & -0.196079 & 4.136024 & -3.298412 \\
C & -0.956903 & 3.480877 & -4.289483 \\
C & -3.998898 & 5.683545 & -4.458057 \\
C & -5.098044 & 6.104725 & -4.808774 \\
C & -6.404111 & 6.571608 & -5.244000 \\
S & -7.821350 & 6.174118 & -4.106357 \\
C & -7.994493 & 4.351432 & -4.383189 \\
C & -6.919651 & 3.555749 & -3.809399 \\
C & -5.928747 & 2.967567 & -3.372647 \\
C & -4.701294 & 2.392679 & -2.921364 \\
C & -4.109696 & 1.291146 & -3.625544 \\
C & -2.882935 & 0.781787 & -3.234950 \\
C & -2.167122 & 1.336766 & -2.130371 \\
C & -2.757929 & 2.439205 & -1.409267 \\
C & -4.022476 & 2.941848 & -1.824032 \\
C & -2.040566 & 3.008804 & -0.313740 \\
C & -0.795457 & 2.518769 & 0.050209 \\
C & -0.212054 & 1.434294 & -0.659004 \\
C & -0.885037 & 0.856725 & -1.728187 \\
H & 0.776122 & 3.724756 & -2.984123 \\
H & -6.432421 & 7.679244 & -5.309175 \\
H & -8.099478 & 4.163741 & -5.465374 \\
H & -2.345964 & 6.722351 & -2.579292 \\
H & -4.459847 & 3.797729 & -1.292709 \\
H & -0.116336 & 5.802784 & -1.893643 \\
H & -0.578331 & 2.557246 & -4.755169 \\
H & -2.434362 & -0.056131 & -3.787228 \\
H & -4.641482 & 0.867172 & -4.489407 \\
H & -8.967200 & 4.109023 & -3.922827 \\
H & -2.806605 & 3.473406 & -5.444089 \\
H & -0.434117 & 0.018657 & -2.281305 \\
H & 0.780007 & 1.059857 & -0.364385 \\
H & -0.240704 & 2.977994 & 0.905083 \\
H & -2.480315 & 3.863952 & 0.229066 \\
H & -6.652242 & 6.182931 & -6.251168
\end{tabular}

Alkyne_anionA

38

Free energy $=-1244.671789$ Hartree

$\begin{array}{llll}\text { C } & -5.407120 & -0.068641 & -5.235096\end{array}$

$\begin{array}{llll}\text { C } & -4.732485 & 0.281220 & -4.033103\end{array}$

$\begin{array}{llll}\text { C } & -3.312034 & 0.333655 & -4.040757\end{array}$

$\begin{array}{llll}\text { C } & -2.597971 & 0.061306 & -5.216276\end{array}$

$\begin{array}{llll}\text { C } & -3.279999 & -0.267596 & -6.403053\end{array}$

$\begin{array}{llll}\text { C } & -4.683956 & -0.337854 & -6.404070\end{array}$

$\begin{array}{llll}\text { C } & -5.492019 & 0.615383 & -2.871781\end{array}$

$\begin{array}{llll}\text { C } & -6.210165 & 0.939204 & -1.923211\end{array}$

$\begin{array}{llll}\text { C } & -7.161568 & 1.445148 & -0.963380\end{array}$

$\begin{array}{llll}\text { S } & -7.958767 & 3.103504 & -1.585979\end{array}$

$\begin{array}{llll}\text { C } & -8.630079 & 2.751627 & -3.163117\end{array}$

$\begin{array}{llll}\text { C } & -7.851567 & 2.800659 & -4.300400\end{array}$

$\begin{array}{llll}\text { C } & -7.133983 & 2.873230 & -5.329825\end{array}$

$\begin{array}{llll}\text { C } & -6.203332 & 2.784664 & -6.376177\end{array}$

$\begin{array}{llll}\text { C } & -6.528181 & 2.353124 & -7.689178\end{array}$

$\begin{array}{llll}\text { C } & -5.539507 & 2.189330 & -8.692739\end{array}$

$\begin{array}{llll}\text { C } & -4.153346 & 2.497957 & -8.395835\end{array}$

$\begin{array}{llll}\text { C } & -3.840552 & 2.967088 & -7.077174\end{array}$

$\begin{array}{llll}\text { C } & -4.813687 & 3.109743 & -6.111779\end{array}$

$\begin{array}{llll}\text { C } & -5.846597 & 1.699773 & -10.006150\end{array}$

$\begin{array}{llll}\text { C } & -4.851590 & 1.518644 & -10.961902\end{array}$

$\begin{array}{llll}\text { C } & -3.493709 & 1.820927 & -10.662302\end{array}$

$\begin{array}{llll}\text { C } & -3.162269 & 2.304827 & -9.394931\end{array}$

$\begin{array}{llll}\text { H } & -2.720591 & -0.444826 & -7.334188\end{array}$

$\begin{array}{llll}\mathrm{H} & -6.702430 & 1.706490 & 0.014518\end{array}$

$\begin{array}{llll}\mathrm{H} & -9.657708 & 2.345976 & -3.210217\end{array}$

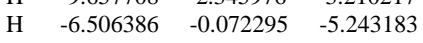

$\begin{array}{llll}\mathrm{H} & -7.572389 & 2.090901 & -7.924437\end{array}$

$\begin{array}{llll}\mathrm{H} & -5.222520 & -0.560281 & -7.337297\end{array}$

$\begin{array}{llll}\text { H } & -1.497700 & 0.122602 & -5.211387\end{array}$ 


$\begin{array}{cccc}\mathrm{H} & -2.789413 & 3.191569 & -6.832351 \\ \mathrm{H} & -4.544642 & 3.437490 & -5.096326 \\ \mathrm{H} & -7.989125 & 0.727100 & -0.782460 \\ \mathrm{H} & -2.780438 & 0.607956 & -3.116455 \\ \mathrm{H} & -6.895172 & 1.456423 & -10.246509 \\ \mathrm{H} & -5.117137 & 1.133823 & -11.960151 \\ \mathrm{H} & -2.713140 & 1.670538 & -11.424778 \\ \mathrm{H} & -2.113573 & 2.540821 & -9.146964\end{array}$

Alkyne_anionB

Free energy $=-1244.67183$ Hartree

$\begin{array}{llll}\text { C } & -2.466561 & 4.440785 & -5.322336\end{array}$

$\begin{array}{llll}\text { C } & -2.878211 & 5.294650 & -4.247042 \\ \text { C } & -1.967005 & 5.436680 & -3.147444\end{array}$

$\begin{array}{llll}\text { C } & -0.739440 & 4.762327 & -3.130623\end{array}$

$\begin{array}{llll}\text { C } & -0.739440 & 4.762327 & -3.130623 \\ \text { C } & -0.357954 & 3.919822 & -4.196917\end{array}$

$\begin{array}{llll}\text { C } & -1.237561 & 3.771937 & -5.289273\end{array}$

$\begin{array}{llll}\text { C } & -4.107054 & 5.983892 & -4.274777\end{array}$

$\begin{array}{llll}\text { C } & -5.183996 & 6.623237 & -4.282952\end{array}$

$\begin{array}{llll}\text { C } & -6.394678 & 7.301300 & -4.297722\end{array}$

$\begin{array}{llll}\text { S } & -6.928651 & 8.289785 & -2.988850\end{array}$

$\begin{array}{llll}\text { C } & -6.167428 & 10.216172 & -3.247908\end{array}$

$\begin{array}{llll}\text { C } & -5.111752 & 10.159558 & -4.197018\end{array}$

$\begin{array}{llll}\text { C } & -4.305575 & 9.787794 & -5.060873\end{array}$

$\begin{array}{llll}\text { C } & -3.375478 & 9.319531 & -6.026818\end{array}$

$\begin{array}{llll}\text { C } & -2.371505 & 8.400815 & -5.663419\end{array}$

$\begin{array}{llll}\text { C } & -1.462229 & 7.878763 & -6.625478\end{array}$

$\begin{array}{llll}\text { C } & -1.563543 & 8.305192 & -8.005281\end{array}$

$\begin{array}{llll}\text { C } & -2.586706 & 9.244545 & -8.354732\end{array}$

$\begin{array}{llll}\text { C } & -3.466248 & 9.733291 & -7.404314\end{array}$

$\begin{array}{llll}\text { C } & -0.457068 & 6.925029 & -6.272877\end{array}$

$\begin{array}{llll}\text { C } & 0.411515 & 6.418601 & -7.232217\end{array}$

$\begin{array}{llll}\text { C } & 0.318652 & 6.845875 & -8.586502\end{array}$

$\begin{array}{llll}\text { C } & -0.651674 & 7.771702 & -8.962948\end{array}$

$\mathrm{H} \quad 0.611483 \quad 3.398710 \quad-4.181747$

$\begin{array}{llll}\mathrm{H} & -6.981132 & 7.302239 & -5.236727\end{array}$

$\begin{array}{llll}\mathrm{H} & -5.851621 & 10.472370 & -2.216866\end{array}$

$\begin{array}{llll}\mathrm{H} & -3.132884 & 4.327640 & -6.191388\end{array}$

$\begin{array}{llll}\mathrm{H} & -2.299085 & 8.061837 & -4.621112\end{array}$

$\mathrm{H} \quad-0.951769 \quad 3.133736 \quad-6.141509$

$\begin{array}{llll}\mathrm{H} & -0.062394 & 4.899904 & -2.270974\end{array}$

$\begin{array}{llll}\mathrm{H} & -2.669063 & 9.574641 & -9.403259\end{array}$

$\begin{array}{llll}\mathrm{H} & -4.254465 & 10.446995 & -7.690590\end{array}$

$\begin{array}{llll}\mathrm{H} & -7.022426 & 10.851035 & -3.556153\end{array}$

$\begin{array}{llll}\mathrm{H} & -2.248584 & 6.094633 & -2.311190\end{array}$

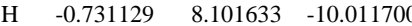

$\begin{array}{llll}\mathrm{H} & 1.012081 & 6.437344 & -9.338417\end{array}$

$\begin{array}{llll}\mathrm{H} & 1.167174 & 5.671630 & -6.942680\end{array}$

$\begin{array}{llll}\mathrm{H} & -0.396973 & 6.580734 & -5.229326\end{array}$

\section{A = 6-methoxynaphthyl, $B=$ phenyl}

Bispropargyl

43

Free energy $=-1359.593524$ Hartree

$\begin{array}{llll}\text { C } & -2.007494 & 1.519145 & -0.966243\end{array}$

$\begin{array}{llll}\text { C } & -3.192744 & 2.233141 & -0.650129\end{array}$

$\begin{array}{llll}\text { C } & -3.250157 & 2.959320 & 0.569200\end{array}$

$\begin{array}{llll}\text { C } & -2.158355 & 2.955414 & 1.447029\end{array}$

$\begin{array}{llll}\text { C } & -0.990063 & 2.239175 & 1.128458\end{array}$

$\begin{array}{llll}\text { C } & -0.920643 & 1.523905 & -0.079790\end{array}$

$\begin{array}{llll}\text { C } & -4.330195 & 2.196474 & -1.517687\end{array}$

$\begin{array}{llll}\text { C } & -5.337818 & 2.153653 & -2.220653\end{array}$

$\begin{array}{llll}\text { C } & -6.544569 & 2.104998 & -3.034536\end{array}$

$\begin{array}{llll}\text { S } & -8.060260 & 2.823816 & -2.237005\end{array}$

$\begin{array}{llll}\text { C } & -8.575319 & 1.410074 & -1.139778\end{array}$

$\begin{array}{llll}\text { C } & -7.610528 & 1.092682 & -0.101080\end{array}$

$\begin{array}{llll}\text { C } & -6.696597 & 0.871373 & 0.691870\end{array}$

$\begin{array}{llll}\text { C } & -5.574242 & 0.651233 & 1.545231\end{array}$

$\begin{array}{llll}\text { C } & -5.464019 & 1.328596 & 2.809760\end{array}$

$\begin{array}{llll}\text { C } & -4.329523 & 1.193557 & 3.590414\end{array}$

$\begin{array}{llll}\text { C } & -3.229979 & 0.380348 & 3.165583\end{array}$

$\begin{array}{llll}\text { C } & -3.344272 & -0.324120 & 1.907077\end{array}$

$\begin{array}{llll}\text { C } & -4.520406 & -0.173143 & 1.124804\end{array}$

$\begin{array}{llll}\text { C } & -2.243501 & -1.119760 & 1.473258\end{array}$

$\begin{array}{llll}\text { C } & -1.077812 & -1.216170 & 2.221052\end{array}$

$\begin{array}{llll}\text { C } & -0.962601 & -0.503452 & 3.454489\end{array}$

$\begin{array}{llll}\text { C } & -2.033187 & 0.269268 & 3.917644\end{array}$

$\begin{array}{llll}\text { O } & 0.154864 & -0.520957 & 4.235595\end{array}$

$\begin{array}{llll}\text { C } & 1.283756 & -1.285587 & 3.815482\end{array}$

$\begin{array}{llll}\mathrm{H} & -0.143739 & 2.220254 & 1.832016\end{array}$

$\begin{array}{llll}\mathrm{H} & -6.420526 & 2.699892 & -3.965306\end{array}$

$\begin{array}{llll}\mathrm{H} & -8.781882 & 0.526596 & -1.781526\end{array}$

$\begin{array}{llll}\mathrm{H} & -4.174635 & 3.494101 & 0.830799\end{array}$

$\begin{array}{llll}\mathrm{H} & -4.583751 & -0.679344 & 0.150226\end{array}$

$\begin{array}{llll}\mathrm{H} & -2.228021 & 3.498948 & 2.40155\end{array}$

$\begin{array}{llll}\mathrm{H} & -0.015455 & 0.948682 & -0.326856\end{array}$

$\begin{array}{llll}\mathrm{H} & -4.253431 & 1.730398 & 4.548709\end{array}$

$\begin{array}{llll}\mathrm{H} & -6.292711 & 1.973038 & 3.139496\end{array}$

$\begin{array}{rrrr}\mathrm{H} & -9.547680 & 1.742557 & -0.717740 \\ \mathrm{H} & -1.957424 & 0.948433 & -1.905510 \\ \mathrm{H} & -1.923820 & 0.809068 & 4.869996 \\ \mathrm{H} & -0.244696 & -1.824939 & 1.845480 \\ \mathrm{H} & -2.318073 & -1.650981 & 0.511972 \\ \mathrm{H} & 2.057637 & -1.145009 & 4.593222 \\ \mathrm{H} & 1.043438 & -2.368983 & 3.735068 \\ \mathrm{H} & 1.684160 & -0.929955 & 2.840290 \\ \mathrm{H} & -6.778055 & 1.065591 & -3.351762\end{array}$

Alkyne_anionA

Free energy $=-1359.085643$ Hartree

$\begin{array}{llll}\text { C } & -2.246249 & -1.055890 & 5.965757\end{array}$

$\begin{array}{llll}\text { C } & -3.549006 & -1.164499 & 5.414651\end{array}$

$\begin{array}{llll}\text { C } & -3.899946 & -2.321272 & 4.612584\end{array}$

$\begin{array}{llll}\text { C } & -2.889729 & -3.314366 & 4.416217\end{array}$

C $\quad-1.614756 \quad-3.189970 \quad 4.962980$

$\begin{array}{llll}\text { C } & -1.281652 & -2.047009 & 5.749910\end{array}$

$\begin{array}{llll}\text { C } & -4.555814 & -0.160951 & 5.612553\end{array}$

$\begin{array}{llll}\text { C } & -5.810878 & -0.279955 & 5.048427\end{array}$

$\begin{array}{llll}\text { C } & -6.173604 & -1.409711 & 4.213052\end{array}$

$\begin{array}{llll}\text { C } & -5.195551 & -2.420301 & 4.039199\end{array}$

$\begin{array}{llll}\text { C } & -7.431691 & -1.435068 & 3.587050\end{array}$

$\begin{array}{llll}\text { C } & -8.503870 & -1.373917 & 2.937622\end{array}$

$\begin{array}{llll}\text { C } & -9.703899 & -1.357274 & 2.248172\end{array}$

$\begin{array}{llll}\mathrm{S} & -10.764930 & 0.014901 & 2.257458\end{array}$

$\begin{array}{llll}\text { C } & -10.252926 & 1.209787 & 0.724097\end{array}$

$\begin{array}{llll}\text { C } & -8.843201 & 1.127561 & 0.486060\end{array}$

$\begin{array}{llll}\text { C } & -7.653046 & 0.801406 & 0.426658\end{array}$

$\begin{array}{llll}\text { C } & -6.288908 & 0.393137 & 0.492786\end{array}$

$\begin{array}{llll}\text { C } & -5.630628 & -0.226694 & -0.604632\end{array}$

$\begin{array}{llll}\text { C } & -4.314757 & -0.694260 & -0.471277\end{array}$

$\begin{array}{llll}\text { C } & -3.630767 & -0.561453 & 0.753833\end{array}$

$\begin{array}{llll}\text { C } & -4.268293 & 0.057504 & 1.843907\end{array}$

$\begin{array}{llll}\text { C } & -5.578503 & 0.537149 & 1.716431\end{array}$

$\begin{array}{llll}\text { O } & -0.049232 & -1.840395 & 6.323768\end{array}$

$\begin{array}{llll}\text { C } & 0.963607 & -2.816776 & 6.124998\end{array}$

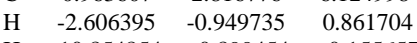

$\begin{array}{llll}\mathrm{H} & -10.854854 & 0.899454 & -0.155657\end{array}$

$\begin{array}{llll}\mathrm{H} & -9.933495 & -2.196443 & 1.563484\end{array}$

$\begin{array}{llll}\mathrm{H} & -6.172644 & -0.350932 & -1.555161\end{array}$

$\begin{array}{llll}\mathrm{H} & -5.431808 & -3.289520 & 3.405441\end{array}$

$\begin{array}{llll}\mathrm{H} & -3.821068 & -1.178159 & -1.329262\end{array}$

$\begin{array}{llll}\mathrm{H} & -3.755612 & 0.141200 & 2.815024\end{array}$

$\begin{array}{llll}\mathrm{H} & -4.310418 & 0.721005 & 6.226942\end{array}$

$\begin{array}{llll}\mathrm{H} & -6.565086 & 0.506776 & 5.209919\end{array}$

$\begin{array}{llll}\mathrm{H} & -10.599103 & 2.205885 & 1.070612\end{array}$

$\begin{array}{llll}\mathrm{H} & -6.087813 & 0.995744 & 2.574946\end{array}$

H $\quad-3.130734 \quad-4.200482 \quad 3.807054$

$\begin{array}{llll}\mathrm{H} & -0.873025 & -3.979591 & 4.776607\end{array}$

$\begin{array}{llll}\mathrm{H} & -1.972992 & -0.177232 & 6.571790\end{array}$

$\begin{array}{llll}\mathrm{H} & 1.860632 & -2.452955 & 6.661497\end{array}$

$\begin{array}{llll}\mathrm{H} & 0.674971 & -3.809399 & 6.539859\end{array}$

$\begin{array}{llll}\mathrm{H} & 1.216908 & -2.943368 & 5.047620\end{array}$

Alkyne_anionB

42

Free energy $=-1359.094426$ Hartree

$\begin{array}{cccc}\text { C } & -4.022451 & 2.120134 & 3.141969 \\ \text { C } & -4.671134 & 3.359939 & 2.822906 \\ \text { C } & -3.820782 & 4.498692 & 2.626056 \\ \text { C } & -2.430372 & 4.401647 & 2.763544 \\ \text { C } & -1.817094 & 3.175761 & 3.091567 \\ \text { C } & -2.634227 & 2.039271 & 3.273724 \\ \text { C } & -6.070244 & 3.453116 & 2.688399 \\ \text { C } & -7.318128 & 3.590196 & 2.641058 \\ \text { C } & -8.686366 & 3.790209 & 2.598972 \\ \text { S } & -9.851991 & 2.576375 & 3.035085 \\ \text { C } & -10.264527 & 1.565001 & 1.380032 \\ \text { C } & -9.054112 & 0.996521 & 0.859426 \\ \text { C } & -7.881396 & 0.691254 & 0.612722 \\ \text { C } & -6.487746 & 0.480640 & 0.439246 \\ \text { C } & -5.656903 & 1.568402 & 0.105084 \\ \text { C } & -4.244553 & 1.432065 & 0.068720 \\ \text { C } & -3.639217 & 0.150654 & 0.360622 \\ \text { C } & -4.503178 & -0.955724 & 0.648107 \\ \text { C } & -5.878260 & -0.797827 & 0.694621 \\ \text { C } & -3.385490 & 2.545061 & -0.170960 \\ \text { C } & -2.004412 & 2.425070 & -0.110377 \\ \text { C } & -1.413231 & 1.162251 & 0.191771 \\ \text { C } & -2.224493 & 0.042758 & 0.400383 \\ \text { O } & -0.062420 & 0.967639 & 0.305107 \\ \text { C } & 0.801325 & 2.093855 & 0.191942 \\ \text { H } & -0.723055 & 3.101125 & 3.192317 \\ \text { H } & -10.741049 & 2.287517 & 0.685665 \\ \text { H } & -4.640274 & 1.215162 & 3.245524 \\ \text { H } & -6.106273 & 2.555569 & -0.071899 \\ \text { H } & -2.173356 & 1.063105 & 3.494285\end{array}$




$\begin{array}{cccc}\mathrm{H} & -1.811152 & 5.299883 & 2.601709 \\ \mathrm{H} & -4.053226 & -1.938037 & 0.865613 \\ \mathrm{H} & -6.525802 & -1.651025 & 0.949238 \\ \mathrm{H} & -11.020865 & 0.815635 & 1.697793 \\ \mathrm{H} & -4.283073 & 5.463552 & 2.363880 \\ \mathrm{H} & -1.747410 & -0.919188 & 0.643832 \\ \mathrm{H} & -1.381380 & 3.316140 & -0.259261 \\ \mathrm{H} & -3.837395 & 3.529423 & -0.365368 \\ \mathrm{H} & 1.827169 & 1.717356 & 0.365317 \\ \mathrm{H} & 0.755897 & 2.555587 & -0.820230 \\ \mathrm{H} & 0.565787 & 2.871121 & 0.952526 \\ \mathrm{H} & -9.068085 & 4.749097 & 2.198378\end{array}$

\section{A = 2-pyridyl, $B$ = phenyl}

\section{Bispropargyl}

Free energy $=-1107.70718$ Hartree

$\begin{array}{llll}\mathrm{N} & 1.371312 & 4.010077 & -0.259786\end{array}$

$\begin{array}{llll}\text { C } & 2.411464 & 4.429668 & -1.026308\end{array}$

$\begin{array}{llll}\text { C } & 2.299445 & 4.629800 & -2.427092\end{array}$

$\begin{array}{llll}\text { C } & 1.065502 & 4.397959 & -3.044407\end{array}$

$\begin{array}{llll}\text { C } & -0.013396 & 3.968610 & -2.254341\end{array}$

$\begin{array}{llll}\text { C } & 0.197657 & 3.790482 & -0.874954\end{array}$

$\begin{array}{llll}\text { C } & 3.659049 & 4.708686 & -0.372891\end{array}$

$\begin{array}{llll}\text { C } & 4.742528 & 5.012601 & 0.119847\end{array}$

$\begin{array}{llll}\text { C } & 6.017582 & 5.450890 & 0.663429\end{array}$

$\begin{array}{llll}\mathrm{S} & 6.071251 & 7.247938 & 1.132401\end{array}$

$\begin{array}{llll}\text { C } & 5.942681 & 8.047636 & -0.539139\end{array}$

$\begin{array}{llll}\text { C } & 4.600329 & 8.046065 & -1.101497\end{array}$

$\begin{array}{llll}\text { C } & 3.453818 & 7.996415 & -1.540941\end{array}$

$\begin{array}{llll}\text { C } & 2.107086 & 7.869412 & -2.005781\end{array}$

$\begin{array}{llll}\text { C } & 1.774418 & 8.090369 & -3.367421\end{array}$

$\begin{array}{llll}\text { C } & 0.455165 & 7.911971 & -3.810976\end{array}$

$\begin{array}{llll}\text { C } & -0.550341 & 7.516241 & -2.910690\end{array}$

$\begin{array}{llll}\text { C } & -0.230910 & 7.298471 & -1.557264\end{array}$

$\begin{array}{llll}\text { C } & 1.083031 & 7.472897 & -1.103512\end{array}$

$\mathrm{H} \quad-1.582064 \quad 7.368336 \quad-3.264866$

$\begin{array}{llll}\mathrm{H} & -1.005361 & 3.783209 & -2.691996\end{array}$

$\begin{array}{llll}\mathrm{H} & 6.259314 & 4.920258 & 1.609014\end{array}$

$\begin{array}{llll}\mathrm{H} & 6.671313 & 7.557412 & -1.220742\end{array}$

$\begin{array}{llll}\mathrm{H} & 3.168361 & 4.986865 & -2.997830\end{array}$

$\begin{array}{llll}\mathrm{H} & 1.341030 & 7.278510 & -0.052282\end{array}$

$\begin{array}{llll}\mathrm{H} & 0.942925 & 4.567790 & -4.124722\end{array}$

$\begin{array}{llll}\mathrm{H} & -1.010676 & 6.975606 & -0.850799\end{array}$

$\begin{array}{llll}\mathrm{H} & -0.634261 & 3.455635 & -0.229657\end{array}$

$\begin{array}{llll}\mathrm{H} & 0.210253 & 8.082035 & -4.870883\end{array}$

$\begin{array}{llll}\mathrm{H} & 2.563152 & 8.392360 & -4.072748\end{array}$

$\begin{array}{llll}\mathrm{H} & 6.308228 & 9.083957 & -0.371730\end{array}$

$\begin{array}{llll}\mathrm{H} & 6.845166 & 5.247299 & -0.049494\end{array}$

Alkyne_anionA

31

Free energy $=-1107.211575$ Hartre

$\begin{array}{llll}\text { C } & 7.682903 & -2.975151 & 1.872969\end{array}$

$\begin{array}{llll}\text { C } & 6.908416 & -1.866234 & 1.434548\end{array}$

$\begin{array}{llll}\text { C } & 5.705996 & -2.113998 & 0.717680\end{array}$

$\begin{array}{llll}\text { C } & 5.281775 & -3.428008 & 0.474272\end{array}$

$\begin{array}{llll}\text { C } & 6.053745 & -4.518192 & 0.916642\end{array}$

$\begin{array}{llll}\text { C } & 7.258104 & -4.286069 & 1.610129\end{array}$

$\begin{array}{llll}\text { C } & 7.273151 & -0.523363 & 1.754145\end{array}$

$\begin{array}{llll}\text { C } & 7.503994 & 0.652925 & 2.044899\end{array}$

$\begin{array}{llll}\text { C } & 7.544961 & 1.997859 & 2.554787\end{array}$

$\begin{array}{llll}\mathrm{S} & 5.758545 & 2.603510 & 3.094488\end{array}$

$\begin{array}{llll}\text { C } & 4.963915 & 1.252428 & 3.863049\end{array}$

$\begin{array}{llll}\text { C } & 4.188437 & 0.364599 & 3.150120\end{array}$

$\begin{array}{llll}\text { C } & 3.446245 & -0.425120 & 2.512347\end{array}$

$\begin{array}{llll}\text { C } & 2.739462 & -1.382950 & 1.768961\end{array}$

$\begin{array}{llll}\text { C } & 2.219963 & -2.573597 & 2.390196\end{array}$

$\begin{array}{llll}\text { C } & 1.575702 & -3.533011 & 1.614876\end{array}$

$\begin{array}{llll}\text { C } & 1.427594 & -3.320632 & 0.223174\end{array}$

$\begin{array}{llll}\text { C } & 1.944879 & -2.121311 & -0.300216\end{array}$

$\begin{array}{llll}\mathrm{N} & 2.573868 & -1.174873 & 0.412369\end{array}$

$\begin{array}{llll}\mathrm{H} & 5.716507 & -5.549163 & 0.725266\end{array}$

$\mathrm{H} \quad 0.930936 \quad-4.055246 \quad-0.42835$

$\begin{array}{llll}\mathrm{H} & 5.174609 & 1.057264 & 4.931474\end{array}$

$\begin{array}{llll}\mathrm{H} & 8.224445 & 2.087273 & 3.429315\end{array}$

$\begin{array}{llll}\mathrm{H} & 2.359544 & -2.717818 & 3.472830\end{array}$

$\begin{array}{llll}\mathrm{H} & 5.088791 & -1.267895 & 0.384883\end{array}$

$\begin{array}{llll}\mathrm{H} & 1.191461 & -4.453753 & 2.084309\end{array}$

$\begin{array}{llll}\mathrm{H} & 4.325666 & -3.596373 & -0.046018\end{array}$

$\begin{array}{llll}\mathrm{H} & 1.843810 & -1.915124 & -1.384114\end{array}$

$\begin{array}{llll}\mathrm{H} & 7.867739 & -5.135145 & 1.957359\end{array}$

$\begin{array}{llll}\text { H } & 8.615649 & -2.792888 & 2.428436\end{array}$

$\begin{array}{llll}\mathrm{H} & 7.846490 & 2.751378 & 1.796048\end{array}$

Alkyne_anionB

Free energy $=-1107.206413$ Hartree

N $\quad-0.003277 \quad 3.670837 \quad-1.919508$

$\begin{array}{cccc}\mathrm{C} & 0.827702 & 4.681625 & -1.534254 \\ \mathrm{C} & 0.570724 & 6.041775 & -1.864304 \\ \mathrm{C} & -0.553556 & 6.356763 & -2.635059 \\ \mathrm{C} & -1.406163 & 5.315241 & -3.038902 \\ \mathrm{C} & -1.084847 & 4.000799 & -2.644946 \\ \mathrm{C} & 2.032510 & 4.372940 & -0.831776 \\ \mathrm{C} & 3.137290 & 4.271304 & -0.285035 \\ \mathrm{C} & 4.521586 & 4.226291 & 0.043736 \\ \mathrm{~S} & 5.603602 & 5.159620 & -1.437508 \\ \mathrm{C} & 4.718657 & 5.088852 & -2.918555 \\ \mathrm{C} & 3.814029 & 6.064671 & -3.309001 \\ \mathrm{C} & 3.018732 & 6.946552 & -3.710001 \\ \mathrm{C} & 2.026717 & 7.871399 & -4.091493 \\ \mathrm{C} & 1.731679 & 9.026024 & -3.293037 \\ \mathrm{C} & 0.681171 & 9.889670 & -3.627068 \\ \mathrm{C} & -0.109353 & 9.662893 & -4.774205 \\ \mathrm{C} & 0.184861 & 8.549170 & -5.589802 \\ \mathrm{C} & 1.226204 & 7.672121 & -5.264586 \\ \mathrm{H} & -0.936037 & 10.343847 & -5.028621 \\ \mathrm{H} & -2.299088 & 5.511039 & -3.651571 \\ \mathrm{H} & 4.921497 & 3.196190 & 0.139959 \\ \mathrm{H} & 4.791130 & 4.170981 & -3.533715 \\ \mathrm{H} & 1.277921 & 6.816903 & -1.543022 \\ \mathrm{H} & 1.426039 & 6.793650 & -5.897629 \\ \mathrm{H} & -0.739877 & 7.399438 & -2.936820 \\ \mathrm{H} & -0.417348 & 8.356833 & -6.493248 \\ \mathrm{H} & -1.743229 & 3.163712 & -2.943978 \\ \mathrm{H} & 0.473490 & 10.756911 & -2.978468 \\ \mathrm{H} & 2.334116 & 9.210180 & -2.389470 \\ \mathrm{H} & 4.802494 & 4.828788 & 0.931368\end{array}$

\section{$A$ = 2-pyridyl, B = 4-methoxyphenyl} Bispropargyl

Free energy $=-1222.130703$ Hartree

$\begin{array}{llll}\text { C } & -0.019729 & 2.860624 & -0.233626\end{array}$

$\begin{array}{llll}\text { C } & 0.579614 & 1.846500 & -1.025267\end{array}$

$\begin{array}{llll}\mathrm{N} & -0.132447 & 1.067288 & -1.880390\end{array}$

$\begin{array}{llll}\text { C } & -1.454558 & 1.292136 & -1.969456\end{array}$

$\begin{array}{llll}\text { C } & -2.137637 & 2.278122 & -1.234471\end{array}$

$\begin{array}{llll}\text { C } & -1.396190 & 3.079836 & -0.349845\end{array}$

$\begin{array}{llll}\text { C } & 2.000365 & 1.643915 & -0.952640\end{array}$

$\begin{array}{llll}\text { C } & 3.223814 & 1.563003 & -0.874644\end{array}$

$\begin{array}{llll}C & 4.673562 & 1.479641 & -0.771589\end{array}$

$\begin{array}{llll}\text { S } & 5.448762 & 2.753984 & 0.332395\end{array}$

$\begin{array}{llll}\text { C } & 5.536150 & 4.204723 & -0.834566\end{array}$

$\begin{array}{llll}\text { C } & 4.239151 & 4.647600 & -1.314795\end{array}$

$\begin{array}{llll}\text { C } & 3.091953 & 4.881663 & -1.692352\end{array}$

$\begin{array}{llll}\text { C } & 1.741563 & 5.039396 & -2.124285\end{array}$

$\begin{array}{llll}\text { C } & 1.218804 & 4.188650 & -3.131454\end{array}$

$\begin{array}{llll}\text { C } & -0.129207 & 4.242223 & -3.504914\end{array}$

$\begin{array}{llll}\text { C } & -0.997674 & 5.154356 & -2.864954\end{array}$

$\begin{array}{llll}\text { C } & -0.484168 & 6.031718 & -1.879516\end{array}$

$\begin{array}{llll}\text { C } & 0.861259 & 5.975682 & -1.515184\end{array}$

$\begin{array}{llll}\text { O } & -2.331094 & 5.250409 & -3.113698\end{array}$

$\begin{array}{llll}\text { C } & -2.902013 & 4.411688 & -4.117951\end{array}$

$\begin{array}{llll}\mathrm{H} & -3.221736 & 2.417884 & -1.361478\end{array}$

$\begin{array}{llll}\mathrm{H} & 4.979648 & 0.505359 & -0.333010\end{array}$

$\begin{array}{llll}\mathrm{H} & 6.207702 & 3.934633 & -1.678518\end{array}$

$\begin{array}{llll}\mathrm{H} & 0.605347 & 3.475223 & 0.429263\end{array}$

$\begin{array}{llll}\mathrm{H} & 1.879920 & 3.446018 & -3.601179\end{array}$

$\begin{array}{llll}\mathrm{H} & -1.883666 & 3.876191 & 0.232422\end{array}$

$\begin{array}{llll}\mathrm{H} & -0.499098 & 3.543748 & -4.267043\end{array}$

$\begin{array}{llll}\mathrm{H} & -2.010446 & 0.647456 & -2.674422\end{array}$

$\begin{array}{llll}\mathrm{H} & -1.175002 & 6.738202 & -1.395913\end{array}$

$\begin{array}{llll}\mathrm{H} & 1.244045 & 6.646885 & -0.731358\end{array}$

$\begin{array}{llll}\mathrm{H} & -3.979350 & 4.657552 & -4.150329\end{array}$

$\begin{array}{llll}\mathrm{H} & -2.780409 & 3.334942 & -3.867667\end{array}$

$\mathrm{H} \quad-2.452366 \quad 4.605865 \quad-5.116700$

$\begin{array}{llll}\mathrm{H} & 6.054495 & 4.990597 & -0.243721\end{array}$

$\begin{array}{llll}\mathrm{H} & 5.152636 & 1.540847 & -1.772912\end{array}$

Alkyne_anionA

Free energy $=-1221.635532$ Hartree

$\begin{array}{llll}\mathrm{C} & -0.440231 & -2.889480 & 4.569306 \\ \mathrm{C} & -0.743151 & -3.782999 & 3.480275 \\ \mathrm{~N} & -2.049116 & -3.992889 & 3.088995 \\ \mathrm{C} & -3.016103 & -3.341553 & 3.755267 \\ \mathrm{C} & -2.809771 & -2.457508 & 4.828486 \\ \mathrm{C} & -1.470642 & -2.228830 & 5.226824 \\ \mathrm{C} & 0.298423 & -4.435840 & 2.799386 \\ \mathrm{C} & 1.306229 & -4.870423 & 2.186168 \\ \mathrm{C} & 2.413405 & -5.367039 & 1.533427 \\ \mathrm{~S} & 4.037780 & -4.994471 & 2.065440 \\ \mathrm{C} & 4.505813 & -3.297884 & 1.229158 \\ \mathrm{C} & 3.505572 & -2.299530 & 1.515780 \\ \mathrm{C} & 2.513224 & -1.633448 & 1.822230 \\ \mathrm{C} & 1.299229 & -0.971255 & 2.165204\end{array}$




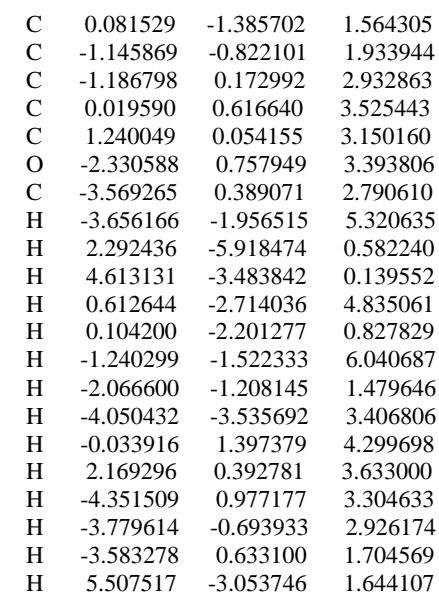

\begin{tabular}{lrrr}
\multicolumn{5}{l}{ Alkyne_anionB } & \\
35 & & & \\
Free energy $=-1221.625564$ & Hartree \\
N & -0.756578 & -0.821497 & 2.164610 \\
C & -0.478750 & -2.134519 & 2.410257 \\
C & -1.477015 & -3.055824 & 2.837831 \\
C & -2.781533 & -2.594244 & 3.039412 \\
C & -3.063185 & -1.237952 & 2.808647 \\
C & -2.017182 & -0.407195 & 2.364755 \\
C & 0.865206 & -2.596977 & 2.273450 \\
C & 1.990213 & -3.107577 & 2.277295 \\
C & 3.234147 & -3.776611 & 2.517943 \\
S & 3.460491 & -4.061840 & 4.483235 \\
C & 2.047409 & -4.879192 & 5.062397 \\
C & 0.897919 & -4.187013 & 5.427500 \\
C & -0.121679 & -3.551604 & 5.780915 \\
C & -1.266878 & -2.762269 & 6.033494 \\
C & -2.514708 & -3.326952 & 6.452243 \\
C & -3.653893 & -2.535816 & 6.626013 \\
C & -3.609281 & -1.140955 & 6.405607 \\
C & -2.385458 & -0.552873 & 6.018783 \\
C & -1.243473 & -1.347063 & 5.841090 \\
O & -4.794346 & -0.455898 & 6.573946 \\
C & -4.796636 & 0.938569 & 6.311350 \\
H & -4.067021 & -0.824152 & 2.985468 \\
H & 4.129611 & -3.191510 & 2.219028 \\
H & 2.013746 & -5.983978 & 4.992705 \\
H & -1.195166 & -4.096310 & 3.047194 \\
H & -0.309322 & -0.877398 & 5.498006 \\
H & -3.562237 & -3.276115 & 3.408445 \\
H & -2.311732 & 0.526273 & 5.822310 \\
H & -2.213245 & 0.663396 & 2.169030 \\
H & -4.611602 & -2.986994 & 6.930489 \\
H & -2.579560 & -4.413919 & 6.616878 \\
H & -5.831692 & 1.291400 & 6.482406 \\
H & -4.508243 & 1.166694 & 5.259153 \\
H & -4.111470 & 1.495991 & 6.990864 \\
H & 3.277691 & -4.780101 & 2.047639 \\
& & &
\end{tabular}

A = 1-benzyl-3-indolyl, B = Phenyl Bispropargyl

50

Free energy $=-1493.228154$ Hartree

$\begin{array}{lccr}\mathrm{C} & 6.488342 & 6.791769 & 2.580579 \\ \mathrm{C} & 5.694702 & 6.203765 & 1.560802 \\ \mathrm{C} & 5.837341 & 6.668134 & 0.227230 \\ \mathrm{C} & 6.738775 & 7.698954 & -0.070245 \\ \mathrm{C} & 7.517038 & 8.279969 & 0.947703 \\ \mathrm{C} & 7.388505 & 7.820913 & 2.271899 \\ \mathrm{C} & 4.740940 & 5.191338 & 1.893914 \\ \mathrm{C} & 3.897188 & 4.364536 & 2.233381 \\ \mathrm{C} & 2.890585 & 3.407037 & 2.669377 \\ \mathrm{~S} & 2.728468 & 3.234030 & 4.512470 \\ \mathrm{C} & 1.634681 & 4.698588 & 4.895535 \\ \mathrm{C} & 2.201985 & 5.979134 & 4.514765 \\ \mathrm{C} & 2.779700 & 6.974294 & 4.075983 \\ \mathrm{C} & 3.442313 & 8.056504 & 3.452011 \\ \mathrm{C} & 3.357038 & 8.395112 & 2.039938 \\ \mathrm{C} & 4.253361 & 9.485713 & 1.821086 \\ \mathrm{~N} & 4.856497 & 9.791176 & 3.035964 \\ \mathrm{C} & 4.371298 & 8.943703 & 4.009175 \\ \mathrm{C} & 2.647565 & 7.849823 & 0.950467 \\ \mathrm{C} & 2.834904 & 8.412877 & -0.318855 \\ \mathrm{C} & 3.712184 & 9.512274 & -0.513286 \\ \mathrm{C} & 4.434132 & 10.065970 & 0.552297 \\ \mathrm{C} & 5.848133 & 10.838175 & 3.221405 \\ \mathrm{C} & 5.293813 & 12.241203 & 3.024707\end{array}$

$\begin{array}{cccc}\text { C } & 6.120281 & 13.258513 & 2.505819 \\ \mathrm{C} & 5.629776 & 14.566878 & 2.349165 \\ \mathrm{C} & 4.302237 & 14.868726 & 2.702240 \\ \mathrm{C} & 3.469909 & 13.855735 & 3.213231 \\ \mathrm{C} & 3.963240 & 12.549757 & 3.373309 \\ \mathrm{H} & 8.218338 & 9.095192 & 0.709750 \\ \mathrm{H} & 1.464234 & 4.621966 & 5.990864 \\ \mathrm{H} & 3.134350 & 2.382301 & 2.314431 \\ \mathrm{H} & 6.367975 & 6.445506 & 3.617132 \\ \mathrm{H} & 7.987733 & 8.276853 & 3.075177 \\ \mathrm{H} & 6.822038 & 8.064714 & -1.104835 \\ \mathrm{H} & 5.211750 & 6.231114 & -0.563867 \\ \mathrm{H} & 6.261345 & 10.727853 & 4.245108 \\ \mathrm{H} & 6.690096 & 10.656630 & 2.519841 \\ \mathrm{H} & 7.157920 & 13.023462 & 2.217392 \\ \mathrm{H} & 6.285651 & 15.351759 & 1.941318 \\ \mathrm{H} & 3.914337 & 15.891018 & 2.572373 \\ \mathrm{H} & 2.428034 & 14.083364 & 3.487018 \\ \mathrm{H} & 3.306075 & 11.756612 & 3.763557 \\ \mathrm{H} & 4.737383 & 9.009676 & 5.039965 \\ \mathrm{H} & 1.889410 & 3.656502 & 2.254317 \\ \mathrm{H} & 1.982074 & 6.987170 & 1.105202 \\ \mathrm{H} & 2.296520 & 7.996332 & -1.184119 \\ \mathrm{H} & 3.834981 & 9.933021 & -1.523157 \\ \mathrm{H} & 5.119388 & 10.912422 & 0.403300 \\ \mathrm{H} & 0.652656 & 4.533783 & 4.401688\end{array}$

Alkyne_anionA

49

Free energy $=-1492.713941$ Hartree

C $\quad 5.222169 \quad 5.552226 \quad-8.132324$

$\begin{array}{llll}\text { C } & 5.591360 & 6.924600 & -8.178369\end{array}$

$\begin{array}{llll}\text { C } & 5.401767 & 7.716189 & -7.011570\end{array}$

$\begin{array}{llll}\text { C } & 4.862395 & 7.152068 & -5.847981\end{array}$

$\begin{array}{llll}\text { C } & 4.493198 & 5.793474 & -5.818016\end{array}$

$\begin{array}{llll}\text { C } & 4.683187 & 4.998424 & -6.963007\end{array}$

$\begin{array}{llll}\text { C } & 6.157879 & 7.504238 & -9.352371\end{array}$

$\begin{array}{llll}\text { C } & 6.674180 & 8.034517 & -10.340942\end{array}$

$\begin{array}{llll}\text { C } & 7.342085 & 8.717664 & -11.416697\end{array}$

$\begin{array}{llll}\text { S } & 9.218048 & 9.187325 & -10.977927\end{array}$

$\begin{array}{llll}\text { C } & 9.184409 & 10.153020 & -9.535861\end{array}$

$\begin{array}{llll}\text { C } & 9.062013 & 9.511412 & -8.306655\end{array}$

$\begin{array}{llll}\text { C } & 8.998555 & 8.854203 & -7.241813\end{array}$

$\begin{array}{llll}\text { C } & 8.595808 & 7.853489 & -6.330608\end{array}$

$\begin{array}{llll}\text { C } & 8.475369 & 6.448450 & -6.714517\end{array}$

$\begin{array}{llll}\text { C } & 7.960287 & 5.733300 & -5.586088\end{array}$

$\begin{array}{llll}\mathrm{N} & 7.763671 & 6.642500 & -4.565881\end{array}$

$\begin{array}{llll}\text { C } & 8.177580 & 7.912355 & -4.994584\end{array}$

$\begin{array}{llll}\text { C } & 8.721248 & 5.766783 & -7.921365\end{array}$

$\begin{array}{llll}\text { C } & 8.471591 & 4.387118 & -7.977894\end{array}$

$\begin{array}{llll}\text { C } & 7.987673 & 3.685033 & -6.84161\end{array}$

$\begin{array}{llll}\text { C } & 7.725519 & 4.344492 & -5.632090\end{array}$

$\begin{array}{llll}\text { C } & 7.256210 & 6.315862 & -3.254015\end{array}$

$\begin{array}{llll}\text { C } & 8.263272 & 5.633545 & -2.334338\end{array}$

$\begin{array}{llll}\text { C } & 7.816371 & 5.002225 & -1.154126\end{array}$

$\begin{array}{llll}\text { C } & 8.729792 & 4.389812 & -0.279196\end{array}$

$\begin{array}{llll}\text { C } & 10.105288 & 4.395180 & -0.577680\end{array}$

$\begin{array}{llll}\text { C } & 10.556361 & 5.016897 & -1.755899\end{array}$

$\begin{array}{llll}\text { C } & 9.640988 & 5.632324 & -2.628736\end{array}$

$\begin{array}{llll}\mathrm{H} & 4.074957 & 5.352067 & -4.899480\end{array}$

$\begin{array}{llll}\mathrm{H} & 8.957681 & 11.232636 & -9.629358\end{array}$

$\begin{array}{llll}\mathrm{H} & 6.847967 & 9.678545 & -11.670122\end{array}$

$\begin{array}{llll}\mathrm{H} & 5.389665 & 4.924109 & -9.019449\end{array}$

$\begin{array}{llll}\mathrm{H} & 4.422610 & 3.929090 & -6.940313\end{array}$

H $4.744552 \quad 7.775743 \quad-4.947846$

$\begin{array}{llll}\mathrm{H} & 5.725412 & 8.766401 & -7.028492\end{array}$

$\begin{array}{llll}\mathrm{H} & 6.898284 & 7.254992 & -2.781880\end{array}$

$\begin{array}{llll}\mathrm{H} & 6.356364 & 5.671720 & -3.368513\end{array}$

$\begin{array}{llll}\mathrm{H} & 6.738828 & 4.990343 & -0.918361\end{array}$

$\begin{array}{llll}\mathrm{H} & 8.365088 & 3.902855 & 0.639069\end{array}$

$\begin{array}{llll}\mathrm{H} & 10.822271 & 3.913076 & 0.105194\end{array}$

$\begin{array}{llll}\mathrm{H} & 11.630464 & 5.022183 & -2.001139\end{array}$

$\begin{array}{llll}\mathrm{H} & 9.994425 & 6.115086 & -3.552955\end{array}$

$\begin{array}{llll}\mathrm{H} & 8.069144 & 8.775381 & -4.327637\end{array}$

$\begin{array}{llll}\mathrm{H} & 7.421754 & 8.102259 & -12.339090\end{array}$

$\begin{array}{llll}\mathrm{H} & 9.069999 & 6.335480 & -8.798355\end{array}$

$\begin{array}{llll}\mathrm{H} & 8.649409 & 3.837127 & -8.915897\end{array}$

$\begin{array}{llll}\mathrm{H} & 7.799624 & 2.601586 & -6.914777\end{array}$

H $\quad 7.341865 \quad 3.801229 \quad-4.755563$

Alkyne_anionB

Free energy $=-1492.730832$ Hartree

$\begin{array}{llll}\text { C } & 5.320432 & 12.583268 & -1.466007\end{array}$

$\begin{array}{llll}\text { C } & 5.198777 & 11.303272 & -2.039135\end{array}$

$\begin{array}{llll}\text { C } & 4.061292 & 11.005533 & -2.815347\end{array}$

$\begin{array}{llll}\text { C } & 3.068447 & 11.975643 & -3.029607\end{array}$

$\begin{array}{llll}\text { C } & 3.199677 & 13.256940 & -2.462162\end{array}$

$\begin{array}{llll}\text { C } & 4.326962 & 13.555401 & -1.675892\end{array}$

$\begin{array}{llll}\text { C } & 6.304716 & 10.268112 & -1.912528\end{array}$ 


$\begin{array}{cccc}\text { N } & 7.212384 & 10.507661 & -0.802078 \\ \mathrm{C} & 6.883117 & 10.410519 & 0.544655 \\ \mathrm{C} & 8.008536 & 10.868476 & 1.308981 \\ \mathrm{C} & 9.029591 & 11.261107 & 0.351185 \\ \mathrm{C} & 8.487473 & 11.021807 & -0.915845 \\ \mathrm{C} & 5.690468 & 9.974442 & 1.151272 \\ \mathrm{C} & 5.637856 & 9.982423 & 2.553059 \\ \mathrm{C} & 6.743725 & 10.423184 & 3.328057 \\ \mathrm{C} & 7.926407 & 10.868233 & 2.719343 \\ \mathrm{C} & 10.325748 & 11.801017 & 0.561526 \\ \mathrm{C} & 11.454701 & 12.295229 & 0.609805 \\ \mathrm{C} & 12.783123 & 12.842209 & 0.451825 \\ \mathrm{~S} & 13.042152 & 13.571718 & -1.320852 \\ \mathrm{C} & 12.638260 & 12.294903 & -2.448034 \\ \mathrm{C} & 11.402762 & 12.193558 & -3.052043 \\ \mathrm{C} & 10.301936 & 12.101772 & -3.655999 \\ \mathrm{C} & 9.035699 & 11.916452 & -4.238282 \\ \mathrm{C} & 7.948792 & 12.833265 & -4.010851 \\ \mathrm{C} & 6.684231 & 12.617451 & -4.568082 \\ \mathrm{C} & 6.421122 & 11.485845 & -5.368601 \\ \mathrm{C} & 7.469206 & 10.569392 & -5.602270 \\ \mathrm{C} & 8.743954 & 10.770618 & -5.058710 \\ \mathrm{H} & 5.415041 & 11.312531 & -5.777790 \\ \mathrm{H} & 12.988874 & 13.699566 & 1.129838 \\ \mathrm{H} & 13.407305 & 11.525983 & -2.651875 \\ \mathrm{H} & 9.545970 & 10.039211 & -5.247215 \\ \mathrm{H} & 7.285677 & 9.671394 & -6.216087 \\ \mathrm{H} & 5.872110 & 13.327455 & -4.342962 \\ \mathrm{H} & 8.124391 & 13.710277 & -3.368619 \\ \mathrm{H} & 6.909481 & 10.277087 & -2.845208 \\ \mathrm{H} & 5.870833 & 9.249777 & -1.819666 \\ \mathrm{H} & 6.209635 & 12.827709 & -0.864727 \\ \mathrm{H} & 4.439123 & 14.555022 & -1.227859 \\ \mathrm{H} & 2.422870 & 14.019606 & -2.630964 \\ \mathrm{H} & 2.187108 & 11.730782 & -3.643055 \\ \mathrm{H} & 3.963310 & 10.008385 & -3.273003 \\ \mathrm{H} & 8.947378 & 11.205830 & -1.894726 \\ \mathrm{H} & 13.572672 & 12.077197 & 0.616222 \\ \mathrm{H} & 8.778783 & 11.220684 & 3.321769 \\ \mathrm{H} & 6.664399 & 10.421923 & 4.426968 \\ \mathrm{H} & 4.719980 & 9.645513 & 3.059819 \\ \mathrm{H} & 4.829816 & 9.652743 & 0.546524\end{array}$

\section{A = 1-benzyl-3-indolyl, B = 4-methoxyphenyl}

Bispropargyl

54

Free energy $=-1607.652501$ Hartree

$\begin{array}{llll}\text { C } & 9.931467 & 6.358362 & -3.900202\end{array}$

$\begin{array}{llll}\text { C } & 9.214450 & 5.192314 & -3.564025\end{array}$

$\begin{array}{llll}\text { C } & 9.926991 & 4.024112 & -3.225201\end{array}$

$\begin{array}{llll}\text { C } & 11.332767 & 4.024006 & -3.20809\end{array}$

$\begin{array}{llll}\text { C } & 12.042132 & 5.192485 & -3.540127\end{array}$

$\begin{array}{llll}\text { C } & 11.336645 & 6.359015 & -3.888682\end{array}$

$\begin{array}{llll}\text { C } & 7.693308 & 5.182909 & -3.543153\end{array}$

$\begin{array}{llll}\mathrm{N} & 7.093055 & 6.083738 & -4.516492\end{array}$

$\begin{array}{llll}\text { C } & 7.153636 & 5.899509 & -5.891995\end{array}$

$\begin{array}{llll}\text { C } & 6.393463 & 6.940611 & -6.508743\end{array}$

$\begin{array}{llll}\text { C } & 5.874372 & 7.774420 & -5.436417\end{array}$

$\begin{array}{llll}\text { C } & 6.336950 & 7.204026 & -4.243916\end{array}$

$\begin{array}{llll}\text { C } & 7.810889 & 4.905610 & -6.639081\end{array}$

$\begin{array}{llll}\text { C } & 7.666228 & 4.949424 & -8.03265\end{array}$

$\begin{array}{llll}\text { C } & 6.894516 & 5.961461 & -8.661727\end{array}$

$\begin{array}{llll}\text { C } & 6.261330 & 6.962212 & -7.912333\end{array}$

$\begin{array}{llll}\text { C } & 5.036021 & 8.903895 & -5.59648\end{array}$

$\begin{array}{llll}\text { C } & 4.257981 & 9.830406 & -5.826054\end{array}$

$\begin{array}{llll}\text { C } & 3.246079 & 10.814176 & -6.173049\end{array}$

$\begin{array}{llll}\text { S } & 1.480276 & 10.273355 & -5.924119\end{array}$

$\begin{array}{llll}\text { C } & 1.301049 & 9.007774 & -7.272840\end{array}$

$\begin{array}{llll}\text { C } & 1.960920 & 7.739699 & -7.002059\end{array}$

$\begin{array}{llll}\text { C } & 2.548818 & 6.693241 & -6.735759\end{array}$

$\begin{array}{llll}\text { C } & 3.315676 & 5.550159 & -6.354261\end{array}$

$\begin{array}{llll}\text { C } & 3.663204 & 5.350201 & -4.990076\end{array}$

$\begin{array}{llll}\text { C } & 4.503704 & 4.302691 & -4.613704\end{array}$

$\begin{array}{llll}\text { C } & 5.030258 & 3.425086 & -5.588835\end{array}$

$\begin{array}{llll}\text { C } & 4.654914 & 3.576722 & -6.940475\end{array}$

$\begin{array}{llll}\text { C } & 3.811235 & 4.632291 & -7.311769\end{array}$

$\begin{array}{llll}\text { O } & 5.918723 & 2.491512 & -5.13295\end{array}$

$\begin{array}{llll}\text { C } & 6.471417 & 1.564067 & -6.066483\end{array}$

H $\quad 3.325340 \quad 11.729046 \quad-5.546823$

$\begin{array}{llll}\mathrm{H} & 0.201331 & 8.882297 & -7.378087\end{array}$

$\begin{array}{llll}\mathrm{H} & 3.289746 & 6.054695 & -4.233127\end{array}$

$\begin{array}{llll}\mathrm{H} & 4.797310 & 4.162707 & -3.562640\end{array}$

$\begin{array}{llll}\mathrm{H} & 5.051398 & 2.911413 & -7.717683\end{array}$

$\begin{array}{llll}\mathrm{H} & 3.557249 & 4.770651 & -8.372881\end{array}$

$\begin{array}{llll}\mathrm{H} & 7.321416 & 5.480408 & -2.541086\end{array}$

\begin{tabular}{llll}
$\mathrm{H}$ & 7.316551 & 4.155250 & -3.734075 \\
\hline
\end{tabular}

$\begin{array}{llll}\mathrm{H} & 9.374979 & 3.103772 & -2.974136\end{array}$

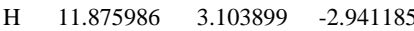

$\begin{array}{llll}\mathrm{H} & 13.143601 & 5.192464 & -3.533327\end{array}$

$\begin{array}{cccc}\mathrm{H} & 11.883647 & 7.276190 & -4.157981 \\ \mathrm{H} & 9.382820 & 7.270319 & -4.184835 \\ \mathrm{H} & 6.158182 & 7.516970 & -3.209015 \\ \mathrm{H} & 1.663160 & 9.456654 & -8.223550 \\ \mathrm{H} & 5.655641 & 7.744262 & -8.395512 \\ \mathrm{H} & 6.792834 & 5.956537 & -9.758662 \\ \mathrm{H} & 8.159029 & 4.181527 & -8.649112 \\ \mathrm{H} & 8.411937 & 4.128083 & -6.145759 \\ \mathrm{H} & 3.358969 & 11.139732 & -7.230103 \\ \mathrm{H} & 7.162348 & 0.920106 & -5.491689 \\ \mathrm{H} & 5.681152 & 0.931924 & -6.527995 \\ \mathrm{H} & 7.036484 & 2.081252 & -6.871899\end{array}$

Alkyne_anionA

Free energy $=-1607.139007$ Hartree

$\begin{array}{llll}\text { C } & 11.938920 & 7.140937 & -3.446029\end{array}$

$\begin{array}{llll}\text { C } & 10.815644 & 6.370754 & -3.039384\end{array}$

$\begin{array}{llll}\text { C } & 11.059808 & 5.089480 & -2.467952\end{array}$

$\begin{array}{llll}\text { C } & 12.362694 & 4.615301 & -2.299293\end{array}$

$\begin{array}{llll}\text { C } & 13.467612 & 5.406265 & -2.695945\end{array}$

$\begin{array}{llll}\text { C } & 13.247450 & 6.669131 & -3.282477\end{array}$

$\begin{array}{llll}\text { C } & 9.514681 & 6.936727 & -3.154938\end{array}$

$\begin{array}{llll}\text { C } & 8.463283 & 7.583651 & -3.230159\end{array}$

$\begin{array}{llll}\text { C } & 7.399364 & 8.542197 & -3.170435\end{array}$

$\begin{array}{lll}7.806142 & 9.918455 & -1.750922\end{array}$

$\begin{array}{llll}\text { C } & 9.388494 & 10.554748 & -2.034258\end{array}$

$\begin{array}{llrr}\text { C } & 10.534088 & 9.906781 & -1.570075\end{array}$

$\begin{array}{llll}\text { C } & 11.541638 & 9.326092 & -1.110526\end{array}$

$\begin{array}{llll}\text { C } & 12.617147 & 8.538775 & -0.649734\end{array}$

$\begin{array}{llll}\text { C } & 14.041981 & 8.798781 & -0.812302\end{array}$

$\begin{array}{llll}\text { C } & 14.753692 & 7.685248 & -0.247658\end{array}$

$\begin{array}{llll}\mathrm{N} & 13.822140 & 6.783080 & 0.221303\end{array}$

$\begin{array}{llll}\text { C } & 12.542675 & 7.293933 & -0.007944\end{array}$

$\begin{array}{llll}\text { C } & 14.769029 & 9.843175 & -1.419294\end{array}$

$\begin{array}{llll}\text { C } & 16.171304 & 9.777627 & -1.440608\end{array}$

$\begin{array}{llll}\text { C } & 16.859324 & 8.682370 & -0.850711\end{array}$

$\begin{array}{llll}\text { C } & 16.161264 & 7.626786 & -0.242461\end{array}$

$\begin{array}{llll}\text { C } & 14.128771 & 5.467483 & 0.744861\end{array}$

$\begin{array}{llll}\text { C } & 14.792900 & 5.489411 & 2.113176\end{array}$

$\begin{array}{llll}\text { C } & 14.521193 & 6.526665 & 3.028656\end{array}$

$\begin{array}{llll}\text { C } & 15.114239 & 6.528601 & 4.302467\end{array}$

$\begin{array}{llll}\text { C } & 15.992399 & 5.493433 & 4.675654\end{array}$

$\begin{array}{llll}\text { C } & 16.275000 & 4.459466 & 3.764065\end{array}$

$\begin{array}{llll}\text { C } & 15.680172 & 4.460248 & 2.489651\end{array}$

$\begin{array}{llll}\text { O } & 14.707090 & 4.870982 & -2.462612\end{array}$

$\begin{array}{llll}\text { C } & 15.842375 & 5.547391 & -3.001400\end{array}$

$\begin{array}{llll}\mathrm{H} & 9.499177 & 11.404978 & -2.736483\end{array}$

$\begin{array}{llll}\mathrm{H} & 6.416050 & 8.124481 & -2.864817\end{array}$

$\begin{array}{llll}\mathrm{H} & 10.207482 & 4.473949 & -2.141989\end{array}$

$\begin{array}{llll}\mathrm{H} & 12.557333 & 3.628137 & -1.853625\end{array}$

$\begin{array}{llll}\mathrm{H} & 14.087168 & 7.317907 & -3.561016\end{array}$

$\begin{array}{llll}\mathrm{H} & 11.770430 & 8.149774 & -3.846263\end{array}$

$\begin{array}{llll}\mathrm{H} & 13.174901 & 4.902078 & 0.791165\end{array}$

$\begin{array}{llll}\mathrm{H} & 14.765008 & 4.929542 & 0.009184\end{array}$

$\begin{array}{llll}\mathrm{H} & 15.911736 & 3.654946 & 1.772922\end{array}$

$\begin{array}{llll}\mathrm{H} & 16.970457 & 3.651779 & 4.042629\end{array}$

$\begin{array}{llll}\mathrm{H} & 16.461224 & 5.496497 & 5.671933\end{array}$

$\begin{array}{llll}\mathrm{H} & 14.892288 & 7.345825 & 5.007230\end{array}$

$\begin{array}{llll}\mathrm{H} & 13.844484 & 7.342550 & 2.727922\end{array}$

$\begin{array}{llll}\mathrm{H} & 11.657339 & 6.695394 & 0.231006\end{array}$

$\begin{array}{llll}\mathrm{H} & 7.279727 & 9.110223 & -4.116359\end{array}$

$\begin{array}{llll}\mathrm{H} & 14.227603 & 10.691860 & -1.868374\end{array}$

$\begin{array}{llll}\mathrm{H} & 16.748608 & 10.588904 & -1.912014\end{array}$

$\begin{array}{llll}\mathrm{H} & 17.961040 & 8.660087 & -0.870652\end{array}$

$\begin{array}{llll}\mathrm{H} & 16.691781 & 6.777593 & 0.213812\end{array}$

\begin{tabular}{llll}
$\mathrm{H}$ & 16.724325 & 4.931919 & -2.740025 \\
\hline
\end{tabular}

$\begin{array}{llll}\mathrm{H} & 15.768099 & 5.635334 & -4.108550\end{array}$

$\begin{array}{llll}\mathrm{H} & 15.968834 & 6.558136 & -2.558716\end{array}$

Alkyne_anionB

53

Free energy $=-1607.144443$ Hartree

$\begin{array}{llll}\text { C } & 11.214663 & 5.925744 & -3.219764\end{array}$

$\begin{array}{llll}\text { C } & 10.325358 & 4.842189 & -2.951222\end{array}$

$\begin{array}{llll}\text { C } & 10.903999 & 3.530533 & -2.949574\end{array}$

$\begin{array}{llll}\text { C } & 12.271719 & 3.333865 & -3.137808\end{array}$

$\begin{array}{llll}\text { C } & 13.137550 & 4.432011 & -3.340145\end{array}$

$\begin{array}{llll}\text { C } & 12.592965 & 5.729457 & -3.399350\end{array}$

$\begin{array}{llll}\text { C } & 8.955321 & 5.021365 & -2.654840\end{array}$

$\begin{array}{llll}\text { C } & 7.850964 & 5.065542 & -2.057858\end{array}$

$\begin{array}{llll}\text { C } & 6.605994 & 5.086759 & -1.451169\end{array}$

$\begin{array}{llll}\mathrm{S} & 5.786795 & 3.609051 & -1.019640\end{array}$

$\begin{array}{llll}\text { C } & 6.481033 & 3.039660 & 0.725540\end{array}$

$\begin{array}{llll}\text { C } & 7.922300 & 3.054306 & 0.750091\end{array}$

$\begin{array}{llll}\text { C } & 9.141003 & 3.187713 & 0.604543\end{array}$

$\begin{array}{llll}\text { C } & 10.507155 & 3.505494 & 0.412991\end{array}$

$\begin{array}{llll}\text { C } & 11.020117 & 4.855883 & 0.237072\end{array}$

$\begin{array}{llll}\text { C } & 12.428098 & 4.744822 & 0.022159\end{array}$

$\begin{array}{llll}\mathrm{N} & 12.757726 & 3.396951 & 0.057502\end{array}$ 


$\begin{array}{cccc}\text { C } & 11.613292 & 2.655828 & 0.296384 \\ \mathrm{C} & 10.405587 & 6.122801 & 0.199392 \\ \mathrm{C} & 11.210848 & 7.247802 & -0.025139 \\ \mathrm{C} & 12.613776 & 7.124561 & -0.206059 \\ \mathrm{C} & 13.243933 & 5.873427 & -0.189628 \\ \mathrm{C} & 14.085529 & 2.867841 & -0.216877 \\ \mathrm{C} & 15.128743 & 3.320884 & 0.792236 \\ \mathrm{C} & 14.837273 & 3.338885 & 2.171540 \\ \mathrm{C} & 15.806105 & 3.754553 & 3.099552 \\ \mathrm{C} & 17.080927 & 4.159210 & 2.659021 \\ \mathrm{C} & 17.377190 & 4.149199 & 1.284499 \\ \mathrm{C} & 16.403328 & 3.735641 & 0.357619 \\ \mathrm{O} & 14.489160 & 4.133365 & -3.42534 \\ \mathrm{C} & 15.401458 & 5.217432 & -3.481641 \\ \mathrm{H} & 6.047276 & 2.026737 & 0.873696 \\ \mathrm{H} & 6.184302 & 6.042672 & -1.086628 \\ \mathrm{H} & 10.252224 & 2.670231 & -2.737093 \\ \mathrm{H} & 12.705732 & 2.322442 & -3.090141 \\ \mathrm{H} & 13.237574 & 6.607578 & -3.543262 \\ \mathrm{H} & 10.815474 & 6.951244 & -3.225432 \\ \mathrm{H} & 14.007649 & 1.760936 & -0.218334 \\ \mathrm{H} & 14.393641 & 3.172185 & -1.239231 \\ \mathrm{H} & 16.629811 & 3.744041 & -0.721443 \\ \mathrm{H} & 18.367189 & 4.475517 & 0.929409 \\ \mathrm{H} & 17.839109 & 4.489273 & 3.386317 \\ \mathrm{H} & 15.564036 & 3.767528 & 4.174241 \\ \mathrm{H} & 13.834963 & 3.036626 & 2.514510 \\ \mathrm{H} & 11.659869 & 1.561083 & 0.335186 \\ \mathrm{H} & 9.313118 & 6.199921 & 0.310238 \\ \mathrm{H} & 10.749023 & 8.247066 & -0.071823 \\ \mathrm{H} & 13.217165 & 8.028437 & -0.384334 \\ \mathrm{H} & 14.327609 & 5.771023 & -0.343610 \\ \mathrm{H} & 6.047218 & 3.719465 & 1.490047 \\ \mathrm{H} & 16.417521 & 4.778195 & -3.485954 \\ \mathrm{H} & 15.271607 & 5.825960 & -4.406712 \\ \mathrm{H} & 15.301203 & 5.893249 & -2.600674\end{array}$

\section{$\mathrm{X}=-\mathrm{NH}-$}

\section{A = 4-methoxyphenyl, $B$ = phenyl}

Bispropargyl

38

Free energy $=-863.2699069$ Hartree

\begin{tabular}{cccc}
\multicolumn{4}{c}{ Free energy $=-863.269069$ Hartree } \\
C & 0.263733 & 6.458740 & 1.139743 \\
$\mathrm{C}$ & 0.860489 & 5.451646 & 1.946483 \\
$\mathrm{C}$ & 0.004539 & 4.547419 & 2.625172 \\
$\mathrm{C}$ & -1.387132 & 4.617284 & 2.492042 \\
$\mathrm{C}$ & -1.961517 & 5.603007 & 1.659602 \\
$\mathrm{C}$ & -1.123039 & 6.533463 & 1.000810 \\
$\mathrm{C}$ & 2.275922 & 5.275353 & 2.017069 \\
$\mathrm{C}$ & 3.475293 & 5.002392 & 2.045085 \\
$\mathrm{C}$ & 4.844392 & 4.455772 & 2.012886 \\
$\mathrm{~N}$ & 4.941376 & 2.989263 & 1.943892 \\
$\mathrm{C}$ & 4.450500 & 2.358390 & 0.708843 \\
$\mathrm{C}$ & 2.990898 & 2.336520 & 0.485610 \\
$\mathrm{C}$ & 1.769969 & 2.319336 & 0.336414 \\
$\mathrm{C}$ & 0.351196 & 2.369171 & 0.149073 \\
$\mathrm{C}$ & -0.501430 & 1.396783 & 0.735472 \\
$\mathrm{C}$ & -1.890806 & 1.468541 & 0.555074 \\
$\mathrm{C}$ & -2.455140 & 2.503153 & -0.213595 \\
$\mathrm{C}$ & -1.620274 & 3.477382 & -0.791435 \\
$\mathrm{C}$ & -0.231865 & 3.413829 & -0.616702 \\
$\mathrm{O}$ & -3.295700 & 5.724609 & 1.417978 \\
$\mathrm{C}$ & -4.192159 & 4.836595 & 2.083857 \\
$\mathrm{H}$ & -3.545418 & 2.552633 & -0.360872 \\
$\mathrm{H}$ & 4.823289 & 1.310153 & 0.695478 \\
$\mathrm{H}$ & 5.394087 & 4.882783 & 1.144257 \\
$\mathrm{H}$ & -0.059890 & 0.587616 & 1.337048 \\
$\mathrm{H}$ & 0.449588 & 3.750122 & 3.238878 \\
$\mathrm{H}$ & -2.538989 & 0.707987 & 1.017893 \\
$\mathrm{H}$ & -2.010187 & 3.873837 & 3.005725 \\
$\mathrm{H}$ & -2.058357 & 4.304987 & -1.370206 \\
$\mathrm{H}$ & -1.590203 & 7.298455 & 0.362310 \\
$\mathrm{H}$ & 0.906184 & 7.173060 & 0.603296 \\
$\mathrm{H}$ & -5.207310 & 5.103722 & 1.736552 \\
$\mathrm{H}$ & -4.140775 & 4.956080 & 3.189351 \\
$\mathrm{H}$ & -3.984955 & 3.775888 & 1.823650 \\
$\mathrm{H}$ & 5.406259 & 4.788464 & 2.914372 \\
$\mathrm{H}$ & 0.418814 & 4.187223 & -1.049548 \\
$\mathrm{H}$ & 4.942893 & 2.862341 & -0.152144 \\
$\mathrm{H}$ & 4.464859 & 2.574514 & 2.755921
\end{tabular}

Alkyne_anionA

37
Free energy $=-862.7451555$ Hartree

$\begin{array}{cccc}\mathrm{C} & 3.229481 & 5.009798 & 0.056698 \\ \mathrm{C} & 4.423046 & 4.682728 & -0.653730 \\ \mathrm{C} & 4.307805 & 4.127545 & -1.959052 \\ \mathrm{C} & 3.046660 & 3.886720 & -2.521483 \\ \mathrm{C} & 1.875853 & 4.182822 & -1.793999 \\ \mathrm{C} & 1.976252 & 4.749926 & -0.511325 \\ \mathrm{C} & 5.678415 & 4.843689 & -0.003795 \\ \mathrm{C} & 6.705878 & 4.949917 & 0.678496 \\ \mathrm{C} & 7.654761 & 4.696066 & 1.772583 \\ \mathrm{~N} & 7.170674 & 3.606884 & 2.688815 \\ \mathrm{C} & 5.946800 & 3.858480 & 3.395051 \\ \mathrm{C} & 4.715943 & 3.435349 & 2.955408 \\ \mathrm{C} & 3.610446 & 2.973796 & 2.552327 \\ \mathrm{C} & 2.378601 & 2.533693 & 2.044531 \\ \mathrm{C} & 2.278160 & 1.817530 & 0.802955 \\ \mathrm{C} & 1.049500 & 1.402940 & 0.272884 \\ \mathrm{C} & -0.156710 & 1.676170 & 0.956222 \\ \mathrm{C} & -0.092640 & 2.374156 & 2.184187 \\ \mathrm{C} & 1.130328 & 2.786777 & 2.719025 \\ \mathrm{O} & -1.421584 & 1.315286 & 0.515584 \\ \mathrm{C} & -1.518258 & 0.665463 & -0.738636 \\ \mathrm{H} & 0.886627 & 3.955518 & -2.220405 \\ \mathrm{H} & 8.657042 & 4.412097 & 1.380278 \\ \mathrm{H} & 6.043253 & 4.509189 & 4.284089 \\ \mathrm{H} & 3.312411 & 5.405421 & 1.077602 \\ \mathrm{H} & 3.197810 & 1.636490 & 0.225944 \\ \mathrm{H} & 1.065572 & 4.946266 & 0.074932 \\ \mathrm{H} & 1.044560 & 0.885987 & -0.697306 \\ \mathrm{H} & 2.974514 & 3.444012 & -3.527458 \\ \mathrm{H} & -1.035708 & 2.588008 & 2.713234 \\ \mathrm{H} & 1.144852 & 3.333037 & 3.675337 \\ \mathrm{H} & 7.803983 & 5.611467 & 2.384954 \\ \mathrm{H} & -2.594360 & 0.478404 & -0.920308 \\ \mathrm{H} & -1.118372 & 1.292193 & -1.571050 \\ \mathrm{H} & -0.980177 & -0.311606 & -0.752830 \\ \mathrm{H} & 5.223075 & 3.869911 & -2.514399 \\ \mathrm{H} & 7.053356 & 2.763665 & 2.110185\end{array}$

Alkyne_anionB

41

Free energy $=-862.7541111$ Hartree

C $\quad 3.370173 \quad 3.285640 \quad-1.096343$

$\begin{array}{llll}\text { C } & 4.151910 & 4.498940 & -1.036993\end{array}$

$\begin{array}{llll}\text { C } & 3.586120 & 5.644543 & -1.711689\end{array}$

$\begin{array}{llll}\text { C } & 2.351140 & 5.578988 & -2.357897\end{array}$

$\begin{array}{llll}\text { C } & 1.592649 & 4.384139 & -2.384195\end{array}$

$\begin{array}{llll}\text { C } & 2.134828 & 3.241938 & -1.750889\end{array}$

$\begin{array}{llll}\text { C } & 5.384590 & 4.564615 & -0.384584\end{array}$

$\begin{array}{llll}\text { C } & 6.529151 & 4.619797 & 0.154589\end{array}$

$\begin{array}{llll}\text { C } & 7.739447 & 4.739953 & 0.783230\end{array}$

$\begin{array}{llll}\mathrm{N} & 8.482104 & 5.969629 & 0.852629\end{array}$

$\begin{array}{llll}\text { C } & 9.533568 & 6.166706 & -0.196563\end{array}$

$\begin{array}{llll}\text { C } & 8.959953 & 6.203473 & -1.552809\end{array}$

$\begin{array}{llll}\text { C } & 8.090970 & 6.144391 & -2.431691\end{array}$

$\begin{array}{llll}C & 7.013125 & 5.975014 & -3.345548\end{array}$

$\begin{array}{llll}\text { C } & 6.376858 & 4.706421 & -3.443147\end{array}$

$\begin{array}{llll}\text { C } & 5.228546 & 4.526355 & -4.224594\end{array}$

$\begin{array}{llll}\text { C } & 4.682246 & 5.616224 & -4.93429\end{array}$

$\begin{array}{llll}\text { C } & 5.326080 & 6.876791 & -4.881795\end{array}$

$\begin{array}{llll}\text { C } & 6.466377 & 7.053899 & -4.096181\end{array}$

$\begin{array}{lll}3.547118 & 5.556091 & -5.690792\end{array}$

$\begin{array}{llll}\text { C } & 2.856263 & 4.309378 & -5.770990\end{array}$

$\begin{array}{llll}\mathrm{H} & 0.616834 & 4.342674 & -2.891345\end{array}$

$\begin{array}{llll}\mathrm{H} & 10.267130 & 5.335806 & -0.104609\end{array}$

$\begin{array}{llll}\mathrm{H} & 3.767700 & 2.378717 & -0.612717\end{array}$

$\begin{array}{llll}\mathrm{H} & 6.764889 & 3.870383 & -2.846105\end{array}$

$\mathrm{H} \quad 1.574566 \quad 2.290696 \quad-1.766574$

$\begin{array}{llll}\mathrm{H} & 4.728699 & 3.548986 & -4.217013\end{array}$

$\begin{array}{llll}\mathrm{H} & 1.976002 & 6.476458 & -2.877909\end{array}$

$\begin{array}{llll}\mathrm{H} & 4.889072 & 7.713283 & -5.447943\end{array}$

$\begin{array}{llll}\mathrm{H} & 6.941429 & 8.045486 & -4.038441\end{array}$

$\begin{array}{llll}\mathrm{H} & 10.091605 & 7.103452 & 0.030211\end{array}$

$\begin{array}{llll}\mathrm{H} & 8.255892 & 3.856990 & 1.203827\end{array}$

$\begin{array}{llll}\mathrm{H} & 1.974015 & 4.485620 & -6.414724\end{array}$

$\begin{array}{llll}\mathrm{H} & 3.490694 & 3.519538 & -6.234174\end{array}$

$\begin{array}{llll}\mathrm{H} & 2.516000 & 3.971565 & -4.767627\end{array}$

$\begin{array}{llll}\mathrm{H} & 4.175532 & 6.574289 & -1.743057\end{array}$

$\begin{array}{llll}\mathrm{H} & 7.824022 & 6.756083 & 0.763532\end{array}$

\section{A = 2,4-dimethoxyphenyl, $B$ = phenyl}

Bispropargyl

Free energy $=-977.6900455$ Hartree

$\begin{array}{llll}\text { C } & 0.291936 & 1.295426 & 1.195764\end{array}$

$\begin{array}{llll}\text { C } & 1.244687 & 2.132717 & 0.556669\end{array}$

$\begin{array}{llll}\text { C } & 0.783380 & 3.109595 & -0.364700\end{array}$

$\begin{array}{llll}\text { C } & -0.587487 & 3.243064 & -0.631502\end{array}$

$\begin{array}{llll}\text { C } & -1.521606 & 2.411964 & 0.013154\end{array}$ 


\begin{tabular}{rrrr}
$\mathrm{C}$ & -1.074691 & 1.435871 & 0.922572 \\
$\mathrm{C}$ & 2.640490 & 1.971939 & 0.832496 \\
$\mathrm{C}$ & 3.825690 & 1.773742 & 1.095579 \\
$\mathrm{C}$ & 5.254854 & 1.523759 & 1.371071 \\
$\mathrm{~N}$ & 5.983783 & 0.646557 & 0.441842 \\
$\mathrm{C}$ & 5.655610 & -0.787209 & 0.502389 \\
$\mathrm{C}$ & 4.286303 & -1.149005 & 0.092597 \\
$\mathrm{C}$ & 3.104093 & -1.250471 & -0.233327 \\
$\mathrm{C}$ & 1.730178 & -1.225682 & -0.608906 \\
$\mathrm{C}$ & 0.739899 & -1.945720 & 0.134157 \\
$\mathrm{C}$ & -0.618480 & -1.816727 & -0.189445 \\
$\mathrm{C}$ & -1.023132 & -0.957003 & -1.236608 \\
$\mathrm{C}$ & -0.063151 & -0.256858 & -1.995828 \\
$\mathrm{C}$ & 1.291282 & -0.401396 & -1.669777 \\
$\mathrm{O}$ & 1.208952 & -2.710335 & 1.156539 \\
$\mathrm{C}$ & 0.273583 & -3.423727 & 1.962457 \\
$\mathrm{O}$ & -2.369525 & -0.857785 & -1.418382 \\
$\mathrm{C}$ & -2.854673 & -0.013813 & -2.461157 \\
$\mathrm{H}$ & -2.596918 & 2.519100 & -0.197668 \\
$\mathrm{H}$ & 5.784123 & 2.502176 & 1.397232 \\
$\mathrm{H}$ & 5.835187 & -1.140912 & 1.542134 \\
$\mathrm{H}$ & 1.514095 & 3.757987 & -0.871241 \\
$\mathrm{H}$ & 2.045825 & 0.170432 & -2.229113 \\
$\mathrm{H}$ & -0.930608 & 4.004086 & -1.350343 \\
$\mathrm{H}$ & -0.355361 & 0.425536 & -2.803403 \\
$\mathrm{H}$ & -1.797136 & 0.762949 & 1.408588 \\
$\mathrm{H}$ & -1.403652 & -2.336879 & 0.373395 \\
$\mathrm{H}$ & -3.958559 & -0.069596 & -2.417137 \\
$\mathrm{H}$ & -2.511103 & -0.359330 & -3.461409 \\
$\mathrm{H}$ & -2.535613 & 1.041353 & -2.314315 \\
$\mathrm{H}$ & 6.395990 & -1.326644 & -0.128506 \\
$\mathrm{H}$ & 0.644668 & 0.515979 & 1.887194 \\
$\mathrm{H}$ & 5.357247 & 1.099367 & 2.394576 \\
$\mathrm{H}$ & 5.859091 & 0.986264 & -0.521437 \\
$\mathrm{H}$ & 0.868294 & -3.959089 & 2.725097 \\
$\mathrm{H}$ & -0.437448 & -2.736296 & 2.471737 \\
$\mathrm{H}$ & -0.303127 & -4.163790 & 1.364636 \\
& & & \\
\hline
\end{tabular}

Alkyne_anionA

41

Free energy $=-977.1636806$ Hartree

$\begin{array}{llll}\text { C } & 3.220373 & 1.058184 & -0.021323\end{array}$

$\begin{array}{llll}\text { C } & 3.690901 & 0.147093 & 0.988351\end{array}$

$\begin{array}{llll}\text { C } & 3.103441 & -1.150433 & 0.973757\end{array}$

$\begin{array}{llll}\text { C } & 2.153912 & -1.554458 & 0.018289\end{array}$

$\begin{array}{llll}\text { C } & 1.754696 & -0.651652 & -0.980151\end{array}$

$\begin{array}{llll}\text { C } & 2.278625 & 0.665298 & -0.98138\end{array}$

$\begin{array}{llll}\text { C } & 4.670908 & 0.502492 & 1.961393\end{array}$

$\begin{array}{llll}\text { C } & 5.898205 & 0.307711 & 2.270194\end{array}$

$\begin{array}{llll}\text { C } & 7.133053 & 0.024082 & 2.768115\end{array}$

$\begin{array}{llll}\mathrm{N} & 7.421725 & -1.257293 & 3.380752\end{array}$

$\begin{array}{llll}\text { C } & 7.982760 & -2.284746 & 2.453477\end{array}$

$\begin{array}{llll}\text { C } & 7.147279 & -2.575359 & 1.277036\end{array}$

$\begin{array}{llll}\text { C } & 6.342303 & -2.654817 & 0.345919\end{array}$

$\begin{array}{llll}\text { C } & 5.470193 & -2.698298 & -0.786697\end{array}$

$\begin{array}{llll}\text { C } & 5.175671 & -1.501725 & -1.496511\end{array}$

$\begin{array}{llll}\text { C } & 4.330808 & -1.525395 & -2.611282\end{array}$

$\begin{array}{llll}\text { C } & 3.769313 & -2.738294 & -3.057951\end{array}$

$\begin{array}{llll}\text { C } & 4.045429 & -3.926875 & -2.360036\end{array}$

$\begin{array}{llll}\text { C } & 4.886879 & -3.913887 & -1.235672\end{array}$

$\begin{array}{llll}\text { O } & 0.887139 & -0.948825 & -2.016887\end{array}$

$\begin{array}{llll}\text { C } & 0.317525 & -2.247242 & -2.036633\end{array}$

$\begin{array}{llll}\text { O } & 3.799450 & 2.307004 & 0.011394\end{array}$

$\begin{array}{llll}\text { C } & 3.410434 & 3.252533 & -0.968943\end{array}$

$\begin{array}{llll}\mathrm{H} & 3.101528 & -2.749156 & -3.933268\end{array}$

$\begin{array}{llll}\mathrm{H} & 8.175104 & -3.218129 & 3.030416\end{array}$

$\begin{array}{llll}\mathrm{H} & 8.012103 & 0.675445 & 2.607289\end{array}$

$\begin{array}{llll}\mathrm{H} & 5.585912 & -0.552242 & -1.124779\end{array}$

$\begin{array}{llll}\mathrm{H} & 3.459433 & -1.878132 & 1.719103\end{array}$

$\begin{array}{llll}\mathrm{H} & 4.087777 & -0.583220 & -3.124205\end{array}$

$\begin{array}{llll}\mathrm{H} & 1.789736 & -2.590098 & 0.031317\end{array}$

$\begin{array}{llll}\mathrm{H} & 3.600336 & -4.878085 & -2.693029\end{array}$

$\begin{array}{llll}\mathrm{H} & 1.951149 & 1.341723 & -1.782846\end{array}$

$\begin{array}{llll}\mathrm{H} & 8.978816 & -1.923231 & 2.113926\end{array}$

$\begin{array}{llll}\mathrm{H} & -0.335915 & -2.294484 & -2.929635\end{array}$

\begin{tabular}{llll}
$\mathrm{H}$ & 1.093695 & -3.042503 & -2.115485 \\
\hline
\end{tabular}

$\begin{array}{llll}\mathrm{H} & -0.298427 & -2.447377 & -1.12782\end{array}$

$\begin{array}{llll}\mathrm{H} & 5.099682 & -4.845708 & -0.689689\end{array}$

$\begin{array}{llll}\mathrm{H} & 6.540554 & -1.636566 & 3.755054\end{array}$

$\mathrm{H} \quad 3.978584 \quad 4.179008 \quad-0.759820$

$\begin{array}{llll}\mathrm{H} & 3.651427 & 2.909016 & -2.002012\end{array}$

$\begin{array}{llll}\mathrm{H} & 2.319793 & 3.481881 & -0.924348\end{array}$

Alkyne_anionB

41

Free energy $=-977.1744578$ Hartree

$\begin{array}{llll}\text { C } & 2.409272 & -0.109273 & -0.536836\end{array}$

$\begin{array}{llll}\text { C } & 2.409272 & -0.109273 & -0.536836 \\ \text { C } & 2.591022 & 0.201948 & 0.849959\end{array}$

$\begin{array}{llll}\mathrm{C} & 2.591022 & 0.201948 & 0.849959 \\ \mathrm{C} & 1.434366 & 0.478357 & 1.622343\end{array}$

$\begin{array}{llll}\text { C } & 1.434366 & 0.478357 & 1.622343 \\ \text { C } & 0.148219 & 0.475823 & 1.066326\end{array}$ $\begin{array}{llll}\text { C } & -0.002147 & 0.198122 & -0.305575\end{array}$

$\begin{array}{llll}\text { C } & 1.125795 & -0.119522 & -1.100900\end{array}$

$\begin{array}{llll}\text { C } & 3.879388 & 0.354481 & 1.427173\end{array}$

$\begin{array}{llll}\text { C } & 4.934112 & 0.582532 & 2.032583\end{array}$

$\begin{array}{llll}\text { C } & 5.976174 & 1.376887 & 2.704573\end{array}$

$\begin{array}{llll}\mathrm{N} & 5.669013 & 2.843793 & 2.686462\end{array}$

$\begin{array}{llll}\text { C } & 4.477916 & 3.250987 & 3.381919\end{array}$

$\begin{array}{llll}\text { C } & 3.260228 & 3.404329 & 2.774475\end{array}$

$\begin{array}{llll}\text { C } & 2.167231 & 3.589987 & 2.162125 \\ \text { C } & 0.959919 & 3.746784 & 1.478615\end{array}$

$\begin{array}{llll}\text { C } & 0.959919 & 3.746784 & 1.478615\end{array}$

$\begin{array}{llll}\text { C } & -0.242988 & 4.214191 & 2.127806\end{array}$

$\begin{array}{llll}\text { C } & -1.443312 & 4.361794 & 1.425636\end{array}$

$\begin{array}{llll}\text { C } & -1.534431 & 4.063156 & 0.046415\end{array}$

$\begin{array}{llll}\text { C } & -0.372058 & 3.588697 & -0.607929\end{array}$

$\begin{array}{llll}\text { C } & 0.835353 & 3.433301 & 0.073792\end{array}$

$\begin{array}{llll}\mathrm{O} & -1.195705 & 0.210231 & -0.970529\end{array}$

$\begin{array}{llll}\text { C } & -2.371596 & 0.546882 & -0.234259\end{array}$

$\begin{array}{llll}\text { O } & 3.551004 & -0.368976 & -1.238743\end{array}$

$\begin{array}{llll}\text { C } & 3.449982 & -0.610400 & -2.637066\end{array}$

$\begin{array}{llll}\mathrm{H} & -2.483467 & 4.185000 & -0.498552\end{array}$

$\begin{array}{llll}\mathrm{H} & 6.083893 & 1.064454 & 3.766078\end{array}$

$\begin{array}{llll}\mathrm{H} & -0.201722 & 4.455781 & 3.202324\end{array}$

$\begin{array}{llll}\mathrm{H} & 1.573634 & 0.760464 & 2.674578\end{array}$

$\begin{array}{llll}\mathrm{H} & -2.335796 & 4.722948 & 1.965889\end{array}$

$\begin{array}{llll}\mathrm{H} & -0.707111 & 0.768317 & 1.687237\end{array}$

$\begin{array}{llll}\mathrm{H} & -0.421099 & 3.305009 & -1.672676\end{array}$

$\begin{array}{llll}\mathrm{H} & 0.956449 & -0.336461 & -2.163173\end{array}$

$\begin{array}{llll}\mathrm{H} & 6.970508 & 1.216284 & 2.229866\end{array}$

$\begin{array}{llll}\mathrm{H} & 4.589641 & 3.367279 & 4.476556\end{array}$

$\begin{array}{llll}\mathrm{H} & -3.208543 & 0.510398 & -0.957057\end{array}$

$\begin{array}{llll}\mathrm{H} & -2.565742 & -0.184699 & 0.582600\end{array}$

$\begin{array}{llll}\mathrm{H} & -2.299514 & 1.571349 & 0.191559\end{array}$

$\begin{array}{llll}\mathrm{H} & 1.712743 & 3.017516 & -0.445807\end{array}$

$\begin{array}{llll}\mathrm{H} & 5.580962 & 3.114364 & 1.697141\end{array}$

$\mathrm{H} \quad 4.482277 \quad-0.767320 \quad-3.001553$

$\begin{array}{rrrr}\mathrm{H} & 2.846549 & -1.519685 & -2.858644 \\ \mathrm{H} & 3.002297 & 0.255819 & -3.174540\end{array}$

\section{A = 2-naphthyl, B = phenyl}

Bispropargyl

Free energy $=-902.3513621$ Hartree

$\begin{array}{llll}\text { C } & -2.088358 & 4.502289 & -4.729821\end{array}$

$\begin{array}{llll}\text { C } & -2.602897 & 5.640774 & -4.041213\end{array}$

$\begin{array}{llll}\text { C } & -1.811368 & 6.237178 & -2.989090\end{array}$

$\begin{array}{llll}\text { C } & -0.545332 & 5.660736 & -2.663935\end{array}$

$\begin{array}{llll}\text { C } & -0.074062 & 4.550593 & -3.350627\end{array}$

$\begin{array}{llll}\text { C } & -0.848763 & 3.969712 & -4.394918\end{array}$

$\begin{array}{llll}\text { C } & -3.862992 & 6.229735 & -4.367649\end{array}$

$\begin{array}{llll}\text { C } & -4.315812 & 7.363783 & -3.714680\end{array}$

$\begin{array}{llll}\text { C } & -3.523780 & 7.979652 & -2.686168\end{array}$

$\begin{array}{llll}\text { C } & -2.298006 & 7.399141 & -2.330763\end{array}$

$\begin{array}{llll}\text { C } & -3.923704 & 9.212760 & -2.085686\end{array}$

$\begin{array}{llll}\text { C } & -4.162913 & 10.324381 & -1.616959\end{array}$

$\begin{array}{llll}\text { C } & -4.255109 & 11.690991 & -1.070157\end{array}$

$\begin{array}{llll}\mathrm{N} & -2.975977 & 12.390074 & -0.876322\end{array}$

$\begin{array}{llll}\text { C } & -2.246607 & 12.773187 & -2.095686\end{array}$

$\begin{array}{llll}\text { C } & -1.659177 & 11.685935 & -2.903789\end{array}$

$\begin{array}{llll}\text { C } & -1.196626 & 10.737359 & -3.535830\end{array}$

$\begin{array}{llll}\text { C } & -0.739576 & 9.602950 & -4.280022\end{array}$

$\begin{array}{llll}\text { C } & 0.530181 & 9.019834 & -4.028590\end{array}$

$\begin{array}{llll}\text { C } & 0.940084 & 7.881393 & -4.737277\end{array}$

$\begin{array}{llll}\text { C } & 0.100635 & 7.306348 & -5.707429\end{array}$

$\begin{array}{llll}\text { C } & -1.157799 & 7.878082 & -5.966462\end{array}$

$\begin{array}{llll}\text { C } & -1.577030 & 9.016133 & -5.266066\end{array}$

$\begin{array}{llll}\mathrm{H} & 0.414046 & 6.396569 & -6.240708\end{array}$

$\begin{array}{llll}\mathrm{H} & -1.435903 & 13.473842 & -1.796177\end{array}$

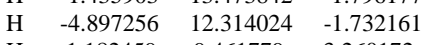

$\begin{array}{llll}\text { H } & 1.182459 & 9.461779 & -3.260172\end{array}$

H $\quad-1.677969 \quad 7.876554 \quad-1.557101$

$\begin{array}{llll}\mathrm{H} & 1.919421 & 7.428804 & -4.519454\end{array}$

$\begin{array}{llll}\mathrm{H} & -1.829929 & 7.418587 & -6.706871\end{array}$

$\begin{array}{llll}\mathrm{H} & -4.470071 & 5.775136 & -5.166773\end{array}$

$\begin{array}{llll}\mathrm{H} & -5.279030 & 7.818141 & -3.989575\end{array}$

$\begin{array}{llll}\mathrm{H} & -4.778811 & 11.660322 & -0.088627\end{array}$

$\begin{array}{llll}\mathrm{H} & -2.572088 & 9.446676 & -5.448692\end{array}$

$\begin{array}{llll}\mathrm{H} & -2.688480 & 4.053815 & -5.537873\end{array}$

$\begin{array}{llll}\mathrm{H} & -0.461674 & 3.092779 & -4.936360\end{array}$

$\begin{array}{llll}\mathrm{H} & 0.907265 & 4.121247 & -3.097047\end{array}$

$\begin{array}{llll}\mathrm{H} & 0.058677 & 6.124965 & -1.868842\end{array}$

$\begin{array}{llll}\mathrm{H} & -2.364325 & 11.828912 & -0.268532\end{array}$

$\begin{array}{llll}\mathrm{H} & -2.936545 & 13.368099 & -2.735226\end{array}$

Alkyne_anionA

39

Free energy $=-901.8343401$ Hartree

$\begin{array}{llll}\text { C } & -3.141735 & -0.989520 & -2.722328\end{array}$

$\begin{array}{llll}\text { C } & -4.069811 & -0.097174 & -3.314874\end{array}$

$\begin{array}{llll}\text { C } & -3.675195 & 0.701259 & -4.468673\end{array}$ 


\begin{tabular}{cccc}
$\mathrm{C}$ & -2.331113 & 0.523268 & -4.957793 \\
$\mathrm{C}$ & -1.446772 & -0.364825 & -4.351937 \\
$\mathrm{C}$ & -1.839763 & -1.133835 & -3.220922 \\
$\mathrm{C}$ & -4.591788 & 1.600749 & -5.055153 \\
$\mathrm{C}$ & -5.927932 & 1.772974 & -4.553789 \\
$\mathrm{C}$ & -6.299731 & 0.943151 & -3.412140 \\
$\mathrm{C}$ & -5.413241 & 0.066594 & -2.826249 \\
$\mathrm{C}$ & -6.856641 & 2.664104 & -5.071057 \\
$\mathrm{C}$ & -7.711745 & 3.535193 & -5.420104 \\
$\mathrm{C}$ & -8.663509 & 4.444649 & -5.759153 \\
$\mathrm{~N}$ & -9.993239 & 4.487395 & -5.210363 \\
$\mathrm{C}$ & -10.346593 & 3.449894 & -4.242036 \\
$\mathrm{C}$ & -9.586862 & 3.470669 & -2.977148 \\
$\mathrm{C}$ & -8.874874 & 3.551946 & -1.976521 \\
$\mathrm{C}$ & -7.953842 & 3.575163 & -0.882975 \\
$\mathrm{C}$ & -6.561839 & 3.421904 & -1.137179 \\
$\mathrm{C}$ & -5.645996 & 3.410074 & -0.075906 \\
$\mathrm{C}$ & -6.090444 & 3.553018 & 1.252733 \\
$\mathrm{C}$ & -7.465168 & 3.713267 & 1.514690 \\
$\mathrm{C}$ & -8.390904 & 3.725147 & 0.461150 \\
$\mathrm{H}$ & -5.367672 & 3.537793 & 2.083551 \\
$\mathrm{H}$ & -11.435118 & 3.525989 & -4.027281 \\
$\mathrm{H}$ & -8.480192 & 5.215766 & -6.528382 \\
$\mathrm{H}$ & -9.465631 & 3.847581 & 0.666346 \\
$\mathrm{H}$ & -4.267965 & 2.198602 & -5.923136 \\
$\mathrm{H}$ & -7.819582 & 3.829175 & 2.551203 \\
$\mathrm{H}$ & -4.573522 & 3.278266 & -0.289739 \\
$\mathrm{H}$ & -5.731547 & -0.526707 & -1.951747 \\
$\mathrm{H}$ & -7.318474 & 1.054396 & -3.006917 \\
$\mathrm{H}$ & -10.183850 & 2.460777 & -4.728863 \\
$\mathrm{H}$ & -6.219467 & 3.302498 & -2.175539 \\
$\mathrm{H}$ & -2.008908 & 1.110468 & -5.834520 \\
$\mathrm{H}$ & -0.425966 & -0.473806 & -4.755392 \\
$\mathrm{H}$ & -1.130472 & -1.830481 & -2.746988 \\
$\mathrm{H}$ & -3.466586 & -1.577098 & -1.845887 \\
$\mathrm{H}$ & -10.211617 & 5.421028 & -4.830459 \\
& & & \\
\hline & & &
\end{tabular}

Alkyne_anionB

39

Free energy $=-901.8343418$ Hartree

$\begin{array}{llll}\text { C } & -2.777920 & 3.832924 & -3.825666 \\ \text { C } & -3.187769 & 5.170525 & -3.552698\end{array}$

$\begin{array}{ll}70525 & -3.552698\end{array}$

$\begin{array}{llll}\text { C } & -2.192318 & 6.222666 & -3.558781\end{array}$

$\begin{array}{llll}\text { C } & -0.830607 & 5.890703 & -3.854620\end{array}$

$\begin{array}{llll}\text { C } & -0.468114 & 4.573561 & -4.119555\end{array}$

$\begin{array}{llll}\text { C } & -1.442902 & 3.537318 & -4.102410\end{array}$

$\begin{array}{llll}\text { C } & -2.589943 & 7.559222 & -3.286891\end{array}$

$\begin{array}{llll}\text { C } & -3.937660 & 7.888277 & -3.029948\end{array}$

$\begin{array}{llll}\text { C } & -4.917032 & 6.837179 & -3.025315 \\ \text { C } & -4.549546 & 5.523546 & -3.275475\end{array}$

$\begin{array}{llll}\text { C } & -4.549546 & 5.523546 & -3.275475 \\ \text { C } & -4.270799 & 9.258689 & -2.813851\end{array}$

$\begin{array}{llll}\text { C } & -4.270799 & 9.258689 & -2.813851\end{array}$

$\begin{array}{llll}\text { C } & -4.386565 & 10.483346 & -2.716567\end{array}$

$\begin{array}{llll}\text { C } & -4.285419 & 11.925824 & -2.425965\end{array}$

$\begin{array}{llll}\mathrm{N} & -3.597641 & 12.251089 & -1.172817\end{array}$

$\begin{array}{llll}\text { C } & -2.225786 & 11.853835 & -1.034078\end{array}$

$\begin{array}{llll}\text { C } & -1.456723 & 11.309282 & -2.014661\end{array}$

$\begin{array}{llll}\text { C } & -0.751958 & 10.799754 & -2.940619\end{array}$

$\begin{array}{llll}\text { C } & -0.119726 & 9.994593 & -3.885351\end{array}$

$\begin{array}{llll}\text { C } & -0.776857 & 9.637821 & -5.127870\end{array}$

$\begin{array}{llll}\text { C } & -0.173819 & 8.781617 & -6.050698\end{array}$

$\begin{array}{llll}\text { C } & 1.109857 & 8.233092 & -5.824450\end{array}$

$\begin{array}{llll}\text { C } & 1.780926 & 8.582150 & -4.627027\end{array}$

$\begin{array}{llll}\text { C } & 1.199151 & 9.432920 & -3.684152\end{array}$

$\begin{array}{llll}\mathrm{H} & 1.572530 & 7.553211 & -6.555955\end{array}$

$\begin{array}{llll}\mathrm{H} & -1.827204 & 12.013623 & -0.016965\end{array}$

$\mathrm{H} \quad-3.723650 \quad 12.411651 \quad-3.256559$

\begin{tabular}{llll}
$\mathrm{H}$ & 1.738061 & 9.673483 & -2.753293 \\
\hline
\end{tabular}

H $\quad-1.832266 \quad 8.353036 \quad-3.273898$

$\begin{array}{llll}\mathrm{H} & 2.782262 & 8.166764 & -4.421897\end{array}$

$\begin{array}{llll}\mathrm{H} & -0.723710 & 8.520882 & -6.971194\end{array}$

$\begin{array}{llll}\mathrm{H} & -5.312309 & 4.727516 & -3.267829\end{array}$

$\begin{array}{llll}\mathrm{H} & -5.969378 & 7.089477 & -2.820423\end{array}$

$\begin{array}{llll}\mathrm{H} & -5.290834 & 12.397953 & -2.403642\end{array}$

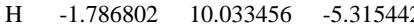

$\begin{array}{llll}\mathrm{H} & -3.535673 & 3.031816 & -3.818199\end{array}$

$\mathrm{H} \quad-1.141137 \quad 2.499850 \quad-4.317124$

$\mathrm{H} \quad 0.580520 \quad 4.330830 \quad-4.35525$

$\begin{array}{llll}\mathrm{H} & -0.083915 & 6.700425 & -3.888886\end{array}$

$\begin{array}{llll}\mathrm{H} & -4.151148 & 11.935328 & -0.363210\end{array}$

\section{$A$ = 6-methoxynaphthyl, $B$ = phenyl}

\section{Bispropargyl}

44

Free energy $=-1016.772431$ Hartree

$\begin{array}{llll}\mathrm{C} & -1.797375 & 2.125928 & -1.128962 \\ \mathrm{C} & -3.032958 & 2.663904 & -0.687668 \\ \mathrm{C} & -3.140839 & 3.178586 & 0.660266 \\ \mathrm{C} & -1.985935 & 3.139559 & 1.495263 \\ \mathrm{C} & -0.783832 & 2.609469 & 1.047511\end{array}$

$\begin{array}{lrrr}\mathrm{C} & -0.688065 & 2.081632 & -0.277294 \\ \mathrm{C} & -4.198908 & 2.689251 & -1.518726 \\ \mathrm{C} & -5.408082 & 3.163866 & -1.041485 \\ \mathrm{C} & -5.530791 & 3.648246 & 0.307502 \\ \mathrm{C} & -4.392157 & 3.663353 & 1.125906 \\ \mathrm{C} & -6.800651 & 4.017664 & 0.846409 \\ \mathrm{C} & -7.874606 & 4.254245 & 1.398984 \\ \mathrm{C} & -9.128482 & 4.331653 & 2.171757 \\ \mathrm{~N} & -9.549096 & 3.088679 & 2.839157 \\ \mathrm{C} & -8.646846 & 2.570446 & 3.879067 \\ \mathrm{C} & -7.398529 & 1.926063 & 3.425933 \\ \mathrm{C} & -6.391325 & 1.363796 & 2.998625 \\ \mathrm{C} & -5.214506 & 0.769299 & 2.441055 \\ \mathrm{C} & -5.209936 & 0.331239 & 1.089715 \\ \mathrm{C} & -4.045756 & -0.200818 & 0.520642 \\ \mathrm{C} & -2.868996 & -0.318058 & 1.282554 \\ \mathrm{C} & -2.864844 & 0.104923 & 2.623405 \\ \mathrm{C} & -4.023383 & 0.642805 & 3.203061 \\ \mathrm{O} & 0.444787 & 1.517354 & -0.785817 \\ \mathrm{C} & 1.603993 & 1.421620 & 0.040519 \\ \mathrm{H} & -1.951411 & -0.716772 & 0.824529 \\ \mathrm{H} & -9.219428 & 1.835153 & 4.489360 \\ \mathrm{H} & -9.030992 & 5.123946 & 2.947315 \\ \mathrm{H} & -6.120814 & 0.451046 & 0.485570 \\ \mathrm{H} & -4.478547 & 4.012978 & 2.165237 \\ \mathrm{H} & -4.050135 & -0.507119 & -0.535968 \\ \mathrm{H} & -1.942994 & 0.030395 & 3.220498 \\ \mathrm{H} & -4.125476 & 2.302566 & -2.547105 \\ \mathrm{H} & -6.300851 & 3.156355 & -1.684967 \\ \mathrm{H} & -9.960158 & 4.657735 & 1.509539 \\ \mathrm{H} & -4.014056 & 0.988349 & 4.247530 \\ \mathrm{H} & -1.701791 & 1.713555 & -2.144546 \\ \mathrm{H} & 0.079880 & 2.583287 & 1.725332 \\ \mathrm{H} & -2.062118 & 3.522698 & 2.524199 \\ \mathrm{H} & 2.380266 & 0.928755 & -0.573815 \\ \mathrm{H} & 1.973551 & 2.422764 & 0.353808 \\ \mathrm{H} & 1.415457 & 0.805639 & 0.947375 \\ \mathrm{H} & -8.403319 & 3.405181 & 4.573664 \\ \mathrm{H} & -9.716587 & 2.358019 & 2.134747\end{array}$

Alkyne_anionA

43

Free energy $=-1016.251747$ Hartree

$\begin{array}{llll}\text { C } & -2.125313 & -1.099387 & 5.517429\end{array}$

$\begin{array}{llll}\text { C } & -3.462410 & -1.322828 & 5.091226\end{array}$

$\begin{array}{llll}\text { C } & -4.073914 & -2.575103 & 5.381500\end{array}$

$\begin{array}{llll}\text { C } & -3.365032 & -3.562834 & 6.079313\end{array}$

$\begin{array}{llll}\text { C } & -2.041848 & -3.330041 & 6.501009\end{array}$

$\begin{array}{llll}\text { C } & -1.426312 & -2.095430 & 6.215955\end{array}$

$\begin{array}{llll}\text { C } & -4.215248 & -0.324190 & 4.398512\end{array}$

$\begin{array}{llll}\text { C } & -4.974148 & 0.467679 & 3.839326\end{array}$

$\begin{array}{llll}\text { C } & -5.938467 & 1.283105 & 3.074615\end{array}$

$\begin{array}{llll}\mathrm{N} & -6.407339 & 0.689160 & 1.822087\end{array}$

$\begin{array}{llll}\text { C } & -7.163157 & -0.535610 & 1.923489\end{array}$

$\begin{array}{llll}\text { C } & -7.448122 & -1.177556 & 3.088262\end{array}$

$\begin{array}{llll}\text { C } & -7.692286 & -1.728509 & 4.205536\end{array}$

$\begin{array}{llll}\text { C } & -7.773304 & -2.348340 & 5.447458\end{array}$

$\begin{array}{llll}\text { C } & -8.478284 & -3.566746 & 5.720867\end{array}$

$\begin{array}{llll}\text { C } & -8.497250 & -4.156162 & 7.008754\end{array}$

$\begin{array}{llll}\text { C } & -7.796227 & -3.520851 & 8.115583\end{array}$

$\begin{array}{llll}\text { C } & -7.098729 & -2.292801 & 7.838172\end{array}$

$\begin{array}{llll}\text { C } & -7.086796 & -1.734154 & 6.579367\end{array}$

$\begin{array}{llll}\text { C } & -9.178992 & -5.385075 & 7.300465\end{array}$

$\begin{array}{llll}\text { C } & -9.178583 & -5.954416 & 8.576131\end{array}$

$\begin{array}{llll}\text { C } & -8.491377 & -5.316138 & 9.646193\end{array}$

$\begin{array}{llll}\text { C } & -7.812872 & -4.112051 & 9.401100\end{array}$

$\begin{array}{llll}\text { O } & -8.434944 & -5.804476 & 10.939344\end{array}$

$\begin{array}{llll}\text { C } & -9.117721 & -7.012873 & 11.227629\end{array}$

$\mathrm{H} \quad-1.491218 \quad-4.108840 \quad 7.051783$

$\begin{array}{llll}\mathrm{H} & -5.497811 & 2.278525 & 2.848373\end{array}$

$\begin{array}{llll}\mathrm{H} & -7.522729 & -0.911771 & 0.949838\end{array}$

$\begin{array}{llll}\mathrm{H} & -1.644851 & -0.133746 & 5.296152\end{array}$

$\mathrm{H} \quad-9.011452 \quad-4.071821 \quad 4.898753$

$\begin{array}{llll}\mathrm{H} & -0.391675 & -1.906654 & 6.542583\end{array}$

$\begin{array}{llll}\mathrm{H} & -3.855228 & -4.523233 & 6.303592\end{array}$

$\begin{array}{llll}\mathrm{H} & -6.555296 & -1.798031 & 8.660810\end{array}$

$\begin{array}{llll}\mathrm{H} & -6.527807 & -0.803791 & 6.392251\end{array}$

$\begin{array}{llll}\mathrm{H} & -6.828361 & 1.466910 & 3.719452\end{array}$

$\begin{array}{llll}\mathrm{H} & -5.109353 & -2.748102 & 5.054091\end{array}$

$\begin{array}{llll}\mathrm{H} & -9.715458 & -5.897791 & 6.484693\end{array}$

$\begin{array}{llll}\mathrm{H} & -9.711529 & -6.902532 & 8.736869\end{array}$

$\begin{array}{llll}\mathrm{H} & -7.281229 & -3.627729 & 10.236468\end{array}$

$\begin{array}{llll}\mathrm{H} & -8.951478 & -7.221453 & 12.302278\end{array}$

$\begin{array}{llll}\mathrm{H} & -10.215027 & -6.932670 & 11.043724\end{array}$

$\begin{array}{llll}\mathrm{H} & -8.725988 & -7.871839 & 10.634446\end{array}$

$\begin{array}{llll}\mathrm{H} & -8.725988 & -7.871839 & 10.634446 \\ \mathrm{H} & -5.617463 & 0.579822 & 1.167303\end{array}$

Alkyne_anionB

43

Free energy $=-1016.254001$ Hartree 


$\begin{array}{rrrr}\mathrm{C} & 1.749997 & 6.354870 & -0.616983 \\ \mathrm{C} & 0.660162 & 5.957486 & -1.484322 \\ \mathrm{C} & 0.009734 & 4.705080 & -1.146517 \\ \mathrm{C} & 0.400154 & 3.957111 & -0.034558 \\ \mathrm{C} & 1.456883 & 4.375851 & 0.807504 \\ \mathrm{C} & 2.122205 & 5.584743 & 0.486226 \\ \mathrm{C} & 0.228751 & 6.731718 & -2.557371 \\ \mathrm{C} & -0.336132 & 7.515523 & -3.382731 \\ \mathrm{C} & -0.952242 & 8.346510 & -4.264771 \\ \mathrm{~N} & -2.142366 & 8.043357 & -5.007941 \\ \mathrm{C} & -2.881625 & 6.825219 & -4.659439 \\ \mathrm{C} & -3.373093 & 6.775953 & -3.268643 \\ \mathrm{C} & -3.576104 & 6.903612 & -2.058224 \\ \mathrm{C} & -3.611156 & 6.956531 & -0.632456 \\ \mathrm{C} & -2.413131 & 6.736266 & 0.077396 \\ \mathrm{C} & -2.379764 & 6.756222 & 1.497852 \\ \mathrm{C} & -3.595802 & 7.021929 & 2.239402 \\ \mathrm{C} & -4.803323 & 7.249749 & 1.497290 \\ \mathrm{C} & -4.813924 & 7.215646 & 0.111164 \\ \mathrm{C} & -1.175078 & 6.510405 & 2.225826 \\ \mathrm{C} & -1.155833 & 6.535177 & 3.618054 \\ \mathrm{C} & -2.353255 & 6.808979 & 4.345384 \\ \mathrm{C} & -3.551845 & 7.045347 & 3.657784 \\ \mathrm{O} & -2.420099 & 6.855571 & 5.712504 \\ \mathrm{C} & -1.238098 & 6.606998 & 6.466689 \\ \mathrm{H} & 1.751098 & 3.782846 & 1.686737 \\ \mathrm{H} & -0.565178 & 9.361985 & -4.458383 \\ \mathrm{H} & -2.199249 & 5.958423 & -4.815010 \\ \mathrm{H} & 2.275712 & 7.299525 & -0.830794 \\ \mathrm{H} & -1.483809 & 6.550625 & -0.476275 \\ \mathrm{H} & 2.949149 & 5.937549 & 1.126635 \\ \mathrm{H} & -0.140276 & 3.022190 & 0.191706 \\ \mathrm{H} & -5.734625 & 7.454718 & 2.049890 \\ \mathrm{H} & -5.752210 & 7.390786 & -0.438120 \\ \mathrm{H} & -3.716995 & 6.707098 & -5.382312 \\ \mathrm{H} & -0.829105 & 4.366648 & -1.775540 \\ \mathrm{H} & -4.464185 & 7.249969 & 4.239185 \\ \mathrm{H} & -0.212087 & 6.334034 & 4.143453 \\ \mathrm{H} & -0.253084 & 6.280047 & 1.667321 \\ \mathrm{H} & -1.526732 & 6.689553 & 7.531754 \\ \mathrm{H} & -0.833652 & 5.586109 & 6.282654 \\ \mathrm{H} & -0.440919 & 7.353144 & 6.250150 \\ \mathrm{H} & -2.774020 & 8.856795 & -5.023725 \\ & & & \end{array}$

\section{A = 2-pyridyl, B = phenyl}

\section{Bispropargyl}

33

Free energy $=-764.887048$ Hartree

$\begin{array}{llll}\text { C } & 1.438151 & 3.709095 & -0.090732\end{array}$

$\begin{array}{llll}\text { C } & 2.496040 & 4.104588 & -0.950351\end{array}$

$\begin{array}{llll}\text { C } & 2.237742 & 4.228588 & -2.342673\end{array}$

$\begin{array}{llll}\text { C } & 0.958003 & 3.971750 & -2.851637\end{array}$

$\begin{array}{llll}\text { C } & 0.958003 & 3.971750 & -2.851637 \\ \text { C } & -0.085127 & 3.584489 & -1.989950\end{array}$

$\begin{array}{llll}\text { C } & 0.160216 & 3.454592 & -0.610999\end{array}$

$\begin{array}{llll}\text { C } & 3.790925 & 4.425698 & -0.434076\end{array}$

$\begin{array}{llll}\text { C } & 4.895544 & 4.779760 & -0.026429\end{array}$

$\begin{array}{llll}\text { C } & 6.217346 & 5.303957 & 0.366453\end{array}$

$\begin{array}{llll}\mathrm{N} & 6.782390 & 6.357528 & -0.489528\end{array}$

$\begin{array}{llll}\mathrm{C} & 6.118442 & 7.668598 & -0.440500\end{array}$

$\begin{array}{llll}\text { C } & 4.755840 & 7.734468 & -1.002268\end{array}$

$\begin{array}{llll}\text { C } & 3.628331 & 7.686797 & -1.488999\end{array}$

$\begin{array}{llll}\text { C } & 2.282493 & 7.539823 & -1.968648\end{array}$

$\begin{array}{llll}\text { C } & 1.251343 & 7.189483 & -1.057363\end{array}$

$\begin{array}{llll}\text { C } & -0.046658 & 7.005294 & -1.545369\end{array}$

$\begin{array}{llll}\text { C } & -0.282372 & 7.181444 & -2.919179\end{array}$

$\begin{array}{llll}\text { C } & 0.801668 & 7.535564 & -3.742155\end{array}$

\begin{tabular}{llll}
$\mathrm{N}$ & 2.056808 & 7.715188 & -3.297065 \\
\hline
\end{tabular}

$\begin{array}{llll}\mathrm{H} & -1.091357 & 3.391911 & -2.392396\end{array}$

$\begin{array}{llll}\mathrm{H} & -1.284055 & 7.037939 & -3.351008\end{array}$

$\begin{array}{llll}\mathrm{H} & 6.765118 & 8.398402 & -0.974665\end{array}$

$\begin{array}{llll}\mathrm{H} & 6.165181 & 5.692124 & 1.407936\end{array}$

$\begin{array}{llll}\mathrm{H} & 1.490317 & 7.041791 & 0.005294\end{array}$

$\begin{array}{llll}\mathrm{H} & 3.047625 & 4.555974 & -3.011063\end{array}$

$\begin{array}{llll}\mathrm{H} & -0.860747 & 6.713211 & -0.865280\end{array}$

$\begin{array}{llll}\mathrm{H} & 0.767999 & 4.091437 & -3.929329\end{array}$

$\begin{array}{llll}\mathrm{H} & 0.645084 & 7.680908 & -4.826372\end{array}$

$\begin{array}{llll}\mathrm{H} & -0.653566 & 3.155741 & 0.067908\end{array}$

$\begin{array}{llll}\mathrm{H} & 1.628884 & 3.615141 & 0.989068\end{array}$

$\begin{array}{llll}\mathrm{H} & 6.946566 & 4.463366 & 0.396671\end{array}$

$\begin{array}{llll}\mathrm{H} & 6.090719 & 8.004802 & 0.619578\end{array}$

$\begin{array}{llll}\mathrm{H} & 6.831565 & 6.026894 & -1.462533\end{array}$

Alkyne_anionA

32

Free energy $=-764.3802359$ Hartree

$\begin{array}{llll}\mathrm{C} & 1.602722 & -0.312567 & 0.221113 \\ \mathrm{C} & 2.995485 & 0.081768 & 0.124218 \\ \mathrm{~N} & 3.710718 & -0.200654 & -1.038111 \\ \mathrm{C} & 3.083858 & -0.853476 & -2.025604\end{array}$ $\begin{array}{llll}\text { C } & 1.743045 & -1.281269 & -2.004723\end{array}$

$\begin{array}{llll}\text { C } & 0.996990 & -0.986080 & -0.831718\end{array}$

$\begin{array}{llll}\text { C } & 3.641792 & 0.737792 & 1.167234\end{array}$

$\begin{array}{llll}\text { C } & 4.265571 & 1.209123 & 2.166669\end{array}$

$\begin{array}{llll}\text { C } & 4.944247 & 1.780927 & 3.199778\end{array}$

$\begin{array}{llll}\mathrm{N} & 6.326229 & 2.190732 & 3.121902 \\ \mathrm{C} & 7.307152 & 1.204293 & 3.637938\end{array}$

$\begin{array}{llll}\text { C } & 7.307152 & 1.204293 & 3.637938\end{array}$

$\begin{array}{llll}\text { C } & 7.335543 & -0.058107 & 2.879433\end{array}$

$\begin{array}{llll}\text { C } & 7.224030 & -1.029309 & 2.130514\end{array}$

$\begin{array}{llll}\text { C } & 7.035958 & -2.132804 & 1.238356\end{array}$

$\begin{array}{llll}\text { C } & 6.125580 & -2.012352 & 0.152752\end{array}$

$\begin{array}{llll}\text { C } & 5.914887 & -3.096220 & -0.712056\end{array}$

$\begin{array}{llll}\text { C } & 6.603326 & -4.310099 & -0.524476\end{array}$

$\begin{array}{llll}\text { C } & 7.511770 & -4.435307 & 0.543844\end{array}$

$\begin{array}{llll}\text { C } & 7.727668 & -3.360811 & 1.420680\end{array}$

$\begin{array}{llll}\mathrm{H} & 6.431410 & -5.158005 & -1.206617\end{array}$

H $\quad \begin{array}{llll}1.302014 & -1.812976 & -2.861168\end{array}$

$\begin{array}{llll}\mathrm{H} & 4.464505 & 1.934047 & 4.184550\end{array}$

$\begin{array}{llll}\mathrm{H} & 7.064687 & 0.998993 & 4.704296\end{array}$

$\begin{array}{llll}\mathrm{H} & 1.042779 & -0.075060 & 1.139685\end{array}$

$\begin{array}{llll}\mathrm{H} & 5.569025 & -1.075266 & -0.017631\end{array}$

$\begin{array}{llll}\mathrm{H} & -0.059887 & -1.291933 & -0.751047\end{array}$

$\begin{array}{llll}\mathrm{H} & 5.194481 & -2.983405 & -1.538262\end{array}$

$\begin{array}{llll}\mathrm{H} & 3.701850 & -1.059380 & -2.923498\end{array}$

$\mathrm{H} \quad 8.053806 \quad-5.381663 \quad 0.699231$

$\begin{array}{llll}\mathrm{H} & 8.430900 & -3.462969 & 2.261894\end{array}$

$\begin{array}{llll}\mathrm{H} & 8.320943 & 1.668028 & 3.637003\end{array}$

$\begin{array}{llll}\mathrm{H} & 6.565986 & 2.388800 & 2.141021\end{array}$

Alkyne_anionB

32

Free energy $=-764.3698632$ Hartree

$\begin{array}{llll}\text { C } & -0.441318 & 3.679144 & -0.634257\end{array}$

$\begin{array}{llll}\text { C } & 0.537385 & 4.460672 & -1.364863\end{array}$

$\begin{array}{llll}\text { C } & 0.041145 & 5.161385 & -2.530744\end{array}$

$\begin{array}{llll}\text { C } & -1.297476 & 5.072754 & -2.919989\end{array}$

$\begin{array}{llll}\text { C } & -2.233696 & 4.300081 & -2.191347\end{array}$

$\begin{array}{llll}\text { C } & -1.773818 & 3.608872 & -1.045841\end{array}$

$\begin{array}{llll}\text { C } & 1.869136 & 4.506334 & -0.961825\end{array}$

$\begin{array}{llll}\text { C } & 3.083162 & 4.434650 & -0.599121\end{array}$

$\begin{array}{llll}\mathrm{C} & 4.373696 & 4.340172 & -0.174069\end{array}$

$\begin{array}{llll}\mathrm{N} & 4.807735 & 3.651342 & 1.016206\end{array}$

$\begin{array}{llll}\text { C } & 3.772031 & 3.088365 & 1.880014\end{array}$

$\begin{array}{llll}\text { C } & 2.995414 & 1.989542 & 1.278228\end{array}$

$\begin{array}{llll}\text { C } & 2.413701 & 1.116214 & 0.632276\end{array}$

$\begin{array}{llll}\text { C } & 1.638102 & 0.282103 & -0.234599\end{array}$

$\begin{array}{llll}\text { C } & 1.092044 & 0.860883 & -1.417302\end{array}$

$\begin{array}{llll}\text { C } & 0.301751 & 0.064160 & -2.252262\end{array}$

$\begin{array}{llll}\text { C } & 0.072650 & -1.278213 & -1.896885\end{array}$

$\begin{array}{llll}\text { C } & 0.661950 & -1.760835 & -0.710741\end{array}$

$\begin{array}{llll}\mathrm{N} & 1.425490 & -1.021347 & 0.109042\end{array}$

$\begin{array}{llll}\mathrm{H} & -3.285664 & 4.234449 & -2.510111\end{array}$

$\mathrm{H} \quad-0.546891 \quad-1.940868 \quad-2.520300$

$\begin{array}{llll}\mathrm{H} & 4.245016 & 2.745079 & 2.826265\end{array}$

$\begin{array}{llll}\mathrm{H} & 5.202684 & 4.801478 & -0.738999\end{array}$

$\begin{array}{llll}\mathrm{H} & 1.303795 & 1.915085 & -1.647963\end{array}$

$\begin{array}{llll}\mathrm{H} & 0.747228 & 5.767605 & -3.121733\end{array}$

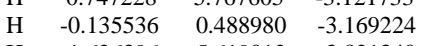

$\begin{array}{llll}\mathrm{H} & -1.626396 & 5.619012 & -3.821340\end{array}$

$\begin{array}{llll}\mathrm{H} & 0.501797 & -2.812672 & -0.408765\end{array}$

$\begin{array}{llll}\mathrm{H} & -2.478002 & 2.993734 & -0.458943\end{array}$

$\begin{array}{llll}\mathrm{H} & -0.103832 & 3.124142 & 0.255008\end{array}$

$\begin{array}{llll}\mathrm{H} & 3.069107 & 3.909894 & 2.150392 \\ \mathrm{H} & 5.516573 & 2.933211 & 0.797244\end{array}$

\section{A = 2-pyridyl, B = 4-methoxyphenyl}

Bispropargyl

37

Free energy $=-879.3101003$ Hartree

$\begin{array}{lrrr}\mathrm{C} & -0.130540 & 2.450831 & -0.212315 \\ \mathrm{C} & 0.533716 & 1.487652 & -1.014675 \\ \mathrm{C} & -0.250256 & 0.704884 & -1.906098 \\ \mathrm{C} & -1.627396 & 0.904868 & -2.010215 \\ \mathrm{C} & -2.269956 & 1.893487 & -1.226514 \\ \mathrm{C} & -1.512692 & 2.653182 & -0.307147 \\ \mathrm{C} & 1.958267 & 1.398630 & -0.980157 \\ \mathrm{C} & 3.187824 & 1.451758 & -0.959822 \\ \mathrm{C} & 4.630412 & 1.742199 & -1.051550 \\ \mathrm{~N} & 4.998238 & 2.882847 & -1.908100 \\ \mathrm{C} & 4.487215 & 4.196423 & -1.489940 \\ \mathrm{C} & 3.058566 & 4.468314 & -1.738338 \\ \mathrm{C} & 1.876700 & 4.676006 & -2.006256 \\ \mathrm{C} & 0.482434 & 4.806984 & -2.327786 \\ \mathrm{~N} & -0.234736 & 5.762003 & -1.679733 \\ \mathrm{C} & -1.543541 & 5.859510 & -1.969284 \\ \mathrm{C} & -2.208940 & 5.038778 & -2.897572 \\ \mathrm{C} & -1.464058 & 4.049420 & -3.562041 \\ \mathrm{C} & -0.098754 & 3.932041 & -3.283115 \\ \mathrm{O} & -3.604304 & 2.051123 & -1.440515\end{array}$




$\begin{array}{rrrr}\mathrm{C} & -4.314978 & 3.006065 & -0.652791 \\ \mathrm{H} & -3.284233 & 5.170753 & -3.089540 \\ \mathrm{H} & 5.081124 & 4.979668 & -2.012356 \\ \mathrm{H} & 5.032428 & 1.932487 & -0.031174 \\ \mathrm{H} & 0.519673 & 3.161637 & -3.764270 \\ \mathrm{H} & 0.461654 & 3.078278 & 0.469509 \\ \mathrm{H} & -1.945491 & 3.369622 & -4.281262 \\ \mathrm{H} & -1.981498 & 3.431576 & 0.309071 \\ \mathrm{H} & -2.103667 & 6.643060 & -1.427230 \\ \mathrm{H} & -2.236026 & 0.317390 & -2.713786 \\ \mathrm{H} & 0.242313 & -0.050413 & -2.537025 \\ \mathrm{H} & -5.365164 & 2.970291 & -0.996491 \\ \mathrm{H} & -3.918282 & 4.033872 & -0.800487 \\ \mathrm{H} & -4.275055 & 2.752980 & 0.430020 \\ \mathrm{H} & 5.175691 & 0.849206 & -1.427371 \\ \mathrm{H} & 4.706338 & 4.329578 & -0.407687 \\ \mathrm{H} & 4.706277 & 2.694288 & -2.876552\end{array}$

\begin{tabular}{lrrr}
\multicolumn{5}{l}{ Alkyne_anionA } \\
36 \\
Free energy = -878.801672 Hartree \\
N & -0.286612 & -2.675438 & 0.635005 \\
$\mathrm{C}$ & 0.669629 & -1.667619 & 0.596918 \\
$\mathrm{C}$ & 0.265907 & -0.282673 & 0.731862 \\
$\mathrm{C}$ & -1.069837 & 0.035017 & 0.926445 \\
$\mathrm{C}$ & -2.036246 & -1.004138 & 0.996218 \\
$\mathrm{C}$ & -1.565271 & -2.319607 & 0.826501 \\
$\mathrm{C}$ & 2.013040 & -1.998851 & 0.418818 \\
$\mathrm{C}$ & 3.230254 & -2.223698 & 0.147703 \\
$\mathrm{C}$ & 4.561957 & -2.418799 & -0.081645 \\
$\mathrm{~N}$ & 5.521778 & -1.348120 & -0.115450 \\
$\mathrm{C}$ & 5.842249 & -0.802092 & -1.471965 \\
$\mathrm{C}$ & 4.656218 & -0.222861 & -2.126775 \\
$\mathrm{C}$ & 3.475685 & 0.100935 & -2.299957 \\
$\mathrm{C}$ & 2.079212 & 0.353524 & -2.421386 \\
$\mathrm{C}$ & 1.533027 & 1.667462 & -2.392254 \\
$\mathrm{C}$ & 0.152154 & 1.869522 & -2.389072 \\
$\mathrm{C}$ & -0.733057 & 0.764456 & -2.402990 \\
$\mathrm{C}$ & -0.210645 & -0.544454 & -2.466842 \\
$\mathrm{C}$ & 1.175567 & -0.742332 & -2.474440 \\
$\mathrm{O}$ & -2.063301 & 1.064070 & -2.346298 \\
$\mathrm{C}$ & -2.998600 & -0.013368 & -2.352187 \\
$\mathrm{H}$ & -3.104616 & -0.798351 & 1.160685 \\
$\mathrm{H}$ & 4.960219 & -3.416339 & -0.344232 \\
$\mathrm{H}$ & 6.259443 & -1.630641 & -2.084992 \\
$\mathrm{H}$ & 1.029600 & 0.503541 & 0.630804 \\
$\mathrm{H}$ & 1.579277 & -1.763856 & -2.462305 \\
$\mathrm{H}$ & -1.377327 & 1.091171 & 1.003508 \\
$\mathrm{H}$ & -0.871664 & -1.420675 & -2.442913 \\
$\mathrm{H}$ & -2.293413 & -3.156888 & 0.848739 \\
$\mathrm{H}$ & -0.275741 & 2.882853 & -2.347257 \\
$\mathrm{H}$ & 2.212346 & 2.532755 & -2.351976 \\
$\mathrm{H}$ & -4.001971 & 0.447823 & -2.287842 \\
$\mathrm{H}$ & -2.846231 & -0.685066 & -1.478523 \\
$\mathrm{H}$ & -2.932823 & -0.607620 & -3.291883 \\
$\mathrm{H}$ & 6.653688 & -0.047165 & -1.368387 \\
$\mathrm{H}$ & 5.158526 & -0.564187 & 0.444077 \\
& & & \\
\hline
\end{tabular}

Alkyne_anionB

Free energy $=-878.7878252$ Hartree

$\begin{array}{lrrr}\mathrm{C} & -0.227141 & -1.058701 & 1.141567 \\ \mathrm{C} & -0.025169 & -2.373720 & 1.692464 \\ \mathrm{C} & -1.138984 & -2.928869 & 2.407990 \\ \mathrm{C} & -2.346904 & -2.234801 & 2.566779 \\ \mathrm{C} & -2.509747 & -0.943302 & 2.019623 \\ \mathrm{C} & -1.429774 & -0.368827 & 1.308797 \\ \mathrm{C} & 1.193660 & -3.058475 & 1.563367 \\ \mathrm{C} & 2.297150 & -3.666531 & 1.479873 \\ \mathrm{C} & 3.467822 & -4.377053 & 1.364836 \\ \mathrm{~N} & 3.662023 & -5.678823 & 1.914032 \\ \mathrm{C} & 4.426217 & -5.766473 & 3.211951 \\ \mathrm{C} & 3.700200 & -5.089881 & 4.295595 \\ \mathrm{C} & 2.765045 & -4.437005 & 4.786241 \\ \mathrm{C} & 1.684070 & -3.592849 & 5.162724 \\ \mathrm{C} & 1.598267 & -2.291434 & 4.574376 \\ \mathrm{C} & 0.465149 & -1.513859 & 4.823758 \\ \mathrm{C} & -0.543211 & -2.025234 & 5.658822 \\ \mathrm{C} & -0.347177 & -3.297265 & 6.235875 \\ \mathrm{~N} & 0.719111 & -4.075906 & 6.003194 \\ \mathrm{O} & -3.656498 & -0.173525 & 2.122412 \\ \mathrm{C} & -4.742662 & -0.706456 & 2.859115 \\ \mathrm{H} & -1.464844 & -1.457939 & 5.856277 \\ \mathrm{H} & 4.607261 & -6.838664 & 3.445888 \\ \mathrm{H} & 4.373057 & -3.912699 & 0.931764 \\ \mathrm{H} & 2.394272 & -1.951168 & 3.899628 \\ \mathrm{H} & -1.023640 & -3.917532 & 2.876845 \\ \mathrm{H} & 0.345286 & -0.536023 & 4.332936 \\ \mathrm{H} & -3.154415 & -2.711495 & 3.141222 \\ \mathrm{H} & -1.115577 & -3.714457 & 6.914474\end{array}$

$\begin{array}{rrrr}\text { H } & -1.559755 & 0.640915 & 0.887471 \\ \mathrm{H} & 0.598042 & -0.582370 & 0.588453 \\ \mathrm{H} & -5.549291 & 0.052356 & 2.833412 \\ \mathrm{H} & -4.473215 & -0.907412 & 3.922962 \\ \mathrm{H} & -5.129502 & -1.654448 & 2.416366 \\ \mathrm{H} & 5.422868 & -5.303859 & 3.048780 \\ \mathrm{H} & 2.744060 & -6.114449 & 2.078101\end{array}$

\section{A = 1-benzyl-3-indolyl, $B$ = phenyl}

Bispropargyl

51

Free energy $=-1150.406859$ Hartree

$\begin{array}{llll}\text { C } & 6.532696 & 6.308724 & 1.688514 \\ \text { C } & 5.446927 & 5.934630 & 0.871555\end{array}$

$\begin{array}{llll}\text { C } & 5.320210 & 6.513118 & -0.407677\end{array}$

$\begin{array}{llll}\text { C } & 6.258676 & 7.456582 & -0.858711\end{array}$

$\begin{array}{llll}\text { C } & 7.335218 & 7.833167 & -0.034619\end{array}$

$\begin{array}{llll}\text { C } & 7.470029 & 7.256226 & 1.240849\end{array}$

$\begin{array}{llll}\text { C } & 4.417412 & 4.937859 & 1.383892\end{array}$

$\begin{array}{llll}\mathrm{N} & 3.844407 & 4.104295 & 0.340500\end{array}$

$\begin{array}{llll}\text { C } & 4.547365 & 3.136662 & -0.367502\end{array}$

$\begin{array}{llll}\text { C } & 3.640419 & 2.521894 & -1.284276\end{array}$

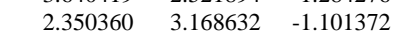

$\begin{array}{llll}\text { C } & 2.536923 & 4.128304 & -0.100009\end{array}$

$\begin{array}{llll}\text { C } & 5.893643 & 2.741266 & -0.264142\end{array}$

$\begin{array}{llll}\text { C } & 6.317607 & 1.690371 & -1.088309\end{array}$

$\begin{array}{llll}\text { C } & 5.424660 & 1.053449 & -1.989677\end{array}$

$\begin{array}{llll}\text { C } & 4.088613 & 1.461503 & -2.097567\end{array}$

$\begin{array}{llll}\text { C } & 1.150181 & 2.781354 & -1.744312\end{array}$

$\begin{array}{llll}\text { C } & 0.172795 & 2.298927 & -2.319043\end{array}$

$\begin{array}{llll}\text { C } & -0.961928 & 1.502552 & -2.821050\end{array}$

$\begin{array}{llll}\mathrm{N} & -1.354365 & 0.341767 & -2.002683\end{array}$

$\begin{array}{llll}\text { C } & -0.329951 & -0.699744 & -1.823037\end{array}$

$\begin{array}{llll}\text { C } & 0.758421 & -0.402474 & -0.872133\end{array}$

$\begin{array}{llll}\text { C } & 1.612609 & -0.106058 & -0.038378\end{array}$

$\begin{array}{llll}\text { C } & 2.601095 & 0.336906 & 0.896175\end{array}$

$\begin{array}{llll}\text { C } & 3.906632 & -0.220451 & 0.908504\end{array}$

$\begin{array}{llll}\text { C } & 4.877446 & 0.268309 & 1.793047\end{array}$

$\begin{array}{llll}\text { C } & 4.568883 & 1.313142 & 2.683416\end{array}$

$\begin{array}{llll}\text { C } & 3.275053 & 1.866586 & 2.685899\end{array}$

$\begin{array}{llll}\text { C } & 2.296977 & 1.385239 & 1.804624\end{array}$

$\begin{array}{llll}\mathrm{H} & 5.338908 & 1.700888 & 3.368425\end{array}$

$\begin{array}{llll}\mathrm{H} & -1.859280 & 2.148953 & -2.938851\end{array}$

$\begin{array}{llll}\mathrm{H} & -0.842029 & -1.631305 & -1.490913\end{array}$

$\begin{array}{llll}\mathrm{H} & 1.292334 & 1.831938 & 1.787637\end{array}$

$\begin{array}{llll}\mathrm{H} & 3.027605 & 2.690246 & 3.373944\end{array}$

$\begin{array}{llll}\mathrm{H} & 5.891465 & -0.158926 & 1.774847\end{array}$

$\begin{array}{llll}\mathrm{H} & 4.155749 & -1.020170 & 0.196316\end{array}$

$\begin{array}{llll}\mathrm{H} & 3.581073 & 5.470483 & 1.881834\end{array}$

$\begin{array}{llll}\mathrm{H} & 4.872538 & 4.278000 & 2.153455\end{array}$

$\begin{array}{llll}\mathrm{H} & 6.647027 & 5.852085 & 2.685115\end{array}$

$\begin{array}{llll}\mathrm{H} & 8.314299 & 7.538611 & 1.889271\end{array}$

$\begin{array}{llll}\mathrm{H} & 8.072161 & 8.571283 & -0.388233\end{array}$

$\begin{array}{llll}\mathrm{H} & 6.150026 & 7.898789 & -1.861106\end{array}$

$\begin{array}{llll}\mathrm{H} & 4.483226 & 6.214259 & -1.059011\end{array}$

$\begin{array}{llll}\mathrm{H} & 1.810872 & 4.815326 & 0.349698\end{array}$

$\mathrm{H} \quad 0.100390 \quad-0.939443 \quad-2.820800$

$\begin{array}{llll}\mathrm{H} & 3.387051 & 0.960931 & -2.782166\end{array}$

$\begin{array}{llll}\mathrm{H} & 5.791537 & 0.220784 & -2.610367\end{array}$

$\begin{array}{llll}\mathrm{H} & 7.361376 & 1.345399 & -1.027195\end{array}$

$\begin{array}{llll}\mathrm{H} & 6.580504 & 3.230786 & 0.441294\end{array}$

$\begin{array}{llll}\mathrm{H} & -0.722868 & 1.135787 & -3.844598\end{array}$

$\begin{array}{llll}\mathrm{H} & -1.673624 & 0.666136 & -1.079851\end{array}$

\begin{tabular}{|c|c|c|c|}
\hline $\begin{array}{l}50 \\
\text { Fre }\end{array}$ & $g y=-1$ & & \\
\hline C & 9.844940 & 4.325532 & -0.748583 \\
\hline $\mathrm{C}$ & 9.646608 & 5.114524 & -1.915781 \\
\hline C & 10.731878 & 5.271805 & -2.827453 \\
\hline C & 11.962901 & 4.654888 & -2.573457 \\
\hline $\mathrm{C}$ & 12.147192 & 3.8 & 052 \\
\hline $\mathrm{C}$ & 11.0 & 3.7 & 219 \\
\hline C & 8.4 & 5.7 & -2 \\
\hline $\mathrm{C}$ & 7.39 & 6.3 & -2 \\
\hline $\mathrm{C}$ & 6.3 & 7.1 & -3 \\
\hline $\mathrm{N}$ & 6.65 & 8.597695 & 688 \\
\hline $\mathrm{C}$ & 7.674155 & 8.983819 & -4.182209 \\
\hline $\mathrm{C}$ & 8.444506 & 8.124996 & -4.919672 \\
\hline $\mathrm{C}$ & 9.138043 & 7.334133 & -5.624543 \\
\hline$r$ & 9.894643 & 6.399937 & -6.338173 \\
\hline C & 9.769703 & 4.940944 & -6.203013 \\
\hline C & 10.725310 & 4.329111 & -7.083476 \\
\hline $\mathrm{N}$ & 11.430549 & 5.331906 & -7.712177 \\
\hline 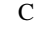 & 10.918504 & 6.582271 & -7.299822 \\
\hline 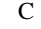 & 8.938263 & 4.127745 & -5.410206 \\
\hline C & 9.044594 & 2.730607 & -5.511379 \\
\hline $\mathrm{C}$ & 9.977613 & 2.139365 & -6.404102 \\
\hline & & & \\
\hline
\end{tabular}




$\begin{array}{cccc}\mathrm{C} & 12.325772 & 5.144497 & -8.830220 \\ \mathrm{C} & 11.632056 & 4.919975 & -10.170502 \\ \mathrm{C} & 10.262330 & 5.204113 & -10.341215 \\ \mathrm{C} & 9.643570 & 5.005810 & -11.588891 \\ \mathrm{C} & 10.385885 & 4.516117 & -12.680020 \\ \mathrm{C} & 11.751666 & 4.222552 & -12.514117 \\ \mathrm{C} & 12.369461 & 4.423150 & -11.266936 \\ \mathrm{H} & 13.115531 & 3.379820 & -1.227729 \\ \mathrm{H} & 7.811759 & 10.077474 & -4.253864 \\ \mathrm{H} & 5.398903 & 7.057310 & -2.540684 \\ \mathrm{H} & 10.577898 & 5.879540 & -3.732031 \\ \mathrm{H} & 12.788260 & 4.782551 & -3.292201 \\ \mathrm{H} & 11.219044 & 3.100920 & 0.399678 \\ \mathrm{H} & 9.013982 & 4.200047 & -0.036520 \\ \mathrm{H} & 12.985171 & 6.038001 & -8.896883 \\ \mathrm{H} & 13.000521 & 4.288107 & -8.614741 \\ \mathrm{H} & 13.438918 & 4.186449 & -11.139740 \\ \mathrm{H} & 12.338727 & 3.829872 & -13.359650 \\ \mathrm{H} & 9.899897 & 4.357398 & -13.655723 \\ \mathrm{H} & 8.572776 & 5.234660 & -11.708111 \\ \mathrm{H} & 9.681528 & 5.586290 & -9.486894 \\ \mathrm{H} & 11.352305 & 7.505358 & -7.699577 \\ \mathrm{H} & 6.057696 & 6.844490 & -4.189926 \\ \mathrm{H} & 8.229179 & 4.600307 & -4.711909 \\ \mathrm{H} & 8.401148 & 2.086583 & -4.891065 \\ \mathrm{H} & 10.038557 & 1.040579 & -6.477455 \\ \mathrm{H} & 11.536564 & 2.460103 & -7.900672 \\ \mathrm{H} & 6.912938 & 8.920175 & -2.269836\end{array}$

Alkyne_anionB

50

Free energy $=-1149.887843$ Hartree

$\begin{array}{llll}\text { C } & 5.893438 & 12.347250 & -0.987588\end{array}$

$\begin{array}{llll}\text { C } & 5.700505 & 11.271199 & -1.933731\end{array}$

$\begin{array}{llll}\text { C } & 4.505562 & 11.356002 & -2.741599\end{array}$

$\begin{array}{llll}\text { C } & 3.578606 & 12.393384 & -2.575568\end{array}$

$\begin{array}{llll}\text { C } & 3.778918 & 13.419467 & -1.622373\end{array}$

$\begin{array}{llll}\text { C } & 4.957221 & 13.373508 & -0.839611\end{array}$

$\begin{array}{llll}\text { C } & 6.631438 & 10.219804 & -2.043466\end{array}$

$\begin{array}{llll}\text { C } & 6.949301 & 9.022574 & -1.703497\end{array}$

$\begin{array}{llll}\text { C } & 7.349524 & 7.784288 & -1.336476\end{array}$

$\begin{array}{llll}\text { N } & 8.144974 & 7.472883 & -0.164017\end{array}$

$\begin{array}{llll}\text { C } & 8.272167 & 8.552147 & 0.824581\end{array}$

$\begin{array}{llll}\text { C } & 6.999972 & 9.070122 & 1.368679\end{array}$

$\begin{array}{llll}\text { C } & 5.866843 & 9.453855 & 1.664167\end{array}$

$\begin{array}{llll}\text { C } & 4.569913 & 9.987232 & 1.882582\end{array}$

$\begin{array}{llll}\text { C } & 3.794443 & 10.051199 & 3.110945\end{array}$

$\begin{array}{llll}\text { C } & 2.570993 & 10.730934 & 2.794702\end{array}$

$\begin{array}{llll}\mathrm{N} & 2.596542 & 11.043733 & 1.440317\end{array}$

$\begin{array}{llll}\text { C } & 3.790405 & 10.609919 & 0.900035\end{array}$

$\begin{array}{llll}\text { C } & 4.015895 & 9.616164 & 4.436025\end{array}$

$\begin{array}{llll}\text { C } & 3.033997 & 9.869468 & 5.405130\end{array}$

$\begin{array}{llll}\text { C } & 1.835754 & 10.556760 & 5.074881\end{array}$

$\begin{array}{llll}\text { C } & 1.588925 & 10.999665 & 3.766068\end{array}$

$\begin{array}{llll}\text { C } & 1.620294 & 11.864625 & 0.743998\end{array}$

$\begin{array}{llll}\text { C } & 1.660675 & 13.335061 & 1.133183\end{array}$

$\begin{array}{llll}\text { C } & 0.563674 & 14.164766 & 0.823461\end{array}$

$\begin{array}{llll}\text { C } & 0.602494 & 15.539673 & 1.112412\end{array}$

$\begin{array}{llll}\text { C } & 1.738776 & 16.099954 & 1.725248\end{array}$

$\begin{array}{llll}\text { C } & 2.832203 & 15.274724 & 2.044394\end{array}$

$\begin{array}{llll}\text { C } & 2.793232 & 13.901576 & 1.749076\end{array}$

$\begin{array}{llll}\mathrm{H} & 3.035228 & 14.216788 & -1.473704\end{array}$

$\begin{array}{llll}\mathrm{H} & 8.806684 & 9.399073 & 0.337907\end{array}$

$\begin{array}{llll}\mathrm{H} & 7.064385 & 6.888284 & -1.916048\end{array}$

$\begin{array}{llll}\mathrm{H} & 4.310030 & 10.556521 & -3.475383\end{array}$

$\begin{array}{llll}\mathrm{H} & 2.664335 & 12.395619 & -3.194878\end{array}$

\begin{tabular}{llll}
$\mathrm{H}$ & 5.131590 & 14.153098 & -0.078741 \\
\hline
\end{tabular}

$\begin{array}{llll}\mathrm{H} & 6.785403 & 12.315564 & -0.341898\end{array}$

$\begin{array}{llll}\mathrm{H} & 1.838358 & 11.779594 & -0.341965\end{array}$

$\begin{array}{llll}\mathrm{H} & 0.603211 & 11.449742 & 0.910682\end{array}$

$\begin{array}{llll}\mathrm{H} & 3.656568 & 13.262949 & 1.987454\end{array}$

$\begin{array}{llll}\mathrm{H} & 3.726588 & 15.702765 & 2.524186\end{array}$

$\begin{array}{llll}\mathrm{H} & 1.769624 & 17.175990 & 1.957970\end{array}$

$\begin{array}{llll}\mathrm{H} & -0.260841 & 16.175942 & 0.860712\end{array}$

$\begin{array}{llll}\mathrm{H} & -0.329033 & 13.730146 & 0.344207\end{array}$

$\begin{array}{llll}\mathrm{H} & 4.024494 & 10.781075 & -0.156116\end{array}$

$\begin{array}{llll}\mathrm{H} & 8.928678 & 8.196496 & 1.648371\end{array}$

$\begin{array}{llll}\mathrm{H} & 4.949188 & 9.091873 & 4.694670\end{array}$

$\begin{array}{llll}\mathrm{H} & 3.193864 & 9.534811 & 6.442165\end{array}$

$\begin{array}{llll}\mathrm{H} & 1.087863 & 10.751074 & 5.859773\end{array}$

$\begin{array}{llll}\mathrm{H} & 0.668745 & 11.545225 & 3.509641\end{array}$

$\begin{array}{llll}\mathrm{H} & 7.747705 & 6.642053 & 0.297531\end{array}$

\section{A = 1-benzyl-3-indolyl, B = 4-methoxyphenyl}

Bispropargyl

55

Free energy $=-1264.831305$ Hartree

C $\quad 10.174193 \quad 6.479942 \quad-3.929503$

$\begin{array}{llll}\text { C } & 9.463094 & 5.301995 & -3.570054\end{array}$ $\begin{array}{llll}\text { C } & 10.212867 & 4.147242 & -3.238176\end{array}$

$\begin{array}{llll}\text { C } & 11.613542 & 4.144479 & -3.299802\end{array}$

$\begin{array}{llll}\text { C } & 12.297881 & 5.308199 & -3.710232\end{array}$

$\begin{array}{llll}\text { C } & 11.567151 & 6.483741 & -3.996339\end{array}$

$\begin{array}{llll}\text { C } & 8.035881 & 5.284770 & -3.643366\end{array}$

$\begin{array}{llll}\text { C } & 6.819829 & 5.310219 & -3.828155\end{array}$

$\begin{array}{llll}C & 5.387146 & 5.447542 & -4.152421\end{array}$

$\begin{array}{llll}5.047117 & 6.438303 & -5.186951\end{array}$

$\begin{array}{lll}5.390075 & 6.080342 & -6.574538\end{array}$

$\begin{array}{llll}6.821813 & 5.939101 & -6.898915\end{array}$

$\begin{array}{llll}8.043670 & 5.833844 & -7.015031\end{array}$

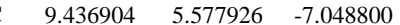

$\begin{array}{llll}\text { C } & 10.065305 & 4.306302 & -6.728341\end{array}$

$\begin{array}{llll}\text { C } & 11.477887 & 4.512179 & -6.794921\end{array}$

$\begin{array}{llll}\mathrm{N} & 11.699083 & 5.842498 & -7.127143\end{array}$

$\begin{array}{llll}\text { C } & 10.483720 & 6.477151 & -7.284305\end{array}$

$\begin{array}{llll}\text { C } & 9.566649 & 3.041565 & -6.355044\end{array}$

$\begin{array}{llll}\text { C } & 10.479037 & 2.015550 & -6.075753\end{array}$

$\begin{array}{llll}\text { C } & 11.878940 & 2.232161 & -6.168458 \\ \text { C } & 12.399430 & 3.482317 & -6.531748\end{array}$

$\begin{array}{llll}\text { C } & 13.017400 & 6.460350 & -7.193855\end{array}$

$\begin{array}{llll}\text { C } & 13.017400 & 6.460350 & -7.193855 \\ \text { C } & 13.873455 & 5.920000 & -8.328381\end{array}$

$\begin{array}{llll}\text { C } & 15.240473 & 5.650902 & -8.119194\end{array}$

$\begin{array}{llll}\text { C } & 16.042559 & 5.164834 & -9.167505\end{array}$

$\begin{array}{llll}\text { C } & 15.480622 & 4.936782 & -10.436118\end{array}$

$\begin{array}{llll}\text { C } & 14.113784 & 5.196581 & -10.649644\end{array}$

$\begin{array}{llll}\text { C } & 13.315808 & 5.685048 & -9.602002\end{array}$

$\begin{array}{llll}\text { O } & 13.650738 & 5.388815 & -3.893275\end{array}$

$\begin{array}{llll}\text { C } & 14.448073 & 4.242653 & -3.597890\end{array}$

$\begin{array}{llll}\mathrm{H} & 4.933248 & 6.847400 & -7.238216\end{array}$

$\begin{array}{llll}\mathrm{H} & 4.826827 & 5.716264 & -3.228229\end{array}$

$\begin{array}{llll}\mathrm{H} & 9.610895 & 7.385312 & -4.197977\end{array}$

$\begin{array}{llll}\mathrm{H} & 12.115435 & 7.386149 & -4.304624\end{array}$

$\begin{array}{llll}\mathrm{H} & 12.155077 & 3.216673 & -3.075712\end{array}$

$\begin{array}{llll}\mathrm{H} & 9.685453 & 3.221624 & -2.965251\end{array}$

$\begin{array}{llll}\mathrm{H} & 12.865887 & 7.553330 & -7.308351\end{array}$

\begin{tabular}{llll}
$\mathrm{H}$ & 13.530536 & 6.302941 & -6.221288 \\
\hline & 15.681118 & 5.819734 & -7.122733
\end{tabular}

$\begin{array}{llll}\mathrm{H} & 15.681118 & 5.819734 & -7.122733\end{array}$

$\begin{array}{llll}\mathrm{H} & 17.108476 & 4.954115 & -8.988310\end{array}$

$\mathrm{H} \quad 16.104870 \quad 4.551078 \quad-11.257062$

$\mathrm{H} \quad 13.665950 \quad 5.013835-11.639050$

$\begin{array}{llll}\mathrm{H} & 12.243802 & 5.878262 & -9.768658\end{array}$

$\begin{array}{llll}\mathrm{H} & 10.441330 & 7.545188 & -7.526444\end{array}$

$\mathrm{H} \quad 4.979177 \quad 4.461960 \quad-4.469795$

$\begin{array}{llll}\mathrm{H} & 8.480226 & 2.884134 & -6.270772\end{array}$

$\begin{array}{llll}\mathrm{H} & 10.107295 & 1.023839 & -5.774501 \\ \mathrm{H} & 12.570583 & 1.404482 & -5.947166\end{array}$

$\begin{array}{llll}\mathrm{H} & 12.570583 & 1.404482 & -5.947166 \\ \mathrm{H} & 13.482692 & 3.655453 & -6.606048\end{array}$

$\begin{array}{cccc}\mathrm{H} & 13.482692 & 3.655453 & -6.606048 \\ \mathrm{H} & 4.867677 & 5.127888 & -6.819025\end{array}$

$\begin{array}{llll}\mathrm{H} & 5.482109 & 7.340024 & -4.948949\end{array}$

$\begin{array}{llll}\mathrm{H} & 15.492634 & 4.523962 & -3.827850\end{array}$

H $\quad 14.373236 \quad 3.960292 \quad-2.524681$

$\begin{array}{llll}\mathrm{H} & 14.158858 & 3.370341 & -4.223184\end{array}$

Alkyne_anionA

Free energy $=-1264.296797$ Hartree

$\begin{array}{llll}\text { C } & 11.542265 & 6.959360 & -3.766599\end{array}$

$\begin{array}{llll}\text { C } & 10.388443 & 6.251948 & -3.374588\end{array}$

$\begin{array}{llll}\text { C } & 10.523116 & 4.925108 & -2.913700\end{array}$

$\begin{array}{llll}\text { C } & 11.787059 & 4.313011 & -2.850965\end{array}$

$\begin{array}{llll}\text { C } & 12.935582 & 5.025013 & -3.244754\end{array}$

$\begin{array}{llll}\text { C } & 12.808636 & 6.349228 & -3.701189\end{array}$

$\begin{array}{llll}\text { C } & 9.002750 & 6.882887 & -3.460148\end{array}$

$\begin{array}{llll}\mathrm{N} & 9.001191 & 8.290715 & -3.778734\end{array}$

$\begin{array}{llll}\text { C } & 8.931262 & 8.845723 & -5.037514\end{array}$

$\begin{array}{llll}\text { C } & 9.116051 & 10.267026 & -4.902158\end{array}$

$\begin{array}{llll}\text { C } & 9.331399 & 10.561424 & -3.484250\end{array}$

$\begin{array}{llll}\text { C } & 9.273446 & 9.306302 & -2.844091\end{array}$

$\begin{array}{llll}\text { C } & 8.714909 & 8.247737 & -6.297744\end{array}$

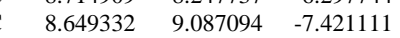

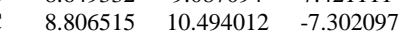

$\begin{array}{llll}9.041548 & 11.082903 & -6.047679\end{array}$

$\begin{array}{llll}\text { C } & 9.486867 & 11.832116 & -2.912145\end{array}$

$\begin{array}{llll}\text { C } & 9.562025 & 12.966324 & -2.362854\end{array}$

$\begin{array}{llll}\text { C } & 9.720343 & 14.172375 & -1.714493\end{array}$

$\begin{array}{llll}\mathrm{N} & 9.839362 & 14.288530 & -0.288721\end{array}$

$\begin{array}{llll}\text { C } & 8.570985 & 14.520040 & 0.490936\end{array}$

$\begin{array}{llll}\text { C } & 7.615894 & 13.409519 & 0.349761\end{array}$

$\begin{array}{llll}\text { C } & 7.174597 & 12.334585 & -0.082483\end{array}$

$\begin{array}{llll}\text { C } & 6.738497 & 11.147214 & -0.729501\end{array}$

$\begin{array}{llll}\text { C } & 6.671303 & 9.886563 & -0.068806\end{array}$

$\begin{array}{llll}\text { C } & 6.366780 & 8.720410 & -0.772756\end{array}$

$\begin{array}{llll}\text { C } & 6.129293 & 8.771259 & -2.168609\end{array}$

$\begin{array}{llll}\text { C } & 6.148320 & 10.013499 & -2.834774\end{array}$

$\begin{array}{llll}\text { C } & 6.452826 & 11.182117 & -2.125258 \\ \text { O } & 5.898726 & 7.567628 & -2.779882\end{array}$

$\begin{array}{llll}\text { C } \quad 5.587836 & 7.565331 & -4.170215\end{array}$

$\begin{array}{llll}\mathrm{H} & 9.628502 & 15.129554 & -2.261137\end{array}$

$\begin{array}{llll}\mathrm{H} & 8.839857 & 14.690442 & 1.557779\end{array}$ 


$\begin{array}{cccc}\mathrm{H} & 6.886323 & 9.832773 & 1.009899 \\ \mathrm{H} & 6.331963 & 7.742392 & -0.268653 \\ \mathrm{H} & 5.995462 & 10.074074 & -3.920495 \\ \mathrm{H} & 6.533071 & 12.138559 & -2.659128 \\ \mathrm{H} & 8.454269 & 6.738813 & -2.504252 \\ \mathrm{H} & 8.399677 & 6.347705 & -4.224841 \\ \mathrm{H} & 9.626332 & 4.366523 & -2.598110 \\ \mathrm{H} & 11.876664 & 3.276588 & -2.489492 \\ \mathrm{H} & 13.927416 & 4.548653 & -3.193308 \\ \mathrm{H} & 13.701979 & 6.915210 & -4.008573 \\ \mathrm{H} & 11.445005 & 7.997468 & -4.118743 \\ \mathrm{H} & 9.285312 & 9.073518 & -1.774172 \\ \mathrm{H} & 8.121839 & 15.468395 & 0.124712 \\ \mathrm{H} & 9.172339 & 12.172972 & -5.945810 \\ \mathrm{H} & 8.748264 & 11.124301 & -8.203667 \\ \mathrm{H} & 8.473469 & 8.642301 & -8.413884 \\ \mathrm{H} & 8.601234 & 7.157263 & -6.401461 \\ \mathrm{H} & 10.253669 & 13.422390 & 0.082390 \\ \mathrm{H} & 5.443083 & 6.506409 & -4.454846 \\ \mathrm{H} & 4.650474 & 8.128811 & -4.379945 \\ \mathrm{H} & 6.411337 & 7.996486 & -4.780654\end{array}$

Alkyne_anionB

54

Free energy $=-1264.306143$ Hartree

$\begin{array}{llll}\text { C } & 11.049964 & 6.018850 & -3.254224\end{array}$

$\begin{array}{llll}\text { C } & 10.144034 & 4.946411 & -3.127323\end{array}$

$\begin{array}{llll}\text { C } & 10.611506 & 3.630863 & -3.318551\end{array}$

$\begin{array}{llll}\text { C } & 11.963214 & 3.388005 & -3.621221\end{array}$

$\begin{array}{llll}\text { C } & 12.861580 & 4.462804 & -3.743224\end{array}$

$\begin{array}{llll}\text { C } & 12.399910 & 5.780114 & -3.559655\end{array}$

$\begin{array}{llll}\text { C } & 8.681621 & 5.194547 & -2.792476\end{array}$

$\begin{array}{llll}\text { N } & 8.127914 & 6.362011 & -3.461790\end{array}$

$\begin{array}{llll}\text { C } & 7.974808 & 6.476535 & -4.837298\end{array}$

$\begin{array}{llll}\text { C } & 7.275615 & 7.693298 & -5.098801\end{array}$

$\begin{array}{llll}\text { C } & 7.016008 & 8.320803 & -3.811952\end{array}$

$\begin{array}{llll}\text { C } & 7.560534 & 7.465660 & -2.848248\end{array}$

$\begin{array}{llll}\text { C } & 8.377787 & 5.609347 & -5.871214\end{array}$

$\begin{array}{llll}\text { C } & 8.046666 & 5.979382 & -7.181558\end{array}$

$\begin{array}{llll}\text { C } & 7.325413 & 7.171790 & -7.453426\end{array}$

$\begin{array}{llll}\text { C } & 6.934041 & 8.035968 & -6.421627\end{array}$

$\begin{array}{llll}\text { C } & 6.290548 & 9.527614 & -3.661614\end{array}$

$\begin{array}{llll}\text { C } & 5.645032 & 10.578604 & -3.724373\end{array}$

$\begin{array}{llll}\text { C } & 4.615157 & 11.594839 & -4.004345\end{array}$

$\begin{array}{llll}\mathrm{N} & 3.336769 & 11.034387 & -4.532816\end{array}$

$\begin{array}{llll}\text { C } & 3.451515 & 10.220756 & -5.726813\end{array}$

$\begin{array}{llll}\text { C } & 3.589635 & 8.864057 & -5.668136\end{array}$

$\begin{array}{llll}\text { C } & 3.581953 & 7.597457 & -5.515810\end{array}$

$\begin{array}{llll}\text { C } & 4.250818 & 6.387021 & -5.216149\end{array}$

$\begin{array}{llll}\text { C } & 4.579739 & 6.054792 & -3.856144\end{array}$

$\begin{array}{llll}\text { C } & 5.283681 & 4.896092 & -3.527538\end{array}$

$\begin{array}{llll}\text { C } & 5.701954 & 3.993760 & -4.530890\end{array}$

$\begin{array}{llll}\text { C } & 5.356256 & 4.261722 & -5.870265\end{array}$

$\begin{array}{llll}\text { C } & 4.637388 & 5.421864 & -6.200764\end{array}$

$\begin{array}{llll}\text { O } & 6.477802 & 2.918791 & -4.104685\end{array}$

$\begin{array}{llll}\text { C } & 7.011163 & 2.062857 & -5.098149\end{array}$

$\begin{array}{llll}\mathrm{H} & 4.388610 & 12.194720 & -3.093282\end{array}$

$\begin{array}{llll}\mathrm{H} & 3.495137 & 10.782859 & -6.678200\end{array}$

$\begin{array}{llll}\mathrm{H} & 4.311086 & 6.770955 & -3.064963\end{array}$

$\begin{array}{llll}\mathrm{H} & 5.563685 & 4.689399 & -2.481976\end{array}$

$\begin{array}{llll}\mathrm{H} & 5.681392 & 3.594216 & -6.681183\end{array}$

$\begin{array}{llll}\mathrm{H} & 4.416861 & 5.627580 & -7.259894\end{array}$

$\mathrm{H} \quad 8.560714 \quad 5.348358 \quad-1.700130$

$\begin{array}{llll}\mathrm{H} & 8.076718 & 4.302751 & -3.064332\end{array}$

$\begin{array}{llll}\mathrm{H} & 9.904587 & 2.788427 & -3.239290\end{array}$

$\begin{array}{llll}\mathrm{H} & 12.313258 & 2.355127 & -3.775485\end{array}$

$\begin{array}{llll}\mathrm{H} & 13.918045 & 4.275652 & -3.991799\end{array}$

$\begin{array}{llll}\mathrm{H} & 13.095685 & 6.627812 & -3.661824\end{array}$

$\mathrm{H} \quad 10.684084 \quad 7.050283 \quad-3.126699$

$\begin{array}{llll}\mathrm{H} & 7.552252 & 7.548835 & -1.754795\end{array}$

$\begin{array}{llll}\mathrm{H} & 6.336799 & 8.941582 & -6.608116\end{array}$

$\begin{array}{llll}\mathrm{H} & 7.056853 & 7.410529 & -8.494940\end{array}$

$\begin{array}{llll}\mathrm{H} & 8.332411 & 5.317658 & -8.014098\end{array}$

$\begin{array}{llll}\mathrm{H} & 8.921680 & 4.679231 & -5.653909\end{array}$

$\begin{array}{llll}\mathrm{H} & 5.007133 & 12.315532 & -4.757128\end{array}$

$\begin{array}{llll}\mathrm{H} & 2.936543 & 10.448543 & -3.786222\end{array}$

$\begin{array}{llll}\mathrm{H} & 7.629530 & 1.308714 & -4.573128\end{array}$

$\begin{array}{llll}\mathrm{H} & 6.213206 & 1.534368 & -5.67058\end{array}$

$\begin{array}{llll}\mathrm{H} & 7.651654 & 2.614502 & -5.825398\end{array}$

\section{$X=-\mathrm{CH} 2-$}

$A=4$-methoxyphenyl, $B$ = phenyl

Bispropargyl
39

Free energy $=-847.2420049$ Hartree

$\begin{array}{lrrr}\mathrm{C} & 0.238274 & 6.467941 & 1.159108 \\ \mathrm{C} & 0.866231 & 5.470068 & 1.950288 \\ \mathrm{C} & 0.049228 & 4.536757 & 2.642737 \\ \mathrm{C} & -1.346553 & 4.602805 & 2.545707 \\ \mathrm{C} & -1.958713 & 5.599951 & 1.763668 \\ \mathrm{C} & -1.160756 & 6.527100 & 1.068713 \\ \mathrm{C} & 2.292720 & 5.379667 & 2.038221 \\ \mathrm{C} & 3.510832 & 5.237475 & 2.125652 \\ \mathrm{C} & 4.961763 & 5.074313 & 2.241751 \\ \mathrm{C} & 5.633707 & 4.162341 & 1.184900 \\ \mathrm{C} & 5.311824 & 2.652994 & 1.314181 \\ \mathrm{C} & 3.917636 & 2.326245 & 1.015339 \\ \mathrm{C} & 2.719393 & 2.179403 & 0.783094 \\ \mathrm{C} & 1.313853 & 2.178093 & 0.526412 \\ \mathrm{C} & 0.766061 & 3.125822 & -0.374809 \\ \mathrm{C} & -0.615572 & 3.235741 & -0.570830 \\ \mathrm{C} & -1.493486 & 2.394223 & 0.146761 \\ \mathrm{C} & -0.962097 & 1.420150 & 1.024704 \\ \mathrm{C} & 0.417353 & 1.313482 & 1.211473 \\ \mathrm{O} & -2.852102 & 2.457202 & 0.073362 \\ \mathrm{C} & -3.442318 & 3.399453 & -0.820000 \\ \mathrm{H} & -3.056633 & 5.649410 & 1.691346 \\ \mathrm{H} & 5.436029 & 6.079406 & 2.185691 \\ \mathrm{H} & 5.564368 & 2.308216 & 2.342460 \\ \mathrm{H} & 0.861753 & 7.191322 & 0.612168 \\ \mathrm{H} & 1.442928 & 3.814569 & -0.901141 \\ \mathrm{H} & -1.633510 & 7.305852 & 0.449466 \\ \mathrm{H} & -0.998297 & 4.009134 & -1.249912 \\ \mathrm{H} & -1.963275 & 3.857529 & 3.070951 \\ \mathrm{H} & -1.661450 & 0.769034 & 1.570076 \\ \mathrm{H} & 0.817672 & 0.566844 & 1.914484 \\ \mathrm{H} & -4.537464 & 3.287382 & -0.710317 \\ \mathrm{H} & -3.158080 & 3.195593 & -1.876729 \\ \mathrm{H} & -3.153991 & 4.442174 & -0.563446 \\ \mathrm{H} & 5.987190 & 2.086244 & 0.634987 \\ \mathrm{H} & 0.528850 & 3.741910 & 3.232312 \\ \mathrm{H} & 5.202106 & 4.687262 & 3.258244 \\ \mathrm{H} & 5.363957 & 4.512048 & 0.166065 \\ \mathrm{H} & 6.732143 & 4.285445 & 1.288924\end{array}$

Alkyne_anionA

Free energy $=-846.7163097$ Hartree

$\begin{array}{llll}\text { C } & 2.711951 & 4.500910 & -0.159057\end{array}$

$\begin{array}{llll}C & 4.010652 & 4.461755 & -0.760569\end{array}$

$\begin{array}{llll}\text { C } & 4.032295 & 4.244089 & -2.179162\end{array}$

$\begin{array}{llll}\text { C } & 2.861393 & 4.010550 & -2.908772\end{array}$

$\begin{array}{llll}\text { C } & 1.600072 & 4.000316 & -2.273196\end{array}$

$\begin{array}{llll}\text { C } & 1.536853 & 4.267699 & -0.889032\end{array}$

$\begin{array}{llll}\text { C } & 5.193786 & 4.623925 & 0.017450\end{array}$

$\begin{array}{llll}\text { C } & 6.085251 & 3.956006 & 0.646981\end{array}$

$\begin{array}{llll}\text { C } \quad 7.025953 & 3.324831 & 1.399915\end{array}$

$\begin{array}{llll}\text { C } & 6.718349 & 2.785816 & 2.776203\end{array}$

$\begin{array}{llll}\text { C } & 6.302914 & 1.272823 & 2.791588\end{array}$

$\begin{array}{llll}\text { C } & 5.093137 & 0.977515 & 2.027452\end{array}$

$\begin{array}{llll}\text { C } & 4.073904 & 0.758082 & 1.372701\end{array}$

$\begin{array}{llll}\text { C } & 2.903456 & 0.466739 & 0.600083\end{array}$

$\begin{array}{llll}\text { C } & 1.929892 & -0.454663 & 1.079254\end{array}$

$\begin{array}{llll}\text { C } & 0.788413 & -0.747219 & 0.317952\end{array}$

$\begin{array}{llll}\text { C } & 0.593249 & -0.134732 & -0.934749\end{array}$

$\begin{array}{llll}\text { C } & 1.549280 & 0.778327 & -1.418818\end{array}$

$\begin{array}{llll}\text { C } & 2.691139 & 1.082231 & -0.662870\end{array}$

$\begin{array}{llll}\text { O } & 0.511387 & 3.688089 & -3.071647\end{array}$

$\begin{array}{llll}\text { C } & -0.748076 & 3.547016 & -2.435222\end{array}$

$\begin{array}{llll}\mathrm{H} & -0.304382 & -0.364209 & -1.530419\end{array}$

$\begin{array}{llll}\mathrm{H} & 6.170464 & 0.919177 & 3.841764\end{array}$

$\begin{array}{llll}\mathrm{H} & 7.966385 & 2.975772 & 0.928460\end{array}$

$\begin{array}{llll}\mathrm{H} & 3.417736 & 1.821033 & -1.031197\end{array}$

$\begin{array}{llll}\mathrm{H} & 2.643286 & 4.676477 & 0.926092\end{array}$

$\begin{array}{llll}\mathrm{H} & 1.397975 & 1.279454 & -2.386801\end{array}$

$\begin{array}{llll}\mathrm{H} & 0.574451 & 4.264118 & -0.356983\end{array}$

$\begin{array}{llll}\mathrm{H} & 0.043233 & -1.460557 & 0.705428\end{array}$

$\begin{array}{llll}\mathrm{H} & 2.904674 & 3.799448 & -3.989844\end{array}$

$\mathrm{H} \quad 5.005093 \quad 4.219596 \quad-2.696571$

$\begin{array}{llll}\mathrm{H} & 7.145702 & 0.676353 & 2.373399\end{array}$

$\begin{array}{llll}\mathrm{H} & -1.470939 & 3.256198 & -3.221639\end{array}$

$\begin{array}{llll}\mathrm{H} & -0.728454 & 2.756311 & -1.648645\end{array}$

$\begin{array}{llll}\mathrm{H} & -1.093585 & 4.498682 & -1.967789\end{array}$

$\begin{array}{llll}\mathrm{H} & 2.082375 & -0.935011 & 2.057730\end{array}$

$\begin{array}{llll}\text { H } & 5.893992 & 3.371516 & 3.239150\end{array}$

$\begin{array}{llll}\mathrm{H} & 7.595351 & 2.867494 & 3.461085\end{array}$

Alkyne_anionB

Free energy $=-846.7236837$ Hartree

$\begin{array}{llll}\text { C } & 3.370348 & 3.156529 & -0.973574\end{array}$

$\begin{array}{llll}\text { C } & 3.986581 & 4.398085 & -0.671516\end{array}$

C $\quad 3.387429 \quad 5.582155 \quad-1.179621$ 


\begin{tabular}{rrrr} 
C & 2.214777 & 5.517178 & -1.940279 \\
$\mathrm{C}$ & 1.598520 & 4.270295 & -2.207822 \\
$\mathrm{C}$ & 2.190950 & 3.083289 & -1.723263 \\
$\mathrm{C}$ & 5.147767 & 4.378059 & 0.165555 \\
$\mathrm{C}$ & 6.105441 & 4.155474 & 0.906376 \\
$\mathrm{C}$ & 7.146186 & 3.727605 & 1.837197 \\
$\mathrm{C}$ & 6.724113 & 2.440363 & 2.635767 \\
$\mathrm{C}$ & 6.436595 & 1.235052 & 1.773610 \\
$\mathrm{C}$ & 5.178779 & 0.831477 & 1.431147 \\
$\mathrm{C}$ & 4.003607 & 0.431190 & 1.161045 \\
$\mathrm{C}$ & 2.736934 & 0.289916 & 0.577310 \\
$\mathrm{C}$ & 1.577325 & 0.972769 & 1.101285 \\
$\mathrm{C}$ & 0.328608 & 0.881459 & 0.482298 \\
$\mathrm{C}$ & 0.138168 & 0.105694 & -0.685692 \\
$\mathrm{C}$ & 1.253068 & -0.589431 & -1.208793 \\
$\mathrm{C}$ & 2.511520 & -0.513520 & -0.601461 \\
$\mathrm{O}$ & 0.438931 & 4.310483 & -2.929343 \\
$\mathrm{C}$ & -0.225300 & 3.074739 & -3.194155 \\
$\mathrm{H}$ & -0.848639 & 0.040991 & -1.169263 \\
$\mathrm{H}$ & 8.082948 & 3.505231 & 1.275907 \\
$\mathrm{H}$ & 3.367744 & -1.049222 & -1.042069 \\
$\mathrm{H}$ & 3.811413 & 2.236255 & -0.566068 \\
$\mathrm{H}$ & 1.138028 & -1.197220 & -2.122824 \\
$\mathrm{H}$ & 1.732036 & 2.096828 & -1.880464 \\
$\mathrm{H}$ & -0.522451 & 1.437105 & 0.911941 \\
$\mathrm{H}$ & 1.740959 & 6.430353 & -2.331021 \\
$\mathrm{H}$ & 3.844086 & 6.560153 & -0.963521 \\
$\mathrm{H}$ & 7.400712 & 4.550766 & 2.544944 \\
$\mathrm{H}$ & 7.303782 & 0.780498 & 1.255103 \\
$\mathrm{H}$ & -1.156064 & 3.331362 & -3.733413 \\
$\mathrm{H}$ & 0.394635 & 2.404704 & -3.831747 \\
$\mathrm{H}$ & -0.476639 & 2.529940 & -2.257354 \\
$\mathrm{H}$ & 1.703411 & 1.600673 & 1.997404 \\
$\mathrm{H}$ & 5.827650 & 2.694403 & 3.242568 \\
$\mathrm{H}$ & 7.553617 & 2.242017 & 3.355977 \\
& & & \\
\hline
\end{tabular}

\section{A = 2,4-dimethoxyphenyl, $B$ = phenyl} Bispropargyl

43

Free energy $=-961.661876$ Hartree

$\begin{array}{lrrr}\mathrm{C} & 0.381438 & 1.350625 & 1.175855 \\ \mathrm{C} & 1.301836 & 2.191119 & 0.471569 \\ \mathrm{C} & 0.772695 & 3.124370 & -0.448214 \\ \mathrm{C} & -0.603473 & 3.261295 & -0.670454 \\ \mathrm{C} & -1.493402 & 2.441574 & 0.052861 \\ \mathrm{C} & -0.998426 & 1.472369 & 0.955849 \\ \mathrm{C} & 2.699297 & 2.175394 & 0.752897 \\ \mathrm{C} & 3.896413 & 2.317172 & 0.993997 \\ \mathrm{C} & 5.289041 & 2.647146 & 1.296209 \\ \mathrm{C} & 5.601960 & 4.160001 & 1.186584 \\ \mathrm{C} & 4.922360 & 5.054027 & 2.253855 \\ \mathrm{C} & 3.470554 & 5.209377 & 2.138055 \\ \mathrm{C} & 2.251495 & 5.345345 & 2.053933 \\ \mathrm{C} & 0.824184 & 5.424762 & 1.969188 \\ \mathrm{C} & 0.184670 & 6.449341 & 1.222667 \\ \mathrm{C} & -1.215024 & 6.498139 & 1.136154 \\ \mathrm{C} & -2.002550 & 5.533747 & 1.791083 \\ \mathrm{C} & -1.378775 & 4.511085 & 2.529436 \\ \mathrm{C} & 0.017656 & 4.454727 & 2.622181 \\ \mathrm{O} & -2.851366 & 2.519341 & -0.030492 \\ \mathrm{C} & -3.422710 & 3.471279 & -0.925551 \\ \mathrm{H} & -3.100945 & 5.572809 & 1.719841 \\ \mathrm{H} & 5.390423 & 6.062681 & 2.212344 \\ \mathrm{H} & 5.544791 & 2.290796 & 2.319862 \\ \mathrm{H} & 0.799976 & 7.201963 & 0.706760 \\ \mathrm{H} & 1.473976 & 3.789102 & -0.973294 \\ \mathrm{H} & -1.696228 & 7.297400 & 0.550461 \\ \mathrm{H} & -0.964894 & 4.030560 & -1.364294 \\ \mathrm{H} & -1.986596 & 3.736230 & 3.021002 \\ \mathrm{H} & -1.730701 & 0.860695 & 1.497478 \\ \mathrm{O} & 0.936144 & 0.481698 & 2.064204 \\ \mathrm{H} & -4.519972 & 3.375959 & -0.820791 \\ \mathrm{H} & -3.137202 & 3.264782 & -1.981345 \\ \mathrm{H} & -3.118986 & 4.508786 & -0.665303 \\ \mathrm{H} & 5.968538 & 2.093784 & 0.609854 \\ \mathrm{H} & 0.506685 & 3.639461 & 3.175526 \\ \mathrm{H} & 5.163284 & 4.654718 & 3.265528 \\ \mathrm{H} & 5.330689 & 4.520661 & 0.171910 \\ \mathrm{H} & 6.699458 & 4.289644 & 1.293285 \\ \mathrm{C} & 0.074329 & -0.356917 & 2.830021 \\ \mathrm{H} & 0.731101 & -0.960689 & 3.483043 \\ \mathrm{H} & -0.621559 & 0.237775 & 3.462528 \\ \mathrm{H} & -0.520143 & -1.037869 & 2.181397 \\ & & & \end{array}$

Alkyne_anionA

42

Free energy $=-961.1343233$ Hartree

$\begin{array}{llll}\text { C } & 3.010403 & 1.057046 & -0.252959\end{array}$

$\begin{array}{llll}\text { C } & 3.284210 & 0.115769 & 0.777299\end{array}$

$\begin{array}{lrrr}\mathrm{C} & 2.433701 & -1.013451 & 0.915774 \\ \mathrm{C} & 1.341953 & -1.186088 & 0.048746 \\ \mathrm{C} & 1.073628 & -0.240348 & -0.960635 \\ \mathrm{C} & 1.911468 & 0.883405 & -1.105792 \\ \mathrm{C} & 4.400781 & 0.363867 & 1.639034 \\ \mathrm{C} & 5.381945 & 0.710249 & 2.296410 \\ \mathrm{C} & 6.528066 & 1.264716 & 3.009208 \\ \mathrm{C} & 6.658315 & 2.819117 & 2.809086 \\ \mathrm{C} & 6.816350 & 3.252499 & 1.373449 \\ \mathrm{C} & 5.776486 & 3.713407 & 0.612446 \\ \mathrm{C} & 4.824448 & 4.212195 & -0.061585 \\ \mathrm{C} & 3.647245 & 4.305506 & -0.830029 \\ \mathrm{C} & 2.358012 & 4.540695 & -0.259352 \\ \mathrm{C} & 1.171529 & 4.560034 & -1.017189 \\ \mathrm{C} & 1.229882 & 4.360972 & -2.407993 \\ \mathrm{C} & 2.489152 & 4.147774 & -3.025856 \\ \mathrm{C} & 3.664470 & 4.122732 & -2.265963 \\ \mathrm{O} & 0.139041 & 4.348048 & -3.262233 \\ \mathrm{C} & -1.145234 & 4.486800 & -2.682903 \\ \mathrm{H} & 0.209015 & -0.377333 & -1.629491 \\ \mathrm{H} & 6.467464 & 1.014002 & 4.094745 \\ \mathrm{H} & 7.762022 & 2.964884 & 0.871463 \\ \mathrm{H} & 3.662667 & 1.937107 & -0.351290 \\ \mathrm{H} & 2.298875 & 4.673341 & 0.832248 \\ \mathrm{H} & 1.709291 & 1.647920 & -1.873539 \\ \mathrm{H} & 0.213681 & 4.722575 & -0.503632 \\ \mathrm{H} & 0.690018 & -2.066831 & 0.165098 \\ \mathrm{H} & 2.501216 & 3.979087 & -4.111498 \\ \mathrm{O} & 4.908283 & 3.883407 & -2.797109 \\ \mathrm{H} & 7.460343 & 0.782352 & 2.636441 \\ \mathrm{H} & -1.878085 & 4.423281 & -3.510507 \\ \mathrm{H} & -1.362775 & 3.678382 & -1.945247 \\ \mathrm{H} & -1.272560 & 5.468337 & -2.167823 \\ \mathrm{H} & 2.635347 & -1.749097 & 1.709876 \\ \mathrm{H} & 5.756809 & 3.296675 & 3.251579 \\ \mathrm{H} & 7.525860 & 3.131737 & 3.439316 \\ \mathrm{C} & 5.009216 & 3.629730 & -4.187686 \\ \mathrm{H} & 6.080678 & 3.451000 & -4.399755 \\ \mathrm{H} & 4.427099 & 2.728485 & -4.491307 \\ \mathrm{H} & 4.660711 & 4.494058 & -4.800452 \\ & & & \end{array}$

\begin{tabular}{lrcc}
\multicolumn{5}{l}{ Alkyne_anionB } & \multicolumn{3}{l}{} \\
42 & & & \\
Free & energy $=-961.1439229$ & Hartree \\
C & 2.551180 & -0.547266 & -0.649636 \\
C & 2.754168 & 0.230531 & 0.550229 \\
C & 1.573419 & 0.855596 & 1.098726 \\
C & 0.325837 & 0.738382 & 0.481803 \\
C & 0.157434 & -0.009052 & -0.707826 \\
C & 1.293394 & -0.650112 & -1.254117 \\
C & 4.018256 & 0.401627 & 1.131507 \\
C & 5.180225 & 0.837823 & 1.403986 \\
C & 6.425561 & 1.278901 & 1.745188 \\
C & 6.678930 & 2.472056 & 2.635114 \\
C & 7.065195 & 3.787481 & 1.865559 \\
C & 6.013259 & 4.209862 & 0.944667 \\
C & 5.052725 & 4.426721 & 0.205891 \\
C & 3.892304 & 4.416439 & -0.627914 \\
C & 3.282871 & 3.174631 & -0.918807 \\
C & 2.106586 & 3.070250 & -1.672241 \\
C & 1.512080 & 4.248998 & -2.165447 \\
C & 2.107093 & 5.510176 & -1.914741 \\
C & 3.283509 & 5.600316 & -1.150886 \\
O & 0.354230 & 4.278966 & -2.891741 \\
C & -0.297021 & 3.036307 & -3.156191 \\
H & -0.828307 & -0.092809 & -1.190606 \\
H & 8.008176 & 3.602690 & 1.300692 \\
H & 3.423381 & -1.040149 & -1.108481 \\
H & 3.737740 & 2.267992 & -0.495975 \\
H & 1.195464 & -1.235236 & -2.184732 \\
H & 1.659292 & 2.077915 & -1.819118 \\
H & -0.542151 & 1.250983 & 0.930670 \\
H & 1.609667 & 6.399126 & -2.322682 \\
O & 3.909555 & 6.774734 & -0.850183 \\
H & 7.298447 & 4.600026 & 2.592812 \\
H & 7.303260 & 0.869755 & 1.206852 \\
H & -1.225915 & 3.282679 & -3.703458 \\
H & 0.333795 & 2.369063 & -3.785789 \\
H & -0.550631 & 2.493086 & -2.219141 \\
H & 1.681405 & 1.460698 & 2.012682 \\
H & 5.775526 & 2.686622 & 3.246732 \\
H & 7.513749 & 2.280491 & 3.351357 \\
C & 3.345452 & 7.990640 & -1.330546 \\
H & 4.004457 & 8.802181 & -0.970320 \\
H & 3.308906 & 8.017501 & -2.442910 \\
H & 2.318862 & 8.154712 & -0.933364 \\
& & &
\end{tabular}

A = 2-naphthyl, B = phenyl

Bispropargyl 
41

\begin{tabular}{|c|c|c|c|}
\hline \multicolumn{4}{|c|}{ Free energy $=-886.3236563$ Hartree } \\
\hline $\mathrm{C}$ & 155 & 5.067117 & 579 \\
\hline $\mathrm{C}$ & -0.223398 & 5.749354 & \\
\hline $\mathrm{C}$ & & & 10987 \\
\hline $\mathrm{C}$ & & & \\
\hline $\mathrm{C}$ & & & \\
\hline $\mathrm{C}$ & -1.4 & 8 & 1.02 \\
\hline $\mathrm{C}$ & 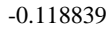 & 1 & \\
\hline $\mathrm{C}$ & & & \\
\hline $\mathrm{C}$ & & & \\
\hline $\mathrm{C}$ & & & \\
\hline $\mathrm{C}$ & & & \\
\hline $\mathrm{C}$ & -2 . & & \\
\hline $\mathrm{C}$ & -3 . & 92 & -2 \\
\hline $\mathrm{C}$ & -3 . & & $-1.2+2$. \\
\hline $\mathrm{C}$ & & & -0 \\
\hline $\mathrm{C}$ & & & \\
\hline $\mathrm{C}$ & & & \\
\hline $\mathrm{C}$ & & & \\
\hline $\mathrm{C}$ & & & \\
\hline $\mathrm{C}$ & & & \\
\hline $\mathrm{C}$ & & & \\
\hline $\mathrm{C}$ & & & \\
\hline $\mathrm{C}$ & & & \\
\hline $\mathrm{H}$ & & & \\
\hline $\mathrm{H}$ & & & \\
\hline $\mathrm{H}$ & & & \\
\hline $\mathrm{H}$ & & & \\
\hline $\mathrm{H}$ & & & -0 . \\
\hline $\mathrm{H}$ & & & -0 . \\
\hline $\mathrm{H}$ & & & \\
\hline $\mathrm{H}$ & & & \\
\hline $\mathrm{H}$ & & & \\
\hline $\mathrm{H}$ & & & \\
\hline $\mathrm{H}$ & & & 400 \\
\hline $\mathrm{H}$ & & & 584 \\
\hline $\mathrm{H}$ & & & \\
\hline $\mathrm{H}$ & & & \\
\hline $\mathrm{H}$ & & & \\
\hline - & & & \\
\hline & & 8.539955 & -4.93 \\
\hline & -0.50105 & 7.793424 & -6.534801 \\
\hline
\end{tabular}

Alkyne_anionA

40

Free energy $=-885.8052871$ Hartree

C $\quad-3.274003 \quad-1.076150 \quad-2.709656$

$\begin{array}{llll}\text { C } & -4.180078 & -0.128536 & -3.260612\end{array}$

$\begin{array}{llll}\text { C } & -3.699320 & 0.793255 & -4.228193\end{array}$

$\begin{array}{llll}\text { C } & -2.354573 & 0.766483 & -4.629390\end{array}$

$\begin{array}{llll}\text { C } & -1.465520 & -0.173831 & -4.075005\end{array}$

$\begin{array}{llll}\text { C } & -1.931842 & -1.091828 & -3.114639\end{array}$

$\begin{array}{llll}\text { C } & -5.542228 & -0.119372 & -2.820401\end{array}$

$\begin{array}{llll}\text { C } & -6.678778 & -0.193402 & -2.356740\end{array}$

$\begin{array}{llll}\text { C } & -8.016584 & -0.319025 & -1.779941\end{array}$

$\begin{array}{llll}\text { C } & -8.642177 & -1.730229 & -1.916479\end{array}$

$\begin{array}{llll}\text { C } & -7.918058 & -2.821402 & -1.149644\end{array}$

$\begin{array}{llll}\text { C } & -6.749602 & -2.635209 & -0.477367\end{array}$

$\begin{array}{llll}\text { C } & -5.658426 & -2.413989 & 0.128348\end{array}$

$\begin{array}{llll}\text { C } & -4.413089 & -2.098752 & 0.661570\end{array}$

$\begin{array}{llll}\text { C } & -3.916450 & -0.732570 & 0.538212\end{array}$

$\begin{array}{llll}\text { C } & -2.677238 & -0.359147 & 1.010072\end{array}$

$\begin{array}{llll}\text { C } & -1.801670 & -1.293710 & 1.663128\end{array}$

$\begin{array}{llll}\text { C } & -2.275178 & -2.662078 & 1.816196\end{array}$

$\begin{array}{llll}\text { C } & -3.549436 & -3.029487 & 1.325290\end{array}$

$\begin{array}{llll}\text { C } & -1.393218 & -3.593067 & 2.470550\end{array}$

$\begin{array}{lrrr}\text { C } & -0.139051 & -3.205081 & 2.933511 \\ \text { C } & 0.315766 & -1.865043 & 2.777452\end{array}$

$\begin{array}{lrrr}\text { C } & 0.315766 & -1.865043 & 2.777452 \\ \text { C } & -0.518492 & -0.932814 & 2.148831\end{array}$

$\begin{array}{llll}\mathrm{C} & -0.518492 & -0.932814 & 2.148831 \\ \mathrm{H} & -0.410767 & -0.190594 & -4.391416\end{array}$

$\begin{array}{llll}\mathrm{H} & -8.692735 & 0.431821 & -2.244980\end{array}$

$\begin{array}{llll}\mathrm{H} & -8.368133 & -3.831163 & -1.195624\end{array}$

$\begin{array}{llll}\mathrm{H} & -4.393790 & 1.530624 & -4.659450\end{array}$

$\begin{array}{llll}\mathrm{H} & -3.883095 & -4.073337 & 1.445661\end{array}$

$\begin{array}{llll}\mathrm{H} & -1.996376 & 1.487371 & -5.381805\end{array}$

$\begin{array}{llll}\mathrm{H} & -1.241628 & -1.825211 & -2.669178\end{array}$

$\begin{array}{llll}\mathrm{H} & -2.333795 & 0.680873 & 0.878077\end{array}$

\begin{tabular}{llll}
$\mathrm{H}$ & -4.558295 & -0.001621 & 0.021134 \\
\hline
\end{tabular}

$\begin{array}{llll}\mathrm{H} & -7.952427 & -0.061356 & -0.698124\end{array}$

$\begin{array}{llll}\mathrm{H} & -3.643245 & -1.785183 & -1.954241\end{array}$

$\begin{array}{llll}\mathrm{H} & -1.730828 & -4.635097 & 2.599307\end{array}$

$\begin{array}{llll}\mathrm{H} & 0.512025 & -3.946723 & 3.426012\end{array}$

$\begin{array}{llll}\mathrm{H} & 1.310264 & -1.568661 & 3.145227\end{array}$

$\begin{array}{llll}\mathrm{H} & -0.182901 & 0.110553 & 2.017441\end{array}$

$\begin{array}{llll}\mathrm{H} & -8.705151 & -1.978736 & -3.005420\end{array}$

$\begin{array}{llll}\mathrm{H} & -9.705018 & -1.648708 & -1.581906\end{array}$

Alkyne_anionB
Free energy $=-885.8020137$ Hartree

\begin{tabular}{lrrr}
$\mathrm{C}$ & -2.688019 & 3.882741 & -4.017256 \\
$\mathrm{C}$ & -3.130704 & 5.209891 & -3.644653 \\
$\mathrm{C}$ & -2.085254 & 6.194147 & -3.472366 \\
$\mathrm{C}$ & -0.736315 & 5.868778 & -3.644826 \\
$\mathrm{C}$ & -0.330949 & 4.560256 & -3.997451 \\
$\mathrm{C}$ & -1.334219 & 3.580812 & -4.178024 \\
$\mathrm{C}$ & -4.479722 & 5.499923 & -3.435742 \\
$\mathrm{C}$ & -5.679060 & 5.686782 & -3.066909 \\
$\mathrm{C}$ & -6.966123 & 5.886914 & -2.666817 \\
$\mathrm{C}$ & -8.031088 & 4.811598 & -2.563296 \\
$\mathrm{C}$ & -7.565429 & 3.383762 & -2.947452 \\
$\mathrm{C}$ & -6.516522 & 2.845812 & -2.083164 \\
$\mathrm{C}$ & -5.582953 & 2.463636 & -1.378954 \\
$\mathrm{C}$ & -4.360462 & 2.189218 & -0.687318 \\
$\mathrm{C}$ & -4.190141 & 1.056120 & 0.178903 \\
$\mathrm{C}$ & -2.972468 & 0.816778 & 0.799612 \\
$\mathrm{C}$ & -1.848565 & 1.677112 & 0.586418 \\
$\mathrm{C}$ & -2.010554 & 2.817802 & -0.288476 \\
$\mathrm{C}$ & -3.274541 & 3.056786 & -0.892765 \\
$\mathrm{C}$ & -0.888450 & 3.667491 & -0.545549 \\
$\mathrm{C}$ & 0.338218 & 3.413793 & 0.056679 \\
$\mathrm{C}$ & 0.494841 & 2.303210 & 0.935452 \\
$\mathrm{C}$ & -0.575367 & 1.450330 & 1.190925 \\
$\mathrm{H}$ & 0.733969 & 4.310112 & -4.120237 \\
$\mathrm{H}$ & -7.258768 & 6.887917 & -2.295486 \\
$\mathrm{H}$ & -7.170616 & 3.409816 & -3.989342 \\
$\mathrm{H}$ & -2.371042 & 7.218395 & -3.182124 \\
$\mathrm{H}$ & -3.406637 & 3.932788 & -1.542790 \\
$\mathrm{H}$ & 0.024375 & 6.653471 & -3.489847 \\
$\mathrm{H}$ & -1.046942 & 2.549092 & -4.443755 \\
$\mathrm{H}$ & -2.855070 & -0.054683 & 1.464491 \\
$\mathrm{H}$ & -5.040743 & 0.376482 & 0.343512 \\
$\mathrm{H}$ & -8.442514 & 2.699673 & -2.958667 \\
$\mathrm{H}$ & -3.449617 & 3.096787 & -4.140463 \\
$\mathrm{H}$ & -0.449740 & 0.582597 & 1.859438 \\
$\mathrm{H}$ & 1.472544 & 2.115069 & 1.406394 \\
$\mathrm{H}$ & 1.198886 & 4.068235 & -0.153880 \\
$\mathrm{H}$ & -1.013319 & 4.508069 & -1.247514 \\
$\mathrm{H}$ & -8.449097 & 4.772959 & -1.526961 \\
$\mathrm{H}$ & -8.910856 & 5.042850 & -3.214208 \\
& & & \\
\hline
\end{tabular}

$A$ = 6-methoxynaphthyl, $B$ = phenyl

Bispropargyl

45

Free energy $=-1000.744793$ Hartree

$\begin{array}{lrrr}\mathrm{C} & -1.775063 & 2.118263 & -1.136270 \\ \mathrm{C} & -3.016777 & 2.659760 & -0.708208 \\ \mathrm{C} & -3.115057 & 3.176354 & 0.610865 \\ \mathrm{C} & -2.002267 & 3.160037 & 1.464867 \\ \mathrm{C} & -0.776585 & 2.627109 & 1.028012 \\ \mathrm{C} & -0.670087 & 2.108557 & -0.275364 \\ \mathrm{C} & -4.126103 & 2.711923 & -1.611793 \\ \mathrm{C} & -5.030795 & 2.785061 & -2.441210 \\ \mathrm{C} & -6.094531 & 2.850244 & -3.445730 \\ \mathrm{C} & -5.620690 & 2.789047 & -4.919500 \\ \mathrm{C} & -4.945366 & 4.080764 & -5.446648 \\ \mathrm{C} & -3.698943 & 4.423592 & -4.762572 \\ \mathrm{C} & -2.691176 & 4.648496 & -4.095232 \\ \mathrm{C} & -1.588030 & 4.828867 & -3.205508 \\ \mathrm{C} & -1.782135 & 5.448617 & -1.963108 \\ \mathrm{C} & -0.736880 & 5.525549 & -1.004252 \\ \mathrm{C} & 0.557325 & 4.958606 & -1.314115 \\ \mathrm{C} & 0.745374 & 4.362049 & -2.601927 \\ \mathrm{C} & -0.288972 & 4.297074 & -3.518860 \\ \mathrm{C} & -0.930745 & 6.106897 & 0.282875 \\ \mathrm{C} & 0.080515 & 6.121571 & 1.233500 \\ \mathrm{C} & 1.349865 & 5.539150 & 0.930614 \\ \mathrm{C} & 1.579653 & 4.981958 & -0.331661 \\ \mathrm{O} & 2.387290 & 5.485358 & 1.815356 \\ \mathrm{C} & 2.209239 & 6.018280 & 3.126655 \\ \mathrm{H} & 0.101020 & 2.637793 & 1.691740 \\ \mathrm{H} & -6.799315 & 2.006279 & -3.268021 \\ \mathrm{H} & -5.660493 & 4.929866 & -5.350655 \\ \mathrm{H} & -1.683336 & 1.739705 & -2.164304 \\ \mathrm{H} & -2.776191 & 5.841616 & -1.703447 \\ \mathrm{H} & 0.291949 & 1.711673 & -0.633090 \\ \mathrm{H} & -2.087965 & 3.583715 & 2.476999 \\ \mathrm{H} & 1.728629 & 3.931384 & -2.848821 \\ \mathrm{H} & -0.135690 & 3.814242 & -4.495537 \\ \mathrm{H} & -4.758072 & 3.964674 & -6.537340 \\ \mathrm{H} & -4.069428 & 3.606436 & 0.950332 \\ \mathrm{H} & 2.564514 & 4.539423 & -0.543760 \\ \mathrm{H} & -0.112192 & 6.565030 & 2.219128 \\ \mathrm{H} & -1.914778 & 6.534680 & 0.529298 \\ \mathrm{H} & 3.165440 & 5.854345 & 3.658067 \\ \mathrm{H} & 1.990266 & 7.108670 & 3.104952 \\ \mathrm{H} & 1.396143 & 5.496363 & 3.678244 \\ \mathrm{H} & -6.696242 & 3.774923 & -3.289946\end{array}$


$\begin{array}{llll}\mathrm{H} & -4.931954 & 1.928258 & -5.051239\end{array}$

$\begin{array}{llll}\mathrm{H} & -6.509774 & 2.592884 & -5.554370\end{array}$

$\begin{array}{cccc}\text { Alkyne_anionA } & & \\ \text { 44 } & & & \\ \text { Free } & \text { energy }=-1000.222104 & \text { Hartree } \\ \text { C } & -2.164728 & -1.103969 & 5.535747 \\ \text { C } & -3.484322 & -1.326111 & 5.073600 \\ \text { C } & -4.157580 & -2.582046 & 5.363871 \\ \text { C } & -3.419792 & -3.548966 & 6.123228 \\ \text { C } & -2.118189 & -3.311540 & 6.568450 \\ \text { C } & -1.474975 & -2.075575 & 6.275996 \\ \text { C } & -5.477883 & -2.806778 & 4.898800 \\ \text { C } & -6.193787 & -1.834532 & 4.132531 \\ \text { C } & -5.493489 & -0.583146 & 3.865940 \\ \text { C } & -4.211146 & -0.347745 & 4.310255 \\ \text { C } & -7.479529 & -2.005190 & 3.623359 \\ \text { C } & -8.613694 & -2.092759 & 3.065602 \\ \text { C } & -9.823113 & -2.146573 & 2.439945 \\ \text { C } & -10.384260 & -1.035822 & 1.570791 \\ \text { C } & -9.549026 & 0.269741 & 1.568441 \\ \text { C } & -8.219493 & 0.133406 & 0.975353 \\ \text { C } & -7.094262 & -0.017769 & 0.502890 \\ \text { C } & -5.758340 & -0.296399 & 0.070761 \\ \text { C } & -5.040353 & -1.364162 & 0.676417 \\ \text { C } & -3.725770 & -1.647816 & 0.281556 \\ \text { C } & -3.101142 & -0.881457 & -0.721024 \\ \text { C } & -3.803060 & 0.177335 & -1.328214 \\ \text { C } & -5.119107 & 0.470909 & -0.939012 \\ \text { O } & -0.192354 & -1.752608 & 6.674509 \\ \text { C } & 0.535028 & -2.706462 & 7.431803 \\ \text { H } & -2.068227 & -1.107728 & -1.028111 \\ \text { H } & -10.112515 & 1.068809 & 1.038118 \\ \text { H } & -10.434227 & -3.066325 & 2.509897 \\ \text { H } & -5.667011 & 1.300559 & -1.411441 \\ \text { H } & -5.962313 & -3.770475 & 5.127094 \\ \text { H } & -3.319530 & 0.780801 & -2.112799 \\ \text { H } & -3.181447 & -2.472335 & 0.767526 \\ \text { H } & -3.716525 & 0.608781 & 4.071052 \\ \text { H } & -6.018536 & 0.176158 & 3.264468 \\ \text { H } & -9.425414 & 0.615010 & 2.620311 \\ \text { H } & -5.533443 & -1.950834 & 1.465486 \\ \text { H } & -3.899833 & -4.512935 & 6.360453 \\ \text { H } & -1.600631 & -4.092599 & 7.144204 \\ \text { H } & -1.656982 & -0.150932 & 5.314270 \\ \text { H } & 1.519655 & -2.251337 & 7.651398 \\ \text { H } & 0.033062 & -2.952428 & 8.396120 \\ \text { H } & 0.697758 & -3.655710 & 6.870568 \\ \text { H } & -11.416910 & -0.760068 & 1.896580 \\ \text { H } & -10.503313 & -1.370476 & 0.509440 \\ & & & \end{array}$

Alkyne_anionB

44

Free energy $=-1000.222019$ Hartree

$\begin{array}{llll}\text { C } & 1.752127 & 6.207090 & -0.687540\end{array}$

$\begin{array}{llll}\text { C } & 0.626515 & 5.906610 & -1.500808\end{array}$

$\begin{array}{llll}\text { C } & -0.252166 & 4.822969 & -1.115533\end{array}$

$\begin{array}{llll}\text { C } & 0.026810 & 4.107606 & 0.087431\end{array}$

$\begin{array}{llll}\text { C } & 1.130136 & 4.421307 & 0.871924\end{array}$

$\begin{array}{llll}\text { C } & 2.009063 & 5.478493 & 0.479407\end{array}$

$\begin{array}{llll}\text { C } & -1.379467 & 4.513260 & -1.922378\end{array}$

$\begin{array}{llll}\text { C } & -1.682708 & 5.258168 & -3.073368\end{array}$

$\begin{array}{llll}\text { C } & -0.807832 & 6.334216 & -3.449985 \\ \text { C } & 0.311840 & 6.640512 & -2.691015\end{array}$

$\begin{array}{llll}\text { C } & 0.311840 & 6.640512 & -2.691015\end{array}$

$\begin{array}{llll}\text { C } & -2.870017 & 4.925251 & -3.800006\end{array}$

$\begin{array}{llll}\text { C } & -3.936064 & 4.510967 & -4.253279\end{array}$

$\begin{array}{llll}\text { C } & -5.201559 & 3.942371 & -4.714730\end{array}$

$\begin{array}{llll}\text { C } & -5.104767 & 2.461100 & -5.162155\end{array}$

$\begin{array}{llll}\text { C } & -4.687499 & 1.486734 & -4.076621\end{array}$

$\begin{array}{llll}\text { C } & -4.446705 & 1.809825 & -2.775089\end{array}$

$\begin{array}{llll}\text { C } & -4.219638 & 2.107889 & -1.562762\end{array}$

$\begin{array}{llll}\text { C } & -3.769602 & 2.514870 & -0.305762\end{array}$

$\begin{array}{llll}\text { C } & -3.891515 & 3.888105 & 0.138323\end{array}$

$\begin{array}{llll}\text { C } & -3.396191 & 4.305124 & 1.375724\end{array}$

$\begin{array}{llll}\text { C } & -2.766333 & 3.402263 & 2.263728\end{array}$

$\begin{array}{llll}\text { C } & -2.646391 & 2.051263 & 1.861068\end{array}$

$\begin{array}{llll}\text { C } & -3.126490 & 1.611456 & 0.623455\end{array}$

$\begin{array}{llll}\text { O } & 3.116225 & 5.846235 & 1.193167\end{array}$

$\begin{array}{llll}\text { C } & 3.431013 & 5.140973 & 2.390618\end{array}$

$\begin{array}{llll}\mathrm{H} & -2.374083 & 3.742150 & 3.234049\end{array}$

$\mathrm{H} \quad-4.489503 \quad 0.450753 \quad-4.412288$

$\begin{array}{llll}\mathrm{H} & -5.932804 & 4.010969 & -3.876458\end{array}$

$\begin{array}{llll}\mathrm{H} & -3.004874 & 0.555862 & 0.330721\end{array}$

$\begin{array}{llll}\mathrm{H} & -2.037045 & 3.680655 & -1.636390\end{array}$

$\begin{array}{llll}\mathrm{H} & -2.155624 & 1.323780 & 2.530580\end{array}$

$\begin{array}{llll}\mathrm{H} & -3.494239 & 5.367803 & 1.656201\end{array}$

$\begin{array}{llll}\mathrm{H} & 0.974420 & 7.467546 & -2.994163\end{array}$

$\begin{array}{llll}\mathrm{H} & -1.038728 & 6.917734 & -4.354565\end{array}$

$\begin{array}{llll}\mathrm{H} & -5.616303 & 4.553197 & -5.546763\end{array}$

$\begin{array}{llll}\mathrm{H} & -4.357259 & 4.614684 & -0.545916\end{array}$

$\begin{array}{rrrr}\mathrm{H} & 2.436378 & 7.024729 & -0.962648 \\ \mathrm{H} & 1.306296 & 3.857979 & 1.797989 \\ \mathrm{H} & -0.666673 & 3.310736 & 0.401477 \\ \mathrm{H} & 4.356314 & 5.600335 & 2.784755 \\ \mathrm{H} & 2.626383 & 5.237788 & 3.153922 \\ \mathrm{H} & 3.618884 & 4.060854 & 2.199432 \\ \mathrm{H} & -4.401013 & 2.404565 & -6.029122 \\ \mathrm{H} & -6.103338 & 2.194439 & -5.591249\end{array}$

\begin{tabular}{|c|c|c|c|}
\hline \multicolumn{4}{|c|}{ A = 2-pyridyl, B = phenyl } \\
\hline \multicolumn{4}{|c|}{ Bispropargyl } \\
\hline & & & \\
\hline \multicolumn{4}{|c|}{ Free energy $=-748.8597586$ Hartree } \\
\hline $\mathrm{C}$ & 1.363530 & 3.656521 & -0.122198 \\
\hline $\mathrm{C}$ & 2.463769 & 4.094070 & -0.906461 \\
\hline $\mathrm{N}$ & 2.380488 & 4.266164 & -2.251683 \\
\hline $\mathrm{C}$ & 1.202675 & 3.996187 & -2.840004 \\
\hline $\mathrm{C}$ & 0.061274 & 3.550450 & -2.149848 \\
\hline $\mathrm{C}$ & 0.149476 & 3.377868 & -0.758236 \\
\hline $\mathrm{C}$ & 3.728903 & 4.338106 & -0.272025 \\
\hline $\mathrm{C}$ & 4.787435 & 4.4 & 0.336967 \\
\hline $\mathrm{C}$ & 6.046467 & & 1.073824 \\
\hline $\mathrm{C}$ & 6.823421 & & \\
\hline $\mathrm{C}$ & 6.13 & & \\
\hline $\mathrm{C}$ & 4.93 & & \\
\hline $\mathrm{C}$ & 3.9 & & \\
\hline $\mathrm{C}$ & & & \\
\hline $\mathrm{C}$ & 2.71 & & \\
\hline $\mathrm{C}$ & 1.54 & 0. & \\
\hline $\mathrm{C}$ & 0.41 & -0.0 & -1 \\
\hline $\mathrm{C}$ & 0.445209 & -0.1 & 0.2 \\
\hline $\mathrm{C}$ & 1.604704 & 0.24 & 0.973269 \\
\hline $\mathrm{H}$ & -0.504454 & -0.2 & -1.685765 \\
\hline $\mathrm{H}$ & -0.869412 & 3.3 & -2.695708 \\
\hline $\mathrm{H}$ & 6.706499 & 5.30 & 0.56 \\
\hline $\mathrm{H}$ & 5.883509 & 2.61 & 3.100347 \\
\hline $\mathrm{H}$ & 1.490397 & 3.51 & 0.960 \\
\hline $\mathrm{H}$ & 3.593765 & 1.151 & -1.68 \\
\hline $\mathrm{H}$ & -0.713099 & 3.015867 & -0.178953 \\
\hline $\mathrm{H}$ & 1.526016 & 2 & 05 \\
\hline $\mathrm{H}$ & 1.161 & 6 & -3 \\
\hline I & -0 & & \\
\hline $\mathrm{H}$ & & & 2.071885 \\
\hline $\mathrm{H}$ & 6.870974 & & 2.323265 \\
\hline $\mathrm{H}$ & 5.845034 & 4.992405 & 2.084161 \\
\hline $\mathrm{H}$ & 7.805478 & 3.475484 & 1.683958 \\
\hline $\mathrm{H}$ & 7.032170 & 2.813818 & 0.221102 \\
\hline
\end{tabular}

Alkyne anionA

Free energy $=-748.3484678$ Hartree

$\begin{array}{llll}\text { C } & 1.834955 & 0.794556 & -0.368662\end{array}$

$\begin{array}{llll}\text { C } & 1.834955 & 0.794556 & -0.368662 \\ \text { C } & 2.936037 & 0.168595 & 0.279813\end{array}$

$\begin{array}{llll}\text { C } & 2.936037 & 0.168595 & 0.279813 \\ \text { C } & 3.198876 & -1.203507 & 0.022483\end{array}$

$\begin{array}{llll}\text { C } & 2.381518 & -1.929744 & -0.858051\end{array}$

$\begin{array}{llll}\text { C } & 1.291303 & -1.305665 & -1.494046\end{array}$

$\begin{array}{llll}\text { C } & 1.026311 & 0.055271 & -1.245052\end{array}$

$\begin{array}{llll}\text { C } & 3.752273 & 0.942797 & 1.165160\end{array}$

$\begin{array}{llll}\text { C } & 4.387970 & 1.711119 & 1.884088\end{array}$

$\begin{array}{llll}\text { C } & 5.130928 & 2.651305 & 2.720963\end{array}$

$\begin{array}{llll}\text { C } & 6.321830 & 3.341009 & 2.010482\end{array}$

$\begin{array}{llll}\text { C } & 5.937247 & 4.298460 & 0.899488\end{array}$

$\begin{array}{llll}\text { C } & 4.698635 & 4.436334 & 0.354422\end{array}$

$\begin{array}{llll}\text { C } & 3.550031 & 4.573701 & -0.166260\end{array}$

$\begin{array}{llll}C & 2.326743 & 4.750388 & -0.803626\end{array}$

$\begin{array}{llll}\mathrm{N} & 1.223030 & 3.993498 & -0.414756\end{array}$

$\begin{array}{llll}\text { C } & 0.058785 & 4.189595 & -1.049482\end{array}$

$\begin{array}{llll}\text { C } & -0.163159 & 5.107622 & -2.092183 \\ \text { C } & 0.953205 & 5.885081 & -2.504775\end{array}$

$\begin{array}{llll}\text { C } & 0.953205 & 5.885081 & -2.504775\end{array}$

$\begin{array}{llll}\text { C } & 2.180477 & 5.717619 & -1.878185\end{array}$

$\begin{array}{llll}\mathrm{H} & 0.651790 & -1.879273 & -2.183820\end{array}$

$\begin{array}{llll}\mathrm{H} & -1.152829 & 5.208040 & -2.562012\end{array}$

$\begin{array}{llll}\mathrm{H} & 6.762628 & 4.914547 & 0.493715\end{array}$

$\begin{array}{llll}\mathrm{H} & 4.425164 & 3.435753 & 3.076728\end{array}$

$\begin{array}{llll}\mathrm{H} & 3.058036 & 6.309219 & -2.18359\end{array}$

$\begin{array}{llll}\mathrm{H} & 1.636583 & 1.869920 & -0.186766\end{array}$

$\begin{array}{llll}\mathrm{H} & 0.850291 & 6.619078 & -3.322496\end{array}$

$\begin{array}{llll}\mathrm{H} & 0.178932 & 0.553031 & -1.743498\end{array}$

$\begin{array}{llll}\mathrm{H} & -0.780243 & 3.552592 & -0.702698\end{array}$

$\begin{array}{llll}\mathrm{H} & 2.598974 & -2.992139 & -1.052269\end{array}$

$\begin{array}{llll}\mathrm{H} & 4.053311 & -1.690961 & 0.516856\end{array}$

$\begin{array}{llll}\mathrm{H} & 5.502115 & 2.123265 & 3.627341\end{array}$

$\begin{array}{llll}\mathrm{H} & 7.011199 & 2.539879 & 1.642043\end{array}$

$\begin{array}{llll}\mathrm{H} & 6.906022 & 3.877746 & 2.796520\end{array}$

Alkyne_anionB

Free energy $=-748.3378984$ Hartree

$\begin{array}{llll}\text { C } \quad-0.496700 & 3.739757 & -0.532584\end{array}$ 


$\begin{array}{rrrr}\mathrm{C} & 0.522593 & 4.332857 & -1.327095 \\ \mathrm{~N} & 0.280421 & 4.858249 & -2.558325 \\ \mathrm{C} & -0.980516 & 4.799148 & -3.020756 \\ \mathrm{C} & -2.055270 & 4.230658 & -2.311615 \\ \mathrm{C} & -1.800719 & 3.691459 & -1.038480 \\ \mathrm{C} & 1.862080 & 4.358195 & -0.811878 \\ \mathrm{C} & 2.941936 & 4.260194 & -0.232219 \\ \mathrm{C} & 4.202706 & 4.122729 & 0.492202 \\ \mathrm{C} & 4.147479 & 4.613425 & 1.961506 \\ \mathrm{C} & 3.243886 & 3.799615 & 2.869911 \\ \mathrm{C} & 2.389299 & 2.828668 & 2.437321 \\ \mathrm{C} & 1.607570 & 1.933426 & 1.997575 \\ \mathrm{C} & 0.727899 & 1.015935 & 1.423186 \\ \mathrm{C} & -0.245192 & 0.268640 & 2.188421 \\ \mathrm{C} & -1.129681 & -0.624252 & 1.575256 \\ \mathrm{C} & -1.111089 & -0.842257 & 0.177564 \\ \mathrm{C} & -0.165370 & -0.125844 & -0.593126 \\ \mathrm{C} & 0.728734 & 0.770397 & -0.003869 \\ \mathrm{H} & -1.812158 & -1.547214 & -0.296610 \\ \mathrm{H} & -3.063693 & 4.211035 & -2.752523 \\ \mathrm{H} & 5.005777 & 4.665561 & -0.052353 \\ \mathrm{H} & 3.247628 & 4.085766 & 3.939046 \\ \mathrm{H} & -0.236155 & 3.325968 & 0.452275 \\ \mathrm{H} & -0.287591 & 0.422368 & 3.279807 \\ \mathrm{H} & -2.606737 & 3.229461 & -0.447898 \\ \mathrm{H} & -1.857467 & -1.168300 & 2.202704 \\ \mathrm{H} & -1.151050 & 5.230527 & -4.024008 \\ \mathrm{H} & -0.129951 & -0.269727 & -1.687376 \\ \mathrm{H} & 1.443598 & 1.332483 & -0.625266 \\ \mathrm{H} & 4.485899 & 3.045053 & 0.487550 \\ \mathrm{H} & 5.199227 & 4.611724 & 2.339801 \\ \mathrm{H} & 3.854073 & 5.694093 & 1.948399\end{array}$

\section{$A$ = 2-pyridyl, $B$ = 4-methoxyphenyl}

Bispropargyl

38

Free energy $=-863.2839729$ Hartree

$\begin{array}{llll}\mathrm{N} & -0.073902 & 2.573340 & -0.102979\end{array}$

$\begin{array}{llll}\text { C } & 0.507217 & 1.583294 & -0.830534\end{array}$

$\begin{array}{llll}\text { C } & -0.239979 & 0.717098 & -1.672373\end{array}$

$\begin{array}{llll}\text { C } & -0.239979 & 0.717098 & -1.672373 \\ \text { C } & -1.626565 & 0.879792 & -1.749379\end{array}$

$\begin{array}{llll}\text { C } & -2.230756 & 1.904746 & -1.000769\end{array}$

$\begin{array}{llll}\text { C } & -1.407312 & 2.713609 & -0.197713\end{array}$

$\begin{array}{llll}\text { C } & 1.927718 & 1.402337 & -0.715780\end{array}$

$\begin{array}{llll}\text { C } & 3.125918 & 1.142012 & -0.633125\end{array}$

$\begin{array}{llll}\text { C } & 4.555211 & 0.837067 & -0.567161\end{array}$

$\begin{array}{llll}\text { C } & 4.960466 & -0.561514 & -1.097923\end{array}$

$\begin{array}{llll}\text { C } & 4.625936 & -1.742139 & -0.151115\end{array}$

$\begin{array}{llll}\text { C } & 3.197197 & -1.896198 & 0.120390\end{array}$

$\begin{array}{llll}\text { C } & 1.981545 & -1.898967 & 0.304786\end{array}$

$\begin{array}{llll}\text { C } & 0.576377 & -1.732167 & 0.502015\end{array}$

$\begin{array}{llll}\text { C } & -0.385579 & -2.478844 & -0.231247\end{array}$

$\begin{array}{llll}\text { C } & -1.748511 & -2.200798 & -0.112681\end{array}$

$\begin{array}{llll}\text { C } & -2.197991 & -1.168056 & 0.744179\end{array}$

$\begin{array}{llll}\text { C } & -1.259302 & -0.444187 & 1.511830\end{array}$

$\begin{array}{llll}\text { C } & 0.106473 & -0.724726 & 1.382802\end{array}$

$\begin{array}{llll}\text { O } & -3.539137 & -0.933943 & 0.747399\end{array}$

$\begin{array}{llll}\text { C } & -4.055098 & 0.067236 & 1.623476\end{array}$

$\begin{array}{llll}\mathrm{H} & -3.318079 & 2.071670 & -1.031673\end{array}$

$\begin{array}{llll}\mathrm{H} & 5.101061 & 1.609782 & -1.153121\end{array}$

$\begin{array}{llll}\mathrm{H} & 5.169302 & -1.603097 & 0.811068\end{array}$

$\begin{array}{llll}\mathrm{H} & 0.274735 & -0.083178 & -2.222087\end{array}$

$\begin{array}{llll}\mathrm{H} & 0.835516 & -0.124296 & 1.946007\end{array}$

$\begin{array}{llll}\mathrm{H} & -2.231591 & 0.206758 & -2.375709\end{array}$

$\begin{array}{llll}\mathrm{H} & -1.576403 & 0.367585 & 2.179763\end{array}$

$\begin{array}{llll}\mathrm{H} & -1.854469 & 3.524723 & 0.405636\end{array}$

$\begin{array}{llll}\mathrm{H} & -2.495402 & -2.760355 & -0.695207\end{array}$

$\begin{array}{llll}\mathrm{H} & -0.046840 & -3.269045 & -0.918682\end{array}$

$\begin{array}{llll}\mathrm{H} & -5.148267 & 0.091202 & 1.457177\end{array}$

$\begin{array}{llll}\mathrm{H} & -3.630273 & 1.068946 & 1.395706\end{array}$

$\begin{array}{llll}\mathrm{H} & -3.851911 & -0.178278 & 2.689952\end{array}$

$\begin{array}{llll}\mathrm{H} & 5.032898 & -2.679102 & -0.591807\end{array}$

$\begin{array}{llll}\mathrm{H} & 4.910500 & 0.956109 & 0.481665\end{array}$

$\begin{array}{llll}\mathrm{H} & 4.488643 & -0.731005 & -2.088110\end{array}$

$\begin{array}{llll}\mathrm{H} & 6.058333 & -0.559598 & -1.259088\end{array}$

\section{Alkyne_anionA}

37

Free energy $=-862.7713665$ Hartree

$\begin{array}{lrrr}\mathrm{C} & -0.384195 & -2.489291 & -0.126736 \\ \mathrm{C} & 0.603169 & -1.665809 & 0.479610 \\ \mathrm{C} & 0.164604 & -0.542492 & 1.227500 \\ \mathrm{C} & -1.194719 & -0.230778 & 1.348604 \\ \mathrm{C} & -2.159176 & -1.042196 & 0.713962 \\ \mathrm{C} & -1.742250 & -2.184546 & -0.009195 \\ \mathrm{C} & 2.003762 & -1.874492 & 0.292033 \\ \mathrm{C} & 3.225042 & -1.904574 & 0.139147 \\ \mathrm{C} & 4.638817 & -1.681450 & -0.150061 \\ \mathrm{C} & 4.846884 & -0.384195 & -1.019729\end{array}$

$\begin{array}{lrrr}\mathrm{C} & 4.336176 & 0.892693 & -0.394036 \\ \mathrm{C} & 3.040132 & 1.300418 & -0.519522 \\ \mathrm{C} & 1.836883 & 1.656578 & -0.690966 \\ \mathrm{C} & 0.463404 & 1.861037 & -0.828731 \\ \mathrm{C} & -0.321866 & 0.981597 & -1.671727 \\ \mathrm{C} & -1.687238 & 1.182997 & -1.805714 \\ \mathrm{C} & -2.306831 & 2.265216 & -1.125324 \\ \mathrm{C} & -1.477212 & 3.063639 & -0.317114 \\ \mathrm{~N} & -0.156210 & 2.902488 & -0.149453 \\ \mathrm{O} & -3.503210 & -0.804632 & 0.734816 \\ \mathrm{C} & -3.975588 & 0.334315 & 1.453051 \\ \mathrm{H} & -3.384017 & 2.472425 & -1.214982 \\ \mathrm{H} & 5.005194 & 1.414346 & 0.316984 \\ \mathrm{H} & 5.204395 & -1.570695 & 0.802403 \\ \mathrm{H} & 0.180346 & 0.133592 & -2.161379 \\ \mathrm{H} & 0.915735 & 0.126679 & 1.669079 \\ \mathrm{H} & -2.286770 & 0.493511 & -2.423449 \\ \mathrm{H} & -1.484196 & 0.683366 & 1.882768 \\ \mathrm{H} & -1.928813 & 3.908293 & 0.243521 \\ \mathrm{H} & -2.509504 & -2.808276 & -0.492061 \\ \mathrm{H} & -0.069467 & -3.367593 & -0.711293 \\ \mathrm{H} & -5.075030 & 0.346239 & 1.326676 \\ \mathrm{H} & -3.547185 & 1.274454 & 1.042682 \\ \mathrm{H} & -3.734207 & 0.263958 & 2.537982 \\ \mathrm{H} & 5.080106 & -2.562949 & -0.671345 \\ \mathrm{H} & 4.345430 & -0.563071 & -1.996571 \\ \mathrm{H} & 5.938612 & -0.313319 & -1.227828\end{array}$

Alkyne_anionB

Free energy $=-862.7573978$ Hartree

$\begin{array}{llll}\text { C } & -0.125584 & -0.868024 & 1.099537\end{array}$

$\begin{array}{llll}\mathrm{C} & -0.032225 & -2.279706 & 1.226871\end{array}$

$\begin{array}{llll}\mathrm{N} & -1.074321 & -3.050367 & 1.643301\end{array}$

$\begin{array}{llll}\text { C } & -2.226451 & -2.419968 & 1.934142\end{array}$

$\begin{array}{llll}\text { C } & -2.420467 & -1.029810 & 1.824353\end{array}$

$\begin{array}{llll}\text { C } & -1.340600 & -0.238602 & 1.390549\end{array}$

$\begin{array}{llll}\text { C } & 1.214723 & -2.898526 & 0.875009\end{array}$

$\begin{array}{llll}\text { C } & 2.340804 & -3.244099 & 0.518495\end{array}$

$\begin{array}{llll}\text { C } & 3.676285 & -3.529328 & 0.010544\end{array}$

$\begin{array}{llll}\text { C } & 4.140312 & -2.473808 & -1.062446\end{array}$

$\begin{array}{llll}\text { C } & 4.217376 & -1.054921 & -0.559535\end{array}$

$\begin{array}{llll}\text { C } & 3.217188 & -0.139500 & -0.744473\end{array}$

$\begin{array}{llll}\text { C } & 2.311324 & 0.715647 & -0.982882\end{array}$

$\begin{array}{llll}\text { C } & 1.122679 & 1.467796 & -0.938142\end{array}$

$\begin{array}{llll}\text { C } & -0.042206 & 1.095948 & -1.694662\end{array}$

$\begin{array}{llll}\text { C } & -1.257866 & 1.787751 & -1.593935\end{array}$

$\begin{array}{llll}\text { C } & -1.377441 & 2.909144 & -0.741707\end{array}$

$\begin{array}{llll}\text { C } & -0.239589 & 3.323399 & -0.010152\end{array}$

$\begin{array}{llll}\text { C } & 0.972043 & 2.634012 & -0.104806\end{array}$

$\begin{array}{llll}\text { O } & -2.530781 & 3.655356 & -0.562994\end{array}$

$\begin{array}{llll}\text { C } & -3.698321 & 3.232879 & -1.244034\end{array}$

$\begin{array}{llll}\mathrm{H} & -3.397596 & -0.586074 & 2.069525\end{array}$

$\begin{array}{llll}\mathrm{H} & 3.712248 & -4.560629 & -0.412971\end{array}$

$\begin{array}{llll}\mathrm{H} & 5.042676 & -0.818269 & 0.140211\end{array}$

$\begin{array}{llll}\mathrm{H} & 0.745712 & -0.302485 & 0.738430\end{array}$

$\begin{array}{llll}\mathrm{H} & 0.014993 & 0.204864 & -2.339230\end{array}$

$\begin{array}{llll}\mathrm{H} & -1.438271 & 0.850947 & 1.254535\end{array}$

$\begin{array}{llll}\mathrm{H} & -2.117957 & 1.429723 & -2.178317\end{array}$

$\begin{array}{llll}\mathrm{H} & -3.058837 & -3.064082 & 2.273216\end{array}$

$\begin{array}{llll}\mathrm{H} & -0.333692 & 4.203268 & 0.647001\end{array}$

$\begin{array}{llll}\mathrm{H} & 1.832906 & 2.970376 & 0.494657\end{array}$

$\mathrm{H} \quad-4.506976 \quad 3.931963 \quad-0.955405$

$\begin{array}{llll}\mathrm{H} & -4.001105 & 2.197789 & -0.957740\end{array}$

$\begin{array}{llll}\mathrm{H} & -3.576382 & 3.263929 & -2.352768\end{array}$

$\begin{array}{llll}\mathrm{H} & 4.401110 & -3.516716 & 0.855800\end{array}$

$\begin{array}{llll}\mathrm{H} & 3.439025 & -2.537529 & -1.922918\end{array}$

$\begin{array}{llll}\mathrm{H} & 5.129654 & -2.835991 & -1.428810\end{array}$

A = 1-benzyl-3-indolyl, $B$ = phenyl

Bispropargyl

52

Free energy $=-1134.378699$ Hartree

$\begin{array}{llll}\text { C } & 6.438337 & 6.426041 & 1.782327\end{array}$

$\begin{array}{llll}\text { C } & 5.447344 & 5.919374 & 0.901077\end{array}$

$\begin{array}{llll}\text { C } & 5.411485 & 6.401332 & -0.434413\end{array}$

$\begin{array}{llll}\text { C } & 6.343638 & 7.352147 & -0.871939\end{array}$

$\begin{array}{llll}\text { C } & 7.325087 & 7.843330 & 0.008647\end{array}$

$\begin{array}{llll}\text { C } & 7.367383 & 7.375077 & 1.334759\end{array}$

$\begin{array}{llll}\text { C } & 4.521004 & 4.913569 & 1.322103\end{array}$

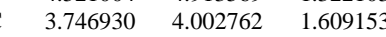

$\begin{array}{llll}\text { C } & 2.805898 & 2.923035 & 1.914186\end{array}$

$\begin{array}{llll}\text { C } & 2.230068 & 2.183405 & 0.678930\end{array}$

$\begin{array}{llll}\text { C } & 3.203684 & 1.188109 & -0.001564\end{array}$

$\begin{array}{llll}\text { C } & 4.430046 & 1.804560 & -0.507502\end{array}$

$\begin{array}{llll}\text { C } & 5.421958 & 2.453005 & -0.839170\end{array}$

$\begin{array}{llll}\text { C } & 6.570526 & 3.260286 & -1.030723\end{array}$

$\begin{array}{llll}\text { C } & 7.535419 & 3.592071 & 0.005775\end{array}$

$\begin{array}{llll}\text { C } & 8.459839 & 4.518515 & -0.567404\end{array}$ 


\begin{tabular}{cccc}
$\mathrm{N}$ & 8.075213 & 4.742800 & -1.883335 \\
$\mathrm{C}$ & 6.952832 & 3.987900 & -2.162579 \\
$\mathrm{C}$ & 7.669838 & 3.226104 & 1.360561 \\
$\mathrm{C}$ & 8.725277 & 3.774255 & 2.101191 \\
$\mathrm{C}$ & 9.646472 & 4.678226 & 1.509210 \\
$\mathrm{C}$ & 9.528512 & 5.063842 & 0.167164 \\
$\mathrm{C}$ & 8.764322 & 5.634760 & -2.800148 \\
$\mathrm{C}$ & 10.146080 & 5.142589 & -3.207263 \\
$\mathrm{C}$ & 10.430982 & 3.764606 & -3.286480 \\
$\mathrm{C}$ & 11.701133 & 3.322378 & -3.694063 \\
$\mathrm{C}$ & 12.701908 & 4.253303 & -4.027126 \\
$\mathrm{C}$ & 12.425895 & 5.630018 & -3.945101 \\
$\mathrm{C}$ & 11.156030 & 6.070751 & -3.532934 \\
$\mathrm{H}$ & 8.061079 & 8.584990 & -0.339726 \\
$\mathrm{H}$ & 2.665709 & 0.671371 & -0.827182 \\
$\mathrm{H}$ & 1.957161 & 3.346351 & 2.497231 \\
$\mathrm{H}$ & 4.658240 & 5.997950 & -1.126716 \\
$\mathrm{H}$ & 6.309214 & 7.706876 & -1.913798 \\
$\mathrm{H}$ & 8.143064 & 7.741685 & 2.024122 \\
$\mathrm{H}$ & 6.486285 & 6.043887 & 2.812077 \\
$\mathrm{H}$ & 8.121202 & 5.754961 & -3.696219 \\
$\mathrm{H}$ & 8.839672 & 6.638613 & -2.329951 \\
$\mathrm{H}$ & 10.947016 & 7.151002 & -3.459612 \\
$\mathrm{H}$ & 13.206040 & 6.365803 & -4.195733 \\
$\mathrm{H}$ & 13.697144 & 3.905900 & -4.344971 \\
$\mathrm{H}$ & 11.911632 & 2.242535 & -3.749062 \\
$\mathrm{H}$ & 9.652651 & 3.033334 & -3.016739 \\
$\mathrm{H}$ & 6.478904 & 4.040585 & -3.149374 \\
$\mathrm{H}$ & 3.292361 & 2.185211 & 2.592955 \\
$\mathrm{H}$ & 6.940880 & 2.539021 & 1.816801 \\
$\mathrm{H}$ & 8.844543 & 3.503204 & 3.161720 \\
$\mathrm{H}$ & 10.465514 & 5.091758 & 2.117964 \\
$\mathrm{H}$ & 10.236177 & 5.769064 & -0.292168 \\
$\mathrm{H}$ & 3.471238 & 0.388354 & 0.726675 \\
$\mathrm{H}$ & 1.337771 & 1.609849 & 1.007533 \\
$\mathrm{H}$ & 1.879822 & 2.929866 & -0.064180 \\
& & & \\
\hline
\end{tabular}

Alkyne_anionA

51

Free energy $=-1133.843527$ Hartree

$\begin{array}{llll}\text { C } & 10.405840 & 3.899887 & -2.927093 \\ \text { C } & 10.088633 & 5.268911 & -3.037610\end{array}$

$\begin{array}{llll}\text { C } & 10.088633 & 5.268911 & -3.037610\end{array}$

$\begin{array}{llll}\text { C } & 11.044527 & 6.148408 & -3.588954\end{array}$

$\begin{array}{llll}\text { C } & 12.290571 & 5.668769 & -4.029612\end{array}$

$\begin{array}{llll}\text { C } & 12.598231 & 4.299822 & -3.920770\end{array}$

$\begin{array}{llll}\text { C } & 11.651624 & 3.418087 & -3.366789\end{array}$

$\begin{array}{llll}\text { C } & 8.734408 & 5.801481 & -2.579864\end{array}$

$\begin{array}{llll}\mathrm{N} & 8.027497 & 4.931045 & -1.672233\end{array}$

$\begin{array}{llll}\text { C } & 8.343158 & 4.747475 & -0.344064\end{array}$

$\begin{array}{llll}\text { C } & 7.485082 & 3.718192 & 0.171331\end{array}$

$\begin{array}{llll}\text { C } & 6.608995 & 3.269835 & -0.909088\end{array}$

$\begin{array}{llll}\text { C } & 6.996187 & 4.036421 & -2.019216\end{array}$

$\begin{array}{llll}\text { C } & 9.298826 & 5.395033 & 0.463492\end{array}$

$\begin{array}{llll}\text { C } & 9.371795 & 5.020872 & 1.813106\end{array}$

$\begin{array}{llll}\text { C } & 8.518064 & 4.018274 & 2.346352\end{array}$

$\begin{array}{llll}\text { C } & 7.580270 & 3.365336 & 1.531786\end{array}$

$\begin{array}{llll}\text { C } & 5.620629 & 2.235875 & -0.782522\end{array}$

$\begin{array}{llll}\text { C } & 4.342728 & 2.184864 & -0.635811\end{array}$

$\begin{array}{llll}\text { C } & 3.010453 & 2.055701 & -0.406922 \\ \text { C } & 2.369388 & 2.158022 & 0.968625\end{array}$

$\begin{array}{llll}\text { C } & 2.369388 & 2.158022 & 0.968625\end{array}$

$\begin{array}{llll}\text { C } & 3.349780 & 2.571034 & 2.096128\end{array}$

$\begin{array}{llll}\text { C } & 4.002068 & 3.859631 & 1.866773\end{array}$

$\begin{array}{llll}\mathrm{C} & 4.609262 & 4.892137 & 1.589314 \\ \mathrm{C} & 5.374204 & 5.992974 & 1.092830\end{array}$

$\begin{array}{llll}\mathrm{C} & 5.374204 & 5.992974 & 1.092830 \\ \mathrm{C} & 5.309893 & 6.310871 & -0.291139\end{array}$

$\begin{array}{llll}\text { C } & 5.309893 & 6.310871 & -0.291139 \\ \text { C } & 6.983427 & 7.330829 & -0.820920\end{array}$

$\begin{array}{llll}\text { C } & 6.983427 & 8.056580 & 0.011864\end{array}$

$\begin{array}{llll}\text { C } & 7.049416 & 7.753629 & 1.384295\end{array}$

$\begin{array}{llll}\text { C } & 6.251246 & 6.734879 & 1.924731\end{array}$

$\begin{array}{llll}\mathrm{H} & 7.620385 & 8.849459 & -0.411594\end{array}$

$\mathrm{H} \quad 2.310165 \quad 1.953610 \quad-1.259443$

$\begin{array}{llll}\mathrm{H} & 2.820569 & 2.569643 & 3.075421\end{array}$

$\mathrm{H} \quad 4.659640 \quad 5.708056 \quad-0.940645$

$\begin{array}{llll}\mathrm{H} & 6.070232 & 7.548235 & -1.899934\end{array}$

$\begin{array}{llll}\mathrm{H} & 7.745041 & 8.303924 & 2.036967\end{array}$

\begin{tabular}{llll}
$\mathrm{H}$ & 6.325971 & 6.475683 & 2.991334 \\
\hline
\end{tabular}

H $\quad 8.083262 \quad 5.978134 \quad-3.462946$

$\begin{array}{llll}\mathrm{H} & 8.865826 & 6.796295 & -2.099563\end{array}$

$\begin{array}{llll}\mathrm{H} & 10.810425 & 7.223084 & -3.669997\end{array}$

\begin{tabular}{llll}
$\mathrm{H}$ & 13.028806 & 6.368553 & -4.452825 \\
\hline
\end{tabular}

$\mathrm{H} \quad 13.575405 \quad 3.922779 \quad-4.260824$

$\begin{array}{llll}\mathrm{H} & 11.886111 & 2.346023 & -3.270170\end{array}$

H $\quad 9.666329 \quad 3.212216 \quad-2.487115$

$\begin{array}{llll}\mathrm{H} & 6.599124 & 4.039718 & -3.041236\end{array}$

$\begin{array}{llll}\mathrm{H} & 4.152152 & 1.801300 & 2.171032\end{array}$

$\begin{array}{llll}\mathrm{H} & 6.904734 & 2.594444 & 1.934604\end{array}$

$\begin{array}{llll}\mathrm{H} & 8.598604 & 3.751111 & 3.412420\end{array}$

$\begin{array}{llll}\mathrm{H} & 10.099447 & 5.521463 & 2.472360\end{array}$

$\begin{array}{llll}\mathrm{H} & 9.955686 & 6.177112 & 0.052940\end{array}$

$\begin{array}{llll}\mathrm{H} & 1.528608 & 2.894417 & 0.953167\end{array}$
H $\quad 1.897009 \quad 1.195804 \quad 1.295392$

\begin{tabular}{|c|c|c|c|}
\hline $\begin{array}{l}\text { Alk } \\
51 \\
\text { Free }\end{array}$ & _anionB & $7 \mathrm{H}$ & rtree \\
\hline $\mathrm{C}$ & 5.771276 & 13.116796 & -1.198293 \\
\hline C & 5.489231 & 5137 & \\
\hline $\mathrm{C}$ & & & \\
\hline $\mathrm{C}$ & & & \\
\hline $\mathrm{C}$ & 3.66 & 13.998 & \\
\hline $\mathrm{C}$ & 4.86 & & \\
\hline $\mathrm{C}$ & & & \\
\hline $\mathrm{N}$ & 6.2 & & \\
\hline $\mathrm{C}$ & 6.5 & & \\
\hline $\mathrm{C}$ & 6.0 & & \\
\hline $\mathrm{C}$ & 5.4 & & \\
\hline C & 5.590964 & 1 & \\
\hline C & 7.113260 & 10.521348 & 89023 \\
\hline $\mathrm{C}$ & 7.2 & & -5.955465 \\
\hline $\mathrm{C}$ & & & \\
\hline $\mathrm{C}$ & & & \\
\hline C & & & \\
\hline $\mathrm{C}$ & & & \\
\hline $\mathrm{C}$ & & & \\
\hline $\mathrm{C}$ & & & \\
\hline $\mathrm{C}$ & & & \\
\hline $\mathrm{C}$ & & & \\
\hline C & & & \\
\hline C & & & \\
\hline $\mathrm{C}$ & & & \\
\hline $\mathrm{C}$ & & & \\
\hline C & 3.5 & & 511 \\
\hline C & 2.72 & & -0. \\
\hline $\mathrm{C}$ & & & 50 \\
\hline 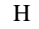 & & & \\
\hline $\mathrm{H}$ & & & \\
\hline $\mathrm{H}$ & & & \\
\hline $\mathrm{H}$ & & & \\
\hline $\mathrm{H}$ & & & \\
\hline $\mathrm{H}$ & & & \\
\hline I & & & \\
\hline I & & & \\
\hline $\mathrm{H}$ & & & \\
\hline I & & & \\
\hline I & & & \\
\hline $\mathrm{H}$ & 2.9 & & 205 \\
\hline $\mathrm{H}$ & 5.096 & & 3135 \\
\hline $\mathrm{H}$ & $6.707^{\prime}$ & 13.2 & -0.635769 \\
\hline $\mathrm{H}$ & 5.269317 & & -1.024698 \\
\hline $\mathrm{H}$ & 3.149 & & \\
\hline $\mathrm{H}$ & 5.779171 & & \\
\hline $\mathrm{H}$ & 6.889 & 88 & -7.5 \\
\hline $\mathrm{H}$ & & 0.966373 & -6.623969 \\
\hline $\mathrm{H}$ & & 11.478376 & \\
\hline $\mathrm{H}$ & & & \\
\hline & & 2.674623 & -2.467249 \\
\hline
\end{tabular}

\section{A = 1-benzyl-3-indolyl, B = 4-methoxyphenyl}

Bispropargyl

56

Free energy $=-1248.803062$ Hartree

$\begin{array}{lccc}\mathrm{C} & 11.048556 & 6.269066 & -3.559534 \\ \mathrm{C} & 10.016823 & 5.334269 & -3.340510 \\ \mathrm{C} & 10.256278 & 3.972730 & -3.616338 \\ \mathrm{C} & 11.502194 & 3.554819 & -4.113174 \\ \mathrm{C} & 12.525166 & 4.494517 & -4.338881 \\ \mathrm{C} & 12.295274 & 5.853500 & -4.060302 \\ \mathrm{C} & 8.662584 & 5.798580 & -2.826135 \\ \mathrm{~N} & 8.041995 & 4.854917 & -1.907686 \\ \mathrm{C} & 8.521028 & 4.578098 & -0.634562 \\ \mathrm{C} & 7.607033 & 3.674469 & -0.009408 \\ \mathrm{C} & 6.552058 & 3.405026 & -0.973116 \\ \mathrm{C} & 6.872152 & 4.150008 & -2.113628 \\ \mathrm{C} & 9.668650 & 5.058705 & 0.021862 \\ \mathrm{C} & 9.876430 & 4.637116 & 1.342484 \\ \mathrm{C} & 8.967843 & 3.757224 & 1.987319 \\ \mathrm{C} & 7.834858 & 3.270641 & 1.321963 \\ \mathrm{C} & 5.398654 & 2.618828 & -0.723108 \\ \mathrm{C} & 4.424258 & 1.959973 & -0.361449 \\ \mathrm{C} & 3.233628 & 1.265143 & 0.130201 \\ \mathrm{C} & 2.160000 & 2.173006 & 0.781796 \\ \mathrm{C} & 2.599779 & 2.891486 & 2.083357 \\ \mathrm{C} & 3.552271 & 3.984299 & 1.879054 \\ \mathrm{C} & 4.363175 & 4.877189 & 1.640509 \\ \mathrm{C} & 5.337770 & 5.836262 & 1.223244 \\ \mathrm{C} & 6.406949 & 6.234117 & 2.062384 \\ \mathrm{C} & 7.427139 & 7.075943 & 1.596020 \\ \mathrm{C} & 7.400510 & 7.532390 & 0.261291 \\ \mathrm{C} & 6.315356 & 7.184936 & -0.574702\end{array}$




$\begin{array}{cccc}\mathrm{C} & 5.303361 & 6.349227 & -0.102760 \\ \mathrm{O} & 8.385465 & 8.281856 & -0.322043 \\ \mathrm{H} & 2.758054 & 0.704104 & -0.705282 \\ \mathrm{H} & 1.690433 & 3.286006 & 2.590672 \\ \mathrm{H} & 4.486265 & 6.046171 & -0.773257 \\ \mathrm{H} & 6.305548 & 7.557797 & -1.609344 \\ \mathrm{H} & 8.260073 & 7.326880 & 2.264991 \\ \mathrm{H} & 6.459816 & 5.842154 & 3.088382 \\ \mathrm{H} & 7.961103 & 5.951141 & -3.671752 \\ \mathrm{H} & 8.763696 & 6.778098 & -2.311162 \\ \mathrm{H} & 10.875965 & 7.333856 & -3.330804 \\ \mathrm{H} & 13.093345 & 6.594074 & -4.226208 \\ \mathrm{H} & 13.502011 & 4.166167 & -4.726485 \\ \mathrm{H} & 11.676375 & 2.488141 & -4.325164 \\ \mathrm{H} & 9.460325 & 3.234312 & -3.428912 \\ \mathrm{H} & 6.320158 & 4.252195 & -3.054827 \\ \mathrm{H} & 3.032540 & 2.144621 & 2.788059 \\ \mathrm{H} & 7.117085 & 2.601669 & 1.821394 \\ \mathrm{H} & 9.158386 & 3.456978 & 3.029125 \\ \mathrm{H} & 10.760372 & 4.999329 & 1.890120 \\ \mathrm{H} & 10.369362 & 5.739068 & -0.482698 \\ \mathrm{H} & 3.546262 & 0.490452 & 0.867949 \\ \mathrm{H} & 1.285715 & 1.533255 & 1.025602 \\ \mathrm{H} & 1.813844 & 2.927850 & 0.044888 \\ \mathrm{C} & 9.497100 & 8.684393 & 0.476671 \\ \mathrm{H} & 10.165284 & 9.260896 & -0.189968 \\ \mathrm{H} & 9.177725 & 9.331842 & 1.323057 \\ \mathrm{H} & 10.049201 & 7.808205 & 0.880641\end{array}$

Alkyne_anionA

55

Free energy $=-1248.268402$ Hartree

\begin{tabular}{|c|c|c|c|}
\hline $\mathrm{C}$ & 11.863738 & 6.801898 & -3.225483 \\
\hline & 10.572506 & 6.215692 & -3.292175 \\
\hline & 10.481432 & 4.844461 & -3.648948 \\
\hline & 11.622948 & 4.080794 & -3.919468 \\
\hline & 12.897967 & 4.685114 & -3.860034 \\
\hline & 13.008867 & 6.049608 & -3.513654 \\
\hline & 9.371981 & 6.956566 & -3.058488 \\
\hline & 8.277090 & 50 & 90 \\
\hline & 6.9 & & \\
\hline & & & \\
\hline & & & \\
\hline & & 14 & \\
\hline & 7.8 & & \\
\hline & 9.16 & 5.0 & 571 \\
\hline & 9.66 & 3.68 & -6.8 \\
\hline & 11.087252 & 3.778312 & -7.069579 \\
\hline & 11.448434 & 5.096705 & -6.920638 \\
\hline & 10.300415 & 5.852979 & -6.639194 \\
\hline & 9.056623 & 2.413534 & -6.891404 \\
\hline & 9.838 & 1.279007 & -7.168504 \\
\hline & 11.232438 & 376 & 549 \\
\hline & 11.8 & 106 & -7 \\
\hline & 12. & 553 & -7. \\
\hline & 13. & & \\
\hline & 14. & & \\
\hline & 15. & & \\
\hline & & & \\
\hline & & & \\
\hline C & 12. & & \\
\hline $\mathrm{O}$ & 14.0 & 4.0 & -4 \\
\hline C & 14.0 & 2.6 & -4 \\
\hline $\mathrm{H}$ & 5.338510 & 5.7 & -4 \\
\hline $\mathrm{H}$ & 6.941567 & 9.114541 & -2.566120 \\
\hline $\mathrm{H}$ & 11.962207 & 4765 & -2.957575 \\
\hline $\mathrm{H}$ & 14.011259 & 6.502809 & -3.476765 \\
\hline $\mathrm{H}$ & 11.502979 & 3.035748 & -4.232298 \\
\hline $\mathrm{H}$ & 9.486045 & 4.389738 & -3.752829 \\
\hline $\mathrm{H}$ & 12.807507 & 6.640561 & -6.672102 \\
\hline $\mathrm{H}$ & 13.496175 & 5.020857 & -6.473863 \\
\hline $\mathrm{H}$ & 15.286623 & 5.016078 & -8.080864 \\
\hline $\mathrm{H}$ & 16.045306 & 5.043625 & 7342 \\
\hline H & 14.4 & 996 & 961 \\
\hline 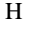 & 12.0 & 746 & 452 \\
\hline$H$ & & & \\
\hline $\mathrm{H}$ & & 743 & 367 \\
\hline $\mathrm{H}$ & & 7.523681 & 243 \\
\hline $\mathrm{H}$ & 7.97 & 2.326986 & -6.707873 \\
\hline $\mathrm{H}$ & 9.365966 & 0.284082 & -7.207599 \\
\hline $\mathrm{H}$ & 11.821538 & 0.498890 & -7.655005 \\
\hline $\mathrm{H}$ & 12.945006 & 2.748677 & -7.603626 \\
\hline 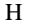 & 5.112800 & 8.252120 & -4.000441 \\
\hline $\mathrm{H}$ & 6.616208 & 8.420310 & -4.952604 \\
\hline $\mathbf{H}$ & 15.070250 & 2.312467 & -4.556743 \\
\hline & 13.633706 & 2.094829 & -3.479325 \\
\hline & & 2.5034 & -5.253194 \\
\hline
\end{tabular}

Free energy $=-1248.276398$ Hartree

C $\quad \begin{array}{lll}11.101722 & 5.997620 & -3.550183\end{array}$

$\begin{array}{llll}\text { C } & 10.196081 & 4.914651 & -3.272247\end{array}$

$\begin{array}{llll}\text { C } & 10.745545 & 3.598721 & -3.410881\end{array}$

$\begin{array}{llll}\text { C } & 12.060482 & 3.377436 & -3.849314\end{array}$

$\begin{array}{llll}\text { C } & 12.905475 & 4.464645 & -4.148634\end{array}$

$\begin{array}{llll}\text { C } & 12.411757 & 5.776988 & -3.975230\end{array}$

$\begin{array}{llll}\text { C } & 8.861130 & 5.175850 & -2.895502\end{array}$

$\begin{array}{llll}\text { C } & 7.661111 & 5.551087 & -3.078652\end{array}$

$\begin{array}{llll}\text { C } & 6.386943 & 6.037392 & -3.184696\end{array}$

$\begin{array}{llll}\text { C } & 6.122179 & 7.511123 & -3.393070\end{array}$

$\begin{array}{llll}\text { C } & 6.097587 & 7.985841 & -4.892845\end{array}$

$\begin{array}{llll}\text { C } & 7.335713 & 7.654123 & -5.593425\end{array}$

$\begin{array}{llll}\text { C } & 8.420106 & 7.253995 & -6.019467\end{array}$

$\begin{array}{llll}9.575497 & 6.558006 & -6.455823\end{array}$

$\begin{array}{llll}\text { C } & 9.663131 & 5.107816 & -6.513515\end{array}$

$\begin{array}{llll}\text { C } & 10.987491 & 4.783904 & -6.935999\end{array}$

$\begin{array}{llll}\mathrm{N} & 11.677152 & 5.975708 & -7.120152\end{array}$

$\begin{array}{llll}\text { C } & 10.832432 & 7.035716 & -6.836180\end{array}$

$\begin{array}{llll}\text { C } & 8.753118 & 4.078713 & -6.200920\end{array}$

$\begin{array}{llll}\text { C } & 9.176491 & 2.750855 & -6.347018\end{array}$

$\begin{array}{llll}\text { C } & 10.486960 & 2.442852 & -6.798426\end{array}$

$\begin{array}{llll}\text { C } & 11.414445 & 3.451065 & -7.093828\end{array}$

$\begin{array}{llll}\text { C } & 13.102488 & 6.066555 & -7.399048\end{array}$

$\begin{array}{llll}\text { C } & 13.501086 & 5.436727 & -8.723908\end{array}$

$\begin{array}{llll}\text { C } & 14.700227 & 4.702703 & -8.822704\end{array}$

$\begin{array}{llll}\text { C } & 15.090478 & 4.128112 & -10.045414\end{array}$

$\begin{array}{llll}\text { C } & 14.276251 & 4.273518 & -11.182831\end{array}$

$\begin{array}{llll}\text { C } & 13.071971 & 4.996571 & -11.088974\end{array}$

$\begin{array}{llll}\text { C } & 12.687837 & 5.573812 & -9.867506\end{array}$

$\begin{array}{llll}\text { O } & 14.198773 & 4.357648 & -4.655087\end{array}$

$\begin{array}{llll}\text { C } & 14.672256 & 3.061140 & -4.972462\end{array}$

$\begin{array}{llll}\text { H } & 5.894723 & 9.081808 & -4.944700\end{array}$

$\begin{array}{llll}\mathrm{H} & 5.538396 & 5.342455 & -3.337269\end{array}$

$\begin{array}{llll}\mathrm{H} & 10.720600 & 7.027418 & -3.473047\end{array}$

$\begin{array}{llll}\mathrm{H} & 13.070679 & 6.625073 & -4.222244\end{array}$

$\begin{array}{llll}\mathrm{H} & 12.400870 & 2.341975 & -3.994329\end{array}$

$\begin{array}{llll}\mathrm{H} & 10.093500 & 2.731660 & -3.224289\end{array}$

$\begin{array}{llll}\mathrm{H} & 13.372899 & 7.142544 & -7.393541\end{array}$

$\mathrm{H} \quad 13.666326 \quad 5.584756 \quad-6.571450$

$\begin{array}{llll}\mathrm{H} & 15.329157 & 4.573147 & -7.926700\end{array}$

$\begin{array}{llll}\mathrm{H} & 16.029995 & 3.556750 & -10.106722\end{array}$

$\begin{array}{llll}\mathrm{H} & 14.574762 & 3.817923 & -12.139755\end{array}$

$\mathrm{H} \quad 12.425306 \quad 5.107780 \quad-11.973787$

$\begin{array}{llll}\mathrm{H} & 11.737922 & 6.126450 & -9.789634\end{array}$

$\begin{array}{llll}\mathrm{H} & 11.201108 & 8.066348 & -6.892010\end{array}$

$\begin{array}{llll}\mathrm{H} & 7.756944 & 4.338779 & -5.811823\end{array}$

$\begin{array}{llll}\mathrm{H} & 8.488056 & 1.928544 & -6.095281\end{array}$

$\begin{array}{llll}\mathrm{H} & 10.790368 & 1.388579 & -6.899694\end{array}$

$\begin{array}{llll}\mathrm{H} & 12.433786 & 3.215980 & -7.429731\end{array}$

$\begin{array}{llll}\mathrm{H} & 5.243858 & 7.489447 & -5.407928\end{array}$

H $\quad 5.141296 \quad 7.813002 \quad-2.954406$

$\begin{array}{llll}\mathrm{H} & 6.901880 & 8.111884 & -2.872907\end{array}$

$\mathrm{H} \quad 15.684235 \quad 3.187160 \quad-5.403755$

$\begin{array}{llll}\mathrm{H} & 14.746507 & 2.408308 & -4.071249\end{array}$

$\begin{array}{llll}\mathrm{H} & 14.020015 & 2.549403 & -5.718198\end{array}$

Alkyne_anionB 